\title{
CONTINUATION OF CROSSCUTTING TECHNOLOGY DEVELOPMENT AT CAST
}

\author{
FINAL TECHNICAL REPORT
}

\author{
Report Period \\ October 1, 2005 to March 31, 2012 \\ Project Director / Principal Investigator \\ Roe-Hoan Yoon \\ Compiled by \\ Christopher E Hull
}

Issued September 13, 2012

DOE Award Number:

DE-FC26-05NT42457

\section{Center for Advanced Separation Technologies \\ Virginia Polytechnic Institute \& State University \\ Blacksburg, Virginia 24061-0258}

National Research Center for Coal \& Energy

West Virginia University

Morgantown, WV 26506-6064

Dept. of Metallurgical Engineering

University of Utah

Salt Lake City, UT 84112-0114

NMBGR

New Mexico Inst. of Mining \& Technology

Socorro, NM 87801
Department of Mining Engineering

University of Kentucky

Lexington, KY 40506-0107

School of Mines \& Engineering

Montana Tech of The Univ. of Montana Butte, MT 59701

Metallurgical \& Materials Engineering University of Nevada, Reno Reno, NV 89557-0042 


\section{DISCLAIMER}

This report was prepared as an account of work sponsored by an agency of the United States Government. Neither the United States Government nor any agency thereof, nor any of their employees, makes any warranty, express or implied, or assumes any legal liability or responsibility for the accuracy, completeness, or usefulness of any information, apparatus, product, or process disclosed, or represents that its use would not infringe privately owned rights. Reference herein to any specific commercial product, process, or service by trade name, trademark, manufacturer, or otherwise does not necessarily constitute or imply its endorsement, recommendation, or favoring by the United States Government or any agency thereof. The views and opinions of authors expressed herein do not necessarily state or reflect those of the United States Government or any agency thereof. 


\section{EXECUTIVE SUMMARY}

This Final Technical Report describes progress made on the sub-projects awarded in the Cooperative Agreement DE-FC26-05NT42457: Continuation of Crosscutting

Technology Development at CAST.

The final reports for each sub-project are attached in the appendix. 


\section{TABLE OF CONTENTS}

TABLE OF CONTENTS

INTRODUCTION

Solid-Solid Separation $\quad 5$

Enhanced Flotation Performance Through Column Froth Enrichment (KY006) 5

Measurement of Surface Forces Between Hydrophobic Surfaces (VA016) 6

Alternative Materials for Dense Medium Separations (KY004) 7

Novel Design of Dm Cyclones to Improve Coal Cleaning Efficiency with Large Eddy Simulation (Les).

(UT010)

Improving Coal Flotation Recovery Using Cavitation Concepts (KY007) 9

Surface Force Measurement Between Hydrophobic Surfaces (VA020) 10

On Improving the Hydrophobicity of Oxidized Coals (WV024) 10

Solid-Liquid Separation $\quad 11$

Warm Air Drying of Fine Particles. (WV021) 11

Proof-of-Concept Testing of a Fine Coal Cleaning \& Dewatering System (VA019) 11

Chemical/Biological Extraction $\quad 12$

Thiosulfate as a Replacement for Cyanide in the Presence of Activators (NV003) 12

Recovery of Gold from Thiosulphate Leach Liquor Using Activated Carbon (MT005) 12

Mass Balance on Mercury During Gold Ore Processing. (NV004) 13

Evaluation of Integrated Halide-Based Extraction and Recovery of Copper from Chalcopyrite Ore.

(UT008) 14

Stabilizing Ruthenium Dioxide for Lower Overpotential Oxygen Evolution Anodes (UT009) 14

Leaching of Chalcopyrite with Sodium Hypochlorite (WV018) 15

Mercury Removal from Coal by Leaching with SO2 (WV019) 16

Enhancement of High Sodium Coals: Sodium Removal Technology Development (MT008) 16

Modeling and Control $\quad 17$

Development of a 3D Lattice-Boltzmann Model for Fluid Flow Simulation under Partially-Saturated

Conditions in Packed Particle Beds. (UT007) 17

Development of a Turbulent Flotation Model and a Computer Simulator (VA015) 18

Development of a Comprehensive Model for the Simulation, Scale-Up and Design of Large Flotation Machines (VA018) 18

Characrerization, Analysis and Simulation of Fine Coal Filtration. (UT011) 18

\section{Environmental Control}

Removal of Selenium (Se) from Coal Mine Wastewater by GAC-Based Iron-Containing Adsorbents. (WV020)

Mercury Removal from Clean Coal Processing Air Stream (MT007)

Determination of Factors Affecting the Separation of Potentially Hazardous Trace Elements and their

Behavior in Coal Tailings Impoundments (KY005)

Selenium Removal from Mine Influenced Water (MIW) Using Nano-Magnetite (WV022) 21

Development of Biochemical Techniques for Extraction of Mercury from Coal Impoundments (WV023)

Development of Techniques for Online Monitoring of Individual Gas Constituents in Coal-Derived Synthesis Gas and Flue Gas (NM006) 
Hydrate $\quad 23$

Gas-Gas Separation by Selective Hydrate Formation - Laying the Foundation (VA022) 23

$\begin{array}{ll}\text { EXPERIMENTAL } & 25\end{array}$

RESULTS, DISCUSSION \& CONCLUSION 26

$\begin{array}{ll}\text { APPENDICES } & 27\end{array}$

APPENDIX 1 - Enhanced Flotation Performance Through Column Froth Enrichment (KY006)

APPENDIX 2- Measurement of Surface Forces Between Hydrophobic Surfaces (VA016)

APPENDIX 3 - Alternative Materials for Dense Medium Separations (KY004)

APPENDIX 4 - Novel Design of DM Cyclones to Improve Coal Cleaning Efficiency with Large Eddy

Simulations (LES) (UT010)

APPENDIX 5- Improving Coal Flotation Recovery Using Cavitation Concepts (KY007)

APPENDIX 6 - Surface Force Measurement Between Hydrophic Surfaces (VA0020

APPENDIX 7 - On Improving the Hydrophobicity of Oxidized Coal (WV024)

APPENDIX 8 - Warm Air Drying of Fine Particles (WV021)

APPENDIX 9 - Proof-of-Concept Testing of a Fine Coal Cleaning \& Dewatering System (VA019)

APPENDIX 10 - Thiosulfate as a Replacement for Cyanide in the Presence of Activators (NV003)

APPENDIX 11- Recovery of Gold from Thiosulphate Leach Liquor Using Activated Carbon (MT005)

APPENDIX 12- Mass Balance on Mercury During Gold Ore Processing. (NV004)

APPENDIX 13- Evaluation of Integrated Halide-Based Extraction and Recovery of Copper from

Chalcopyrite Ore. (UT008)

APPENDIX 14 - Stabilizing Ruthenium Dioxide for Lower Overpotential Oxygen Evolution Anodes

(UT0009)

APPENDIX 15- Leaching of Chalcopyrite with Sodium Hypochlorite (WV018)

APPENDIX 16 - Mercury Removal from Coal by Leaching with SO2 (WV019)

APPENDIX 17- Enhancement of High Sodium Coals: Sodium Removal Technology Development

(MT008)

APPENDIX 18-Development of a 3D Lattice-Boltzman Model for Fluid Flow Simulation Under Partially-Saturated Conditions in Packe Particle Beds. (UT007)

APPENDIX 19 - Development of a Turbulent Flotation Model Model and Computer Simulator(VA015)

APPENDIX 20 - Development of a Comprehensive Model for the Simulation, Scale-Up and Design of Large Flotation Machines (VA018)

APPENDIX 21 - Characterization, Analysis and Simulation of Fine Coal Filtration (UT011)

APPENDIX 22 - Removal of Selenium (Se) from Coal Mine Wastewater by GAC-Based Iron-

Containing Adsorbents. (WV020)

APPENDIX 23- Mercury Removal from Clean Coal Processing Air Stream (MT007)

APPENDIX 24 - Determination of Factors Affecting the Separation of Potentially Hazardous Trace

Elements and their Behavior in Coal Tailings Impoundments (KY005)

APPENDIX 25 - Selenium Removal from Mine Influenced Water (MIW) Using Nano-Magnetite

(WV022)

APPENDIX 26- Development of Biochemical Techniques for Extraction of Mercury from Coal Impoundments (WV023)

APPENDIX 27- Development of Techniques for Online Monitoring of Individual Gas Constituents in Coal-Derived Synthesis Gas and Flue Gas (NM006)

APPENDIX 28- Gas-Gas Separation by Selective Hydrate Formation (VA022) 


\section{INTRODUCTION}

The U.S. is the largest producer of mining products in the world. In 2003, U.S. mining operations produced $\$ 57$ billion worth of raw materials that contributed a total of $\$ 564$ billion to the nation's wealth. Despite these contributions, the mining industry has not been well supported with research and development funds as compared to mining industries in other countries. To overcome this problem, the Center for Advanced Separation Technologies (CAST) was established to develop technologies that can be used by the U.S. mining industry to create new products, reduce production costs, and meet environmental regulations. Originally set up by Virginia Tech and West Virginia University, this endeavor has been expanded into a seven-university consortium Virginia Tech, West Virginia University, University of Kentucky, University of Utah, Montana Tech, New Mexico Tech and University of Nevada, Reno - that is supported through U.S. DOE Cooperative Agreement No. DE-FC26-05NT42457: Continuation of Crosscutting Technology Development at CAST.

Much of the research to be conducted with Cooperative Agreement funds will be longerterm, high-risk, basic research and will be carried out in five broad areas:

a) Solid-solid separation

b) Solid-liquid separation

c) Chemical/Biological Extraction

d) Modeling and Control, and

e) Environmental Control.

Distribution of funds is handled via competitive solicitation of research proposals through Site Coordinators at the seven member universities. These were first reviewed and ranked by a group of technical reviewers (selected primarily from industry). Based on these reviews, and an assessment of overall program requirements, the CAST Technical Committee made an initial selection/ranking of proposals and forwarded these to the DOE/NETL Project Officer for final review and approval. The successful projects are listed below by category, along with brief abstracts of their aims and objectives.

\section{Solid-Solid Separation}

\section{Enhanced Flotation Performance Through Column Froth Enrichment (KY006)}

Principal Investigators: Rick Q. Honaker and Daniel Tao, University of Kentucky Period of Performance: October 1, 2006-September 30, 2009 (1-Year Project)

Froth flotation is a process that separates particles based on their differences in physical and surface chemistry properties. For fine feed material that is comprised of particles having wide degrees of floatability, selectivity is often optimized by maximizing the differences in the flotation rates between the particles targeted for recovery in the froth concentrate and those needed to report to the underflow stream. The benefits of differential flotation rates are realized in the collection zone of a flotation system where 
bubble-particle collision and attachment occurs. However, selectivity between particles of varying floatability can be significantly enhanced in the froth zone through a reflux mechanism that circulates detached particles back to the collection zone. Particle detachment in the froth zone occurs due to bubble coalescence, which leads to an insufficient amount of bubble surface area to carry all of the material recovered in the collection zone. Based on previous research, the detachment process is selective in that particles having a lower degree of floatability (or hydrophobicity) are preferentially released from the bubble surfaces. Selectivity through the detachment process can be improved by the addition of a more hydrophobic material into the froth zone and possibly by recycling a portion of the flotation concentrate, thereby enriching the flotation froth.

In a recent study, froth zone and overall flotation recovery values were quantified for particles in an anthracite coal that were characterized as having wide differences in floatability potential. The unique aspect of the coal was the presence of 'bone' material in the high density fractions which had a relatively high degree of floatability. As a result, the minimum product grade achieved by froth flotation was about 15\% despite washability data indicating the potential for achieving a product containing nearly $3 \%$ ash. Highly floatable material was added directly into the froth zone while treating the anthracite coal and then removed from the product and tailing samples using density fractionation. The enriched froth phase reduced the product ash content of the anthracite product by 5 absolute percentage points while maintaining coal recovery at the same level. These results support the findings of previous fundamental research conducted on hematite flotation with the addition of hydrophobic silica directly into the froth phase.

The proposed two-year project will further investigate the fundamentals of the detachment process as well as develop unique methods of commercial application that may be more useful for sulfide and precious mineral industries. Plastic material that contains an amount of magnetite that makes the plastic easily recoverable by a lowintensity magnetic separator will be directly added in the flotation froth through the wash water distributor of a flotation column. By varying the formulations of the plastic, surface hydrophobicity and thus floatability can be controlled. Initial tests will involve the flotation of silica that will be methylated to achieve varying degrees of floatability. External refluxing of a portion of the flotation froth will also be investigated using the methylated silica. To evaluate the commercial benefits, the improved selectivity achieved by froth enrichment using external refluxing and the addition of the magnetic material will be accessed for the flotation of coal, sulfide minerals and phosphate.

\section{Measurement of Surface Forces Between Hydrophobic Surfaces (VA016)}

Principal Investigators: Roe-Hoan Yoon, Virginia Tech

Period of Performance: October 1, 2006-September 30, 2010 (1-Year Project)

Froth flotation is the most widely used method of separating fine particles in the mining industry. The process is based on rendering a selected mineral hydrophobic, so that it can be separated from other minerals that are hydrophilic. Thus, control of particle 
hydrophobicity is critically important in flotation. However, the nature of the hydrophobicy is not fully understood, particularly in view of the forces acting between two hydrophobic particles suspended in water. In the proposed work, surface force measurements will be conducted using an atomic force microscope (AFM). The force measurements will be conducted with gold, sulfide minerals, and rutile after coating them with thiols, xanthates, and anionic and cationic surfactants. In addition, the surfaces will be characterized in terms of their acid-base properties, infrared spectra, and AFM images. The results will be useful for better understanding the nature of hydrophobicity and, hence, furthering the flotation technology.

\section{Alternative Materials for Dense Medium Separations (KY004)}

Principal Investigators: Rick Honaker, University of Kentucky

Period of Performance: October 1, 2006-September 30, 2009 (1-Year Project)

Approximately 640 million tons of coal are treated annually in the U. S. to remove impurities that could cause negative environmental effects during combustion and to upgrade heating value. Dense medium processes, which are used to clean approximately $65 \%$ of the total processed coal in the U. S., employ ultrafine magnetite suspensions to separate the coal from the mineral matter. As a result of a recent closure of the only domestic magnetite source and a growing demand from the Chinese coal industry that is exhausting supplies on the international market, the U.S. coal industry is facing increased magnetite costs and a lack of confidence in future supplies. The proposed 2-year project will evaluate and develop alternative materials that can be used in the dense medium process.

Alternatives to ultrafine magnetite used in dense medium separators include a vast source of coarse magnetite, steel production slag, magnetic material from combustion residue and fine reject material that comprises the feed coal. Coarse magnetite is generally perceived as providing inferior separation performances. However, recent research indicates that coarse magnetite containing greater than $30 \%+100$ excellent separation performances in dense medium cyclones. Similar performances were also reported from a full-scale study conducted in Australia involving the use of magnetic material from combustion residue.

The associated fine mineral matter that exists within the raw coal may also provide a material that can be successfully used in formulating dense medium as a sole source or as a supplement to magnetite. The findings of previous research concluded that the use of fine coal rejects results in inferior separation performance due to an elevated medium viscosity. However, it is proposed to investigate a nominal 150 x 25 fine reject stream of an operating plant. Varying amounts of -25 magnetite will be added to the coarser fraction in an effort to identify an optimum particle size distribution and blend, respectively.

The proposal requests funds for a two-year study that will investigate the aforementioned alternatives for dense medium material. The test program will be conducted using a 152- 
mm (6-in) dense medium cyclone to treat $10 \times 1 \mathrm{~mm}$ coal in a closed loop system that exists in the University of Kentucky processing laboratory. The criteria used to determine adequacy of each alternative include separation performance, efficiency and economics.

\section{Novel Design of Dm Cyclones to Improve Coal Cleaning Efficiency with Large Eddy Simulation (Les). (UTO10)}

Principal Investigator: Raj K Rajamani, University of Utah

Period of Performance: October 1, 2006-September 30, 2010 (2-Year Project)

The past two decades have seen a number of studies examining the efficiency of dense media cyclones (DMC). Some noteworthy advances were made when researchers (Chedgy et al., Napier-Munn, Rao et al., Davis, King and Juckes, Klima, He and Laskowski) pursued empirical and phenomenological models relating partition curve to the design and feed variables. Then came, the use of density tracers to measure the performance of large DMC operating in plants (Wood et al). Simultaneously, in the 1990s several groups (Diakowski and Williams, Davidson, Rajamani et al.) were applying fluid dynamic principles or Navier Stokes equations to understand the size classification efficiency of hydrocyclones. These works have advanced the state-of-theart in many aspects and are able predict performance of plant scale hydrocyclones (up to $660 \mathrm{~mm}$ diameter). In the meantime, commercial computational fluid dynamic software packages such as FLUENT, CFX and CFD-ACE have progressed so much so that solving complex flow problems is much easier than in the past.

The project objective is to pick up the computational fluid dynamic track from hydrocyclones and apply it to the DMC's intrinsic efficiency problem directly. From the past studies, we know that the intrinsic efficiency of DMC is interplay of the size classification of media (magnetite stream) and partitioning of coal particle (density and size wise) simultaneously happening in the interior of a DMC. While we've known some of the inherent problems, such as short circuit to the overflow and misplacement of clean coal, we do not fully understand the specific gravity regions within the DMC or the region where effective separation takes place. CFD is capable of producing a map of these regions and therefore one can change design and feed flow variables to enlarge the effective separation region. CFD produces a vast array of information that is next to impossible even with the most expensive instrumentation such as tomography.

The exercise of applying CFD to DMC has a parallel in grinding technology. For a full four decades plants and research groups applied the popular Bond's power equation (empirical model) with some success in ball mills but much less in semi autogenous mills (SAG). Then came a method called discrete element method in the 1990s. This method was quickly adapted to the SAG mill problem (Rajamani et al., Moys et al, and Cleary) and as a result revolutionary design alterations of the internals of the SAG mill came about. These design changes were quickly adopted by the mining industry (Sherman and Rajamani) that realized $10-15 \%$ gain in operational energy efficiency. In a similar way a 
detailed modeling of the DMC with CFD can break open unforeseen design changes, which can lead to a leap in the intrinsic efficiency.

The work plan is to establish the large eddy simulation (LES) turbulence model by predicting published (Rajamani et al 1988,1990,1994,1996,2005) data in DMC. Next, the LES solution will be extended to the size classification of the media. Then published partition curves (King and Juckes, Laskowski) will be predicted with LES. Then we will predict the available plant DMC data (Luttrel et al.). Thus the LES becomes a tool to examine novel designs or a combination of design and operating variables to maximize intrinsic efficiency. The set of feed conditions (media makeup, flow rates and coal/specific gravity distributions) that is compatible for extracting maximum efficiency of DMCs will be explored by simulation. In a like manner, the changes to basic design that would increase efficiency will be explored. In fact, the LES template becomes a plant-wide tool for design and assessment of installed equipment.

\section{Improving Coal Flotation Recovery Using Cavitation Concepts (KY007)}

Principal Investigators: Daniel Tao and Rick Honaker, University of Kentucky Period of Performance: October 1, 2007-September 30, 2010 (2-Year Project)

Froth flotation is the most widely used solid-solid separation process for coal and minerals beneficiation. However, its high process efficiency is limited to a narrow particle size range, which is usually 10-100 $\square \mathrm{m}$ beyond which its efficiency decreases sharply, especially for oxidized coal that is more difficult to float due to weak hydrophobicity.

The particle-bubble collision, attachment and detachment are the most critical steps in the flotation process. The low flotation recovery of fine particles is mainly due to the low probability of bubble-particle collision while the main reason for poor flotation recovery of coarse particles is the high probability of detachment of particles from bubble surface. Fundamental analysis indicated that use of smaller bubbles is the most effective approach to increase the probability of collision and reduce the probability of detachment.

The main goal of the proposed project is to enhance recovery of difficult-to-float coal particles $(-0.15 \mathrm{~mm}$ or -100 mesh), particularly ultrafine ( $<44$ or -325 mesh $\square \mathrm{m}$ ) particles using a flotation column and a mechanical flotation cell featured with a hydrodynamic picobubble generator or an air eductor for feed preconditioning. Preliminary results indicate that the flotation rate of -100 mesh Coalberg coal can be increased by at least $200 \%$ by using an air eductor to pretreat the feed with submicron bubbles. Picobubbles (usually less than $1 \square \mathrm{m}$ ) selectively attach to the hydrophobic coal particles. They are characterized by an inherently high probability of collision with particles and high probability of attachment and low probability of detachment due to their tiny size, low ascending velocity and rebound velocity from the surface, and high surface free energy to be satisfied, and therefore are very effective for enhancing flotation recovery of fine and coarse particles. Other major advantages of the proposed technique include lower collector dosage and air consumption since picobubbles are produced from air naturally 
dissolved in water and they act as the secondary collector on particle surfaces thereby resulting in considerably lower operating costs.

\section{Surface Force Measurement Between Hydrophobic Surfaces (VA020)}

Principal Investigator: Roe-Hoan Yoon, Virginia Tech

Period of Performance: October 1, 2007 - September 30, 2010 (2-Year Project)

Froth flotation is the most widely used method of separating fine particles in the mining industry. The process is based on rendering a selected mineral hydrophobic, so that it can be separated from other minerals that are hydrophilic. Thus, control of particle hydrophobicity is critically important in flotation. However, the nature of the hydrophobicy is not fully understood, particularly in view of the forces acting between two hydrophobic particles suspended in water. In the proposed work, surface force measurements will be conducted using an atomic force microscope (AFM). The force measurements will be conducted with thiol-coated gold surfaces as functions of $\mathrm{pH}, \mathrm{E}_{\mathrm{h}}$, and thiol concentration. Further, the force measurements will be conducted in the presence of electrolytes and solvents that can affect the structure of the water in the thin films between two hydrophobic surfaces. The results will be useful for exploring the nature of hydrophobicity and the origin of hydrophobic force.

\section{On Improving the Hydrophobicity of Oxidized Coals (WV024)}

Principal Investigator: Mohindar Seehra, West Virginia University Period of Performance: October 1, 2007 - September 30, 2010 (2-Year Project)

Physical cleaning of fine coals by froth floation depends on the different hydrophobic/hydrophilic characteristics of surfaces of particles of coal and minerals to be separated. The process works efficiently only if coal particles are hydrophobic and minerals are hydrophilic. Some coals are hydrophilic (e.g. Illinois \#6) and other coals become hydrophilic upon oxidation (e.g. Upper Freeport). Determining the factors which govern the hydrophobic/hydrophilic characteristics of coals at the microscopic/atomic level may lead to development of new strategies for improving the hydrophobicity of coals.

It is well known that water is a polar molecule and polar materials which can form hydrogen bonds with $\mathrm{H} 2 \mathrm{O}$ (e.g. alcohols containing $\mathrm{OH}$ groups) are miscible with water. Non-polar materials such as alkanes, oils and fats are hydrophobic and it is for these reasons that oils have often been tested to improve the hydrophobicity of coals. Previous studies have shown that oxygen content, surface moisture and mineral matter can have significant effect on the undesirable wettability of coals.

In our research, we will focus on determining the difference between the hydrophilic and hydrophobic coals in terms of their surface $\mathrm{OH}$ groups, moisture content, and oxidation behavior using infrared (IR) spectroscopy combined with thermogravimetric analysis (TGA), zeta potential measurements and electron spin resonance (ESR) spectroscopy of 
active sites on the surfaces of coal. Since presence of surface OH groups may be a key to understanding hydrophilicity of coals, IR spectroscopy of deutrated samples with D2O should allow us to locate the surface OH, OD, HDO, and bound H2O and D2O molecules. The investigations of the formation of surface $\mathrm{OH}$ groups through oxidation and hydration of the active sites on the coal surface and subsequent elimination of these hydroxyl groups from the surfaces of the coal particles using appropriate chemical treatments may be the key to converting hydrophilic coals to hydrophobic coals. To achieve this goal through these studies is the primary aim of this work. A successful outcome from this project will have a major positive impact on the economics of coal industry.

The Argonne coal bank contains eight well-characterized coal samples sealed in N2, including Illinois \#6 and Upper Freeport coals. We will purchase these coals for the proposed studies, although additional coals (hydrophobic and hydrophilic) may also be tested later in the project in consultation with the Yoon-Luttrell team at Virginia Tech. The needed experimental facilities of IR spectroscopy, TGA and ESR spectroscopy are available in our laboratory.

\section{Solid-Liquid Separation}

Warm Air Drying of Fine Particles. (WV021)

Principal Investigator: Eric K Johnson and Bruce S. Kang, West Virginia University Period of Performance: October 1, 2006-September 30, 2010 (2-Year Project)

The goal of this research project is to evaluate the concept of a Warm Air Dryer of Fine Particles, WADFP. This concept employs low pressure steam to maintain the temperatures of the surfaces and the drying air below safe limits. Drying takes place in a fluidized bed riser where there is significant interaction between the particles and the air. Applications of this system include drying waste coal from ponds and drying lignite at a power plant site. The WADFP system has been constructed and was purchased by WVU for testing.

\section{Proof-of-Concept Testing of a Fine Coal Cleaning \& Dewatering System (VA019)}

Principal Investigator: Gerald Luttrell and Roe-Hoan Yoon, Virginia Tech

Period of Performance: October 1, 2007 - September 30, 2010 (2-Year Project)

Fine coal streams are currently upgraded using independent unit operations for ash removal and dewatering that are generally less efficient and more costly than processes used to treat coarse coal. To address this shortcoming, researchers at the Center for Advanced Separation Technologies (CAST) have developed an innovative separation technology that can simultaneously remove both mineral matter and surface moisture from coal in a single processing step. This revolutionary process works with higher rank coals that are naturally hydrophobic and have a strong affinity for non-polar liquids. The process is thermodynamically spontaneous and, hence, requires no energy other than that 
used to agitate the coal slurry and recover/recycle the spent non-polar liquid. Data obtained from batch laboratory tests indicate that this innovative process has the potential to reject essentially all of the free mineral matter and surface moisture from fine coal particles in a cost-effective manner. The objective of the work outlined in this proposal is to design, construct and test a small-scale continuous unit that can be used to provide critical operating data that is needed to develop a full-scale commercial unit.

\section{$\underline{\text { Chemical/Biological Extraction }}$}

Thiosulfate as a Replacement for Cyanide in the Presence of Activators (NV003) Principal Investigator: Maurice C. Fuerstenau, University of Nevada, Reno Period of Performance: October 1, 2006-September 30, 2009 (2-Year Project)

The United States is the third largest producer of gold in the world, and Nevada is by far the largest gold-producing state in the country. In processing gold and other precious metals, cyanide is used universally as a lixiviant. It is effective and reasonably low cost and has been used for greater than a century commercially. It is an extremely toxic reagent, however, and considerable care must be exercised to prevent personal injury and harm to the environment during and after metal processing. To obviate the downsides of the use of cyanide, considerable research has been conducted with other lixiviants that are not as toxic as cyanide to establish if a replacement for cyanide can be found. These include the halides (chlorine, iodine and bromine), thiourea, thiosulfate and polysulfides. Of these reagents thiosulfate is the most promising. The problem with this reagent, however, is the stability of thiosulfate in solution. Oxidative conditions are essential for the dissolution of gold and under these conditions, some thiosulfate is oxidized to tetrathionate, and its effectiveness is greatly reduced. The development of reaction conditions is needed in which the kinetics of gold dissolution is increased, while the losses of thiosulfate by oxidation are minimized. In this investigation the use of activators in gold dissolution will be studied, and the interaction between the various parameters involved in thiosulfate leaching of gold will be carefully investigated.

\section{Recovery of Gold from Thiosulphate Leach Liquor Using Activated Carbon (MT005)}

Principal Investigator: Courtney A. Young and Larry G. Twidwell, Montana Tech Period of Performance: October 1, 2006-September 30, 2009 (2-Year Project)

Gold is generally recovered from the ore by a cyanide leaching process. This procedure is used because of its simplicity and ability to work effectively at low concentrations. However, cyanide itself is highly toxic. Furthermore, it also leaches relatively nonspecifically causing other metals to go into solution as well. These metal cyanide complexes can also be toxic. Resulting leach solutions have been accidentally released from various process facilities causing environmental damage. Consequently, particularly over the last decade, alternatives to cyanide leaching have been sought. 
Particular attention has been given to thiosulfate; however, recovery of the gold from the thiosulfate leach presents a problem. Conventional carbon adsorption does not work so more expensive recovery processes have been investigated with resin adsorption being the most common. The research we propose to undertake investigates a novel use of activated carbon by pre-adsorbing cuprous cyanide at its surface and using it to exchange with gold in a metal-exchange, cementation-type reaction:

$$
\mathrm{Au}(\mathrm{S} 2 \mathrm{O} 3) 23-+\mathrm{Cu}(\mathrm{CN}) 2-\text { ads } \rightarrow \mathrm{Au}(\mathrm{CN}) 2-\text { ads + } \mathrm{Cu}(\mathrm{S} 2 \mathrm{O} 3) 23-
$$

Resulting cuprous thiosulfate is recycled for further gold leaching with the copper serving as catalyst to the leaching process. Likewise, the adsorbed gold cyanide product can be eluted using a subsequent conventional gold recovery process common in traditional cyanide operations:

$$
\mathrm{Au}(\mathrm{CN}) 2-\text { ads }+\mathrm{CN}-\rightarrow \text { Csite }+\mathrm{Au}(\mathrm{CN}) 2-+\mathrm{CN}-
$$

Consequently, the novel process is believed to be cost-effective and will ultimately allow thiosulfate leaching to be a viable alternative to cyanide. Because direct investigation of these adsorption reactions are masked by the location of the carbon adsorption sites in commercial activated carbons, we propose to study them in-situ using Raman spectroscopy.

We also propose to investigate using coal-based metathesis technologies to generate carbon alternatives to activated carbon. In this regard, carbon nanotubes, because of their graphite- like structure, is expected to have similar adsorption capacities and could therefore prove to be an extremely effective high surface-area alternative with high adsorptive capacity. Although nanotubes are currently expensive, their production from coal and other cheap carbon sources could be economically equivalent to coconut shellbased activated carbons.

\section{Mass Balance on Mercury During Gold Ore Processing. (NV004)}

Principal Investigators: Maurice C. Fuerstenau and Manoranjan Misra, University of Nevada, Reno

Period of Performance: October 1, 2006-September 30, 2010 (2-Year Project)

The United States is the third largest producer of gold in the world, and Nevada is by far the largest producing state in the country. The gold mines in Nevada contain significant amounts of mercury. As a matter of fact, mercury is produced as a byproduct from the mines in Nevada, and these mines are the largest source of new mercury in the U.S. Current demand for mercury is 150-200 tons/years in this country; the mercury produced in Nevada is 30-50 tons /year. Gold mines are required to measure mercury emissions once per year. A systematic determination of mercury losses in the various unit operations involved in gold ore milling/processing is needed. Such data, not presently available, are important to the industry itself, regulatory agencies and the public. 


\section{Evaluation of Integrated Halide-Based Extraction and Recovery of Copper from Chalcopyrite Ore. (UTO08)}

Principal Investigator: Michael L. Free, University of Utah

Period of Performance: October 1, 2006-September 30, 2009 (2-Year Project)

Extraction and recovery of copper from chalcopyrite is one of the most important processes in the nonferrous metals industry. It is currently performed using mining, mineral processing, smelting, converting, and electrorefining at an energy cost of more than 50 million Btu per metric ton of copper. Most of the energy for copper production is consumed by mineral processing, which produces a concentrate precursor for smelting. Much of the energy consumed by the mineral processing and smelting could be saved by replacing these processes with halide leaching and electrowinning.

Research has demonstrated the technical viability of halide leaching using chalcopyrite concentrates. However, most of the potential energy savings associated with halide leaching and electrowinning is linked to its use in ore leaching, which eliminates most of the mineral processing energy consumption that is needed to produce a concentrate. Investigations of chalcopyrite ore leaching using halide solutions under ambient conditions associated with commercial ore leaching are clearly lacking. Furthermore, the important link between chalcopyrite ore leaching and electrowinning using halide media in an integrated flow sheet has not been adequately explored. Consequently, there is a need to perform investigations involving chalcopyrite ore leaching and electrowinning of copper in an integrated flow sheet. The proposed project will involve experiments and data analysis that are designed to accomplish this need, which is a precursor to commercial utilization that could save as much as 30 million Btu per metric ton of copper.

\section{Stabilizing Ruthenium Dioxide for Lower Overpotential Oxygen Evolution Anodes (UT009)}

Principal Investigator: Dr. Michael S. Moats, University of Utah

Period of Performance: October 1, 2006-September 30, 2009 (2-Year Project)

With the increasing use of hydrometallurgical processes to extract metals from minerals, electrowinning is becoming a preferred process to produce high purity non-ferrous products. Unfortunately, electrowinning is a fairly energy intensive process using 1.8-3.3 $\mathrm{kWh} / \mathrm{kg}$ of metal produced. It would thus be advantageous to reduce the power consumption of this process as identified by the CAST Roadmap. This can be achieved in one of four ways - change the anodic reaction, reduce the oxygen overpotential at the anode, decrease the spacing between electrodes and/or remove current inefficiencies.

Borrowing technology from the chemical industry, it may be possible to switch from a lead anode to a dimensionally stable anode (titanium coated with a precious metal oxide coating). The use of a dimensional stable anode would be advantageous by: 1) allowing ferrous oxidation as the anodic reaction, 2) lowering the oxygen overpotential and 3) decreasing electrode spacing and improving current efficiency because lead contamination would not be present to contaminate the cathode and cause dendritic formations. All of these benefits could radically change the electrowinning process and 
reduce the power consumption of the process by $10-50 \%$ while improving cathode quality and removing hazardous lead by-products.

Unfortunately, the costs of these anodes have been prohibitive to date. To reduce the cost of these electrodes, "stabilized" ruthenium dioxide electrocatalysts have been invented. These electrocatalysts are presently under evaluation in commercial copper electrowinning cells. While these electrocatalysts hold promise, they have been developed on an empirical basis and the fundamental nature of these "stabilized" ruthenium dioxide electrocatalysts are not understood. This project will investigate the relationship between the compositions of ruthenium dioxide based electrocatalysts and their physical structure and electrochemical performance. Correlation of these properties across a range of compositions will provide a solid foundation for future electrocatalyst developments. This may lead to the implementation of a low overpotential anode for electrowinning and could lead to crosscutting technology for most industrial electrochemical processes, such as chlorine electrolysis, electrogalvanizing and metal plating. This view is by ELTECH Systems Corporation, who is supporting this research.

\section{Leaching of Chalcopyrite with Sodium Hypochlorite (WV018)}

Principal Investigators: Eung Ha Cho and Ray Y. K. Yang, West Virginia University Period of Performance: October 1, 2006-September 30, 2009 (2-Year Project)

It is known that chalcopyrite is very difficult to leach with ferric sulfate or ferric chloride because its leaching rates are very low. This may be the reason that there is no commercial process being used in industry to date for leaching of the mineral with these reagents. This proposed method involves two-stage leaching of chalcopyrite: In the first stage, the mineral will be converted to probably cupric hydroxide with hypochlorite at high temperature and high $\mathrm{pH}$. In the second stage, the converted mineral will be leached to cupric ion with sulfuric acid at room temperature.

A preliminary experiment was conducted by the PI to leach chalcopyrite with a 0.5 molar hypochlorite solution at $90 \mathrm{oC}$ and $\mathrm{pH} 12.9$ for one hour. This leach mineral was leached again in the second stage with a 0.5 molar sulfuric acid solution at room temperature for one hour. It was found that the leaching conversion with these two stages reached $42.6 \%$. This is a very high conversion and would be 23-47 times as high as those with ferric sulfate or ferric chloride. This fast leaching rate may be due to the fact that no sulfur layer is formed during the reaction through which the leaching rate would be limited by diffusion of the reagent, and that a specific reaction mechanism plays a role despite of the weak oxidation power of hypochlorite.

It is proposed in this project that chalcopyrite will be converted to probably cupric hydroxide with hypochlorite at high temperature and high $\mathrm{pH}$ in the first stage and the converted mineral will be leached with sulfuric acid at room temperature in the second stage. The objectives are to determine the most practical leaching conditions in both stages and further to determine the feasibility of this technology as applied in developing a commercial process. 


\section{Mercury Removal from Coal by Leaching with SO2 (WV019)}

Principal Investigator: Eung Ha Cho, West Virginia University

Period of Performance: October 1, 2006-September 30, 2009 (2-Year Project)

Mercury from coal-fired utilities is the most health hazardous air pollutant and thus its emission will likely be regulated by law in the future. There are some technologies currently available to control its emission. One of them is considered as the "standard technology" and is based on adsorption of mercury vapor on activated carbon. However, since this technology is very expensive, other technologies are in great need to be explored and developed. This proposal is in this end and deals with pre-combustion treatment of coal to remove mercury content.

This proposal proposes to remove minute amounts of mercury from coal using a gas stream containing SO2 and oxygen which simulates the flue gas from coal-fired power plants. Since the mercury content in coal is minute (e.g., $0.1 \mathrm{ppm}$ ), it may not be extractable economically using generally known oxidizing reagents such as ferric iron, oxygen and chlorine. However, this proposed method may have advantage over currently available technologies because it will use a waste gas mixture of the flue gas stream, and thus can become more economically feasible.

SO2 is a reducing reagent but when it is combined with oxygen in an acid solution, it becomes a strong oxidizing reagent. It has been proven that this reagent can leach coal pyrite effectively. Thus, since more than half of the mercury in the coal may exist in the form of solid solution with pyrite, the mercury will be liberated from the coal pyrite and subject to leaching by the same oxidizing reagents. Also, the mercury associated with coal organically has a chance to be extracted because of the strong oxidizing power of the reagents.

The main objective of this proposal is to determine the technical feasibility for this technology to be developed into a commercial process.

\section{Enhancement of High Sodium Coals: Sodium Removal Technology Development (MT008)}

Principal Investigator: Jay McCloskey, Montana Tech

Period of Performance: October 1, 2008-September 30, 2010 (2-Year Project)

Montana Tech of The University of Montana (Montana Tech) and its Center for Advanced Minerals and Metallurgical Processing (CAMP) are pleased to submit this proposal which is designed to support the Clean Coal Industry. The proposed scope-ofwork will focus on the evaluation and development of sodium removal and sodium recovery technologies needed for the beneficiation of high sodium coals to be used for coal gasification applications. The proposed work will be directed to support the development and expansion of the coal industry particularly in the State of Montana's 
Tongue River Basin and adjacent areas of southeastern Montana, including the Otter Creek coal deposits.

The Project Team will consist of representatives and graduate students from the following Montana Tech/CAMP and other Departments and Affiliations:

i. $\quad$ Center for Advanced Minerals and Metallurgical Processing (CAMP)

ii. Metallurgical and Materials Engineering Department

iii. Chemistry \& Geochemistry Department

v. Montana Enviromet

Great Northern Properties (GNP) has agreed to provide matching funds for this Project and representatives from GNP have provided support and insight as to the issues surrounding sodium and how the presence of sodium influences the coal industry in Montana. High sodium coal samples from various locations will be used in this study. The information gained here will be applicable to the Otter Creek reserves, to reserves currently being mined at Spring Creek and Decker and to other coals with high sodium content.

\section{Modeling and Control}

Development of a 3D Lattice-Boltzmann Model for Fluid Flow Simulation under Partially-Saturated Conditions in Packed Particle Beds. (UT007)

Principal Investigators: Jan Miller and Michael Sukop, University of Utah

Period of Performance: October 1, 2006-September 30, 2009 (2-Year Project)

Heap leaching is a process extensively used by the mining industry to recover metals from large quantities of marginal grade materials. Currently, heap leaching finds important applications in the recovery of copper and gold from their ores because of its low cost, short construction time, operational simplicity, good performance, and environmental advantages. Characterization and analysis of heap leaching systems should involve the study of both the chemistry/biochemistry and mechanics of the heap leaching system. Optimum design of the chemistry, utilization of reactants (acids, air, microorganisms, bacteria population, iron), is limited by our current understanding of the mechanics of the system. Mechanics of the heap leaching system include such issues as particle breakage/exposure/liberation, particle agglomeration/pretreatment, and fluid flow phenomena inside the packed bed of particles which constitute the heap.

The proposed research has been designed to develop software capable of simulating the fluid flow of a leaching solution through a packed bed of particles under partiallysaturated conditions. The software will use the Lattice Boltzmann Method (LBM), which has numerous advantages over other approaches. The proposed research involves consideration of the mechanics of the heap leaching system as influenced by particle size distribution, pore-network structures, solution flow rate, oxygen flow rate, and moisture content. In a broader sense, this research is part of a long range program using X-Ray Microtomography (XMT) to determine to what extent the rate and practical recovery from heap leaching is limited by: (1) mineral exposure, (2) fluid flow/transport 
phenomena and (3) chemistry. It is expected that the results from the proposed research will help to design heap leaching operations for more effective utilization of our mineral resources.

\section{Development of a Turbulent Flotation Model and a Computer Simulator (VA015)}

Principal Investigators: Roe-Hoan Yoon and Gerald H. Luttrell, Virginia Tech

Period of Performance: October 1, 2006-September 30, 2010 (2-Year Project)

Flotation is the most widely used method of separating fine particles in the mining industry. The method of using air bubbles was awarded a U.S. patent in 1905, exactly 100 years ago. Yet, there are no flotation models incorporating both surface chemistry (e.g., hydrophobicity, epptential, and surface tension) and hydrodynamic (e.g., bubble size, particle size, energy dissipation) parameters under turbulent flow conditions. It is, therefore, proposed to develop a comprehensive flotation model incorporating practically all of the process variables employed in flotation practice. The turbulent flotation model will be able to describe the events taking place in both the pulp and froth phases. Laboratory experiments will be carried out to verify the model, and model will be transformed into a compute simulator that can predict complicated flotation circuits incorporating rougher, scavenger and cleaner operations.

\section{Development of a Comprehensive Model for the Simulation, Scale-Up and Design of Large Flotation Machines (VA018)}

Principal Investigators: Roe-Hoan Yoon Demetri Telionis, Gerald H. Luttrell, Pavlos Vlachos and Saad Ragab, Virginia Tech

Period of Performance: October 1, 2007-September 30, 2010 (2-Year Project)

Flotation is the most widely used method of separating fine particles in the mining industry. It is a complex process, involving three phases, i.e., solid, liquid, and gas, which makes it difficult to model from first principles. Therefore, most flotation models developed in the past are empirical, with no predictive and diagnostic capabilities. Further, the model parameters include only the hydrodynamic parameters such as particle size, bubble size, pulp densities, etc. Therefore, researchers at Virginia Tech have been developing a model that can predict flotation using both hydrodynamic and surface chemistry parameters under turbulent flow conditions. In the present work, the model will be further developed by incorporating a bubble generation model. The results of the proposed work will be used to design a $300 \mathrm{~m}$ flotation machine, which in turn will be used to validate the flotation model.

Characrerization, Analysis and Simulation of Fine Coal Filtration. (UT011)

Principal Investigators: Jan Miller and C. L. Lin, University of Utah

Period of Performance: October 1, 2008-September 30, 2010 (2-Year Project) 
Fine coal filtration and dewatering are of great importance to the coal industry due to its significant impact in the quality, shipping and handling of the coal product. High moisture content in the coal product reduces its heating value, increases costs, and reduces the coke yield in the case of metallurgical coal. In this regard, it is of significant importance to improve our fundamental understanding of water removal from the pore network structures present in filtration cakes.

In order to gain a better understanding of the complex transport phenomena that occur in the porous media, a study of the effect of three-dimensional pore geometry on the effective transport properties of the porous media is necessary. At present, the information from the microscopic pore geometry analysis is not detailed enough to provide an accurate prediction of transport properties from model simulation. Techniques and methodology for a detailed description of the three-dimensional pore geometry analysis of a completely interconnected porous system is needed.

The proposed research work is aimed to study fluid transport phenomena in fine coal filter cake using x-ray microtomography techniques to characterize the complex threedimensional pore geometry. The 3D porous structure captured by XMT analysis will be coupled with the Lattice Boltzmann Method (LBM) to simulate and to establish a fundamental relationship between pore microstructure and filtration operation variables.

The proposed research program will provide a wealth of new information that will be of benefit to investigators working on fine coal dewatering. Procedures for the determination of a detailed 3-D interconnected pore structure from x-ray microtomography measurements will be established. Analysis of the pore connectivity in a packed bed of particles should allow for a detailed description of fluid flow and transport in the filter cake structure. We expect to be able to study the effect of pressure drop, cake pore geometry, surface tension and contact angle on the cake resistance and the breakthrough pressure which are so significant in the cake cracking phenomena of major importance in fine coal dewatering process.

\section{Environmental Control}

Removal of Selenium (Se) from Coal Mine Wastewater by GAC-Based IronContaining Adsorbents. (WV020)

Principal Investigators: Dianchen Gang, West Virginia University Institute of Technology and Lance Lin, West Virginia University

Period of Performance: October 1, 2006-September 30, 2010 (2-Year Project)

One of the major challenges facing coal and metal mining industries today is to address public concerns of environmental pollution associated with the mining activities. Oxidation of pyrites and other metal sulfides in coal and metal mine tailings can generate acid mine drainage (AMD) that may contain high concentrations of sulfate, iron, and many toxic elements such as selenium (Se) and arsenic. The overall objective of this project is to develop a novel and cost effective technology to remove Se from wastewater including AMD. This study focuses on selenate (SeO42-) and selenite (SeO32-) because 
they are highly soluble in oxidizing conditions and normally not removed by many of the treatment processes designed for divalent heavy metals such as lime neutralization and precipitation. Furthermore, selenate (SeO42-) and selenite (SeO32-) cannot be effectively removed by traditional anion-exchange process designed for anions removal due to the very similar chemical properties between selenate (SeO42-) and sulfate (SO42-). We propose to develop an iron-containing granular activated carbon for removing selenium from mining wastewater. The proposed work is consistent with the research roadmap developed by CAST for the chemical separations and environmental control issues. Developing innovative technologies for effectively remove Se from mining wastewater could reduce environmental impacts associated with the mining activities and benefit the mining companies and eventually sustain and promote coal usage as an integrated component of the Nation's energy policy and ensure the Nation's energy security.

\section{Mercury Removal from Clean Coal Processing Air Stream (MT007)}

Principal Investigators: Kumar Ganesan, Montana Tech

Period of Performance: October 1, 2007-September 30, 2010 (2-Year Project)

Mercury emissions from coal fired power plants contribute to about 50 tons of the anthropogenic emissions in the US. The US EPA has set mercury emission limits for coal fired power plants. The US DOE has been intensely involved in developing technologies to control mercury emissions. In general, mercury is present in elemental, ionic and particulate bound forms in a coal fired flue gas. The technologies currently under review for commercialization are focused on oxidizing the elemental mercury in a more readily soluble ionic form. These technologies merely convert mercury from one form to another form and distribute the transformed mercury into water and solid waste streams including fly ash byproducts. This can potentially cause serious problems in the future due to mercury being released into the environment through byproducts and wastestreams containing the transformed mercury. Therefore, long term solution for mercury must be focused on eliminating mercury from entering the environment rather than transforming and transferring it into waste streams and byproducts. This proposal is to remove mercury from a pre-combustion thermal treatment clean coal process using metallic filters. The WRI's patented coal cleaning process stream will be tested with Montana Tech's metallic mercury filters. Metallic filters will be developed at Montana Tech's mercury research laboratory for conditions suitable for the sweep gas stream of a coal cleaning process stream. These filters initially will be tested with synthetic gas to simulate the sweep gas stream. The selected filters then will be tested in the field at the Western Research Institute's patented clean coal thermal treatment process. This facility is in Laramie, Wyoming. The test is expected to last two weeks. The filtration system for the field test will be designed with a residence time of $0.1-1.0 \mathrm{sec}$ for a flow rate of $40-50 \mathrm{lpm}$. Mercury concentrations will be measured using the portable mercury tracker instrument. Montana Tech has developed and tested metallic filters (patent pending) for mercury removal from coal fired power plant flue gas. The metallic mercury filters have been proven to remove elemental mercury at or above $90 \%$ efficiency in a coal fired power plant. 
Determination of Factors Affecting the Separation of Potentially Hazardous Trace Elements and their Behavior in Coal Tailings Impoundments (KYO05)

Principal Investigators: Frank E. Huggins, Naresh Ahah, Gerald P. Huffman, University of Kentucky

Period of Performance: October 1, 2006-September 30, 2009 (2-Year Project)

A number of trace elements in coal are of significant environmental concern. Such potentially hazardous elements include $\mathrm{As}, \mathrm{Cr}, \mathrm{Se}$, and $\mathrm{Hg}$, and are regulated under either the 1990 Clean Air Act Amendments or the 1976 Resource Conservation and Recovery Act or both. Coal cleaning is performed principally to minimize the amount of pyrite and other mineral matter entering pulverized coal combustion. At the same time, hazardous trace elements associated with these minerals are also removed from coal combustion and hence their environmental impact during combustion is thereby reduced. However, such trace elements tend to be concentrated in the waste tailings from coal separation technologies, which normally are disposed of under water in coal waste impoundments. Here, trace elements may pose other environmental problems because both major and trace elements may be leached and mobilized. Consequently, the waters in such impoundments have to be isolated from the local groundwater in order to avoid possible contamination of local drinking water supplies. Despite concerns about solubilized trace elements in coal impoundments, relatively little is known about the concentrations and specific chemical forms of such trace elements that can be leached from coal minerals and mobilized in these waters. More data are clearly needed to assess the environmental hazard from mobilized ions in coal tailings impoundments.

The behavior of trace elements in both coal separation technologies and tailings disposal scenarios depends on their modes of occurrence and associations with major minerals or macerals in the coal or coal fractions. Such information is rarely determined, but is of vital importance to our understanding of trace elements in these processes. In this proposal, we intend to determine the mineral or chemical forms (mode of occurrence) and association of a number of critical trace elements (As, Cr, Se, Hg, etc.) in coals and coal fractions using a comprehensive array of conventional and advanced characterization methods. Our intention is to collaborate with other CAST-supported investigations of advanced coal separation technologies that can supply the clean coal and tailings fractions needed for this investigation. The information obtained will then be used (i) to determine the efficiency of various coal cleaning methods for specific trace element removal; (ii) to interpret the behavior of specific elemental forms and associations in coal tailings exposed to water in laboratory simulations of storage ponds; and (iii) to assess the actual environmental impact posed by specific trace elements in coal tailings impoundments.

Selenium Removal from Mine Influenced Water (MIW) Using Nano-Magnetite (WV022)

Principal Investigators: Xianchao Wei, West Virginia University 
Period of Performance: October 1, 2007-September 30, 2010 (2-Year Project)

This study will address the limitation of current adsorption processes in selenium removal from mine influenced waters (MIWs), i.e., the inadequacy of selenate (Se6+) removal. This proposal suggests an innovative technology that can simultaneously remove both selenite (Se4+) and Se6+ from MIWs using nano-magnetite as an adsorbent. Mining operations and the subsequent processing and utilization of coal and minerals result in the release of selenium into MIWs. Management of selenium in MIWs is becoming a great challenge for the mining industry in response to increasing public concerns, more stringent environmental regulations, and the difficulty in removing selenium to levels that meet current discharge criteria. Consequently, there is an urgent need to develop costeffective selenium removal processes. To date, selenium removal by adsorption using iron oxides/hydroxides is the most promising process. However, this process is not effective in removing Se6+. The inadequacy of Se6+ removal can significantly limit its application in MIW treatment because both Se6+ and Se4+ are commonly present in MIWs.

The scope of this research is consistent with CAST roadmap on environmental control regarding MIW issues. Natural magnetite was previously tested for selenium removal by other researchers. Notably, as opposed to other iron oxides/hydroxides, magnetite can adsorb Se6+ more effectively than Se4+. Due to its nano size, large specific surface area, numerous active sites for adsorption, and relatively low synthesis cost, nano-magnetite is an attractive alternative to natural magnetite. Batch tests will be carried out using nanomagnetite as an adsorbent to study adsorption kinetics, isotherms, and adsorptive thermodynamics in conjunction with studies on the effects of $\mathrm{pH}$, temperature, concentration, and contact time for selenium removal from synthetic and actual MIWs. In order to facilitate the large-scale application of nano-magnetite for selenium adsorption and to develop a continuous process, the impregnation of diatomaceous earth with nanomagnetite will also be investigated.

\section{Development of Biochemical Techniques for Extraction of Mercury from Coal Impoundments (WV023)}

Principal Investigators: Jay Weidemann, Richard Squire and Milson Luce, West Virginia University Institute of Technology.

Period of Performance: October 1, 2007-September 30, 2009 (2-Year Project)

The West Virginia University Institute of Technology, Department of Chemistry Project, "Development of Biochemical Techniques for the Extraction of Mercury from Fine Coal Particle Solutions", includes three objectives: 1) development of a biochemical or molecular biology technique to facilitate the conversion of mercury compounds in coal containing solutions to its most water-soluble form of mercury, 2) development of very efficient and novel ligands that preferentially bind to mercury even in the presence of the much higher concentrations of other metals, and 3) the isolation of mercury from the mercury bound ligand solution. The results of this work will be the basis for the development of a novel process for the extracting mercury from coal impoundment water 
in a "zero waste" manner.

Development of Techniques for Online Monitoring of Individual Gas Constituents in Coal-Derived Synthesis Gas and Flue Gas (NM006)

Principal Investigators: Peng Zhang, New Mexico Tech and Ning Liu, Petroleum Recovery Research Center.

Period of Performance: October 1, 2008-September 30, 2010 (2-Year Project)

The objectives of the proposed research program are: 1) Development of a mass spectrometer-based technique to rapidly detect and continuously monitor gas constituents in synthesis gas (syngas) and flue gas; and 2) demonstration of the effectiveness of the technique through field tests.

In-situ detection of synthesis gas composition using such a mass spectrometer can be achieved with a specially designed bypassing flow loop that continuously conducts a small fraction of synthesis gas from its main stream to be measured by the spectrometer. A heat-exchanger and pressure reducer can be applied to adjust the temperature and pressure of the incoming gas to a suitable range for the mass spectrometer. Solid particles such as ashes and tars in the synthesis gas can be removed by appropriate gas filters. Major research tasks in this project will include the design of a laboratory flow loop model, characterization of the system accuracy, and response time to the individual gas constituents, as well as the mixture of the syngas, and finally, the scaling-up of the lab model for field testing in an actual coal-fired power plant. This in-situ syngas detection system, once completed, could be adapted to monitor the compositions in flue gas exhausted from a power plant, a process which is at high demand for successful carbon dioxide capture and sequestration. This proposed two-year project will be led by the principal investigator (PI), Dr. Peng Zhang, from the Chemistry Department, and coprincipal investigator, Dr. Ning Liu, from the Petroleum Recovery Research Center (PRRC), both at New Mexico Institute of Mining and Technology.

\section{Hydrate}

Gas-Gas Separation by Selective Hydrate Formation - Laying the Foundation (VA022)

Principal Investigators: Roe-Hoan Yoon, Jialin Wang and Gerald Luttrell, Virginia Tech Period of Performance: October 1, 2008-September 30, 2010 (2-Year Project)

Gas-gas separation is an important part of producing hydrogen from coal. At present, the separation is achieved using the membrane, pressure-swing adsorption (PSA), and cryogenic distillation methods. In this project, the possibility of separating different gases by selective hydrate formation will be explored. In principle, this method can be of high capacity and simple to operate. In this project, we are planning to develop methods of minimizing the pressure requirement and improving the kinetics for hydrate formation. The basic information obtained in the present work will be used to achieve separation 
between $\mathrm{CO}_{2}$ and nitrogen $\left(\mathrm{N}_{2}\right)$ and methane $\left(\mathrm{CH}_{4}\right)$ and $\mathrm{CO}_{2}$ using the selective hydrate formation technique. 


\section{EXPERIMENTAL}

The CAST initiative is comprised of a diverse group of subprojects, most of which are multistage, task-oriented developmental projects that cannot be conveniently categorized by the traditional reporting criteria required by the DOE Uniform Reporting

Requirements. For example, several of the projects have required the construction of unique test equipment, others the generation of simulation models, etc., as preliminary tasks in the overall execution of the project. As such, they are more appropriately described and discussed as "Project Tasks" within the context of the individual Technical Reports. These reports will be attached to this document as Appendices and should be referred to for this information. 


\section{RESULTS, DISCUSSION \& CONCLUSION}

The CAST initiative is comprised of a diverse group of subprojects, most of which are multistage, task-oriented developmental projects that cannot be conveniently categorized by the traditional reporting criteria required by the DOE Uniform Reporting

Requirements. For example, several of the projects have required the construction of unique test equipment, others the generation of simulation models, etc., as preliminary tasks in the overall execution of the project. As such, the presentation of results discussion and conclusion are more appropriately described and discussed within the context of the individual Technical Reports. These reports are attached to this document as Appendices and should be referred to for this information. 
APPENDICES 
APPENDIX 1 - Enhanced Flotation Performance Through Column Froth Enrichment (KY006) 


\section{FINAL REPORT}

Contract Title and Number:

Continuation of Crosscutting Development at CAST

(DE-FC26-05NT42457)
Period of Performance:

Starting Date: 10/1/2005

Ending Date: 9/30/10
Sub-Recipient Project Title:

Enhanced Flotation Performance Through Column

Froth Enrichment

Principal Investigators:

Honaker, Tao

Contact Address:

University of Kentucky

234B Mining and Mineral Resources

Lexington KY 40506

Subcontractor Address:

No subcontracts issued.
Report Information:

Type: Final

Number: 6

Period: 10/01/05-9/30/09

Date: $\quad$ 12/15/09

Code: KY006-FINAL

Contact Information:

Phone: (859) 257-1108

Fax: (858) 323-1962

E-Mail: rhonaker@engr.uky.edu

Subcontractor Information:

Phone:

Fax:

E-Mail:

\section{ABSTRACT}

Froth flotation is the most common process used for cleaning coal having a particle size below 150 micrometers. Although the process has been well researched and commercially applied for more than a century, several problems still exist including the flotation of weakly hydrophobic coals, the flotation of coarse (+150 micrometers) and ultrafine (-25 micrometers) coal, and selectivity for difficult-to-clean coals. In this investigation, feed pretreatment using hydrodynamic cavitation, the addition of hydrophobic plastic material and external refluxing a portion of the froth concentrate was evaluated to enhance flotation performance. Cavitation pretreatment nearly doubled the flotation rate and improved the combustion recovery achieved in a flotation column from $71 \%$ to $76 \%$. Using hydrophobic plastic particles in the feed and/or the froth phase improved flotation rates from $1.04 \mathrm{~min}^{-1}$ to $1.31 \mathrm{~min}^{-1}$ which provided an increase in combustible recovery by about 3 absolute percentage points under the same test conditions. Finally, enrichment of the selective detachment mechanism in the froth zone by providing external reflux had a positive impact on the selective detachment process and provided a reduction in the product ash content of nearly 3 absolute percentage points while maintaining the same recovery value. The improvement was realized at low and intermediate feed rates. 


\section{DISCLAIMER}

This report was prepared as an account of work sponsored by an agency of the United States Government. Neither the United States Government nor any agency thereof, nor any of their employees, make any warranty, express or implied, nor assume any legal liability or responsibility for the accuracy, completeness, or usefulness of any information, apparatus, product, or process disclosed, or represents that its use would not infringe privately owned rights. Reference herein to any specific commercial product, process, or service by trade name, trademark, manufacturer, or otherwise does not necessarily constitute or imply endorsement, recommendation, or favoring by the United States Government or any agency thereof. The views and opinions of authors expressed herein do not necessarily state or reflect those of the United States Government or agency thereof. 


\section{TABLE OF CONTENTS}

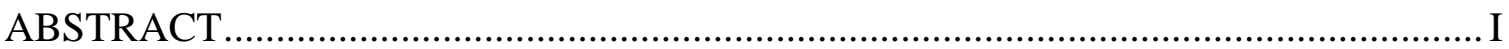

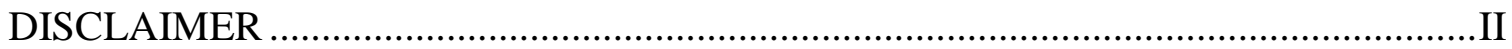

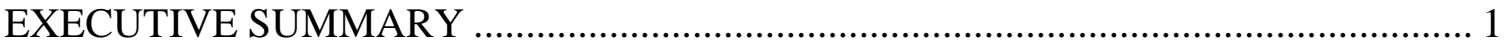

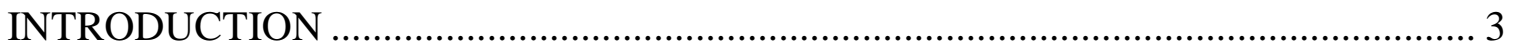

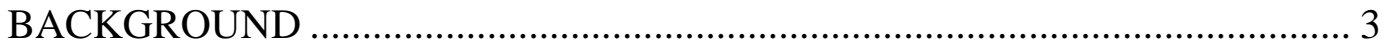

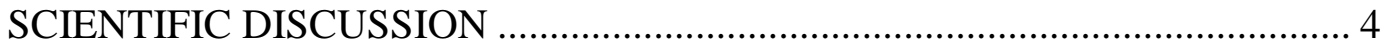

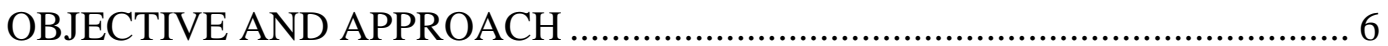

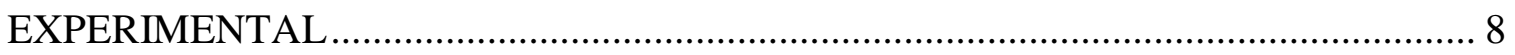

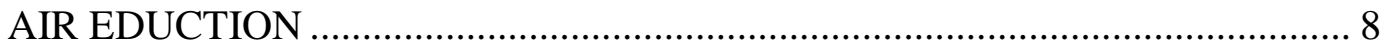

Sample Characterization ......................................................................... 8

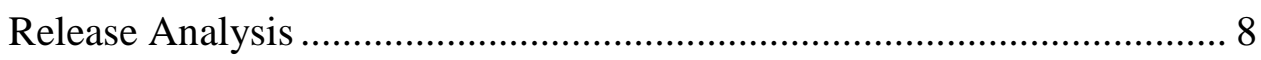

Flotation Rate Test............................................................................. 9

Column Flotation Test ........................................................................... 10

Air Eductor Pretreatment .......................................................................... 11

In-Plant Conventional Flotation Tests ........................................................ 12

MAGNETIC PLASTIC EVALUATION ………………………………......... 13

Sample Characterization ........................................................................... 13

Magnetic Plastic Material ..................................................................... 14

Contact Angle Analysis ........................................................................... 16

Zeta Potential Measurements ................................................................. 18

Density Fraction Test ............................................................................ 20

Flotation Rate Test................................................................................. 20

Column Flotation Test ........................................................................... 20

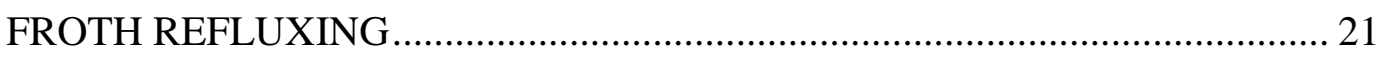

RESULTS AND DISCUSSIONS ....................................................................... 24

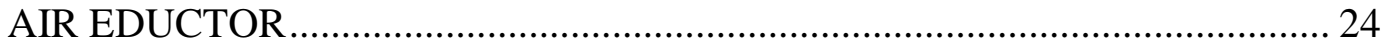

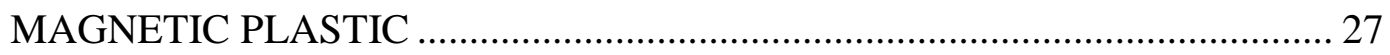

Conventional Flotation Tests ................................................................... 27

Repetitive Performance Evaluation ............................................................ 31 
Effect of Coal Type.............................................................................. 34

Effect of $\mathrm{pH}$................................................................................ 36

Particle Size-by-Size Flotation .................................................................. 40

Column Flotation Tests.......................................................................... 43

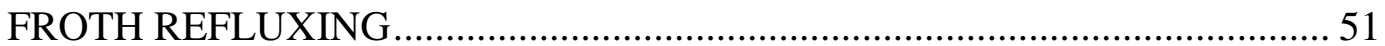

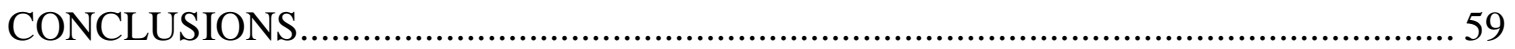

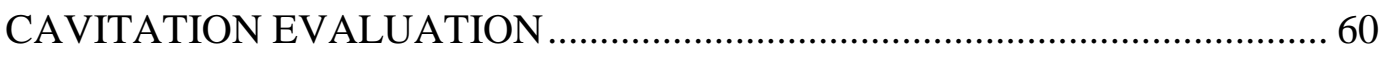

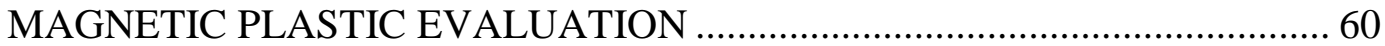

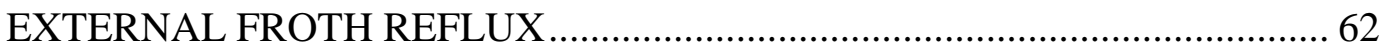

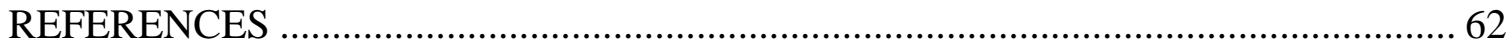

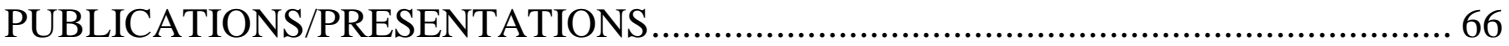




\section{EXECUTIVE SUMMARY}

For over a century, froth flotation has been used as the primary process to recovery coal finer than 150 micrometers. The process exploits differences in surface hydrophobicity between particles in the feed stream to achieve a separation. In contrast to the hydrophilicity of most reject particles, coal particles are naturally hydrophobic but vary in their degree of hydrophobicity within the coal and from coal-to-coal. Although past research has resolved many of the issues that have hampered the widespread use of froth flotation within the coal industry, improvements are still needed including the ability to recover difficult-to-float coals, enhanced selectivity for coals contains a relative large amount of mixed-phase particles and coal pyrite, and increased throughput capacity.

In this investigation, improving selectivity was the primary objective. The proposed methods involved i) the addition of magnetic hydrophobic plastic material directly into the froth zone and ii) enriching the froth zone by refluxing a portion of the froth concentrate back into the froth zone. In both methods, the increased concentration of highly hydrophobic particles crowd the bubble surfaces and cause selective detachment of the more weakly hydrophobic, high ash particles. However, an important finding of the research was that the plastic addition had a greater impact on enhancing coal flotation recovery and this impact became a primary focus of the study. The magnetic characteristic of the plastic allows recovery using a low-intensity magnetic separator. Another method that was discovered to enhance recovery was the pretreatment of the flotation feed through a cavitation tube. As such, all three methods were evaluated to assess and quantify the impact on both flotation recovery and selectivity.

Two coal samples with distinctly different characteristics were used for the flotation experiments. Pittsburgh No. 8 coal is well liberated and thus easy-to-clean in regards to ash reduction. However, the coal contains a significant amount of pyritic sulfur that is weakly-to-moderately hydrophobic. As such, pyrite rejection is typically limited in a froth flotation process. The other flotation feed sample was collected from an active preparation plant that treats coal from the Coalburg seam. Coalburg coal tends to be difficult-to-float and contains a relatively large amount of mixed-phase particles, which results in feed containing particles having a wide range of floatability characteristics.

The impact of adding hydrophobic plastic particles $\left(\theta=84^{\circ}\right)$ directly into the flotation feed slurry of a semi-batch Denver flotation cell was evaluated at concentrations of $2 \%$, $5 \%$ and $10 \%$ weight as related to the amount of dry coal solids in the feed. At a $5 \%$ concentration, combustible recovery increased by 15 absolute percentage points after 1 minute of flotation time when treating the Pittsburgh No. 8 coal. A total recovery improvement of 20 absolute percentage points was realized when using a $10 \%$ plastic concentration. In effort to ensure that the observed improvements were valid, the tests with no plastic material and $10 \%$ plastic addition were repeated five times under identical conditions. By averaging the flotation performances, a flotation rate increase from 1.04 $\min ^{-1}$ to $1.31 \mathrm{~min}^{-1}$ was obtained which corresponds to a $26 \%$ improvement. The corresponding recovery gain was 11 absolute percent points. 
As previously described, the Coalburg seam coal is difficult to float. After 1 minute of flotation time, only $61 \%$ of the coal was recovered from the flotation cell. The flotation rate was a relatively low $0.51 \mathrm{~min}^{-1}$. However, adding the plastic particles directly in the feed resulted in a flotation rate improvement to $0.74 \mathrm{~min}^{-1}$, which resulted in a $77 \%$ recovery of the combustible material.

Using the Pittsburgh No. 8 flotation feed sample, the performance improvement resulting from the plastic particle addition was evaluated as a function of coal particle size. A minimum recovery improvement of 5 absolute percentage points was obtained for particle sizes smaller than 25 micrometer. Recovery improvement increased with particle size to a maximum increase of 35 absolute percentage points for 150 micrometer particles. Additional tests were conducted on individual particle size fractions in separate tests with and without the addition of plastic particles. Very little improvement in the recovery of the coarse particles was observed from the individual size fraction tests. Based on these results, it is hypothesized that the plastic material may be reducing the drainage of the water surrounding the bubble surfaces and thus reducing bubble coalescence and maintaining coarse particle attachment due to high bubble surface area flux rates.

Experiments were also conducted at $\mathrm{pH}$ values of 3.9 and 10.1. Without the addition of plastic particles, flotation recovery was suppressed to values below 35\%. By adding the plastic particles, coal recovery returned to the values achieved under neutral $\mathrm{pH}$ conditions which were around 85\%.

In the continuous column flotation tests, the positive impact on coarse particle recovery was also observed. Feed solid concentrations were varied to assess the carrying-capacity of the column when treating the Pittsburgh No. 8 coal. When plastic particles were continuously added through the wash water distribution ring, the product mass flux rate increased and then became flat at a critical feed mass flux rate which indicates a positive impact on coarse particle recovery. Overall recovery improvements were generally less than the conventional cell results and varied as a function of volumetric feed rate and the amount of plastic particles added. At a 5\% plastic concentration, recovery improvement was from around 4 absolute percentage points at feed rates between $400 \mathrm{ml} / \mathrm{min}$ to 800 $\mathrm{ml} / \mathrm{min}$ for the treatment of Pittsburgh No. 8 coal. For Coalburg coal, the recovery improvement was around 7 absolute percentage points at a feed rate of $600 \mathrm{ml} / \mathrm{min}$.

Further investigations involved the evaluation of the impact of pre-aeration of the feed material through a cavitation tube. Recent investigations found that the bubble-particle attachment rate is increased when small air bubbles pre-exist on the coal particles prior to exposure to the bubbles generated from conventional flotation systems. For the Coalburg coal, pre-aeration using a cavitation tube resulted in an 18 absolute percentage point improvement in recovery after one minute of flotation time in a laboratory Denver cell. Flotation rate was improved from $0.65 \mathrm{~min}^{-1}$ to $1.25 \mathrm{~min}^{-1}$. 


\section{INTRODUCTION}

\section{Background}

The froth flotation process is comprised of two separate and distinctly different zones, i.e., the collection zone and the froth zone. In the collection zone, the separation of the valuable minerals from the non-valuables is achieved based on the bubble-particle attachment process. Due to hydraulic entrainment, a portion of the non-valuable minerals is carried from the collection zone into the froth zone with the mineral-bubble aggregates. Selectivity of the process can be enhanced in the froth zone by providing drainage of the feed pulp and utilizing the selective detachment of the more weak hydrophobic particles as a result of bubble coalescence and the resulting bubble surface area reduction. The importance of the latter sub-process is the subject of recent investigations (van Deventer et al., 2004; Honaker and Ozsever, 2003; Ata et al., 2002; Ralston et al., 1999; Falutsu, 1994; Hewitt et al., 1994; Yianatos et al., 1988). On the other hand, decades of research focused on the froth flotation process have resulted in a clear understanding of the processes and sub-processes involved in the selectivity achieved in the collection zone (Yoon and Mao, 1996; Mao and Yoon, 1997).

Moys (1978) and later Yianatos et al. (1988) confirmed by experimental evidence that the detachment process is selective. From Yianatos et al. (1988), the detachment rate for chalcopyrite under a given set of conditions was $0.30 \mathrm{~min}^{-1}$ whereas pyrite obtained a higher rate at $0.41 \mathrm{~min}^{-1}$. As such, differential detachment rates can be exploited to improve the selectivity between hydrophobic species. Schultz et al. (1991) obtained an improved concentrate grade and recovery in a column by introducing the feed (Alabama shale, $d_{90}=23$ microns) into the froth zone.

Detailed investigations utilizing modified column apparatus designs have provided promising quantifiable evidence of the selective detachment and drop-back processes (Rubio, 1996; Ata et al., 2002; Honaker and Ozsever, 2003). For example, Ata et al. (2002) used a unique cell design to show that highly hydrophobic particles added directly into the froth zone can selectively replace particles of lower hydrophobicity. Specifically, the addition of hydrophobic silica particles in the froth reduced the recovery of hematite particles that were added in the collection zone by 10 percentage points. Based on this finding, it is plausible that the addition of highly hydrophobic particles in the froth zone could assist in the selective rejection of the more weakly hydrophobic material (e.g., low grade particles) reporting from the collection zone, thereby enhancing the overall selectivity between particles of varying hydrophobicity.

The current project exploited the selective detachment process to enhance the selectivity between particles having varying degrees of floatability. External refluxing and the addition of hydrophobic material directly into the froth zone was investigated as a means to improve recovery and selectivity. 


\section{$\underline{\text { Scientific Discussion }}$}

The separation of the desired mineral from the other mineral components in the collection zone is based on differential flotation rates. The collection zone flotation rate of a mineral, $k_{c i}$, can be quantified by the expression:

$k_{c_{i}}=\frac{3}{2} \frac{V_{g}}{D_{b}} P_{c} P_{a} P_{d}$

in which $V_{g}$ is the superficial gas velocity, $D_{b}$ the bubble diameter, $P_{c}$ the probability of collision, $P_{a}$ the probability of attachment and $P_{d}$ the probability of detachment. As shown in Eq. (1), a change in gas velocity or bubble size affects the flotation rate of each mineral species equally and thus does not improve selectivity. Furthermore, bubbleparticle collision is not a selective process and should be maintained at maximum efficiency to ensure high recovery of the floatable mineral. The probability of detachment is a function of both particle size and density and thus may play a minor role in selectivity. However, the bubble-particle attachment process as quantified by $P_{a}$ is the principle mechanism defining the ability to effectively separate minerals in the collection zone of a froth flotation process.

After bubble-particle collision and attachment, the aggregate moves upward through the collection zone and into the froth zone. The transition into the froth zone is characterized by an increase in the air fraction from around $20 \%-30 \%$ to $70 \%-80 \%$ (Finch and Dobby, 1990). In the froth zone, the water filling the voids between the bubbles drain back into the collection zone and the water layer surrounding each bubble thins. Upon approach of the liquid-air interfaces, bubbles coalesce thereby causing a reduction in bubble surface area. If the reduced amount of bubble surface area available in the froth zone is insufficient to carry the solids reporting from the collection zone, particles detach and potentially move with the fluid into the collection zone. As such, froth zone recovery may have a significant influence on overall flotation recovery.

To assess the effect on overall flotation recovery for a given mineral, linear analysis concepts developed by Meloy (1983) can be applied to the mass transport processes illustrated in Figure 1. As proposed by Finch and Dobby (1990), an expression for the overall flotation recovery $R_{O}$ for a mineral component $i$ can be derived as a function of the collection zone recovery $R_{C}$ and froth zone recovery $R_{F}$ (Eq. 2), i.e.,

$$
R_{O}=\frac{R_{C} R_{F}}{R_{C} R_{F}+\left(1-R_{C}\right)}
$$




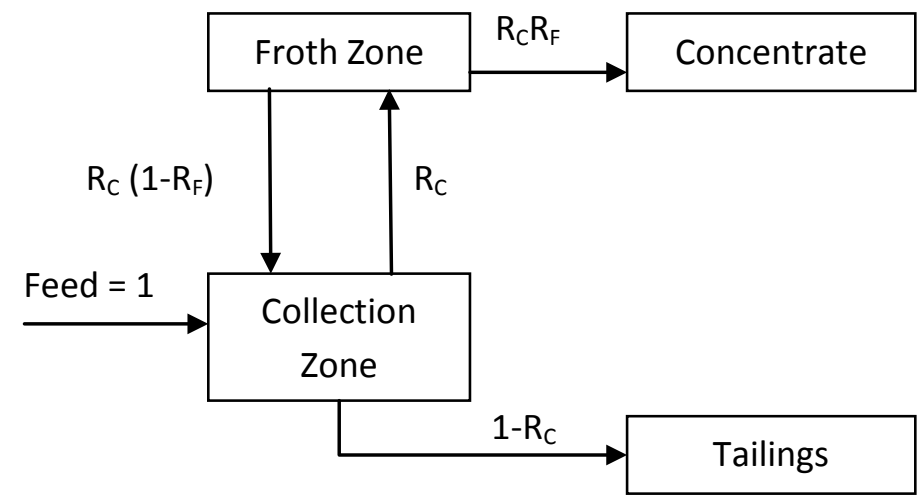

Figure 1. Interaction between zones in a column flotation cell.

One should note that, if $R_{F}$ equals $100 \%, R_{O}$ is equivalent to $R_{C}$.

An analysis of Eq. (2) reveals that froth zone recovery has a relatively small impact on the overall recovery of particles that have a collection zone recovery. Highly floatable particles that are detached in the froth zone are efficiently recovered in the collection zone. However, weakly hydrophobic particles have a low collection zone recovery and thus are less likely to be recovered in the collection zone. As a result, reductions in froth zone recovery have a significant impact on the overall recovery of particles that have moderate-to-weak flotation characteristics. Therefore, the differential effect of froth zone recovery values between particles of varying floatability indicates an additional selectivity mechanism provided by the reflux action between the collection and froth zones.

For systems that contain multiple mineral species or un-liberated particles with varying degrees of hydrophobicity, the selectivity of the detachment process in the froth zone also enhances the overall separation performance achieved by froth flotation as discussed in the previous section. Particles with a low degree of surface hydrophobicity are likely to be weakly bound to the bubble surface and thus will be preferentially detached. By adding particles directly into the froth phase that have a higher degree of hydrophobicity than the low grade particles, selectivity can be enhanced as a result of selective detachment. Figure 2 shows the improvement in ash reduction achieved for an anthracite coal when a more hydrophobic material is added into the froth at concentrations of $5 \%$, $10 \%$ and $15 \%$ of the collection zone feed. The added material was removed from the product and tailing samples by density fractionation. At a given recovery value, the product ash content was reduced from $19 \%$ to nearly $14 \%$ at a concentration equal to $15 \%$ of the feed mass flow rate. 


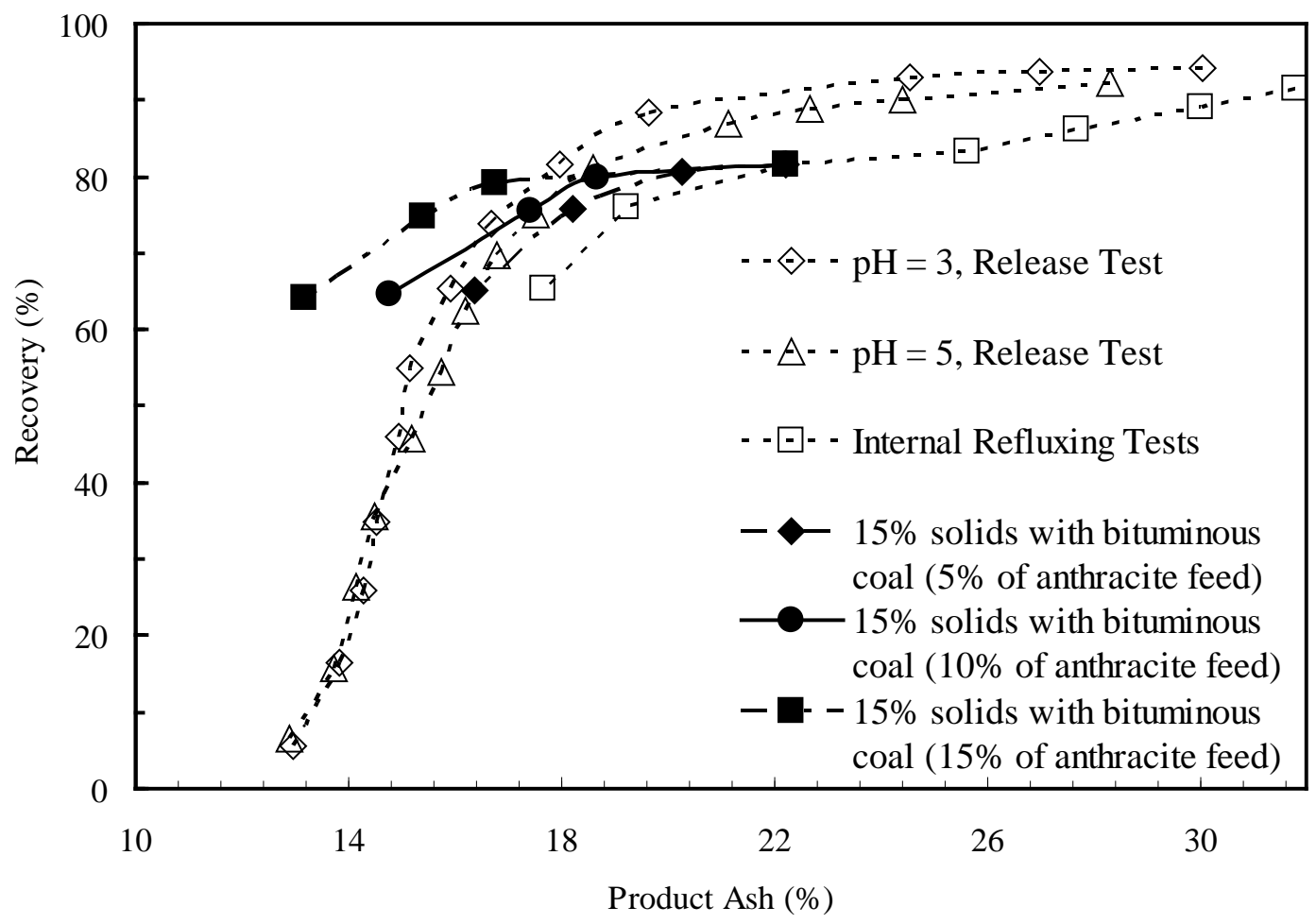

Figure 2. Improvement in the separation performance achieved on anthracite coal.

\section{Objective and Approach}

The main objective of this research focused on the selective detachment process and the hydrodynamic cavitation technology in an effort to enhance the performance of column flotation when cleaning ultrafine coal. Enhanced flotation by the addition of hydrophobic material or external refluxing into the froth zone will promote selective detachment of particles having varying degrees of floatability. To improve recovery, gas nuclei or picobubbles on a particle surface will favor activation of flotation by improving surface hydrophobicity and thus bubble-solid attachment. By using available fundamental relationships, full quantification of the different enhancement alternatives will be performed over a range of operating parameters.

It was hypothesized that the addition of highly hydrophobic particles into the froth zone will increase competition among particles having different degrees of floatability. Thus, the introduction of material with a higher degree of hydrophobicity than the original feed material will result in the detachment of weakly hydrophobic particles, which typically have high ash content. The most hydrophobic particles will be selectively recovered. The selectivity will improve grade without a significant reduction of recovery. A naturally hydrophobic plastic product that contains a significant amount of magnetite and thus recoverable by low intensity magnetic separator was used as froth enrichment material. 
The plastic material is currently provided by Eriez Manufacturing Company. The study involved conventional flotation cell and column flotation experiments with and without the addition of magnetic material in the feed or froth phase.

The same principles were also exploited by refluxing a minor portion of froth concentrate back through the wash water and into the froth phase. As a result, concentration of highly hydrophobic particles occurs which should stimulate the selectivity of the detachment process. Bubble coalescence in the froth phase decreases the amount of bubble surface area needed to recover all of the collected particles. The shock energy of the coalescence event and the lack of bubble surface area cause particle detachment.

In addition, the action of pretreatment feed slurry to promote picobubble formation on the surface of hydrophobic solid will improve flotation rate and thus flotation recovery by increasing the probability of attachment. The advantages will be more prevalent for weakly floatable coals. Effectiveness of this innovative process was evaluated to assess the upgrading potential under different operating conditions. In this particular study, a Coalburg sample obtained from an operating coal preparation plant was used in a column flotation cell, pilot bench-type cells and a continuous column flotation unit.

The specific objectives of the investigation were:

- To develop and demonstrate the potential use of naturally hydrophobic magnetic plastic material to enhance the selectivity achieved when treating coal by froth flotation. Characterization of the plastic material was achieved by evaluating recovery efficiency for a range of magnetic plastic particle sizes and quantifying the surface characteristics. Flotation performance and selectivity detachment was analyzed over a range of conditions (solids concentrations, feed volumetric flow rate, particles size and $\mathrm{pH}$ ).

- To investigate the potential selectivity benefits associated with recycling a portion of the froth product back to the froth phase in a continuous column flotation. The potential separation enhancement will be quantified.

- To demonstrate advantages in flotation performance by treating a weakly floatable and high middling Coalburg feed slurry with a hydrodynamic cavitation technique. The performance enhancement achieved in a continuous column flotation system will be determined over a range of operating conditions. 


\section{EXPERIMENTAL Air Eduction}

\section{Sample Characterization}

Three 208-liter drums of coal slurry were collected from a West Virginia coal preparation plant treating coal from the Coalburg seam. Representative flotation feed at the West Virginia preparation plant was obtained on three consecutive days. The slurry contained $5 \%$ solids by weight. The Coalburg slurry sample was used to investigate the impacts of using hydrodynamic cavitation on coal recovery in a froth flotation process. The flotation feed was comprised of about $84 \%$ minus $44 \mu m$ (325 mesh) as shown in Table 1 . The material finer than $44 \mu \mathrm{m}$ contained the largest amount of combustible (floatable) material with $66.6 \%$ of the total amount.

Table 1. Particle size distribution and quality characteristics of a West Virginia preparation plant flotation feed.

\begin{tabular}{|c|c|c|c|c|c|c|}
\hline \multirow{2}{*}{$\begin{array}{c}\text { Particle Size } \\
\text { Fraction } \\
(\mu \mathrm{m})\end{array}$} & \multicolumn{3}{|c|}{ Incremental (\%) } & \multicolumn{3}{c|}{ Cumulative (\%) } \\
\cline { 2 - 7 } & Weight & $\begin{array}{c}\text { Ash } \\
\text { Content }\end{array}$ & $\begin{array}{c}\text { Coal } \\
\text { Distribution }\end{array}$ & Weight & $\begin{array}{c}\text { Ash } \\
\text { Content }\end{array}$ & $\begin{array}{c}\text { Coal } \\
\text { Distribution }\end{array}$ \\
\hline+149 & 1.41 & 5.66 & 3.08 & 1.41 & 5.66 & 3.08 \\
$149 \times 44$ & 14.98 & 12.05 & 30.34 & 16.39 & 11.50 & 33.42 \\
-44 & 83.61 & 60.74 & 66.58 & 100.00 & 52.67 & 100.00 \\
\hline Total & 100.00 & 52.67 & 100.00 & & & \\
\hline
\end{tabular}

\section{Release Analysis}

Release analysis is the procedure used to obtain the best possible separation performance achievable by any froth flotation process. As such, the performance of a flotation process can be monitored and improved with the idea to approach the theoretical flotation response curve given by the release analysis curve for a certain feed material. This goal is analogous to the gravity-based washability analysis. Generally, the release analysis test is carried out in conventional laboratory flotation cells and conducted in two-phases with distinctly different goals.

The first stage separates the hydrophobic material away from the hydrophilic material by doing multiple cleaning phases from the initial feed. The sample was introduced in a laboratory Denver flotation cell of 1 liter capacity at a $5 \%$ feed solid content by weight. After mixing collector (Fuel Oil No. 2) and frother (ShurFlot 948) was injected at minimum doses. The fuel oil was added and conditioned for 5 minutes prior to the 
addition of the frother. Flotation was continuously performed in phase I to float all the hydrophobic material from the cell. When needed, frother was added to allow a continuation of flotation. The product was continuously collected in a separate container. When all of the floatable material was collected, the remaining material was placed in a separate container which was labeled as tailings. The floated material was placed back into the flotation cell and refloated. This process was repeated three times to ensure remove of all the hydrophilic particles from the hydrophobic particles.

The second stage has the goal of separating particles into fractions of degrees of surface hydrophobicity. For coal, the more hydrophobic coal contains the least amount of ashforming material with the ash content increasing with a decline in hydrophobicity. Fractionated samples are obtained by controlling air flow rate and rotator revolutions under starvation conditions. Lower air flow rates and rotator revolutions lead to higher floatable particles reporting first until no more particles are able to float (sample 1). Then an incremental increase in air flow rate and/or rotator revolution was allowed to obtain the next most floatable particles, which contained a larger amount of ash (sample 2). A progressive increase in air rate or rotation speed assures the total flotation of the next set of more floatable particles by a fractionation process (sample 3, 4, etc.). The process stops when no more particles can float. The sample were filtered, dried and weighed followed by analysis of the ash content.

\section{Flotation Rate Test}

The flotation rate tests followed the ASTM D5114 procedure with Fuel Oil No.2 as collector and an alcohol-based frother currently used at a West Virginia preparation plant. The collector-to-frother dosage ratio was maintained at 2:1. The coal slurry was treated through an air eductor in an effort to nucleate fine bubbles on the surface of the weakly hydrophobic coal particles prior to flotation tests.

The laboratory semi-batch flotation system was a Denver flotation unit equipped with a 1-liter cell. Flotation was conducted using natural air source. The sample was conditioned with collector for 15 minutes before adding frother, which assured correct adsorption of surfactants without overlapping. The flotation concentrate samples were collected as a function of time. Samples were filtered, dried, weighed and analyzed for ash content using the appropriate ASTM procedures.

In summary, three tests were performed:

Test 1: $\quad$ Rate test using current frother and fuel oil.

Test 2: $\quad$ Rate test on feed slurry using air eductor treatment. Slurry was recycled through the eductor through several cycles.

Test 3: Rate test on feed slurry processed through an air eductor using a single pass. Feed was not recycled back through the eductor. 


\section{Column Flotation Test}

Laboratory flotation tests were performed using a continuously operated flotation column over a range of feed volumetric flow rates which provided a range of retention times. The inlet feed slurry was located at $45 \mathrm{~cm}$ below the overflow lip. The flotation column used the Microcel bubble generator which consisted of a static mixer and a pump configuration to produce the bubbles. The length of the collection and froth zones typically used in the test program were $210 \mathrm{~cm}$ and $30 \mathrm{~cm}$, respectively. With a diameter of $5 \mathrm{~cm}$, the length-to-diameter ratio was around 50:1 which provided near plug-flow conditions. Wash water was added in the froth zone at a depth that was $1 / 3$ of the froth zone height below the overflow lip. A summary of the table operating parameters is provided in Table 2.

Table 2. Operative column flotation conditions use for the entire experimental program test.

Feed rate

Feed solids concentration by weight

Bias factor

Collector/Frother ratio

Froth depth

Superficial gas velocity

Volumetric wash water
$200 \mathrm{ml} / \mathrm{min}$ to $1000 \mathrm{ml} / \mathrm{min}$

$5 \%$

0.7

2:1

$30 \mathrm{~cm}$

$2 \mathrm{~cm} / \mathrm{s}$

$400 \mathrm{ml} / \mathrm{min}$

The tailing flow was adjusted with a control valve attached to the tailing pipe. A microprocessor series 2600 Love Controls received signals from a pressure transducer located at the bottom of the column. The signal adjusts the air pressure that controls the underflow valve whose flow is based on the desired froth level. Conventional air bubbles were generated by processing a portion of the tailing stream through a static mixer at a pressure of $1.36 \mathrm{~atm}$ (20 psi). Frother and air are injected into the stream prior to the static mixer. The output of the static mixer was located inside the cell and near the bottom.

A period of time equivalent to three particle retention times was allowed to achieve steady-state conditions. After reaching steady-state, samples of the feed product and tailing streams were collected simultaneously. The samples were filtered, dried, weighed and analyzed for ash content. 


\section{Air Eductor Pretreatment}

In the study of flotation performance enhancement using hydrodynamic cavitation, feed pretreatment with picobubbles was carried out with the use of a venturi tube. The venture tube consists of a gradual contraction from the pipe diameter $(D)$ to the throat diameter (d) and then a gradual expansion to the original pipe diameter. The high flow velocity leads to hydrodynamic cavitation due to momentarily reduction of vapor pressure in the liquid.

Prior to each test, the feed slurry was conditioned for 15 minutes with Fuel Oil No. 2 which was used to enhance the hydrophobicity of the coal surfaces. Conditioning was conducted in a sump that was equipped with a mixer and four baffles placed vertically and separated by an equal distance along the circumference of the sump.

After conditioning, the slurry was processed through an air eductor to generate micron size bubbles on the coal surfaces. Two different air eductor sizes were used, i.e., $1.27-\mathrm{cm}$ (1/2-in) inner diameter for the laboratory conventional and flotation column tests and 3.8$\mathrm{cm}$ diameter unit for the pilot-scale conventional flotation bank. The slurry was pumped through both air eductors at a volumetric flow rate that provided a pressure of $2.72 \mathrm{~atm}$ (40 psi). Air was injected into the slurry prior to the eductor at a rate of $1 \mathrm{ml} / \mathrm{min}$.

As shown in Figure 3, the process in each experiment involved pretreatment of the conditioned feed through an air eductor. The aerated feed slurry was then pumped to a feed tank which utilized a recirculating line to ensure suspension of all solids. A peristaltic pump was used to draw a pre-determined amount of feed into the flotation column at a point just below the pulp-froth interphase. As a result, the pre-treated coal particles initially move downward in the cell against a rising flow of air bubbles. Upon collision and attachment, the air bubble-particle aggregate rises to the top where they are collected in an overflow launder. The hydrophilic particles remain unattached and flow with the majority of the pulp to the underflow (tailings) stream. 


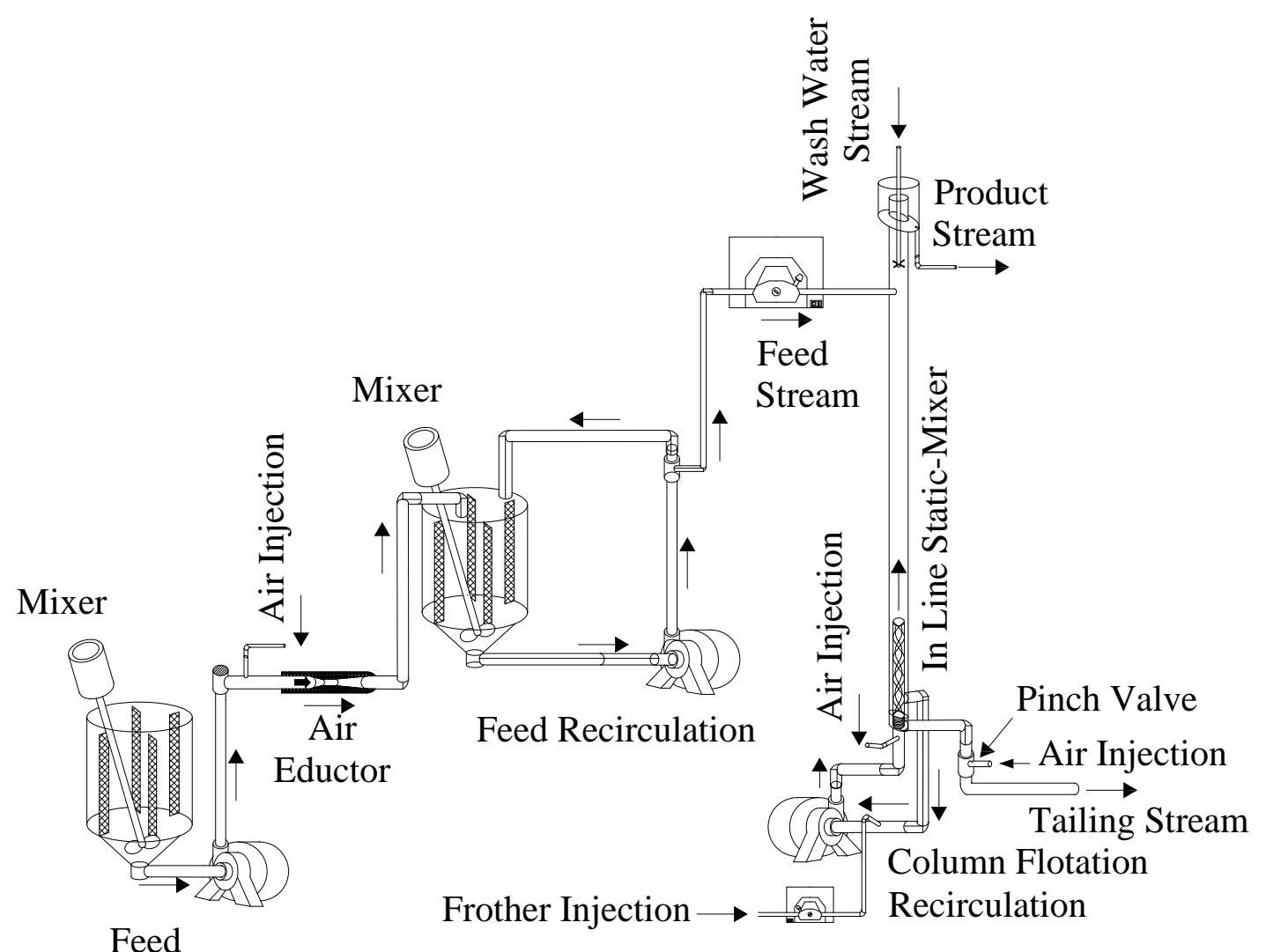

Figure 3. Schematic of the column flotation circuit with the air eductor device.

\section{In-Plant Conventional Flotation Tests}

A 10 liter pilot-scale conventional flotation bank (Figure 4) was used in the West Virginia processing facility to evaluate the performance improvement provided by the use of an air eductor to pretreat the flotation feed. The test was conducted on the fullscale conventional bank currently operating in the West Virginia facility over a period of about 19 hours. A total of 11 tests were performed; 6 air eductor under various air rates, 2 standard tests and 3 tests involving another collector. The frother type was maintained the same and the fuel oil addition was turned off during the period of time that the special collector was being added.

The plant feed during the first three test periods was comprised of Coalburg and Dorothy seam coals at a mass flow rate of $850 \mathrm{tph}$. The solids content in the flotation feed averaged $5.32 \%$ by weight while the mass flow rate to the full-scale flotation bank was determined to be around 67 tph based on a volumetric flow rate of 18,560 liter per minute. 


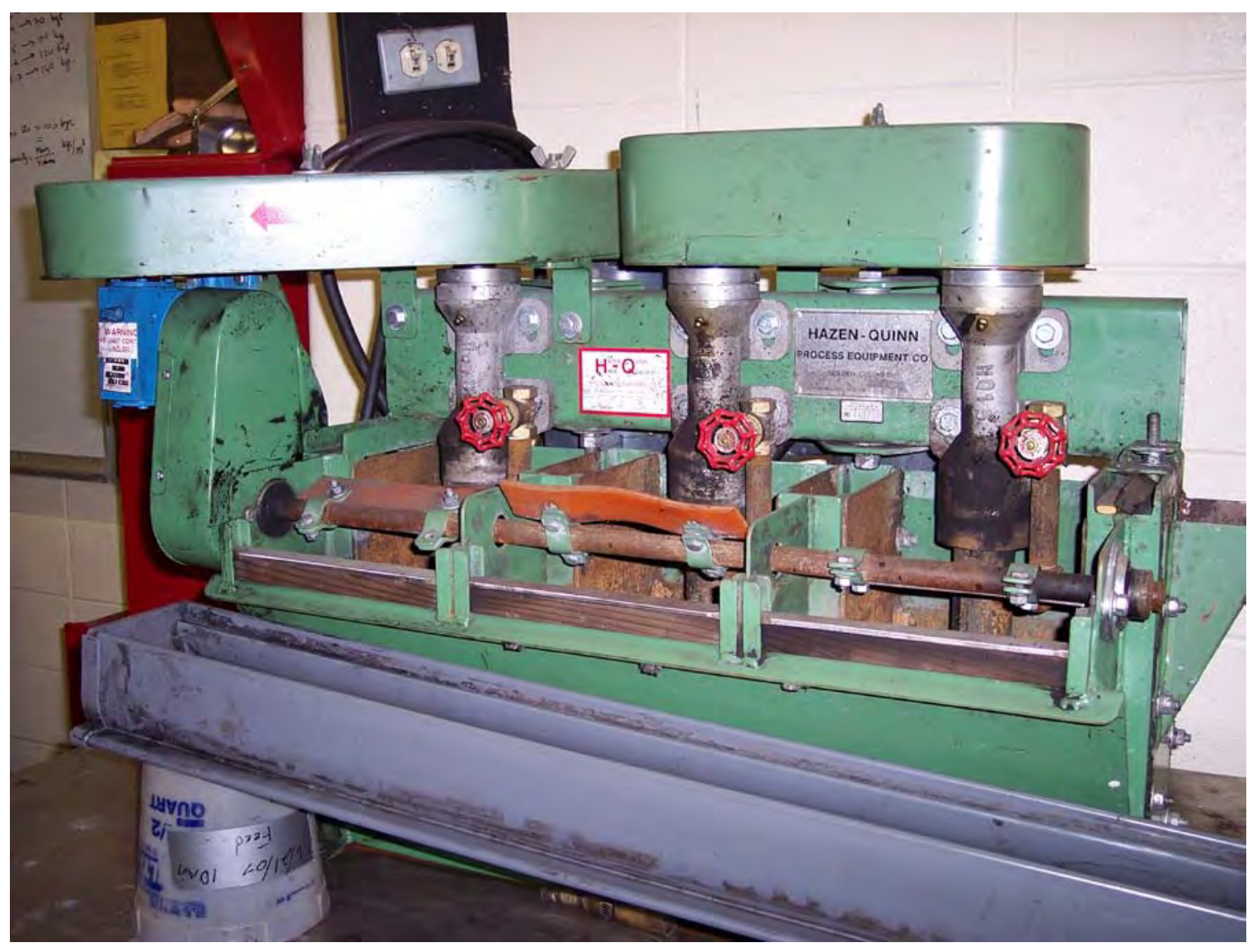

Figure 4. Conventional flotation bank cell used in plant with air eductor.

\section{Magnetic Plastic Evaluation}

\section{Sample Characterization}

As part of the magnetic plastic research, two different bituminous coal samples were independently used to evaluate the impact of selective detachment in a continuous column flotation by the addition of hydrophobic magnetic plastic material. These two samples were Pittsburgh No. 8 and Coalburg seam coal. The Pittsburgh No. 8 coal was very floatable and contained a relatively high amount of coal pyrite while the Coalburg coal had a relatively high content of middling (mixed-phase) particles in a range of $10 \%$ to $20 \%$.

Representative samples of Pittsburgh No.8 and Coalburg coal from operative coal preparation plants in Blacksville and Logan W.V. (USA), respectively, were collected. Both dry samples of minus $8 \mathrm{~cm}$ (3 inch) particle size were stored in drums. Upon arrival to the laboratory, each coal type was crushed with a jaw crusher to obtain $100 \%$ minus 
$1.27 \mathrm{~cm}(1 / 2$-inch) particle size. In a dry room, each coal type was spread out on a clean floor surface for 24 hours to remove excess of moisture and finally combined and quartered to obtain a homogenous representative coal sample that was used during the entire experimental program. In the mean time, the samples were stored into hermetic sealed drums to prevent contamination or prolonged exposure to air. Fresh and intact properties of the coal surface were always considered a high priority. Therefore, the sample was pulverized to minus $177 \mu \mathrm{m}$ ( 80 mesh) just prior to the flotation test.

Characterization of the coal samples was performed by taking representative samples from the storage drum to be prepared and pulverized at minus $177 \mu \mathrm{m}$ and then subjected to analytical analyses for ash content, volatile matter, fixed carbon, total sulfur and caloric value as shown in Table 3.

Table 3. General characterization of Pittsburgh No. 8 and Coalburg sample feed 100\% minus $177 \mu \mathrm{m}$.

\begin{tabular}{l|c|c}
\hline \multicolumn{1}{c|}{ Coal Characterization } & Pittsburgh No. 8 & Coalburg \\
\hline Ash Content (\%) & 16.3 & 36.12 \\
Total Sulfur (\%) & 3.07 & 0.68 \\
Volatile Matter (\%) & 34.11 & 23.21 \\
Calorific Value (Btu/lb) & 12,562 & 9,123 \\
Moisture (\%) & 0.66 & 2.40 \\
\hline
\end{tabular}

\section{Magnetic Plastic Material}

Eriez Equipment Company currently produces a plastic product that contains a significant amount of magnetite. The naturally hydrophobic plastic material is magnetic and thus recoverable by low intensity magnetic separators. The magnetic plastic material arrived as dry sample with a particle size of $100 \%$ minus $0.635 \mathrm{~cm}$ (1/4 inch). A representative sample was collected and ground to obtain material having a particle size less than $177 \mu \mathrm{m}$ which was used in the evaluation of the magnetic strength of the magnetic plastic material by using a Davis tube at full magnetic strength of 4000 Gauss. The results demonstrated that $96 \%$ of sample was reported to the product from a total sample of 28 grams, which indicates the magnetic strength of the plastic material.

In addition, a surface characterization of the magnetic plastic material was performed using a scan electrode image machine (S.E.M) at $500 \mu \mathrm{m}$ from a pulverized sample that was usually utilized during the entire program test. Figure 5 shows a granular, irregular and porous surface with an apparently narrow particle size distribution. It seems like most of the magnetite particles are well coated with the plastic resin after the grinding process. 


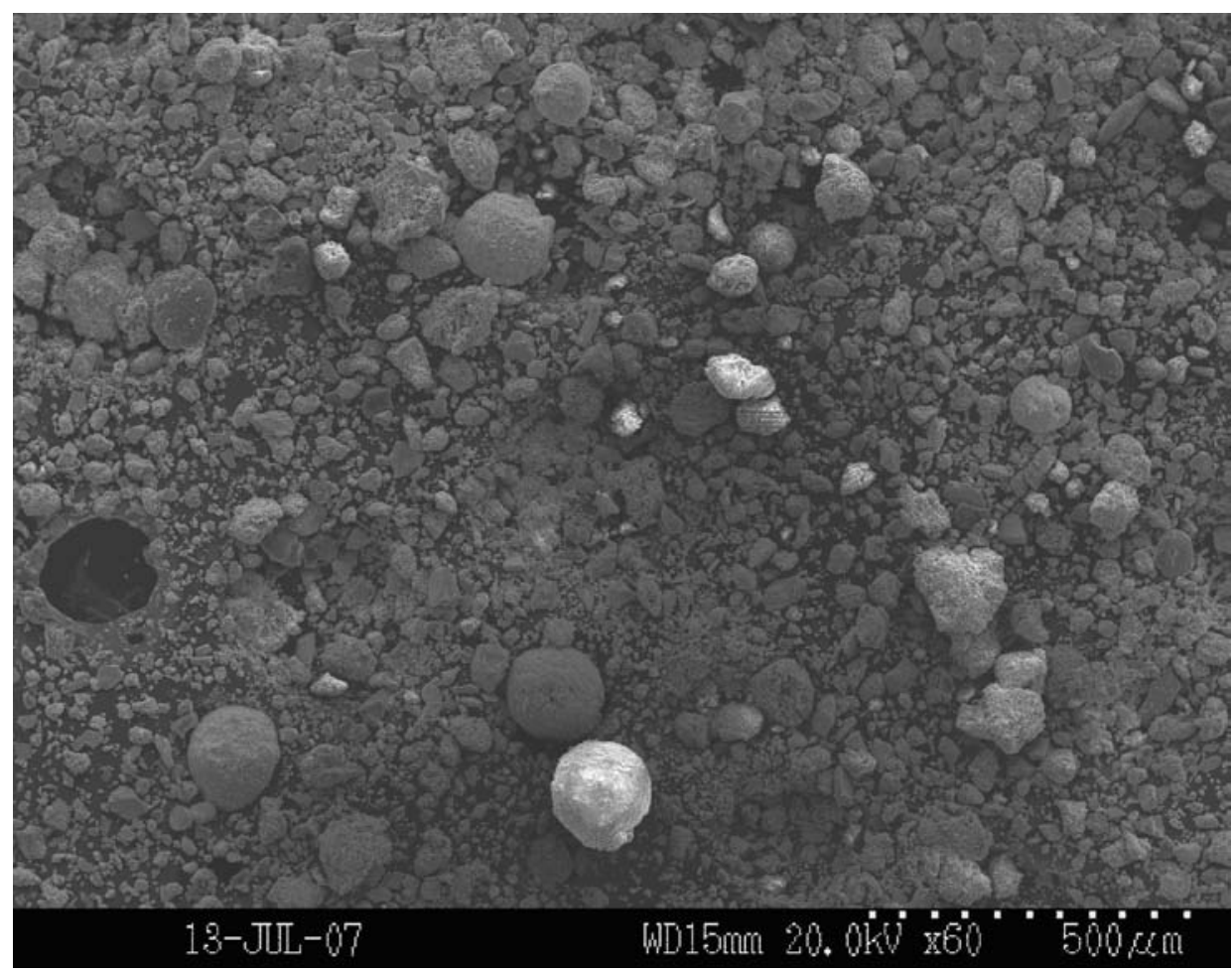

Figure 5. SEM picture of minus $177 \mu$ m particle size of magnetic plastic material at $500 \mu \mathrm{m}$ optical resolution.

An Eriez magnetic separator (Figure 6) was used to recover the magnetic plastic material from the coal slurry. The magnetic separator machine creates a magnetic field in the core of the apparatus where a chamber is located; the chamber is connected with two pieces of pipe line to let the slurry sample pass through the system by gravity action. When the combined slurry of coal and magnetic plastic material pass through a maximum magnetic field focus in the chamber, the magnetic plastic particles are attached by the magnetic gradient fields to the perforated metallic plates locate inside the chamber while the nonmagnetic particles follow water stream path through the chamber. Water is then flushed into the system to assure that all particles have passed through the chamber and only magnetic plastic were attached. Afterward, in another empty and clean container, the adhered magnetic plastic particles are recovered by turning off the magnetic field and flushing water into the chamber to capture the magnetic plastic. The separation process is repeated multiple times to obtain a complete separation. The samples were filtered, dried and weighed to assess magnetic recovery and general performances.

By experimental analysis, it was found that particle size has an important effect on magnetic plastic recovery with an optimum range being minus $177 \mu \mathrm{m}$ particle size material. Lower or higher particle size distributions than the optimum range considerably decrease the magnetic plastic recovery. Thus, lower particles size distribution follows stream water lines while magnetic plastic with higher particles size distribution do not 
attach in the magnetic chamber. Also experimental observation indicates that three passages through the separator are required to ensure maximum recovery of the plastic material. In the first separation process, the magnetic separator was able to recover $87.6 \%$ of the total magnetic sample. A second repetition enhanced recovery to $96.1 \%$ and a final stage resulted in $99.95 \%$ of the magnetic material being recovered.

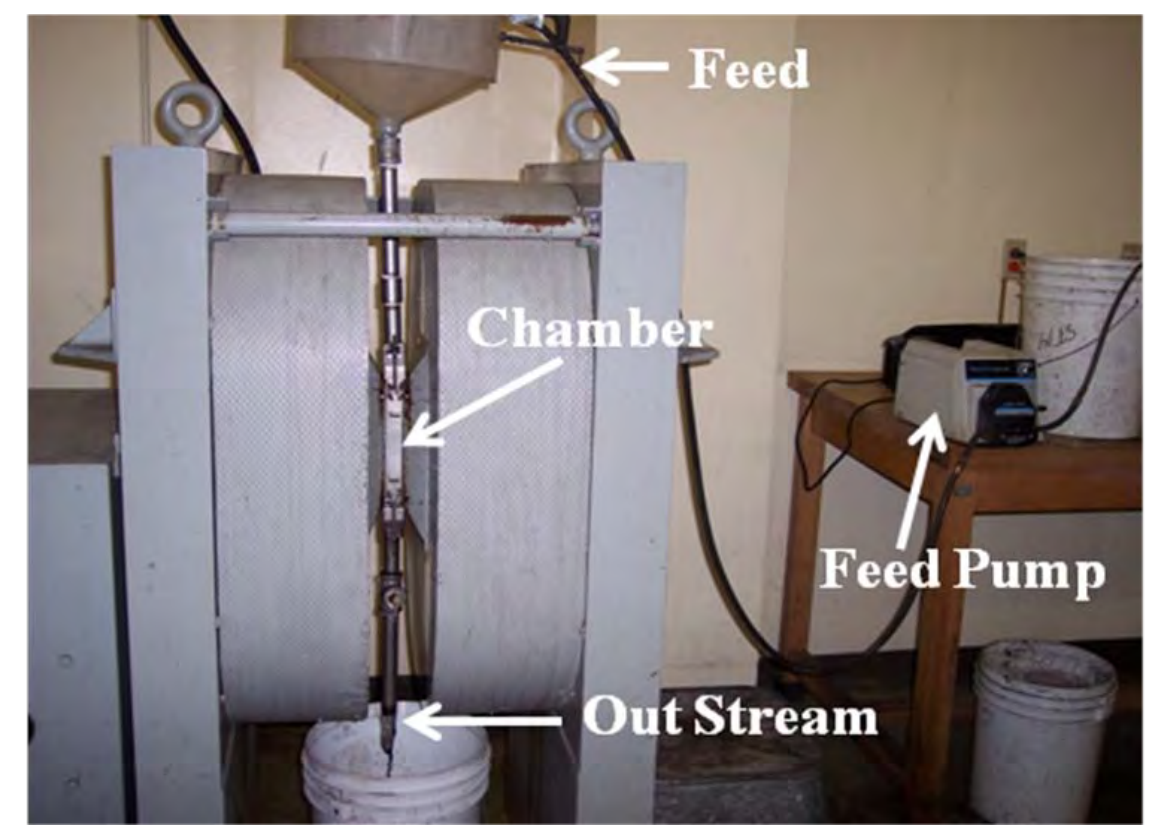

Figure 6. Eriez magnetic plastic separator showing the chamber and machine set up.

\section{Contact Angle Analysis}

The sessile drop method was used to measure the contact angle for the coal and plastic material. Measurements were conducted using a Rame-Hart contact angle goniometer Model 100-00. The model consists of a goniometer with two rotatable cross hairs for measuring contact angle, a specimen stage and a variable intensity illuminator. All the instruments were mounted on an optical bench. The set up for measurement of contact angle is shown in Figure 7. 


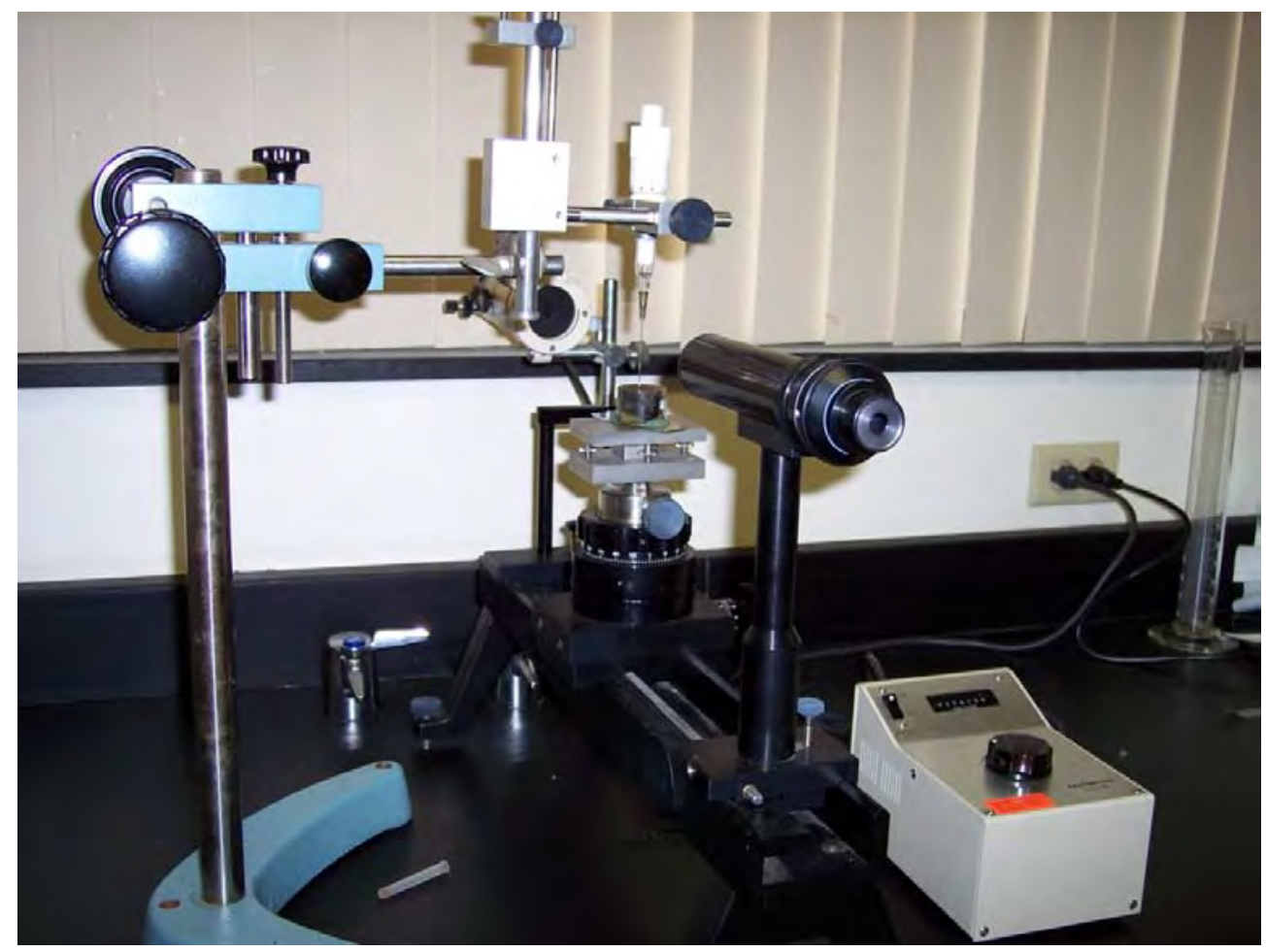

Figure 7. Goniometer used in the determination of contact angles.

The samples of approximately $1 \mathrm{~cm} \times 1.0 \mathrm{~cm}$ x $0.5 \mathrm{~cm}$ size were mounted on an epoxy resin. The mounted specimen was then polished on a Buehler polishing machine using 60, 240, 400 and 600 grit silicon carbide papers. Final polishing was done on a microcloth using an aqueous suspension of 0.05 microns Micropolish Alumina B. As polishing can have a drastic effect on the value of the contact angle, the polishing procedure was kept constant for all measurements. After polishing, the specimen was washed with distilled water and submerged in a microwave bath for a couple of minutes to remove any adhering alumina.

The contact angles of coal and magnetic plastic were measured with distilled water and Methylene Iodide at neutral $\mathrm{pH}$. The equilibrium contact angle on both sides of the water droplet was measured in three different positions. The standard deviation in measuring a single contact angle was $\pm 3^{\circ}$. All measurements were conducted at $25^{\circ} \pm 2^{\circ} \mathrm{C}$. Results in Table 4 clearly indicates that the magnetic plastic surfaces are much more hydrophobic than the surfaces of the Pittsburgh No. 8 coal. 
Table 4. Contact angle measurements with distilled water and methylene iodide.

\begin{tabular}{|c|c|c|c|c|}
\hline \multirow{2}{*}{$\begin{array}{c}\text { Contact Angle } \\
\text { (Degrees) }\end{array}$} & \multicolumn{2}{|c|}{ Distilled Water } & \multicolumn{2}{|c|}{ Methylene Iodide } \\
\cline { 2 - 5 } & Left & Right & Left & Right \\
\hline Mag. Plastic & 81.7 & 84.4 & 61.6 & 62.2 \\
Pittsburgh No. 8 & 54.6 & 55.3 & 41.8 & 42.6 \\
\hline
\end{tabular}

\section{Zeta Potential Measurements}

The surface charge of the Pittsburgh No. 8 coal and magnetic plastic material were studied using a Brookhaven Zeta Plus system (Figure 8). The procedure consisted in prepare enough solution with double distilled water with potassium chloride at $10^{-3} \mathrm{M}$ concentration (4 lt.) for the entire set of experiments. Also, 2 liters of solutions of hydrochloric acid (HCL 25\% concentration) at $3.25 \mathrm{M}$ concentration and sodium hydroxide $(\mathrm{NaOH})$ at $1.73 \mathrm{M}$ concentration were prepared to change the $\mathrm{pH}$ of the potassium chloride solution.

The coal samples and magnetic plastic material were pulverized and wet screened to a particle size below 25 $\mu$ m (500 mesh). For each solid type, six beakers were prepared by the addition of 0.1 grams of solid into a $50 \mathrm{ml}$ potassium chloride solution at $10^{-3} \mathrm{M}$ concentration and agitated until solid dispersion was ensured. Next, the $\mathrm{pH}$ was adjusted by the addition of hydrochloric acid or sodium hydroxide to obtain $\mathrm{pH}$ values within the range of 2 to 12 . The samples were left to reach an equilibrium $\mathrm{pH}$ for approximately 24 hours. 


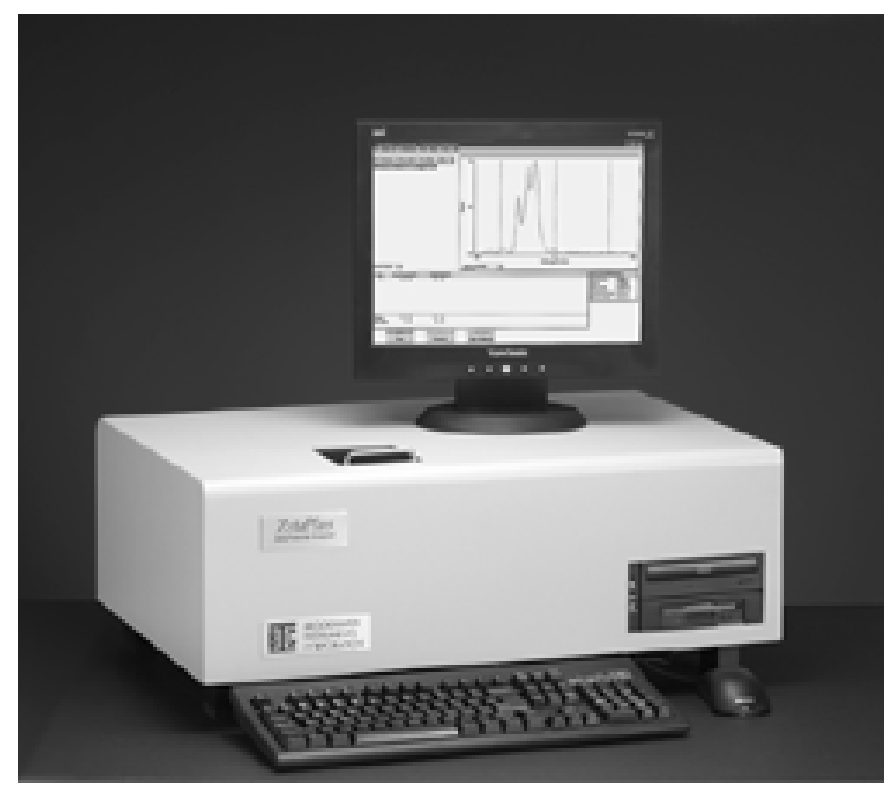

Figure 8. Brookhaven zeta potential analyzer.

Later, representative sample solution of $1.5 \mathrm{ml}$ were taken from each beaker and analyzed by using the Brookhaven ZetaPlus system. Ten (10) measurements were obtained and a final average value determined. The effect of slurry $\mathrm{pH}$ on zeta potential of the coal samples and the magnetic plastic particles is shown in Figure 9. For $\mathrm{pH}$ values greater than about 3, the surface charge is negative for all three solids and the magnitude is significant at $\mathrm{pH}$ values greater than 6 .

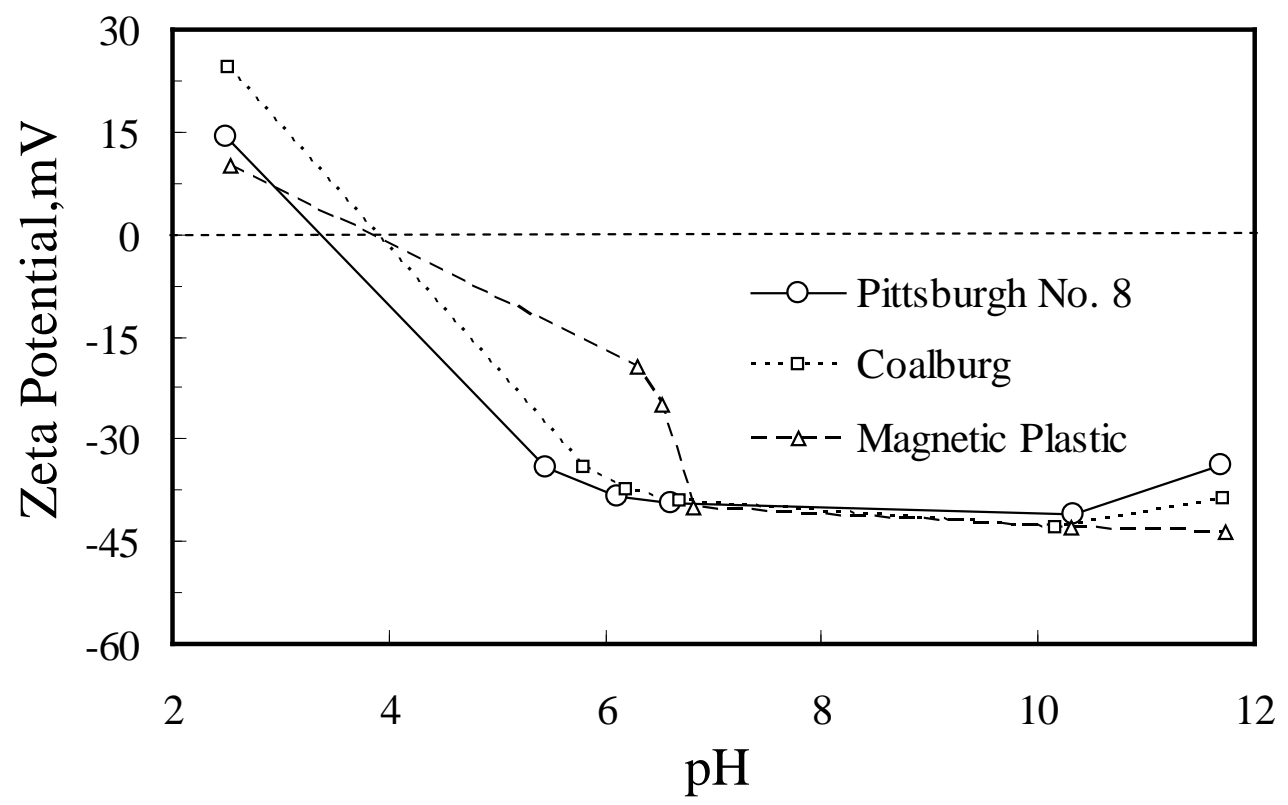

Figure 9. Zeta potential as a function of $\mathrm{pH}$ for the coal and magnetic plastic material. 


\section{Density Fraction Test}

The density fraction test was used to develop a washability curve to estimate the theoretical maximum mass yield and recovery expected while achieving a given product grade for a certain coal. The separation performances predict the theoretical performance, which means the recovery achieved while producing a given product quality can not be greater than the recovery achieved by density fractionation.

The density fraction tests were performed on the Pittsburgh No. 8 and Coalburg sample for particles smaller than $177 \mu \mathrm{m}$. LMT (Lithium Meta-Tungstate) was used as the heavy liquid to increase the density of the solution (specific density of $2.95 \mathrm{RD}$ ) with distilled water. The required medium density (i.e. 1.3, 1.4, 1.5, 1.6, 1.75 and 1.9 RD) was achieved by mixing LMT and distilled water in different beakers. The procedure followed the ASTM D4371 standard. The coal was submerged in the lowest density medium first. After allowing sufficient time for particle separation in the medium, the floated material was removed using a hand held screen. The material that sank to the bottom was recovered by filtration. The sink material was then submerged in the medium with the next highest density. This process was repeated through all of the mediums having a density range from $1.3 \mathrm{RD}$ to $1.9 \mathrm{RD}$. The float products and final tailings were rinsed, filtered, dried and weighed. Each sample was analyzed for ash content using the ASTM D3172 standard procedure.

\section{Flotation Rate Test}

As part of the coal characterization, flotation rate measurements were performed for the Pittsburgh No.8 and Coalburg coal samples with and without the addition of magnetic plastic material. Flotation rate tests were performed in the same manner as described in Section 3.1.3. However, the amount of magnetic plastic material added in the test $(2 \%$, $5 \%$ or $10 \%$ by weight) was subtracted from the total of 215 grams solid to obtain the net amount of coal remaining to be utilized which maintained the solid concentration at 5\% by weight. After collecting the samples, coal was separated from the magnetic plastic material using the Eriez high gradient magnetic separator (Figure 6). After removing the plastic material, the floated coal fractions and final tailings were filtered, dried and weighed and subsequently analyzed for ash content.

\section{Column Flotation Test}

To assess the impact of adding the magnetic plastic material on separation performance, experiments were conducted in a laboratory flotation column. The tests were performed on both the Pittsburgh No. 8 and Coalburg coal samples using the specific parameter settings in Table 5. The ShurFlot 948 was used as the polyglycol frother. 
Table 5. Specific parameter values used for the magnetic plastic addition effects on column flotation performance.

Feed rate

Feed solids concentration by weight

\% of Plastic Added Relative to Feed

Frother concentration (ShurFlot 948)

Collector concentration (Fuel oil No. 2)
$200 \mathrm{ml} / \mathrm{min}$ to $1400 \mathrm{ml} / \mathrm{min}$

$2 \%$ to $12 \%$

$5 \%-10 \%$

15 ppm

$0.45 \mathrm{~kg} / \mathrm{ton}$

Two sets of column flotation tests were performed, the first set established a baseline performance without the use of the magnetic plastic material. These baseline tests were immediately followed by a series of tests that studied the impact of adding various amounts of plastic particles into the froth phase or directly in the feed.

Magnetic plastic material was added into the column flotation in two different ways. The first method was by mixing the external material directly into the feed slurry. In the second method, the injection was in the froth phase. In this case, an auxiliary sump was set up to hold the external material to be pumped into the froth zone. A pump helped to maintain a homogeneous mixture of the plastic particles in water. After obtaining steady state condition in the column flotation, the magnetic plastic material was injected into the froth phase through the wash water as depicted in Figure 10. The injection rate was 100 $\mathrm{ml} / \mathrm{min}$ of magnetic plastic slurry at $5 \%$ or $10 \%$ by weight and $300 \mathrm{ml} / \mathrm{min}$ of water to maintain the same overall volume of wash water that was supplied in the baseline column tests.

\section{Froth Refluxing}

As previously discussed, a method that could induce selective detachment is enrichment of the froth zone by recycling a portion of the froth product back into the froth. The experimental program conducted to investigate the use of external froth refluxing utilized the same fine coal samples as those in the magnetic plastic experiments. A flotation column was used in the circuit shown in Figure 11. Using a Y-connector with a valve on the stream reporting to the final product, the amount of froth concentrate was controlled and material routed to a sump. A pump was used to recycle a controlled amount of the froth product back to the column froth through the wash water system. The rate of injection of slurry into the froth zone was $100 \mathrm{ml} / \mathrm{min}$ plus $300 \mathrm{ml} / \mathrm{min}$ of fresh water to keep the same volume of wash water that was used in the baseline tests. Also, $200 \mathrm{ml} / \mathrm{min}$ of fresh water was required in the launder to flush down the reported concentrate through a pipe line where the Y-tube pipe provides a split of the concentrate stream. The process was totally continuous including the refluxing of the froth product. A period equivalent to three residence times was allowed to reach steady-state prior to the collection of the feed, product and tailing samples. The samples were filtered, dried, weighed and analyzed for ash and total sulfur content. 


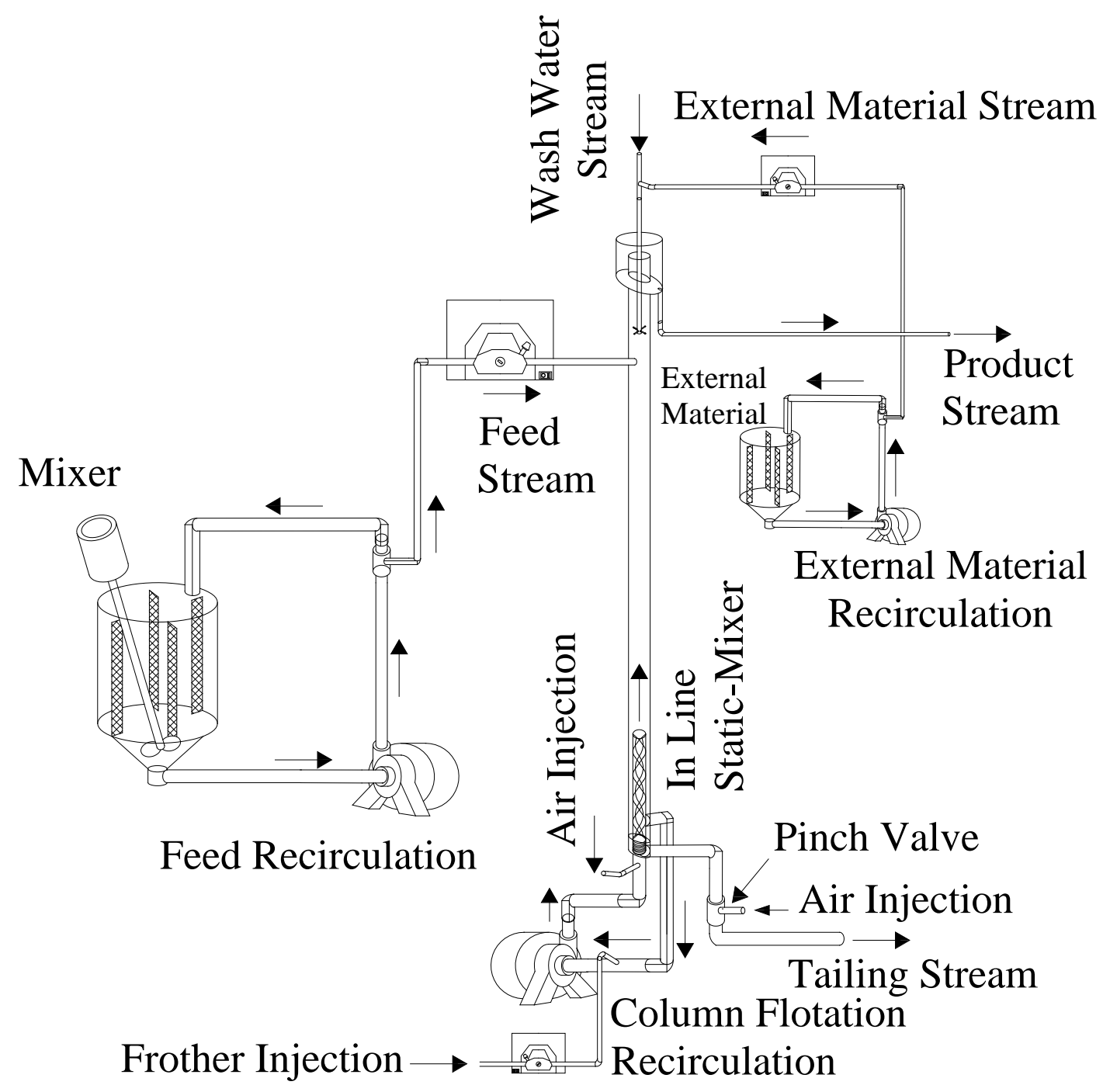

Figure 10. Column flotation circuit used to evaluate the performance benefits of the addition into the froth zone. 


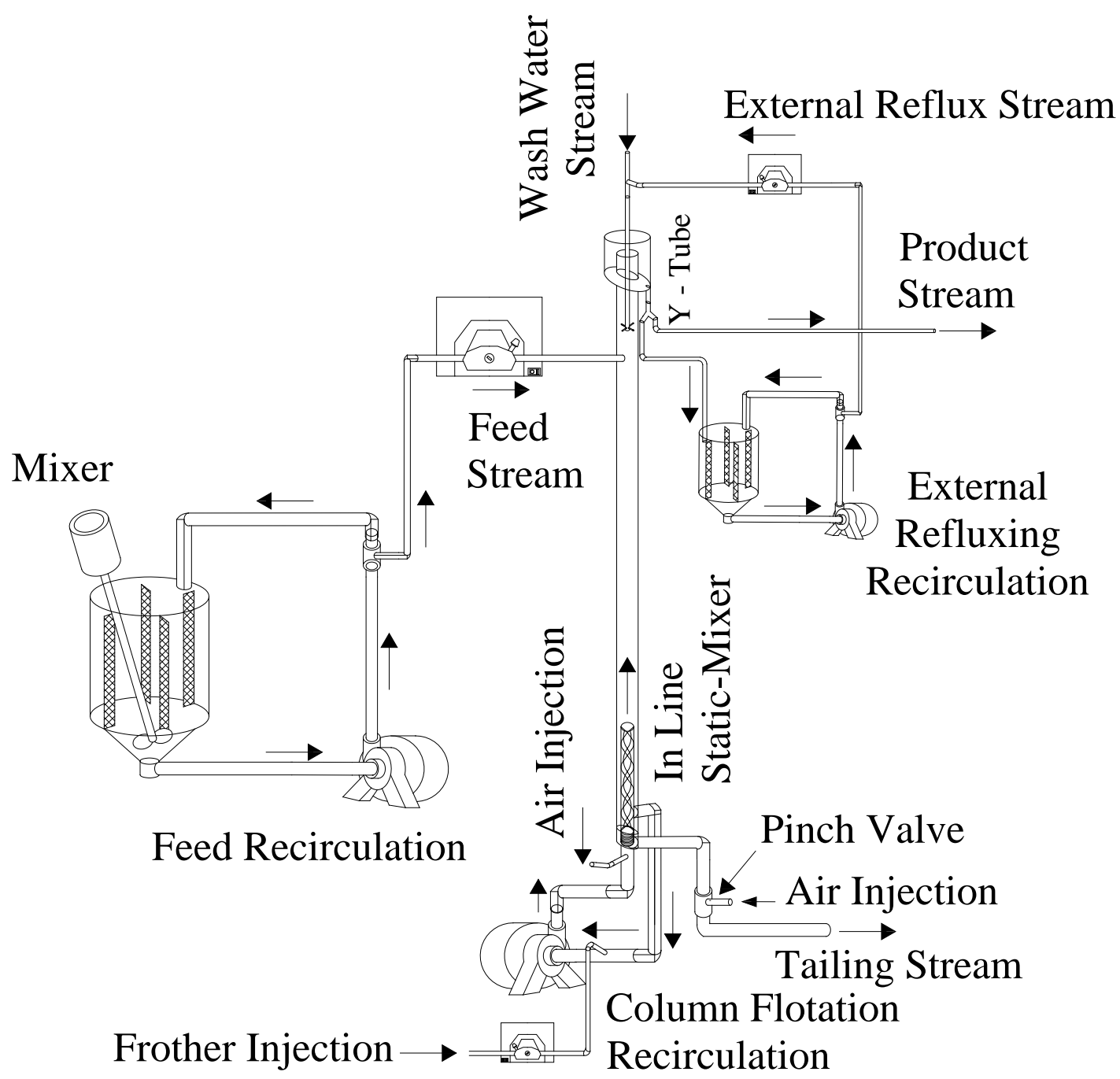

Figure 11. Column flotation used to study the impact of using external froth refluxing to improve separation. 


\section{RESULTS AND DISCUSSIONS}

\section{$\underline{\text { Air Eductor }}$}

Extensive evaluation of the Coalburg feed slurry shows that feed pretreatment through an air eductor has the potential to enhance flotation performance in a conventional cell. In the past, emphasis focused on developing new collectors for low floatability coal. However, a more practical technique that uses an air eductor mechanism has many potential benefits. Figure 12 summarizes the flotation performances achieved in a conventional flotation cell when treating Coalburg coal. After 60 seconds of flotation time, a combustible recovery of about $62 \%$ was achieved. When the feed was treated through a cavitation tube either one or recycled through several times, the recovery after 60 seconds was $80 \%$ which represents an increase of $18 \%$ absolute percentage points.

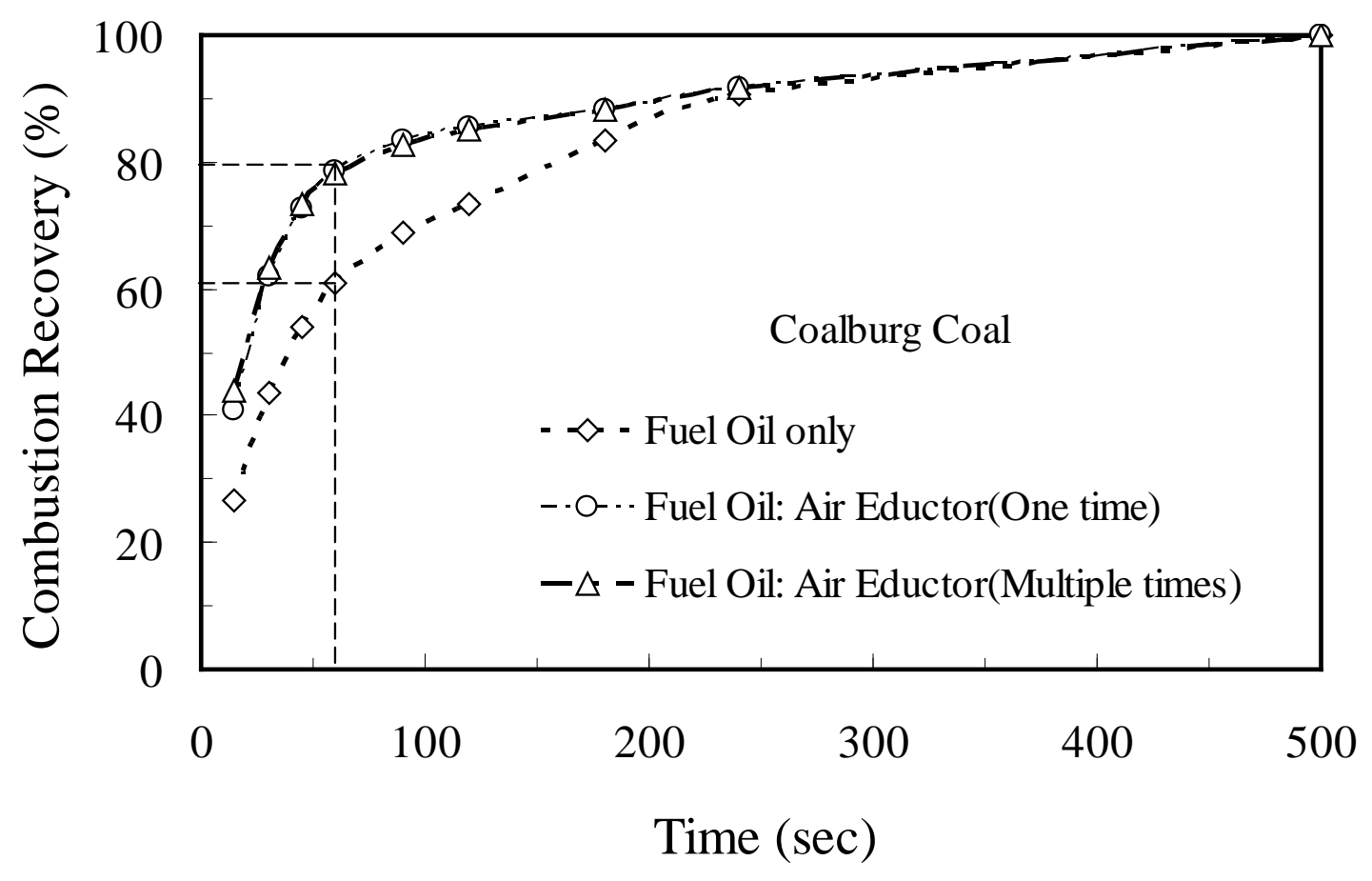

Figure 12. Effects of air eductor performance with the Coalburg seam coal sample in a laboratory conventional flotation cell.

Assuming perfect mixing and first-order kinetics conditions, the flotation rate constants were determined and tabulated for one minute residence time as presented in Table 6 . The flotation rate during the first minute of flotation nearly doubled as a result of using the air eductor. The mechanism for the improvement may be due to the nucleation of the micron-sized air bubbles onto the surface of the weakly hydrophobic coal surface. Evidence provided by Krasocoska and Malysa (2006) indicates that a surface that is 
coated with micron-sized bubbles results in a significant reduction in the induction time upon colliding with a conventional bubble size. As a result, the probability of attachment and thus flotation rate is increased. Similar results in flotation recovery improvement were reported by Attallo et al. (2000) and Zhou et al. (1997).

Table 6. Improvements in flotation rate and recovery using data obtained after one minute of flotation for the Coalburg coal.

\begin{tabular}{|l|c|c|}
\hline \multicolumn{1}{|c|}{$\begin{array}{c}\text { Test } \\
\text { Identification }\end{array}$} & $\begin{array}{c}\text { Flotation } \\
\text { Rate } \\
\left(\mathrm{min}^{-1}\right)\end{array}$ & $\begin{array}{c}\text { Flotation } \\
\text { Recovery } \\
(\%)\end{array}$ \\
\hline Fuel Oil only & 0.65 & 61.0 \\
Air Eductor (Single Pass) & 1.25 & 78.4 \\
Air Eductor (Multiple Pass) & 1.24 & 78.2 \\
\hline
\end{tabular}

Multiple passes of the feed slurry through the air eductor did not further improve the flotation constant rate as indicated in Table 6. However, Figure 13 shows the potential for improving selectivity by passing the slurry through the air eductor multiple times. The improvement is noted by the shift to the left of the recovery-product ash curve. The selectivity improvement may have occurred due to the selective removal of air bubbles from the more weakly hydrophobic particles that contain higher qualities of ash-forming minerals.

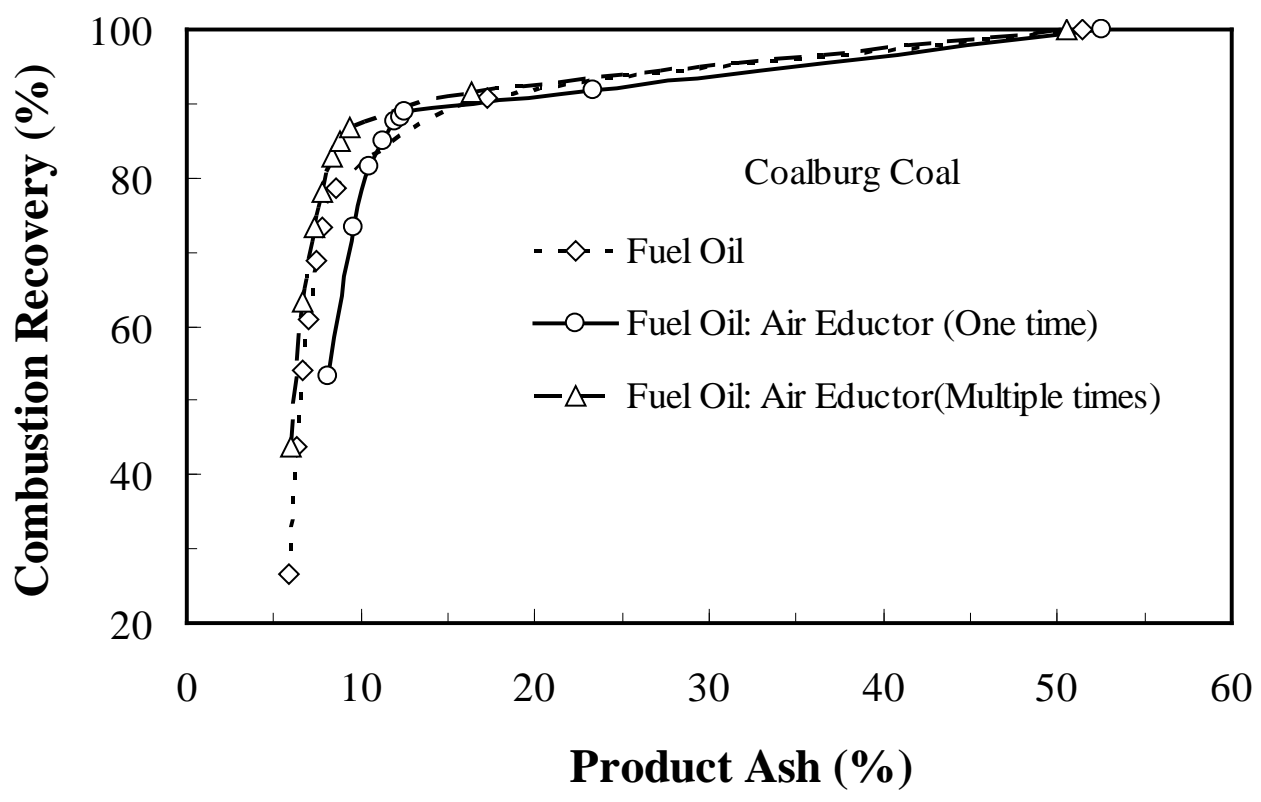

Figure 13. Effect on performance achieves with air eductor for a Coalburg coal sample obtained from flotation rate tests in a conventional flotation cell. 
Considering the notable positive effect observed in the laboratory conventional flotation cells, column flotation tests were performed to evaluate performance on a laboratory flotation column. As indicated in Figure 14, no appreciable difference was obtained from the flotation column tests at the low and high volumetric feed flow rates. However, when moderate volumetric flow rates, the cavitation process improved recovery and reduced the product ash content. A five absolute percentage point improvement in recovery was achieved when the volumetric feed flow rate was between 400 and $600 \mathrm{ml} / \mathrm{min}$.

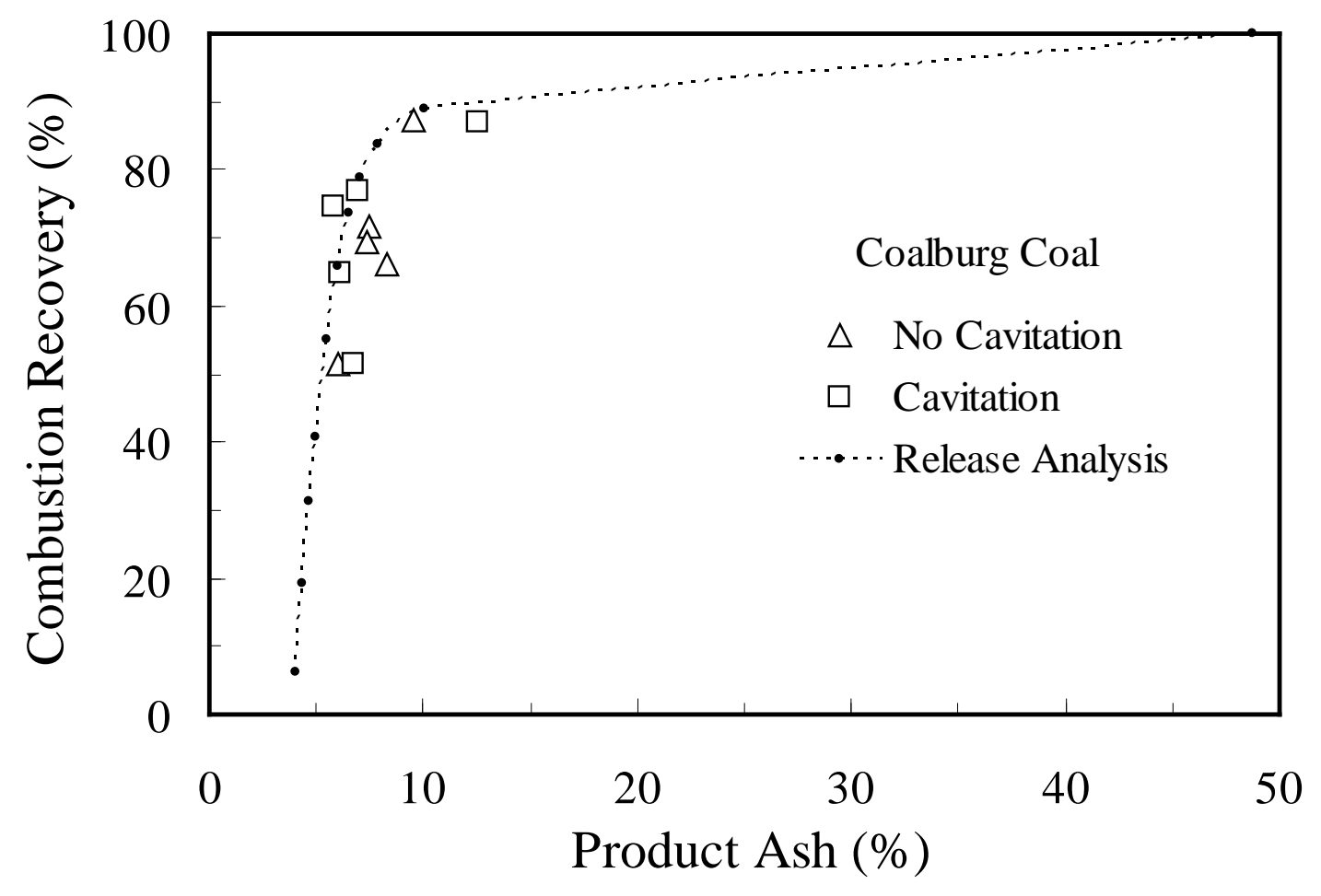

Figure 14. Effects on flotation performance with air eductor utilization for a Coalburg coal preparation plant sample.

In tests investigating the impact of the air eductor feed pretreatment, little-to-no gain in recovery was observed while varying flotation time in a column as shown in Figure 15. This may be due to the experimental set-up which processed the feed stream through a pump and sump after the cavitation tube and before the flotation column. 


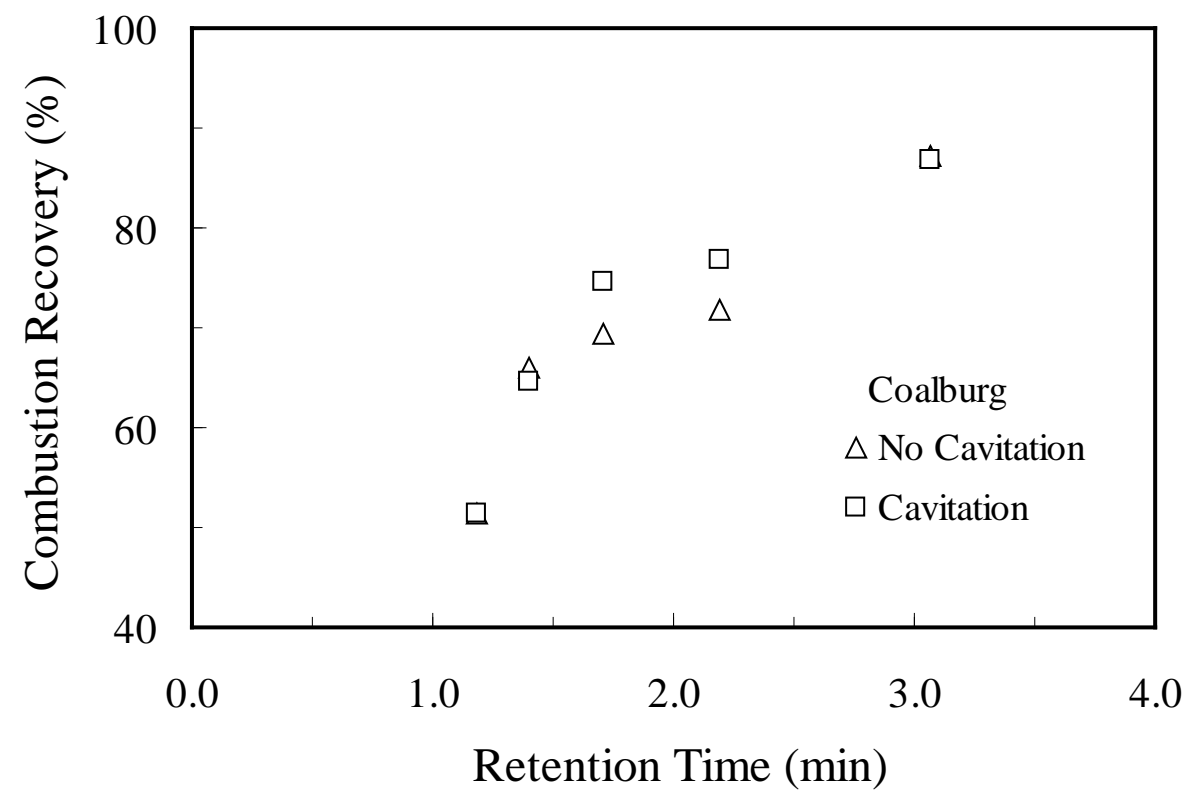

Figure 15. Effects of air eductor on flotation performance under different retention time conditions with a column flotation.

\section{$\underline{\text { Magnetic Plastic }}$}

\section{Conventional Flotation Tests}

The separation performance improvements resulting from the addition of magnetic plastic particles were initially evaluated in a laboratory conventional flotation cell. The plastic particles were mixed into the feed slurry at concentrations expressed as a percentage of the total solid mass. The flotation tests were performed as a function of time on two different coals samples and varying operating conditions.

The impact of magnetic plastic concentration on flotation performance was evaluated over at concentration values of $0 \%, 2 \%, 5 \%$ and $10 \%$ by weight. Figure 16 shows the recovery improvement achieved as a function of time and plastic particle concentration when treating Pittsburgh No. 8 coal. Increasing the plastic concentration from $2 \%$ to 5\% by weight enhanced the combustible recovery by about 15 absolute percentage points. Doubling the concentration to $10 \%$ by weight further increased recovery by another 5 absolute percentage points. Evidence presented in this chapter will point to the high degree of hydrophobicity of the plastic particles $\left(\theta_{\mathrm{w}}=84^{0}\right)$ as the reason for the recovery improvement. 


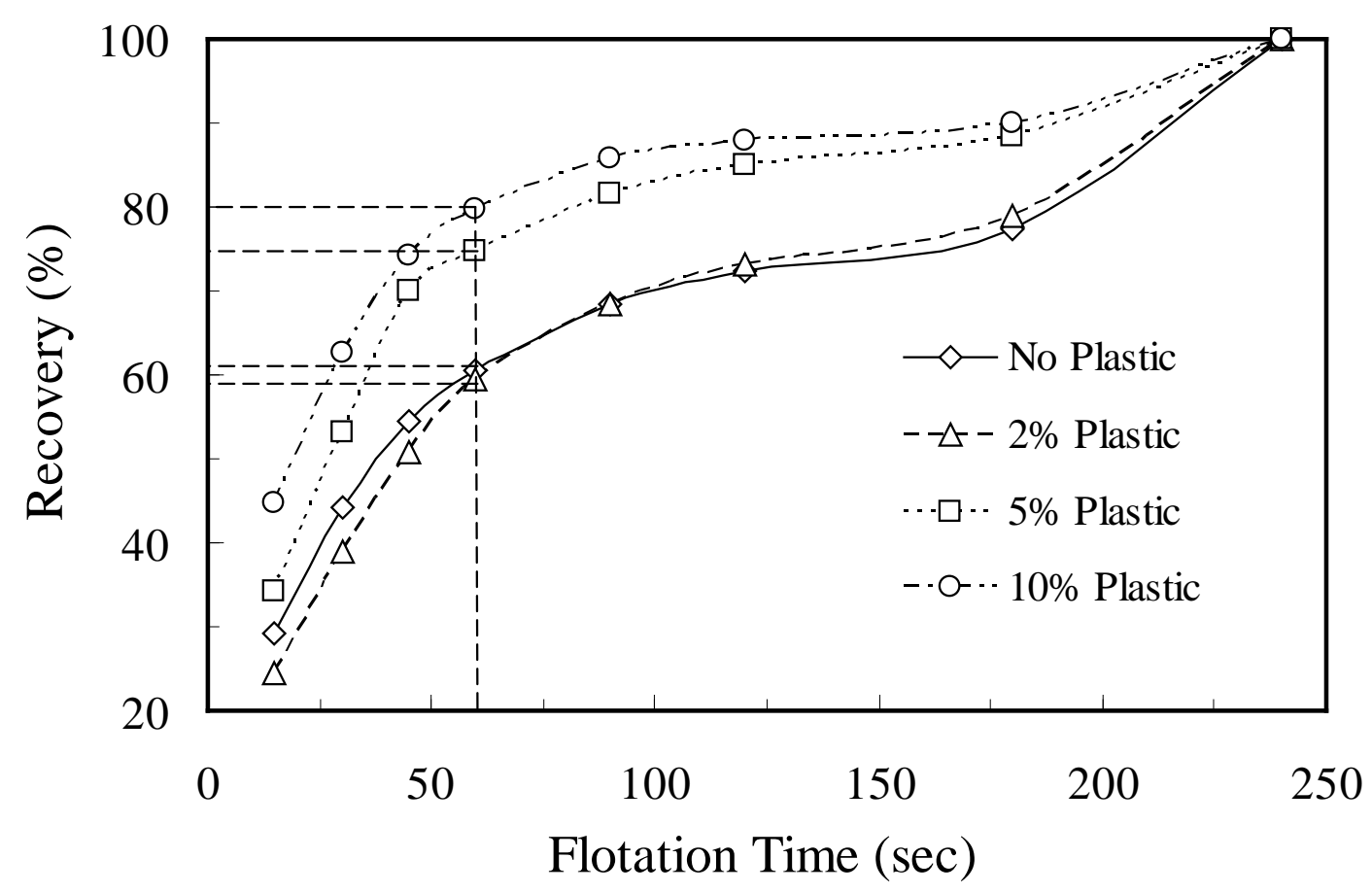

Figure 16. Recovery improvements achieved as a function of flotation time and varying amounts of plastic particles when treating Pittsburgh No. 8 coal in a conventional

flotation cell.

The flotation rates were determined from the data presented in Figure 16. The natural flotation rate of the Pittsburgh No. 8 coal was found to be $0.58 \mathrm{~min}^{-1}$. By adding the plastic particles at a $5 \%$ by weight concentration, the flotation rate was increased to 0.93 $\mathrm{min}^{-1}$, which equates to a $75 \%$ enhancement.

The absolute percentage improvement in recovery as a function of plastic particle concentration is more clearly shown in Figure 17. It is evident that the $10 \%$ plastic concentration has a significant impact on recovery with a 15 absolute percentage point gain after just 15 second of flotation. The maximum recovery improvement occurs after 45 seconds of flotation where a 20 absolute percentage point gain is realized. 


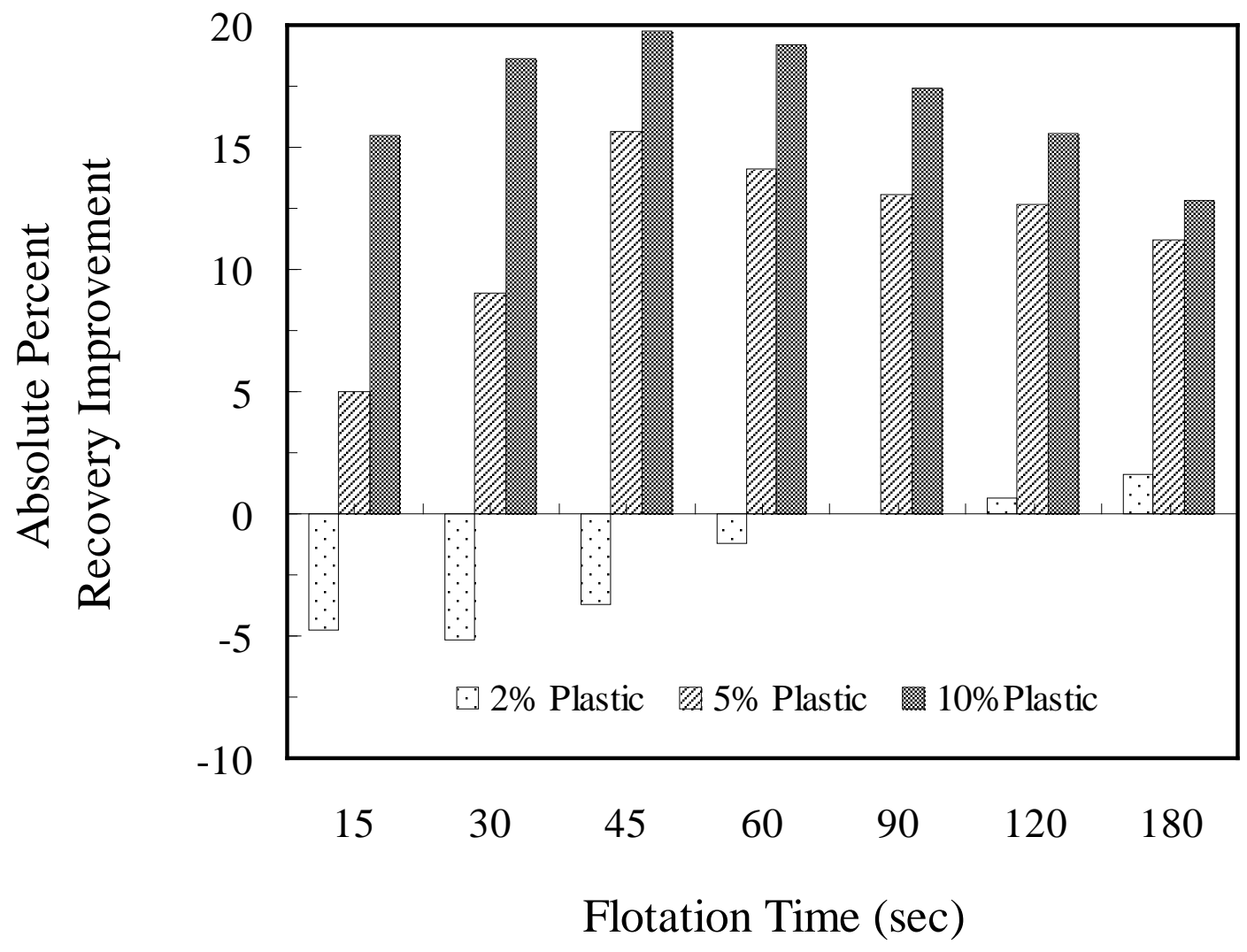

Figure 17. Absolute percent recovery improvement as a function of magnetic plastic concentration in a conventional flotation cell; Pittsburgh No. 8 coal.

The initial tests reveal that no improvement in selectivity was realized when adding the plastic particles. As shown in Figure 18, the ash contents in the froth concentrates were increased with the $5 \%$ and $10 \%$ additions. The finding indicates that the plastic particles may be preferentially enhancing the floatability of the higher ash (middles) particles or reducing drainage of the water containing entrained clay particles surrounding the air bubbles. Also, there is a correlation between the amount of magnetic plastic addition and enhancement in flotation performance as it is described in Table 7. 


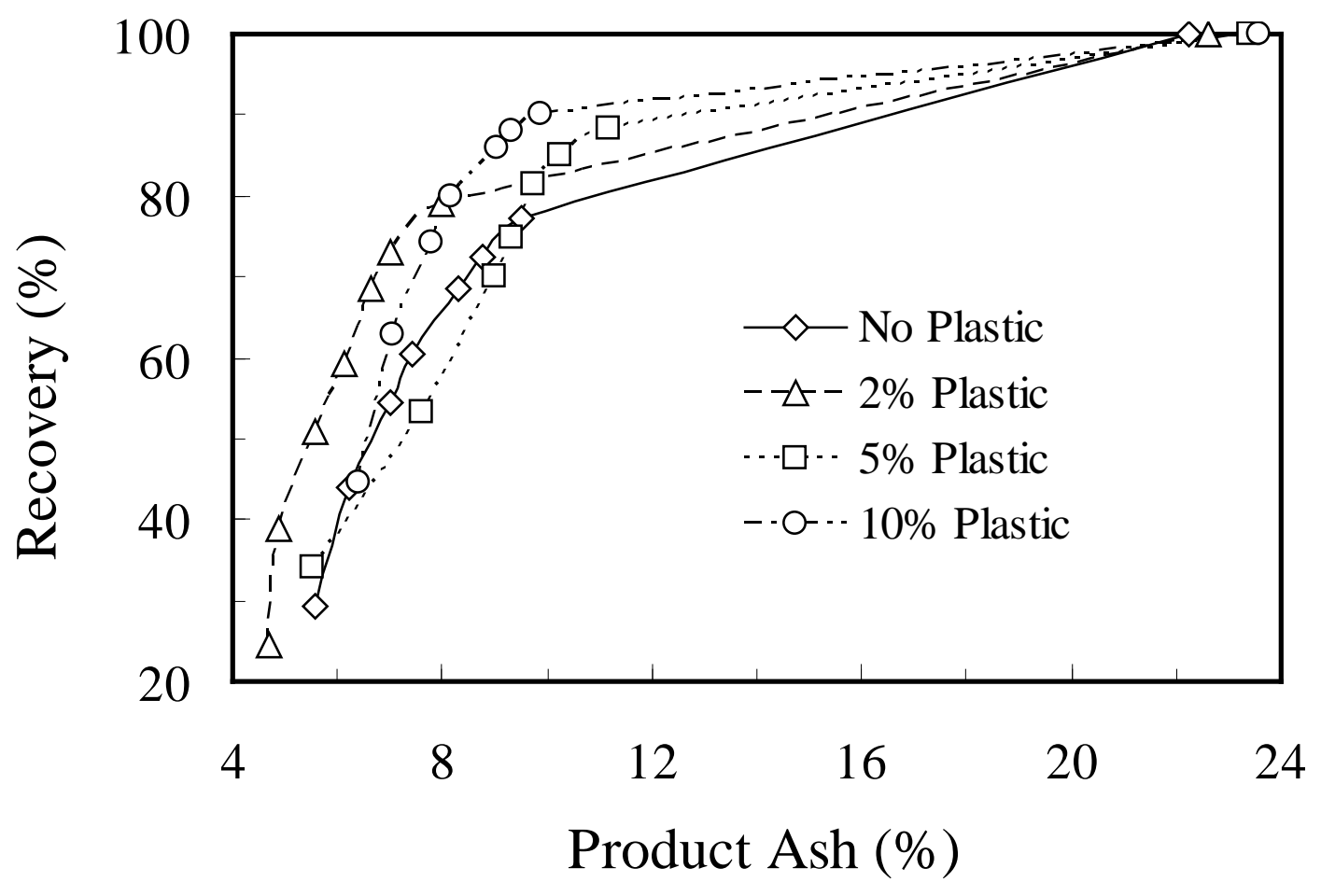

Figure 18. Selectivity performances for varying amounts of magnetic plastic material in a conventional flotation cell treating Pittsburgh No. 8 coal.

Table 7. Flotation performance with addition of $2 \%, 5 \%$ and $10 \%$ magnetic plastic material after one minute of flotation in a conventional cell.

\begin{tabular}{|l|c|c|}
\hline \multicolumn{1}{|c|}{$\begin{array}{c}\text { Test } \\
\text { Identification }\end{array}$} & $\begin{array}{c}\text { Flotation } \\
\text { Rate } \\
\left(\mathrm{min}^{-1}\right)\end{array}$ & $\begin{array}{c}\text { Flotation } \\
\text { Recovery } \\
(\%)\end{array}$ \\
\hline 0\% Plastic Material & 0.58 & 60.6 \\
\hline 2\% Plastic Material & 0.60 & 59.3 \\
\hline 5\% Plastic Material & 0.93 & 74.7 \\
\hline 10\% Plastic Material & 0.95 & 79.8 \\
\hline
\end{tabular}

The recovery of the magnetic particles in the flotation process was also measured as a function of flotation time. The majority of the plastic particles are recovered within the first 15 seconds of flotation and nearly 100\% are recovered in the first minute of flotation (Figure 19). This finding indicates that any benefit in the flotation of coal should occur within a retention time of one minute. 


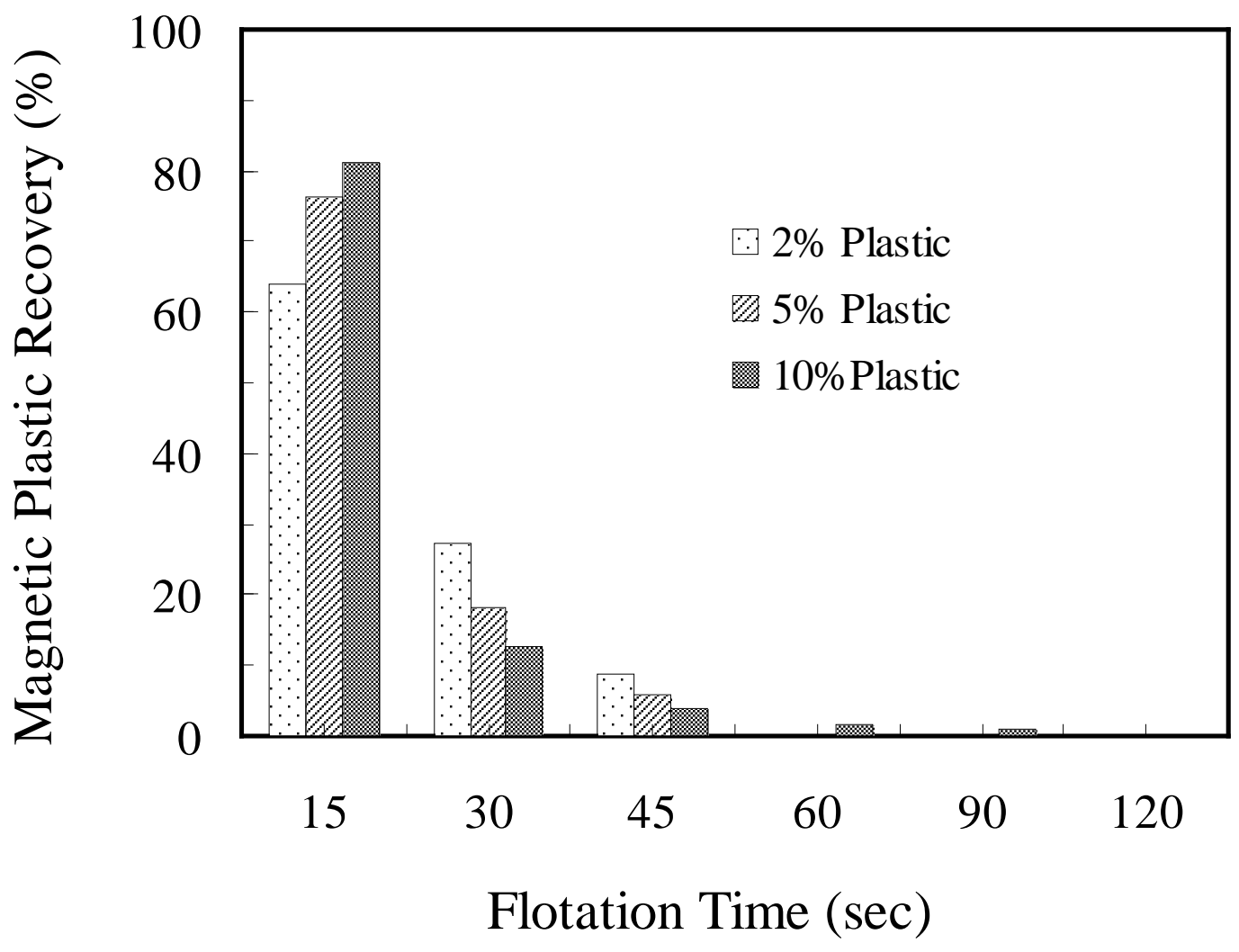

Figure 19. Recovery of magnetic plastic material from flotation kinetic rate test at different magnetic plastic concentration (2\%, 5\% and 10\%).

\section{Repetitive Performance Evaluation}

After the favorable results were obtained, the next evaluation assessed the repeatability of the improved performances at plastic concentrations of $0 \%$ and $10 \%$. The tests were performed under the same conditions five times to obtain statistical evidence of the phenomena. The average results from the analysis are shown in Figure 20. The same positive impact with the use of magnetic plastic material was observed with an improvement of 11 absolute percent point in recovery and almost a 30\% rise in the flotation rate constant as show in Table 8. A small loss in overall recovery was observed but significant improvement in flotation performance was obtained. 


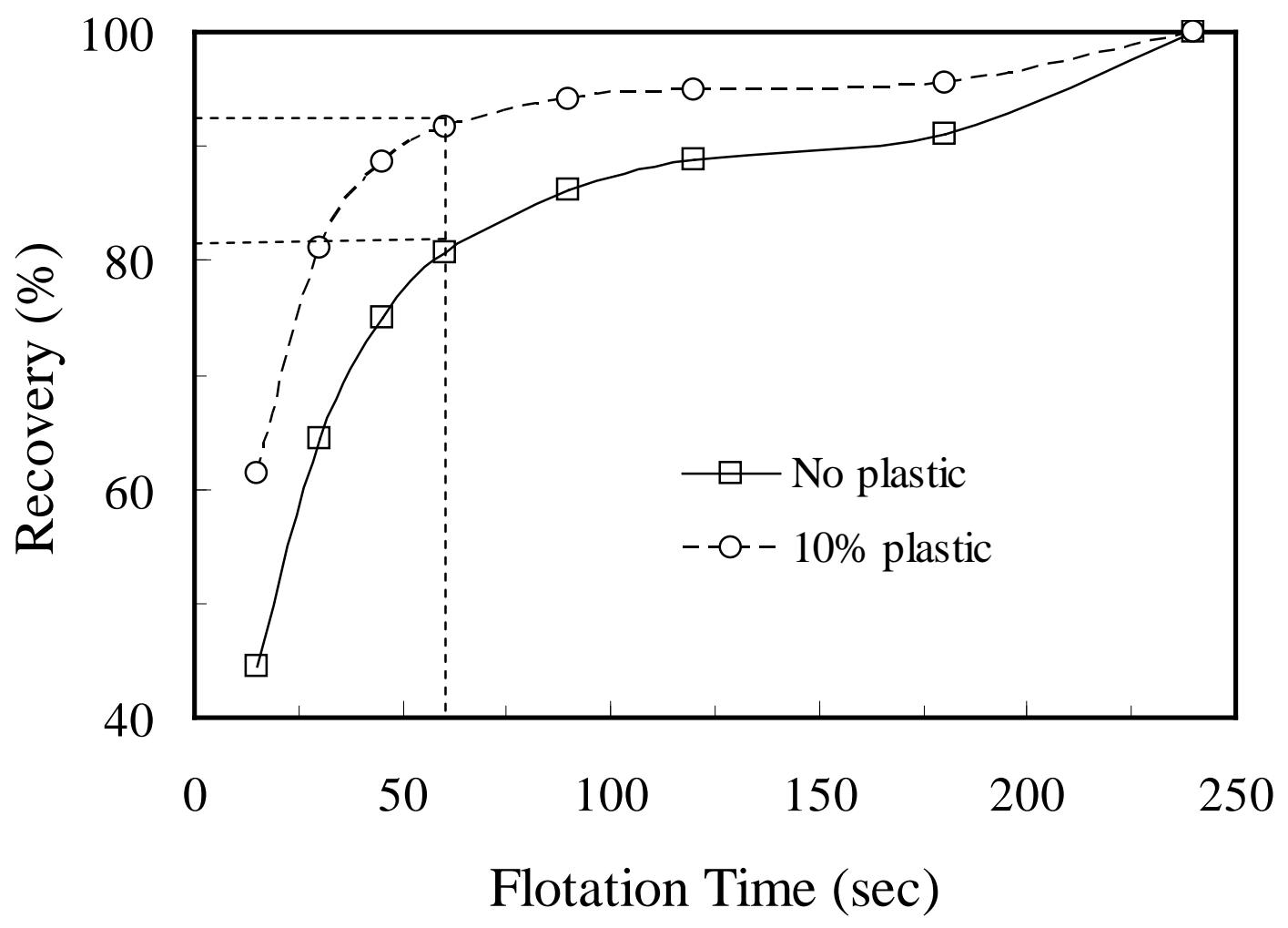

Figure 20. Average recovery improvements achieved after repeating the flotation test under the same conditions with $10 \%$ magnetic plastic and no magnetic plastic for a total of five times; Pittsburgh No. 8 coal.

The recovery data achieved within the first minute of flotation were used to determine the flotation rate. From the average recovery data collected from five test repetitions, the flotation rate of the Pittsburgh No. 8 coal was increased from $1.04 \mathrm{~min}^{-1}$ to $1.31 \mathrm{~min}^{-1}$ by the addition of the magnetic plastic particles at a concentration of $10 \%$ by weight as shown in Table 8 . The rate was increased by $26 \%$.

Table 8. Flotation performances with five times repetition at $0 \%$ and $10 \%$ magnetic plastic material using a residence time of one minute.

\begin{tabular}{|l|c|c|}
\hline \multicolumn{1}{|c|}{$\begin{array}{c}\text { Test } \\
\text { Identification }\end{array}$} & $\begin{array}{c}\text { Flotation } \\
\text { Rate } \\
\left(\mathrm{min}^{-1}\right)\end{array}$ & $\begin{array}{c}\text { Flotation } \\
\text { Recovery } \\
(\%)\end{array}$ \\
\hline No Plastic & 1.04 & 80.7 \\
10\% Plastic & 1.31 & 91.7 \\
\hline
\end{tabular}

Figure 21 addresses the impact on selectivity realized with the addition of $10 \%$ magnetic plastic material compared to the baseline achieved without the addition of the magnetic plastic particles. As typical, higher recovery values are associated with an increase in ash 
content. The results represent the average data obtained from five experimental test repetitions with $0 \%$ and 10\% magnetic plastic material, when treating Pittsburgh No. 8 coal.

The steep portion of the curve was obtained within the first 45 seconds of flotation which is the time period that most of the plastic particles report to the froth concentrates. As shown in Figure 20, this is also the same period of time in which the largest recovery gain is achieved which also leads to elevated product ash content (Figure 21). Considering the individual incremental ash in both cases for each sample at the same period of time, higher incremental ash content is observed with the use of magnetic plastic material. Thus, the sloped curve in the first 45 second of flotation without adding magnetic plastic material is $31 \%(\Delta H / \Delta L)$ against $21 \%\left(\Delta H^{\prime} / \Delta L^{\prime}\right)$ with addition of magnetic plastic material. However, the lower slope curve is corresponds to the higher recovery values observed within the first 15 seconds of flotation when using the magnetic plastic material. The first 45 seconds of regular flotation without magnetic plastic material has the same effect as 30 seconds of flotation with the magnetic plastic material. The majority of the impact is concentrated within the first 15 seconds of flotation. That is the same period during which the majority of the plastic particles report to the flotation concentrates.

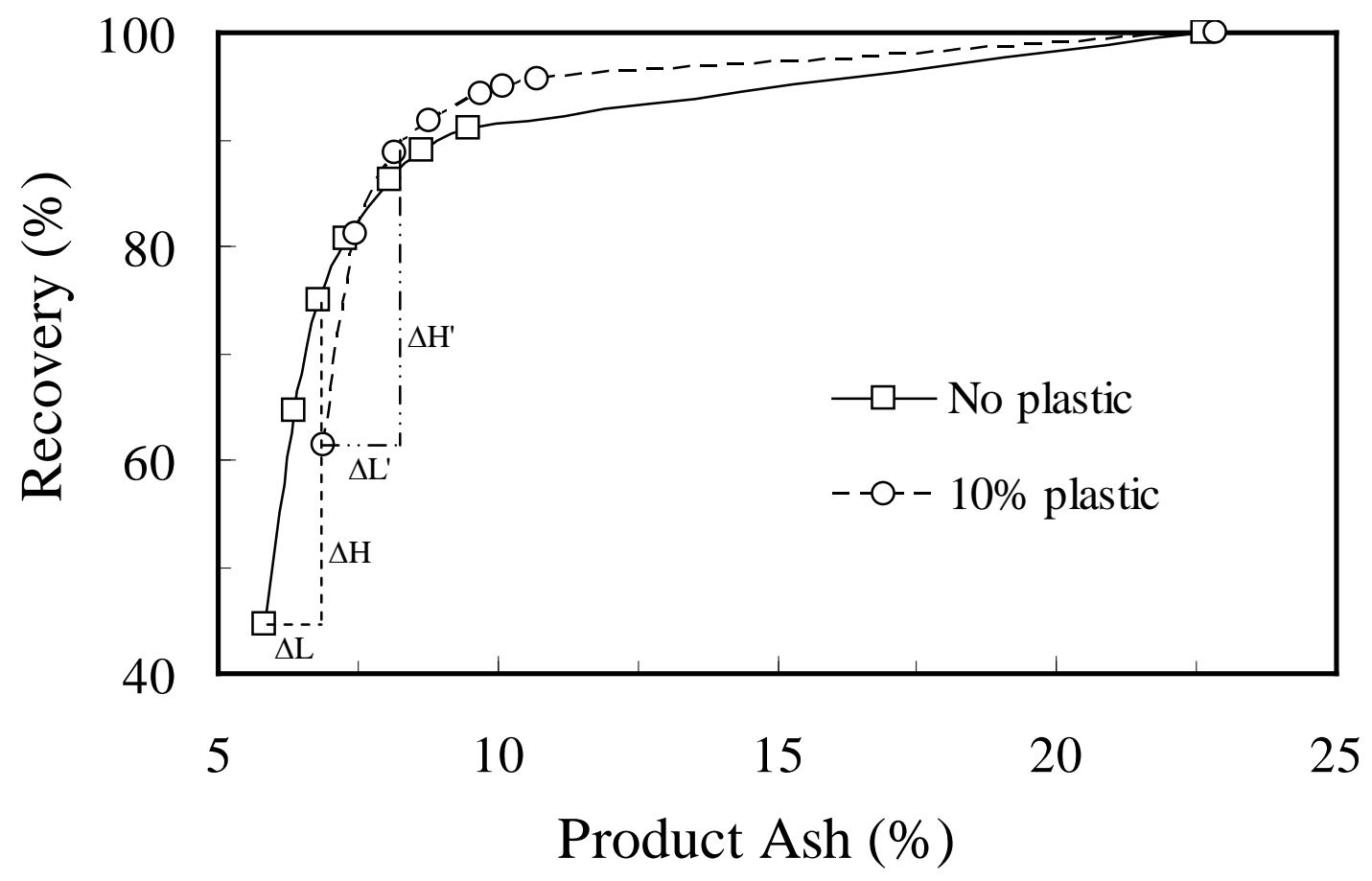

Figure 21. Average improvements in flotation performance achieved by the additional of magnetic plastic material; five experimental repetitions.

The impact of magnetic plastic material on the reduction of pyrite when treating Pittsburgh No. 8 coal samples is minimal based on the data given of Figure 22. The data 
clearly show the improvement in recovery, however, absolute reduction in total sulfur does not indicate a clear impact from the plastic particle addition. It is unclear whether these results reflect the incomplete liberation of the pyrite or the possibility that the plastic particles could adsorb onto the weakly hydrophobic pyrite thereby elevating their surface hydrophobicity. Entrainment could provide a countering effect for any positive selectivity gains (Kawatra and Eisele, 1996).

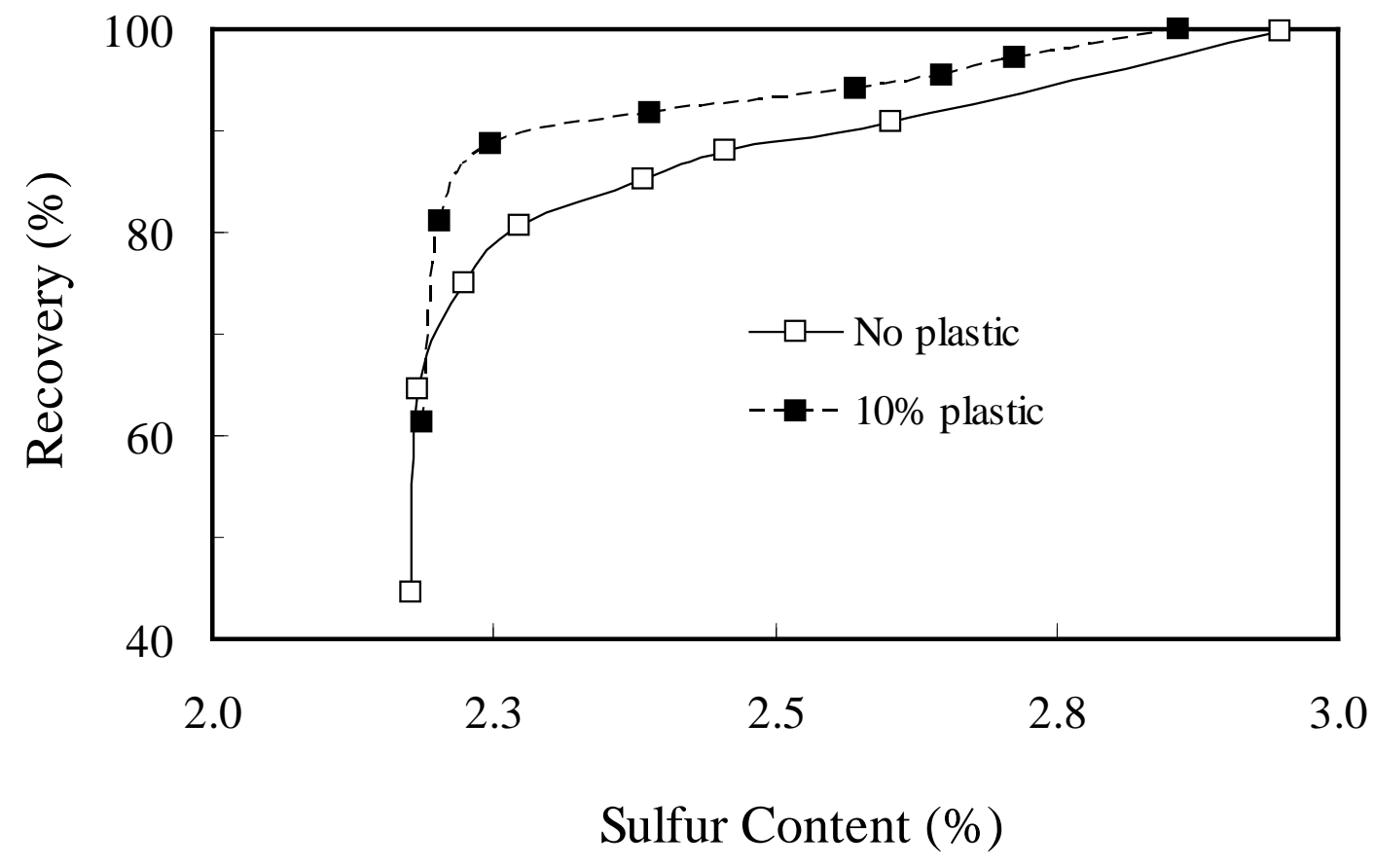

Figure 22. Effect of $10 \%$ magnetic plastic material on sulfur content. Average result of five flotation test.

\section{Effect of Coal Type}

The Coalburg coal seam in the central Appalachia coalfields is well known as a difficultto-clean coal. This characteristic is mainly due to the amount of middling (mixed phase), unliberated particles in the coal, which is in contrast with the Pittsburgh No. 8 coal. The Coalburg coal also has poor floatability characteristics, which is reflected in the low flotation rate value of $0.51 \mathrm{~min}^{-1}$.

The addition of the plastic particles into the Coalburg flotation feed slurry at a $10 \%$ by weight concentration significantly improved the recovery rate. However, the improvement was smaller than that realized for the Pittsburgh No. 8 coal. As shown in Figure 23, coal recovery after one minute of flotation improved from $61.1 \%$ to $77.4 \%$ by the addition of the plastic particles in a conventional cell. As shown in Table 9, the flotation rate increased from $0.51 \mathrm{~min}^{-1}$ to $0.74 \mathrm{~min}^{-1}$ which is a $145 \%$ improvement. 


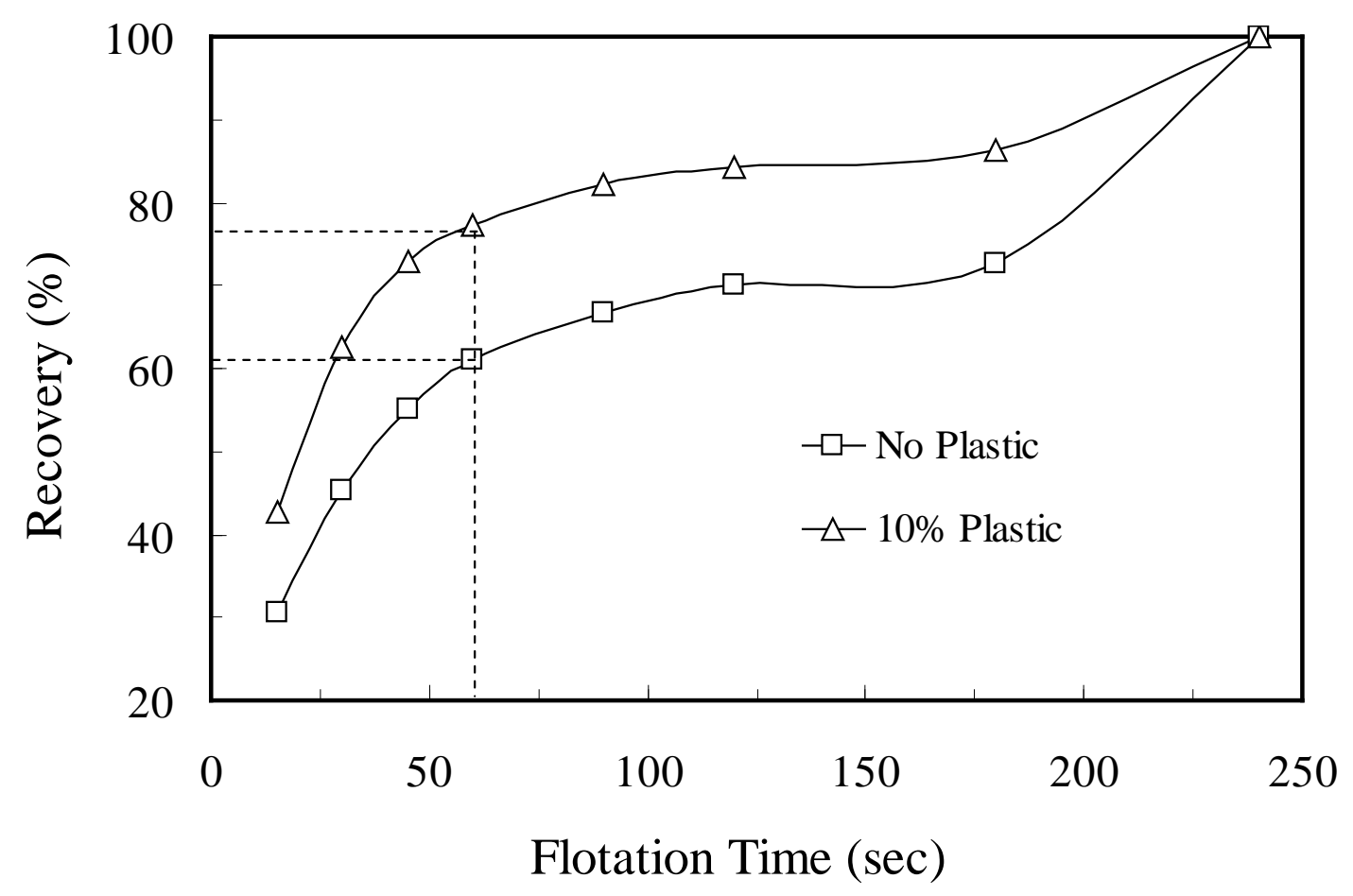

Figure 23. Flotation recovery rate improvement achieved when adding $10 \%$ by weight of plastic particles to the Coalburg flotation feed in a conventional cell.

Table 9. Flotation rate improvements achieved by the addition of plastic particles at a $10 \%$ by weight concentration to Coalburg coal feed slurry.

\begin{tabular}{|c|c|c|}
\hline $\begin{array}{c}\text { Test } \\
\text { Identification }\end{array}$ & $\begin{array}{c}\text { Flotation } \\
\text { Rate } \\
\left(\mathrm{min}^{-1}\right)\end{array}$ & $\begin{array}{c}\text { Flotation } \\
\text { Recovery } \\
(\%)\end{array}$ \\
\hline No Plastic, Coalburg & 0.51 & 61.1 \\
10\% Plastic, Coalburg & 0.74 & 77.4 \\
\hline
\end{tabular}

The selectivity of the flotation process was also enhanced by the plastic particles. As shown in Figure 24, the recovery increase varied as a function of product ash which is indicative of a selectivity improvement. When producing a product containing $15 \%$ ash, coal recovery was increased by 10 absolute percentage points. 


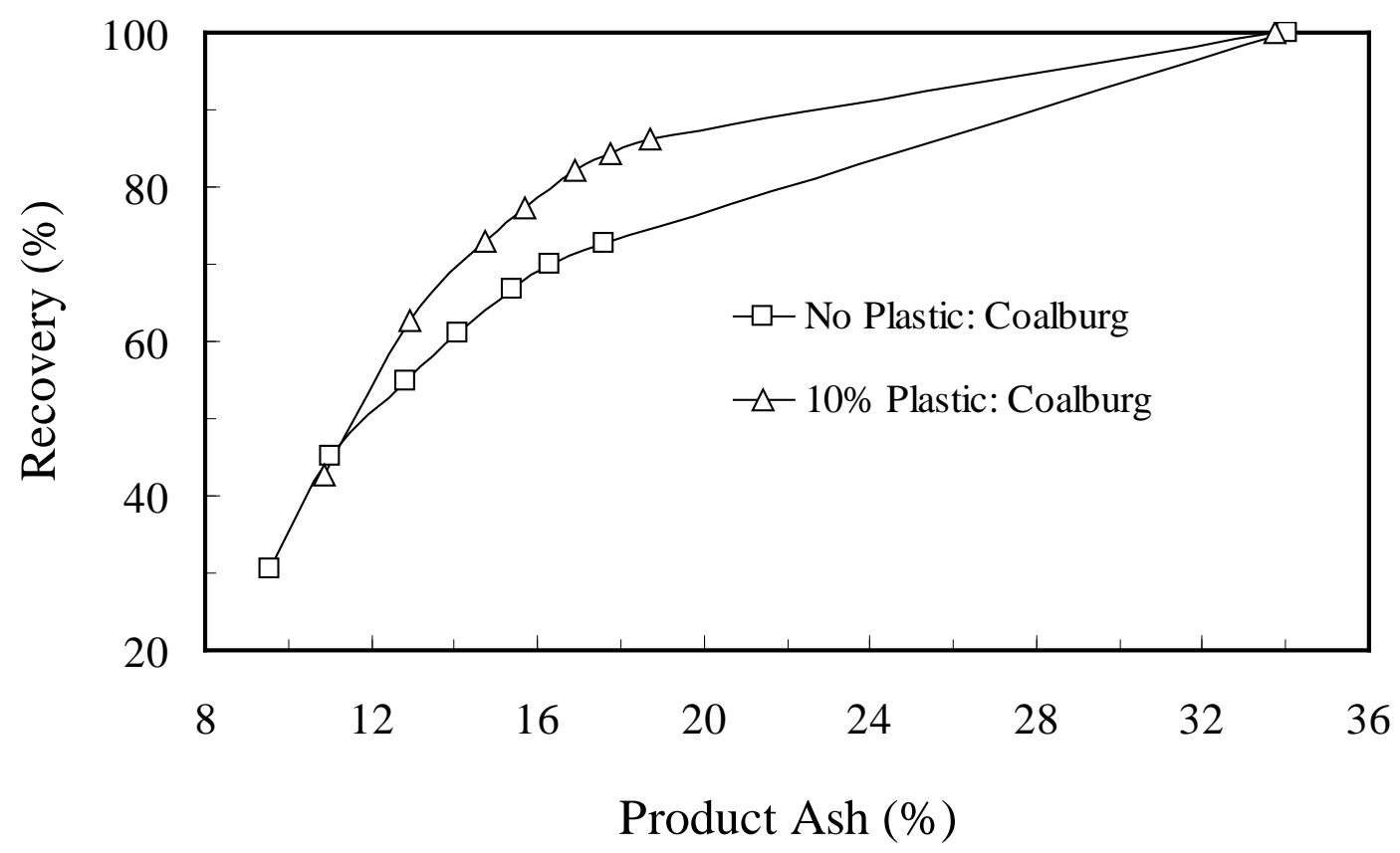

Figure 24. Selectivity performance improvement achieved by the addition of plastic particles at a $10 \%$ by weight concentration to a conventional cell flotation feed containing Coalburg coal.

\section{Effect of $p H$}

As previously discussed, the electrostatic charge of the particle and bubble surfaces plays a significant role in bubble-particle attachment. The zeta potential, which is an approximation of the surface charge, varies as a function of $\mathrm{pH}$ for both the Pittsburgh No. 8 and plastic particles. In general, the electrostatic charge is negative and increases in magnitude as the $\mathrm{pH}$ is elevated from 3 to 10 . Obviously, higher surface charges lead to greater repulsive forces which could slow or prevent attachment to a bubble. Optimum recovery typically occurs under $\mathrm{pH}$ conditions that provide neutrally charged coal surfaces.

As shown in Figure 25, the best separation performances were achieved under the neutral $\mathrm{pH}$ conditions, which likely corresponded to a $\mathrm{pH}$ value slightly lower than 7.0 for the Pittsburgh No. 8 coal. The flotation recovery obtained under $\mathrm{pH}$ values of 3.9 and 10.1 were significantly depressed as indicated by recovery values of only $32.8 \%$ after 1 minute of flotation, which is about 27 percentage points less than that achieved at the neutral $\mathrm{pH}$ conditions. At $\mathrm{pH} 3.9$, the electrostatic charge on the coal particles was relatively low so the poor recovery was unexpected. However, it is consistent with previously reported coal flotation results. 


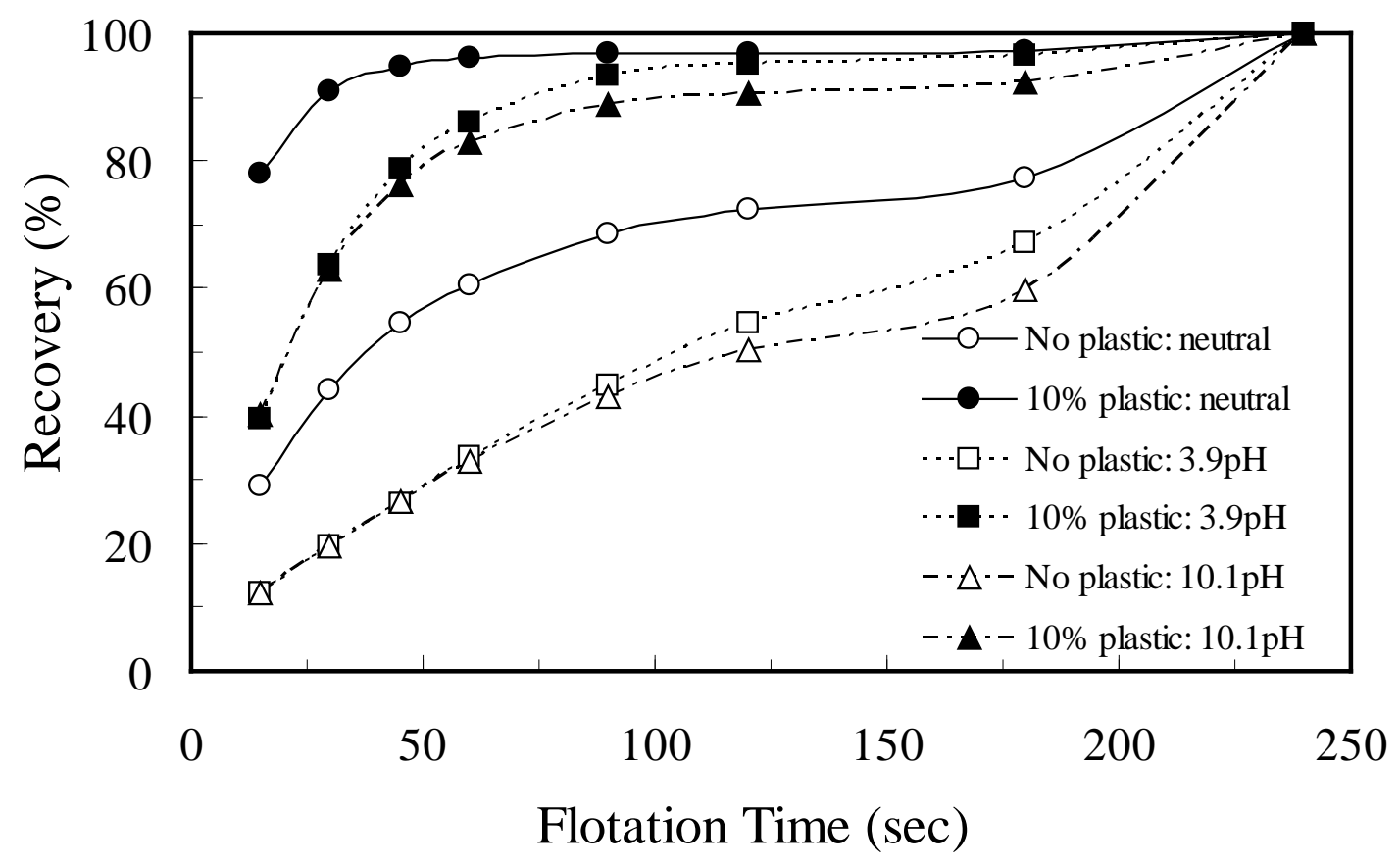

Figure 25. Impact of slurry $\mathrm{pH}$ on the recovery improvements realized when adding plastic particles to a conventional flotation feed containing Pittsburgh No. 8 coal.

By adding the plastic particle at a concentration of $10 \%$ by weight, flotation recovery after one minute increased by nearly 50 absolute percentage points under the $\mathrm{pH}$ conditions of 3.9 and 10.1. The recovery improvement is directly a result of the flotation rate rise from $0.30 \mathrm{~min}^{-1}$ to $1.51 \mathrm{~min}^{-1}$ at $\mathrm{pH} 3.9$ and from $0.30 \mathrm{~min}^{-1}$ to $1.33 \mathrm{~min}^{-1}$ at $\mathrm{pH}$ 10.1 (Table 4.5). The explanation is likely linked to the high hydrophobic plastic particles. A surface with a high degree of surface hydrophobicity produces a highly attractive interactive force with other hydrophobic particles that can overcome the electrostatic repulsive forces. 
Table 10. Flotation rate improvements achieved under varying $\mathrm{pH}$ conditions when adding plastic particles to Pittsburgh No. 8 coal at a concentration of $10 \%$ by weight.

\begin{tabular}{|c|c|c|c|}
\hline \multicolumn{2}{|c|}{ Test Identification } & $\begin{array}{c}\text { Flotation } \\
\text { Rate }\left(\mathrm{min}^{-1}\right)\end{array}$ & $\begin{array}{c}\text { Flotation } \\
\text { Recovery (\%) }\end{array}$ \\
\hline \multirow{2}{*}{ Neutral } & No Plastic & 0.66 & 60.5 \\
& $10 \%$ Plastic & 1.32 & 96.0 \\
\hline \multirow{2}{*}{ pH 3.9 } & No Plastic & 0.30 & 32.8 \\
& $10 \%$ Plastic & 1.51 & 85.8 \\
\hline \multirow{2}{*}{ pH 10.1 } & No Plastic & 0.30 & 32.8 \\
& $10 \%$ Plastic & 1.33 & 82.7 \\
\hline
\end{tabular}

The addition of the plastic material also appears to provide a selectivity improvement at the $\mathrm{pH}$ conditions of 3.9 and 10.1. As shown in Figure 26, the performance improvement appears totally due to recovery gains for the neutral $\mathrm{pH}$ values of 3.9 and 10.1 shift to the left and upward when the plastic particles are added, which indicates a reduction in the product ash content while also elevating coal recovery. The coal recovery gains are more clearly highlighted as a function of flotation time in Figure 27 for the three $\mathrm{pH}$ conditions studied. The neutral $\mathrm{pH}$ condition had the largest gain in the shortest time (15 seconds) while the largest overall improvement was realized under $3.9 \mathrm{pH}$ after 45 seconds of flotation.

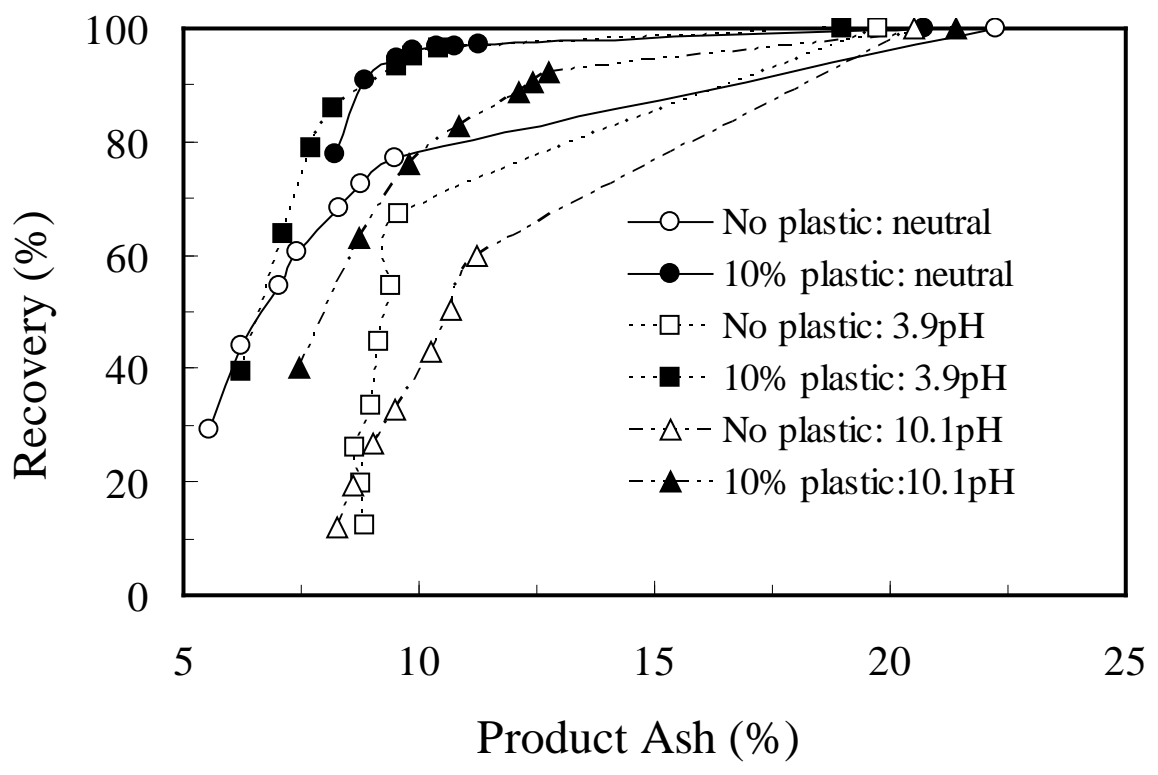

Figure 26. Selectivity performance improvements achieved by the addition of the plastic particles at a concentration of $10 \%$ by weight in a conventional flotation cell; Pittsburgh No. 8 coal. 


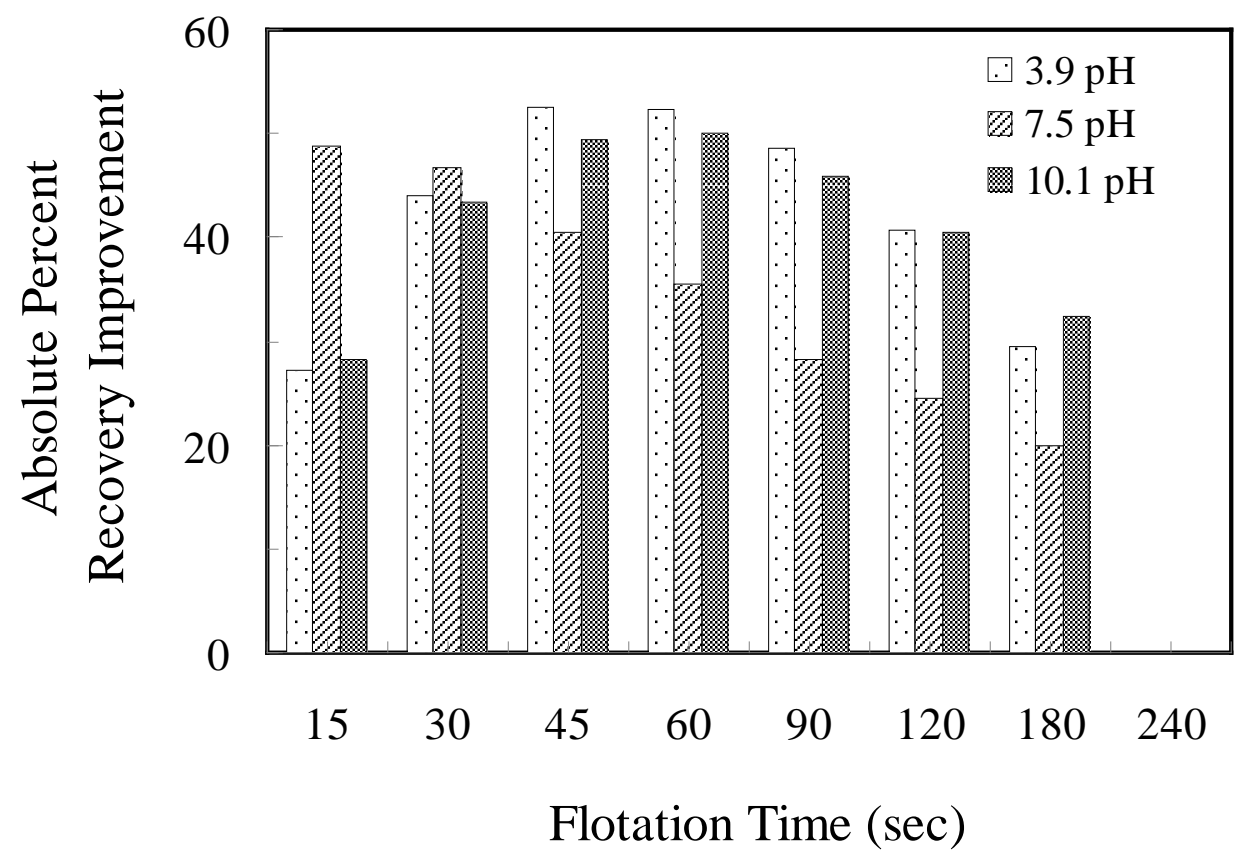

Figure 27. Realized from the addition of plastic particles at a $10 \%$ concentration in a conventional cell when treating Pittsburgh No. 8 coal.

Interestingly, the slurry $\mathrm{pH}$ also had an effect on the recovery time of the plastic particles in the conventional flotation cell. Under neutral $\mathrm{pH}$ conditions, nearly $98 \%$ of the plastic was recovered from the flotation cell within the first 60 seconds of flotation process as shown in Figure 28. After the first 15 seconds, nearly $80 \%$ of the plastic was recovered as compared to only $45 \%$ of the coal. The plastic particle recovery was only slightly depressed under $\mathrm{pH} 10.1$ with about $70 \%$ recovery after 15 seconds of flotation. However, under $\mathrm{pH} 3.9$, plastic recovery was only about $45 \%$ after 15 seconds of flotation time and increments representing $5 \%$ to $10 \%$ absolute percentage units of the total plastic particle concentration reported in all time increments up to 240 seconds ( 4 minutes). The acidity of the slurry could have resulted in chemical changes that negatively affected floatability although significant coal recovery improvements were observed. 


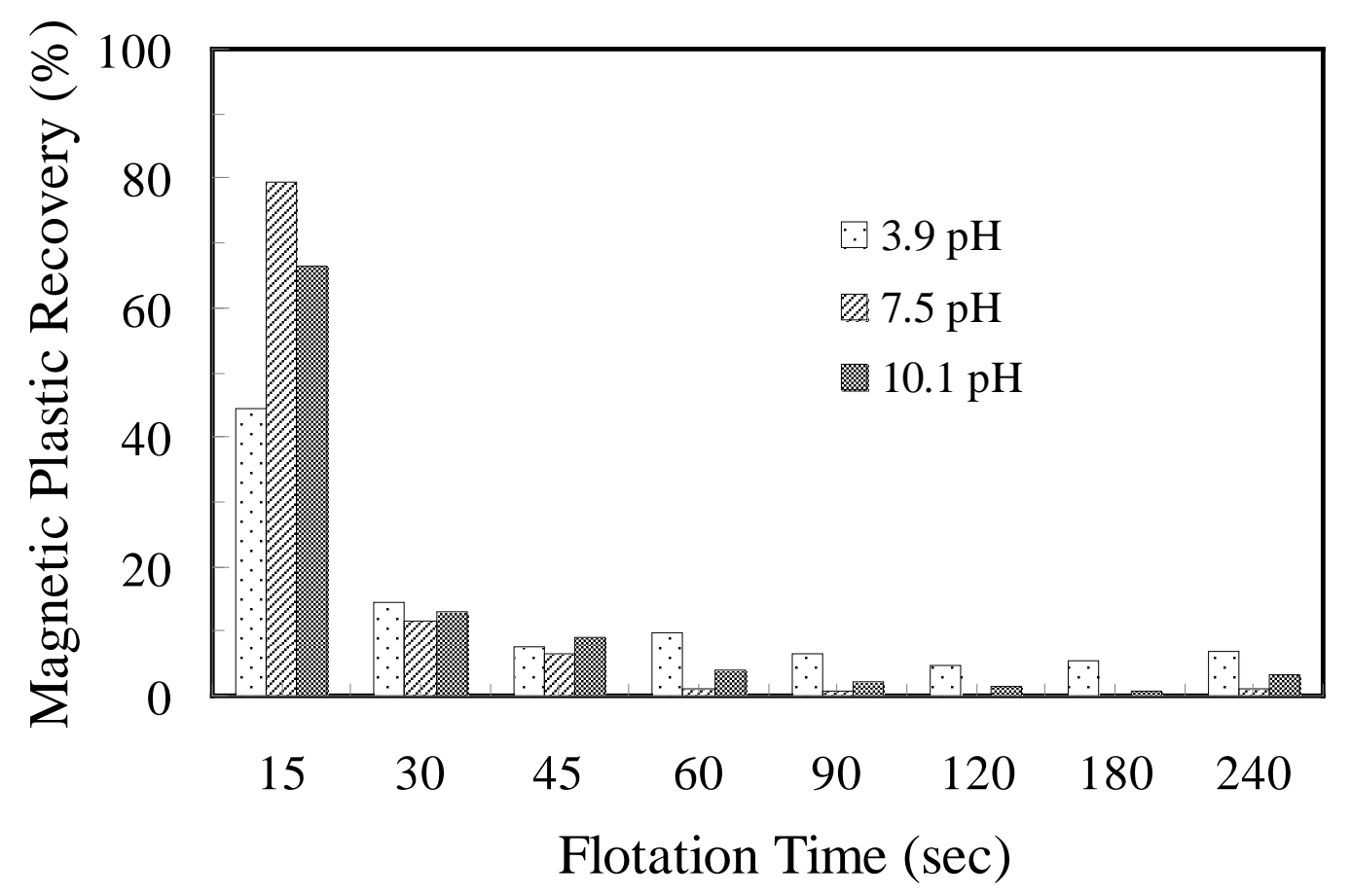

Figure 28. Recovery distribution of magnetic plastic material from the flotation rate tests performed on Pittsburgh No. 8 coal over a range of $\mathrm{pH}$ values.

\section{Particle Size-by-Size Flotation}

Froth flotation performance varies significantly as a function of particle size. In fact, a lower and upper particle size exists and the absolute value of the limits varies from material-to-material. For coal, the typical reported limits are 50 to 500 microns. The lower limit is a result of inefficiencies in the bubble-particle collision process while the upper size limit is associated with the detachment of particles from the bubble surfaces.

To determine the impact of the magnetic plastic material on particle size-by-size performance, a flotation rate test was performed on the Pittsburgh No. 8 coal. Using a $10 \%$ concentration of plastic particles in the feed stream, the weight recovery achieved on the +100 mesh fraction of the total feed was significantly increased as shown in Figure 29. After one minute of flotation, weight recovery was elevated by nearly 30 absolute percentage points. 


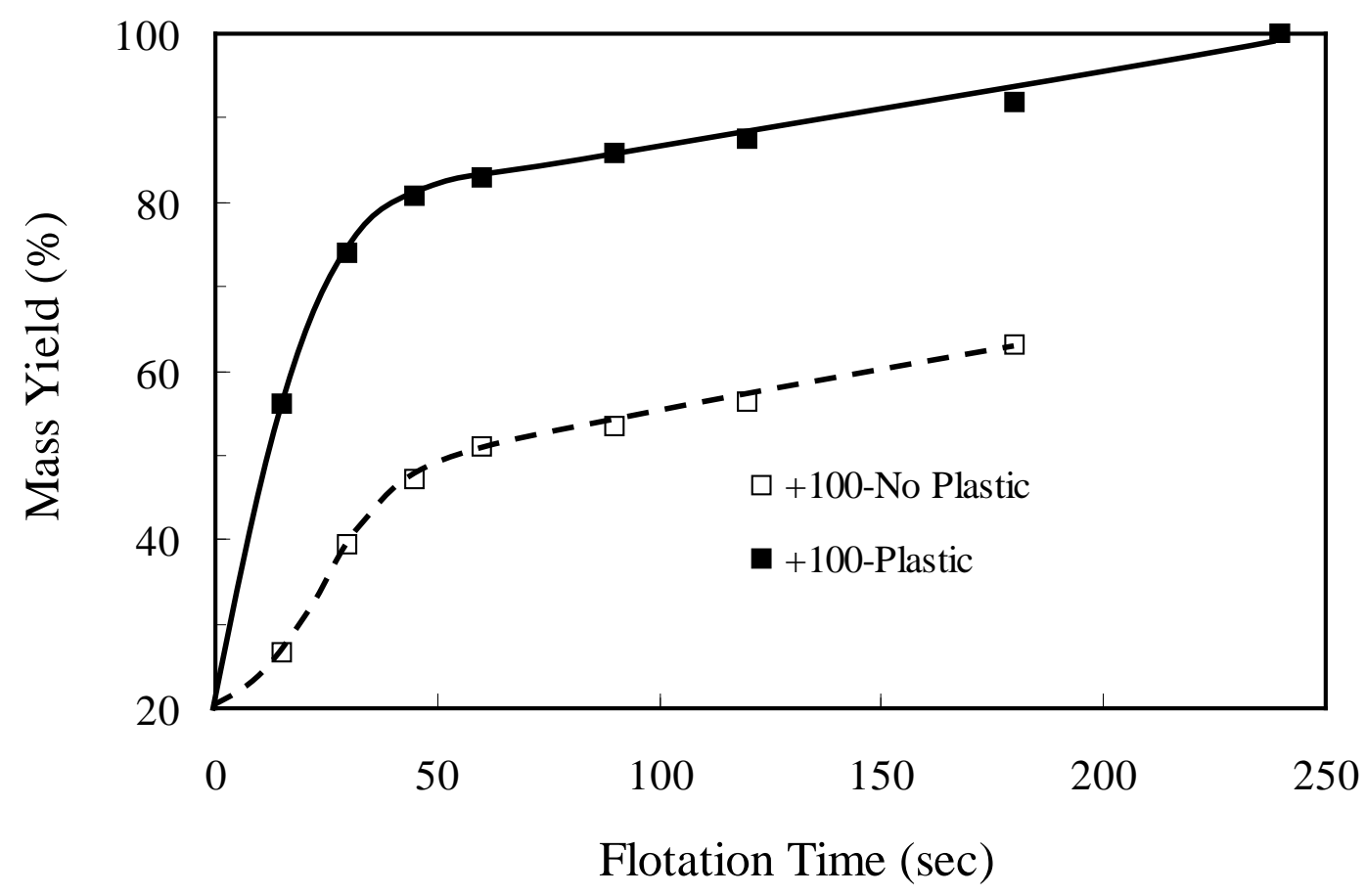

Figure 29. Improvements in the mass recovery rate of the +100 mesh Pittsburgh No. 8 coal by the addition of plastic particles in a conventional flotation cell.

The weight recovery achieved after one minute for each particle size fraction was assessed to quantify the potential change in the lower and upper particle size limits. As shown in Figure 30, mass recovery was increased throughout the particle size range by at least 5 absolute percentage points as a result of the plastic particle addition. However, the impact was much greater for the particles having a size larger than $0.10 \mathrm{~mm}$. A possible explanation is that the presence of more hydrophobic solids on the surface of the air bubbles causes drainage resistance of the water film surrounding an air bubble. As a result, bubble coalescence is reduced thereby maintaining high bubble surface air flux through the froth zone. The availability of bubble surface area reduces preferential detachment of the coarse particles within the froth zone. 


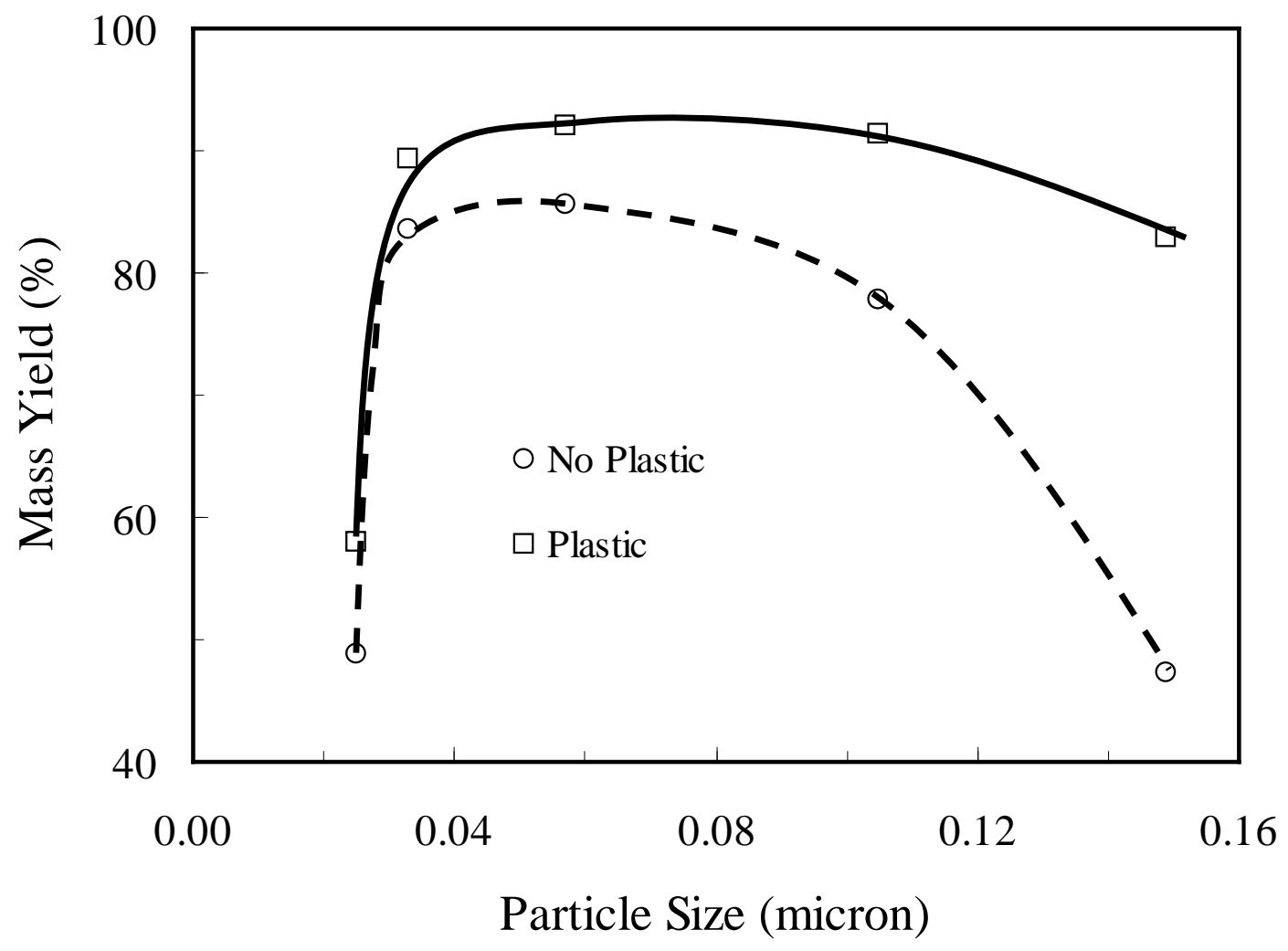

Figure 30. Particle size-by-size mass recovery improvements achieved on Pittsburgh No.

8 coal by the addition of plastic particles at a concentration $10 \%$ by weight in a conventional flotation cell.

Low flotation rates for the coarse particles typically occur due to higher collector adsorption rates of the finer particles and preferential detachment resulting from overloaded surfaces. Tests were performed to determine if the positive impact of the plastic particle addition was maintained if the coarse particles were floated separately from the fine particles. The Pittsburgh No. 8 coal was screened to obtain size fractions of particles greater than 200 mesh, 200 x 500 mesh and finer than 500 mesh. Each particle size fraction was floated separately.

As shown in Figure 31 and Table 11, the flotation rates of the coarser size fractions were much greater when the fractions were floated separately as compared to floating the same size fraction together. Furthermore, the addition of the plastic particles had no positive effect on coarse particle flotation. However, a 5 absolute percentage point increase was obtained for the material finer than 25 microns (500 mesh). In this case, the plastic particles could be either i) serving as a carrier for the fine coal particles and/or ii) the plastic particles act as a seed for hydrophobic agglomeration and the larger coal-plastic agglomerates are more readily collected by the conventionally-sized bubbles. 


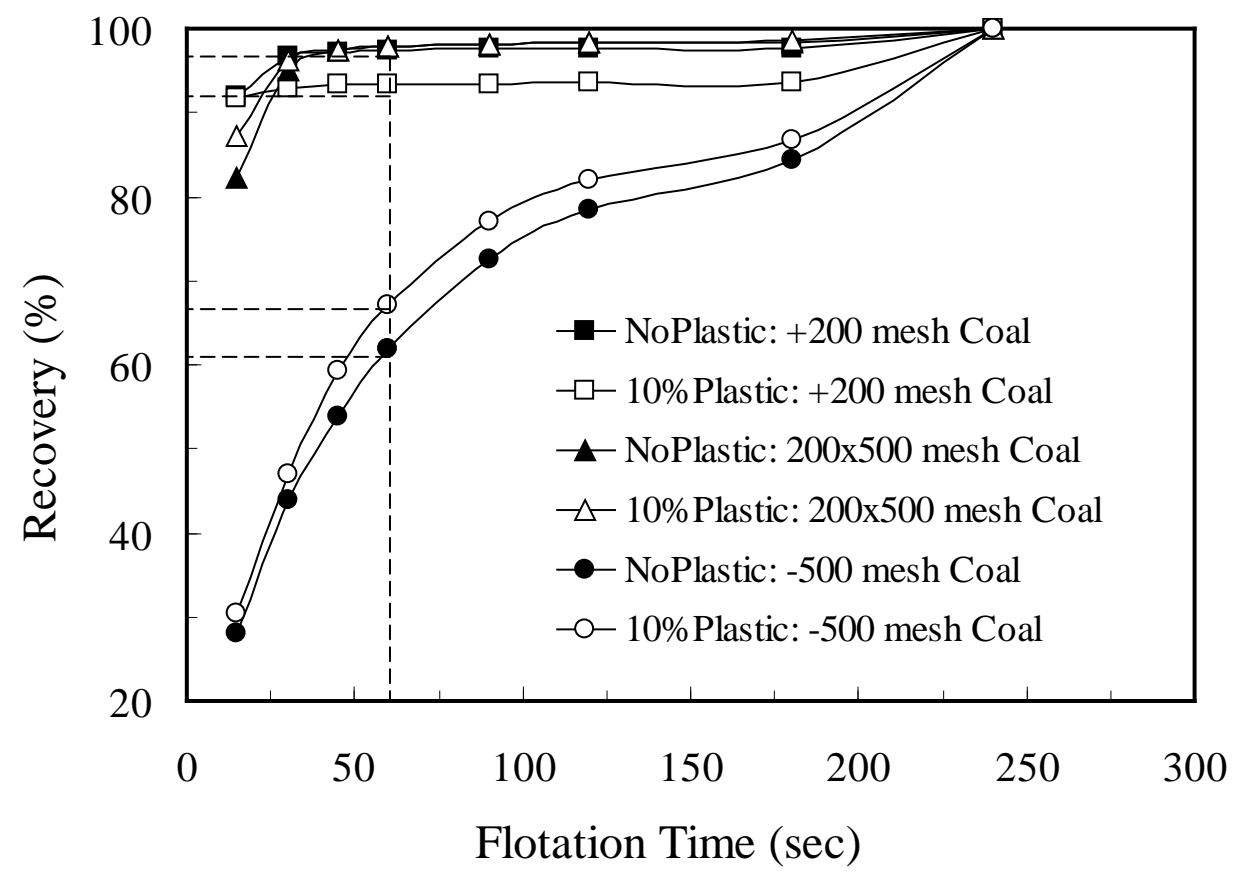

Figure 31. Recovery improvements from the flotation of individual particle size fractions in a conventional flotation cell with and without the addition of plastic particles;

Pittsburgh No. 8 coal.

Table 11. A comparison of the flotation rates and recovery values achieved with and without the addition of plastic particles when floating each size fraction separately; conventional cell, Pittsburgh No. 8 coal.

\begin{tabular}{|c|c|c|c|}
\hline $\begin{array}{c}\text { Particle size } \\
\mu \mathrm{m}\end{array}$ & $\begin{array}{c}\text { External } \\
\text { Material } \\
\text { Addition }\end{array}$ & $\begin{array}{c}\text { Flotation } \\
\text { Rate } \\
\left(\mathrm{min}^{-1}\right)\end{array}$ & $\begin{array}{c}\text { Flotation } \\
\text { Recovery } \\
(\%)\end{array}$ \\
\hline \multirow{2}{*}{+75} & No Plastic & 2.31 & 97.3 \\
& $10 \%$ Plastic & 2.25 & 93.4 \\
\hline $75 \times 25$ & No Plastic & 2.54 & 97.8 \\
& 10\% Plastic & 2.53 & 97.8 \\
\hline \multirow{2}{*}{-25} & No Plastic & 0.59 & 61.9 \\
& $10 \%$ Plastic & 0.71 & 67.0 \\
\hline
\end{tabular}

\section{Column Flotation Tests}

An investigation was performed to quantify the overall performance impacts of adding the magnetic plastic particles in a continuously-operated, laboratory flotation column. A major difference between the conventional cell and the flotation column is the 
hydrodynamic conditions which are mostly due to the geometric dimensions of the units. The high length-to-diameter ratio of a laboratory column provides near plug-flow conditions which are optimum for recovery. Therefore, the overall recovery improvements were expected to be lower from the laboratory column unit since the conditions are more ideal than those realized in conventional flotation cells. Since the initial focus of the study was aimed at improving selective detachment in the froth phase, the majority of the column experiments involved injection of the plastic particles through the was water distributer in the froth.

All flotation systems have a limitation on the amount of product that can be conveyed to the collection launder. This limit is typically referred to as the carrying capacity. To determine the carrying capacity, the feed solids concentration is increased while holding the volumetric feed rate constant. As the feed solid content is increased, the product mass rate also rises linearly at a rate that reflects the amount of floatable material entering in the feed stream as shown in Figure 32. When the bubble surfaces reporting to the top of the cell are completely full, the product mass rate reaches a maximum. A further elevation of the feed solids concentration results in a decrease in the product mass flow rate due to selective detachment of the coarse particles, which have more overall mass than the fine particles that remain on the bubble.

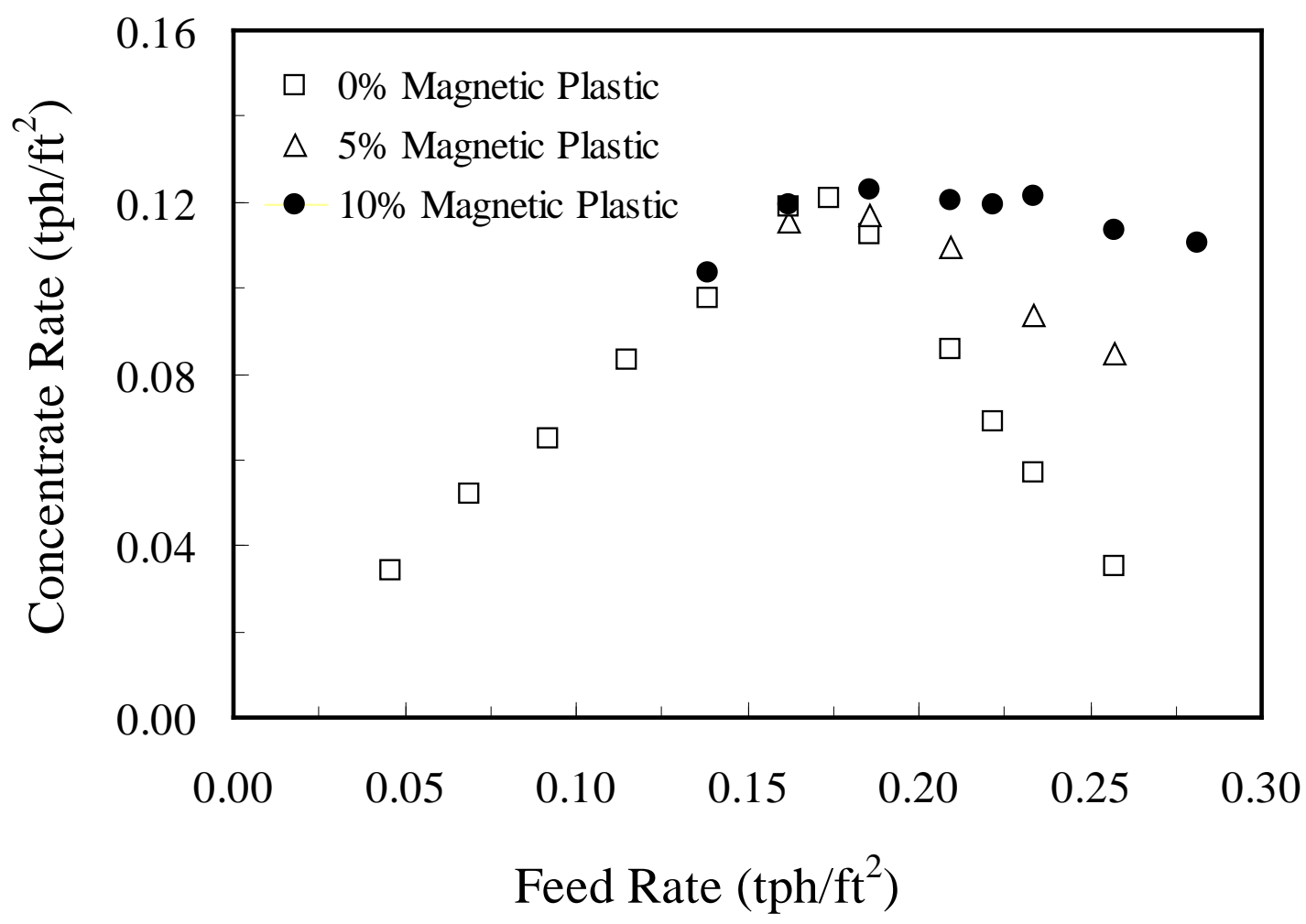

Figure 32. Enhanced carrying capacity characteristics realized by the addition of varying concentrations of magnetic plastic material into the froth phase of a flotation column;

Pittsburgh No. 8 coal. 
As shown in Figure 32, the injection of plastic particles at a concentration of $10 \%$ by weight into the froth phase reduced the detachment of coarse particles as indicated by a near constant product mass rate after reaching carrying capacity. This finding indicates that either the hydrophobic plastic particles are stabilizing the froth and thereby increasing the available surface area or the preferential detachment of coarse particles has been significant reduced. Since the concentrate mass rate reaches a maximum, it is more likely that the selectively detachment of coarse particles is being prevented. A possible explanation is that the plastic particles are coating the coarse coal thereby enhancing the hydrophobicity of the coal surfaces.

The plastic addition had no positive impact on the selectivity achieved on the basis of ash reduction as shown in Figure 33. The range in recovery and product ash values was obtained by varying the volumetric feed flow rate while maintaining the feed solid concentration at $7.5 \%$ by weight. Although the performances are not equal under the same operating conditions, the general recovery versus product ash relationships are nearly equal for the tests not involving plastic addition and those for which plastic was added at $5 \%$ by weight concentration.

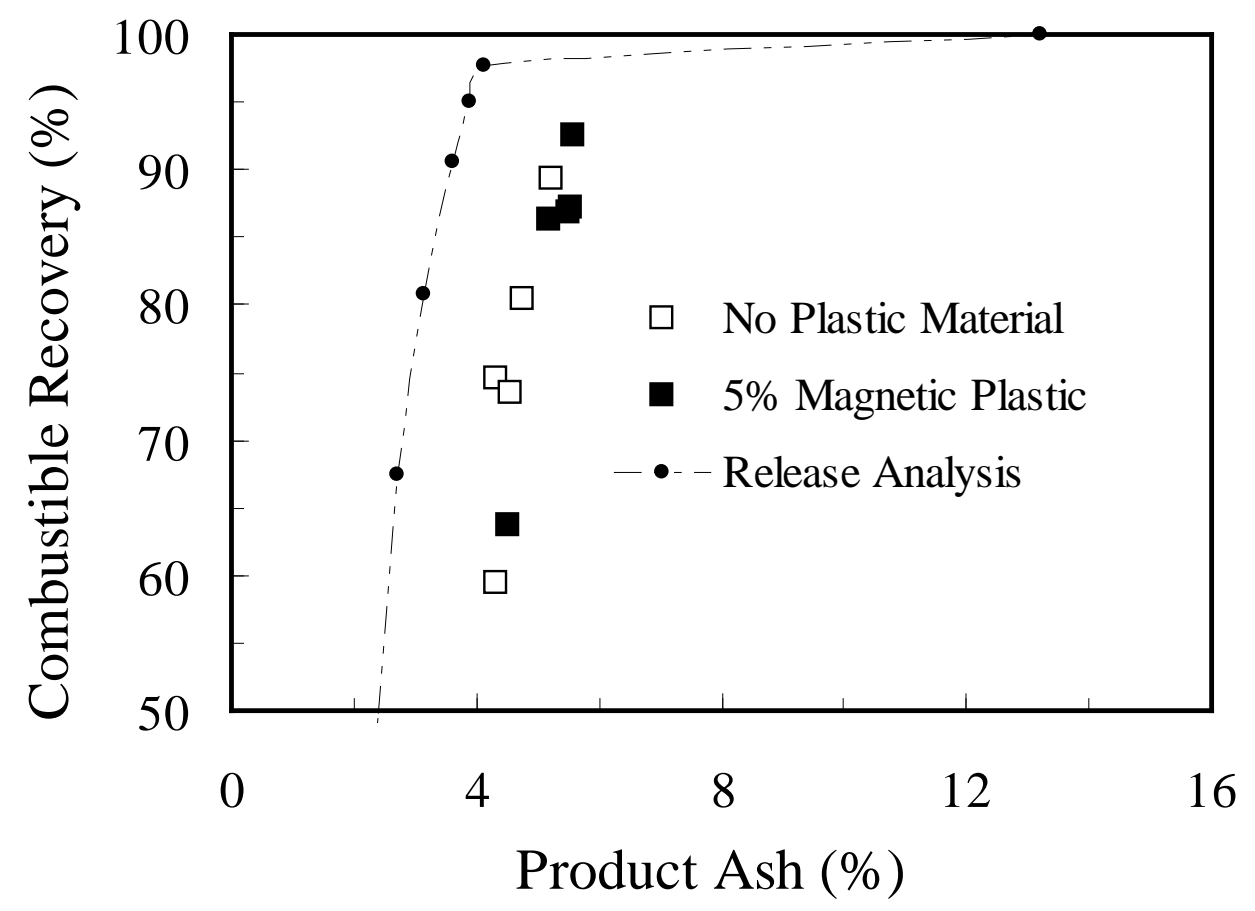

Figure 33. Effect of magnetic plastic material injection on the selectivity of a column flotation froth under elevated solid loading conditions; 7.5\% feed solid concentration; Pittsburgh No. 8 coal.

The performance data in Figure 33 are plotted as a function of volumetric feed rate in Figure 34. At a given feed rate, the combustible recovery is increased by the addition of the plastic material. The recovery gain reaches a maximum of ground 13 absolute 
percentage points in the feed rate range of $600-800 \mathrm{ml} / \mathrm{min}$. This improvement compares well with those obtained from the conventional cell results. However, a negative impact is a slight elevation in ash content in the froth concentrate from around $4.0 \%$ to $5.5 \%$ under equivalent feed rates.

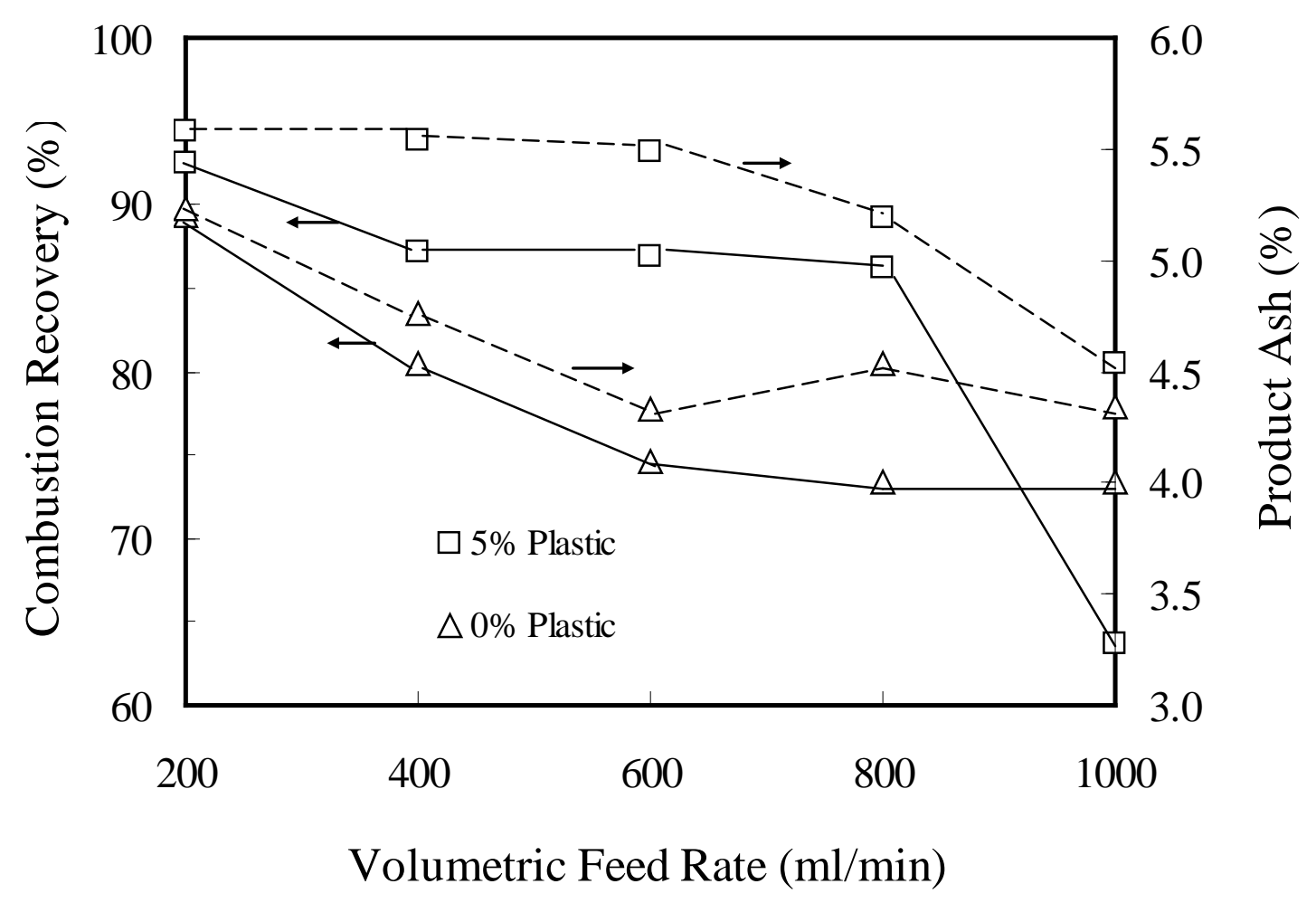

Figure 34. Separation performance improvements achieved as a function of the feed volumetric flows rate when adding $5 \%$ by weight plastic particles into the froth phase of a flotation column treating Pittsburgh No. 8 coal; 7.5\% feed solids concentration.

Two methods of injecting the magnetic plastic particles were studied, i.e., i) adding the particles through the wash water which is distributed in the froth and ii) mixing the plastic particles into the feed coal slurry and injecting into the collection zone of the column. The performances shown in Figure 35 indicate that adding the plastic particles directly into the froth zone provides better selectivity. 


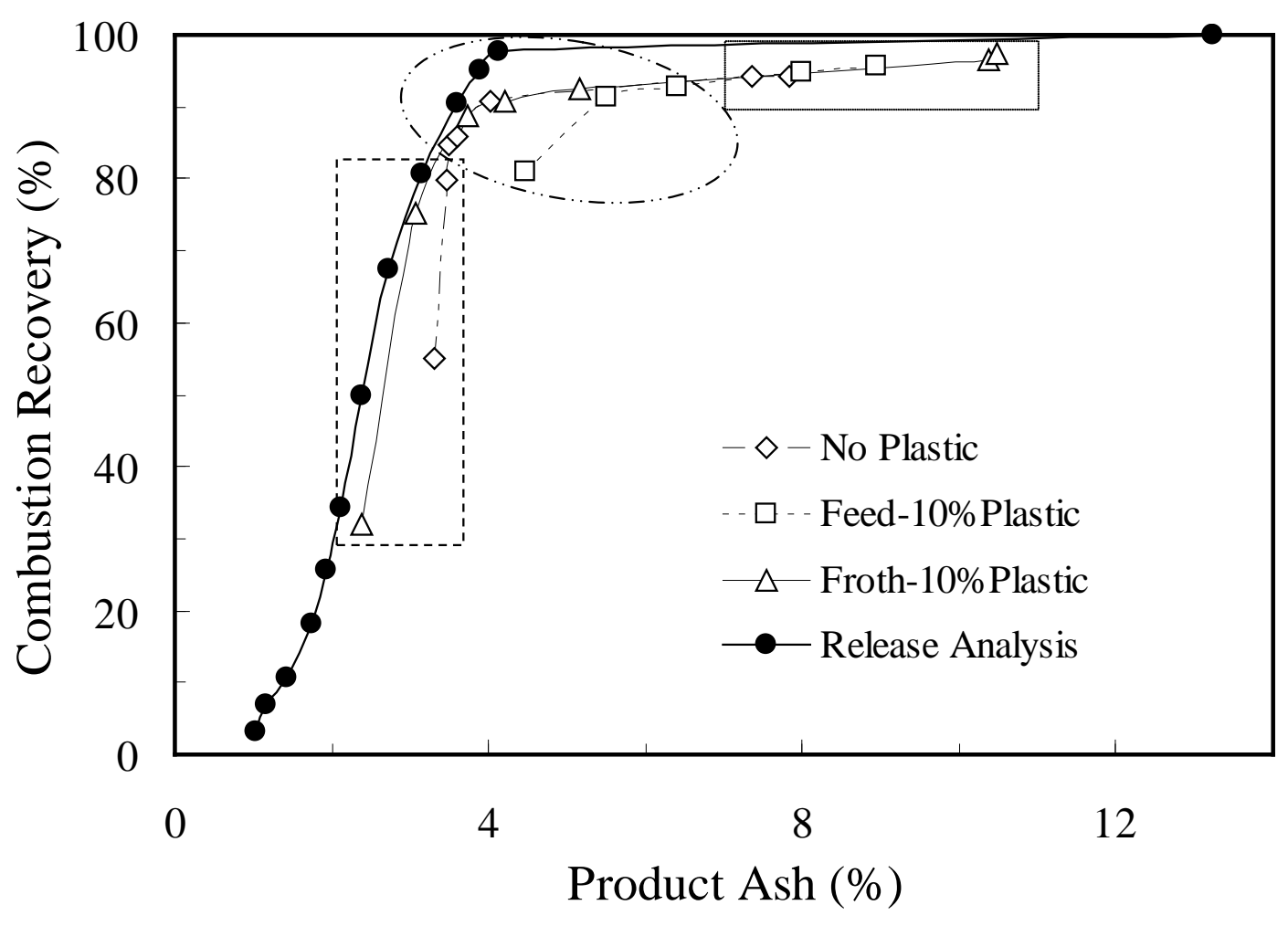

Figure 35. A selectivity improvement comparison between tests in which the plastic particles were added in the froth or in the feed stream of a flotation column at $10 \%$ by weight concentration; Pittsburgh No. 8 coal.

The recovery improvement that was realized from using a $10 \%$ by weight concentration of plastic particles was significantly lower than that achieved with a 5\% concentration. As shown in Figure 36, a recovery gain ranging from 2 to 5 absolute percentage points was obtained for volumetric feed rates below about $1050 \mathrm{ml} / \mathrm{min}$, which interestly corresponds to the feed rates providing the performances near the elbow of the recovery versus product ash curve in Figure 35 . Above the $1050 \mathrm{ml} / \mathrm{min}$ feed rate, recovery drops significantly below the values achieved using no plastic particles. It is also noted that product ash contents were significantly higher when adding plastic particles below the critical feed rate. Overall, the results were inferior to those achieved using a plastic particle concentration of $5 \%$ by weight of the total feed mass flow rate. 


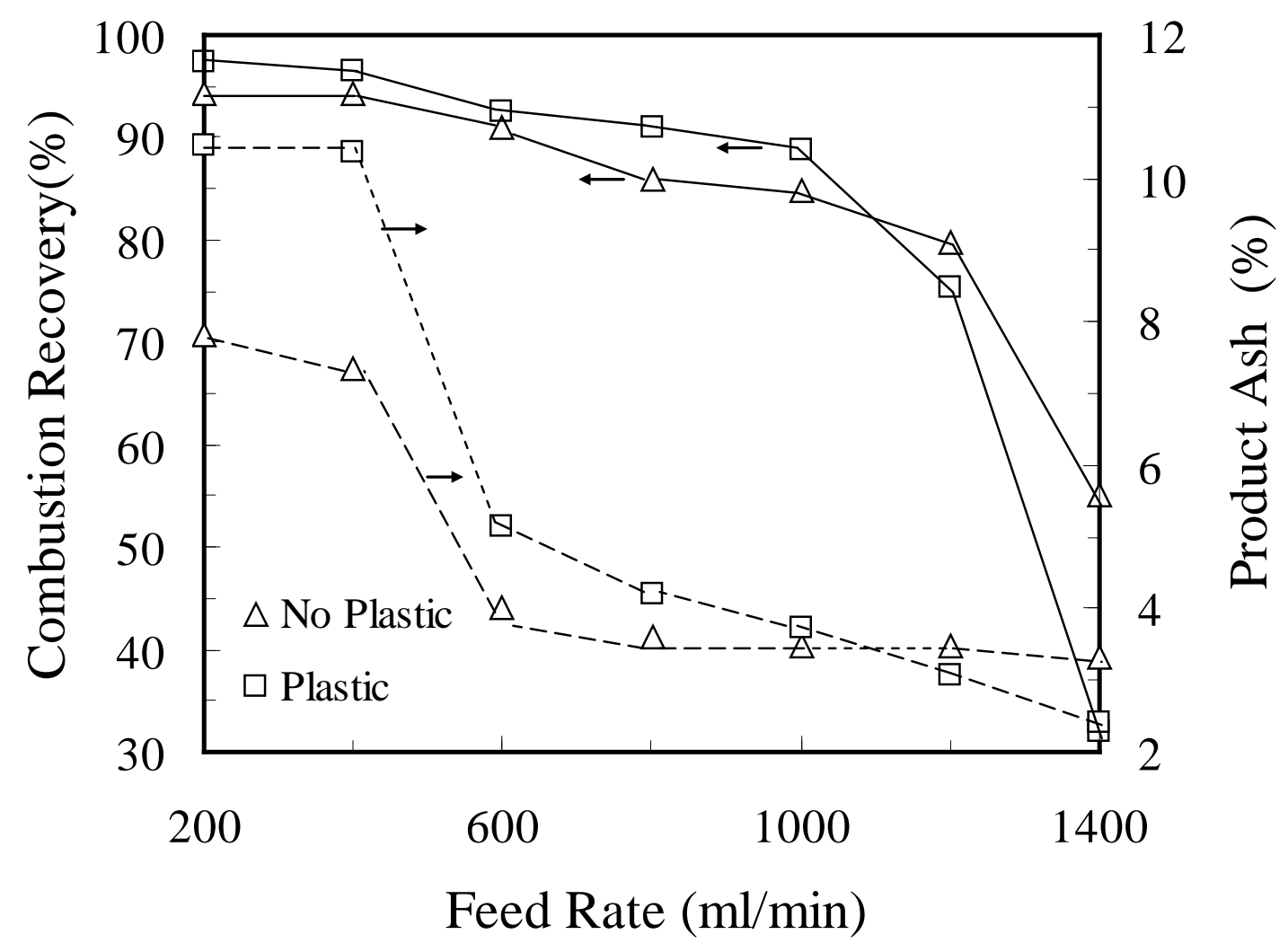

Figure 36. Separation performances as a function of volumetric feed rate to a flotation column when adding plastic particles into the froth phase at a concentration of $10 \%$ of the column feed; Pittsburgh No. 8 coal.

In the conventional cell tests, the plastic material was added directly in the feed and significant recovery improvement was realized. The performances achieved by the flotation column when adding the plastic particles in the column feed stream also indicate an increase in recovery at volumetric feed rate flow rates equal to or lower than 800 $\mathrm{ml} / \mathrm{min}$. As shown in Figure 37, the maximum recovery improvement was about 5 absolute percent points when using a plastic particle concentration of $10 \%$ by weight as compared to recovery values achieved using no plastic particle addition. 


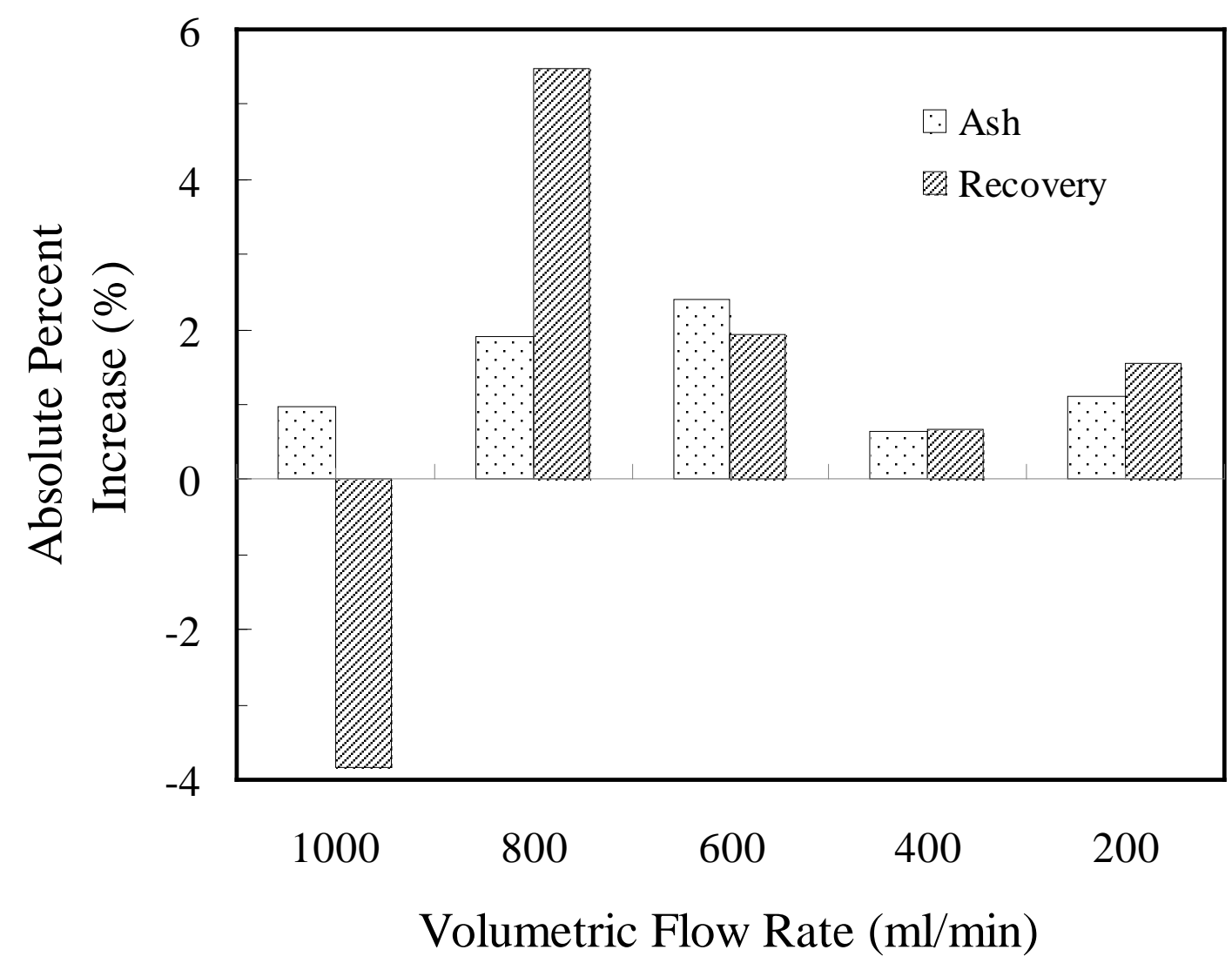

Figure 37. Change in ash content and recovery performance after addition of $10 \%$ magnetic plastic material into the feed slurry in a column flotation respect to baseline performs without addition of external material; Pittsburgh No. 8 coal.

The recovery of the magnetic plastic particles was also somewhat affected by the feed volumetric flow rate. Figure 38 compares the plastic recovery values achieved by adding the plastic particles in the froth phase or the feed stream at a $10 \%$ by weight concentration. As expected, plastic recovery was significantly higher when adding the particles in the froth phase, especially at volumetric feed rates greater than $800 \mathrm{ml} / \mathrm{min}$. The recovery values were nearly $100 \%$ at flow rates below $800 \mathrm{ml} / \mathrm{min}$.

The separation performances achieved on the difficult-to-clean Coalburg coal using the flotation column and plastic particles are shown in Figure 39. Very little if any improvements were realized when using a plastic particle concentration of $10 \%$ by weight of the feed. However, the same results plotted as a function of volumetric feed rate in Figure 40 indicate that a 3 to 5 absolute percentage point improvement in recovery was achieved at feed rates between 600 and $800 \mathrm{ml} / \mathrm{min}$ with minimal effect on product ash content. 


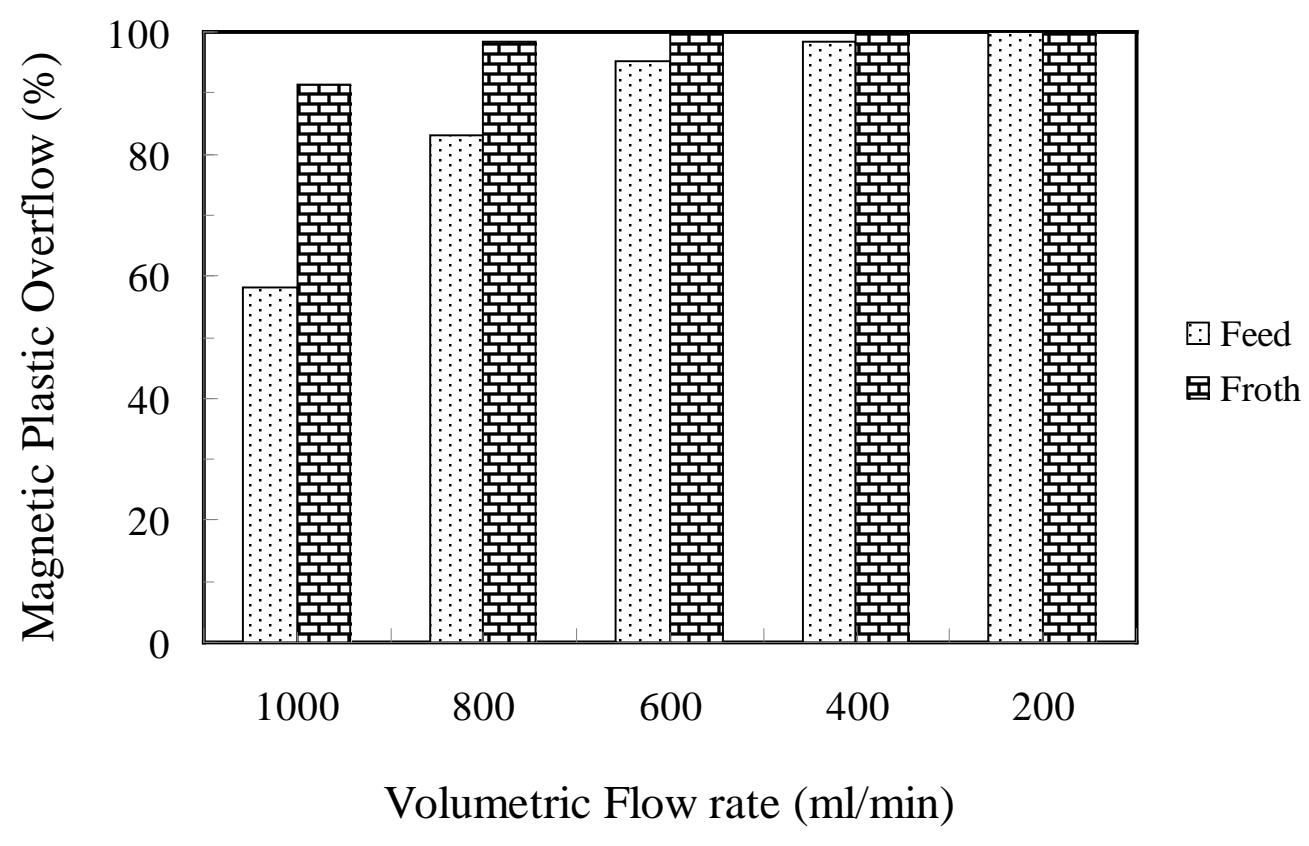

Figure 38. Comparison of the magnet plastic recovery performance achieved when injecting into the froth phase or the feed stream of a flotation column; $10 \%$ plastic particle concentration by weight.

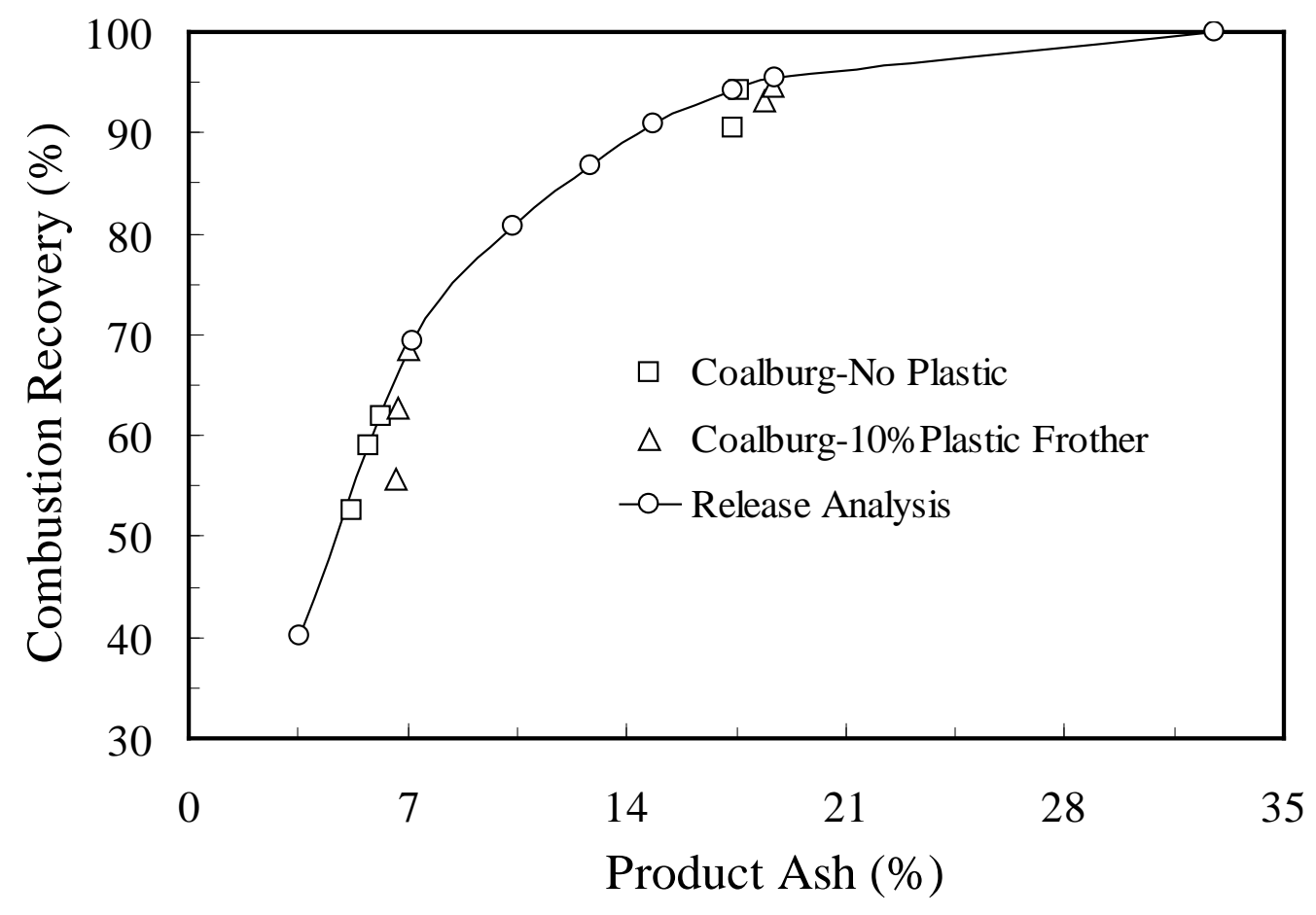

Figure 39. Separation performance achieved when treating Coalburg coal in a flotation column with a $10 \%$ by weight plastic particle injection into the froth phase. 


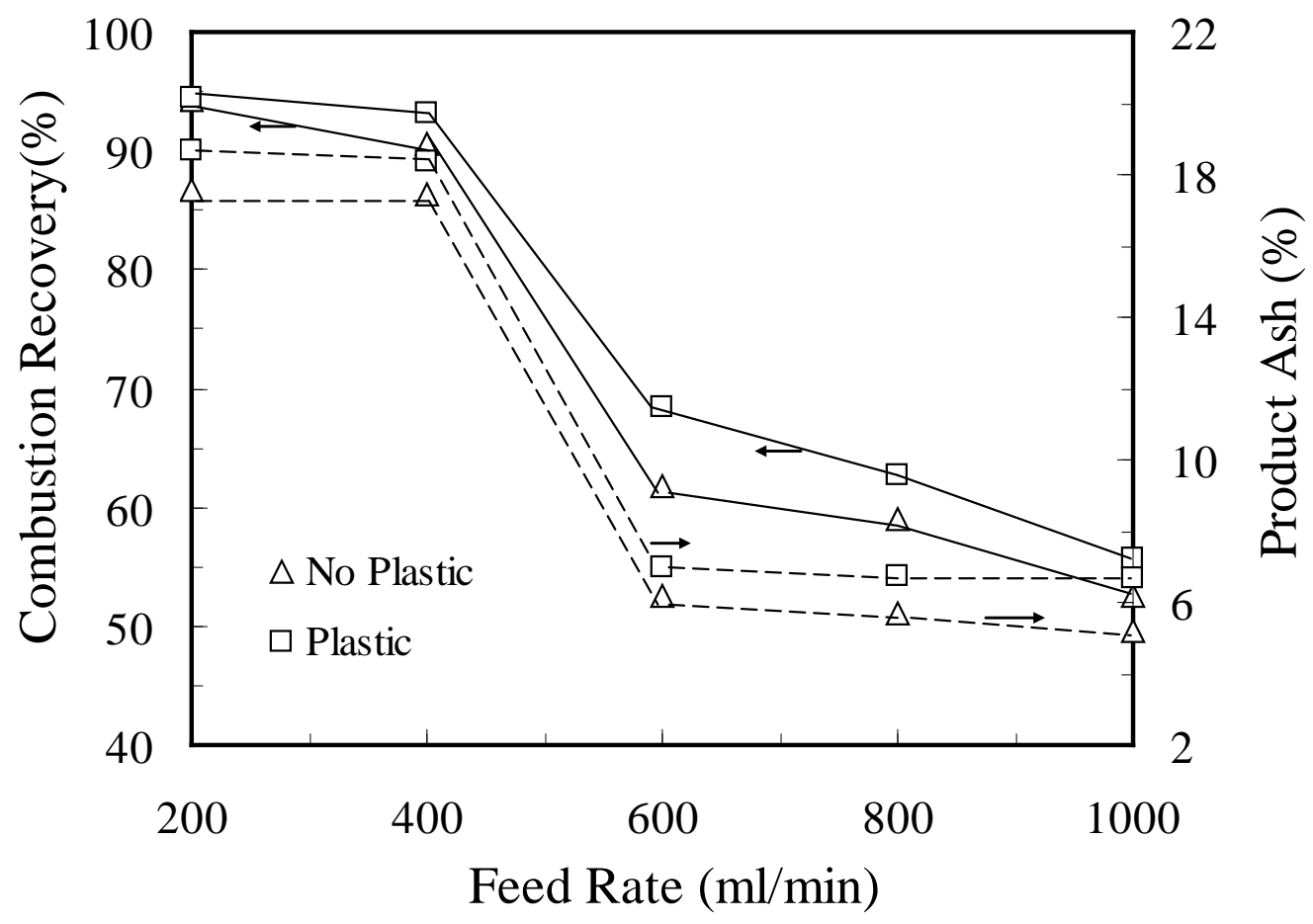

Figure 40. Separation performance achieved as a function of volumetric feed rate when treating Coalburg coal in a flotation column using $10 \%$ by weight plastic particle concentration.

\section{Froth Refluxing}

As previously discussed, selective detachment occurs in the froth zone as a result of bubble coalescence and the subsequent reduction in the amount of surface area per unit of time reporting to the final product. When the amount of particles being conveyed by the bubbles exceed the bubble surface area available, particles are detached. Those particles with a weak binding energy with the bubble surface are preferentially detached. The weak binding energy is likely due to poor surface hydrophobicity which is typically associated with high ash particles. Thus, by promotion of the conditions leading to particle detachment, the selectivity of the process may be improved. Moys (1978) and later Yianatos et al. (1988) have confirmed in previous studies that selective detachment in the froth zone occurs and experiment evidence shows improvements in the product grade.

To activate the selective detachment process, a portion of the froth concentrate stream was continuously collected and recycled through the wash water and into the froth. As a result, the froth was concentrated with high quality, highly hydrophobic coal particles. The flotation column as well as the recycling process was operated continuously. Two test conditions were evaluated in the study: 1 ) low solids loading condition (feed solid content $=2 \%$ by weight) which typically limits selectivity to that achieved on the basis of flotation rate differentials and 2 ) high solids loading (feed solids content $=7.5 \%$ ) which 
achieves selectivity due to both flotation rate differences and selective particle detachment in the froth zone. Froth refluxing was expected to provide the greatest impact under low feed solid conditions since bubble-particle detachment in the froth zone would be minimal due to an abundance of available bubble surface area.

The selectivity achieved by a process is typically indicated by a shift in the recovery versus product grade relationship. As shown in Figure 41, a slight shift to higher recovery and lower product ash values occurred when refluxing a portion of the froth concentrate under the high load condition. The improvements are more easily visualized in Figure 42 as a function of the feed volumetric flow rate. At low feed flow rates which typically provide high recovery values, refluxing reduced the ash content by about 3 absolute percentage points. Since the low flow rates likely provide a low froth loading, detachment is probably limited. Refluxing froth helps to load the froth and encourage the selective detachment of the high ash coal particles. As the feed flow was increased beyond $500 \mathrm{ml} / \mathrm{min}$, the ash reduction benefit was reduced to zero and recovery improvements were realized. The effect on recovery was unexpected and may be due to simper randomness. However, the trend was relatively constant. The increased recovery may be result of the concentration of highly hydrophobic particles and the positive impact on froth stability (more bubble surface area) as well as agglomeration of the coal particles.

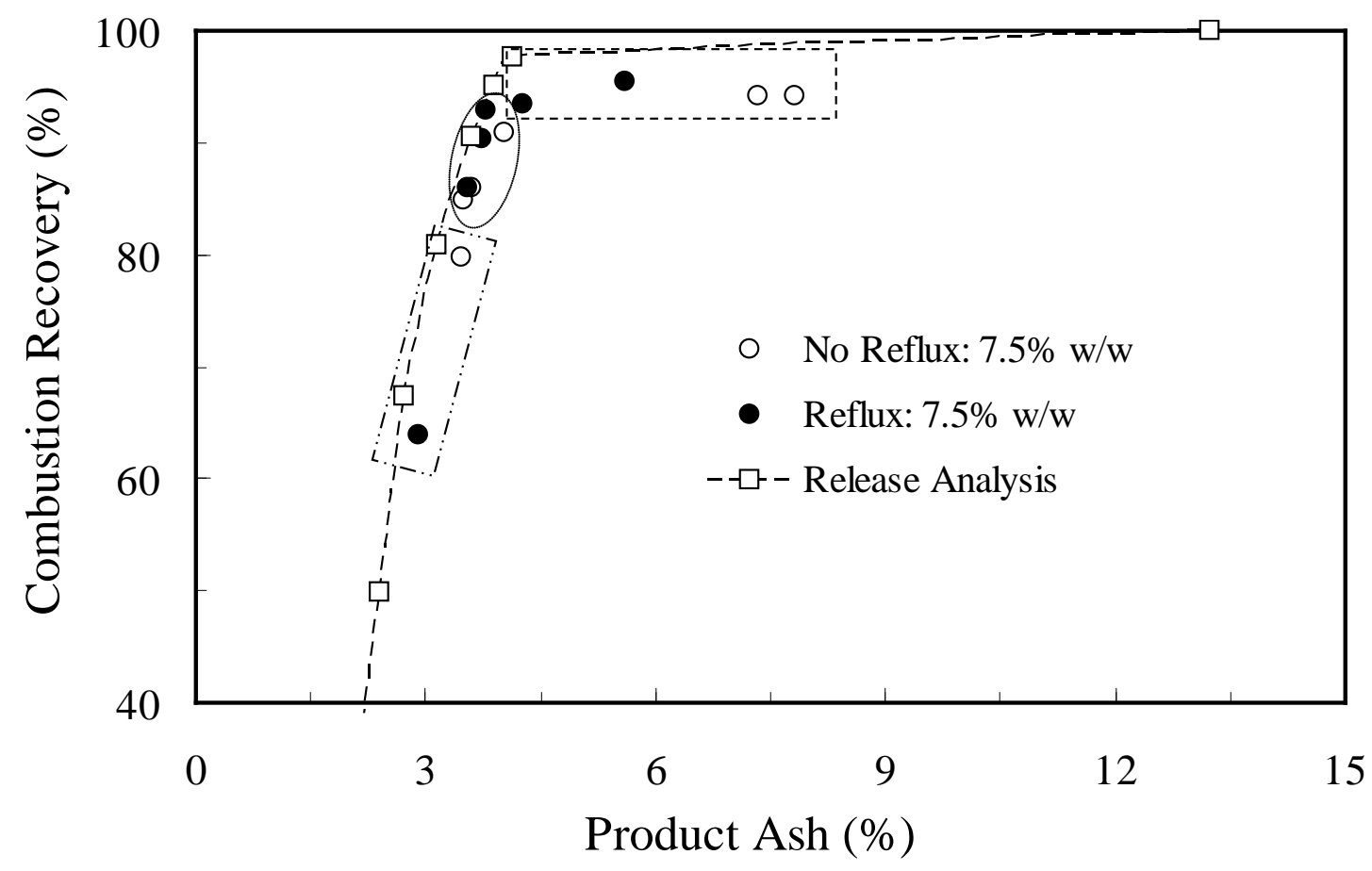

Figure 41. Comparison between continuous column flotation with and without external reflux material at 7.5\% solid content; Pittsburgh No. 8. 


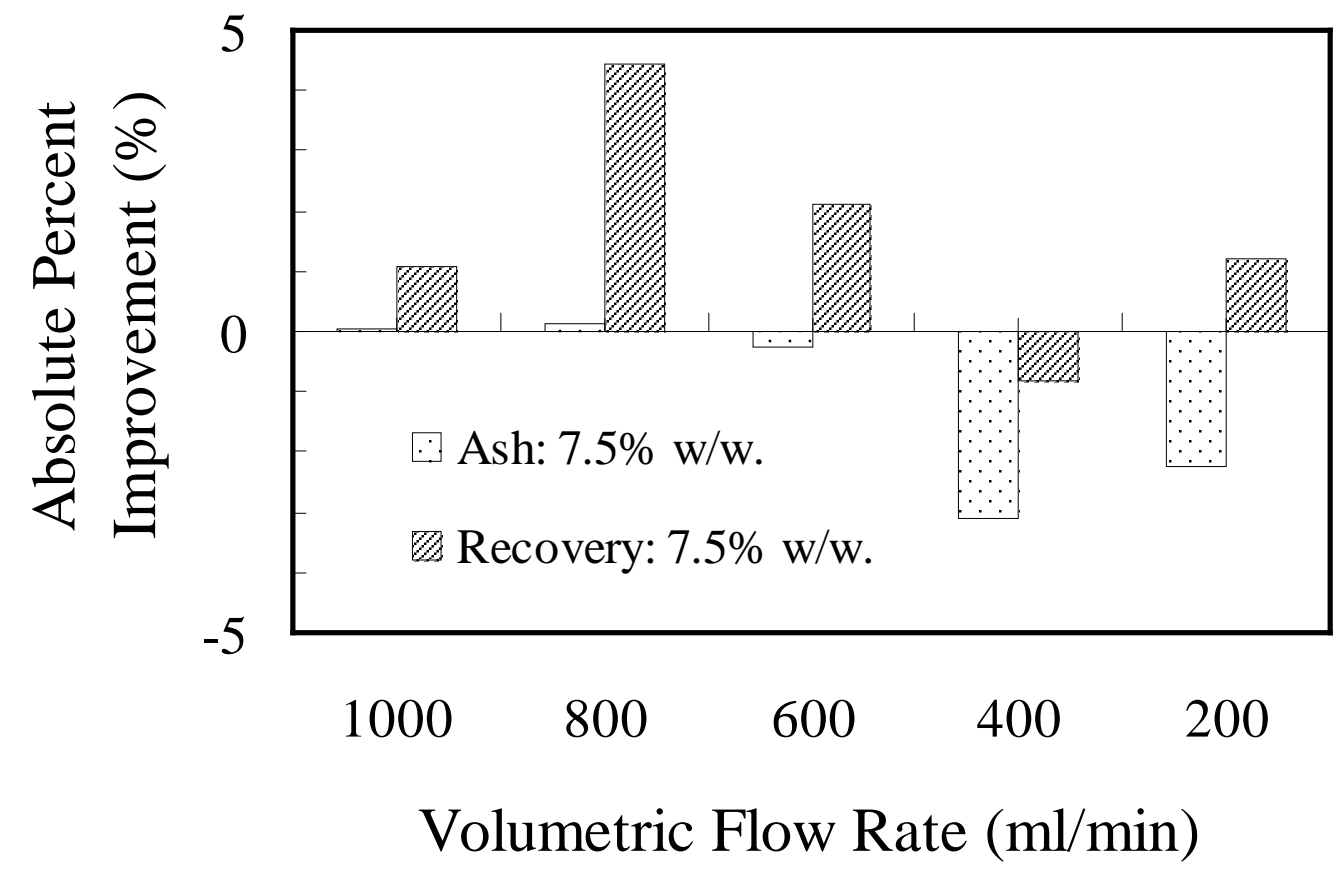

Figure 42. Change in ash content and recovery performance after external reflux into the froth zone in a column flotation respect to baseline performs without refluxing at $7.5 \%$ solid content; Pittsburgh No. 8 coal.

Column flotation tests conducted under low feed solids loading conditions resulted in a similar trend in regards to ash content reduction when employing froth reflux. As shown in Figure 43, the recovery versus product ash curve achieved using refluxing was slightly shifted toward higher recovery and lower ash values as compared to the performance realized when the external froth reflux was not employed. This trend is similar to that described under the high solids conditions in Figure 41. However, the ash content reduction was lower. The ash content was decreased by only 1.5 absolute percentage points at flow rates below $600 \mathrm{ml} / \mathrm{min}$. At higher feed flow rates, a slight ash reduction was realized as shown in Figure 44 but recovery was negatively affected. The overall impact of froth refluxing under low feed solids conditions was minimal which may be due to the availability of bubble surface area even when refluxing a portion of the product. A higher level of refluxing may be needed to realize the impacts on performance under the low solids conditions. 


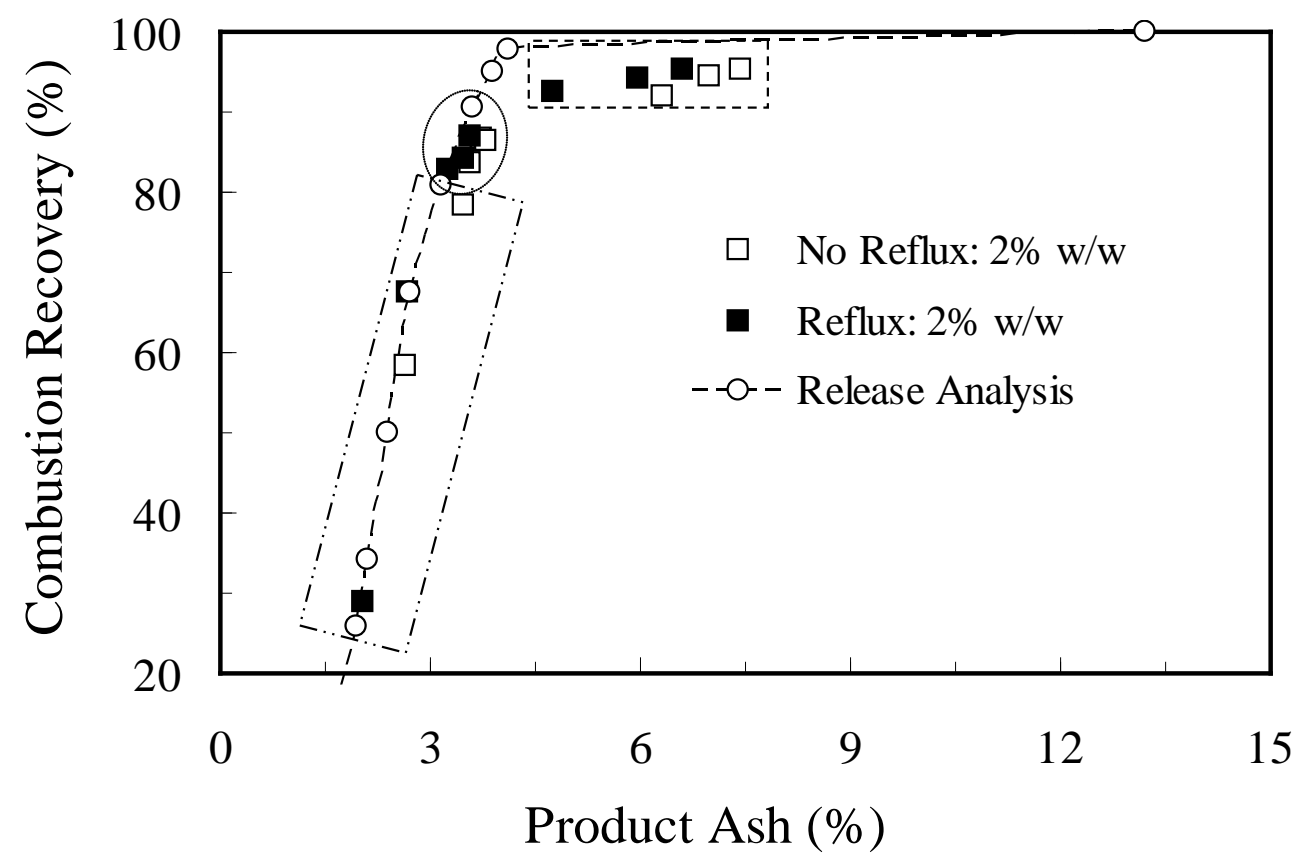

Figure 43. Comparison between continuous column flotation with and without external reflux material at $2 \%$ solid content; Pittsburgh No. 8.

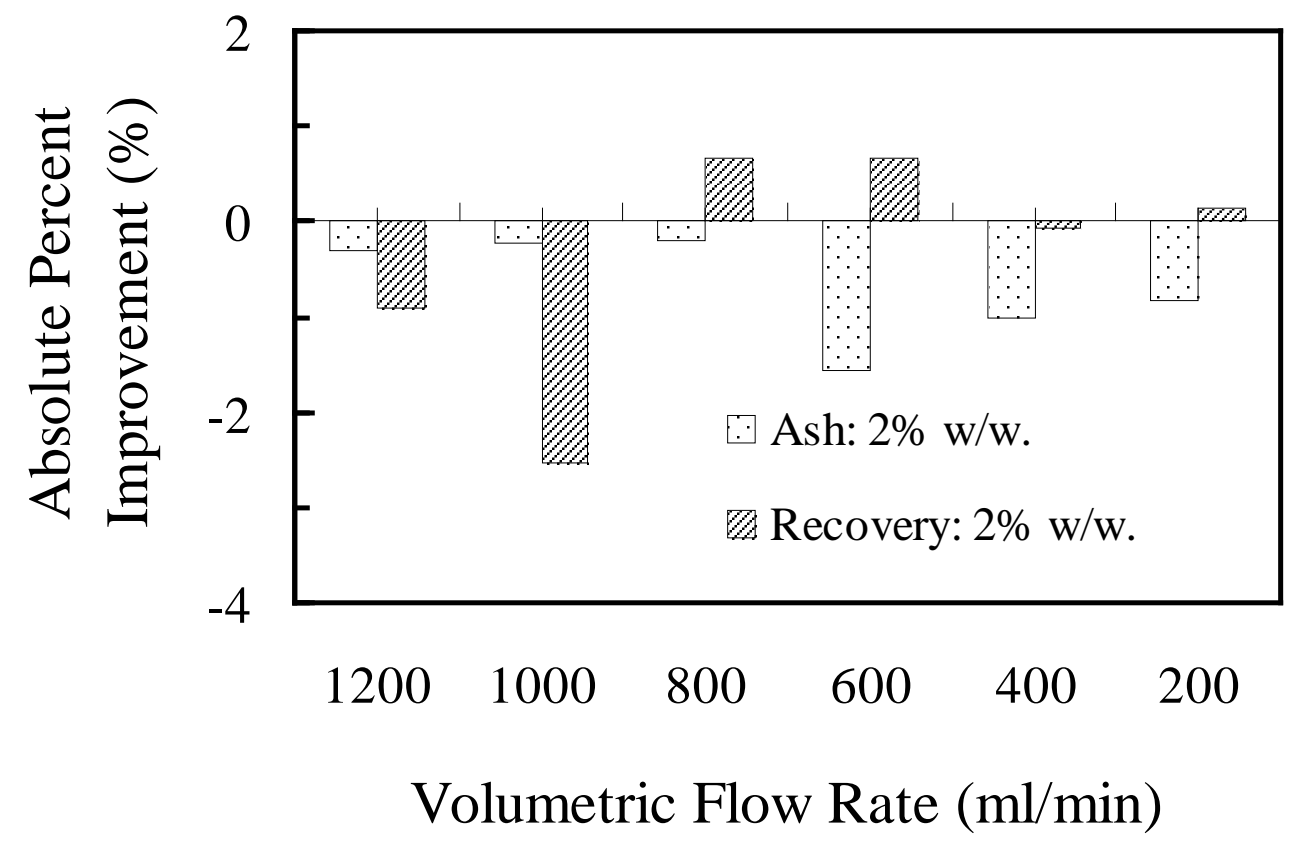

Figure 44. Change in ash content and recovery performance after external reflux into the froth zone in a column flotation respect to baseline performs without refluxing at $2.0 \%$ solid content; Pittsburgh No. 8 coal. 
Coal pyrite is typically weakly-to-moderately hydrophobic naturally. When fuel oil is added, the pyrite floatability increases significantly. The froth reflux action was expected to provide a significant sulfur content reduction benefit for the Pittsburgh No. 8 coal which contains a relatively large amount of pyrite. However, according to the results in Figure 45, no improvement in the sulfur reduction was achieved by froth refluxing in tests conducted under high solids loading conditions. Similar results were also obtained using a $2 \%$ feed solids concentration (Figure 46). The lack of improved selectivity with respect to sulfur reduction may be due to liberation issues and/or the hydrophobic nature of the coal pyrite.

A similar test program was performed on the Coalburg coal with nearly equal results. As described previously, the Coalburg coal contains a significant quantity of middling particles, which are particles comprised of both coal and mineral matter and typically have a low degree of surface hydrophobicity. Thus, selectivity improvements were expected to be greater for the Coalburg coal as compared to that achieved from the treatment of Pittsburgh No. 8 coal.

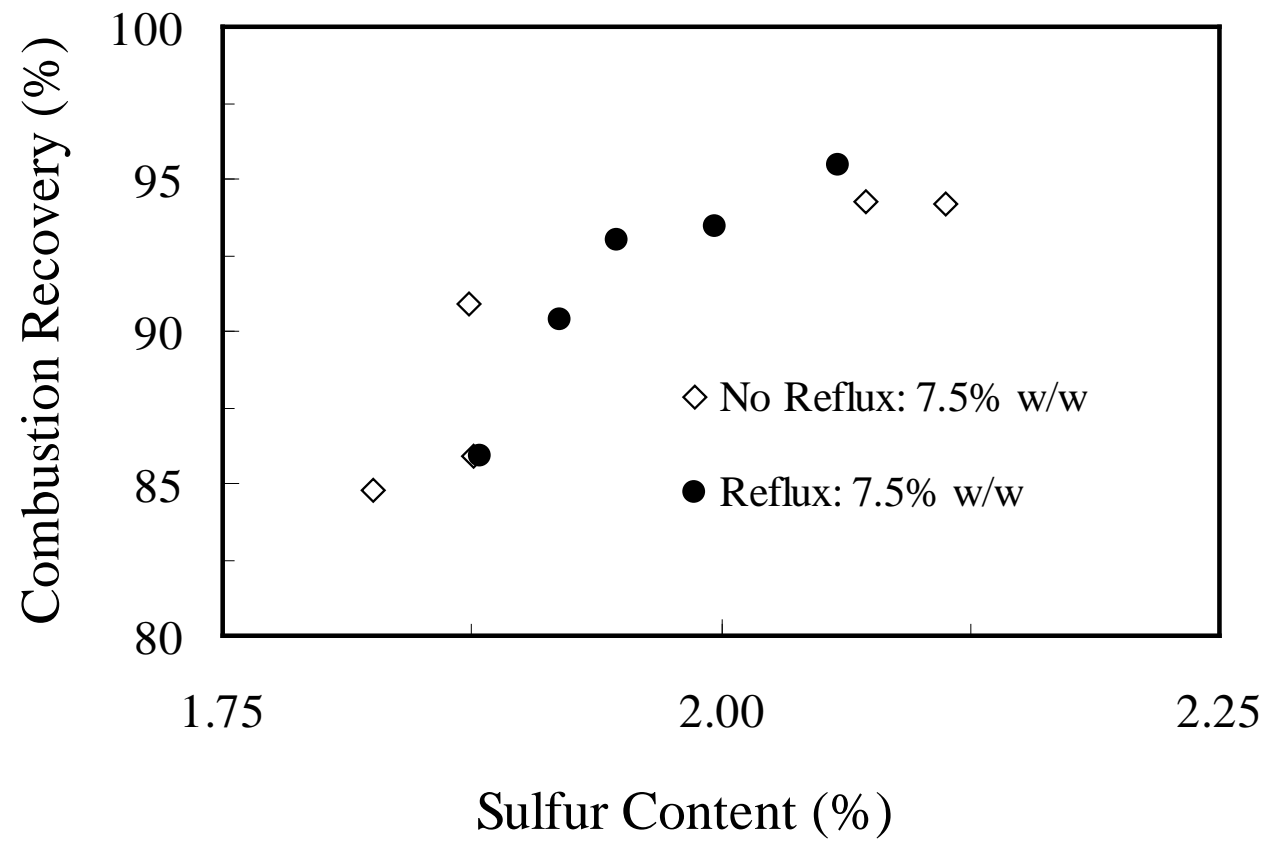

Figure 45. Sulfur reduction comparison from results obtained with and without external froth reflux in a flotation column using a 7.5\% feed solids content; Pittsburgh No. 8 coal. 


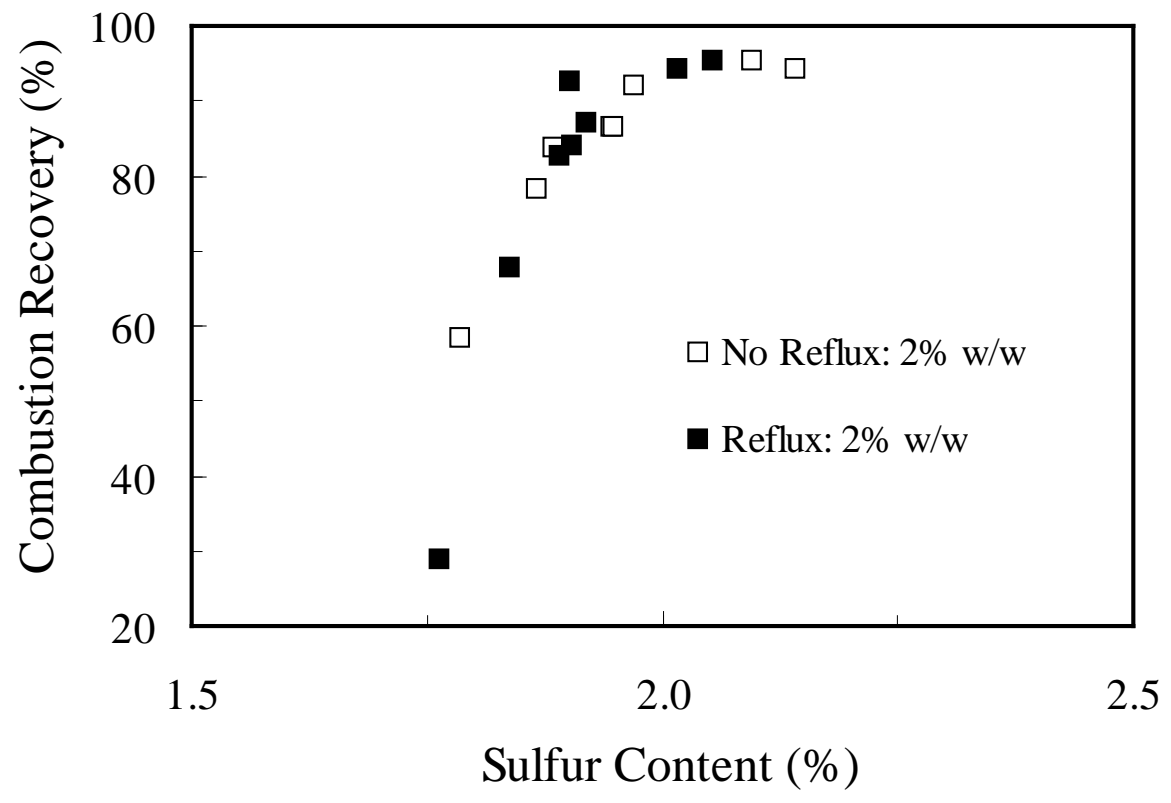

Figure 46. Sulfur reduction performance comparison of results obtained with and without external reflux material in a flotation column being fed at $2 \%$ solids content by weight; Pittsburgh No. 8 coal.

As shown in Figure 47, the recovery versus product ash relationship obtained when refluxing a portion of the froth slightly shifted to lower product ash and recovery values. Figure 48 shows the improvements in both combustible recovery and product ash over the range of volumetric feed rates tested at a feed solids concentration of $7.5 \%$ by weight. The improvement trends were nearly identical to those observed from the data generated when cleaning Pittsburgh No. 8 coal. Ash reductions of 3 to 4 absolute percentage points were achieved at feed rates below $600 \mathrm{ml} / \mathrm{min}$ while little or no improvements were obtained at higher feed flow rates. Positive recovery improvements were obtained at feed rates of $600 \mathrm{ml} / \mathrm{min}$ and higher which was the same finding realized from the Pittsburgh No. 8 coal. 


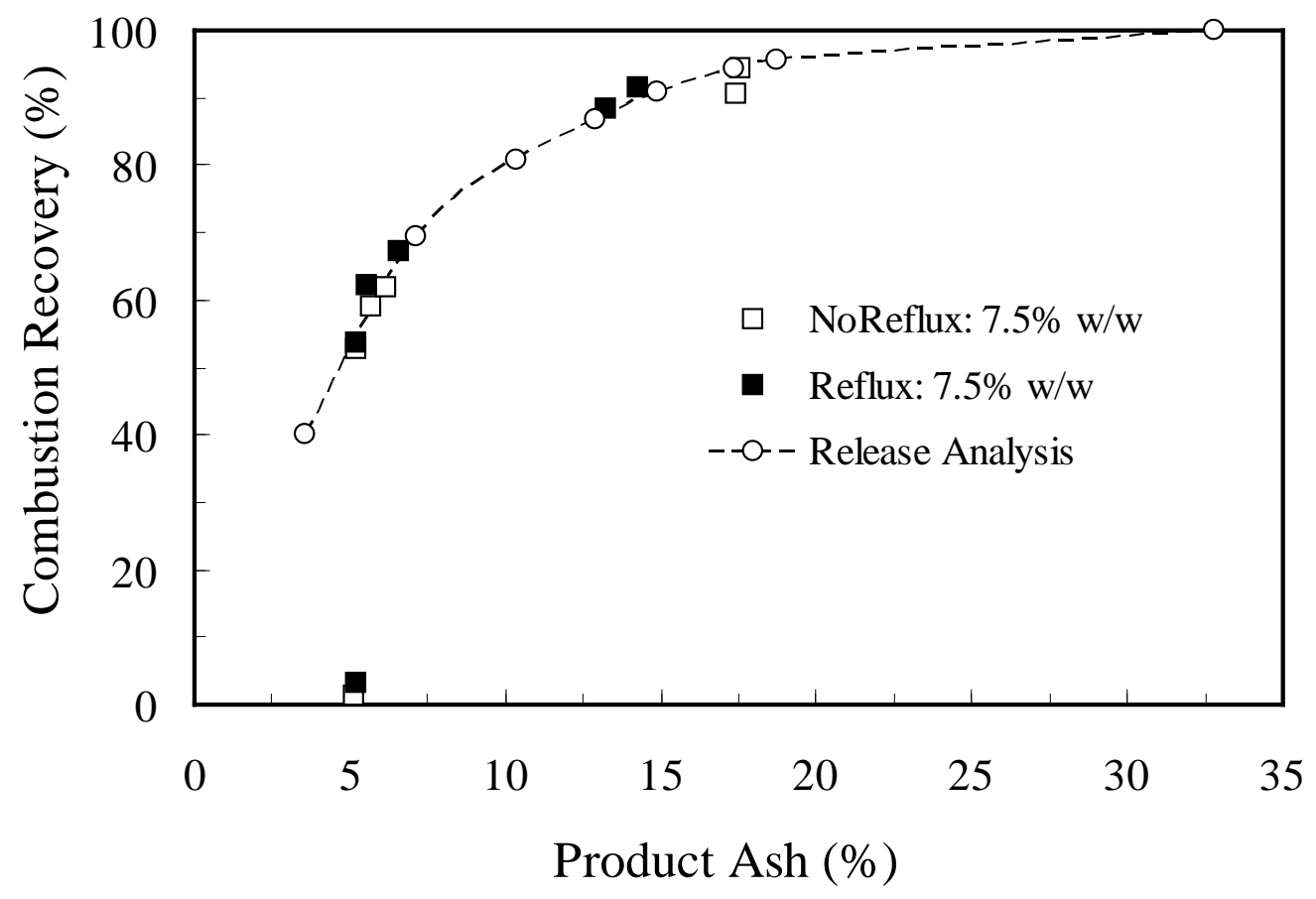

Figure 47. Comparison between separation performances achieved with and without external froth reflux in a flotation column at 7.5\% feed solid content; Coalburg coal.

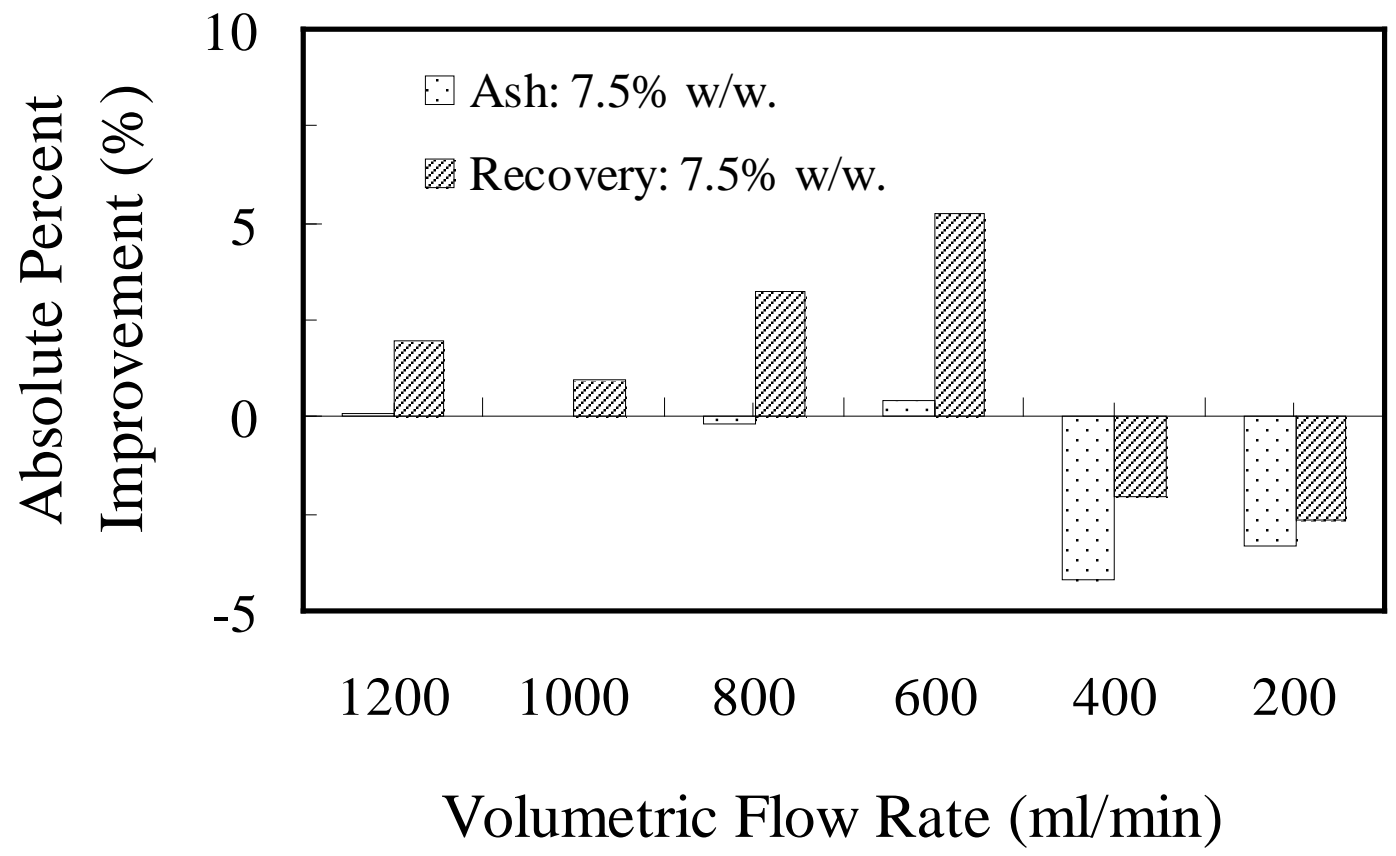

Figure 48. Recovery and product ash content improvements achieved when using external froth refluxing in a flotation column at a feed solids content of $7.5 \%$ by weight; Coalburg coal. 
The results from the $2 \%$ feed solid tests also resemble those achieved on the Pittsburgh No. 8 coal. Figure 49 and 50 clearly indicate ash reductions were achieved up to 4 absolute percentage points at a feed rate of $400 \mathrm{ml} / \mathrm{min}$. The ash reduction was reduced to almost zero as the feed rate was increased above $400 \mathrm{ml} / \mathrm{min}$. No recovery improvement was achieved while a significant reduction was realized at the highest feed rate tested.

Overall, the impact of froth refluxing on separation performance was relatively consistent for the two coal samples and the two solids loading conditions evaluated. The improvement in ash reduction of 3 to 4 absolute percentage points occurred under low feed flow rates which typically are associated with high collection zone recovery values. No recovery improvement was expected; however, elevated recovery values were observed for both coal samples at feed rates above a critical level when using a feed solids concentration of $7.5 \%$ by weight. Surprisingly, no improvement in sulfur reduction resulted from external froth refluxing.

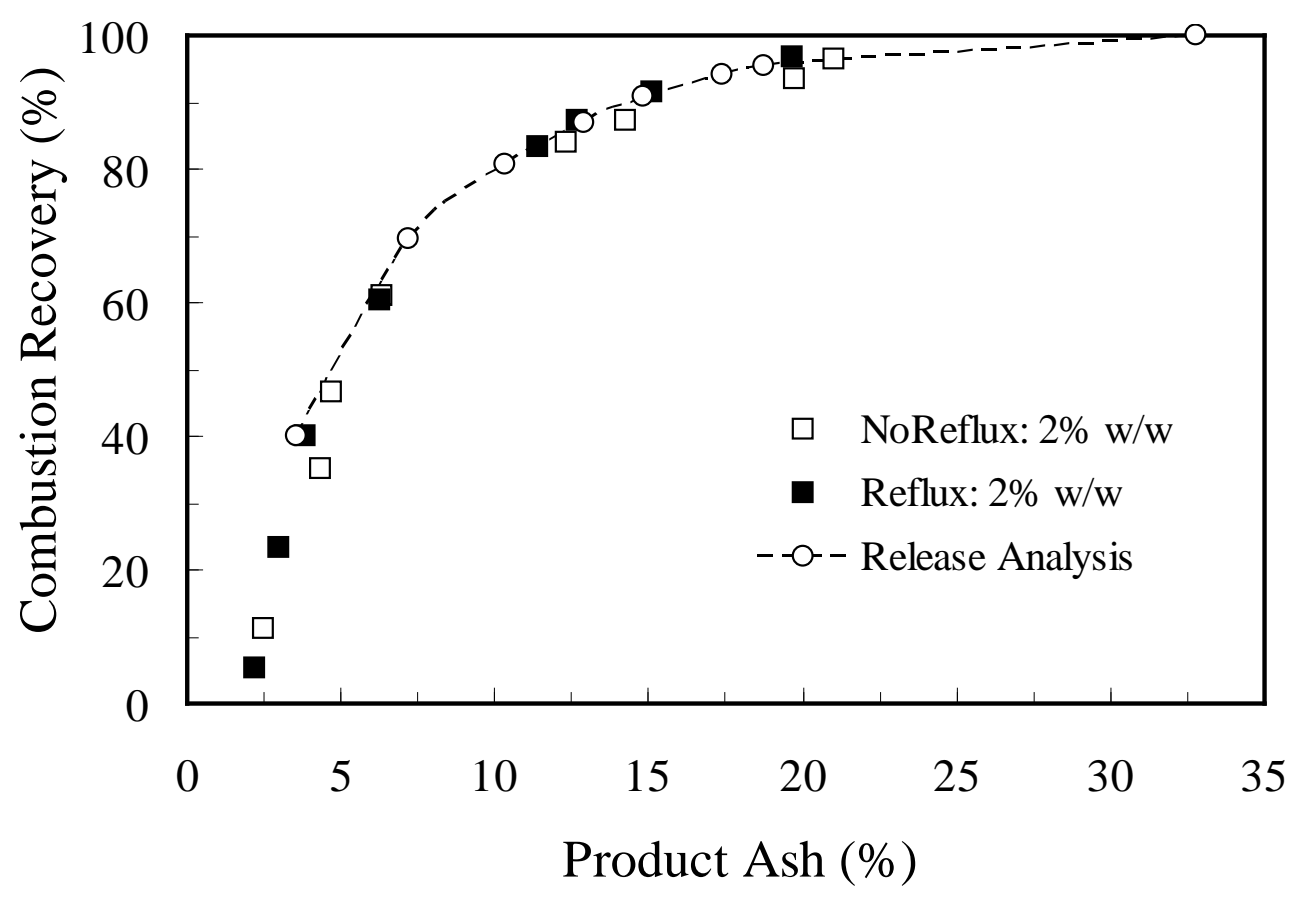

Figure 49. Comparison between separation performances achieved with and without external froth reflux material in a flotation column at $2 \%$ feed solid content; Coalburg coal. 


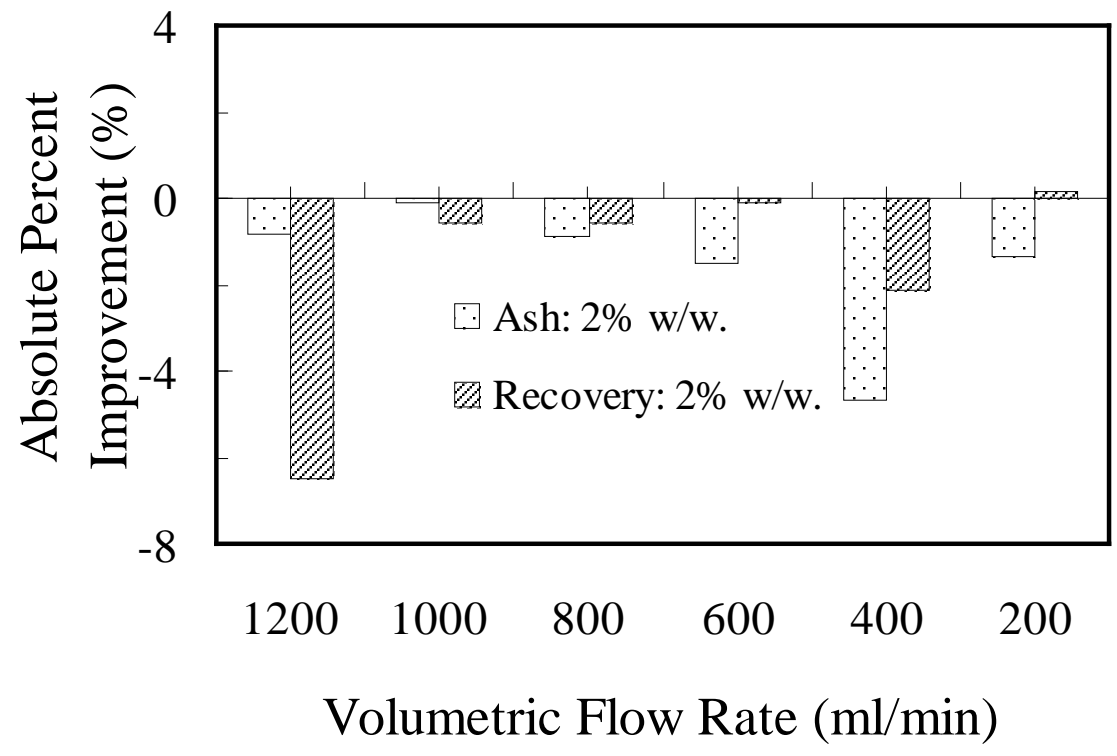

Figure 50. Recovery and product ash content improvements realized from the use of the external froth refluxing with a feed solids content of $2 \%$ by weight; Coalburg coal.

\section{CONCLUSIONS}

In the present study, several techniques to improve flotation performance were explored for the treatment of coal finer than 150 microns, including coal with poor flotation characteristics. Three novel concepts were studied: i) pretreatment of feed coal with hydrodynamic cavitation, ii) injection of magnetic plastic material into the flotation process, and iii) external refluxing to promote selective detachment in the froth zone to improve selectivity. The first technique exploited the picobubble formation phenomena on hydrophobic particles surfaces by hydrodynamic cavitation in an effort to enhance the hydrophobicity of weakly hydrophobic surfaces. The second and third methods used physical and chemical properties of the particles to produce differential flotation rates and selective detachment. Utilization of the selective detachment mechanism and differential flotation rates are the main fundamental principles that determine the overall separation performance.

For the cavitation study, Coalburg seam coal was used for which about $80 \%$ of the material was below 25 microns. The sample was pretreated one or multiple times in the cavitation device before the flotation process. Several flotation rate tests, laboratory column flotation and pilot scale conventional flotation tests in an operating plant were performed.

For the magnetic plastic and froth reflux studies, Pittsburgh No. 8 and Coalburg seam coal samples were utilized. The coal and magnetic plastic material had a particle size that was less than 177 microns. Experiments were carried initially to obtain a baseline 
performance level under standard conditions with no refluxing or plastic additions. For the studies involving the magnetic plastic, flotation rate tests were conducted while varying $\mathrm{pH}$, particle size and the concentration of the added plastic. Subsequently, continuous column flotation tests were performed at optimum parameter levels identified from previous studies. Finally, tests were conducted to evaluate the impact of refluxing a portion of the froth concentrate back to froth zone in a continuous column flotation process. Column flotation tests were performed at different feed solid concentrations and volumetric feed flow rates.

The conclusions obtained from this investigation are provided as follows:

\section{Cavitation Evaluation}

1. The use of an air eductor may be a very inexpensive method to improve coal flotation rate. Micron-sized bubbles are thought to aid in particle attachment to the conventionally-sized bubbles in the flotation cell thereby improving flotation probability. The micron-size bubbles agglomerate fine particles by bridging particles together to the point that the effective size of the agglomerates are able to collide with regular air bubbles more efficiently. Also, the interaction between the bubble coated particle and the conventional bubble is more attractive which reduces the adhesion time and thereby increases flotation rate and recovery, especially for weakly hydrophobic solids.

2. Incorporating a cavitation tube in the feed line to a conventional flotation cell nearly doubled the flotation rate which improved combustible recovery by 18 percentage points. An optimum feed velocity was detected above which recovery decreased. Differences in selectivity were found by using one or multiple repetitions of pretreating the feed slurry. The impact of treatment using the cavitation tube may be greater for particles having a low degree of hydrophobiciy, such as the Coalburg coal. An additional study is recommended to investigate the impact on coal of varying degrees of hydrophobicity.

3. A pilot-scale flotation test was performed side-by-side with a conventional flotation bank in an operating preparation plant. The air eductor test results were superior to those from the full-scale bank. However, the air eductor was not operated properly and thus no real conclusions could be drawn from the pilot-scale study.

\section{Magnetic Plastic Evaluation}

4. The addition of hydrophobic magnetic plastic at a $5 \%$ concentration by weight into feed coal slurry containing moderately hydrophobic Pittsburgh No. 8 coal increased the flotation rate from $0.58 \mathrm{~min}^{-1}$ to $0.93 \mathrm{~min}^{-1}$ under standard conditions. The increase in flotation rate enhanced combustible recovery by about 18 percentage points after one minute of flotation. 
5. For the more weakly hydrophobic Coalburg coal, the flotation rate was improved from $0.51 \mathrm{~min}^{-1}$ to $0.74 \mathrm{~min}^{-1}$ which provided a recovery increase of about 16.3 percentage points after 1 minute of flotation in a conventional cell. The fundamental explanations for the magnetic plastic impact on recovery may include:

a. The hydrophobic plastic particles limit drainage of the water surrounding each air bubble. As a result, bubble coalescence is reduced thereby maximizing the bubble surface area flux rate. The elevation in the amount of surface area available through the froth phase limits preferential detachment of the coarse and weakly hydrophobic particles;

b. Attachment of the plastic material onto the surface of the coal particles thereby yielding a more hydrophobic surface for the bubble-particle attachment process;

c. The hydrophobic plastic material serves as a seed for agglomeration due to strong hydrophobic interaction energies. The large agglomerates are more easily collected in the flotation process;

d. The hydrophobic plastic serves as additional surface area that can be used for the convergence of coal particles into the froth product.

6. It is well known that coal recovery in froth flotation is typically optimum at $\mathrm{pH}$ values around the isoelectric point. Indeed, experiments conducted at $\mathrm{pH}$ values of 3.9 and 10.1 produced flotation rates of around $0.30 \mathrm{~min}^{-1}$ when treating Pittsburgh No. 8 coal. However, after the addition of $10 \%$ magnetic plastic particles, the rate was significantly enhanced to $1.3 \mathrm{~min}^{-1}$ plastic particles at both $\mathrm{pH}$ conditions. Again, this is believed to be due to the strong hydrophobic interactions associated with the plastic particles. The corresponding recovery increase under both conditions was greater than 50 absolute percentage points, which is highly remarkable.

7. The original concept associated with the use of plastic particles involved enrichment of the froth phase to induce selective detachment and, thus improve selectivity. For the Pittsburgh No. 8 coal, very little selectivity improvement was observed from the laboratory conventional cell tests. However, selectivity was significantly improved by the presence of the plastic particle from the treatment of the Coalburg coal. While producing a $15 \%$ ash concentrate, the plastic material increased recovery by nearly 10 absolute percentage points in a conventional cell. The finding can be explained by the large quantity of middling (unliberated) material present in the Coalburg coal.

8. Test results indicate that the coal recovery improvement observed from the addition of the hydrophobic plastic is preferentially associated with the coarse particles, especially those having a particle size equal to $0.10 \mathrm{~mm}$ or greater. This finding 
tends to support the hypothesis that the plastic particles are attaching to the coal surfaces and enhancing their hydrophobicity.

9. This hypothesis was further substantiated by column flotation experiments in which carrying capacity were evaluated. Typically, the product mass flow rate increases and then reaches a maximum at a given feed mass flow rate. Beyond the critical mass flow rate, product mass flow decreases as a result of selective detachment of coarse coal particles. When plastic particles are added directly into the froth, the product mass flow did not decrease but maintained a constant value with an increase in feed flow rate. The only plausible explanation is that the plastic particles reduce the detachment rate of the coarse coal particles. This event occurs due to a stronger binding force between the bubble and the plastic coated coal particles.

10. In the continuous column flotation tests, improvements in recovery were generally less than the conventional cell results and varied as a function of volumetric feed rate and the amount of plastic particles added. At a 5\% concentration, recovery improvement was from around 4 absolute percentage points at feed rates between $400 \mathrm{ml} / \mathrm{min}$ to $800 \mathrm{ml} / \mathrm{min}$ for the treatment of Pittsburgh No. 8 coal. For Coalburg coal, the recovery improvement was around 7 absolute percentage points at a feed rate of $600 \mathrm{ml} / \mathrm{min}$. Lower recovery improvements in the flotation column were expected due to the difference in hydrodynamics conditions between flotation column (i.e. near plug flow) and conventional cells (i.e. near perfectly-mixed). The addition of the magnetic plastic provided no improvement in selectivity.

\section{External Froth Reflux}

11. By refluxing a portion of the froth concentrate back to the froth phase through the wash water, significantly lower product ash contents were achieved under kinetic limiting conditions (low volumetric flow rates). At the same low feed rate, product ash content decreased from around $7 \%$ to nearly $4 \%$ while maintaining recovery at a near constant level for the Pittsburgh No. 8 coal. Similar findings were obtained in tests involving the Coalburg coal.

12. Near the elbow of the recovery-product ash curve, very little to no improvements were observed from froth refluxing. However, at ash content lower than those near the elbow of the curve, the recovery losses due to the froth reflux were evident.

\section{REFERENCES}

Alexander, D.J., Franzidis, J.P. and Manlaping, E.V., 2003, Froth Recovery Measurement in Plant Scale Flotation Cells. Minerals Engineering, 16 (11), 1197-1203.

Ata, S, Ahmed, N. and Jameson, G J, 2002, Collection of Hydrophobic Particles in the Froth Phase, International Journal Of Mineral Processing, 64:101-122. 
Ata, S., Ahmed, N. and Jameson, G.J., 2004, The Effect of Hydrophobicity on The Drainage of Gangue Minerals in Flotation Froths. Mineral Engineering, 17(7-8), 897-901.

Attalla, M., Chao, C. and Nicol, S.K., 2000, The Role of Cavitation in Coal Flotation. Proceedings of the Eight Australian Coal Preparation Conference; Port Stephens, Nov 1216; 337-350.

Bisshop, J.P. and White, M.E., 1976, Study of Particle Entrainment in Flotation Froths. Transactions of The Institution of Mining and Metallurgy, Section C: Mineral Processing and Extractive Metallurgy 85, 191- 194.

Cheng, T. and Holthman, P., 1995, The Particle Detachment Process in Flotation. Minerals Engineering 8 (8), 883-891.

Cilek, E.C. and Yilmazer, B.Z., 2003, Effects of Hydrodynamics Parameters on Entrainment and Flotation, Minerals Engineering, 16 (8), 745-756.

Dippenaar, A., 1982, The Destabilization of Froth by Solids. I. The Mechanism of Film Rupture. International Journal Mineral Processing, 9, 1-14.

Dippenaar, A., 1982, The Destabilization of Froth by Solids. II. The Rate-Determining Step. International Journal Mineral Processing, 9, 15-22.

Falutsu, M., and Dobby, G.S., 1989, Direct Measurement of Froth Drop-Back and Collection Zone Recovery in a Laboratory Flotation Column. Minerals Engineering, 2, 377-386.

Falutsu, M., 1994, Column Flotation Froth Characteristics-Stability of The BubbleParticle System, International Journal of Mineral Processing, 40: 25-243.

Finch, J. A. and Dobby, G. S., 1990, Column Flotation, Pergamon Press: Oxford.

Finch, J.A., Xiao, J., Hardie, C., and Gomez, C.O., 2000, Gas Dispersion Properties: Bubbles Surface Area Flux and Gas Holdup. Minerals Engineering, 13: 365-372.

George, P., Nguyen, A.V., and Jameson, G.J., 2004, Assessment of True Flotation and Entrainment in the Flotation of Submicron Particles by Fine Bubbles. Minerals Engineering, 17 (7-8), 847-853.

Hemmings, C.E., 1981, On the Significance of Flotation Froth Liquid Lamella Thickness Trans. Instn Min. Metall. (Sect. C: Mineral Process. Extr. Metall.) 90, C96.

Honaker, R. Q. and Ozsever, A. V., 2003, Evaluation of the Selective Detachment Process in Flotation Froth, Minerals Engineering, 16(10): 975-982. 
Honaker, R.Q., Ozsever, A.V. and Parekh, B.H., 2006, Selective Detachment Process in Column Flotation Froth, Minerals Engineering, 19(6-8), 687-695.

Johansson, G., and Pugh, R.J., 1992, The Influence of Particle Size and Hydrophobicity on the Stability of Mineralized Froths. International Journal Mineral Processing. 34 (12), $1-21$.

Laplante A.R., Kaya, M., and Smith, H.W., 1989, The Effect of Froth on Flotation Kinetics- A Mass Transfer Approach, In Frothing In Flotation (Laskowski, J.S., Ed.), Gordon and Breach Science Publishers, 77-99.

Malysa, K., 1998, Water Contents and Distribution in Flotation Froths. In: Frothing in Flotation II. Cordon and Breach, Laskowski, J.S., Woodburn, E.T. (Eds.), New York, pp 81-108.

Mathe, Z.T., Harris, M.C. and O’Connor, C.T., and Franzidis, J.P., 1998, Review of Froth Modeling in Steady State Flotation Systems. Minerals Engineering, 11 (5), 397421.

Mathe, Z.T., Harris, M.C., O’Connor, C.T., 2000, A Review of Methods to Model the Froth Phase in Non-Steady Flotation Systems. Minerals Engineering, 13 (2), 127-140.

Moys, M.H., 1978, A Study of a Plug-Flow Model for Flotation Froth Behaviour. International Journal Mineral Processing. 5: 21-38.

Moys, M.H., 1984, Residence Time Distributions and Mass Transport in the Froth Phase of The Flotation Process. International Journal Mineral Processing. 13: 117-142.

Neethling, S.J. and Cilliers, J.J., 1999, Visual Kinematic Model of Flowing Foams Incorporating Coalescence. Powder Technology, 101,249-256.

Neethling, S.J., and Cilliers, J.J., 2002, The Entrainment of Gangue into a Flotation Froth. International Journal of Mineral Processing, 64(2-3), 123-134.

Neethling, S.J., and Cilliers, J.J., 2003, Modelling Flotation Froths. International Journal of Mineral Processing, 72(1-4), 267-287.

Nguyen, A.V., Harvey, P.A., and Jameson, G.J., 2003, Influence of Gas Flow Rate and Frothers on Water Recovery in a Froth Column. Minerals Engineering, 16(11), 11431147.

Ozsever, A. V, 2005, Evaluation of the Selective Detachment Process in Froth Flotation. PhD. Thesis. University of Kentucky. 
Perez, R., and Villar, R.D., 1997. Estimation of Bias and Entrainment in Flotation Columns Using Conductivity Measurements. Canadian Metallurgical Quarterly, 36 (5), 299-307.

Ralston, J., Fornasiero, D and Hayes, R, 1999. Bubble-Particle Attachment and Detachment in Flotation, International Journal of Mineral Processing, 56(1-4),133-164.

Ralston, J. and Dukhin, S.S., 1999. The Interaction between Particles and Bubbles. Colloid Surface, 151, 3-14.

Savassi, O.N., Alexander, D.J., Johnson, N., Franzidis, J.P., and Manlaping, E.V., 1997. Measurement of Froth Recovery of Attached Particles in Industrial Flotation Cells. In: Sixth Mill Operators’ Conference. Madang, Papua New Guinea, pp. 149-156.

Savassi, O.N., Alexander, D.J., Franzidis, J.P., and Manlaping, E.V., 1998. An Empirical Model for Entrainment in Industrial Flotation Plants. Mineral Engineering, 11(3), 243256.

Savassi, O.N., 1998. Direct Estimation of the Degree of Entrainment and Froth Recovery of Attached Particles in Industrial Flotation Cells. Department of Mining, Minerals and Material Engineering, JKMRC. PhD Thesis, University of Queensland, Brisbane, p.393.

Seaman, D., Franzidis, J. and Manlaping E., 2004. Bubble Load Measurement in the Pulp Zone of Industrial Flotation Machines- A New Device For Determining the Froth Recovery of Attached Particles. International Journal of Mineral Processing 74 (1-2), 113.

Subrahmanyam, T.V. and Forssberg, E., 1988. Froth Stability, Particles Entrainment and Drainage in Flotation - A Review. International Journal of Mineral Processing, 23(1-2), 33-53.

Sulman, H.L., Picard H.F.K. and Ballot J., 1905. British patent 7,803, April 12; Duplicated as U.S. Patent 835,120, May 29.

Tao, D., 2004. Role of Bubble Size in Flotation of Coarse and Fine Particles- A review, Separation Science and Technology, 39 (4):741-760.

Van Deventer, J. S. J., Van Dyk, W.A., and Lorenzen, L., 2000. The Separation of Coarse Particles by a Moving Froth Bed. In Proceedings of the $21^{\text {st }}$ International Mineral Processing Congress, Rome. Italy, July 23-28. Amsterdam: Elsevier, C7-1 - C7-8.

Van Deventer, J. S. J., Van Dyk, W.A., Lorenzen, L., and Feng, D., 2000a. The Dynamic Behavior of Particles in Flotation Froths. Part I: Model. Minerals Engineering 15:635645. 
Van Deventer, J. S. J., Van Dyk, W.A., Lorenzen, L., and Feng, D., 2000b. The Dynamic Behavior of Particles in Flotation Froths. Part II: Density Tracer Tests. Minerals Engineering 15:647-657.

Van Deventer, J. S. J., Van Dyk, W.A., Lorenzen, L., and Feng, D., 2000c. The Dynamic Behavior of Particles in Flotation Froths. Part III: Ore particles. Minerals Engineering 15:659-665.

Van Deventer, J. S. J., Feng, D. and Burger, A. J., 2004. Transport phenomena at the Pulp-Froth Interface in a Flotation Column: II Detachment, International Journal of Mineral Processing, 74(1-4): 217-231.

Van Dyk, W.A., van Deventer, J.S.J., and Lorenzen, L., 1995. The Dynamic Behavior of Coarse Particles in Flotation Froths. In Proceeding of the $19^{\text {th }}$ International Minerals Processing Congress. Volume 3. Littleton, CO: SME, 99-103.

Vera, M.A., Franzidis, J.P. and Manlapig, E.V., 1998. Simultaneous Determination of Collection Zone Rate Constant and Froth Zone Recovery Factor. Frothing in Flotation II, Gordon and Breach Science Publications. Amsterdam.

Vera, M.A., Mathe, Z.T., Franzidis, J.P., Harris, M.C., Manlaping, E.V., and O’Connor, C.T., 2002. The Modeling of Froth Zone Recovery in Batch and Continuously Operated Laboratory Flotation Cells. International Journal of Mineral Processing, 64 (2-3), 135151.

Xu, M., Uribe-Salas A. and Finch J.A., 1991. Maximum Gas and Bubble Surface Rates in Column Flotation. International Journal of Mineral Processing, 32, 233-250.

Yianatos, J. B., Finch, J. A. and Laplante, A. R., 1986. Holdup Profile and Bubble Size Distribution of Flotation Column Froths, Can. Met. Q., 25 (1): 23-29.

Yianatos, J. B., Finch, J. A. and Laplante, A. R., 1988. Selectivity in Column Flotation Froths, International Journal of Mineral Processing, 23: 279-292.

Zhou, Z.A., Xu, Z.,. Finch, J.A., Hu, H. and Rao, S.R., 1997. Role of Hydrodynamic Cavitation in Fine Particle Flotation. International Journal Mineral Processing, 51, 139149.

\section{PUBLICATIONS/PRESENTATIONS}

Honaker, R. Q., C. Munoz and M. Mankosa, "Enhancing Coal Flotation Performance Using Recyclable Hydrophobic Particles,” $137^{\text {th }}$ SME Annual Meeting, Salt Lake City, Colorado, February 24 - 27, 2008. 
APPENDIX 2- Measurement of Surface Forces Between Hydrophobic Surfaces (VA016) 


\section{FINAL TECHNICAL REPORT}

Contract Title and Number:

Continuation in Crosscutting Technology Development

at CAST. (DE-FC26-05NT42457)

Sub-Recipient Project Title:

Measurement of Surface Forces between Hydrophobic

Surfaces

Principal Investigators:

Roe-Hoan Yoon

Contact Address:

146 Holden Hall

Blacksburg, VA 24060

Subcontractor Address:
Period of Performance:

Starting Date: 07/26/06

Ending Date: $09 / 30 / 10$
Report Information:

Type: Final

Number:

Period: $\quad$ 07/26/06-9/30/10

Date:

Code: VA016-FINAL

Contact Information:

Phone: 5402314508

Fax: $\quad 5402313948$

E-Mail: ryoon@vt.edu

Subcontractor Information:

Phone:

Fax:

E-Mail:

$\underline{\text { Disclaimer }}$

This report was prepared as an account of work sponsored by an agency of the United States Government. Neither the United States Government nor any agency thereof, nor any of their employees, make any warranty, express or implied, nor assume any legal liability or responsibility for the accuracy, completeness, or usefulness of any information, apparatus, product, or process disclosed, or represents that its use would not infringe privately owned rights. Reference herein to any specific commercial product, process, or service by trade name, trademark, manufacturer, or otherwise does not necessarily constitute or imply endorsement, recommendation, or favoring by the United States Government or any agency thereof. The views and opinions of authors expressed herein do not necessarily state or reflect those of the United States Government or agency thereof. 


\section{ABSTRACT}

In 1982, Israelachvili and Pashley reported the first measurements of a hitherto unknown attractive force between two mica surfaces hydrophobized in cetyltrimethylammonium bromide (CTAB) solutions. Follow-up experiments conducted by many investigators confirmed their results, while others suggested that the "hydrophobic force" is an artifact due to nanobubbles (or cavitation). Evidences for the latter included the discontinuities (or steps) in the force versus distance curves and the pancake-shaped nanobubbles seen in atomic force microscopic (AFM) images. Recent measurements conducted in degassed water showed, however, smooth force versus distance curves, indicating that the hydrophobic force is not an artifact due to nanobubbles. ${ }^{1,2}$

Still other investigators ${ }^{3,4}$ suggested that the long-range attraction observed between hydrophobic surfaces is due to the correlation between the patches of adsorbed ionic surfactant and the patches of unoccupied surface. For this theory to work, it is necessary that the charged patches be laterally mobile to account for the strong attractive forces observed in experiment. In an effort to test this theory, AFM force measurements were conducted with gold substrates hydrophobized by self-assembly of alkanethiols and xanthates of different chain lengths. The results showed long-range attractions despite the fact that the hydrophobizing agents chemisorb on gold and, hence, the adsorption layer is immobile.

When the gold surfaces were hydrophobized in a $1 \times 10^{-3} \mathrm{M}$ thiol-in-ethanol solution for an extended period of time, the force curves exhibited steps. These results indicate that the long-range attractions are caused by the coalescence of bubbles, as was also reported by Ederth. ${ }^{5}$ The steps disappeared, however, when the species adsorbed on top of the chemisorbed monolayer were removed by solvent washing, or when the gold substrates were hydrophobized in a $1 \times 10^{-5} \mathrm{M}$ solution for a relatively short period of time.

AFM force measurements were also conducted between gold substrates coated with short-chain thiols and xanthates to obtain hydrophobic surfaces with water contact angles $(\theta)$ of less than $90^{\circ}$. Long-range attractions were still observed despite the fact that cavitation is thermodynamically not possible.

Having shown that hydrophobic force is not due to coalescence of pre-existing bubbles, cavitation, or correlation of charged patches, the next set of force measurements was conducted in ethanol-water mixtures. The attractive forces became weaker and shorterranged than in pure water and pure ethanol. According to the Derjaguin "s approximation ${ }^{6}$, an attractive force arises from the decrease in the excess free energy $\left(\gamma^{\mathrm{f}}\right)$ of the thin film between two hydrophobic surfaces. ${ }^{7}$ Thus, the stronger hydrophobic forces observed in pure water and pure ethanol can be attributed to the stronger cohesive energy of the liquid due to stronger $\mathrm{H}$ bonding. Further, the increase in hydrophobic force with decreasing separation between two hydrophobic surfaces indicates that the $\mathrm{H}$-bonded structure becomes stronger in the vicinity of hydrophobic surfaces.

The force measurements conducted at different temperatures in the range of $10-40^{\circ} \mathrm{C}$ showed that the hydrophobic attraction between macroscopic surfaces causes a decrease in film entropy $\left(S^{f}\right)$, which confirms that the hydrophobic force is due to the structuring of water in the thin film between two hydrophobic surfaces. The results showed also that the hydrophobic interaction entails a reduction in the excess film enthalpy $\left(\mathrm{H}^{\mathrm{f}}\right)$, which may be associated with the formation of partial (or full) clathrates formed in the vicinity of 
hydrophobic surfaces. The presence of the clathrates is supported by the recent finding that the density of water in the vicinity of hydrophobic surfaces is lower than in the bulk. ${ }^{8}$

\section{References}

1. Meyer, E. E.; Lin, Q.; Israelachvili, J. N., Langmuir 2005, 21, 256-259.

2. $\quad$ Zhang, J.; Yoon, R.-H.; Mao, M.; Ducker, W. A., Langmuir 2005, 21, 5831-5841.

3. Miklavic, S. J.; Chan, D. Y. C.; White, L. R.; Healy, T. W., J. Phys. Chem. 1994, 98, 9022-9032.

4. Meyer, E. E.; Lin, Q.; Hassenkam, T.; Oroudjev, E.; Israelachvili, J. N., Proc. Nat. Acad. Sci. U.S.A 2005, 102, 6839-6842.

5. $\quad$ Ederth, T.; Claesson, P.; Liedberg, B., Langmuir 1998, 14, 4782-4789.

6. Derjaguin, B. V., Kolloid Zeits 1934, 69, 155-164.

7. Eriksson, J. C.; Ljunggren, S.; Claesson, P. M., J. Chem. Soc., Faraday Trans. 2 1989, 85, (3), 163-176.

8. Doshi, D. A.; Watkins, E. B.; Israelachvili, J. N.; Majewski, J., Proc. Nat. Acad. Sci. U.S.A 2005, 102, 9458-9462. 


\section{Table of Contents}

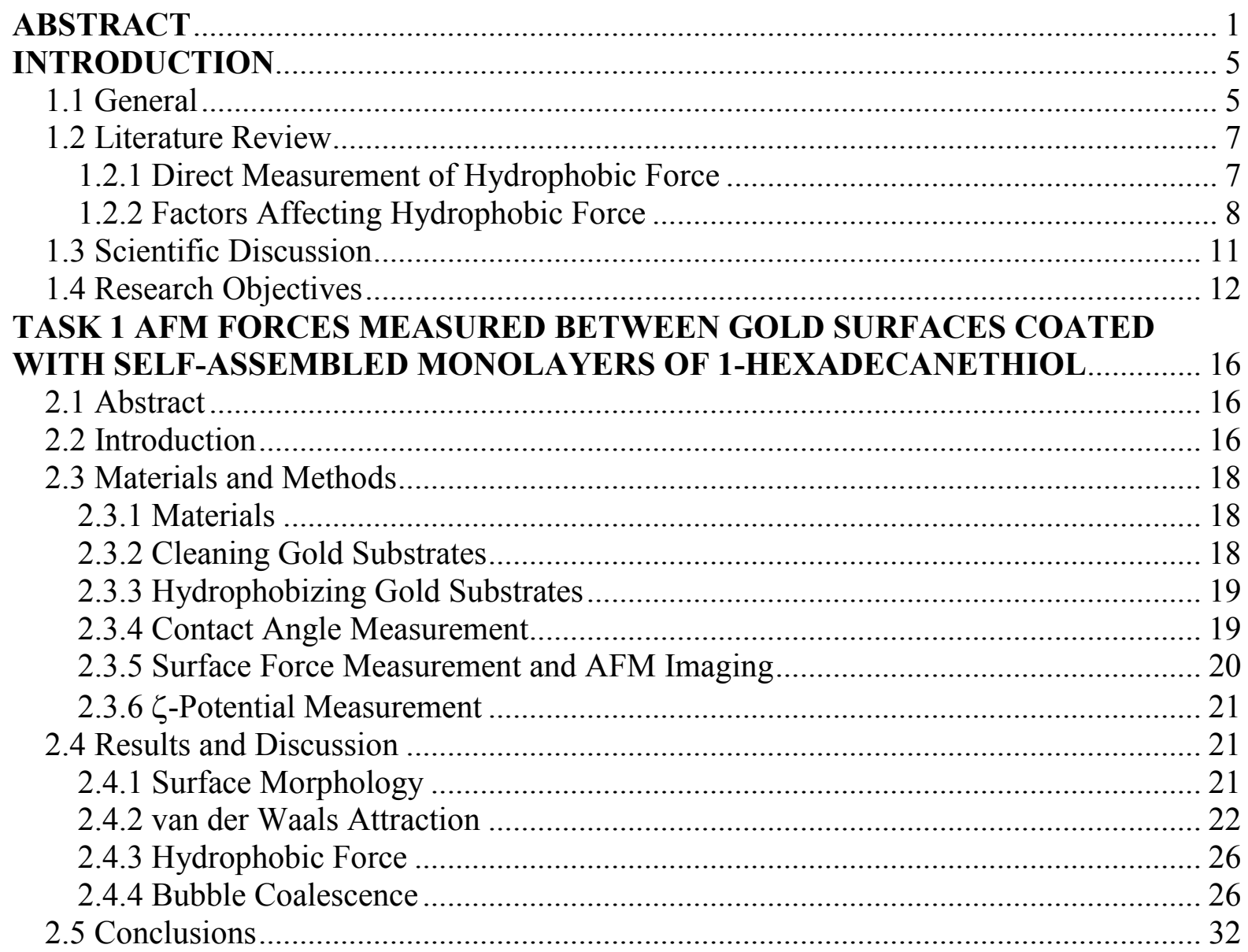

TASK 2 SURFACE FORCE MEASUREMENTS BETWEEN GOLD SURFACES HYDROPHOBIZED WITH ALKANETHIOLS OF DIFFERENT CHAIN LENGTHS

3.1 Abstract

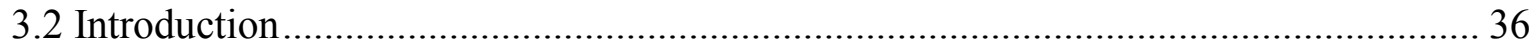

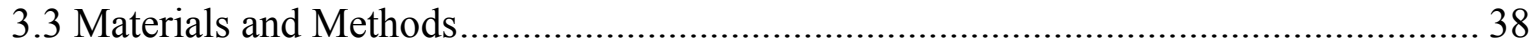

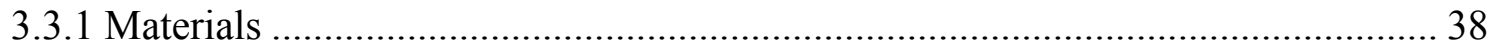

3.3.2 Hydrophobizing Gold Surfaces....................................................................... 39

3.3.3 Contact Angle Measurement.................................................................................. 40

3.3.4 AFM Surface Force Measurement.................................................................... 40

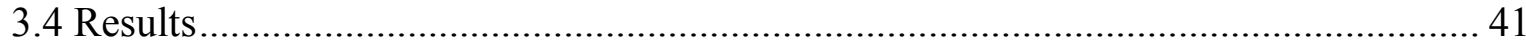

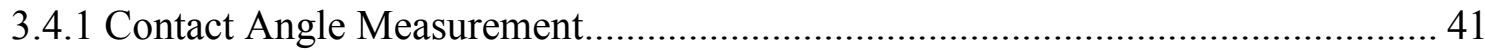

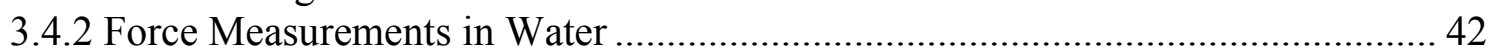

3.4.3 Force Measurements in $\mathrm{NaCl}$ Aqueous Solutions ............................................... 46

3.4.4 Force Measurements in the Presence of $\mathrm{C}_{12} \mathrm{TACl}$ Surfactant.................................. 48

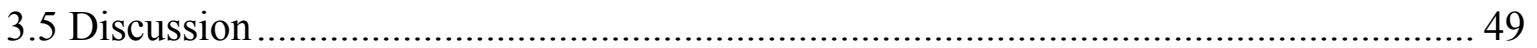

3.5.1 The Existence of the Long-Range Hydrophobic Force ....................................... 49

3.5.2 Effect of Surface Adsorption on the Hydrophobic Force ....................................... 51

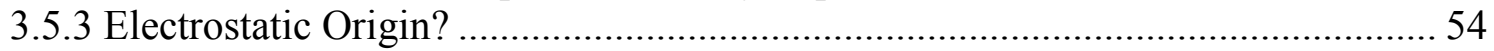

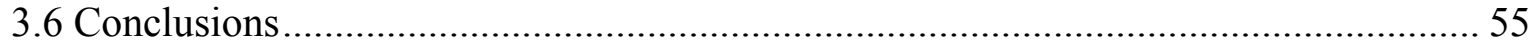




\section{TASK 3 SURFACE FORCE BETWEEN GOLD SURFACES IN XANTHATE}

SOLUTIONS AND ITS IMPLICATION IN FLOTATION ....................................... 59

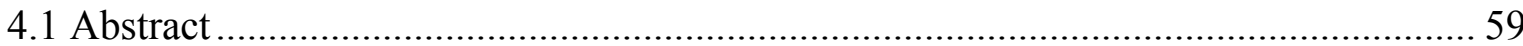

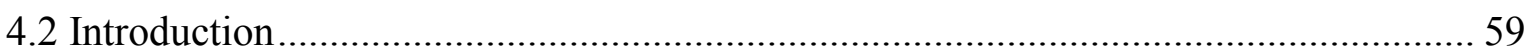

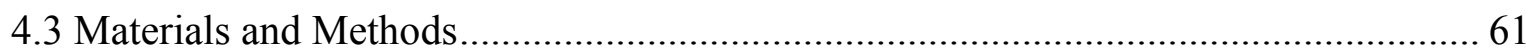

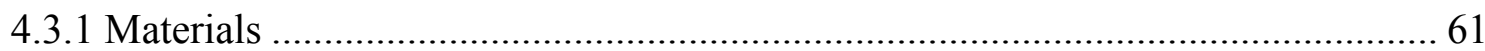

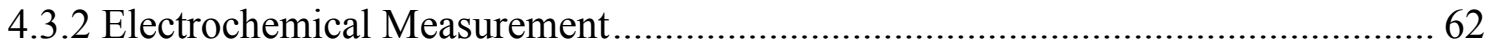

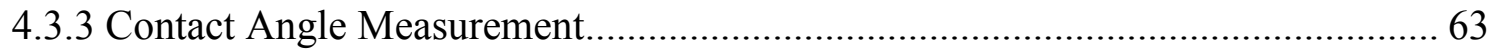

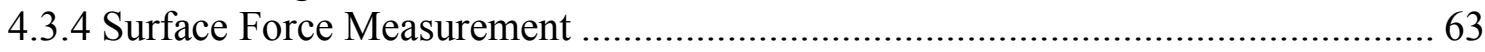

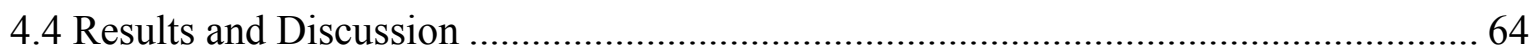

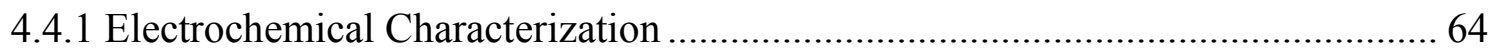

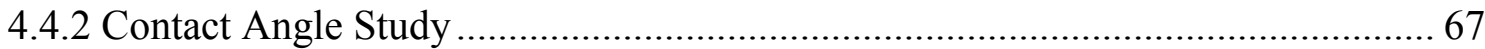

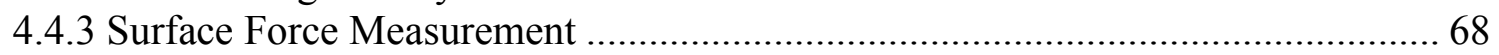

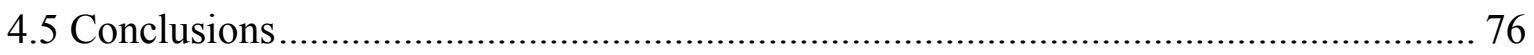

TASK 4 SURFACE FORCES BETWEEN HYDROPHBIZED GOLD SURFACES SUBMERGED IN ALCOHOLS AND IN WATER-ETHANOL MIXTURES ............ 78

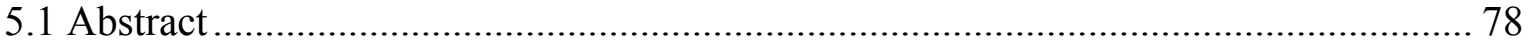

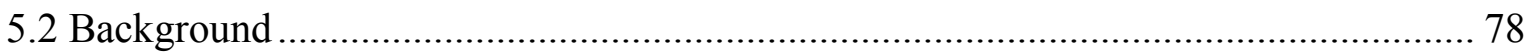

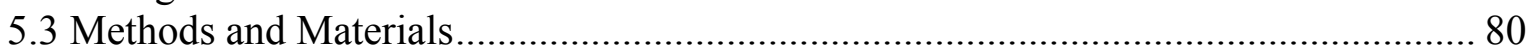

5.3.1 Surface Force Measurements by Means of AFM .......................................... 80

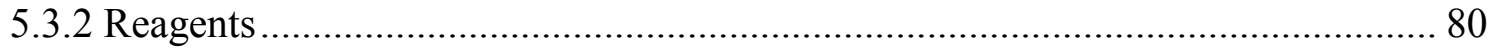

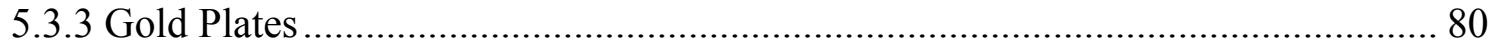

5.3.4 Gold Probes............................................................................................. 81

5.3.5 Preparation of Hydrophobic Surfaces ........................................................... 81

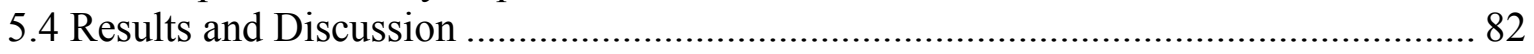

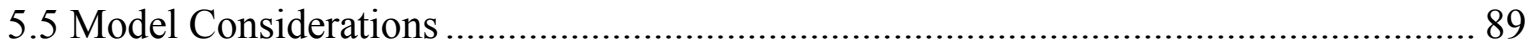

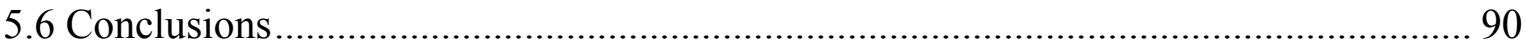

TASK 5 HYDROPHOBIC ATTRACTION ORIGINATES FROM CHANGES IN

WATER STRUCTURE: THERMODYNAMIC EVIDENCE …............................... 92

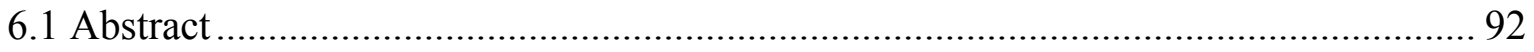

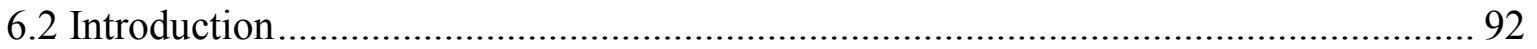

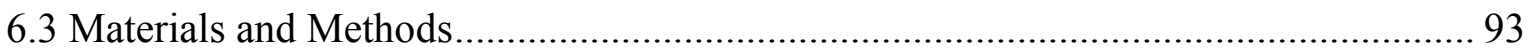

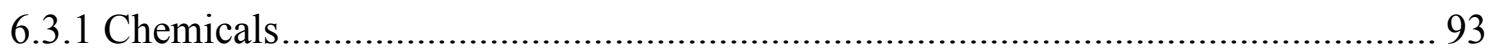

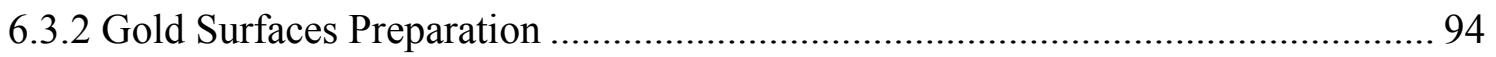

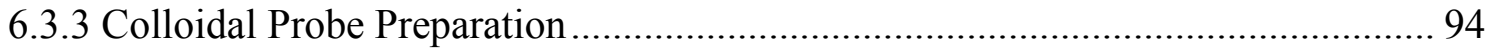

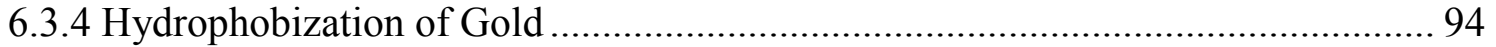

6.3.5 AFM Force Measurement .......................................................................... 95

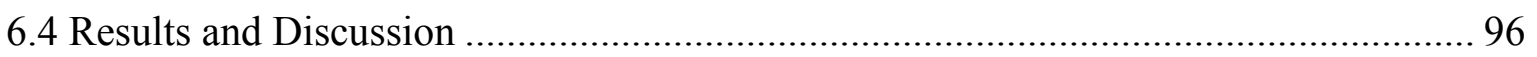

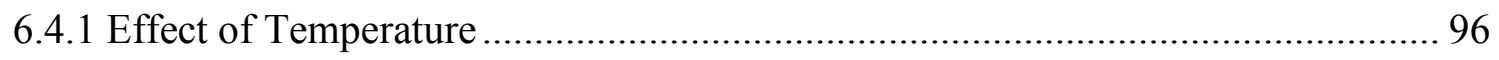

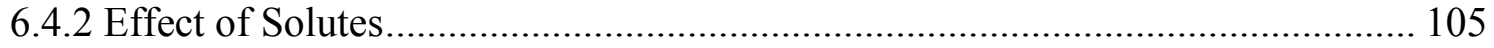

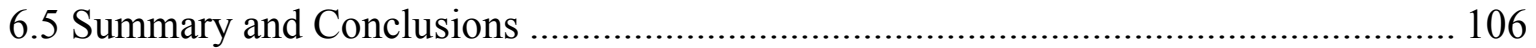

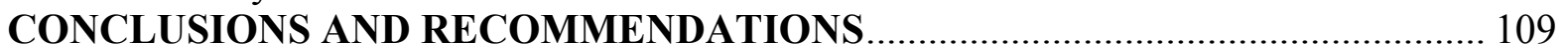

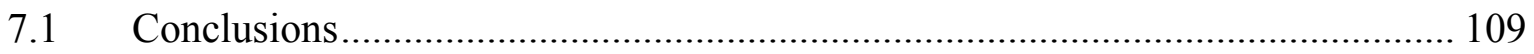

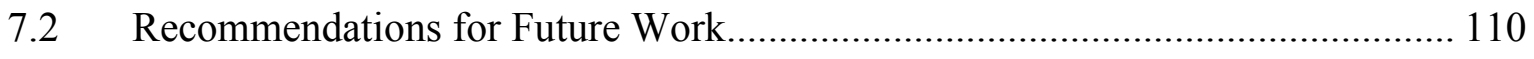

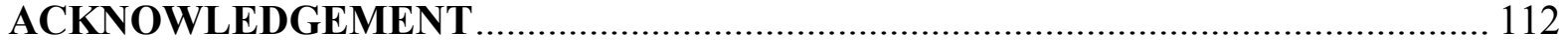




\section{INTRODUCTION}

\subsection{General}

Froth flotation is the most widely used solid-solid separation process employed in the mining industry. The process is designed to separate hydrophobic particles from hydrophilic ones. Therefore, control of particle hydrophobicity is most important in flotation. For this reason, the early days of flotation research was focused on developing various reagents (collectors) that can be used to hydrophobize different minerals. During these early days of research, contact angle was used as the measure of hydrophobicity. However, it is a thermodynamic property and, hence, doses not give kinetic information. On the other hand, flotation is a kinetic process, and the industry strives to improve flotation rate and, thereby, to maximize recovery and throughput.

In colloid chemistry, the kinetics of coagulation can be predicted by the classical DLVO theory ${ }^{1,2}$, which considers two surface forces, namely, repulsive electrical doublelayer force $\left(F_{e}\right)$ and attractive van der Waals dispersion force $\left(F_{d}\right)$. These two surface forces are considered additive. Thus, the total interaction force $\left(F_{t}\right)$ between two particles can be given as follows:

$$
F_{t}=F_{e}+F_{d}
$$

Typically, a plot (Figure 1.1) of Equation 1.1 shows a maximum repulsive force $\left(F_{\max }\right)$ at a critical separation distance $\left(H_{c r}\right)$ between two colloidal particles. If $F_{\max }$ is large,

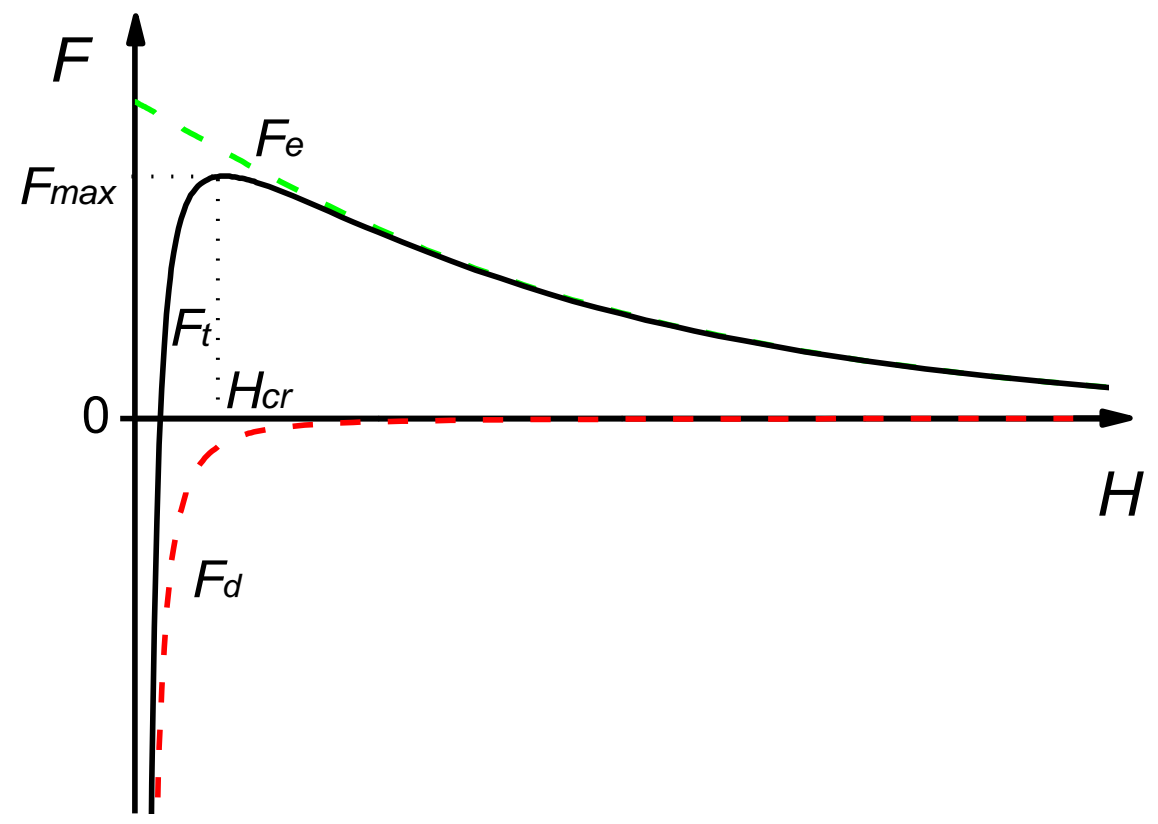

Figure 1.1 Surface interaction $(F)$ versus distance $(H)$ diagram for particle-particle interaction, with $F_{\max }$ representing the energy barrier. 
coagulation of the particles are slow; and if $F_{\max }$ is small, the coagulation is fast. As such, the DLVO theory is useful for describing the interactions between small mineral particles with limited degree of hydrophilicity ${ }^{3}$ in water (e.g., coagulation and dispersion), but not for the interactions between hydrophobic air bubbles and hydrophobic particles (i.e., flotation). In the latter, both $F_{e}$ and $F_{d}$ are repulsive under most conditions where flotation is carried out ${ }^{4}$. Thus, the DLVO theory cannot be used to describe flotation processes that are spontaneous and fast in most cases.

The DLVO theory is a theoretical model, which was verified in direct surface force measurements conducted between microscopic surfaces ${ }^{5}$. However, the force measurements conducted between two hydrophobic surfaces exhibited an additional attractive force, which is naturally referred to as "hydrophobic force" . Many investigators showed subsequently that the hydrophobic forces were 10 to 100 times larger than the van der Waals force ${ }^{7}$. It was shown also that coagulation of hydrophobic particles can be modeled much better by recognizing the existence of the hydrophobic forces as follows ${ }^{8,9}$ :

$$
F_{t}=F_{e}+F_{d}+F_{h}
$$

where $F_{h}$ represents the hydrophobic force term. It was shown that Equation 1.2, which is referred to as extended DLVO theory, can also be used for modeling the bubble-particle interactions in flotation ${ }^{10-12}$. Under most flotation conditions, both $F_{e}$ and $F_{d}$ are repulsive, as has already been noted. In such cases, $F_{h}$ is the only driving force for bubble-particle attachment, as shown in Figure 1.2, and it is not possible to model flotation without

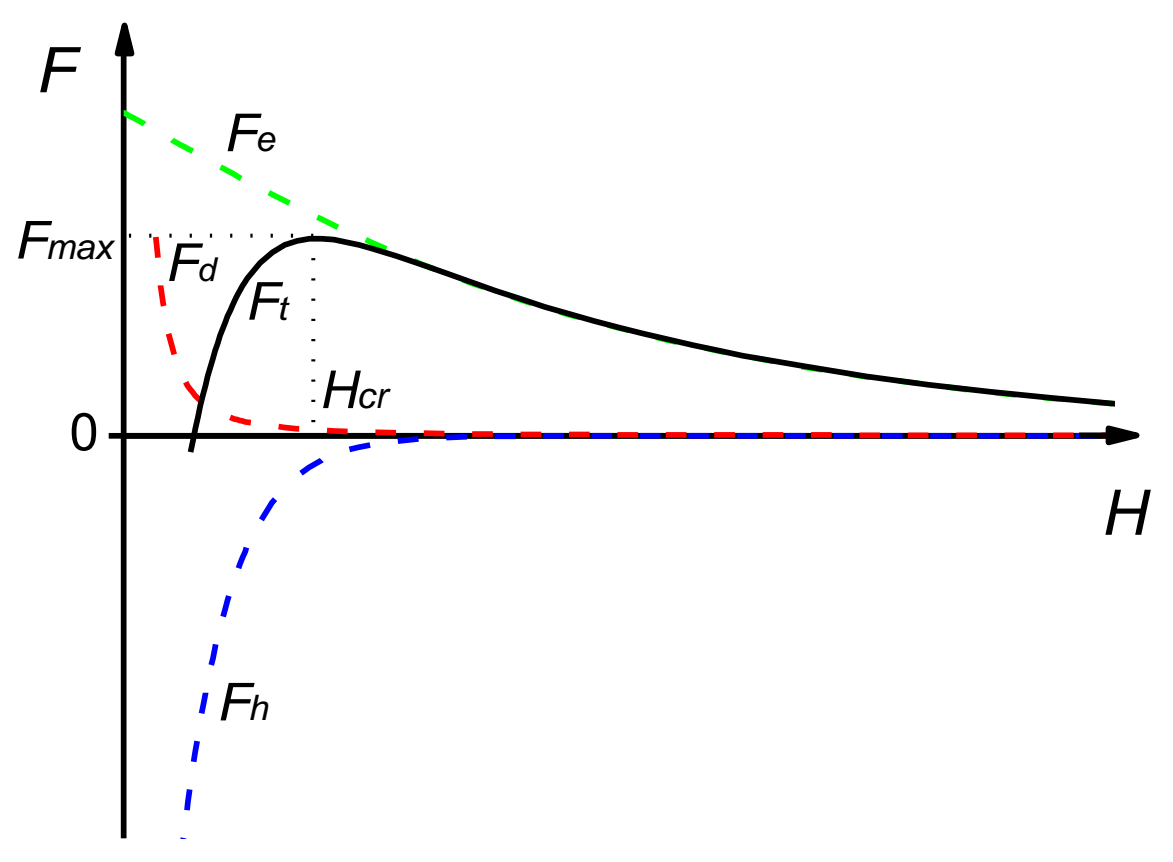

Figure 1.2 Surface interaction $(F)$ versus distance $(H)$ diagram for bubble-particle interaction, with $F_{h}$ representing the hydrophobic force and $F_{\max }$ the energy barrier. 
recognizing the existence of the hydrophobic force.

However, there has been a great deal of controversy regarding the existence and the origin of the hydrophobic force. Some investigators suggested that it is due to nano-size bubbles nucleating on hydrophobic surfaces ${ }^{13}$ or cavities ${ }^{14}$, while others believe that the socalled hydrophobic force is actually an electrostatic attraction ${ }^{15-18}$. It is, thus, the objective of the present work to conduct surface force measurements between hydrophobic surfaces and determine the possible origin(s) of the long-range attractions observed.

\subsection{Literature Review}

\subsubsection{Direct Measurement of Hydrophobic Force}

Initially, most of the force measurements were conducted using surface force apparatus (SFA), which was limited to measurements between two curved semitransparent mica sheets. In the SFA technique, surface force was measured between two crossed cylinders of radius of $R_{1}$ and $R_{2}$ as a function of separation distance $H$. The surfaces force was obtained by measuring the deflection of a spring, and the separation distance was accurately determined using an interferometer. One can relate the measured force $F(H)$ to the free energy of interaction per unit area $W(H)$ between surface $\mathbf{1}$ and $\mathbf{2}$, as follows:

$$
F(H)=2 \pi\left(\frac{R_{1} R_{2}}{R_{1}+R_{2}}\right) W(H)
$$

which is known as Derjaguines approximation ${ }^{19}$.

Rabinovich and Yoon ${ }^{20,21}$ used atomic force microscope (AFM) to measure the hydrophobic forces between a glass sphere with a radius of $R$ and a flat silica plate (with $R=$ $\infty$ ), which made it easier to measure the hydrophobic forces between a varieties of macroscopic solids, e.g., polypropylene ${ }^{22}$, polystyrene ${ }^{23}, \mathrm{ZnS}^{24}$, gold ${ }^{25}$, etc. The AFM uses a photodetector to detect the deflection of a cantilever spring resulting from the interaction of a colloidal particle attached to the apex of the cantilever with a flat substrate which sits on a piezoelectric scanner. The surface interaction can be calculated from the deflection of the cantilever using Hooke"s law and the known spring constant of the cantilever. The movement of the surfaces relative to one another is accurately controlled by the piezoelectric scanner. The force versus distance data obtained using AFM were presented by normalizing the measured forces by the radius $R$ of the sphere (glass) used for the measurement. According to the Derjaguin approximation (Equation 1.3), the normalized force $F(H) / R$ could be related to the free energy of interaction $W(H)$ per unit area between the sphere and flat surfaces. For the sphere-plate geometry, Equation (1.3) is reduced to:

$$
F(H) / R=2 \pi W(H)
$$

The results should be independent of the force-measuring technique, and one can directly compare the results obtained using different methods. However, surface force measurements are difficult when one or both surfaces are deformable. 
Since no theory is able to explain all the experimental results, the measured hydrophobic forces were usually represented by an empirical force $\operatorname{law}^{26,27}$ :

$$
F / R=C \exp (-H / D)
$$

where $h$ is the closest separation distance between two surfaces, and $C$ and $D$ are the fitting parameters for the experimental data. The first parameter characterizes the strength of the force, while the second parameter characterizes its distance range. The second parameter is often referred to as decay length. In general, the stronger the hydrophobic force is, the more negative the value of $C$ and the larger the value of $D$ becomes. form $^{20,28,29}$ :

The force data were also fitted by a double exponential force law in the following

$$
F / R=C_{1} \exp \left(-H / D_{1}\right)+C_{2} \exp \left(-H / D_{2}\right)
$$

in which $C_{1}$ and $C_{2}$ represent the magnitudes of the short- and long-range hydrophobic forces, respectively, and $D_{1}$ and $D_{2}$ are the respective decay lengths.

Another way of representing the hydrophobic forces measured in experiment is to use a power $\operatorname{law}^{20}$ :

$$
F / R=-K_{131} / 6 H^{2}
$$

where $K_{131}$ is the hydrophobic force constant between two solids 1 in water 3. Equation (1.7) has only one fitting parameter as compared to two in Equation 1.5 and four in Equation 1.6. Equation 1.7 is of the same form as the van der Waals dispersion force; therefore, the value of $K_{131}$ is directly comparable with the Hamaker constant $\left(A_{131}\right)$.

\subsubsection{Factors Affecting Hydrophobic Force}

In 1982, Israelachivili and Pashley ${ }^{6}$ published a seminal paper in Nature, which showed the presence of a third surface force. They measured an additional attractive force between two mica surfaces hydrophobized by cetyltrimethylammonium bromide (CTAB). Naturally, the non-DLVO attractive force was named hydrophobic force, which was not only stronger than the van der Waals force but was also longer-ranged, that is, the force was discernable at longer separation distances. The work of Israelachvili and Pashley created interests among many colloid chemists, with numerous follow-up measurements on different types of hydrophobic surfaces using a range of different methods of hydrophobization, as has been reviewed recently ${ }^{7,13,30-33}$.

A wide range of factors affecting the hydrophobic force have been investigated, which are summarized as follows:

1. Type of hydrophobic surface. Christenson and Claesson ${ }^{7}$ presented a state of the art review of the hydrophobic force from an experimental perspective. Recognizing the large variation of the measured interaction is dependent on the type of hydrophobic surface, they classified the non-DLVO attractive forces observed in water between hydrophobic surfaces into three different categories. (I) Stable hydrophobic surfaces, e.g., polymerized Langmuir-Blodgett (LB) films deposited on mica ${ }^{34}{ }^{35}$, bulk polymer 
surfaces $^{22}$, show a fairly short-range but strongly attractive force, much stronger than the van der Waals force. (II) Very hydrophobic surfaces, e.g., silica or glass surfaces made hydrophobic by silylation ${ }^{36-38}$, gold surfaces made hydrophobic by self-assembly of thiol $^{25,39,40}$, give an attraction of variable strength and range caused by the presence of small bubbles sporadically adhering to hydrophobic surfaces. (III) Results obtained with a variety of hydrophobic surfaces, e.g., LB films of surfactants or lipids on mica or silica $^{28,41-43}$, in-situ adsorbed surfactant on silica ${ }^{38,44-47}$, appear to give rise to a very-long range attractive force with exponential decay.

2. Surface hydrophobicity. Rabinovich and $\operatorname{Yoon}^{20}$ measured the hydrophobic force between a silica bead and a silica plate surfaces, which were hydrophobized with varying amounts of octadecylchlorosilane (OTS) or trimethylchlorosilane (TMS) as a means of controlling contact angle. The range of the measured hydrophobic force depended on the contact angle, varying from approximately $30 \mathrm{~nm}$ for the surface contact angle $\theta_{a}=88^{\circ}$ to over $100 \mathrm{~nm}$ for $\theta_{a}=115^{\circ}$. Yoon and Ravishankar ${ }^{48}$ measured hydrophobic forces between mica surfaces in dodecylammonium chloride $(\mathrm{DAHCl})$ solutions in the presence of dodecanol or octanol. The results showed that when $\theta_{a}<90^{\circ}$, only short range hydrophobic forces were measured with decay lengths of around $1.3 \mathrm{~nm}$. Long-ranged hydrophobic forces, resulting from the formation of domains of close-packed hydrocarbon chains, were observed at $\theta_{a} \geq 90^{\circ}$. Ederth and Liedberg ${ }^{49}$ investigated the influence of wetting properties on the long-range hydrophobic interaction between gold surfaces hydrophobized by self-assembly of methyl- and hydroxyl-functionalized alkanethiols. The results showed that whenever the advancing water contact angle on the hydrophobized gold surfaces exceeded $90^{\circ}$, long-range attraction appeared and the attractive force curves had steps or discontinuities. When the contact angle was lower than $90^{\circ}$, the interaction was a van der Waals force.

3. Salt effect. Claesson et al. ${ }^{28}$ first investigated the electrolyte effect, and they found that the interaction between water-stable hydrophobic LB monolayers on mica was weakly dependent on $\mathrm{KBr}$ concentration up to a concentration of $0.01 \mathrm{M}$. Christenson et al. ${ }^{50}$ studied the effect of divalent electrolyte $(0.01 \mathrm{M}$ magnesium sulfate sand $0.1 \mathrm{M}$ magnesium sulfate solutions) on the hydrophobic attraction between two mica surfaces coated with LB film of dimethyldioctadecylammonium bromide (DDOABr). They found that the magnitude of the hydrophobic attraction was reduced with increasing electrolyte concentration but remained much larger than the van der Waals force. Parker et al. ${ }^{36}$ conducted surface force measurements using chemically fluorinated glass surfaces in the presence of $\mathrm{KBr}$ and $\mathrm{NaCl}$. They found that no reduction in force upon addition of electrolyte, except at very high salt concentrations $(5 \mathrm{M} \mathrm{NaCl})$ where the strength of the attractive forces increased slightly. Meagher and $\mathrm{Craig}^{22}$ measured the hydrophobic interaction between two polypropylene surfaces in $\mathrm{NaCl}$ solutions. The results showed that increasing $\mathrm{NaCl}$ concentration up to $1 \mathrm{M}$ had little or no effect on the range of the interaction. Craig et al ${ }^{51}$ demonstrated that the hydrophobic attraction was undiminished between silica surfaces in soluble cetylpyridinium chloride in the presence of $0.1 \mathrm{M}$ $\mathrm{NaCl}$. Kekicheff and Spalla ${ }^{17}$ measured surface force between electrically neutral glass surfaces in aqueous solution of cetyltrimethylammonium bromide (CTAB) at $p \mathrm{H}$ about 5.7. It was found that the long-range attractive force decayed with the addition of $\mathrm{KBr}$ $\left(10^{-5}-10^{-2} \mathrm{M}\right)$. Zhang et al. ${ }^{46}$ conducted AFM force measurements between silica sphere and fused-silica plate in aqueous octadecyltrimethylammonium chloride $\left(\mathrm{C}_{18} \mathrm{TACl}\right)$ 
solutions. The results showed the attractive force was screened by an added electrolyte $(\mathrm{NaCl})$.

4. Dissolved gas. Meagher and $\mathrm{Craig}^{22}$ used a modified AFM to measure the hydrophobic interaction between polypropylene surfaces in $\mathrm{NaCl}$ solutions. They showed that the measured attraction in dilute $\mathrm{NaCl}$ solutions was reduced upon removal of the gas. Rabinovich and Yoon $^{21}$ demonstrated that attractive interaction between OTS-coated silica surfaces in water became considerably stronger when the water was saturated with argon. Craig et al. ${ }^{52}$ measured the hydrophobic forces between silica surfaces hydrophobized by adsorption of cetylpyridinium chloride (CPC). The effect of dissolved gas on the measured interaction was studied. It was shown that the removal of dissolved gas decreased the range and magnitude of the attraction at long range. Mahnke et al. ${ }^{53}$ investigated the influence of dissolved gas on the interactions between silica surfaces hydrophobized by either dehydroxylation or methylation. They found degassing caused a significant reduction in large jump distance $(>25 \mathrm{~nm}$ ), but not in the smaller jump distance. For dehydroxylated silica surfaces interacting in $\mathrm{CO}_{2}$ saturated solution, they found the jump distance were considerably larger than in the presence of air or argon. Considine $e t \mathrm{al}^{54}$ measured the surface forces between two polystyrene latex spheres in aqueous solution containing different amount of gas. They found that the range of the attraction decreased significantly when the level of dissolved gas in the water was reduced. Sakamoto et al. ${ }^{55}$ conducted force measurements between silica surfaces in aqueous $\mathrm{C}_{18} \mathrm{TACl}$ solutions in the presence and absence of dissolved gas. They concluded that long-range attractive force was not observed in carefully degassed solutions. Zhang et al. ${ }^{46}$ repeated the experiment of Sakamoto et al., however, they found instead that the long-range hydrophobic force still existed in degassed solution. Stevens et $a l^{56}$ studied the effects of degassing on the long-range attractive force between hydrophobic amorphous fluoropolymer surfaces. They found the range of the attraction was significantly decreased in degassed water, but the range and magnitude of the attraction remained greater than the van der Waals attraction. The effect of dissolved gas on the hydrophobic attraction between dimethyldioctadecylammonium bromide(DODAB) coated mica was studied by Meyer et al..$^{57}$ using a surface forces apparatus (SFA). Removal of dissolved gas was seen to reduce the range of the attraction while the short-range attraction $(<25 \mathrm{~nm})$ remained unchanged.

5. Temperature effect. Tsao et al. ${ }^{29}$ measured the attractive forces between two hydrophobized mica surfaces immersed in water at three different temperatures in the range $25-50^{\circ} \mathrm{C}$. The results showed that a very long range attractive force changed dramatically with temperature, the attraction decreasing with increasing temperature. They attributed the decrease in attractive force to the change of the state of the hydrocarbon chains on the surface when the temperature was increased. Parker et al. ${ }^{36}$ investigated the effect of temperature using stable surfaces prepared by covalent modification of silica with fluorocarbon silane. It was shown that there was a significant increase in the strength of the interaction measured between silane-coated mica surfaces when the temperature was increased from room temperature to $41^{\circ} \mathrm{C}$.

6. Ethanol effect. Parker $\mathrm{et} \mathrm{al.}{ }^{36}$ showed that the addition of ethanol reduced the strength of the long range forces between silane-coated silica surfaces. Kokkoli et al. ${ }^{58}$ also showed that addition of ethanol to water resulted in a decrease in the strength of the hydrophobic attraction between self-assembled monolayers of hexadecanethiol on gold. As the 
ethanol concentration was increased further ( $75 \%$ mole fraction), the hydrophobic attraction became comparable to the van der Waals force. Ederth ${ }^{40}$ showed that the attractive force measured between two hexadecanethiol-coated gold surfaces in water containing $12.5 \%$ ethanol (by weight) was slightly shorter-ranged than that in pure water. As the ethanol concentration was increased to $20 \%$, the attractive force measured was the van der Waals force. Nguyen et al. ${ }^{37}$ measured attraction between silanated glass surfaces in water-ethanol mixtures. The results showed that the strong attractive force decreased with an increase in the ethanol content and disappeared in pure ethanol.

7. Chain order. Rabinovich et al..$^{59}$ investigated the dependence of hydrophobic force on the chain order for mica surfaces coated by double-chain surfactant (dimethyldioctadecylammonium, DDOA) monolayers. It was found the hydrophobic force increased with the ordering the hydrocarbon chains. It was thus suggested that the hydrophobic force was related to the hydrocarbon chains ordering, which in turn may affect the structure of vicinal water.

\section{$\underline{1.3 \text { Scientific Discussion }}$}

Many research groups around the world conducted surface force measurements with different types of hydrophobic surfaces, and showed the existence of long-range attractions. However, the results are far from being consistent. Therefore, many investigators cast doubts about the long-range force being the real hydrophobic force. Nevertheless, various theoretical models and ideas have been rendered to explain the experimental results. Eriksson et al. ${ }^{60}$ carried out a theoretical analysis to suggest that the long range hydrophobic attraction is due to the enhanced hydrogen bonding of the water molecules in the vicinity of a hydrophobic solid. Some other investigators considered that they are due to surface-induced perturbation in the adjacent fluid ${ }^{61}$, ion-ion correlations ${ }^{62}$, mobile charged patches ${ }^{15}$, correlated in plane dipoles $^{48,63}$, lack of stability of hydrophobic surface groups ${ }^{64}$, and rearrangement of the charged patchy bilayers ${ }^{33}$. However, these models cannot be reconciled with all of the experimental data, particularly with regards to salt effect. In particular, Miklavic et al. ${ }^{15}$ predicted that the attraction due to charge correlation should decay exponentially with a decay length equal to one-half of the Debye length $\left(\kappa^{-1}\right)$. Although there are some experiment results consistent with this theory ${ }^{61,62}$, more and more experimental data show that the force is independent of ionic strength ${ }^{22,36,51}$.

More recently, many investigators showed evidence that the non-DLVO attractions observed during the force measurements are artifacts introduced during the measurement. Tyrrell and Attard ${ }^{65,66}$ and Ishida et al. ${ }^{67}$ showed that the "so-called" hydrophobic force is caused by the small bubbles (nanobubbles) preexisting on hydrophobic surfaces. Christenson and Claesson ${ }^{14}$ and Yaminsky and Ninham ${ }^{68}$ suggested that the very long range forces measured are a consequence of cavitations between microscopic hydrophobic surfaces. The most frequently quoted evidences for the air-bubbles were the discontinuities (or steps) observed in force versus distance curves $^{36}$, each step representing coalescence of nanobubbles. When two bubbles coalesce, gas bridges (or cavities) are formed between two surfaces, which give rise to capillary forces. Tyrell and Attard ${ }^{66}$ and Yang et al. ${ }^{69}$ actually showed AFM images of the nanobubbles formed on hydrophobic surfaces. It is interesting that the nanobubbles were flat, which, according to the authors, was necessary to minimize the gas pressure inside the nanobubbles. 
Seemingly definitive evidence for the microscopic bubbles theory was given by Sakamoto et al. ${ }^{55}$, who conducted AFM force measurements between a glass sphere and silica plate immersed in octadecyltrimethylammonium chloride $\left(\mathrm{C}_{18} \mathrm{TACl}\right)$ solutions. They found that the long-range attractions were observed only when the measurements were conducted in air-saturated solutions and not in carefully degassed solutions. This observation leads the authors to the conclusion that "long-range attraction never appears in completely air-free $\mathrm{C}_{18} \mathrm{TACl}$ solutions".

This theory seems appealing, because the existence of the bubbles solves the range problem. The apparent ranges of the forces depend on the heights of bubbles with varying dimensions. However, a primary setback for this theory is that very small air bubbles in the bulk water phase are short-lived ${ }^{70}$. Stevens et al. ${ }^{56}$ showed the range and strength of the attraction between hydrophobic amorphous fluoropolymer surfaces in deaerated water remain significantly greater than the van der Waals force. Meyer et al. ${ }^{57}$ conducted SFA force measurements between mica surfaces coated by a Langmuir-Blodgett (LB)-deposited hydrophobic monolayer of double-chain cationic surfactant (DODAB) both in the presence and absence of dissolved gases. In the presence of dissolved gases, they observed long-range attractive forces, while in degassed solutions only short-range attraction forces were observed. The authors concluded, therefore, that "true" hydrophobic force exists even in degassed solutions. They stated further that the longer range attractive force may be unrelated to hydrophobicity. On the other hand, Zhang et al. ${ }^{46}$ observed long-range attractive forces with a decay length as large as $36 \mathrm{~nm}$ even after degassing a $\mathrm{C}_{18} \mathrm{TACl}$ solution.

Thus, the questions concerning the existence of the hydrophobic force and its origins remain controversial. It has been shown that the classical DLVO theory cannot explain the coagulation of strongly hydrophobic particles and the coalescence of air bubbles at low surfactant concentrations $8,9,71,72$. Both of these phenomena are important in flotation as they affect particle and bubble size distributions. Also, modeling bubble-particle interactions is not possible without recognizing the existence of hydrophobic force ${ }^{10-12}$. Furthermore, most of the surface force measurements conducted in the past was made between mica and silica surfaces using surfactants that are not commonly used for flotation.

\subsection{Research Objectives}

The main objective of these studies is to examine the existence of the long range hydrophobic forces between thiol- and xanthate-coated gold surfaces in air-equilibrated water. The factors that may affect the hydrophobic force will be investigated. The results will be used to study the nature of hydrophobicity and the origin of hydrophobic force.

The specific objectives for this research are:

1. Conduct AFM force measurements between two gold macroscopic surfaces in aqueous solution to determine the Hamaker constant of gold (Chapter 2).

2. Conduct force measurements with gold surfaces pretreated by ex-situ adsorption of alkanethiols as functions of surfactant concentration and immersion time (Chapter 2 and Chapter 3).

3. Conduct force measurements with gold surfaces pretreated by ex-situ adsorption of alkanethiols as function of chain length (Chapter 3). 
4. Conduct force measurements with gold surfaces pretreated by in-situ adsorption of water-soluble xanthate surfactants as functions of surfactant concentration and immersion time (Chapter 4).

5. Conduct force measurements in the presence of various solutes such as inorganic electrolytes, surfactants and alcohols that can affect the properties (e.g., structure) of the thin water films between two hydrophobic surfaces (Chapter 3, Chapter 4 and Chapter 5).

6. Measure the surface forces at different temperatures to determine the changes in film entropies $\left(\Delta \mathrm{S}^{\mathrm{f}, \mathrm{ex}}\right)$ and enthalpies $\left(\Delta \mathrm{H}^{\mathrm{f} \text { ex }}\right)$ across the film thickness (Chapter 6$)$.

\section{References}

1. $\quad$ Derjaguin, B. V.; Laudau, L., Acta Physicochim. U. R. S. S. 1941, 14, 622-633.

2. Verwey, E. J. W.; Overbeek, J. T. G., Theory of the Stability of Lyophobic Colloids. Elsevier: Amsterdam, 1948.

3. Derjaguin, B. V.; Churaev, N. V., Colloids Surf. 1989, 41, 223-237.

4. $\quad$ Laskowski, J.; Kitchener, J. A., J. Colloid Interface Sci. 1969, 29, 670-679.

5. Israelachvili, J. N., Surf. Sci. Rep. 1992, 14, 109-159.

6. Israelachvili, J. N.; Pashley, R. M., Nature 1982, 300, 341-342.

7. Christenson, H. K.; Claesson, P. M., Adv. Colloid Interface Sci. 2001, 91, 391-436.

8. $\quad \mathrm{Xu}, \mathrm{Z}$.; Yoon, R.-H., J. Colloid Interface Sci. 1989, 132, (2), 532-541.

9. $\quad \mathrm{Xu}, \mathrm{Z}$.; Yoon, R.-H., J. Colloid Interface Sci. 1990, 134, (2), 427-434.

10. Yoon, R.-H.; Mao, L., J. Colloid Interface Sci. 1996, 181, 613-626.

11. Yoon, R.-H. In Hydrodynamic and Surface Forces in Bubble-Particle Interactions, XVII International Mineral Processing Congress, Dresden, Germany, 1991; Aufbereitungs-Technik: Dresden, Germany, 1991; pp 474-485.

12. Mao, L.; Yoon, R.-H., Int. J. Miner. Process 1997, 51, 171-181.

13. Attard, P., Adv. Colloid Interface Sci. 2003, 104, 75-91.

14. Christenson, H. K.; Claesson, P. M., Science 1988, 239, (4838), 390-392.

15. Miklavic, S. J.; Chan, D. Y. C.; White, L. R.; Healy, T. W., J. Phys. Chem. 1994, 98, 9022-9032.

16. Podgornik, R., J. Chem. Phys 1989, 91, (9), 5840-5849.

17. Kekicheff, P.; Spalla, O., Phys. Rev. Lett. 1995, 75, (9), 1851-1855.

18. Meyer, E. E.; Lin, Q.; Hassenkam, T.; Oroudjev, E.; Israelachvili, J. N., Proc. Nat. Acad. Sci. U.S.A 2005, 102, 6839-6842.

19. Derjaguin, B. V., Kolloid Zeits 1934, 69, 115-164.

20. Rabinovich, Y. I.; Yoon, R.-H., Langmuir 1994, 10, 1903-1909.

21. Rabinovich, Y. I.; Yoon, R.-H., Colloids and Surfaces 1994, 93, 263-273.

22. Meagher, L.; Craig, V. S. J., Langmuir 1994, 10, 2736-2742.

23. Vinogradova, O. I.; Yakubov, G. E., J. Chem. Phys. 2001, 114, (18), 8124-8131.

24. Muster, T. H.; Toikka, G.; Hayes, R. A.; Prestidge, C. A.; Ralston, J., Colloids and Surf., A 1996, 106, 203-211.

25. Wang, J.; Yoon, R.-H., Langmuir 2008, 24, 7889-7896.

26. Israelachvili, J.; Pashley, R., Nature 1982, 300, 341-342.

27. Christenson, H. K.; Claesson, P. M.; Berg, J.; Herder, P. C., J. Phys. Chem. 1989, 93, 1472-1478. 
28. Claesson, P. M.; Blom, C. E.; Herder, P. C.; Ninham, B. W., J. Colloid Interface Sci. 1986, 114, (1), 234-242.

29. Tsao, Y.-H.; Yang, S. X.; Evans, D. F.; Wennerstrom, H., Langmuir 1991, 7, 31543159.

30. Spalla, O., Curr. Opin. Colloid Interface Sci. 2000, 5, 5-12.

31. Eriksson, J. C.; Yoon, R.-H., The Nature of Hydrophobic Attraction Forces. In Froth Flotation A Century of Innovation, Fuerstenau, M. C.; Jameson, G.; Yoon, R.-H., Eds. Society for Mining, Metallurgy, and Exploration, Inc.: 2006; pp 133-178.

32. Eriksson, J. C.; Yoon, R.-H., Hydrophobic Attraction in the Light of Thin-Film Thermodynamics. In Colloid Stability: The Role of Surface Forces, Tadros, T. F., Ed. WILEY-VCH Verlag GmbH \& Co. KGaA: Weinheim, Germany, 2007; Vol. 1, pp 99-132.

33. Meyer, E. E.; Rosenberg, K. J.; Israelachvili, J., Proc. Nat. Acad. Sci. U.S.A 2006, 103, 15739-15746.

34. Wood, J.; Sharma, R., Langmuir 1994, 10, 2307-2310.

35. Wood, J.; Sharma, R., Langmuir 1995, 11, (12), 4797-4802.

36. Parker, J. L.; Claesson, P. M.; Attard, P., J. Phys. Chem. 1994, 98, 8468-8480.

37. Nguyen, A. V.; Nalaskowski, J.; Miller, J. D.; Butt, H.-J., Int. J. Miner. Process. 2003, 72, 215-225.

38. Ishida, N.; Kinoshita, N.; Miyahara, M.; Higashitani, K., J. Colloid Interface Sci. 1999, 216, 387-393.

39. Ederth, T.; Claesson, P.; Liedberg, B., Langmuir 1998, 14, 4782-4789.

40. Ederth, T., J. Phys. Chem. B 2000, 104, 9704-9712.

41. Hato, M., J. Phys. Chem. 1996, 100, 18530-18538.

42. Kurihara, K.; Kunitake, T., J. Am. Chem. Soc. 1992, 114, 10927-10933.

43. Lin, Q.; Meyer, E. E.; Tadmor, M.; Israelachvili, J. N.; Kuhl, T. L., Langmuir 2005, 21, 251-255.

44. Parker, J. L.; Yaminsky, V. V.; Claesson, P. M., J. Phys. Chem. 1993, 97, 7706-7710.

45. Rutland, M. W.; Parker, J. L., Langmuir 1994, 10, 1110-1121.

46. Zhang, J.; Yoon, R.-H.; Mao, M.; Ducker, W. A., Langmuir 2005, 21, 5831-5841.

47. Zhang, J.; Yoon, R.-H.; Eriksson, J. C., Colloids Surf., A 2007, 300, 335-345.

48. Yoon, R.-H.; Ravishankar, S. A. R., J. Colloid Interface Sci. 1996, 179, 391-402.

49. Ederth, T.; Liedberg, B., Langmuir 2000, 16, 2177-2184.

50. Christenson, H. K.; Fang, J.; Ninham, B. W.; Parker, J. L., J. Phys. Chem. 1990, 94, 8004-8006.

51. Craig, V. S. J.; Ninham, B. W.; Pashley, R. M., Langmuir 1998, 14, (12), 3326-3332.

52. Craig, V. S. J.; Ninham, B. W.; Pashley, R. M., Langmuir 1999, 15, 1562-1569.

53. Mahnke, J.; Stearnes, J.; Hayes, R. A.; Fornasiero, D.; Ralston, J., Phys. Chem. Chem. Phys. 1999, 1, 2793-2798.

54. Considine, R. F.; Hayes, R. A.; Horn, R. G., Langmuir 1999, 15, 1657-1659.

55. Sakamoto, M.; Kanda, Y.; Miyahara, M.; Higashitani, K., Langmuir 2002, 18, 57135719.

56. Stevens, H.; Considine, R. F.; Drummond, C. J.; Hayes, R. A.; Attard, P., Langmuir 2005, 21, 6399-6405.

57. Meyer, E. E.; Lin, Q.; Israelachvili, J. N., Langmuir 2005, 21, 256-259.

58. Kokkoli, E.; Zukoski, C. F., J. Colloid Interface Sci. 1999, 209, 60-65. 
59. Rabinovich, Y. I.; Guzonas, D. A.; Yoon, R.-H., Langmuir 1993, 9, 1168-1170.

60. Eriksson, J. C.; Ljunggren, S.; Claesson, P. M., J. Chem. Soc., Faraday Trans. 2 1989, 85, (3), 163-176.

61. Attard, P., J. Phys. Chem 1989, 93, 6441-6444.

62. Spalla, O.; Belloni, L., Phys. Rev. Lett. 1995, 74, (13), 2515-2518.

63. Tsao, Y.-H.; Evans, D. F.; Wennerstrom, H., Science 1993, 262, (5133), 547-550.

64. Christenson, H. K.; Yaminsky, V. V., Colloids Surf., A 1997, 129-130, 67-74.

65. Tyrrell, J. W. G.; Attard, P., Phys. Rev. Lett. 2001, 87, (17), 176104-1.

66. Tyrrell, J. W. G.; Attard, P., Langmuir 2002, 18, 160-167.

67. Ishida, N.; Sakamoto, M.; Miyahara, M.; Higashitani, K., J. Colloid Interface Sci. 2002, 253, 112-116.

68. Yaminsky, V. V.; Ninham, B. W., Langmuir 1993, 9, 3618-3624.

69. Yang, J.; Duan, J.; Fornasiero, D.; Ralston, J., J. Phys. Chem. B 2003, 107, 61394147.

70. Ljunggren, S.; Eriksson, J. C., Colloids and Surf., A 1997, 129-130, 151-155.

71. Wang, L.; Yoon, R.-H., Langmuir 2004, 20, 11457-11464.

72. Yoon, R.-H.; Aksoy, B. S., J. Colloid Interface Sci. 1999, 211, 1-10. 


\section{TASK 1 AFM FORCES MEASURED BETWEEN GOLD SURFACES COATED WITH SELF-ASSEMBLED MONOLAYERS OF 1-HEXADECANETHIOL ${ }^{*}$}

\section{$\underline{2.1 \text { Abstract }}$}

An atomic force microscope (AFM) was used to measure the forces between gold surfaces with and without hydrophobizing them by the self-assembly of 1-hexadecanethiol. The forces measured between bare gold surfaces were fitted to the Derjaguin-LandauVerwey-Overbeek (DLVO) theory with a Hamaker constant of $1.2 \times 10^{-20} \mathrm{~J}$, which was close to the value determined using the methylene iodide contact angle method but was lower than that calculated using the Lifshitz theory. When the surfaces were hydrophobized in a $1 \times 10^{-2}$ $\mathrm{mM}$ thiol-in-ethanol solution for 10 minutes, the measured forces exhibited a long-range force with a decay length of $35 \mathrm{~nm}$. Despite its high water contact angle $\left(105^{\circ}\right)$, the force curve was smooth and exhibited no steps. When the surfaces were hydrophobized in a $1 \mathrm{mM}$ thiol solution for longer than 6 hours, however, the force curves exhibited steps, indicating that the long-range attractions were caused by bridging bubbles. When the measurements were conducted after washing the substrates with organic solvents, the steps disappeared and long-range attractive force appeared. In the presence of ethanol, the water contact angle decreased below $90^{\circ}$, the attraction became weaker, and the force curves became smooth. On the basis of the results obtained in the present work, possible mechanisms for the long-range attractions are discussed.

\subsection{Introduction}

In colloid chemistry, the Derjaguin-Landau-Verwey-Overbeek (DLVO) theory has been used extensively to predict the kinetics of coagulation. It was derived to predict the stability of lyophobic colloids, that is, particles with low or no affinity with the dispersion medium, on the basis of a balance between the van der Waals (attractive) force and the electrical double layer (repulsive) force. Thus, the theory should work well with hydrophobic colloids when the medium is water. However, many hydrophobic particles ${ }^{1,2}$ and surfaces ${ }^{3-8}$ exhibit attractions that are much stronger than the van der Waals attractions. In this regard, Derjaguin and Churaev" suggested that the DLVO theory should be applicable for "particles or surfaces having some limited degree of hydrophilicity". They recognized that hydrophobic attractions are detected experimentally when advancing contact angles $\left(\theta_{a}\right)$ are greater than $64^{\circ},{ }^{3}$ while hydrophilic repulsions are detectable at $\theta_{a}<15^{\circ} .{ }^{10}$ It appears, therefore, that the DLVO theory has a limited applicability for colloids with a relatively narrow range of contact angles.

The DLVO theory faces even more difficult challenges when one wishes to predict the stability of the wetting films formed on hydrophobic surfaces. Thermodynamically, wetting films are unstable at $\theta>0^{\circ}$, and rupture instantaneously once the film thickness is reduced to a critical value $\left(H_{\mathrm{cr}}\right)$. Blake and Kitchener ${ }^{11}$ measured the values of $H_{\text {cr }}$ to be in the range of 6-220 nm, while, according to the DLVO theory, no rupture should occur as both the double-layer and van der Waals forces are repulsive in wetting films. ${ }^{12}$ Thus, one

\footnotetext{
* Reproduced with permission from [10.1021/la800276r]. Copyright [2008] American Chemical Society.
} 
way of explaining the rupture of wetting films would be to consider the possibility of a nonDLVO force destabilizing the wetting film. Laskowski and Kitchener ${ }^{13}$ speculated the presence of a long-range "hydrophobic influence" operating in the flotation of methylated silica, while Blake and Kitchener" 11 suggested the presence of "hydrophobic force" in wetting films.

Israelachvili and Pashley ${ }^{14}$ were the first to actually measure the hydrophobic forces in the thin water films between two mica surfaces coated with cetyltrimethylammonium bromide (CTAB) using the surface force apparatus (SFA). They not only were stronger than the van der Waals force but also were longer-ranged. The work of Israelachvili and Pashley created interests among many colloid chemists and physicists, with numerous follow-up publications. ${ }^{8,15-19}$ Recognition of the hydrophobic force also made it possible to model the coagulation of hydrophobic particles ${ }^{1,2}$ and the bubble-particle adhesion occurring during flotation. $^{20-22}$

There has been a great deal of controversy, however, regarding the existence and the nature of the hydrophobic force. Some investigators consider that it is due to the changes in water structure in the vicinity of hydrophobic solids, ${ }^{16,23-25}$ while others believe that it is an electrical effect due to the correlation between charged patches ${ }^{26,27}$ or between large dipoles $^{28,29}$ formed on solid surfaces, ${ }^{27,} 28$ metastability of the water films between hydrophobic surfaces, 5,30 and bridging of preexisting nanobubbles. ${ }^{31-33}$ Recently, the nanobubble theory gained momentum as many investigators showed the discontinuities (or steps) in their force versus distance curves, ${ }^{33,34}$ Tyrell and Attard ${ }^{31}$ and Yang et al. ${ }^{35}$ actually showed the AFM images of the nanobubbles formed on hydrophobic surfaces. Interestingly, the nanobubbles were flat, which was necessary to minimize the gas pressure inside the bubbles.

Seemingly definitive evidence for the nanobubble theory was presented by Sakamoto et al. ${ }^{36}$ who conducted AFM force measurements between a glass sphere and a silica plate immersed in octadecyltrimethylammonium chloride $\left(\mathrm{C}_{18} \mathrm{TACl}\right)$ solutions. They observed long-range attractions when the measurements were conducted in air-saturated solutions but not in carefully degassed solutions. These observations led to a statement that "long-range attraction never appears in completely air-free $\mathrm{C}_{18} \mathrm{TACl}$ solutions". However, the repeat experiments conducted by Zhang et al. ${ }^{17}$ on the same system showed that long-range attractions with decay lengths of $36 \mathrm{~nm}$ were observed in degassed solutions. Meyers et al. ${ }^{19}$, ${ }^{37}$ also conducted force measurements between mica surfaces coated with a double-chain (C18) cationic surfactant both in the presence and absence of dissolved gases. In the former, they observed a long-range attractive force, while in the latter only a short-range attraction was observed. It was concluded, therefore, that only the short-range force observed at separations below $10 \mathrm{~nm}$ was the "true" hydrophobic force, while the long-range force observed above $20 \mathrm{~nm}$ was unrelated to hydrophobicity. Meyers et al. ${ }^{27}$ suggested that the long-range attraction was due to the electrostatic attraction between the positively charged patches of cationic surfactants and the negatively charged bare mica surface. This explanation was similar to the charged-patch model of Miklavic et al., ${ }^{26}$ according to which the patches must be mobile to account for the long-range attractions observed in surface force measurements.

In the present work, AFM force measurements were conducted between gold substrates hydrophobized by the self-assembly of 1-hexadecanethiol $\left(\mathrm{C}_{16} \mathrm{SH}\right)$. It is well known that $n$-alkanethiols form stable monolayers with the terminal $-\mathrm{CH}_{3}$ groups in contact 
with the aqueous phase, providing hydrophobic surfaces whose surface free energy (19 $\mathrm{mJ} / \mathrm{m}^{2}$ ) is lower than any hydrocarbon surfaces studied to date. ${ }^{38}$ Further, the strong covalent $\mathrm{Au}-\mathrm{S}$ bonding provides robust and immobile hydrophobic monolayer, which will preclude the possibility of mobile hydrophobic groups ${ }^{39,40}$ or mobile charged patches ${ }^{19,26,27}$ creating the long-range forces.

Ederth $^{40}$ and Ederth et al., ${ }^{41}$ used a bimorph surface force apparatus (MASIF) to measure the forces between $\mathrm{C}_{16} \mathrm{SH}$-coated gold surfaces in water. The force curves obtained by these investigators showed steps, which led to their conclusion that the "excess" force was due to bubble coalescence. They concluded that the excess force was observed only when advancing contact angle $\left(\theta_{a}\right)$ exceeded $90^{\circ}$ and that no attraction beyond the van der Waals attraction was observed when $\theta_{a}<90^{\circ}$. It has been found in the present work that strong and long-range hydrophobic forces can still be observed without the steps when gold substrates are contacted with $\mathrm{C}_{16} \mathrm{SH}$-in-ethanol solutions of lower concentrations and for shorter contact times than employed by Ederth and his co-workers. Further, the steps disappear when gold substrates hydrophobized at higher concentrations for a long period of time are washed with appropriate solvents.

\section{$\underline{2.3 \text { Materials and Methods }}$}

\subsubsection{Materials}

\section{a) Regents}

A Nanopure II (Barnstead IA) water purification system was used to obtain doubledistilled and deionized water with a resistivity of $18.2 \mathrm{M} \Omega / \mathrm{cm}$. To remove particulates, a submicron Postfilter $(0.2 \mu \mathrm{m}$ pore size $)$ from Fisher Scientific was used in conjunction with the Nanopure water system. 1-hexadecanethiol $\left(\mathrm{C}_{16} \mathrm{SH}, 97 \%\right)$ from TCI dissolved in 200 proof ethanol (AAPER Alcohol) was used to hydrophobize the gold surfaces. Sulfuric acid (98\%) from VMR International and hydrogen peroxide $\left(\mathrm{H}_{2} \mathrm{O}_{2}, 29.0-32.0 \%\right)$ from Alfa Aesar were used to clean gold plates.

\section{b) Gold Plates}

Gold microspheres and gold-coated glass slides were used for AFM surface force measurements. The gold-coated glass slides were obtained by depositing pure gold on glass using a vacuum evaporator. A $50 \AA$ chromium layer was deposited first on the glass prior to coating it with a thin-layer ( $500 \AA$ ) of gold. The chromium coating was necessary to achieve strong bonding between gold and substrate. The coatings produced without the chromium adhesive layer were easily removed in acid solutions.

c) Gold Spheres

Gold spheres were produced by melting a gold micro-powder $(1.5-3.0 \mu \mathrm{m},>99.96 \%$, Alfa Aesar) in a furnace. The powder was placed in an alumina crucible, and heated until the temperature was raised above its melting point $\left(1,064.18^{\circ} \mathrm{C}\right)$. It was kept at $1,100^{\circ} \mathrm{C}$ for 15 minutes and then cooled down slowly. The furnace was flushed with nitrogen to provide an oxygen-free atmosphere. The gold spheres obtained in this manner had a wide range of sizes. Only those with diameters of 15-20 $\mu \mathrm{m}$ were selected for AFM force measurements.

\subsubsection{Cleaning Gold Substrates}


To obtain high-quality thiol monolayers on gold, a substrate must be cleaned thoroughly prior to immersing it in a thiol solution. ${ }^{42,} 43$ There are several cleaning procedures reported in the literature, including the methods of using piranha solution, ${ }^{38,44,45}$ chromic acid, ${ }^{46,}{ }^{47} \mathrm{UV} /$ ozone treatment, ${ }^{43,48}$ etc. In the present work, the gold plates were cleaned first by immersing them in a boiling piranha solution $\left(1: 2 \mathrm{H}_{2} \mathrm{O}_{2} / \mathrm{H}_{2} \mathrm{SO}_{4}\right)$ for 20 minutes, and then washing it with nanopure water for 1 minute, followed by ethanol wash for 2 minutes. After the cleaning, the gold plate was immediately contacted with a thiol solution for hydrophobization. The piranha solution reacts violently with organic matter, especially when it is hot, and is extremely corrosive. Therefore, it is known to oxidize gold surfaces. ${ }^{42}$ In the present work, the gold surfaces cleaned in the manner described above exhibited zero water contact angles, possibly due to the formation of gold oxide $\left(\mathrm{Au}_{2} \mathrm{O}_{3}\right)$. After rinsing it with ethanol for 2 minutes, the contact angle was increased to $65^{\circ}$. It has been reported that the gold oxide is unstable at ambient and can be readily reduced by ethanol. ${ }^{42,43}$

For the case of gold spheres, cleaning was done after they had been glued onto cantilever springs. To prevent the glues from being destroyed by the piranha solution, each gold sphere was flushed with ethanol, irradiated by UV irradiation $(\lambda=254 \mathrm{~nm})$ for 2 hours, and then rinsed with ethanol again.

\subsubsection{Hydrophobizing Gold Substrates}

The gold plates and spheres cleaned in the manner described above were hydrophobized by contacting them in $\mathrm{C}_{16} \mathrm{SH}$-in-ethanol solutions. The specific interaction between sulfur and gold allowed the surfactant molecules to form robust self-assembled monolayers. ${ }^{38}$ The kinetics of adsorption varied with the surfactant concentration. ${ }^{49}$ In the present work, the hydrophobization was carried out in $10^{-2}$ or $1 \mathrm{mM} \mathrm{C}{ }_{16} \mathrm{SH}$-in-ethanol solutions at room temperature. It was shown that good monolayers can be formed at a concentration as low as $10^{-2} \mathrm{mM}$, given a sufficient contact time. ${ }^{38}$ After the hydrophobization, the gold substrates were washed with ethanol and then dried in a nitrogen gas stream. For a given force measurement, a set of gold plate and sphere was immersed in a thiol solution for a predetermined length of time, so that the hydrophobicity of the two macroscopic surfaces would be the same. A gold sphere was glued onto a cantilever spring before being immersed into a thiol solution. Reversing the order made it difficult to glue the sphere onto a spring.

\subsubsection{Contact Angle Measurement}

Ederth ${ }^{40}$ showed that $\mathrm{C}_{16} \mathrm{SH}$-coated gold exhibited very small contact angle hysteresis $(\Delta \cos \theta=0.10)$. Therefore, equilibrium water contact angles were measured on the hydrophobized gold plates using a goniometer (Ramé-Hart, Inc) under ambient conditions. Droplets of water (or ethanol solutions) of 1-2 mm diameter were placed on a horizontally placed plate by means of a syringe. The angles were measured on each side of a droplet. The measurements were conducted on a total of five droplets placed on different locations of a gold plate, and the results were averaged. The sessile drop technique was also used to measure the contact angles of methylene iodide on gold. The result was used to calculate the Hamaker constant of gold in water. ${ }^{18}$ 


\subsubsection{Surface Force Measurement and AFM Imaging}

Surface force measurements were conducted using a Nanoscope III (Digital Instruments, Inc., Santa Barbara, CA) atomic force microscope (AFM) equipped with a standard fluid cell and a scanner "E". All the AFM force measurements were carried out in a manner described by Zhang et al. ${ }^{17}$ Rectangular non-contact silicon cantilevers (dlevers, Model: 1930-00, Veeco Probes) were used for the force measurements. Their spring constants $(k)$ were determined using the Cleveland method. ${ }^{50}$ In each experiment, a gold sphere was glued onto a cantilever with EPON 1004 resin (Shell Chemical Co) using a homemade 3D micromanipulator under an Olympus BH-2 light microscope. The force measurements were conducted immediately after the thiol monolayers were formed on gold

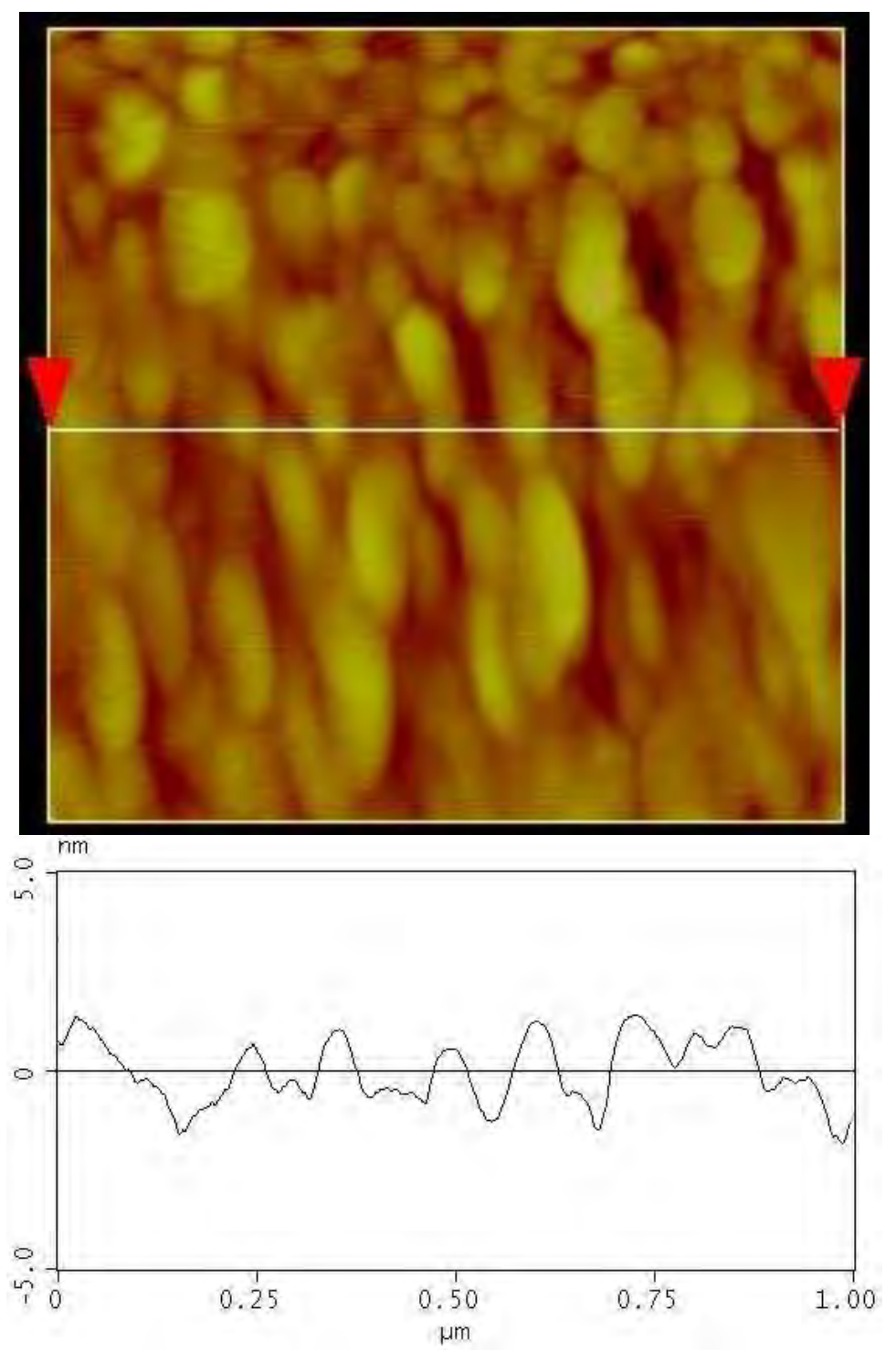

Figure 2.1. An AFM image (and its cross section) of the evaporated gold film on Cr-coated glass after treatment with piranha solution. 
substrates. AFM images of a clean bare gold plate were taken using triangular $\mathrm{Si}_{3} \mathrm{~N}_{4}$ cantilevers with nominal $k=0.12 \mathrm{Nm}^{-1}$. The image was obtained in the height mode.

\subsection{6 ל-Potential Measurement}

A Pen Kem Model 501 Lazer Zee meter was used to measure the electrophoretic mobilities of gold spheres $(1.5-3.0 \mu \mathrm{m})$. Micro-spheres of gold were suspended in water or in $\mathrm{NaCl}$ solutions by means of an ultrasonic vibration. The mobilities were converted to $\zeta$ potentials using the Smoluchowski equation. The $\zeta$-potentials reported in this communication represent the averages of at least five measurements.

\subsection{Results and Discussion}

\subsubsection{Surface Morphology}

Figure 2.1 shows an AFM image of the surface of a clean gold plate as obtained using

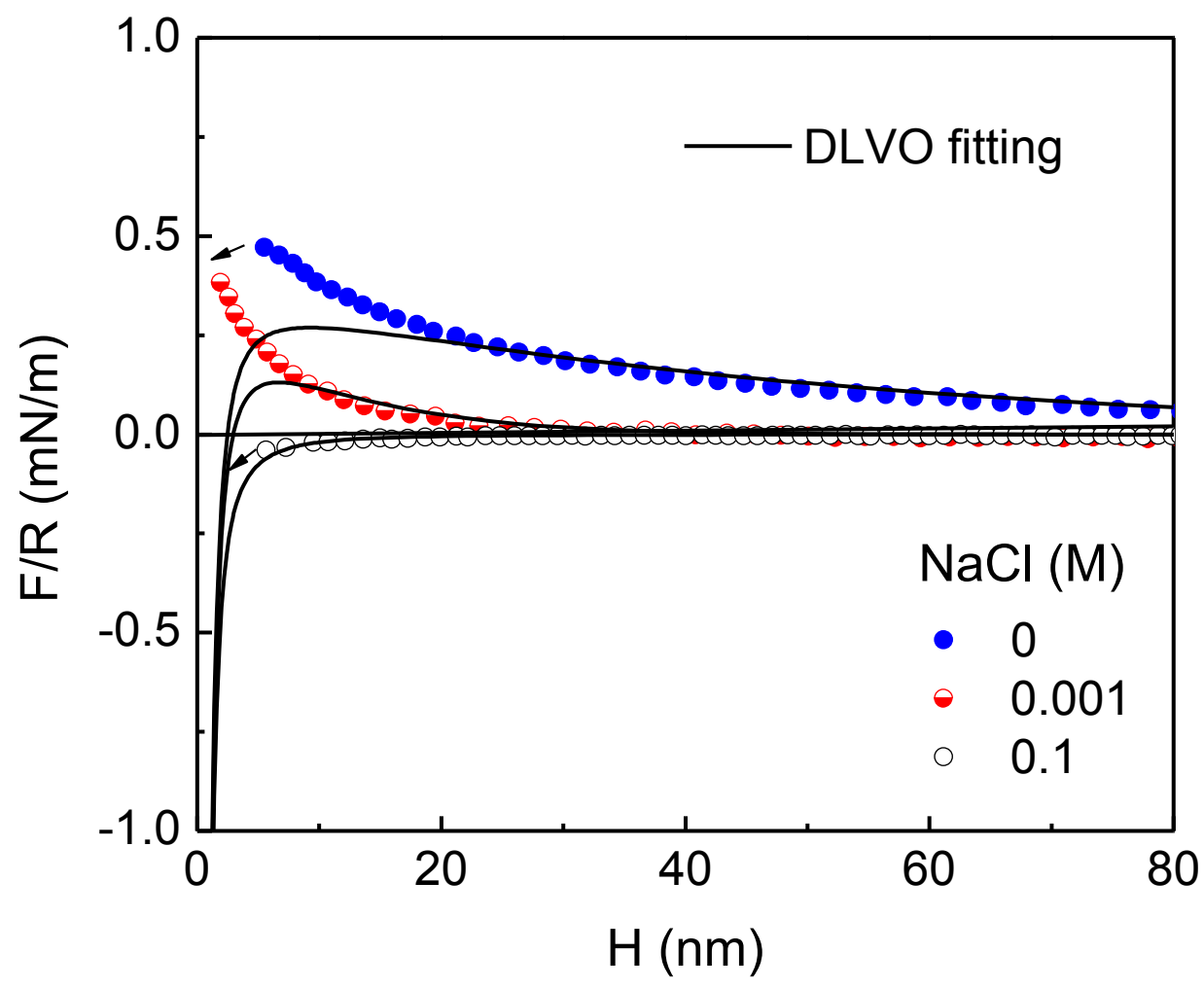

Figure 2.2. Surface forces measured between a microscopic gold sphere and a gold-coated glass plate in water, $1 \mathrm{mM}$ and $100 \mathrm{mM} \mathrm{NaCl}$ solutions. The data points have been fitted to the DLVO theory under conditions of constant potential using the following parameters: for water, $\psi_{0}=-55 \mathrm{mV}, \kappa^{-1}=424 \AA$; for $1 \mathrm{mM} \mathrm{NaCl}, \psi_{0}=-24 \mathrm{mV}, \kappa^{-1}=93 \AA$; for $100 \mathrm{mM} \mathrm{NaCl}$, $\psi_{0}=-8 \mathrm{mV}, \kappa^{-1}=9 \AA . \mathrm{A}_{131}=1.2 \times 10^{-20} \mathrm{~J}$. 
a regular silicon nitride tip. The surface consisted of different grains, with the maximum peak-to-valley distance of $3.3 \mathrm{~nm}$ and the root mean square (RMS) roughness of $0.8 \mathrm{~nm}$ over an area of $1 \times 1 \mu \mathrm{m}^{2}$.

\subsection{2 van der Waals Attraction}

Figure 2.2 shows the AFM force curves $(F / R$ versus $H)$ obtained between bare gold surfaces immersed in 0,1 , and $100 \mathrm{mM} \mathrm{NaCl}$ solutions. The force curves obtained in pure water and at $1 \mathrm{mM} \mathrm{NaCl}$ solution showed net repulsive forces over the entire separation distances investigated, while the results obtained at $100 \mathrm{mM}$ showed no repulsive forces until the separation distance was reduced below approximately $20 \mathrm{~nm}$. The solid lines represent the experimental data fitted to the DLVO theory. The fitting parameters were: $A_{131}$ (Hamaker constant $)=1.2 \times 10^{-20} \mathrm{~J}, \psi_{0}($ surface potential $)=-55 \mathrm{mV}$, and $\kappa^{-1}$ (Debye length $)=42.4 \mathrm{~nm}$ in pure water. At $1 \mathrm{mM} \mathrm{NaCl}, \psi_{0}$ decreased to $-24 \mathrm{mV}$ and $\kappa^{-1}$ to $9.3 \mathrm{~nm}$ due to double-layer compression. As the $\mathrm{NaCl}$ concentration was increased to $100 \mathrm{mM}, \psi_{0}$ decreased further to -8 $\mathrm{mV}$ and $\kappa^{-1}$ to $0.9 \mathrm{~nm}$. The double-layer potentials were calculated using the constant potential model of Oshima et al. ${ }^{51}$ The values of $\psi_{0}$ obtained from the curve fitting exercise were close to the $\zeta$-potentials measured in the present work as shown in Table 2.1.

Table 2.1. Comparison of the $\zeta$-potentials of Gold Spheres and the DLVO and the DLVO Potentials in Water and $\mathrm{NaCl}$ solutions

\begin{tabular}{cccc}
\hline Gold Substrate & $\begin{array}{c}\text { NaCl Concentration } \\
(\mathrm{mM})\end{array}$ & $\begin{array}{c}\zeta \text {-potential } \\
(\mathrm{mV})\end{array}$ & $\begin{array}{c}\text { DLVO Potential } \\
(\mathrm{mV})\end{array}$ \\
\hline \multirow{3}{*}{ Uncoated } & 0 & -56.9 & -55 \\
& 1 & -24.2 & -24 \\
& 100 & -8.0 & -8 \\
\hline $\mathrm{C}_{16}$ SH Coated & 0 & -57.2 & - \\
\hline
\end{tabular}

${ }^{1}$ from AFM force curves in Figure 2.2

It may be of interest to compare the value of $A_{131}$ determined from curve fitting in the manner described above with that determined using a different method. According to the combining rule, ${ }^{52}$

$$
A_{131}=\left(\sqrt{A_{11}}-\sqrt{A_{33}}\right)^{2}
$$

where $A_{131}$ is the Hamaker constant of material $\mathbf{1}$ in medium $\mathbf{3}$ (water), $A_{11}$ the same in vacuo, and $A_{33}$ is the Hamaker constant of water in vacuo. It has been shown that $A_{\mathrm{ii}}$ can be determined for a variety of polar and apolar materials using the following relation: ${ }^{53}$

$$
A_{i i}=24 \pi d_{0}^{2} \gamma_{i}^{L W}
$$

in which $l_{0} \approx 1.57 \AA$. Substituting Equation 2.2 into Equation 2.1, one obtains that 


$$
A_{131}=1.86 \times 10^{-21}\left(\sqrt{\gamma_{1}^{L W}}-\sqrt{\gamma_{3}^{L W}}\right)^{2}
$$

in which $\gamma_{1}{ }^{\mathrm{LW}}$ is the dispersion component of the surface tension of gold and $\gamma_{3}{ }^{\mathrm{LW}}$ is the same for water.

In the present work, the value of $\gamma_{1}{ }^{\mathrm{LW}}$ was determined using the following relation: ${ }^{18}$

$$
\gamma_{1}^{L W}=\left[\left(1+\cos \theta_{m}\right) \gamma_{m} / 2 \sqrt{\gamma_{m}^{L W}}\right]^{2}
$$

in which $\theta_{\mathrm{m}}$ is the contact angle of methylene iodide on gold, and $\gamma_{\mathrm{m}}$ and $\gamma_{\mathrm{m}}{ }^{\mathrm{LW}}$ are the surface tension of methylene iodide and its apolar component, respectively. The values of $\theta_{\mathrm{m}}$ for the gold-coated glass surfaces used in the present work gave the value of $6^{\circ}$ as shown in Table 2.2. By substituting this and the values of $\gamma_{\mathrm{m}}=50.8 \mathrm{mN} / \mathrm{m}^{18}$ and $\gamma_{\mathrm{m}}{ }^{\mathrm{LW}}=48.5 \mathrm{mN} / \mathrm{m}^{18}$ into Equation 2.4, one obtains $\gamma_{1}^{\mathrm{LW}}=52.9 \mathrm{mN} / \mathrm{m}$. This value is comparable to those reported by Fowkes $^{54}(60-120 \mathrm{mN} / \mathrm{m})$ and the value of $121.6 \mathrm{mN} / \mathrm{m}$ reported by Thelen. ${ }^{55}$

Table 2.2. Surface Tension and Methylene Iodide Contact Angle Data Used to Determine the Hamaker Constant for Gold in Water.

\begin{tabular}{cccccc}
\hline & $\begin{array}{c}\gamma \\
(\mathrm{mN} / \mathrm{m})\end{array}$ & $\begin{array}{c}\gamma^{\mathrm{LW}} \\
(\mathrm{mN} / \mathrm{m})\end{array}$ & $\begin{array}{c}\gamma^{\mathrm{AB}} \\
(\mathrm{mN} / \mathrm{m})\end{array}$ & $\begin{array}{c}\theta_{\mathrm{m}} \\
(\text { degree })\end{array}$ & $\begin{array}{c}A_{131} \\
(\mathrm{~J})\end{array}$ \\
\hline methylene iodide $^{1}$ & 50.8 & 48.5 & 2.3 & 6 & \\
Water $^{2}$ & 72.8 & 21.8 & 51 & & \\
gold & & 52.9 & & & $1.26 \times 10^{-20}$ \\
\hline gold-water-gold & & & &
\end{tabular}

${ }^{1}$ ref $18 ;{ }^{2}$ ref 53

By substituting the value of $\gamma_{1}{ }^{\mathrm{LW}}=52.9 \mathrm{mN} / \mathrm{m}$ obtained in the present work and the value of $\gamma_{3}{ }^{\mathrm{LW}}=21.8 \mathrm{mN} / \mathrm{m}^{53}$ into Equation 2.3 , one obtains $A_{131}=1.26 \times 10^{-20} \mathrm{~J}$, which is close to that $\left(A_{131}=1.2 \times 10^{-20} \mathrm{~J}\right)$ obtained from curve fitting. Both of these values are a little lower than that $\left(4 \times 10^{-20} \mathrm{~J}\right)$ obtained by Ederth ${ }^{40}$ from AFM force measurement. On the other hand, Biggs and Mulvaney ${ }^{47}$ conducted AFM force measurement between bare gold surfaces, that is, gold-coated silica sphere and gold plate, and obtained a value of $A_{131}=2.5 \times$ $10^{-19} \mathrm{~J}$, which was close to those $\left(2.5 \sim 4 \times 10^{-19} \mathrm{~J}\right)$ calculated using the Lifshitz theory. Biggs et $a l .{ }^{56}$ used these values to fit the AFM force curves obtained in different $\mathrm{NaCl}$ solutions. However, the fits were relatively poor at short separations. In later experiments, Kane and Mulvaney ${ }^{44}$ used a considerably lower value of $A_{131}\left(=1 \times 10^{-19} \mathrm{~J}\right)$ to fit their AFM force data. Further, Ducker and Senden ${ }^{57}$ could not fit their AFM force curves obtained between gold-coated silica sphere and gold-coated mica surface to the DLVO theory using the value of $A_{131}=3.5 \times 10^{-19} \mathrm{~J}$ from the Lifshitz theory. Giesbers et al. ${ }^{45}$ also showed that the force curves obtained for the gold-gold interactions in water exhibited very weak van der Waals interactions.

That different researchers reported different Hamaker constants for gold-gold interactions may be attributed to the differences in coating thickness, contamination level, and surface roughness. Owing to the large Hamaker constants, as calculated from the Lifshitz theory, gold surfaces can be readily contaminated. Ducker and Senden ${ }^{57}$ attributed the failure 
to fit their data with $A_{131}=3.5 \times 10^{-19} \mathrm{~J}$ to the possible adsorption of organic materials on gold. It has actually been shown that the adsorption of citrate mediates the van der Waals interaction between gold surfaces. ${ }^{58,59}$ The same explanation could be extended to silversilver interactions. The AFM force measurements conducted between silver-coated glass plate and silica sphere gave a Hamaker constant of $2 \times 10^{-20} \mathrm{~J}$, which was much smaller than the value of $3 \times 10^{-19} \mathrm{~J}$ calculated from the Lifshitz theory. ${ }^{60}$

According to Considine and Drummond, ${ }^{61}$ surface roughness attenuates short-range forces such as van der Waals, steric, and hydration forces. Bhattacharjee et al. ${ }^{62}$ showed also that random distribution of asperities can also reduce the interaction energies substantially.

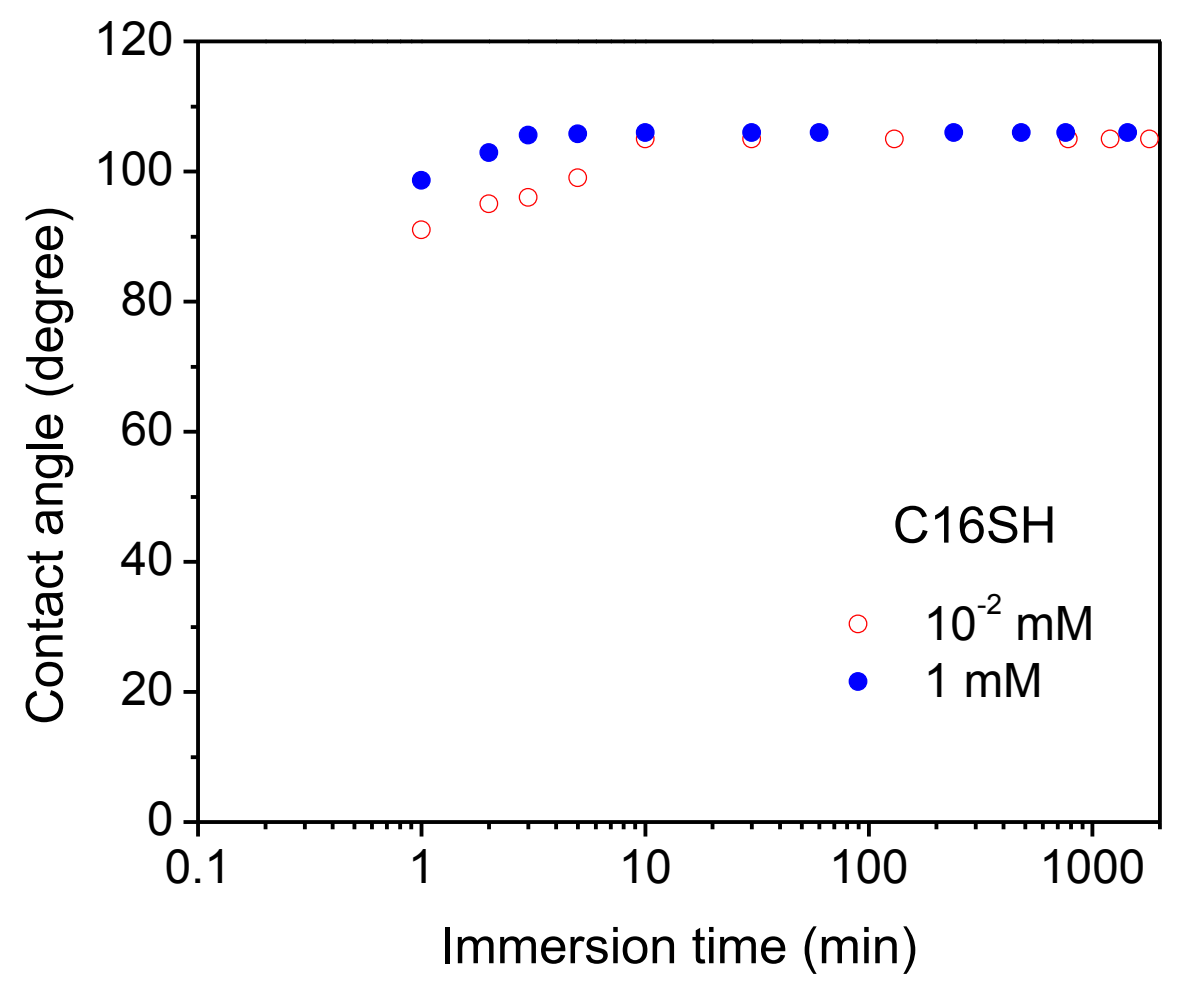

Figure 2.3. Changes in equilibrium water contact angle $(\theta)$ on gold plate as a function of immersion time in 0.01 and $1 \mathrm{mM} 1$-hexadecanethiol-in-ethanol solutions. 

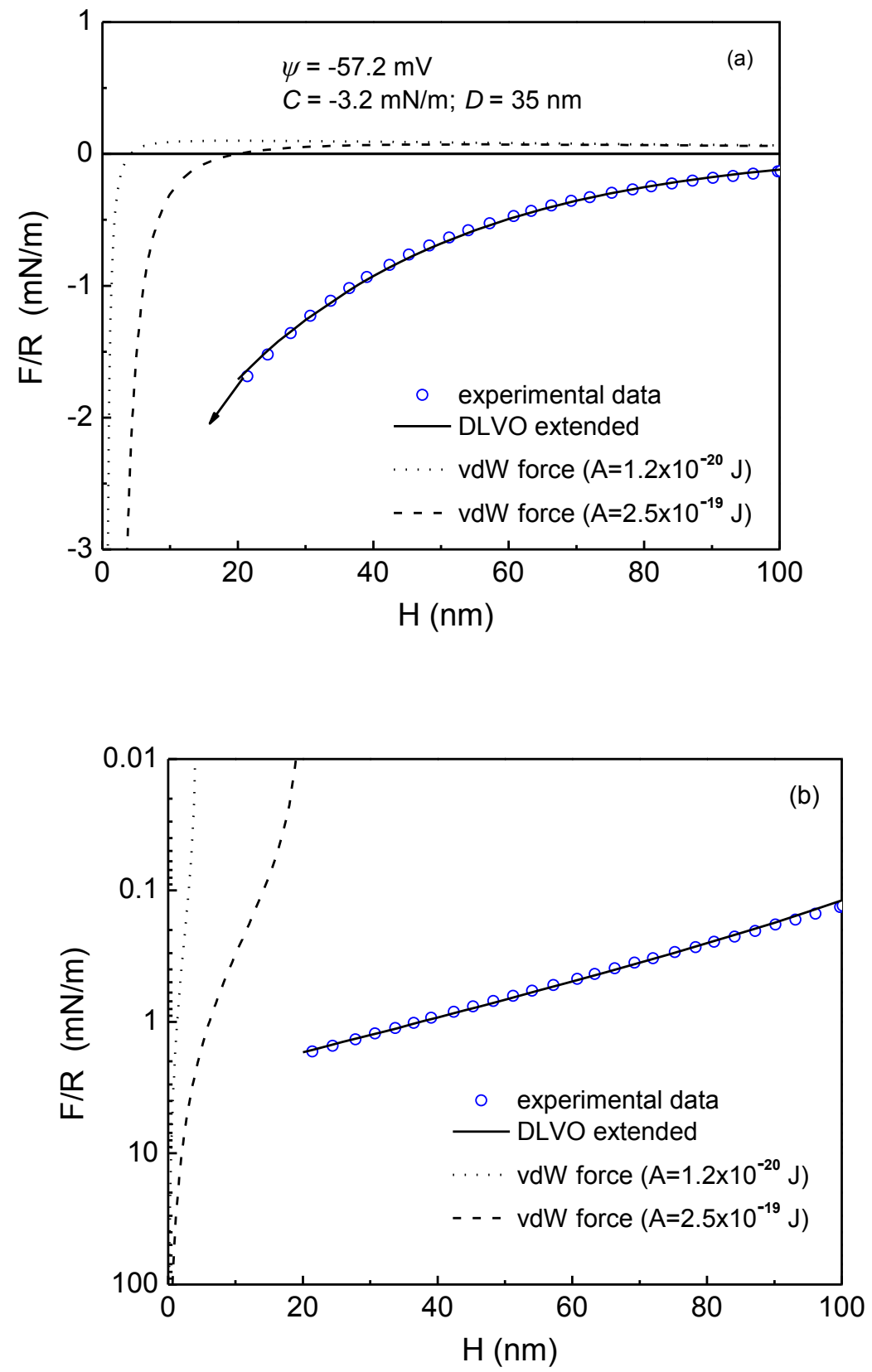

Figure 2.4. (a) A normalized AFM force curve obtained in water between gold surfaces hydrophobized in a $1 \times 10^{-2} \mathrm{mM} \mathrm{C}_{16} \mathrm{SH}$-in-ethanol solution for 10 minutes. The dashed and dotted lines represent the force curves fitted to the DLVO theory with $A_{131}=1.2 \times 10^{-20} \mathrm{~J}$ and $A_{131}=2.5 \times 10^{-19} \mathrm{~J}$, respectively, for the gold-water-gold system. The solid line represents the experimental data fitted to the DLVO theory extended to include the contributions from the hydrophobic force. The hydrophobic force is represented by the single-exponential force law $(F / R=C \exp (-H / D))$ with $C=-3.2 \mathrm{mN} / \mathrm{m}$ and $D=35 \mathrm{~nm}$ as fitting parameters. (b) The same data plotted on a log-log scale to show the goodness of the fit. 


\subsubsection{Hydrophobic Force}

Samples of gold spheres and plates were hydrophobized by immersing them in a $1 \times$ $10^{-5} \mathrm{M} \mathrm{C}_{16} \mathrm{SH}$-in-ethanol solution for 10 minutes. Figure 2.3 shows the equilibrium water contact angles measured on these samples at different immersion times. As shown, the contact angle reached a maximum of $105^{\circ}$ after 10 minutes of immersion time. Thus, the 10minute immersion time should be sufficient to complete the formation of a monolayer.

Figure 2.4 shows a force versus distance curve obtained with a gold sphere and a gold plate hydrophobized in the matter described above. The measured forces were net attractive and long-ranged $(0 \sim 80 \mathrm{~nm})$, with the two surfaces jumping into contact at $H \approx 22 \mathrm{~nm}$ where the force gradient exceeded the spring constant. The dashed and dotted lines represent the van der Waals force curves with $A_{131}=1.2 \times 10^{-20}$ and $A_{131}=2.5 \times 10^{-19} \mathrm{~J}$, respectively, while the solid line represents the DLVO theory extended to include the contribution from the hydrophobic force. The force curve was smooth indicating that the long-range attraction was not caused by nanobubbles. Figure $2.4 \mathrm{~b}$ shows the same experimental results plotted in a semi-log plot to show that the long-range attraction can be fitted to a single-exponential force law with a decay length of $35 \mathrm{~nm}$. Thus, the long-range attractive force decays exponentially, and is much stronger and longer-ranged than the van der Waals forces considered in the present work.

It is well known that the forces measured between hydrophobic surfaces vary a great deal depending on the measuring apparatus used and the methods of hydrophobization employed. The measured forces reported in the literature have been classified into three groups, ${ }^{8,19}$ which include i) the short range, but strongly attractive, forces that are typically observed between robust hydrophobic surfaces formed by the adsorption of chemisorbing surfactants, ${ }^{63,64}$ ii) the long-range, exponentially decaying forces observed between surfaces coated with physisorbed surfactants, ${ }^{65-68}$ and iii) the attractive forces of random strengths and ranges, the force curves showing steps due to bubble coalescence. The third group of forces is observed typically with hydrophobic surfaces with very high contact angles. ${ }^{31-33,69}$

It would be difficult to classify the results obtained in the present work to any one of the three groups. Since $\mathrm{C}_{16} \mathrm{SH}$ chemisorbs on gold and forms robust hydrophobic surfaces, they should belong to the first group. However, the forces measured in the present work are of very long-range and decay exponentially without steps.

\subsubsection{Bubble Coalescence}

Ederth $^{40}$ and Ederth et al. ${ }^{41}$ used a bimorph surface force apparatus (MASIF) to measure the forces between $\mathrm{C}_{16} \mathrm{SH}$-coated gold surfaces in water and ethanol/water mixtures. They observed "excess" (long-range) attractions at $\theta>90^{\circ}$, but the force curves showed steps at separations in the range of $20-50 \mathrm{~nm}$. They concluded, therefore, that the excess attractions were caused by the coalescence of bubbles on the surface. It should be pointed out, however, that Ederth et al. ${ }^{41}$ conducted the force measurements after immersing the substrates in $1 \mathrm{mM}$ $\mathrm{C}_{16} \mathrm{SH}$-in-ethanol solutions for more than 15 hours. These conditions represented 2 orders of magnitude higher thiol concentration and longer contact time than employed in the present work to obtain the results shown in Figure 2.4.

Figure 2.5a shows the AFM force measurements conducted in the present work after hydrophobizing the gold substrates under the same conditions as employed by Ederth et al. ${ }^{41}$ 

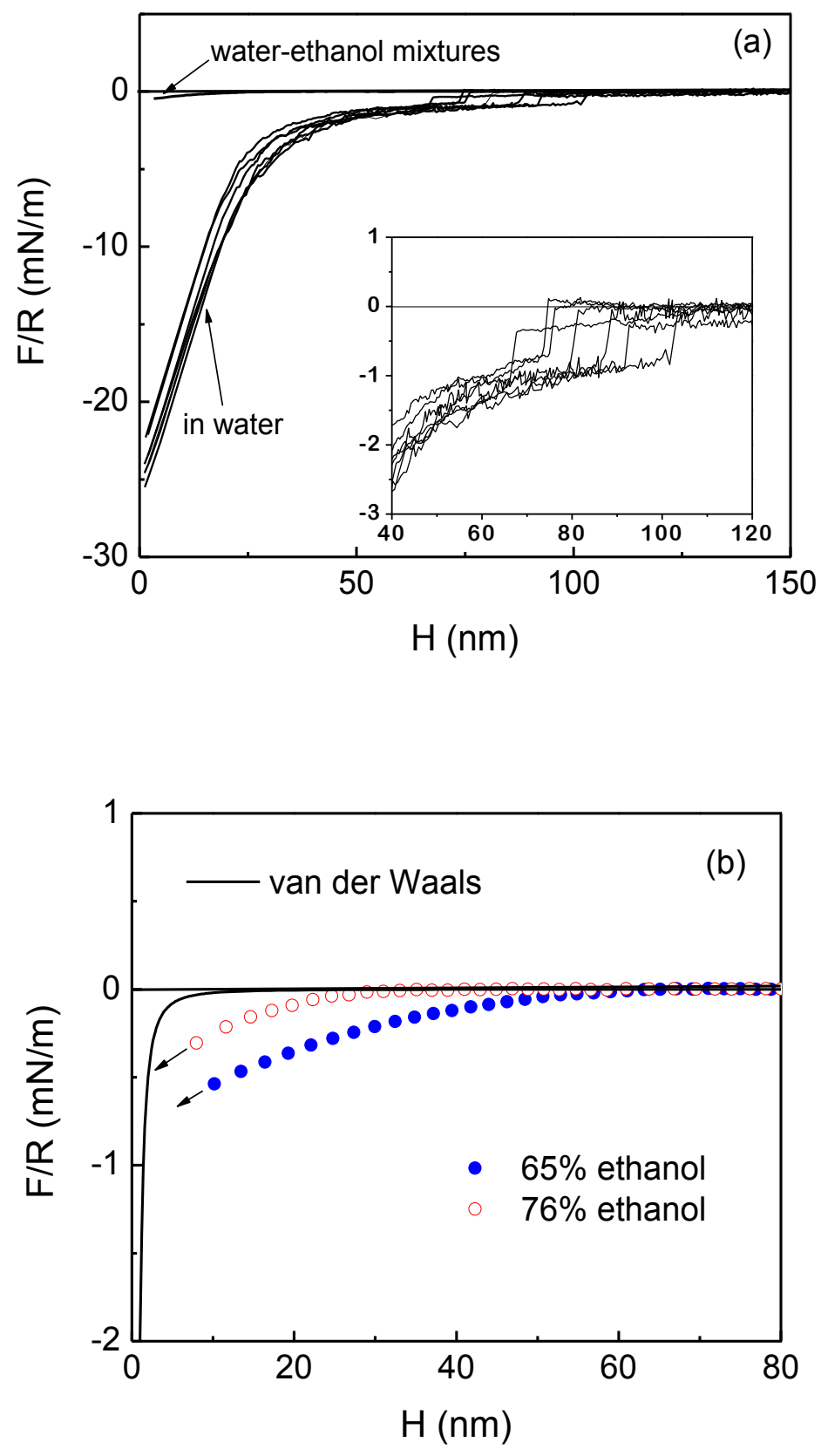

Figure 2.5. (a) Normalized forces measured in pure water and ethanol/water mixtures between gold surfaces contacted with a $1 \mathrm{mM} \mathrm{C} \mathrm{C}_{16} \mathrm{SH}$-in-ethanol solution for at least 15 hours; (b) The contact angles of gold in the ethanol-water mixtures were 44 and $39^{\circ}$ in 65 and $76 \%$ ethanol, respectively.

The equilibrium water contact angle was $106^{\circ}$. The force curves obtained in water show steps, as reported by these investigators, but not in ethanol/water mixtures. In aqueous 
solutions containing 65 and $76 \%$ ethanol by volume, the steps disappeared completely as shown in Figure 2.5b. This was not surprising because the contact angles were less than $90^{\circ}$, that is, 44 and $39^{\circ}$ in 65 and $76 \%$ ethanol-in-water solutions, respectively. Under these conditions, bubble nucleation is thermodynamically not possible. Therefore, the net-attractive forces observed in the ethanol/water mixtures should be considered real hydrophobic forces. Note here that the magnitudes of the attractive forces measured in the ethanol/water mixtures were substantially less than in pure water, which may be attributed to the likelihood that the network of hydrogen bonds in solution became weaker in the presence of ethanol. Effects of ethanol on the AFM force measurements will be discussed further in detail in another communication.

The results presented in Figure 2.5a show that nanobubbles can indeed be formed when gold substrates are hydrophobized at a high thiol concentration for a long period of time. It is possible that the thiol monolayer can have defects (or pits) in which air bubbles can nucleate. It is possible that at lower concentrations (e.g., $\left.1 \times 10^{-2} \mathrm{mM} \mathrm{C}_{16} \mathrm{SH}\right)$ and shorter contact times smoother coatings are formed, which are less likely to trap nanobubbles and hence give rise to force curves with no steps, as shown in Figure 2.4. Sakamoto et al. ${ }^{36}$ also showed steps in their AFM force curves obtained with silica surfaces coated with $\mathrm{C}_{18} \mathrm{TACl}$

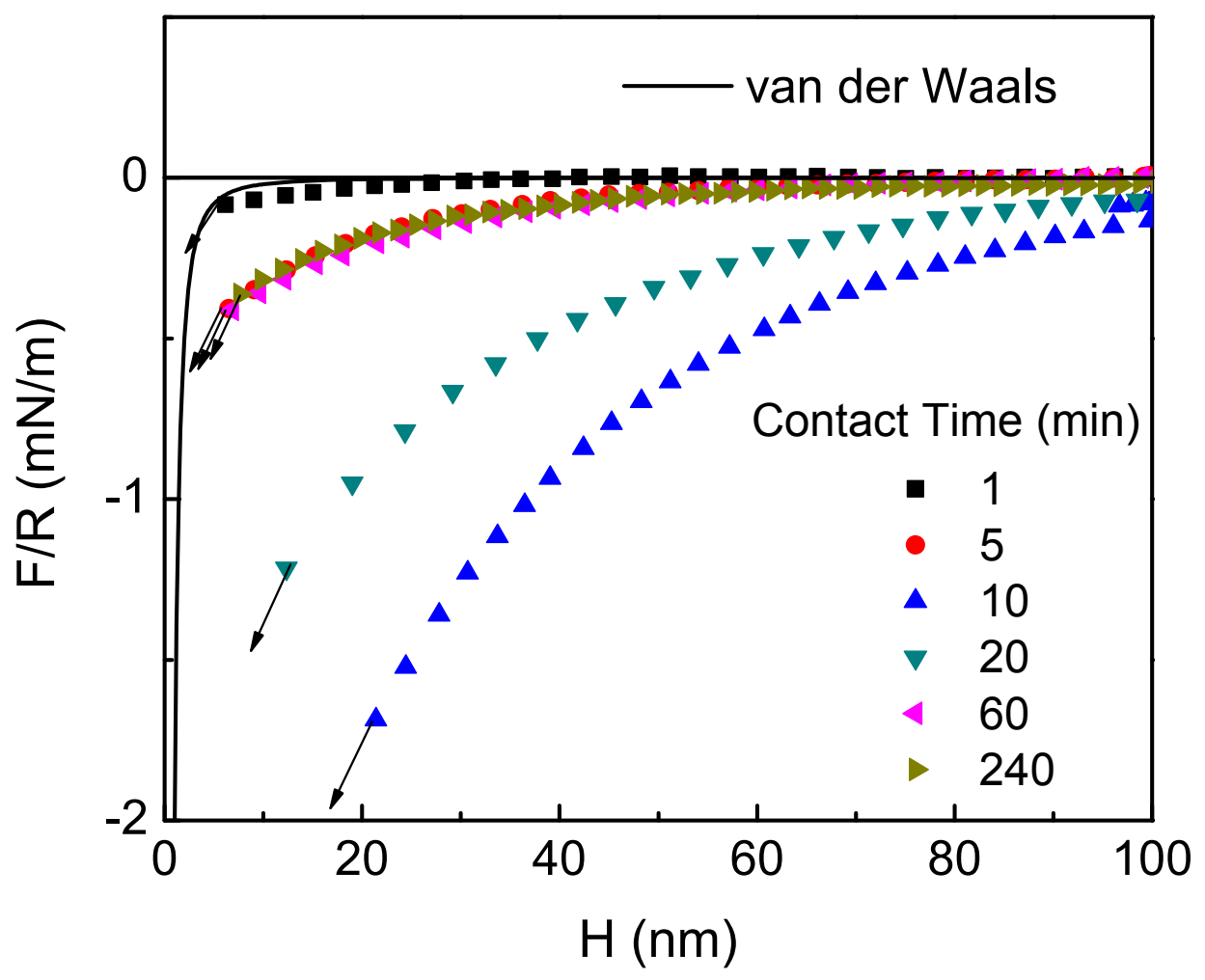

Figure 2.6. Effects of the contact time between gold substrates and a $0.01 \mathrm{mM} \mathrm{C}_{16} \mathrm{SH}$-inethanol solution on the AFM forces measured in pure water. 
solutions, and concluded that the long-range attractions were due to bridging bubbles. In support of this claim, they showed that the long-range attractions disappeared when the solution was degassed. On the other hand, Zhang et al. ${ }^{17}$ showed that long-range attractions were still observed in degassed solutions. However, the long-range attractions observed in degassed solutions were an order of magnitude weaker than those measured by Sakamoto et $a l .{ }^{36}$ Thus, the long-range attractions observed by Zhang et al. ${ }^{17}$ were considered true hydrophobic forces while those measured by Sakamoto et al. ${ }^{36}$ were actually capillary forces. Likewise, the long-range attraction observed in the present work between gold surfaces hydrophobized at $1 \times 10^{-2} \mathrm{mM} \mathrm{C}_{16} \mathrm{SH}$ for 10 minutes may also be considered a true hydrophobic force.

Figure 2.6 shows a set of AFM force curves obtained with the gold surfaces immersed in a $1 \times 10^{-2} \mathrm{mM} \mathrm{C}_{16} \mathrm{SH}$-in-ethanol solution for different periods of time. As the contact time was increased from 1 to 10 minutes, the measured forces became stronger, reached a maximum, and then decreased as the contact time was further increased. Also, none of the force curves shows steps, indicating that the attractive forces were not created by bubble coalescence. Thus, all of the force curves shown in Figure 2.6 may be considered to
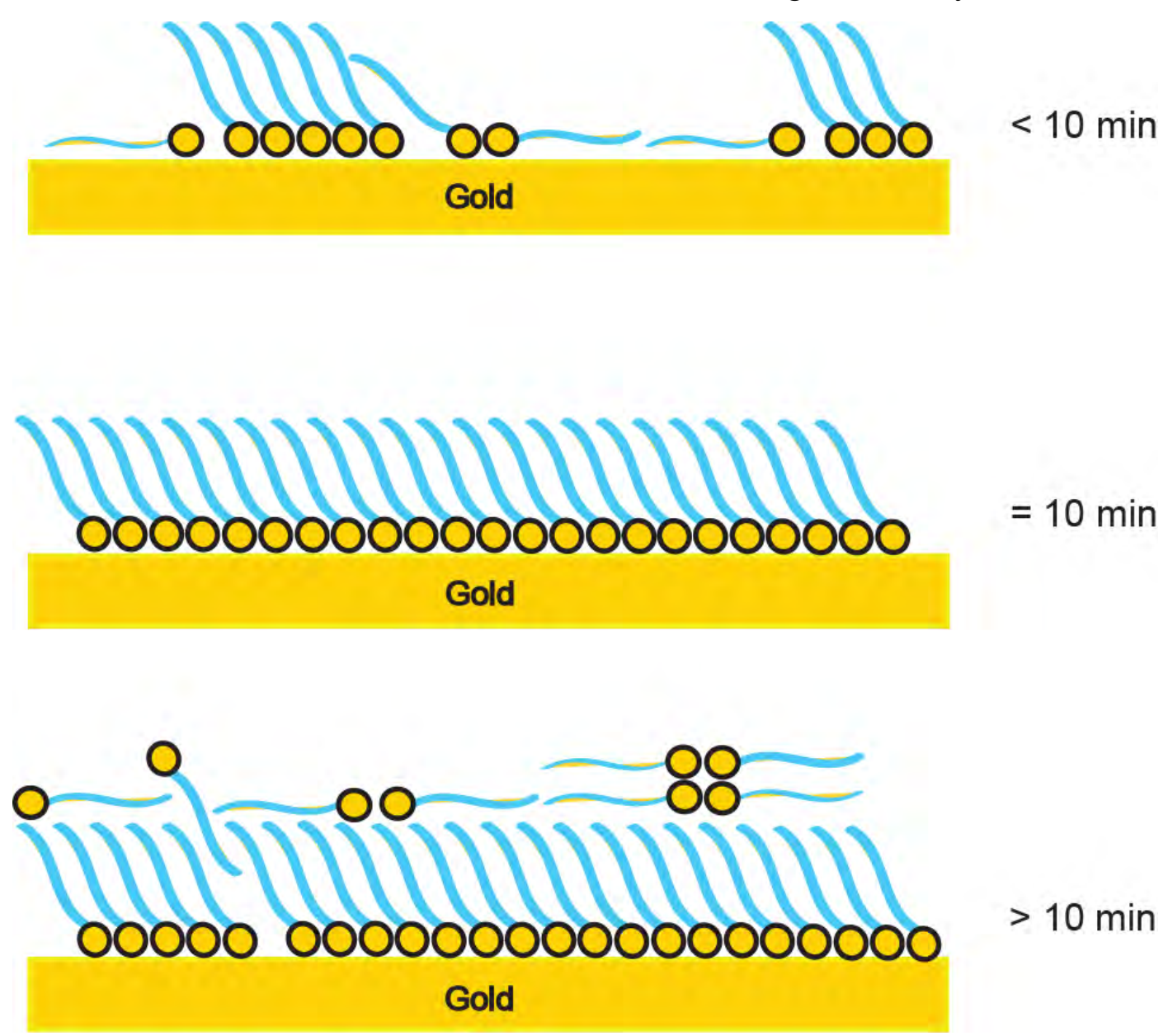

Figure 2.7. A model for the adsorption of $\mathrm{C}_{16} \mathrm{SH}$ on gold. The surface coverage and orientation are shown to change with the contact time in a $10^{-2} \mathrm{mM}$ thiol-in-ethanol solution. 
represent true hydrophobic attractions.

The forces measured after 240 minutes of contact time were substantially lower than the maximum observed after only 10 minutes of contact time, as shown in Figure 2.6. It was found, however, that the maximum force was fully restored after washing the substrates with appropriate organic solvents. The washing procedure involved flushing the AFM cell, mounted with both the plate and sphere, first with ethyl ether and then with ethanol for a few seconds each, followed by flushing with a sufficient amount of nanopure water. It is likely that after a long contact time, the $\mathrm{C}_{16} \mathrm{SH}$ adsorption resulted in a multi-layer coating, and that solvent washing removed only the species adsorbing on top of the first monolayer. The solvents would not remove the monolayer of the chemisorbed thiol. Thus, the solvent washing would expose the $-\mathrm{CH}_{3}$ groups, which should increase the hydrophobicity and, hence, the long-range attraction (or hydrophobic force).

It is well known that alkyl xanthates and thionocarbamates form multi-layers on sulfide minerals (e.g., $\mathrm{Cu}_{2} \mathrm{~S}$, and copper-activated $\mathrm{ZnS}$ ) and precious metals (e.g., $\mathrm{Au}, \mathrm{Ag}$, and $\mathrm{Au}-\mathrm{Ag}$ alloys), and that the species adsorbing in the multi-layers are metal xanthates. ${ }^{70-73}$ It is known also that the head groups of various metal xanthates have varying degrees of hydrophobic character, depending on the difference in electronegativities of the sulfur and metal ions. ${ }^{74}$ Xanthates are commonly used as hydrophobizing reagents (collectors) in the base metals flotation industry, and they behave similarly as $n$-alkanethiols. Thus, gold surfaces coated with multi-layers of $\mathrm{C}_{16}$-thiol should still be hydrophobic. In fact, the water contact angles does not change with increasing contact time in $1 \times 10^{-2} \mathrm{mM} \mathrm{C}_{16} \mathrm{SH}$-in-ethanol solutions, as shown in Figure 2.3, which makes it difficult to explain the restoration of the full hydrophobic force by the solvent washing. One possible explanation would be that thiolcoated gold surfaces become smoother after the solvent washing. It has been shown that smoother surfaces gives stronger attractive forces. ${ }^{75}$

Figure 2.7 may depict a mechanism for the adsorption of $\mathrm{C}_{16} \mathrm{SH}$ on gold at a relatively low concentration (e.g., $1 \times 10^{-2} \mathrm{mM}$ ). At a short contact time, the surface coverage will be low, which may be responsible for a weak hydrophobic force. After an optimum contact time of 10 minutes, a close-packed monolayer is formed, which gives rise to a maximum hydrophobic force as shown in Figure 2.6. At a longer contact time, the hydrophobic force diminishes possibly due to the exposure of the -SH or -SAu groups toward the aqueous phase, or the surface roughness created by multi-layer coatings. When a gold substrate is hydrophobized at a very high concentration (e.g., $1 \mathrm{mM}$ ), for a very long time (e.g., overnight), the surface roughness may be greatly increased. The force curves obtained under such conditions (see Figure 2.5a and Ederth et al. ${ }^{41}$ ) show steps, indicating that nanobubbles are trapped in between the aspirates and the valleys of rough surfaces. Solvent washing would remove the species adsorbed on top of monolayers, provide a smooth hydrophobic surface, and hence create a strong long-range attraction. In order to explore this possibility, a set of gold sphere and plate were hydrophobized in a $1 \mathrm{mM} \mathrm{C}_{16} \mathrm{SH}$-in-ethanol solution overnight (12 hours) in the same manner as Ederth et al., ${ }^{41}$ and then washed with ethanol and subsequently with pentane. The residual solvents were evaporated off the surface in a nitrogen gas stream. The force measurements were then conducted a Nanoscope IVa, which was equipped with a standard fluid cell and a scanner "J". Just before the measurement, the gold substrates were washed again by flushing the liquid cell with pure ethanol for a few seconds and subsequently with a plenty of nanopure water. As shown in Figure 2.8, the measured force was net negative and the force curve was smooth with no 
steps. It appears, therefore, that the solvent washing replaced the capillary force with a true hydrophobic force.

It has been shown in the present work that the long-range attractions observed in the present work between thiol-coated gold surfaces are much larger than the van der Waals force, and that they are not caused by the preexisting bubbles on the surface. If the long range attraction with a decay length of $35 \mathrm{~nm}$ cannot be attributed to a hydrophobic attraction, one might consider the possibility that it originates from an electrostatic attraction between charged patches. ${ }^{26}$ For this theory to work, however, it is necessary that the patch sizes be large (larger than the size of hemimicelles) and mobile. ${ }^{76}$ The latter is unlikely in view of the fact that thiols chemisorb on gold. ${ }^{77}$ Further, the thiolated gold has almost the same $\zeta$ potential as bare gold as shown in Table 2.1. What is left then would be the possibility that the long-range attraction is related to the changes in water structure around hydrophobic surfaces. A problem with this approach is that the computer simulations show that the surface-induced water structure can be extended up to several layers of water molecules only. ${ }^{78-80}$ As suggested by Ninham, ${ }^{81}$ the hydrophobic force may be related to the gas

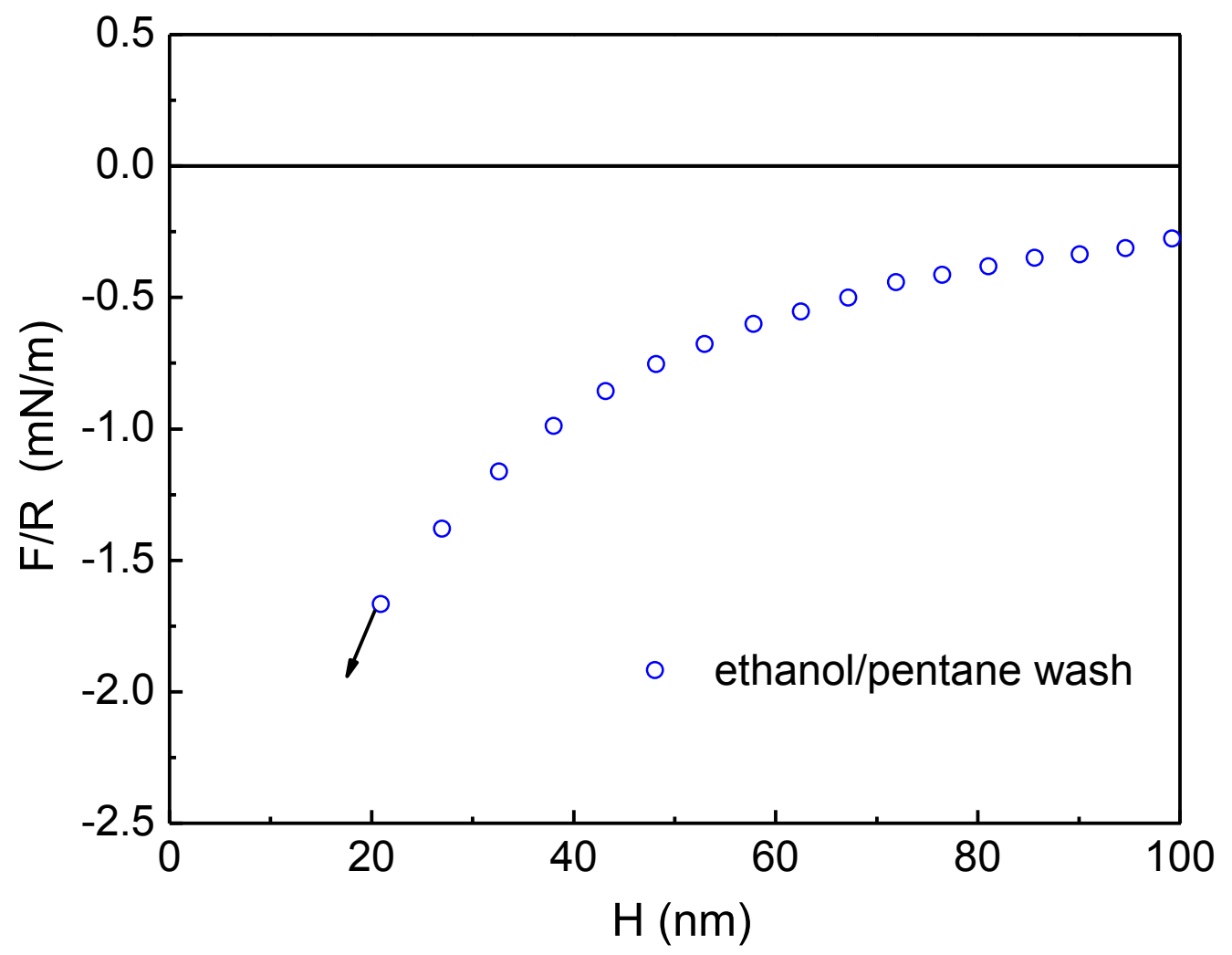

Figure 2.8. An AFM force curve obtained between gold surfaces immersed in a $1 \mathrm{mM}$ $\mathrm{C}_{16} \mathrm{SH}$-in-ethanol solution for 12 hours and then washed with organic solvents before the measurements. The solvent washing eliminated the steps shown in Figure 2.5a and gave rise to a smooth attractive force curve. 
molecules (oxygen and nitrogen) dissolved in water. At 1 atm, the concentration of the dissolved gas molecules is $5 \times 10^{-2} \mathrm{M}$, above which the DLVO theory breaks down and the Hofmeister effects begin to show up. It is possible that the dissolved gas molecules, which are hydrophobic and are likely to concentrate near hydrophobic surfaces, may promote the structuring of water at larger distances away from hydrophobic surfaces. In degassed solutions, the surface-induced structuring may not be extended too far into the solution, thereby making the hydrophobic force disappear, ${ }^{82}$ or only the short-range hydrophobic force remain. $^{37}$

Although the force measurements conducted by Zhang et al. ${ }^{17}$ in degassed solutions showed long-range forces, it would be difficult to claim that the AFM cell was completely sealed off from the ambient during the measurement. Thus, the long-range force observed by Zhang et al. could have been affected by a small amount of dissolved gases still present in the system. Pashley et $a .^{82-85}$ showed recently that hydrocarbon oils can be readily emulsified in water without a surfactant when the mixture was thoroughly $(>97 \%)$ degassed. These investigators suggested that degassing eliminated the hydrophobic force, making the emulsions to be stabilized by the double-layer force as predicted by the DLVO theory.

The charged-patch model ${ }^{26,27}$ may be useful for explaining the long-range forces observed between mica or silica surfaces coated with physisorbing cationic surfactants. ${ }^{27,86 \text {, }}$ 87 It is difficult, however, to relate the mechanism to the gases dissolved in solution, while the long-range force observed (at $H>20 \mathrm{~nm}$ ) in air-saturated solutions disappears upon degassing. 37

\section{$\underline{2.5 \text { Conclusions }}$}

The Hamaker constant for the gold-gold interactions in water was determined to be $1.2 \times 10^{-20} \mathrm{~J}$ by fitting the AFM force curves obtained between bare gold surfaces to the DLVO theory. This value is close to that $\left(1.26 \times 10^{-20} \mathrm{~J}\right)$ determined using the methylene iodide contact angle method. However, these values are substantially lower than those (2.5 4 $\times 10^{-19} \mathrm{~J}$ ) obtained from the Lifshitz theory. The lower Hamaker constants measured in the present work may be due to possible contamination of the gold surface, which may be difficult to avoid in view of the large Hamaker constant of the heavy metal.

AFM force measurements were also conducted using gold surfaces hydrophobized by the self-assembly of 1-hexadecanethiol. When the surfaces were hydrophobized in a $1 \mathrm{mM}$ thiol-in- ethanol solution longer than 6 hours, the measured forces were net-attractive and long-ranged. However, the force curves exhibited steps, indicating that the measured forces were due to bridging bubbles. Bubble nucleation was warranted as the water contact angles of the thiolated gold surfaces were over $90^{\circ}$. These results were in agreement with those of Ederth et $a l .{ }^{40}$ When the AFM force measurements were conducted in ethanol/water mixtures, the contact angles became less than $90^{\circ}$, and hence prevented bubble nucleation and gave rise to smooth force curves without steps. The measured forces were weaker than in pure water, but were still net attractive, long-ranged, and stronger than the van der Waals force.

When the gold substrates were hydrophobized in a dilute $\left(1 \times 10^{-2} \mathrm{mM}\right)$ thiol solution at a relatively short contact time (10 minutes), a long-range attractive force which decayed exponentially with a decay length of $35 \mathrm{~nm}$ was obtained. The force curve exhibited no steps indicating that the long-range attraction was not due to bridging bubbles. Steps appeared 
when the gold substrates were hydrophobized in a strong $(1 \mathrm{mM})$ thiol solution at a long contact time (overnight). When the force measurements were conducted after washing the substrates with appropriate solvents, the steps in force curves disappeared and long-range hydrophobic forces appeared.

The results obtained in the present work show that the long-range attraction observed between thiolated gold surfaces in water and ethanol solutions are not due to bridging nanobubbles or electrostatic attraction between charged patches. This leaves the possibility that the long-range attraction is caused by the changes in water structure near hydrophobic surfaces. Further work is needed to obtain stronger evidence that the long-range hydrophobic forces are indeed of structural origin.

\section{References}

1. Xu, Z.; Yoon, R.-H., J. Colloid Interface Sci. 1989, 132, (2), 532-541.

2. $\quad \mathrm{Xu}, \mathrm{Z}$; Yoon, R.-H., J. Colloid Interface Sci. 1990, 134, (2), 427-434.

3. Israelachvili, J. N.; Pashley, R. M., J. Colloid Interface Sci. 1984, 98, (2), 500-514.

4. $\quad$ Pashley, R. M.; Mcguiggan, P. M.; Ninham, B. W., Science 1985, 229, 1088-1089.

5. Claesson, P. M.; Christenson, H. K., J. Phys. Chem 1988, 92, 1650-1655.

6. $\quad$ Rabinovich, Y. I.; Derjaguin, B. V., Colloids Surf. 1988, 30, 243-251.

7. $\quad$ Parker, J. L.; Cho, D. L.; Claesson, P. M., J. Phys. Chem. 1989, 93, 6121-6125.

8. Christenson, H. K.; Claesson, P. M., Adv. Colloid Interface Sci. 2001, 91, 391-436.

9. $\quad$ Derjaguin, B. V.; Churaev, N. V., Colloids Surf. 1989, 41, 223-237.

10. Churaev, N. V., Usp. Kolloidn, Khim., FAN, Tashkent 1987, 70-78.

11. Blake, T. D.; Kitchener, J. A., J. Chem. Soc., Faraday Trans. 1 1972, 68, 1435-1442.

12. Hough, D. B.; White, L. R., Adv. Colloid Interface Sci. 1980, 14, 3-41.

13. Laskowski, J.; Kitchener, J. A., J. Colloid Interface Sci. 1969, 29, 670-679.

14. Israelachvili, J. N.; Pashley, R. M., Nature 1982, 300, 341-342.

15. Rabinovich, Y. I.; Yoon, R.-H., Langmuir 1994, 10, 1903-1909.

16. Eriksson, J. C.; Ljunggren, S.; Claesson, P. M., J. Chem. Soc., Faraday Trans. 2 1989, 85, (3), 163-176.

17. Zhang, J.; Yoon, R.-H.; Mao, M.; Ducker, W. A., Langmuir 2005, 21, 5831-5841.

18. Yoon, R.-H.; Pazhianur, R., Colloids and Surf., A 1998, 144, 59-69.

19. Meyer, E. E.; Rosenberg, K. J.; Israelachvili, J., Proc. Nat. Acad. Sci. U.S.A 2006, 103, 15739-15746.

20. Yoon, R.-H., Aufbereit. Tech 1991, 32, 474-485.

21. Yoon, R.-H.; Mao, L., J. Colloid Interface Sci. 1996, 181, 613-626.

22. Mao, L.; Yoon, R.-H., Int. J. Miner. Process 1997, 51, 171-181.

23. Eriksson, J. C.; Yoon, R.-H., Hydrophobic Attraction in the Light of Thin-Film Thermodynamics. In Colloid Stability: The Role of Surface Forces, Tadros, T. F., Ed. WILEY-VCH Verlag GmbH \& Co. KGaA: Weinheim, Germany, 2007; Vol. 1, pp 99-132.

24. Yoon, R.-H.; Wang, L., Hydrophobic Forces in Foam Films. In Colloid Stability. The Role of Surface Forces-Part I, Tadros, T. F., Ed. Wiley-VCH: 2007; Vol. 1, pp 161186.

25. Eriksson, J. C.; Henriksson, U., Langmuir 2007, 23, 10026-10033. 
26. Miklavic, S. J.; Chan, D. Y. C.; White, L. R.; Healy, T. W., J. Phys. Chem. 1994, 98, 9022-9032.

27. Meyer, E. E.; Lin, Q.; Hassenkam, T.; Oroudjev, E.; Israelachvili, J. N., Proc. Nat. Acad. Sci. U.S.A 2005, 102, 6839-6842.

28. Tsao, Y.-H.; Evans, D. F.; Wennerstrom, H., Science 1993, 262, (5133), 547-550.

29. Yoon, R.-H.; Ravishankar, S. A. R., J. Colloid Interface Sci. 1996, 179, 391-402.

30. Christenson, H. K.; Claesson, P. M., Science 1988, 239, 390-392.

31. Tyrrell, J. W. G.; Attard, P., Langmuir 2002, 18, 160-167.

32. Attard, P.; Moody, M. P.; Tyrrell, J. W. G., Physica A 2002, 314, 696-705.

33. Parker, J. L.; Claesson, P. M.; Attard, P., J. Phys. Chem. 1994, 98, 8468-8480.

34. Nguyen, A. V.; Nalaskowski, J.; Miller, J. D.; Butt, H.-J., Int. J. Miner. Process. 2003, 72, 215-225.

35. Yang, J.; Duan, J.; Fornasiero, D.; Ralston, J., J. Phys. Chem. B 2003, 107, 61396147.

36. Sakamoto, M.; Kanda, Y.; Miyahara, M.; Higashitani, K., Langmuir 2002, 18, 57135719.

37. Meyer, E. E.; Lin, Q.; Israelachvili, J. N., Langmuir 2005, 21, 256-259.

38. Bain, C. D.; Troughton, E. B.; Tao, Y.-T.; Evall, J.; Whitesides, G. M.; Nuzzo, R. G., J. Am. Chem. Soc. 1989, 111, 321-335.

39. Christenson, H. K.; Yaminsky, V. V., Colloids Surf., A 1997, 129-130, 67-74.

40. Ederth, T., J. Phys. Chem. B 2000, 104, 9704-9712.

41. Ederth, T.; Claesson, P.; Liedberg, B., Langmuir 1998, 14, 4782-4789.

42. Ron, H.; Rubinstein, I., Langmuir 1994, 10, 4566-4573.

43. Ron, H.; Rubinstein, I., J. Am. Chem. Soc. 1998, 120, 13444-13452.

44. Kane, V.; Mulvaney, P., Langmuir 1998, 14, 3303-3311.

45. Giesbers, M.; Kleijin, J. M.; Stuart, M. A. C., J. Colloid Interface Sci. 2002, 252, 138-148.

46. Hillier, A. C.; Kim, S.; Bard, A. J., J. Phys. Chem. 1996, 100, 18808-18817.

47. Biggs, S.; Mulvaney, P., J. Chem. Phys. 1994, 100, (11), 8501-8505.

48. Sondag-Huethorst, J. A. M.; Fokkink, L. G. J., Langmuir 1992, 8, 2560-2566.

49. Karpovich, D. S.; Blanchard, G. J., Langmuir 1994, 10, 3315-3322.

50. Cleveland, J. P.; Manne, S.; Bocek, D.; Hansma, P. K., Rev. Sci. Instrum. 1993, 64, (2), 403-405.

51. Ohshima, H.; Healy, T. W.; White, L. R., J. Colloid Interface Sci. 1982, 89, 484-493.

52. Israelachvili, J. N., Proc. R. Soc. London, Ser. A 1972, 331, 39-55.

53. Oss, C. J. v., Interfacial Forces in Aqueous Media. 2 ed.; CRC Press: 2006.

54. Fowkes, F. M., Ind. Eng. Chem. 1964, 56, (12), 40-52.

55. Thelen, E., J. Phys. Chem. 1967, 71, (6), 1946-1948.

56. Biggs, S.; Mulvaney, P.; Zukoski, C. F.; Grieser, F., J. Am. Chem. Soc. 1994, 116, (20), 9150-9157.

57. Ducker, W. A.; Senden, T. J., Langmuir 1992, 8, 1831-1836.

58. Larson, I.; Chan, D. Y. C.; Drummond, C. J.; Grieser, F., Langmuir 1997, 13, 24292431.

59. Wall, J. F.; Grieser, F.; Zukoski, C. F., J. Chem. Soc., Faraday Trans. 1997, 93, (22), 4017-4020.

60. Dagastine, R. R.; Grieser, F., Langmuir 2004, 20, 6742-6747. 
61. Considine, R. F.; Drummond, C. J., langmuir 2001, 17, 7777-7783.

62. Bhattacharjee, S.; Ko, C.-H.; Elimelech, M., Langmuir 1998, 14, 3365-3375.

63. Parker, J. L.; Claesson, P. M., Langmuir 1994, 10, 635-639.

64. Parker, J. L.; Claesson, P. M.; Wang, J.-H.; Yasuda, H. K., Langmuir 1994, 10, 27662773.

65. Claesson, P. M.; Blom, C. E.; Herder, P. C.; Ninham, B. W., J. Colloid Interface Sci. 1986, 114, (1), 234-242.

66. Lin, Q.; Meyer, E. E.; Tadmor, M.; Israelachvili, J. N.; Kuhl, T. L., Langmuir 2005, 21, 251-255.

67. Christenson, H. K.; Fang, J.; Ninham, B. W.; Parker, J. L., J. Phys. Chem. 1990, 94, 8004-8006.

68. Hato, M., J. Phys. Chem. 1996, 100, 18530-18538.

69. Ishida, N.; Sakamoto, M.; Miyahara, M.; Higashitani, K., Langmuir 2000, 16, (13), 5681-5687.

70. Mielczarski, J. A.; Yoon, R.-H., J. Phys. Chem. 1989, 93, 2034-2038.

71. Mielczarski, J. A.; Yoon, R.-H. In Proceedings of the Engineering Foundation Conference, Palm Coast, Florida, January 10-15, 1989; Moudgil, B. M.; Scheiner, B. J., Eds. United Engineering Trustees, Inc., New York: Palm Coast, Florida, 1989; pp 619-630.

72. Mielczarski, J. A.; Yoon, R.-H., J. Colloid Interface Sci. 1989, 131, (2), 423-432.

73. Mielczarski, J. A.; Yoon, R.-H., Langmuir 1991, 7, 101-106.

74. Leja, J., Surface Chemistry of Froth Flotation. In Plenum Press: Vancouver, 1982; p 510.

75. Walz, J. Y.; Suresh, L.; Piech, M., J. Nanopart. Res. 1999, 1, 99-113.

76. Zhang, J.; Yoon, R.-H.; Eriksson, J. C., Colloids Surf., A 2007, 300, 335-345.

77. Ulman, A., Chem. Rev. 1996, 96, (4), 1533-1554.

78. Fa, K.; Nguyen, A. V.; Miller, J. D., J. Phys. Chem. 2005, 109, (27), 13112-13118.

79. Forsman, J.; Jonsson, B.; Woodward, C. E., J. Phys. Chem. 1996, 100, (36), 1500515010.

80. Sakurai, M.; Tamagawa, H.; Ariga, K.; Kunitake, T.; Inoue, Y., Chem. Phys. Lett. 1998, 289, 567-571.

81. Ninham, B. W., Prog. Colloid Polym. Sci. 2006, 133, 65-73.

82. Maeda, N.; Rosenberg, K. J.; Israelachvili, J. N.; Pashley, R. M., Langmuir 2004, 20, 3129-3137.

83. Pashley, R. M., J. Phys. Chem. B 2003, 107, 1714-1720.

84. Francis, M. J.; Gulati, N.; Pashley, R. M., J. Colloid Interface Sci. 2006, 299, 673677.

85. Francis, M. J.; Pashley, R. M., Colloids Surf., A 2006, 287, 36-43.

86. Tsao, Y.-H.; Evans, D. F.; Wennerstrom, H., Langmuir 1993, 9, 779-785.

87. Claesson, P. M.; Herder, P. C.; Blom, C. E.; Ninham, B. W., J. Colloid Interface Sci. 1987, 118, 68-79. 


\section{TASK 2 SURFACE FORCE MEASUREMENTS BETWEEN GOLD SURFACES HYDROPHOBIZED WITH ALKANETHIOLS OF DIFFERENT CHAIN LENGTHS}

\section{$\underline{3.1 \text { Abstract }}$}

An atomic force microscope (AFM) was used to measure the surface forces between two macroscopic gold surfaces. A microsphere of gold and a gold-coated glass plate were immersed in thiol-in-ethanol $\left(1 \times 10^{-2} \mathrm{mM}\right.$ or $\left.1 \mathrm{mM}\right)$ solutions to hydrophobize the surfaces. The degree of hydrophobization was controlled by varying immersion time and using thiols of different chain lengths. In the present work, alkanethiols, such as ethanethiol $\left(\mathrm{C}_{2} \mathrm{SH}\right), 1$ butanethiol $\left(\mathrm{C}_{4} \mathrm{SH}\right)$, 1-dodecanethiol $\left(\mathrm{C}_{12} \mathrm{SH}\right)$ and 1-hexadecanethiol $\left(\mathrm{C}_{16} \mathrm{SH}\right)$ were used. The equilibrium water contact angle obtained with $\mathrm{C}_{2} \mathrm{SH}$ was $67^{\circ}$. The contact angles were greater than $90^{\circ}$ on gold surfaces coated with the other thiols of longer chain lengths. Regardless of whether the contact angle was larger than $90^{\circ}$ or not, the force curves obtained were smooth and showed no steps, and the measured forces were substantially larger and longer-ranged than the van der Waals force. When the thiol-coated surfaces were exposed for prolonged periods of times in the atmospheric air or contaminated, discontinuities or steps on the force curves were observed. The maximum equilibrium water contact angles obtained with $\mathrm{C}_{4} \mathrm{SH}, \mathrm{C}_{12} \mathrm{SH}$ and $\mathrm{C}_{16} \mathrm{SH}$ were $94^{\circ}, 105^{\circ}$ and $105^{\circ}$, respectively, and the strongest hydrophobic attractions were measured on surfaces exhibiting maximum contact angles. The strongest hydrophobic force measured on $\mathrm{C}_{4} \mathrm{SH}$-coated gold was weaker than those measured on $\mathrm{C}_{12} \mathrm{SH}$ - and $\mathrm{C}_{16} \mathrm{SH}$-coated gold surfaces. The latter two surfaces were indistinguishable with respect to the measured strongest hydrophobic forces.

It was found that the strengths and ranges of the hydrophobic attractive forces decreased in the presence of $\mathrm{NaCl}$ electrolyte and a cationic surfactant $\mathrm{C}_{12} \mathrm{TACl}$. The decay lengths of the hydrophobic forces were not equal to one half of the Debye lengths, contrary to the predictions from the electrostatic models. The results obtained in the present work suggest that the long-range attractions measured may represent true hydrophobic forces originating from the structural change of water in the vicinity of hydrophobic surfaces. Solvable foreign species added to the system, e.g., cationic surfactant $\mathrm{C}_{12} \mathrm{TACl}$ and $\mathrm{NaCl}$, break the hydrogen bond network, and reduce the hydrophobic forces.

\subsection{Introduction}

The classic Derjaguin-Landau-Verwey-Overbeek (DLVO) theory, which combines the effects of the van der Waals attraction and electrostatic double layer repulsion, has been successfully applied to many colloidal systems. However, some colloidal systems comprising hydrophobic particles ${ }^{1,2}$ or oil droplets ${ }^{3}$ exhibit additional attraction which is much stronger than the van der Waals force considered in DLVO theory. Early experiments carried out in 1972 by Blake and Kitchener ${ }^{4}$ on the stability of wetting films sandwiched between an air bubble and hydrophobic silica indicated the existence of a long-range hydrophobic attraction force. In 1982, the hydrophobic force was first measured directly by Israelachvili and Pashley $^{5}$ on two mica surfaces hydrophobized by the in-situ adsorption of hexadecyltrimethylammonium bromide (CTAB) in aqueous solutions using a surface force apparatus (SFA). The pioneering works of Blake and Kitchener, and Israelachvili and Pashley had established that in addition to the traditional DLVO forces, a long-range 
attractive force called "hydrophobic force" operates between hydrophobic surfaces in aqueous media. The hydrophobic force plays an important role in many scientific and technological fields, such as surface and colloid science, biology, and minerals processing. In the field of minerals processing, recognition of the long-range hydrophobic attraction force is important in the modeling the coagulation of hydrophobic particles ${ }^{1,2}$ and the process of bubble-particle attachment in flotation. ${ }^{6-8}$

The significant role of the hydrophobic force in many scientific and technological fields has led to a great deal of study. During the past two decades or more, several instruments, e.g., surface force apparatus (SFA), measurement and analysis of surface interaction force (MASIF), and atomic force microscope (AFM), have been used for the direct measurement of the hydrophobic force. Numerous reports published document the existence of this long-range attractive force. Different research groups used different types of hydrophobic surfaces and a range of hydrophobization methods. However, the results were far from being consistent. The magnitudes and ranges of the forces varied from one experiment to another. Also the origin of the long-range attractive forces has been debated without consensus. Various theoretical models have been brought forward to explain the experimental results. Eriksson et al. ${ }^{9}$ attributed the long-range attractive force to the enhanced hydrogen bonding of the water molecules confined between two hydrophobic surfaces. However, computer simulations ${ }^{10-13}$ showed that the change in the structure of the water near the hydrophobic surface only extend the distance of several molecular diameters, while many experimentalist reported that the force can be measured at the separation distances of up to $80 \mathrm{~nm}$. Other investigators suggested that the long-range hydrophobic attraction is due to the correlations between the charged patches ${ }^{14,15}$ formed as a result of surfactant adsorption, between mobile surface groups ${ }^{16}$ when the two surfaces approach to each other, or between large dipoles associated with the patches of adsorbed surfactants. ${ }^{17,18}$ However, some of these models cannot be reconciled with all the experimental data. In particular, Miklavic et al. ${ }^{14}$ predicted that the attraction due to correlation should be exponentially decaying with decay lengths equal to one half the Debye lengths upon increasing the electrolyte concentration. Although there were some experimental results supporting this theory, more recent experimental data show that the force is independent of ionic strength.

More recently, many investigators proposed that the non-DLVO attractive forces observed from the force measurements are caused by bridging submicroscopic bubbles preexisting on the hydrophobic surfaces ${ }^{19-23}$ or cavities. ${ }^{24-29}$ The most frequently quoted evidence for this was the discontinuities (or steps) observed in force versus distance curves, each step representing the coalescence of nanobubbles. ${ }^{19}$ When two bubbles coalesce, gas bridges (or cavities) are formed between two surfaces, which give rise to capillary forces. This theory seems appealing, because the existence of the bubbles solves the range problem, and the range of the forces depends on the size of the bubbles involved. A primary setback for this theory is that very small air bubbles in bulk water are short-lived. ${ }^{30}$ In addition, experiments showed that long-range hydrophobic attractions were observed for hydrophobic surfaces on which there were no signs of bubbles. ${ }^{31,32}$ Stevens et al. ${ }^{33}$ found that the range and strength of the attraction between two hydrophobic amorphous fluoropolymer surfaces in deaerated water was significantly greater than that of the van der Waals attraction. Meyers et $\mathrm{al}^{34}$ conducted SFA force measurements between mica surfaces coated by a LangmuirBlodgett (LB)-deposited hydrophobic monolayer of DODAB both in the presence and 
absence of dissolved gases. In the presence of dissolved gases, they observed long-range attractive forces, while in degassed solutions only short-range attraction forces (under $\sim 250$ $\AA$ A) were observed. They concluded, therefore, that ,true ${ }^{e e}$ hydrophobic force exists even in degassed solutions.

Ederth and Liedberg ${ }^{35}$ and Ederth $^{23}$ have conducted direct force measurement between thiolated-gold surfaces in water, water/ethanol mixtures by MASIF. The wettability of the surfaces was controlled by changing the mole ratios of 1-hexadecanethiol $\left(\mathrm{C}_{16} \mathrm{SH}\right)$ and 16-hydroxyhexanedecanethiol $\left(\mathrm{HOC}_{16} \mathrm{SH}\right)$, and by changing the mixing ratios between water and ethanol. They showed that when the contact angle of the thiolated-gold surface was larger than $90^{\circ}$, long-range attractions with step-like profiles which were observed due to the coalescences of microscopic air bubbles on the surfaces. When the contact angle was less than $90^{\circ}$, the attraction was actually the same as the van der Waals attraction. In the present work, an AFM force microscope was used to measure the surface forces between two macroscopic gold surfaces in water, aqueous $\mathrm{NaCl}$ electrolytes and cationic surfactant dodecyltrimethylammonium chloride $\left(\mathrm{C}_{12} \mathrm{TACl}\right)$ solutions. We also chose the self-assembled monolayers of thiols on gold as interacting surfaces, because they are robust, well characterized and easy to prepare. Spheres of gold and gold-coated glass plates were immersed in quiescent thiol-in-ethanol solutions to hydrophobize the gold surfaces. The degree of hydrophobization was controlled by varying immersion times or using thiols of different chain lengths. The results are used to test existing theoretical models and discuss the possible origins of the long-range hydrophobic force.

\section{$\underline{3.3 \text { Materials and Methods }}$}

\subsubsection{Materials}

A Millipore direct-Q 3 ultrapure (Millipore, MA) water system was purchased and used to obtain deionized water with a resistivity of $18.2 \mathrm{M} \Omega / \mathrm{cm}$ at $25^{\circ} \mathrm{C}$. Alkanethiol such as ethanethiol $\left(\mathrm{C}_{2} \mathrm{SH}, 98 \%\right.$, TCI America $)$, 1-butanethiol $\left(\mathrm{C}_{4} \mathrm{SH}, 99 \%\right.$, Aldrich), 1dodecanethiol $\left(\mathrm{C}_{12} \mathrm{SH}, 98 \%\right.$, Aldrich $)$ and 1-hexadecanethiol $\left(\mathrm{C}_{16} \mathrm{SH}, 97 \%\right.$, TCI America) dissolved in 200 proof ethanol (AAPER alcohol) were used to hydrophobize the gold surfaces. They were used without further purification. Sulfuric acid (98\%) from Fisher Scientific and hydrogen peroxide $\left(\mathrm{H}_{2} \mathrm{O}_{2}, 29-32 \% \mathrm{w} / \mathrm{w}\right)$ from Alfa Aesar were used as received to clean gold plates. Sodium Chloride (99.999\%, Sigma-Aldrich) was roasted in air at $560{ }^{\circ} \mathrm{C}$ to decompose organic impurities and dissolved in pure water. Dodecyltrimethylammonium chloride $\left(\mathrm{C}_{12} \mathrm{TACl}, 99 \%\right)$ was purchased from Acros Organics Co. Gold wire $(0.0127 \mathrm{~mm}$ dia, $99.9 \%$, Alfa Aesar) was used to make gold microspheres.

Gold microspheres and gold-coated glass plate were used for AFM force measurements. The gold-coated glass plates were produced by depositing pure gold on a clean glass slide using a vacuum evaporator. A $50 \AA$ chromium layer was deposited first on the glass prior to coating it with a thin-layer ( $500 \AA$ ) of pure gold. The chromium coating was necessary to achieve strong bonding between gold and substrate. The AFM image showed that the flat gold surfaces were smooth, with maximum peak-to-valley distance of $3.3 \mathrm{~nm}$. Gold spheres with appropriate diameters were produced according to the procedure developed by Raiteri et al. ${ }^{36}$ The two ends of a thin gold wire were connected to a power supply $(120 \mathrm{~V}, \mathrm{AC})$ and briefly short circuited in a glass tray as shown in Figure 3.1. A small 


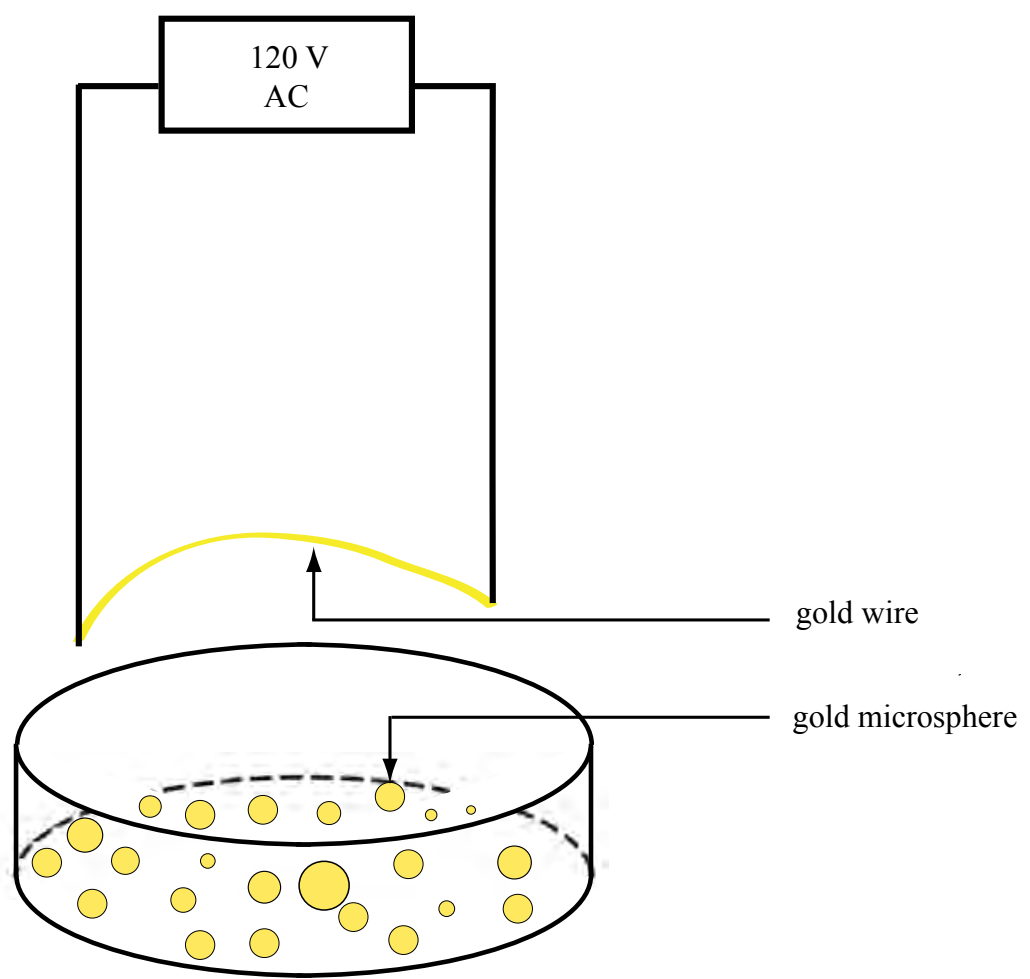

Figure 3.1. Schematic illustration of the method for making gold microspheres.

cloud of gold particles were produced in the spark. In this manner, gold spheres with a wide size range were produced. The spheres with radius of $4.5 \sim 10 \mu \mathrm{m}$ were chosen for the present experiments.

\subsubsection{Hydrophobizing Gold Surfaces}

The cleanliness of the gold surface is critically important in the process of selfassembly of thiol on gold. ${ }^{32,37-40}$ To ensure the high-quality thiol monolayers on gold, gold surfaces must be cleaned thoroughly prior to soaking them in thiol-in-ethanol solutions. In the present work, the gold plate surfaces were cleaned first by immersing them in a boiling $\mathrm{H}_{2} \mathrm{SO}_{4} / \mathrm{H}_{2} \mathrm{O}_{2}(2: 1$ by volume $)$ at $120^{\circ} \mathrm{C}$ for 20 minutes, and then rinsed in ultra-pure water for 1 minute, followed by ethanol wash for 2 minutes to remove the oxides produced in the piranha solution. After cleaning, the gold plates were immediately exposed to quiescent thiol solutions for hydrophobization in a fume hood. For the case of gold spheres, cleaning procedure was performed after they had been glued onto the tips of AFM cantilever springs. To prevent gold spheres from being washed away by strong acid solution, before hydrophobization, each gold sphere was flushed with ethanol, irradiated by a UV lamp $(\lambda=$ $254 \mathrm{~nm}$ ) for 2 hours to remove possible organic contaminants, and then rinsed with ethanol again.

The cleaned gold plates and spheres were hydrophobized by soaking them in thiol-inethanol solutions at room temperature via chemical reaction. To ensure the accuracy of the force measurement, for every set of experiment, only the fresh thiol surfactants were used to prepare thiol-in-ethanol solutions, because the thiol surfactant in ethanol will degrade with time. After hydrophobization, the gold substrates were washed with ethanol and then dried in a nitrogen gas stream. For a given force measurement, a set of gold plate and sphere on 
cantilever was immersed in a thiol solution for a desired period of time, so that the hydorphobicity of the two microscopic surfaces would be the same.

\subsubsection{Contact Angle Measurement}

Equilibrium water contact angles on the hydrophobized gold plates were measured with the sessile drop method using a goniometer (Ramé-Hart, Inc) under ambient conditions. Droplets of pure water of 1-2 mm diameter were placed on the surface of a horizontally placed thiolated-gold plate using a Norm-Ject syringe. The angles were measured on each side of a water droplet. The measurements were conducted using a total of five droplets placed on different locations of a gold surface, and the results were averaged. For the contact angles of thiol-coated gold in $\mathrm{NaCl}$ solutions, aqueous $\mathrm{NaCl}$ solution with different concentrations drops were employed instead of pure water as the contacting liquid.

\subsubsection{AFM Surface Force Measurement}

A Nanoscope III (Digital Instruments, CA) multi-mode atomic force microscope (AFM) equipped with a standard fluid liquid cell and a piezoelectric scanner "E" was used for the force measurements. The surface interactions between a gold-coated glass and a gold microsphere in water medium were measured at room temperature $\left(\sim 22{ }^{\circ} \mathrm{C}\right)$ using the colloidal probe technique. ${ }^{41}$ The separation distance $(H)$ between the spherical probe (gold sphere) and the flat substrate (gold-coated glass) was determined by monitoring the deflection of the cantilever on which the sphere was attached. Triangular silicon nitride (NP20 , Veeco probes) cantilevers with a nominal spring constant $(k)$ of $0.58 \mathrm{~N} / \mathrm{m}$ were used in this study, and their spring constants were determined using the Cleveland method. ${ }^{42}$ For each experiment, a gold sphere was glued onto the end of a cantilever with EPON-1004 resin (Shell Chemical Co.) using a homemade three-dimensional micromanipulator under an optical microscope (Olympus BH-2). The fluid cell made of glass was cleaned in pure water in an ultrasonic cleaner and blow-dried with nitrogen gas before each experiment. The force

measurements were conducted immediately after the thiol monolayers were formed on gold substrates. 


\section{$\underline{3.4 \text { Results }}$}

\subsubsection{Contact Angle Measurement}

Samples of gold spheres and plates were hydrophobized by immersing them in $1 \times$ $10^{-2} \mathrm{mM} \mathrm{C} \mathrm{C}_{16} \mathrm{SH}$-in-ethanol, $\mathrm{C}_{12} \mathrm{SH}$-in-ethanol or $\mathrm{C}_{4} \mathrm{SH}$-in-ethanol solutions for different periods of times. Figure 3.2 shows the water contact angles measured on these samples as functions of immersion time. As shown, the contact angles reached a maximum values of $\theta=$ $105^{\circ}$ with both $\mathrm{C}_{16} \mathrm{SH}$ - and $\mathrm{C}_{12} \mathrm{SH}$-coated gold surfaces. The immersion time of 10 minutes and 2 hours were employed for $\mathrm{C}_{16} \mathrm{SH}$ and $\mathrm{C}_{12} \mathrm{SH}$, respectively. With the $\mathrm{C}_{4} \mathrm{SH}$-coated gold surfaces, the maximum contact angle was $\theta=94^{\circ}$. The contact time employed in this case was 5 hours. It appears that the contact angles reached a maximum more quickly as the hydrocarbon chain length was increased, which is in line with the studies by Bain $e t$ al. ${ }^{43}$. These investigators found that the kinetics of adsorption is faster for longer chain thiols. This finding was attributed to the fact that van der Waals interaction between the hydrocarbon

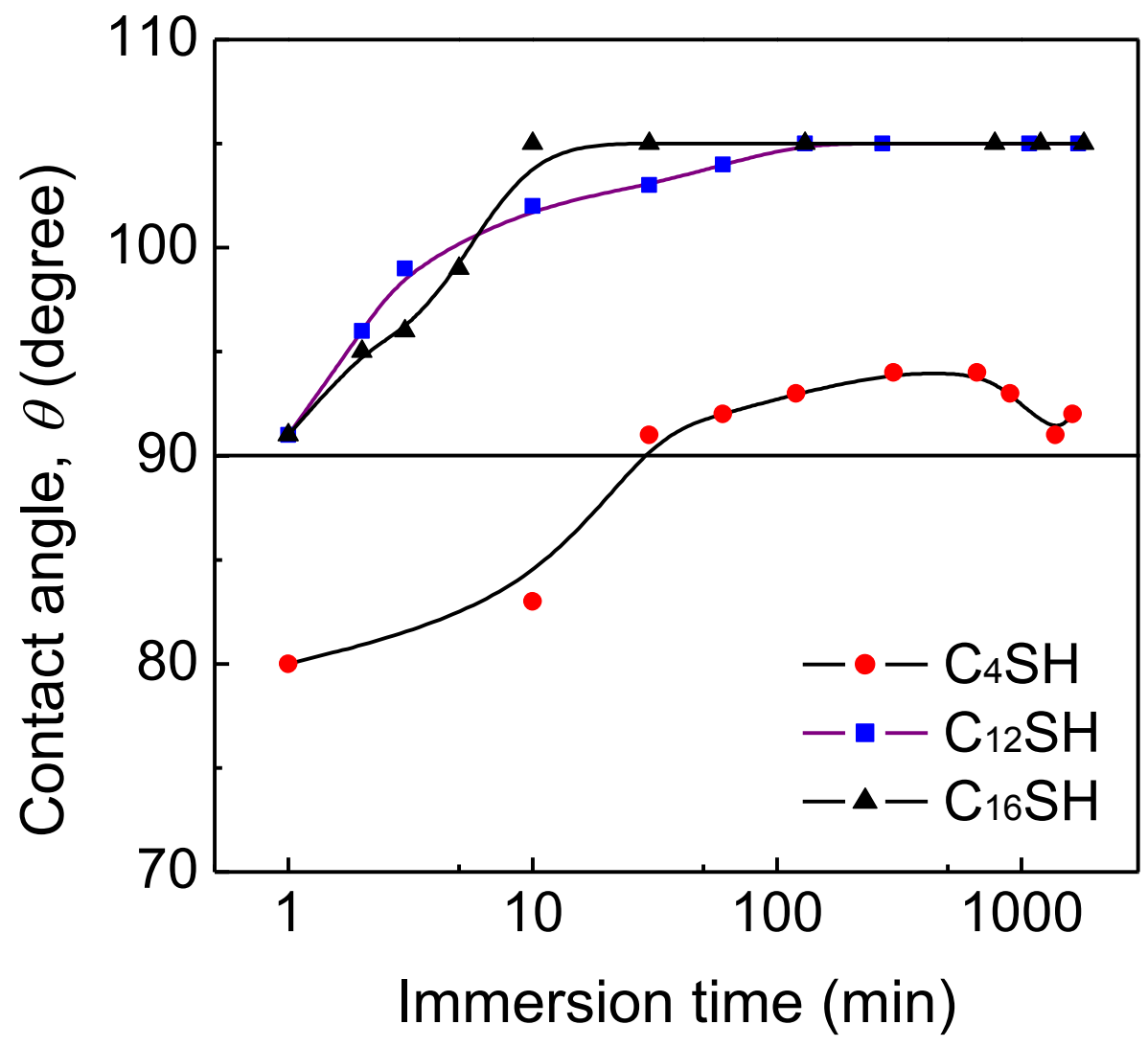

Figure 3.2. Changes in the equilibrium water contact angles $(\theta)$ on gold plates as functions of immersion time in $1 \times 10^{-2} \mathrm{mM} \mathrm{C}_{4} \mathrm{SH}-, \mathrm{C}_{12} \mathrm{SH}-$, and $\mathrm{C}_{16} \mathrm{SH}$-in-ethanol solutions. 
chains of alkanethiol increased with chain length. ${ }^{44}$ With the C-12 and C-16 thiols only 1 minute dipping time was sufficient to obtain contact angles above $90^{\circ}$.

\subsubsection{Force Measurements in Water}

Figure 3.3 shows the surface forces $(F / R)$ measured between $\mathrm{C}_{12} \mathrm{SH}$-coated gold microspheres (with radius $R$ ) and gold-coated glass plates in water as functions of the closest distance $(H)$ separating the two macroscopic surfaces. The gold substrates were immersed in $1 \times 10^{-2} \mathrm{mM} \mathrm{C}_{12} \mathrm{SH}$-in-ethanol solutions for different period of time. The trend is the same as observed on $\mathrm{C}_{16} \mathrm{SH}^{32}$ that is, the attractive force increases with immersion time, and then decreases. All the forces measured were stronger and longer-ranged than the van der Waals force. The van der Waals force curve shown in Figure 3.3 was plotted based on the Hamaker constant $\left(\mathrm{A}_{131}=1.2 \times 10^{-20} \mathrm{~J}\right)$ determined experimentally. ${ }^{32}$ At the immersion time of 1 minute, the force was only slightly stronger than the van der Waals force. However, the attractive force was detected at a separation distance $H \approx 30 \mathrm{~nm}$. At the immersion time of 30 minutes, the strength and range of the attraction increased with the attractive forces being

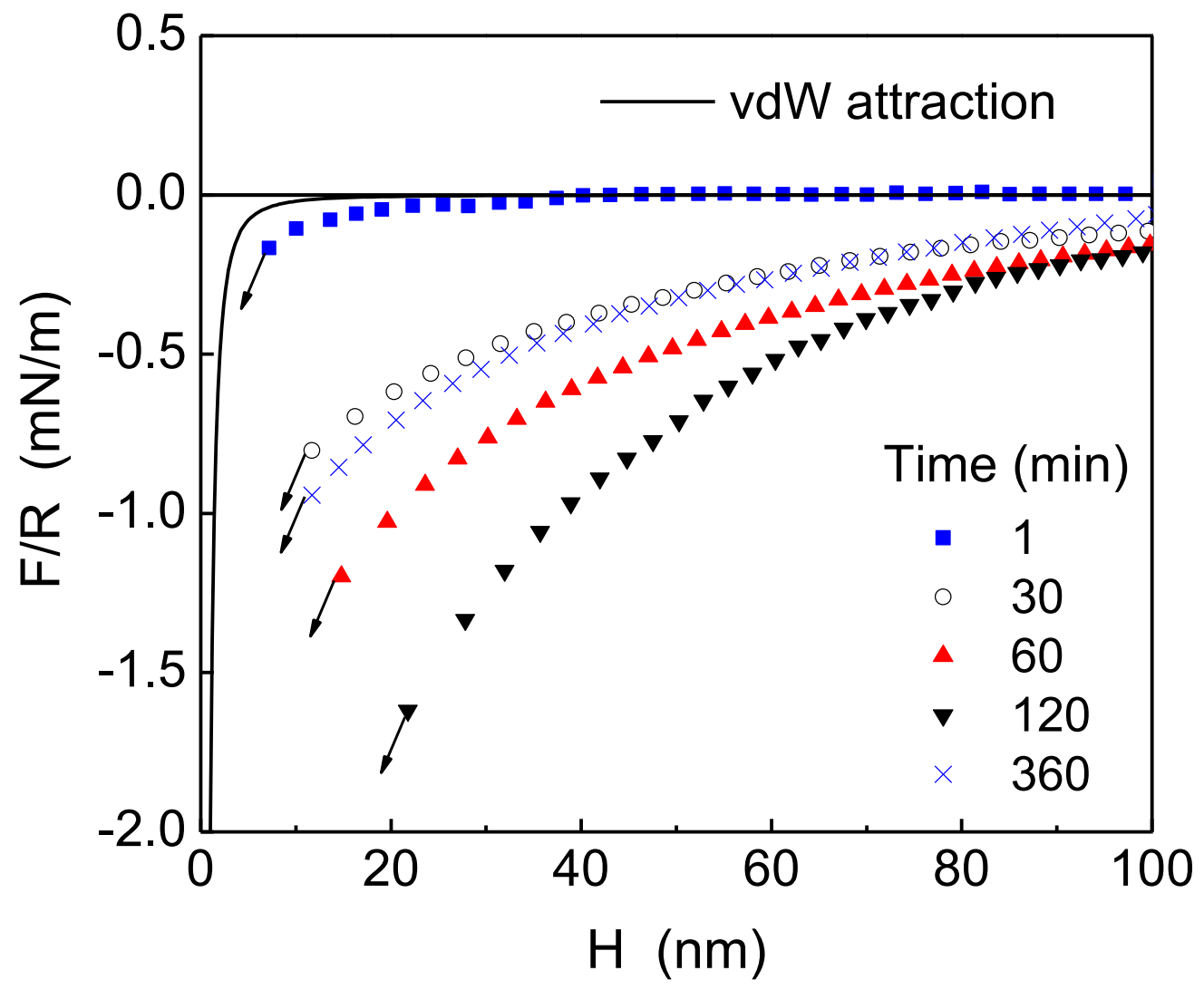

Figure 3.3. Effects of the immersion times of gold substrates in a $1 \times 10^{-2} \mathrm{mM} \mathrm{C}_{12} \mathrm{SH}-\mathrm{in}$ ethanol solution on the AFM forces measured in pure water. The solid line represents the non-retarded van der Waals force acting between two gold surfaces in water. 
detected at $H>100 \mathrm{~nm}$. At the immersion time of 60 minutes, the strength of the force further increased. The strongest attraction was obtained at the 120 minutes immersion time. As the immersion time was further increased to 360 minutes, both the strength and the range of the attraction were reduced.

As shown in Figure 3.2, the equilibrium water contact angles $(\theta)$ on $\mathrm{C}_{16} \mathrm{SH}$-coated gold were $91^{\circ}, 99^{\circ}, 105^{\circ}, 105^{\circ}, 105^{\circ}$ and $105^{\circ}$ as the immersion times were $1,5,10,20,60$ and 240 minutes. On $\mathrm{C}_{12} \mathrm{SH}$-coated gold, they were $91^{\circ}, 103^{\circ}, 104^{\circ}, 105^{\circ}$ and $105^{\circ}$ at the immersion times of $1,30,60,120$ and 360 minutes, respectively. In view of the force measurements conducted with $\mathrm{C}_{12} \mathrm{SH}$-coated surfaces, from immersion time $t=1$ to 120 minutes, the attraction became gradually stronger with contact angle; beyond $t=120$ minutes, the attraction became weaker, while the contact angle was constant. The vanished attractive forces on $\mathrm{C}_{16} \mathrm{SH}$ - and $\mathrm{C}_{12} \mathrm{SH}$-coated gold prepared with longer immersion time were restored to the original full strengths after rinsing the hydrophobic thiol-coated surfaces in the liquid cell with ether. Based on this observation, it is supposed that the hydrophobic force response is reduced due to trapping of extra thiol molecules with the - $\mathrm{SH}$ groups turned outward to water, thus forming a kind of adlayer. This physisorbed thiol layer could be removed by means of rinsing with ether.

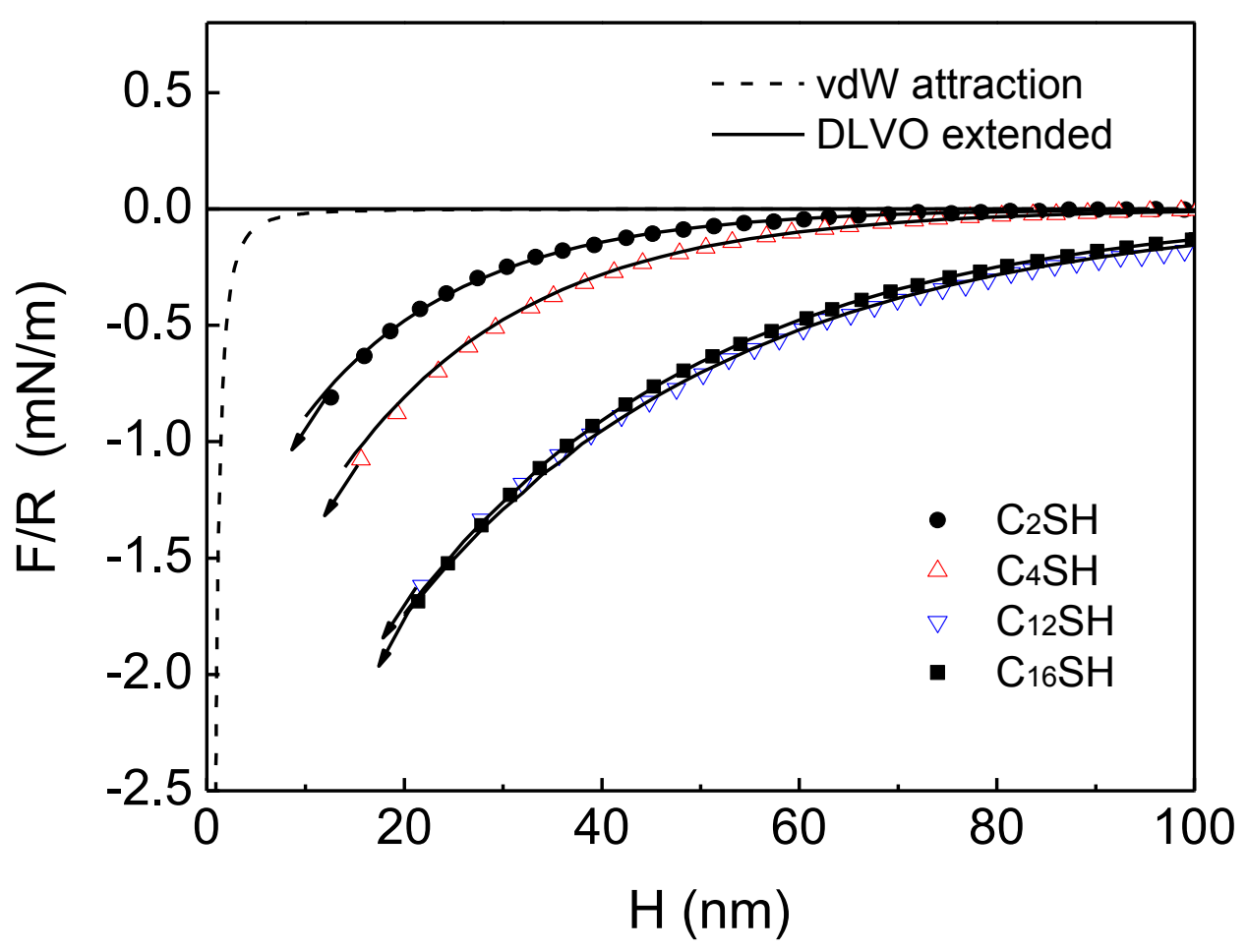

Figure 3.4. Surface forces $(F / R)$ versus separation distances $(H)$ between hydrophobized gold surfaces prepared using alkanethiols with different chain lengths. The solid lines represent the fittings of surface force data points using single-exponential laws (Equations 3.1). The dashed line represents the van der Waals force. 
Figure 3.4 shows the surface force measurements performed in water on gold surfaces hydrophobized by self-assembly of alkanethiols with different chain lengths. The strongest attractive force curves measured which were shown in Figure 3.3 on $\mathrm{C}_{12} \mathrm{SH}$-coated gold surfaces and on $\mathrm{C}_{16} \mathrm{SH}$-coated gold ${ }^{32}$ are re-drawn in Figure 3.4. Also included in Figure 3.4 are surface forces data in water on $\mathrm{C}_{4} \mathrm{SH}$ - and $\mathrm{C}_{2} \mathrm{SH}$-coated gold surfaces. According to the contact angle measurements on $\mathrm{C}_{4} \mathrm{SH}$-coated gold (Figure 3.2), the contact angle approaches the maximum value $\left(\theta=94^{\circ}\right)$ after 5 hours of immersion time. In Figure 3.4, the surface force between $\mathrm{C}_{4} \mathrm{SH}$-coated gold surfaces in water was shown when the $\mathrm{C}_{4} \mathrm{SH}$ coatings were prepared with an immersion time of 5 hours. The attractive force measured on surfaces prepared under this condition should be the strongest according to the relation between contact angle and hydrophobic force (Figure 3.3). The strongest hydrophobic force on $\mathrm{C}_{4} \mathrm{SH}-$ coated gold is measurable at the separation distance $H \approx 70 \mathrm{~nm}$. The surface force between $\mathrm{C}_{2} \mathrm{SH}$-coated gold surfaces in water was measured when the $\mathrm{C}_{2} \mathrm{SH}$ coatings were prepared by immersing a gold microsphere and a gold coated-glass plate in $1 \mathrm{mM}$ solution for an immersion time of 6 hours. The water contact angle measured on $\mathrm{C}_{2} \mathrm{SH}$-coated gold surface prepared under this condition was $\theta=67^{\circ}$, and the measured force between these surfaces was purely attractive within a separation distance $H \approx 60 \mathrm{~nm}$. All the force curves shown in Figure 3.3 and 3.4 are smooth, without showing steps or discontinuities, indicating that the hydrophobic surfaces were relatively free of contaminations or air bubbles. All the attractions are much stronger and longer-ranged than the non-retarded van der Waals force.

All of the surface force curves in Figure 3.4 were fitted to a single-exponential force law, that is:

$$
\frac{F}{R}=C \exp \left(-\frac{H}{D}\right)
$$

where $F / R$ denotes the normalized surface force, $R$ is the radius of the gold sphere, $C$ is a constant and $D$ is the decay length. $H$ is the closest separation distance between two approaching surfaces. Since the hydrophobic force is overwhelmingly larger compared to the double layer repulsive force on thiol-coated gold, ${ }^{32}$ we assume the surfaces of the hydrophobized gold are neutral, thus only the hydrophobic and van der Waals forces are considered in the fittings. The Hamaker constant of $\mathrm{A}_{131}=1.2 \times 10^{-20} \mathrm{~J}^{32}$ as determined by measuring the interaction between two bare gold surface in water and salt solutions was used in this study. The fitting was carried out after subtracting the forces due to van der Waals

Table 3.1. The Parameters Obtained by Fitting the Surface Data between Gold Surfaces Coated by Alkanethiols with Different Chain Lengths in Pure Water with Extended DLVO Theory

\begin{tabular}{cccc}
\hline & $\theta($ degree $)$ & $C(\mathrm{mN} / \mathrm{m})$ & $D(\mathrm{~nm})$ \\
\hline $\mathrm{C}_{2} \mathrm{SH}$ & 67 & -1.6 & 16.5 \\
$\mathrm{C}_{4} \mathrm{SH}$ & 94 & -2.3 & 19 \\
$\mathrm{C}_{12} \mathrm{SH}$ & 105 & -3.2 & 33 \\
$\mathrm{C}_{16} \mathrm{SH}$ & 105 & -3.3 & 31
\end{tabular}


interaction from the measured forces, and the values for $C$ and $D$ were derived and are shown in Table 3.1. As shown, the decay length $D$ and $C$ values of the attractive force depend on both the chain length and the water contact angle. The decay length $(D=16.5$ to $33 \mathrm{~nm})$ and $C$ value (from 1.6 to $3.2 \mathrm{mN} / \mathrm{m}$ ) increase with chain length (from $n=2$ to 12 ) and contact angle (from $\theta=67^{\circ}$ to $105^{\circ}$ ). For the thiols with chain length of 12 and 16 , the contact angles $\left(\theta=105^{\circ}\right)$ are the same, the attractive forces appear to coincident with each other, and the decay lengths $(D=31$ versus $33 \mathrm{~nm})$ and $C$ values $(3.3$ versus $3.2 \mathrm{mN} / \mathrm{m})$ are similar.

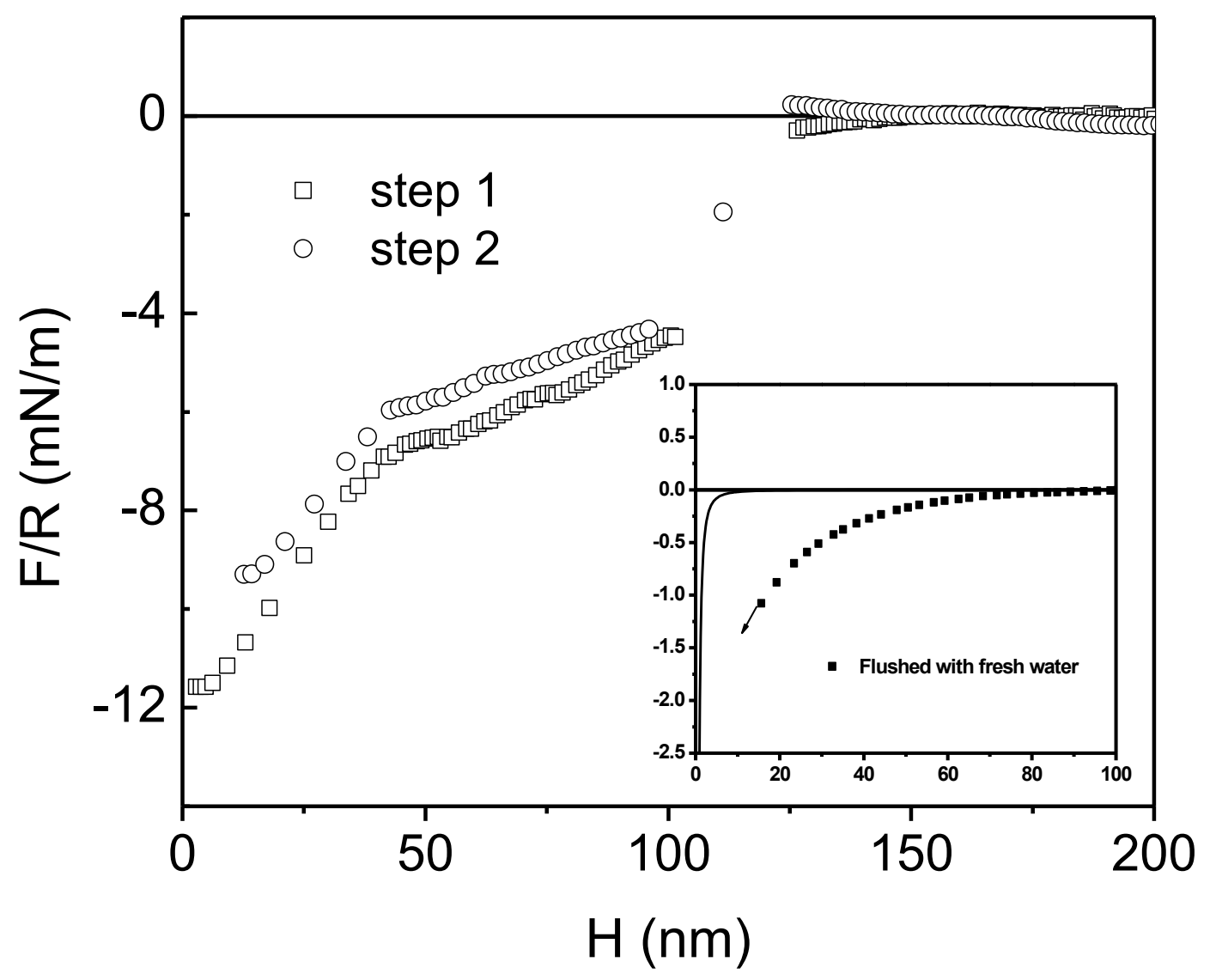

Figure 3.5. Normalized forces $(F / R)$ between gold surfaces which were hydrophobized in a $1 \times 10^{-2} \mathrm{mM} \mathrm{C}_{4} \mathrm{SH}$-in-ethanol solution for 6 hours. The step-like force curves were obtained under the condition that $\mathrm{C}_{4} \mathrm{SH}$-coated gold plate was exposed in air for elongated time prior to the commencement of force measurement. The inset represents the force obtained on the same surfaces flushed with fresh water. The contamination of hydrophobized surfaces or the air bubbles could give rise to the steps or discontinuities shown on the force curves.

Figure 3.5 shows the results of the AFM force measurements conducted between two $\mathrm{C}_{4} \mathrm{SH}$-coated gold surfaces in water. The $\mathrm{C}_{4} \mathrm{SH}$ coatings on gold were prepared in a $1 \times 10^{-2}$ $\mathrm{mM} \mathrm{C}_{4} \mathrm{SH}$-in-ethanol solution for 5 hours. The step-like force curves were obtained under the condition that $\mathrm{C}_{4} \mathrm{SH}$-hydrophobized gold surfaces were exposed in air for a prolonged period of time prior to the commencement of the force measurement. After the AFM liquid cell and the gold surfaces were flushed with fresh water, the extremely large attractions with steps 


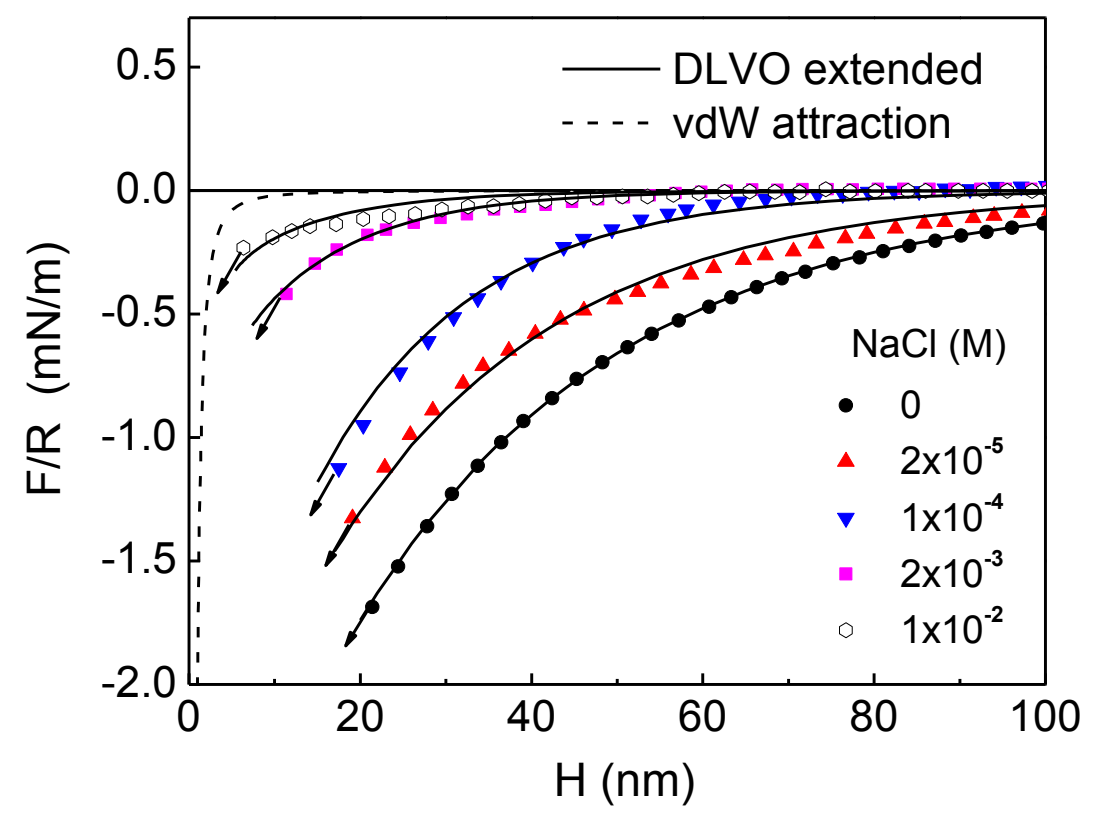

Figure 3.6. Effect of $\mathrm{NaCl}$ on the forces measured between gold surfaces hydrophobized in a $1 \times 10^{-2} \mathrm{mM} \mathrm{C}_{16} \mathrm{SH}$-in-ethanol solution for 10 minutes. The solid lines represent the fittings of surface force data points using single-exponential laws (Equations 3.1). The dashed line represents the van der Waals force.

disappeared, and a much smaller, smooth force curve was obtained. The steps were attributed to the bridging of contaminating air bubble from the air which can easily form on the $\mathrm{C}_{4} \mathrm{SH}-$ coated hydrophobic surfaces during the "drying" coating preparation process.

\subsubsection{Force Measurements in $\mathrm{NaCl}$ Aqueous Solutions}

Figure 3.6 and 3.7 show the effect of $\mathrm{NaCl}$ concentration on the hydrophobic forces measured on $\mathrm{C}_{16} \mathrm{SH}$ - and $\mathrm{C}_{12} \mathrm{SH}$-coated gold surfaces, respectively. The $\mathrm{C}_{16} \mathrm{SH}$-coated surfaces were prepared in a $1 \times 10^{-2} \mathrm{mM} \mathrm{C}_{16} \mathrm{SH}$-in-ethanol solution for 10 minutes, and the $\mathrm{C}_{12} \mathrm{SH}$-coated surfaces were prepared in a $1 \times 10^{-2} \mathrm{mM} \mathrm{C}_{12} \mathrm{SH}$-in-ethanol solution for 2 hours. The forces measured on $\mathrm{C}_{16} \mathrm{SH}$ - and $\mathrm{C}_{12} \mathrm{SH}$-coated gold in pure water were the strongest attractions and of the same magnitude and range. On both the $\mathrm{C}_{16} \mathrm{SH}-$ and $\mathrm{C}_{12} \mathrm{SH}-$ coated gold surfaces, the attractive forces decreased in range and magnitude as the $\mathrm{NaCl}$ concentration was increased from 0 to $1 \times 10^{-2} \mathrm{M}$. As shown in Figure 3.6 and 3.7, the attractive forces measured in the most concentrated $\mathrm{NaCl}$ solution $\left(1 \times 10^{-2} \mathrm{M}\right)$ were still stronger and longer-ranged than the van der Waals force. After finishing the experiments with $\mathrm{NaCl}$, the AFM liquid cell was flushed with fresh water in order to remove the $\mathrm{NaCl}$ electrolyte, and the strong attractive forces in water reappeared. It is demonstrated that the $\mathrm{C}_{16} \mathrm{SH}$ and $\mathrm{C}_{12} \mathrm{SH}$ monolayer coatings on gold were very stable, and the surface properties of these chemisorbed surfactant layers were not changed upon increasing concentration of $\mathrm{NaCl}$. Sessile drop contact angle measurement using $\mathrm{NaCl}$ aqueous drops indicates that the 


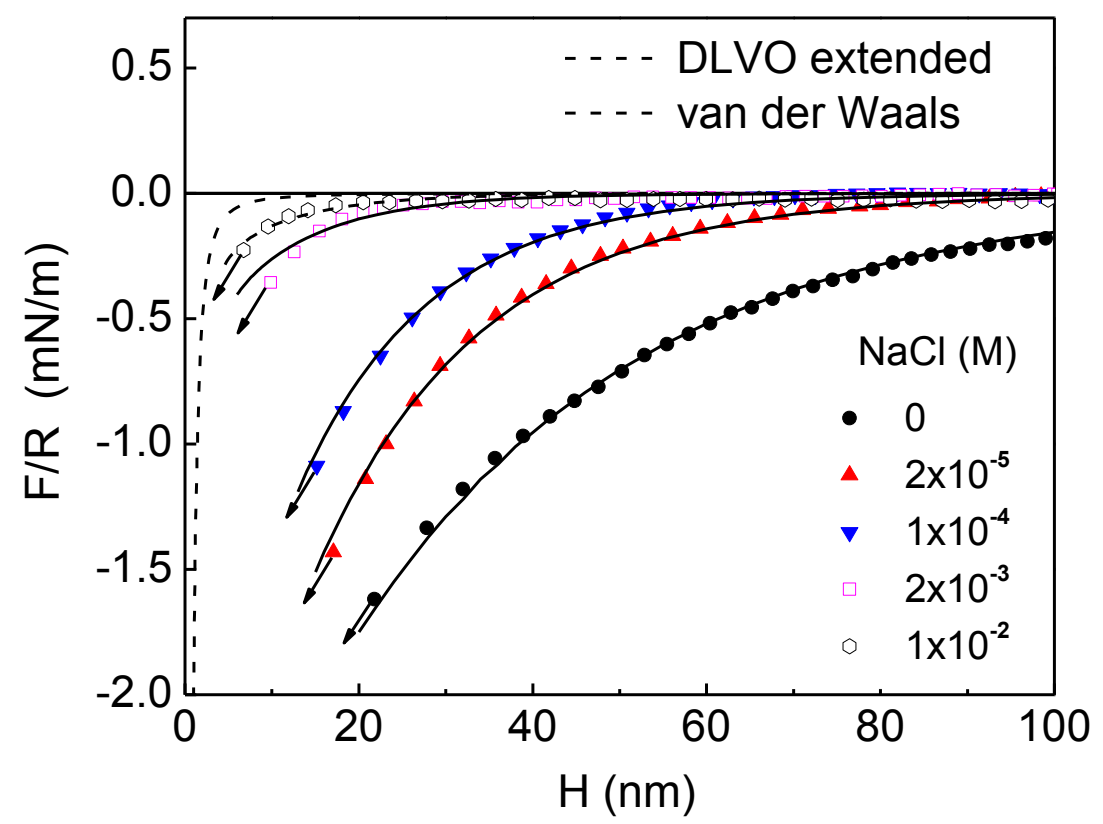

Figure 3.7. Effect of $\mathrm{NaCl}$ on the forces measured between gold surfaces hydrophobized in a $1 \times 10^{-2} \mathrm{mM} \mathrm{C}_{12} \mathrm{SH}$-in-ethanol solution for 2 hours. The solid lines represent the fittings of surface force data points using single-exponential laws (Equations 3.1). The dashed line represents the van der Waals force.

contact angle on the thiol surfaces did not change with the changed concentration of $\mathrm{NaCl}$. It is suggested that the surfaces did not become less hydrophobic in $\mathrm{NaCl}$ solutions.

Table 3.2. Effects of $\mathrm{NaCl}$ on Debye Lengths $\left(\kappa^{-1}\right)$ and Decay Lengths $(D)$ between $\mathrm{C}_{12} \mathrm{SH}$-coated Gold Surfaces and $\mathrm{C}_{16} \mathrm{SH}$-coated Gold Surfaces

\begin{tabular}{cccccc}
\hline & & \multicolumn{2}{c}{$\mathrm{C}_{12} \mathrm{SH}$} & \multicolumn{2}{c}{$\mathrm{C}_{16} \mathrm{SH}$} \\
\cline { 3 - 6 } $\mathrm{NaCl}(\mathrm{mM})$ & $\kappa^{-1}(\mathrm{~nm})$ & $C(\mathrm{mN} / \mathrm{m})$ & $D(\mathrm{~nm})$ & $C(\mathrm{mN} / \mathrm{m})$ & $D(\mathrm{~nm})$ \\
\hline 0 & 134.2 & -3.2 & 33 & -3.3 & 31 \\
0.02 & 67.1 & -3.3 & 19 & -2.8 & 26 \\
0.1 & 30 & -2.8 & 15 & -2.7 & 18 \\
2 & 6.7 & -0.6 & 11 & -0.9 & 13 \\
10 & 3 & -0.3 & 10 & -0.4 & 12 \\
\hline
\end{tabular}

each

ached 13.1). wn in The 1. The the $t_{\text {: }}$ oated valur xactly 
the same. With the concentration of $\mathrm{NaCl}$ increasing from 0 to $10 \mathrm{mM}$, the Debye length decreased from 134.2 to $3 \mathrm{~nm}$. At the same time, both $C$ values (from 3.3 to $0.4 \mathrm{~nm}$ ) and $D$ values (from 31 to $12 \mathrm{~nm}$ ) obtained on $\mathrm{C}_{16} \mathrm{SH}$-coated gold decrease with the increasing concentration of $\mathrm{NaCl}$. On $\mathrm{C}_{12} \mathrm{SH}$-coated gold, the $C$ values decrease from 3.2 to $0.3 \mathrm{~nm}$, the $D$ values decrease from 33 to $10 \mathrm{~nm}$.

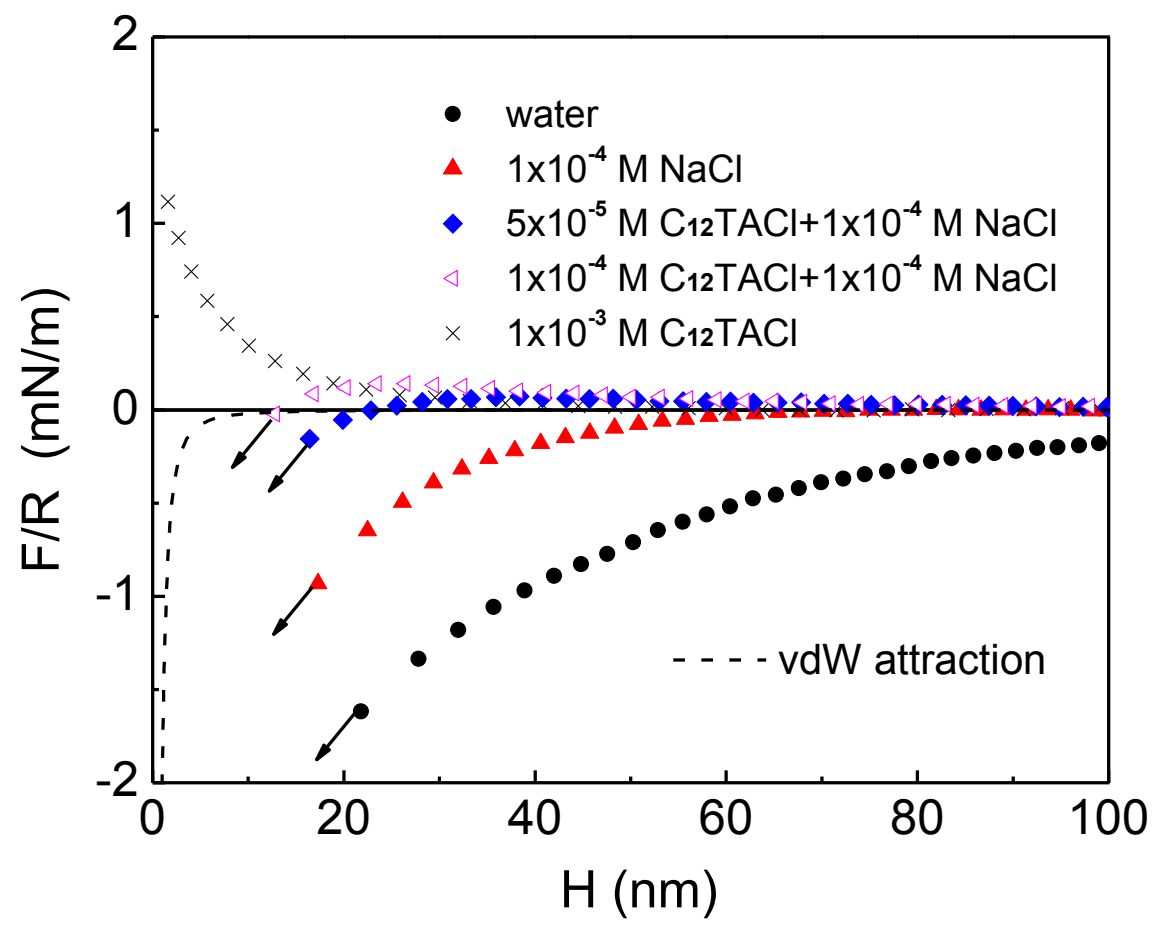

Figure 3.8. The AFM force curves obtained on $\mathrm{C}_{12} \mathrm{SH}$-coated gold surfaces immersed in $\mathrm{NaCl}, \mathrm{C}_{12} \mathrm{TACl}$ solution, and in $\mathrm{NaCl} / \mathrm{C}_{12} \mathrm{TACl}$ mixtures. The dashed line represents the van der Waals force.

\subsubsection{Force Measurements in the Presence of $C_{12}$ TACl Surfactant}

Figure 3.8 shows the surface forces measured between hydrophobic $\mathrm{C}_{12} \mathrm{SH}$-coated gold surfaces in $\mathrm{NaCl}, \mathrm{C}_{12} \mathrm{TACl}$, and $\mathrm{C}_{12} \mathrm{TACl} / \mathrm{NaCl}$ aqueous solutions as functions of separation distance. The arrows indicate the jump distances. A gold microsphere and a goldcoated glass slide were hydrophobized in a $1 \times 10^{-2} \mathrm{mM} \mathrm{C}_{12} \mathrm{SH}$-in-ethanol solution for 2 hours. Using this preparing procedure, the attractive force was the strongest in pure water as indicated in Figure 3.3. In the presence of $5 \times 10^{-5} \mathrm{M} \mathrm{C}_{12} \mathrm{TACl}$ and $1 \times 10^{-4} \mathrm{M} \mathrm{NaCl}$, the force became repulsive at long range and became attractive at short range. The attractive force was dramatically reduced to a much shorter-ranged attraction with a jump distance $H \approx$ $18 \mathrm{~nm}$, and a long-ranged double layer repulsive force operating at separation distance $H>$ $40 \mathrm{~nm}$ appeared, most probably due to the inverse orientation of the $\mathrm{C}_{12} \mathrm{TA}^{+}$ions. ${ }^{45}$ At $1 \times$ $10^{-4} \mathrm{M} \mathrm{C}_{12} \mathrm{TACl}$ and $1 \times 10^{-4} \mathrm{M} \mathrm{NaCl}$, the attractive force became progressively weaker and the surfaces jumped into contact from a distance $H \approx 16 \mathrm{~nm}$; and the double layer repulsive force became even stronger. The attractive force disappeared and the electrostatic repulsion 
dominated at all separation distances as the concentration of $\mathrm{C}_{12} \mathrm{TACl}$ increased to $1 \times 10^{-3}$ M.

\section{$\underline{3.5 \text { Discussion }}$}

\subsubsection{The Existence of the Long-Range Hydrophobic Force}

Although the long-range hydrophobic force has been observed experimentally on different types of hydrophobic surfaces using different surface force measurement techniques, the existence of this strong attractive force is still questioned by many people. In an attempt to explain the long-range attractions of variable strengths and ranges reported so far with a single theory, Hato et al. ${ }^{46}$ and Meyer et $_{\text {al }}{ }^{34}$ suggested that the various long-range attractive forces measured consist of two components; one is the real hydrophobic force, of rather short range $(<20 \mathrm{~nm})$, and the other is the long-range attraction $(>20 \mathrm{~nm})$ which is rather dependent on surface preparation method than the hydrophobicity of the surfaces. Surface force measurements ${ }^{19}, 20,23,35,47-50$ and the surface images ${ }^{21,22,51}$ showed that the long-range attractive force is caused by the bridging of nano or submicro air bubbles preexisting on the two hydrophobic surfaces. Air bubbles adsorb and nucleate at the defective sites on the hydrophobic surface, and steps on the force curve signify the coalescence of two air bubbles. The range of the measured attractive force is determined by the size of the bubble.

Ederth et al..$^{23,} 35$ had conducted surface force measurements on thiol-coated gold surface with a MASIF instrument. The microscopic gold surfaces were hydrophobized in a 1 $\mathrm{mM} \mathrm{C}_{16} \mathrm{SH}$-in-ethanol solution overnight, and the contact angles measured on them were all above $90^{\circ}$. They attributed the steps or discontinuities on the obtained force-separation curves to the bridging of the two air bubbles preexisting on the hydrophobic thiol-coated gold surfaces. When the contact angles on thiol-coated gold were below $90^{\circ}$, which were obtained by using ethanol/water mixtures as solvents or by employing bi-functional thiol with $-\mathrm{OH}$ group on the other end as the co-surfactant, they obtained only the van der Waals force. It was suggested, therefore, that when the surface contact angle was above $90^{\circ}$, there was a long-range attractive force not considered in classic DLVO theory acting between two hydrophobic surfaces, which in turn was caused by the bridging of two air bubbles. At $\theta<$ $90^{\circ}$, only the van der Waals force was measured.

In the present study, the gold substrates were hydrophobized in dilute solutions $(1 \times$ $10^{-2} \mathrm{mM}$ for $\mathrm{C}_{4} \mathrm{SH}, \mathrm{C}_{12} \mathrm{SH}$ and $\mathrm{C}_{16} \mathrm{SH}$ ) of thiol-in-ethanol for significantly shorter period of time (1 minute to several hours). Figure 3.3 and 3.4 shows that all the force curves obtained were smooth and the long-range surface forces measured were net attractive and stronger than van der Waals, regardless of whether the contact angles were above $90^{\circ}$ or not. Figure 3.5 shows the results of the force measurements in the presence and absence of air bubbles. When the $\mathrm{C}_{4} \mathrm{SH}$ hydrophobized gold surfaces were exposed in atmospheric air for a longer period time before the force measurement, preexisting bubbles were present on the surface, which gave rise to capillary forces. The hydrophobic surfaces were dried completely when exposed in air for a long period of time. As the surfaces were immersed in water, the surfaces did not get wet completely, leaving air bubbles on the surfaces. In the absent of air bubbles, or when the air bubbles were flushed away by fresh water, a smooth curve was obtained 
instead of discontinued one, and the measured force was still much stronger than the van der Waals force.

The contact angles on the $\mathrm{C}_{4} \mathrm{SH}-, \mathrm{C}_{12} \mathrm{SH}$ - and $\mathrm{C}_{16} \mathrm{SH}$-coated gold surfaces prepared in this study were all above $90^{\circ}$, meaning that large-scale cavities may be formed due to the metastability of the intervening fluid between the hydrophobic surfaces. ${ }^{24-29}$ The cavitation can occur when the surface tension $\left(\gamma_{12}\right)$ of thiol-coated surface is less than the solid-liquid interfacial tension $\left(\gamma_{13}\right)$.

$$
\Delta G_{c}=\gamma_{12}-\gamma_{13}=\gamma_{23} \cos \theta
$$

Here, $\Delta G_{c}$ is the free energy of cavitation. The subscript 1 refers to solid, 2 refers to air, and 3 refers to liquid. The cavitations are expected to occur as the cavity state is the thermodynamically favored state under situations where the receding contact angle $\theta>$ $90^{\circ} .52$

Figure 3.4 shows surface force measured on $\mathrm{C}_{2} \mathrm{SH}$-coated gold in water. The water equilibrium contact angle on this surface was $67^{\circ}$, which was the mean value for the advancing and receding contacts. According to Equation 3.2, the cavitation is not possible in this case. However, the force measured on $\mathrm{C}_{2} \mathrm{SH}$-coated gold was still much stronger than the van der Waals force. Thus, it is concluded that long-range hydrophobic forces measured on thiol-coated gold are not of bridging air bubbles or cavitation ${ }^{\text {ee }}$ s origin.

Comparing the contact angle measurements (Figure 3.2) with the surface force measurements (Figure 3.3 and 3.4) on the same surfaces, it is found that the hydrophobic force is dependent on the contact angle, which denotes the degree of surface hydrophobicity. For $\mathrm{C}_{12} \mathrm{SH}$ - and $\mathrm{C}_{16} \mathrm{SH}$-coated gold surfaces, the hydrophobic force increased with contact angle, which was varied by changing the immersion time. When the contact angle was controlled by using alkanethiols with different chain lengths, the measured forces showed the same relation with water contact angle. The hydrophobic forces measured on $\mathrm{C}_{12} \mathrm{SH}-$ and $\mathrm{C}_{16} \mathrm{SH}$-coated gold surfaces are the same when they have the same contact angles. Indeed, the hydrophobic force more or less depends on the surface preparation methods. ${ }^{53}$ With the immersion time longer than 10 minutes for $\mathrm{C}_{16} \mathrm{SH}$ coating and 2 hours longer than $\mathrm{C}_{12} \mathrm{SH}$ coating preparations, the contact angles were constant, but the hydrophobic forces diminished with time. As indicated previously, the reduction of the hydrophobic force is due to the physical adsorption of the multilayer of thiols, which can be removed by washing with non-polar solvent ether. The relationship between the contact angle and chain length of alkanethiol is in good agreement with the relation between the chain length and the structure of the SAM monolayer of alkanethiol. The IR spectroscopic and ellipsometric data indicated that self-assembled monolayers of short chain alkanethiols $(n<9)$ are disordered and liquidlike, while the long-chain thiols form a densely packed, crystalline-like monolayers which are free of pin holes. ${ }^{54}$ With increasing chain length, the structure becomes increasingly ordered with higher packing density and coverage, which gives rise to the stronger longrange hydrophobic attractive force. ${ }^{55,56}$ Figure 3.9 shows the fitted decay lengths $D$ and values $C$ for the measured hydrophobic forces plotted against the water contact angles $(\theta)$ of the thiol-coated gold surfaces with which the force measurements have been conducted. The values $C$ and decay lengths $D$ changed little until $\theta$ reached $94^{\circ}$, and then increased sharply 
above this value. The sharp increase in decay length at $\theta \geq 94^{\circ}$ may be due to the changes in the packing density of the hydrocarbon chains and, hence, the degree of ordering. ${ }^{18}$

\subsubsection{Effect of Surface Adsorption on the Hydrophobic Force}

Figure 3.8 shows the surface forces measured in the presence of a cationic surfactant, $\mathrm{C}_{12} \mathrm{TACl}$, which ionizes in water to form charged $\mathrm{C}_{12} \mathrm{TA}^{+}$species. Addition of a very small amount $\left(5 \times 10^{-5} \mathrm{M}\right)$ of $\mathrm{C}_{12} \mathrm{TACl}$ in water dramatically reduced the hydrophobic force between the $\mathrm{C}_{12} \mathrm{SH}$-coated gold surfaces. In the presence of $1 \times 10^{-4} \mathrm{M} \mathrm{NaCl}$ and the cationic surfactant, repulsive forces were observed. The amphiphilic surfactant $\mathrm{C}_{12} \mathrm{TACl}$ can adsorb on the hydrophobic surfaces by hydrocarbon association. Earlier studies ${ }^{45}$ on the surface forces between the mica surfaces hydrophobized with $\mathrm{C}_{12} \mathrm{TACl}$ in the presence of $\mathrm{C}_{12} \mathrm{TACl}$ showed that $\mathrm{C}_{12} \mathrm{TA}^{+}$ions adsorb on hydrophobic surfaces with inverse (or flip-flop) orientation, as depicted in Figure 3.10. The inverse orientation exposes the charged head groups $\left(-\mathrm{N}\left(\mathrm{CH}_{3}\right)_{3}{ }^{+}\right)$toward the aqueous phase, changes the interfacial water structure, and causes the surface to be less hydrophobic. In addition, the charged head groups on both surfaces can generate the electrostatic repulsion. On the $\mathrm{C}_{12} \mathrm{SH}$-coated gold surfaces, the attractive hydrophobic force decreased and the electrostatic repulsive force increased in the presence of $\mathrm{C}_{12} \mathrm{TACl}$. At $1 \times 10^{-3} \mathrm{M}$, the attractive force was completely suppressed, and only the repulsive force was operating at the short separation distance. In the concentration range of $5 \times 10^{-5} \sim 1 \times 10^{-3} \mathrm{M}$, the physically adsorbed $\mathrm{C}_{12} \mathrm{TACl}$ molecules may not be saturated on the hydrophobic surfaces, meaning some molecules were "lying down" while some were "standing up". As the concentration increased, more and more molecules were "standing up" and the surface became much less hydrophobic, causing a decrease in the attractive force. At the same time, the surface charge and hence the potential increased due to

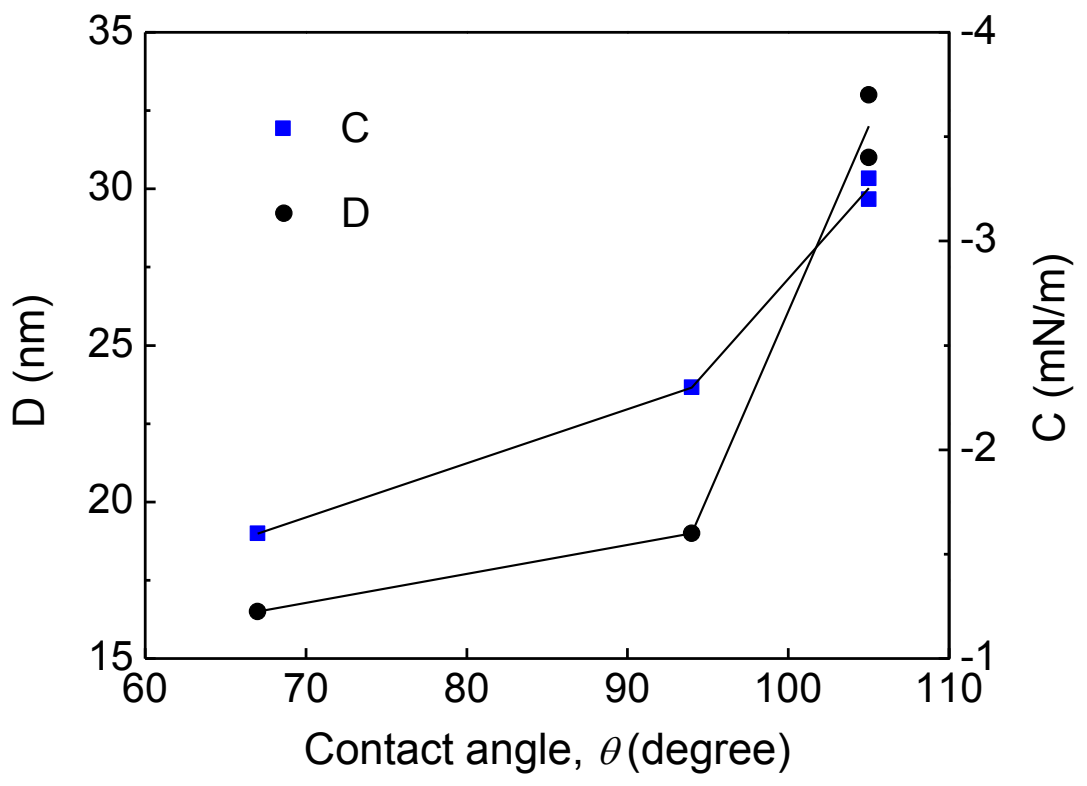

Figure 3.9. Decay length $(D)$ and value $C$ versus contact angle plots for the data obtained on $\mathrm{C}_{2} \mathrm{SH}-, \mathrm{C}_{4} \mathrm{SH}-, \mathrm{C}_{12} \mathrm{SH}$ - and $\mathrm{C}_{16} \mathrm{SH}$-coated gold. 
the adsorption of the $\mathrm{C}_{12} \mathrm{TA}^{+}$species, which gave rise to a stronger electrostatic repulsion.

Also shown in Figure 3.8 are the effects of $\mathrm{NaCl}$. In the presence of $1 \times 10^{-4} \mathrm{M} \mathrm{NaCl}$, the hydrophobic force was greatly reduced. This can be attributed to the possibility that the hydrogen bond network diminished in the presence of $\mathrm{NaCl}$. In the presence of $\mathrm{NaCl}$, the adsorption of the $\mathrm{C}_{12} \mathrm{TA}^{+}$species may increase due to charge neutralization which in turn increases surface activity of a charged surfactant. The residual $\mathrm{C}_{12} \mathrm{TA}^{+}$species left in solution may also contribute to diminishing the H-bonded water structure and decrease the hydrophobic force, as suggested by Zhang et al. ${ }^{45}$

Figure 3.2 shows the kinetics of adsorption of alkanethiols $\left(\mathrm{C}_{4} \mathrm{SH}, \mathrm{C}_{12} \mathrm{SH}\right.$ and $\left.\mathrm{C}_{16} \mathrm{SH}\right)$ of different chain lengths on gold as monitored by the changes in contact angle with time. The data indicates that, the water contact angles of the thiol-coated gold surfaces reached the
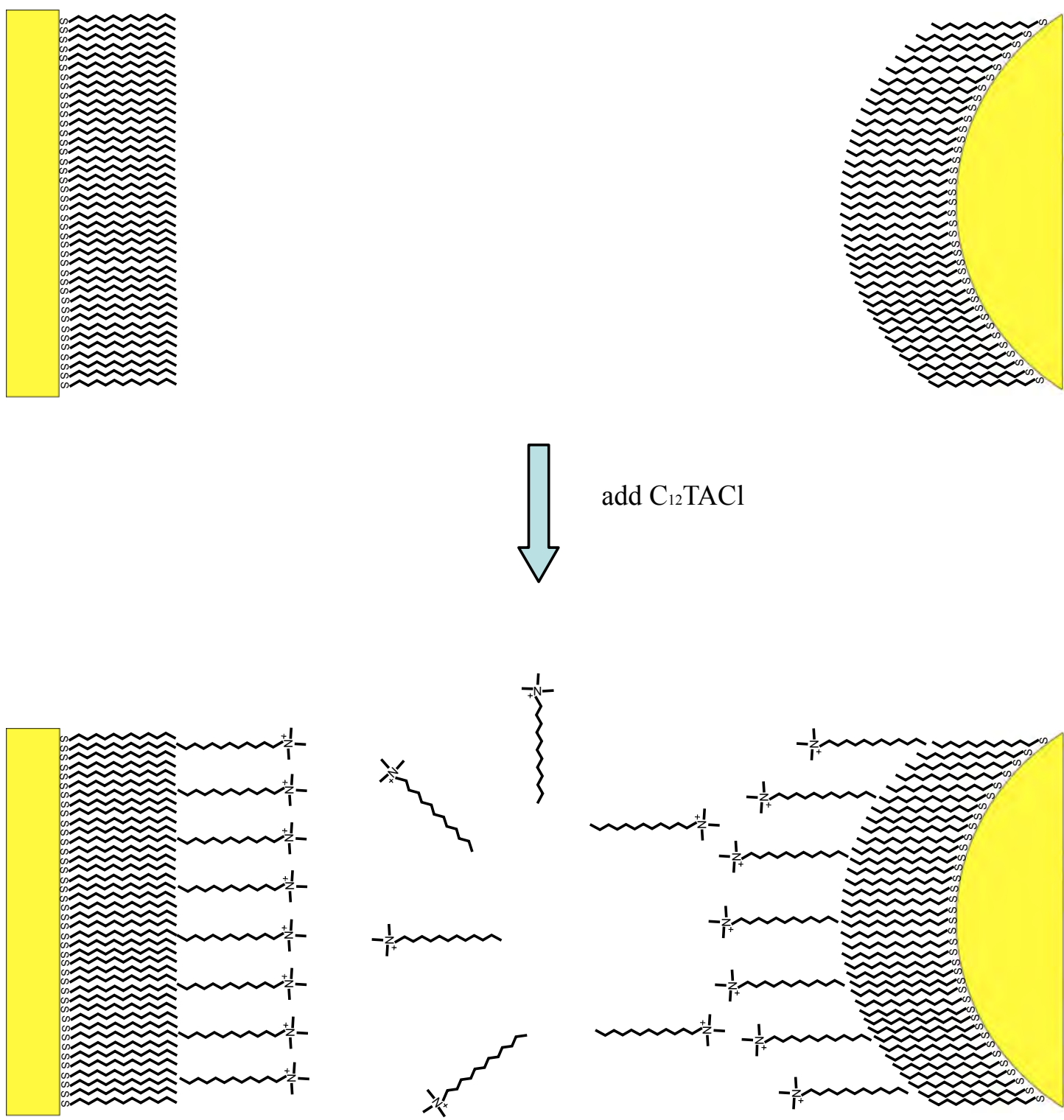

add $\mathrm{C}_{12} \mathrm{TACl}$

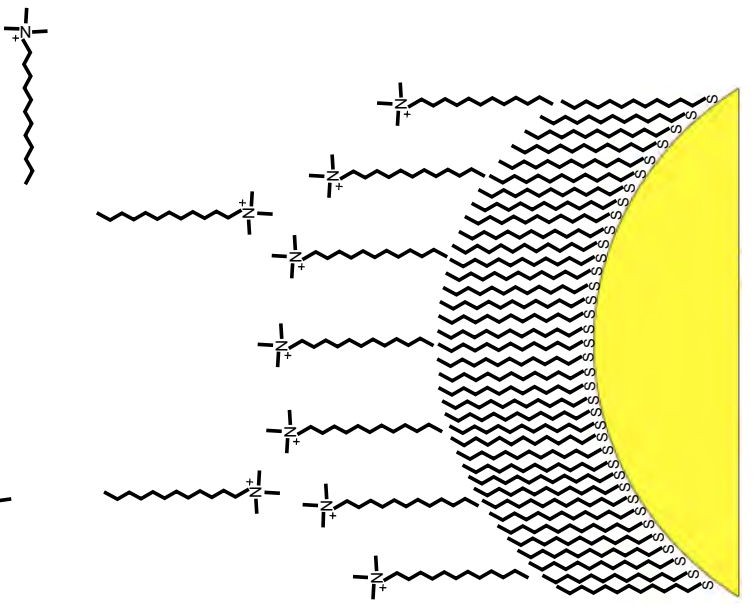

Figure 3.10. Schematic illustration for the effect of adding $\mathrm{C}_{12} \mathrm{TACl}$ in bulk solution. 
maximum after an exposure time of 5 hours, 2 hours and 10 minutes with $\mathrm{C}_{4} \mathrm{SH}, \mathrm{C}_{12} \mathrm{SH}$ and $\mathrm{C}_{16} \mathrm{SH}$, respectively. Thus, it takes less time for an alkanethiol with a longer chain to completely cover the gold surface and form a self-assembled monolayer. There is a large body of information reported in the literature on the kinetics of alkanethiol adsorption on gold. However, the experimental results obtained with various analytical methods regarding the time required for the ordered monolayer formation are conflicting. Bain et al., ${ }^{43}$ measured the kinetics of formation of the self-assembled monolayer (SAM) of 1-octadecanethiol $\left(\mathrm{C}_{18} \mathrm{SH}\right)$ on gold by measuring water contact angles. At thiol concentration below $1 \mathrm{mM}$, it took from hours to days to form a perfectly-ordered monolayer. Pan et al. ${ }^{39}$ reported a contact time of 800 minutes for the monolayer formation in a $5 \mathrm{mM} \mathrm{C}_{12} \mathrm{SH}$ ethanol solution on gold as monitored by a quartz crystal microbalance (QCM) in situ. On the other hand, the X-ray photoelectron spectroscopy (XPS) analysis and scanning tunneling microscopy (STM) imaging by Kawasaki et al. ${ }^{58}$ showed that almost complete monolayer coverage of octanethiol $\left(\mathrm{C}_{8} \mathrm{SH}\right) \mathrm{SAM}$ was obtained well within a few minutes, e.g., in a $1 \times 10^{-2} \mathrm{mM}$ alcoholic solution at room temperature. Campbell and Mutharasan ${ }^{59}$ observed a $\mathrm{C}_{16} \mathrm{SH}$ SAM monolayer formation within 1 hour at various concentrations ( $1 \mathrm{nM}$ to $10 \mathrm{mM})$ using a piezoexcited millimeter-sized gold-coated cantilever sensor. The infrared spectroscopy studies by Truong and Rowntree ${ }^{60}$ indicated a shorter time scale of 1-10 minutes for the ordered $\mathrm{C}_{4} \mathrm{SH}$ SAM formation from a $5 \mathrm{mM}$ solution. Pan et $a l^{39}$. attributed the discrepancies among these reports to the contaminants on the gold surfaces. The pre-adsorbed contaminants can retard the adsorption process, because it is necessary to displace the adsorbed contaminants before thiol adsorption.

The surface force data obtained with $\mathrm{C}_{12} \mathrm{SH}$ (Figure 3.3) and $\mathrm{C}_{16} \mathrm{SH}$-coated gold ${ }^{32}$ show that the hydrophobic forces were the strongest when the water contact angles approached the maximum values after immersion time longer than 1 minute. As the immersion time was extended, the contact angles remained the same. On the other hand, the long-range attractions significantly decreased after longer immersion time. When the thiolcoated surfaces were flushed with non-polar solvent such as ether and then the fresh water was re-introduced in liquid cell, the strongest attractive forces were detected on $\mathrm{C}_{12} \mathrm{SH}$ - and $\mathrm{C}_{16} \mathrm{SH}$-coated gold substrates. It is suggested that the decrease of hydrophobic force was caused by the physical adsorption of the bilayers of thiol. ${ }^{32}$ Apparently, the "ethanol wash" step after hydrophobization step cannot ensure that the physisorbed multilayer is completely removed. The adsorption of a thiol on a gold surface may be represented as follows:

$$
\begin{aligned}
& A u \rightarrow \underline{A u}^{+}+e \\
& \underline{A u}^{+}+R S H \rightarrow \underline{A u}-S R+H^{+}
\end{aligned}
$$

where the underscore refers to the gold species remaining on the surface. Thus, the chemisorption yields the $\mathrm{Au}^{+}$species on the surface, which uptakes the thiol in solution. After a long immersion time, however, some gold species may enter the bulk ethanol solution and form gold-thiol complex, which subsequently adsorb on the top of the chemisorbed thiol monolayer. Although the bi-layer formation does not cause a decrease in water contact angle, the presence of the gold-thiol complex in the adsorption layer can render the surface less ordered, which may cause a decrease in hydrophobic force. When such species are removed by appropriate solvent wash, a more ordered hydrophobic surface is exposed on the surface, which may give rise to a strong hydrophobic force. 


\subsubsection{Electrostatic Origin?}

Based on the early studies ${ }^{31,61-65}$ showing that the hydrophobic forces decrease in range and magnitude in the presence of electrolytes, it was suggested that the hydrophobic force may be a electrostatic force in origin due to surface-induced perturbation in the fluid, ${ }^{66}$ ion-ion correlations,${ }^{67,68}$ mobile charged patches, ${ }^{14}$ correlated in plane dipoles, ${ }^{17}, 18$ instability of hydrophobic surface groups ${ }^{16}$ and rearrangement of the charged patchy bilayers. ${ }^{15}$ The appealing point of this theory is that the classical electrostatic interaction is able to give rise to long-range force of exponential function measured for the hydrophobic attraction.

Recently, more and more surface force measurements were performed using different types of hydrophobic surfaces to determine the dependence of the range and strength of the hydrophobic force on the concentrations of the electrolytes. However, detailed experiments have shown that increased $\mathrm{NaCl}$ concentration up to $1 \mathrm{M}$ has little or no effect on the range of the interaction, ${ }^{69}$ the hydrophobic attraction is undiminished in the presence of concentrated electrolyte $(0.1 \mathrm{M} \mathrm{NaCl}),{ }^{70}$ and electrolyte has negligible effect on the range and strength of the measured force, the strength of the attractive forces increases slightly even at very high salt concentrations. ${ }^{19}$ These results cannot be reconciled with the above models that attribute an electrostatic attraction to the hydrophobic force.

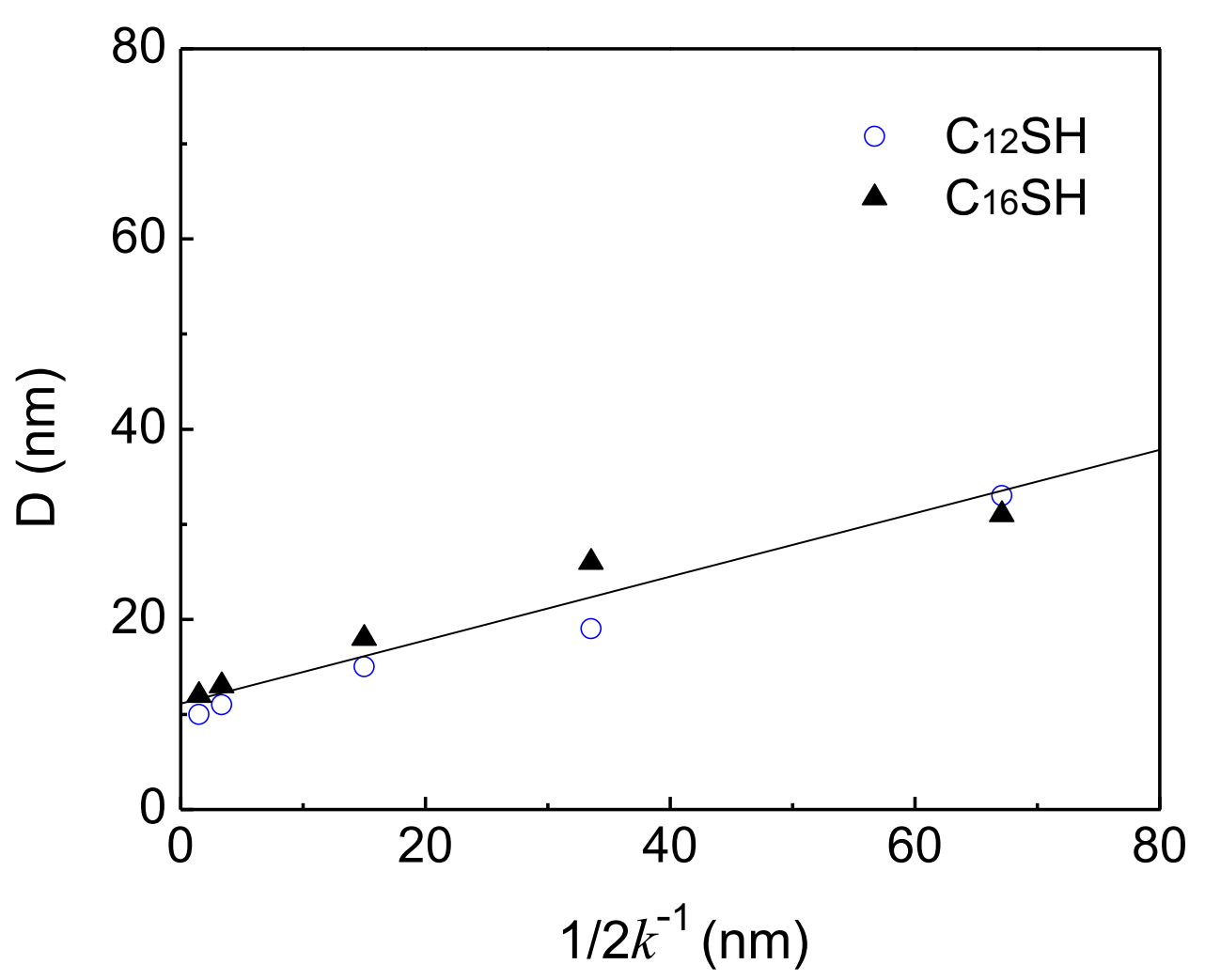

Figure 3.11. Decay length $(D)$ versus the Debye length $\left(\kappa^{-1}\right)$ plots for the data obtained on $\mathrm{C}_{12} \mathrm{SH}$ - and $\mathrm{C}_{16} \mathrm{SH}$-coated gold in $\mathrm{NaCl}$ aqueous solutions with different concentrations. 
Figure 3.6 and 3.7 show the electrolyte $(\mathrm{NaCl})$ effect on the hydrophobic forces measured on $\mathrm{C}_{12} \mathrm{SH}$ - and $\mathrm{C}_{16} \mathrm{SH}$-coated gold surfaces. As shown in Table 3.2, analysis by fitting the force data points with extended DLVO theory which includes the contribution from the hydrophobic force confirms that the value $C$ and decay length $D$ decrease as the concentration of $\mathrm{NaCl}$ increases. The stability of $\mathrm{C}_{12} \mathrm{SH}$ - and $\mathrm{C}_{16} \mathrm{SH}$-coated gold surfaces in $\mathrm{NaCl}$ aqueous solutions was evaluated by surface force and contact angle measurements. The experimental results show the decreased surface forces measured in $\mathrm{NaCl}$ solutions were restored when the $\mathrm{NaCl}$ solutions were replaced by fresh water, and the hydrophobicity did not change in the presence of $\mathrm{NaCl}$ electrolyte. According to the electrostatic models developed by Attard, ${ }^{66}$ Miklavic et al. ${ }^{14}$ Podgornik, ${ }^{67}$ and Spalla and Belloni, ${ }^{68}$ the attraction decreases exponentially with a decay length $D$ equal to the one half of the Debye length $\left(\kappa^{-1}\right)$. In Figure 3.11, the values $D$ obtained at different $\mathrm{NaCl}$ concentrations are plotted versus $1 / 2 \kappa^{-1}$. However, it is shown that the $D$ values are not equal to $1 / 2 \kappa^{-1}$, except the $D$ values obtained in $0.1 \mathrm{mM} \mathrm{NaCl}$ solution, the $D\left(15 \mathrm{~nm}\right.$ for $\mathrm{C}_{12} \mathrm{SH}$ and $18 \mathrm{~nm}$ for $\mathrm{C}_{16} \mathrm{SH}$ surfaces $)$ values are approximately $1 / 2 \kappa^{-1}(30 \mathrm{~nm})$. At the concentration of $\mathrm{NaCl}$ less than $0.1 \mathrm{mM}$, the obtained $D$ values are smaller than the values of $1 / 2 \kappa^{-1}$, while at $\mathrm{NaCl}$ concentration above $0.1 \mathrm{mM}$, the $D$ values are much larger than $1 / 2 \kappa^{-1}$. In addition, the thiol molecules formed immobile and robust monolayers on gold via chemical reaction, which cannot meet the requirement of the theoretical models that assume that the surfaces are covered by movable charges, ${ }^{14}$ mobile hydrophobic surface groups, ${ }^{16}$ or "rolling" bilayer. ${ }^{15}$ This conclusively rules out any electrostatic mechanism on stable thiol-coated gold surfaces.

The very long-range hydrophobic forces observed on thiol-coated surfaces may be explained by the changes in water structure across the thin film between the hydrophobic surfaces. A problem with this approach is that the computer simulations show the surfaceinduced structure can be extended up to only several layers of water molecules. It was argued by Eriksson et $a l .{ }^{71}$ that the classical simulations of Lee et al. ${ }^{10}$ based on a simplistic water potential was not appropriate because it only explored density changes in thin water film. In fact, structural rearrangement of water can occur without volume changes. ${ }^{71}$ Based on the water structure theory, the effects of organic surfactant and inorganic electrolyte ions across water thin film between thiol-coated hydrophobic surfaces on the hydrophobic force can be explained. It is suggested that the cationic surfactant $\mathrm{C}_{12} \mathrm{TACl}$ and $\mathrm{NaCl}$ broke the hydrogen bond network ${ }^{45}$ or the hydrations of $\mathrm{Na}^{+}$and $\mathrm{Cl}^{-}$altered the water structure ${ }^{57,72}$ present in thin films between hydrophobic surface, and thus reducing the hydrophobic force. Ninham ${ }^{73}$ suggested that the hydrophobic force may also be related to the gas molecules dissolved in water, because degassing can stabilized the surfactant-free emulsions ${ }^{74}$ and can reduce the range and magnitude of the hydrophobic attractive force. ${ }^{34}$ It was shown that the solubility of oxygen in water decreases with increasing $\mathrm{NaCl}$ concentration, so the bubble coalescence is inhibited by the presence of $\mathrm{NaCl}$.

\section{$\underline{3.6 \text { Conclusions }}$}

AFM force measurements were conducted using gold surfaces hydrophobized by the self-assembly of alkanethiols with $n=2-16$. We have observed net-attractive and long-range $(\sim 100 \mathrm{~nm})$ forces between thiol-coated surfaces regardless of whether the water contact angle is greater than $90^{\circ}$ or not, and the strengths and ranges of the attractions depends on the surface hydrophobicity. The force curves do not show discontinuities or steps at all 
separation distances except when the prepared thiol-coated hydrophobic surfaces were exposed in air for a prolonged period of time before being immersed in water for force measurement. We have tentatively exposed $\mathrm{C}_{4} \mathrm{SH}$-coated gold surfaces in atmospheric air for prolonged time after they were prepared ex-situ in thiol-in-ethanol solution. The force curves exhibited steps, indicating that the measured forces were due to bridging bubbles, which were trapped on the hydrophobic surfaces when $\mathrm{C}_{4} \mathrm{SH}$-coated surfaces were brought into water. When the air bubbles were washed away using water, the steps in force curves disappeared and long-range hydrophobic forces appeared. When gold surfaces were hydrophobized with $\mathrm{C}_{2} \mathrm{SH}$, the contact angle was less than $90^{\circ}$. In this case, no cavitation is possible. Yet, the AFM force measurement gave a long-range attraction, although it was weaker than those measured with gold surfaces coated with longer chain thiols. That longrange attractions are still observed under conditions cavitation is not thermodynamically possible suggest that hydrophobic force is not due to bubbles formed on hydrophobic surfaces.

The attractive forces measured on $\mathrm{C}_{12} \mathrm{SH}$ - and $\mathrm{C}_{16} \mathrm{SH}$-coated gold surfaces were effectively reduced by electrolyte $(\mathrm{NaCl})$, indicating that they may be of electrostatic origin. For this mechanism to work, it is necessary to assume that the charged patches must be mobile when two surfaces approach each other to maximize the correlation and give rise to a long-range attraction. However, the strong covalent Au-S bonding provides robust and immobile hydrophobic monolayers, which precludes the possibility of charged patches being mobile. Further, there is no reason that thiol adsorption produces charged patches as the thiol chemisorbs on gold.

Our work leaves the possibility that the long-range attraction is caused by the changes in water structure near hydrophobic surfaces. It is possible that the electrolyte $\mathrm{NaCl}$ and surfactant $\mathrm{C}_{12} \mathrm{TACl}$ in solution break the $\mathrm{H}$-bonded water structure confined between two hydrophobic surfaces, and thus, reduce the hydrophobic force.

\section{References}

1. $\quad \mathrm{Xu}, \mathrm{Z}$.; Yoon, R.-H., J. Colloid Interface Sci. 1989, 132, (2), 532-541.

2. $\quad \mathrm{Xu}, \mathrm{Z}$.; Yoon, R.-H., J. Colloid Interface Sci. 1990, 134, (2), 427-434.

3. $\quad$ Demetriades, K.; Coupland, J. N.; McClements, D. J., J. Food Sci. 1997, 62, (3), 462467.

4. Blake, T. D.; Kitchener, J. A., J. Chem. Soc., Faraday Trans. 1 1972, 68, 1435-1442.

5. Israelachvili, J.; Pashley, R., Nature 1982, 300, 341-342.

6. Yoon, R.-H. In Hydrodynamic and Surface Forces in Bubble-Particle Interactions, XVII International Mineral Processing Congress, Dresden, Germany, 1991; Dresden, Germany, 1991; pp 17-31.

7. $\quad$ Yoon, R.-H.; Mao, L., J. Colloid Interface Sci. 1996, 181, 613-626.

8. $\quad$ Mao, L.; Yoon, R.-H., Int. J. Miner. Process 1997, 51, 171-181.

9. Eriksson, J. C.; Ljunggren, S.; Claesson, P. M., J. Chem. Soc., Faraday Trans. 2 1989, 85, (3), 163-176.

10. Lee, C. Y.; McCammon, J. A.; Rossky, P. J., J. Chem. Phys. 1984, 80, (9), 44484455.

11. Forsman, J.; Jonsson, B.; Woodward, C. E., J. Phys. Chem. 1996, 100, (36), 1500515010. 
12. Sakurai, M.; Tamagawa, H.; Ariga, K.; Kunitake, T.; Inoue, Y., Chem. Phys. Lett. 1998, 289, 567-571.

13. Fa, K.; Nguyen, A. V.; Miller, J. D., J. Phys. Chem. 2005, 109, (27), 13112-13118.

14. Miklavic, S. J.; Chan, D. Y. C.; White, L. R.; Healy, T. W., J. Phys. Chem. 1994, 98, 9022-9032.

15. Meyer, E. E.; Lin, Q.; Hassenkam, T.; Oroudjev, E.; Israelachvili, J. N., Proc. Nat. Acad. Sci. U.S.A 2005, 102, 6839-6842.

16. Christenson, H. K.; Yaminsky, V. V., Colloids Surf., A 1997, 129-130, 67-74.

17. Tsao, Y.-H.; Evans, D. F.; Wennerstrom, H., Science 1993, 262, (5133), 547-550.

18. Yoon, R.-H.; Ravishankar, S. A. R., J. Colloid Interface Sci. 1996, 179, 391-402.

19. Parker, J. L.; Claesson, P. M.; Attard, P., J. Phys. Chem. 1994, 98, 8468-8480.

20. Nguyen, A. V.; Nalaskowski, J.; Miller, J. D.; Butt, H.-J., Int. J. Miner. Process. 2003, 72, 215-225.

21. Ishida, N.; Sakamoto, M.; Miyahara, M.; Higashitani, K., J. Colloid Interface Sci. 2002, 253, 112-116.

22. Tyrrell, J. W. G.; Attard, P., Phys. Rev. Lett. 2001, 87, (17), 176104-1.

23. Ederth, T., J. Phys. Chem. B 2000, 104, 9704-9712.

24. Yushchenko, V. S.; Yaminsky, V. V.; Shchukin, E. D., J. Colloid Interface Sci. 1983, 96, (2), 307-314.

25. Yaminsky, V. V.; Ninham, B. W., Langmuir 1993, 9, 3618-3624.

26. Yaminsky, V. V., Colloids and Surf., A 1999, 159, 181-195.

27. Craig, V. S. J.; Ninham, B. W.; Pashley, R. M., Langmuir 1999, 15, 1562-1569.

28. Christenson, H. K.; Claesson, P. M., Science 1988, 239, 390-392.

29. Wood, J.; Sharma, R., Langmuir 1995, 11, (12), 4797-4802.

30. Ljunggren, S.; Eriksson, J. C., Colloids and Surf., A 1997, 129-130, 151-155.

31. Zhang, J.; Yoon, R.-H.; Mao, M.; Ducker, W. A., Langmuir 2005, 21, 5831-5841.

32. Wang, J.; Yoon, R.-H., Langmuir 2008, 24, 7889-7896.

33. Stevens, H.; Considine, R. F.; Drummond, C. J.; Hayes, R. A.; Attard, P., Langmuir 2005, 21, 6399-6405.

34. Meyer, E. E.; Lin, Q.; Israelachvili, J. N., Langmuir 2005, 21, 256-259.

35. Ederth, T.; Liedberg, B., Langmuir 2000, 16, 2177-2184.

36. Raiteri, R.; Preuss, M.; Grattarola, M.; Butt, H.-J., Colloids Surf., A 1998, 136, 191197.

37. Ron, H.; Rubinstein, I., Langmuir 1994, 10, (12), 4566-4573.

38. Ron, H.; Rubinstein, I., J. Am. Chem. Soc 1998, 120, (51), 13444-13452.

39. Pan, W.; Durning, C. J.; Turro, N. J., Langmuir 1996, 12, (18), 4469-4473.

40. Xu, S.; Cruchon-Dupeyrat, S. J. N.; Garno, J. C.; Liu, G.-Y.; Jennings, G. K.; Yong, T.-H.; Laibinis, P. E., J. Chem. Phys. 1998, 108, (12), 5002-5012.

41. Ducker, W. A.; Senden, T. J., Langmuir 1992, 8, (7), 1831-1836.

42. Cleveland, J. P.; Manne, S.; Bocek, D.; Hansma, P. K., Rev. Sci. Instrum. 1993, 64, (2), 403-405.

43. Bain, C. D.; Troughton, E. B.; Tao, Y.-T.; Evall, J.; Whitesides, G. M.; Nuzzo, R. G., J. Am. Chem. Soc. 1989, 111, 321-335.

44. Ulman, A., An Introduction to Ultrathin Organic Films From langmuir-Blodgett to Self-Assembly. Academic Press: Rochester, New York, 1991.

45. Zhang, J.; Yoon, R.-H.; Eriksson, J. C., Colloids Surf., A 2007, 300, 335-345. 
46. Hato, M., J. Phys. Chem. 1996, 100, 18530-18538.

47. Attard, P., Adv. Colloid Interface Sci. 2003, 104, 75-91.

48. Carambassis, A.; Jonker, L. C.; Attard, P.; Rutland, M. W., Phys. Rev. Lett. 1998, 80, (24), 5357-5360.

49. Ishida, N.; Sakamoto, M.; Miyahara, M.; Higashitani, K., Langmuir 2000, 16, (13), 5681-5687.

50. $\quad$ Sakamoto, M.; Kanda, Y.; Miyahara, M.; Higashitani, K., Langmuir 2002, 18, 57135719.

51. Yang, J.; Duan, J.; Fornasiero, D.; Ralston, J., J. Phys. Chem. B 2003, 107, 61396147.

52. Meyer, E. E.; Rosenberg, K. J.; Israelachvili, J., Proc. Nat. Acad. Sci. U.S.A 2006, $103,15739-15746$.

53. Christenson, H. K.; Claesson, P. M., Adv. Colloid Interface Sci. 2001, 91, 391-436.

54. Porter, M. D.; Bright, T. B.; Allara, D. L.; Chidsey, C. E. D., J. Am. Chem. Soc. 1987, 109, (12), 3559-3568.

55. Tsao, Y.-H.; Yang, S. X.; Evans, D. F.; Wennerstrom, H., Langmuir 1991, 7, 31543159.

56. Rabinovich, Y. I.; Guzonas, D. A.; Yoon, R.-H., Langmuir 1993, 9, 1168-1170.

57. Weissenborn, P. K.; Pugh, R. J., J. Colloid Interface Sci. 1996, 184, 550-563.

58. Kawasaki, M.; Sato, T.; Tanaka, T.; Takao, K., Langmuir 2000, 16, (4), 1719-1728.

59. Campbell, G. A.; Mutharasan, R., Langmuir 2005, 21, (25), 11568-11573.

60. Truong, K. D.; Rowntree, P. A., Prog. Surf. Sci. 1995, 50, (1-4), 207-216.

61. Rabinovich, Y. I.; Derjaguin, B. V., Colloids Surf. 1988, 30, 243-251.

62. Claesson, P. M.; Blom, C. E.; Herder, P. C.; Ninham, B. W., J. Colloid Interface Sci. 1986, 114, (1), 234-242.

63. Tsao, Y.-H.; Evans, D. F.; Wennerstrom, H., Langmuir 1993, 9, 779-785.

64. Kekicheff, P.; Spalla, O., Phys. Rev. Lett. 1995, 75, (9), 1851-1855.

65. Christenson, H. K.; Claesson, P. M.; Berg, J.; Herder, P. C., J. Phys. Chem. 1989, 93, 1472-1478.

66. Attard, P., J. Phys. Chem. 1989, 93, (17), 6441-6444.

67. Podgornik, R., J. Chem. Phys 1989, 91, (9), 5840-5849.

68. Spalla, O.; Belloni, L., Phys. Rev. Lett. 1995, 74, (13), 2515-2518.

69. Meagher, L.; Craig, V. S. J., Langmuir 1994, 10, 2736-2742.

70. Craig, V. S. J.; Ninham, B. W.; Pashley, R. M., Langmuir 1998, 14, (12), 3326-3332.

71. Eriksson, J. C.; Yoon, R.-H., Hydrophobic Attraction in the Light of Thin-Film Thermodynamics. In Colloid Stability: The Role of Surface Forces, Tadros, T. F., Ed. WILEY-VCH Verlag GmbH \& Co. KGaA: Weinheim, Germany, 2007; Vol. 1, pp 99-132.

72. Craig, V. S. J.; Ninham, B. W.; Pashley, R. M., Nature 1993, 364, 317-319.

73. Ninham, B. W., Prog. Colloid Polym. Sci. 2006, 133, 65-73.

74. Pashley, R. M., J. Phys. Chem. B 2003, 107, 1714-1720. 


\title{
TASK 3 SURFACE FORCE BETWEEN GOLD SURFACES IN XANTHATE SOLUTIONS AND ITS IMPLICATION IN FLOTATION
}

\author{
$\underline{4.1 \text { Abstract }}$
}

Understanding the wetting behavior of sulfide and precious minerals in xanthate solutions and the surface forces in flotation is essential for improving the process of flotation. Contact angle and surface force measurements were conducted on the potassium amyl xanthate (PAX)-gold system. Monolayer structures of PAX were formed by spontaneous adsorption at different concentrations and contact (or adsorption) times. Captive bubble contact angle measurements showed that chemisorption of PAX on gold was concentration dependent. A monolayer was formed more readily on gold in a $1 \times 10^{-5} \mathrm{M}$ than in a $5 \times 10^{-6}$ M PAX solution. The contact angle measurements conducted in $1 \times 10^{-5} \mathrm{M}$ and $5 \times 10^{-6} \mathrm{M}$ PAX solutions showed a rapid increase in contact angle to a peak value of $94^{\circ}$, followed by a slower period of decreasing contact angle.

Surface forces were measured using an atomic force microscope (AFM) with gold substrates hydrophobized by PAX coatings. The results showed the existence of long-range hydrophobic forces, which were closely related to the surface hydrophobicity. The strongest hydrophobic force with a decay length of $23 \mathrm{~nm}$ was measured when the water contact angle was the largest. The hydrophobic force decreased when the contact angle was decreased due to the physisorption of additional xanthate ions on the underlying monolayer possibly with reverse orientation. It was found that the residual xanthate species left in solution caused a decrease in hydrophobic force, possibly because their presence in solution is detrimental to maximizing the cohesive energy of water.

\section{$\underline{4.2 \text { Introduction }}$}

Froth flotation is the most important solid-solid separation process in the mining industry. Owing to its simplicity, the process is widely used for extracting many minerals from their ores, cleaning coals, and recycling plastics, etc, by exploiting the differences in hydrophobicity. In froth flotation, a stream of small air bubbles is introduced to the bottom of a flotation cell, in which a mixture of finely grounded ore particles is suspended in water. The air bubbles rise to the top of the tank under a buoyancy force. During the process, only the hydrophobic particles adhere to the surface of air bubbles and are carried to the upper surface of the pulp. Rendering a desired mineral particle more hydrophobic than the others determines the selectivity, and is the key to the success of a flotation process. For this reason, during the early stages of flotation research, most of the efforts were focused on hydrophobizing minerals with surfactants (collectors) and monitoring the changes in surface hydrophobicity by measuring contact angles. However, surface chemistry parameters such as contact angle, are thermodynamic properties and do not provide kinetic information. On the other hand, flotation is a kinetic process and the industry strives to improve flotation kinetics and, thus, increase recovery and throughput.

Froth flotation can be described as a first order process with a rate constant

proportional to the probability of particle collection $(P) .{ }^{1}$ The probability of particle collection $(P)$ is represented by: 


$$
P=P_{c} P_{a}\left(1-P_{d}\right)
$$

where $P_{c}$ is the probability of air bubble and mineral particle collision, $P_{a}$ is the probability of bubble particle adhesion, and $P_{d}$ is the probability of detachment. In mineral flotation, it is believed that both the adhesion and detachment processes are determined by the surface force between the air bubble and mineral particle. Derjaguin and Dukhin $^{2,3}$ were the first to describe the bubble particle interaction in flotation by considering surface forces. However, the authors only considered the traditional DLVO force, i.e., van der Waals and electrostatic forces, which cannot explain the strong attraction between air bubbles and hydrophobic mineral particles in flotation. Laskowski and Kitchener ${ }^{4}$ were the first to recognize the existence of the hydrophobic force by studying the interaction across a thin water film between an air bubble and a silicate plate hydrophobized by trimethylchlorosilane (TMCS). The air bubble readily adheres on the hydrophobic silica surface, even though both the van der Waals and electrostatic force in the wetting film are repulsive. Therefore, they speculated the existence of a third force, which is responsible for the bubble particle adhesion. Furthermore, they said the long range hydrophobic influence may be responsible for the flotation. In 1972, Blake and Kitchener ${ }^{5}$ were the first to use the term hydrophobic force in wetting films. Recent studies of hydrophobic forces are focused on thin aqueous films between solid hydrophobic surfaces. Most of the force measurements have been based on employing the surface force apparatus (SFA) and atomic force microscope (AFM). In 1982, an attractive force much larger than the van der Waals force between two curved mica surfaces immersed in hexadecyltrimethylammonium bromide (CTAB) solution was first

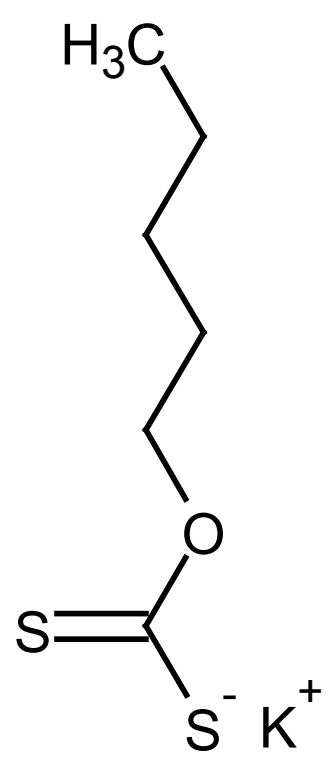

(a)

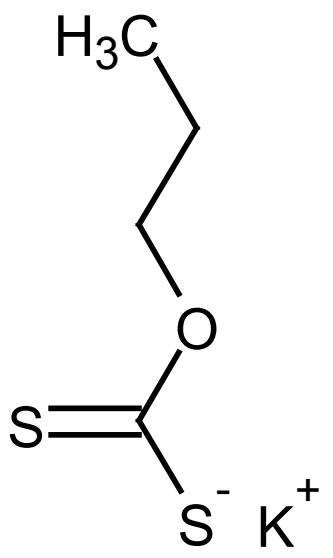

(b)

Figure 4.1. Molecular structure of (a) potassium amyl xantahte (PAX) (b) and potassium ethyl xanthate (KEX). 
measured by Israelachvili and Pashley ${ }^{6}$ using a SFA. They referred to the additional attractive force as a hydrophobic force. More recently, Yoon and Aksoy ${ }^{7}$ and Wang and Yoon ${ }^{8}$ suggested that the hydrophobic forces are also present in soap films.

Xanthates (dithiocarbonates) are used as collectors for sulfide mineral flotation, and are known to chemisorb on them. Xanthates also adsorb to noble metals such as silver, copper and gold, in a manner similar to thiols. ${ }^{9}$ Most of the hydrophobic force measurements conducted in the past was made with mica and silica surfaces using surfactants that are not commonly used for flotation. Therefore, in the present study, it is proposed to conduct AFM force measurements with gold substrates by xanthate adsorption. The results of the present work may be useful for better understanding the mechanisms on the origin of hydrophobic force, and help improve flotation technology.

\section{$\underline{4.3 \text { Materials and Methods }}$}

\subsubsection{Materials}

Potassium amyl xanthate (PAX, $\mathrm{C}_{6} \mathrm{H}_{11} \mathrm{KOS}_{2},>90.0 \%$ ) and potassium ethyl xanthate (KEX, $\mathrm{C}_{3} \mathrm{H}_{5} \mathrm{KOS}_{2},>90.0 \%$ ) were obtained from TCI America. The molecular structures of potassium amyl xanthate and potassium ethyl xanthate are shown in Figure 4.1. The xanthates were purified by dissolution in acetone (HPLC grade, Fisher Scientific, Inc.), filtration and recrystallization with diethyl ether ( $\geq 99.9 \%$, Sigma-Aldrich, Inc.), as described in the literature. ${ }^{10}$ Alkyl xanthate is unstable in water and decomposes to form $\mathrm{CS}_{2}$. The xanthates were recrystallized at least twice prior to use. Sodium perchlorate monohydrate $\left(\mathrm{NaClO}_{4} \cdot \mathrm{H}_{2} \mathrm{O}, 97 \%\right)$, potassium ferrocyanide trihydrate $\left(\mathrm{K}_{4} \mathrm{Fe}(\mathrm{CN})_{6} \cdot 3 \mathrm{H}_{2} \mathrm{O}, 100 \%\right)$ and potassium ferricyanide $\left(\mathrm{K}_{3} \mathrm{Fe}(\mathrm{CN})_{6}, 90 \%\right)$ were obtained from Fisher Scientific, and used for electrochemical study. High purity sodium chloride $(\mathrm{NaCl}, 99.999 \%)$ from Sigma-Aldrich was used as electrolyte. A Nanopure water treatment unit was used to obtain deionized water with a resistivity of $18.2 \mathrm{M} \Omega \mathrm{cm}^{-1}$ at $22{ }^{\circ} \mathrm{C}$. The feed to the water treatment system was double-distilled water. All of the aqueous solutions used in the present study were prepared using nanopure water.

Gold microspheres and gold-coated glass slides were used for AFM surface force measurements. The gold-coated glass slides were obtained by depositing pure gold on glass using a vacuum evaporator. A $50 \AA$ chromium layer was deposited first on the glass prior to coating it with a thin layer (500 $\AA$ ) of gold. The chromium coating was necessary to achieve strong bonding between the gold and glass substrate. The gold coatings produced without the chromium adhesive layer were easily removed in acid solutions.

Gold spheres were produced by melting a gold micropowder $(1.5-3.0 \mu \mathrm{m},>99.6 \%$, Alfa Aesar) in a high temperature furnace. The powder was placed in an alumina crucible and heated until the temperature was raised above its melting point $\left(1064.18^{\circ} \mathrm{C}\right)$. It was kept at $1100{ }^{\circ} \mathrm{C}$ for 15 minutes and then cooled down slowly. The furnace was flushed with nitrogen gas to provide an oxygen-free atmosphere. The gold spheres obtained in this manner had a wide range of sizes. Only those with radius of 3.5-7.5 $\mu \mathrm{m}$ were selected for AFM force measurements.

The gold plates were cleaned first by immersing them in a boiling piranha solution (30:70 $\mathrm{H}_{2} \mathrm{O}_{2} / \mathrm{H}_{2} \mathrm{SO}_{4}$ ) for 20 minutes and then washing them with nanopure water for 1 minute, followed by an ethanol wash for 2 minutes. The gold spheres were cleaned after they 


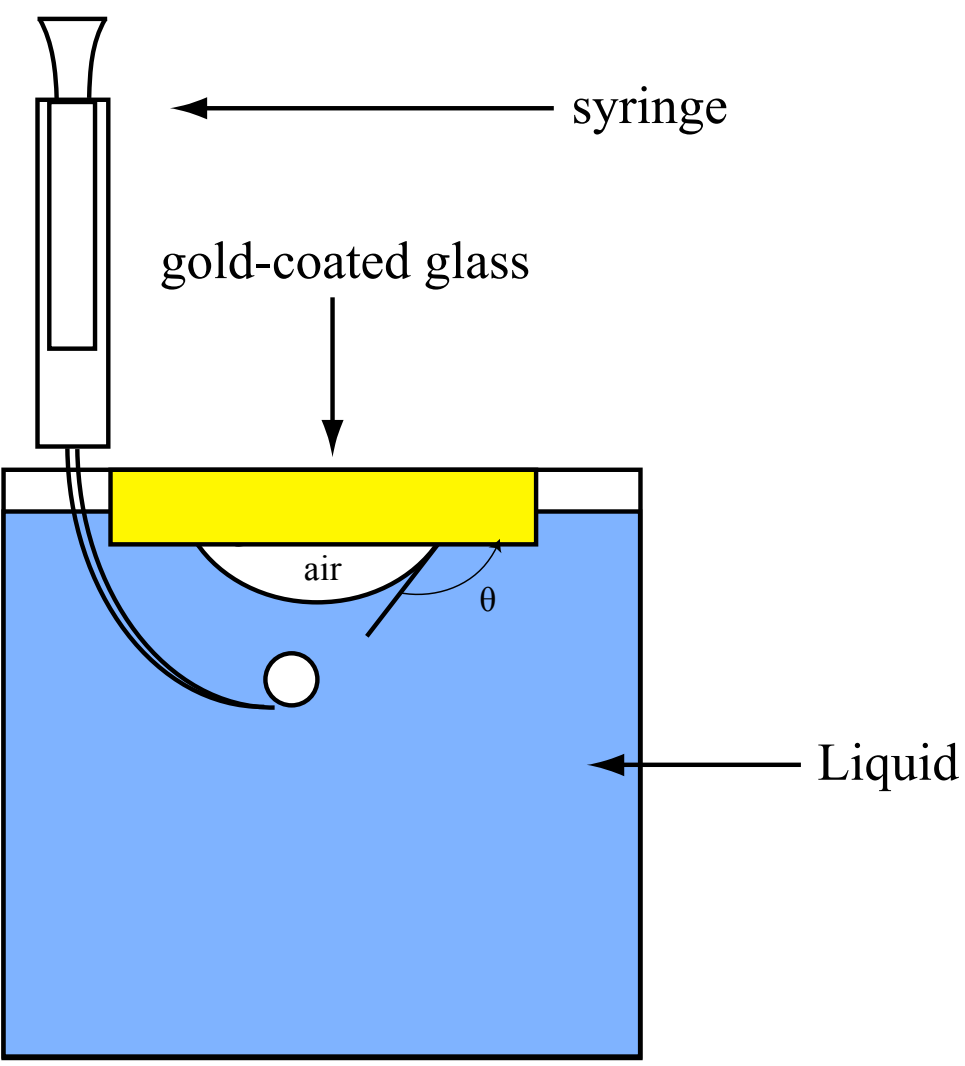

Figure 4.2. A schematic picture of the liquid cell for captive air bubble contact angle measurement.

had been glued onto AFM cantilever springs. Because piranha solution would have destroyed the glue attaching the gold spheres to the AFM cantilever springs, each gold sphere was instead flushed with ethanol, exposed in UV light $(\lambda=254 \mathrm{~nm})$ for two hours, and then rinsed with ethanol again.

\subsubsection{Electrochemical Measurement}

Cyclic voltammetry (CV) experiments were conducted in a standard three-electrode electrochemical cell under ambient conditions. The cell was equipped with a saturated calomel electrode (SCE, $0.242 \mathrm{~V}$ versus $\mathrm{NHE}$ ) as reference electrode and a platinum mesh electrode as counter electrode. A gold-coated glass slide $\left(1 " \times 1.5^{\prime \prime}\right)$ was used as the working electrode, and $1 \times 10^{-1} \mathrm{M} \mathrm{NaClO}_{4}$ as the supporting electrolyte. The potential on the gold electrode was controlled by a potentiostat (model 273A, EG\&G Princeton Applied Research). The cyclic voltammetry experiments were carried out in $1 \times 10^{-1} \mathrm{M} \mathrm{NaClO}_{4}$ solutions containing $1 \times 10^{-4} \mathrm{M} \mathrm{PAX}$ at a scan rate of $250 \mathrm{mV} / \mathrm{s}$ to study the adsorption mechanism. When testing the blocking capability of the xanthate layer on gold, an electrolyte solution containing $1 \times 10^{-1} \mathrm{M} \mathrm{NaClO} 4,1 \times 10^{-5} \mathrm{M} \mathrm{PAX}$, and $1 \times 10^{-2} \mathrm{M}$ $\mathrm{K}_{3} \mathrm{Fe}(\mathrm{CN})_{6} / \mathrm{K}_{4} \mathrm{Fe}(\mathrm{CN})_{6}$ at a scan rate of $200 \mathrm{mV} / \mathrm{s}$ was used. The potentials are reported against the standard hydrogen electrode (SHE), i.e., assuming that the potential is $242 \mathrm{mV}$ more negative than the saturated calomel electrode. 


\subsubsection{Contact Angle Measurement}

Contact angles of gold were measured using the captive air bubble technique in a home-made plastic sample holder, which is shown in Figure 4.2. After the gold-coated glass slides were kept in xanthate solutions for a desired period of time, air bubbles were brought to contact using a syringe. Contact angles were measured by means of a contact angle goniometer (model 100-00 115, Ramé-Hart, Inc., Mountain Lakes, NJ). An average of at least five different individual measurements was used at a given experimental condition.

\subsubsection{Surface Force Measurement}

Surface force measurements were conducted using a nanoscope III (Digital Instruments, Inc., Santa Barbara, CA) atomic force microscope (AFM) equipped with a standard fluid cell and a piezoelectric scanner "E" . All the AFM force measurements were carried out using the colloidal probe technique ${ }^{11,12}$ at room temperature $\left(22 \pm 1{ }^{\circ} \mathrm{C}\right)$. Triangular silicon nitride cantilevers (NP-20, Veeco Probes, Inc.) were used for the force measurements. The spring constant $(k)$ was determined using the resonant frequency technique. ${ }^{13}$ In each experiment, a gold sphere was glued onto a cantilever spring with EPON 1004 resin (Shell Chemical Co.) using a homemade 3-D micromanipulator with a hot plate. The diameter of the gold sphere was measured using an Olympus BH-2 light microscope.

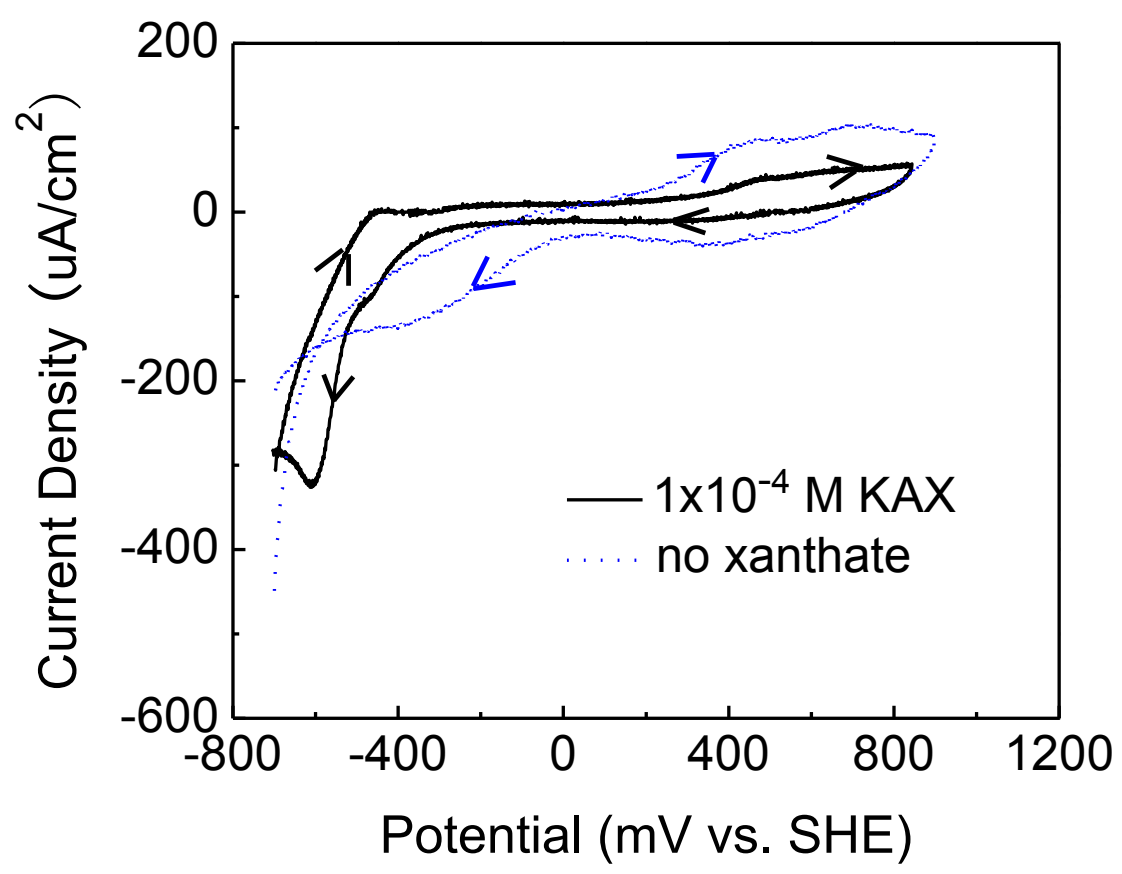

Figure 4.3. Cyclic voltammograms of gold recorded in $1 \times 10^{-1} \mathrm{M} \mathrm{NaClO}_{4}$ aqueous solutions with and without $1 \times 10^{-4} \mathrm{M}$ potassium amyl xanthate $(\mathrm{PAX})$ using a scan rate of $250 \mathrm{mV} / \mathrm{s}$. 
The liquid cell, used to hold the sphere probe, was cleaned in an ultrasonic water bath. All of the measurements were conducted in air-equilibrated solution. The separation distance $(H)$ between the gold sphere and the flat-gold coated glass plate was measured by monitoring the deflection of the cantilever on which the gold sphere was attached. Measured forces $(F)$ were normalized with respect to the radii $(R)$ of the gold spheres.

\subsection{Results and Discussion}

\subsubsection{Electrochemical Characterization}

The key chemical step in a flotation process is the interaction of the organic collectors with a selected mineral; thereby the mineral surface is rendered hydrophobic so that gas bubbles can adhere to them. Electrochemical techniques such as cyclic voltammetry have been used to study the mechanism of interaction between gold and xanthate. ${ }^{14-17}$ In the present study, cyclic voltammetry was used to investigate the interaction and adsorption process between PAX and gold surfaces.

Cyclic voltammograms of the gold working electrode obtained in $1 \times 10^{-1} \mathrm{M} \mathrm{NaClO}_{4}$ solution and $1 \times 10^{-1} \mathrm{M} \mathrm{NaClO}_{4}+1 \times 10^{-4} \mathrm{M}$ PAX mixture solution are shown in Fig. 4.1. Potential scans were performed between -700 and $900 \mathrm{mV}$ at a sweep rate of $250 \mathrm{mV} / \mathrm{s}$. The dotted curve represents the relationship between current density and applied potential for a bare gold electrode in the absence of $1 \times 10^{-4} \mathrm{M}$ PAX, while the cyclic voltammogram for gold in the presence of $1 \times 10^{-4} \mathrm{M}$ PAX is represented by the solid line. As shown in Figure 4.3, the shape of the voltammogram changed dramatically when the xanthate was added. During the anodic scan, in the absence of xanthate, the small anodic current peak at potential around $400 \mathrm{mV}$ is caused by the adsorption of perchloride ions on gold. The anodic peak

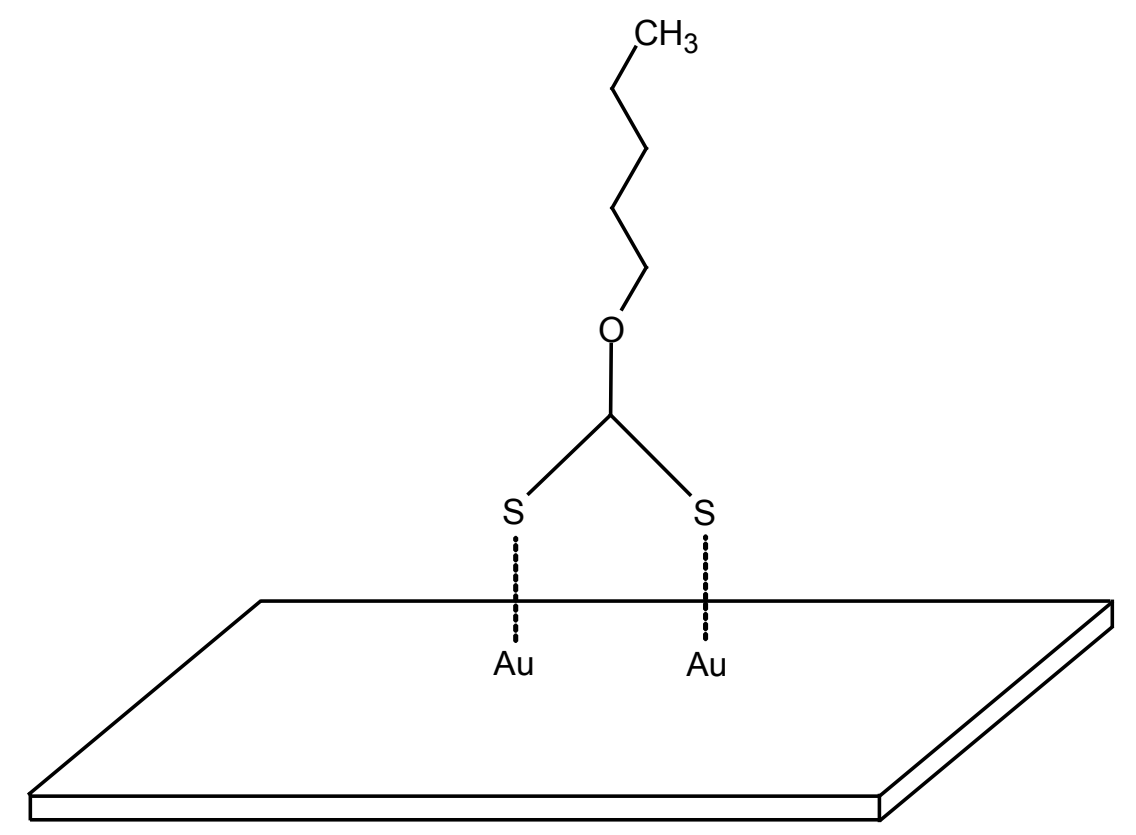

Figure 4.4. Schematic illustration for the arrangement and coordination of amyl xanthate ion on gold. 
observed at potential of about $650 \mathrm{mV}$ is due to the oxidation of gold, which is consistent with previous work. ${ }^{18}$ As the potential was swept in the reverse direction (cathodic scan), the reduction of gold began at a potential about $400 \mathrm{mV}$. In the presence of $1 \times 10^{-4} \mathrm{M}$ PAX, anodic at $450 \mathrm{mV}$ and a cathodic current at $-450 \mathrm{mV}$ were observed. Previous CV studies on the adsorption of potassium ethyl xanthate (KEX) on gold suggest that in the presence of $\mathrm{KEX}$, the chemisorption of xanthate ions $\left(\mathrm{EX}^{-}\right)$begins at $-470 \mathrm{mV}^{15}$ or $-400 \mathrm{mV}^{16}$ and the oxidation of ethyl xanthate to ethyl dixanthogen occurs at $+200 \mathrm{mV}^{15,16}$ during the anodic scan. As the potential was swept in the cathodic direction, the reduction of dixanthogen began at a potential of about $-400 \mathrm{mV}^{15}$ or $-250 \mathrm{mV}^{16}$. Thus, we cannot exclude the possibility that the same types of chemical reaction occurs for potassium amyl xanthate. In the presence of PAX, the anodic current peak at $-450 \mathrm{mV}$ is attributed to the adsorption of amyl xanthate ions on gold, and the peak at $450 \mathrm{mV}$ is due to the oxidation of amyl xanthate ion $\left(\mathrm{X}^{-}\right)$to amyl dixanthogen $\left(\mathrm{X}_{2}\right)$, which is bound to the underlying chemisorbed xanthate. ${ }^{19}$ The cathodic current peak around $-450 \mathrm{mV}$ is due to the reduction of amyl dixanthogen $\left(\mathrm{X}_{2}\right)$ to amyl xanthate ion $\left(\mathrm{X}^{-}\right)$. The cyclic voltammetry experiment shows that the amyl xanthate ions from aqueous solutions spontaneously chemisorb on gold and form a xanthate monolayer.

The chemical interaction of xanthates such as potassium $p$-methyl benzyl xanthate, potassium $p$-trifluoromethyl benzyl xanthate, ethyl and octyl xanthate ions with gold was also studied with infrared reflection absorption spectroscopy (IRAS) and x-ray photoelectron spectroscopy (XPS). ${ }^{20,} 21$ The xanthate monolayers were prepared by immersing gold substrates in 1 to $10 \mu \mathrm{M}$ aqueous solutions of the xanthates for different adsorption times.

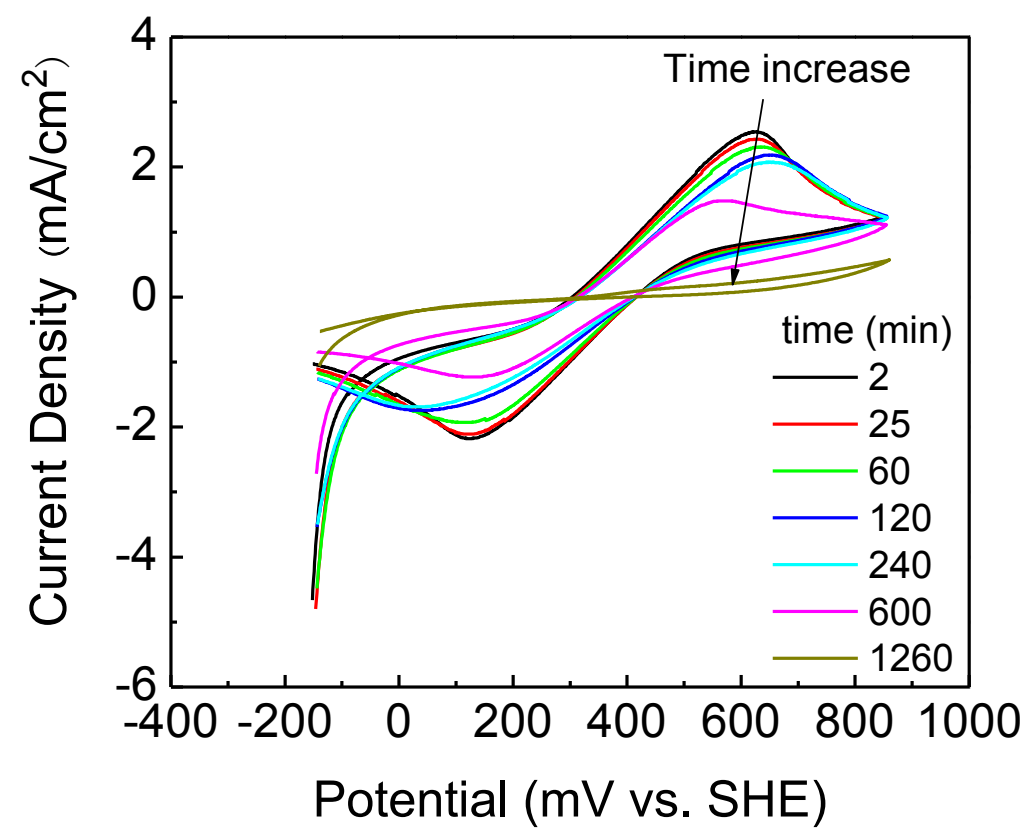

Figure 4.5. Cyclic voltammograms of a gold electrode recorded in $1 \times 10^{-2} \mathrm{M}$ $\mathrm{K}_{3} \mathrm{Fe}(\mathrm{CN})_{6} / \mathrm{K}_{4} \mathrm{Fe}(\mathrm{CN})_{6}, 1 \times 10^{-1} \mathrm{M} \mathrm{NaClO}_{4}$ and $1 \times 10^{-5} \mathrm{M} \mathrm{KAX}$ aqueous solutions at different adsorption times, with scan rate of $200 \mathrm{mV} / \mathrm{s}$ versus an SHE reference electrode. 
The experimental reflection-absorption (R-A) spectra of xanthate ions adsorbed on gold were compared with the calculated R-A spectrum of gold xanthate salts. According to the experimentally obtained peak positions, which were consistent with the calculated values, the chemical structure of the xanthate species on gold were closely related to those of the metal salts. Based on IRAS measurements, it was proposed that the xanthate ions are coordinated to the gold surface through both sulfur atoms (Figure 4. 4). The XPS studies further confirmed a bridge-like coordination for the chemisorbed xanthates.

Figure 4.5 shows cyclic voltammograms of gold in the presence of $1 \times 10^{-5} \mathrm{M}$ PAX in $1 \times 10^{-2} \mathrm{M} \mathrm{K}_{3} \mathrm{Fe}(\mathrm{CN})_{6} / \mathrm{K}_{4} \mathrm{Fe}(\mathrm{CN})_{6}$ and $1 \times 10^{-1} \mathrm{M} \mathrm{NaClO}_{4}$. The scan rate was at $200 \mathrm{mV} / \mathrm{s}$ in both directions. The blocking capability of the amyl xanthate layers on gold formed in $1 \times$ $10^{-5} \mathrm{M}$ amyl xanthate aqueous solution at different immersion times towards the ferri/ferro redox couples was investigated. A cleaned bare gold electrode gave the expected cyclic voltammograms of $\mathrm{K}_{3} \mathrm{Fe}(\mathrm{CN})_{6} / \mathrm{K}_{4} \mathrm{Fe}(\mathrm{CN})_{6}$. The anodic current peak shows the oxidation of $\mathrm{Fe}(\mathrm{CN})_{6}^{-4}$ to $\mathrm{Fe}(\mathrm{CN})_{6}^{-{ }^{-3}}$ on gold; and the cathodic sweep shows the reduction of $\mathrm{Fe}(\mathrm{CN})_{6}^{-3}$ to $\mathrm{Fe}(\mathrm{CN})_{6}^{-4}$. There are no current peaks assigned to the oxidation or desorption of amyl xanthate ions observed. It is known that the electron transfer reaction might be occurring at pinhole sites. ${ }^{22}$ With the immersion time extending from 2 to 1,260 minutes, noticeable decreases in the peak current were observed in the cyclic voltammograms. It appears that the amyl xanthate layeres ability to block the transfer of electrons to the gold electrode surfaces increased with time. After 1,260 minutes of immersion time, the voltammogram was of a sigmoidal line shape, which indicates that the layers had the least pinholes and defects. Thus, the layer was, from an electrochemical point of view, well packed. It is suggested that the organic hydrocarbon part of the xanthate molecule first lies down on the gold surfaces, and

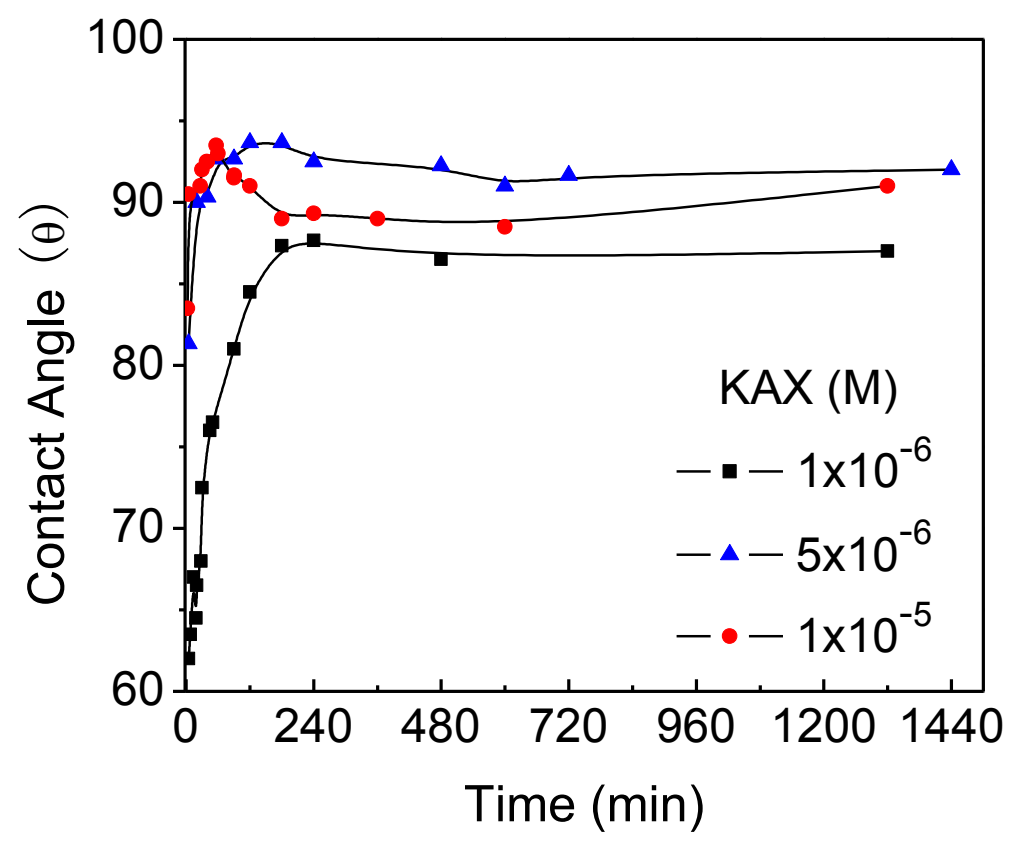

Figure 4.6. Contact angles of amyl xanthate layers on gold surface formed in different xanthate aqueous solutions with varying immersion time. 
erects itself, approaching a conformation normal to the surface with the packing density increasing with reaction time. ${ }^{20}$ It also indirectly indicates that the formation of a closepacked monolayer of amyl xanthate onto gold surface was a rather slow process, which required at least 20 hours.

\subsubsection{Contact Angle Study}

Equilibrium contact angle measurement would be another way to examine the adsorption process of amyl xanthate ions onto gold surfaces. The result of in-situ contact angle measurements on gold in amyl xanthate solutions with the captive bubble technique is shown in Fig. 4.6. The gold-coated glass plates were hydrophobized by in-situ adsorption using a range of aqueous concentration $\left(1 \times 10^{-6}, 5 \times 10^{-6}\right.$ and $\left.1 \times 10^{-5} \mathrm{M}\right)$ of amyl xanthate surfactants. Generally, the contact angle of gold dramatically changed upon contact with the xanthate solution, reaching peak values faster for higher concentrations of amyl xanthate. In $1 \times 10^{-6} \mathrm{M}$ solution, the contact angle of bare gold $\left(62^{\circ}\right)$ reached a maximum value of $87^{\circ}$ after 180 minutes immersion time. In a higher concentration of $5 \times 10^{-6} \mathrm{M}$, a peak value of $94^{\circ}$ was obtained after 120 minutes. When the concentration was further increased to $1 \times 10^{-5}$ $\mathrm{M}$, a peak value of $94^{\circ}$ was obtained after 57 minutes. As discussed before, according to the cyclic voltammetry experiments and literature reports, ${ }^{20,21}$ the xanthate readily adsorb on the gold by chemical reaction just like thiol. The adsorption rate of thiol on gold is influenced by many factors, such as temperature, solvent, concentration, and chain length of the adsorbate. ${ }^{23,24}$ A xanthate monolayer forms with the polar head group in contact with gold, ${ }^{17}$ and the methyl group $\left(-\mathrm{CH}_{3}\right)$ extends toward the aqueous phase, rendering the gold surface hydrophobic.

At higher concentrations $\left(5 \times 10^{-6} \mathrm{M}\right.$ and $\left.1 \times 10^{-5} \mathrm{M}\right)$, the adsorption process was characterized by two distinct phases: a rapid adsorption followed by slower process. Within 5 minutes, the contact angles were close to the peak values. This initial, rapid adsorption was followed by a slower period lasting 1 hour at $1 \times 10^{-5} \mathrm{M}$ PAX and 2 hours at $5 \times 10^{-6} \mathrm{M}$ PAX. The maximum values $\left(94^{\circ}\right)$ were the same for $5 \times 10^{-6} \mathrm{M}$ and $1 \times 10^{-5} \mathrm{M}$, indicating that the a full monolayer of xanthate formed on gold when the contact angle reached the peak value. The contact angles of gold decreased after reaching the maximum values. It is supposed that a physisorbed adlayer of xanthate, which reversely oriented with polar group toward the aqueous phase, was formed on top of a chemisorbed monolayer. According to the $\mathrm{CV}$ experiment conducted in a solution containing $1 \times 10^{-5} \mathrm{M}$ PAX and ferrocyanide/ferricyanide mixture solutions, the blocking capability reached the highest level after an immersion time of 21 hours. While the contact angle measurements show that the hydrophobicity reached maximum value after 1 hour in $1 \times 10^{-5} \mathrm{M}$ xanthate solution. It suggests that after the xanthate completely covered the gold surface, the monolayer took a much longer time ( 21 hours) to reorganize itself and formed a close-packed monolayer. In a very dilute solution of $1 \times 10^{-6} \mathrm{M}$, the contact angle reached maximum value of $87^{\circ}$ and stayed constant as time elapsed. The maximum contact angle on xanthate-coated gold was, however, less than the contact angle of $94^{\circ}$ for the full coverage of xanthate. This indicates that at low concentrations, the entirety of xanthate molecules from the bulk solution migrated to the gold surface without completely covering the gold surfaces. As suggested by Persson et al. ${ }^{20}$ the hydrocarbon chain lay down on the gold surface at low coverage densities, but 
erected itself with a conformation normal to the surface when the coverage densities increased.

According to the Younges equation, the changes of contact angle $\theta$ for gold surface can be affected by changes in the surface tension $\gamma_{\mathrm{lv}}$ of a xanthate solution, the surface tension $\gamma_{\mathrm{sv}}$ of gold, and the tension $\gamma_{\mathrm{sl}}$ of gold/liquid. The Younge ${ }^{\text {ee }}$ equation is shown below:

$$
\cos \theta=\frac{\gamma_{s v}-\gamma_{s l}}{\gamma_{l v}}
$$

When a gold plate was placed in an amyl xanthate solution (e.g., $5 \times 10^{-6} \mathrm{M}$ ), the amyl xanthate ions from the aqueous solution migrated and adsorbed on gold, making the gold surface hydrophobic. Under such conditions, the surface tension $\gamma_{\mathrm{lv}}$ of a xanthate liquid did not change with time. (Xanthate has no surface activity at the air/water interface due to short hydrocarbon chain.) Compared to the surface tension $\gamma_{\mathrm{sv}}$ of xanthate-coated gold, the interfacial tension $\gamma_{\mathrm{sl}}$ at the xanthate-coated gold/liquid interface changed much more with immersion time. The interfacial tension $\gamma_{\mathrm{sl}}$ increased as more amyl xanthate ions adsorbed onto the gold surface with the non-polar methyl group exposed to water, increasing contact angles. After a monolayer was formed on gold, the additional xanthate molecules started to adsorb on the monolayer with inverse orientation, causing a decrease for the interfacial tension $\gamma_{\mathrm{sl}}$, thereby decreasing the hydrophobicity.

\subsubsection{Surface Force Measurement}

Figure 4.7 shows the surface forces measured as a function of immersion time between a flat gold-coated glass slide and a gold microsphere in a $1 \times 10^{-6} \mathrm{M}$ PAX solution.

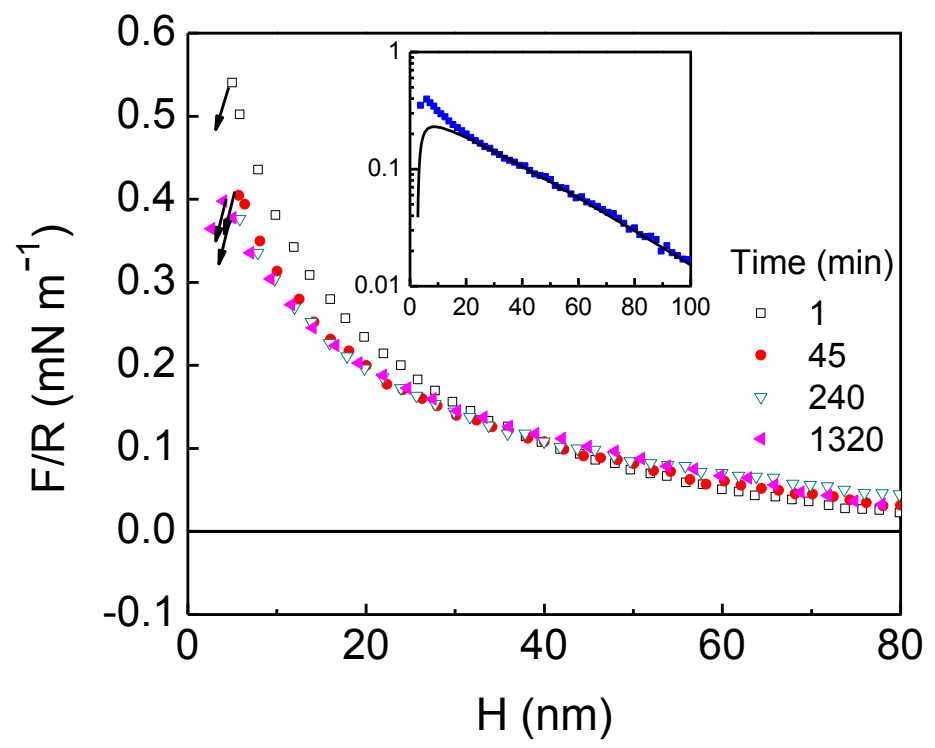

Figure 4.7. Surface forces measured between bare gold in $1 \times 10^{-6} \mathrm{M}$ PAX aqueous solution at different immersion times. 
Fresh solutions were prepared before each set of experiments. The arrows represent the separation distance at which the two surfaces jumped into contact due to attractive forces. The jumps occur when the gradient of the surface force exceeds the spring constant and can be identified by the rapid acceleration of the sphere probe. As shown, there was no hydrophobic force observed for the immersion times investigated, and the surface forces measured in $1 \times 10^{-6} \mathrm{M}$ PAX were traditional DLVO forces, i.e., a short-range van der Waals attractive force and a long-range electrostatic double-layer repulsive force. The repulsive double-layer forces dominated at separation distance $H>10 \mathrm{~nm}$, and the repulsive forces decreased with time. The experimental force versus distance curves recorded for $1 \times 10^{-6} \mathrm{M}$ PAX solution at different immersion times were fitted using classical DLVO theory. To obtain the electrostatic component, the nonlinear Poisson-Boltzmann equation was solved using constant potential boundary condition at the theoretical Debye length $\kappa^{-1}$. The van der Waals component of the interaction was calculated using an experimental Hamaker constant $A_{131}=1.2 \times 10^{-20} \mathrm{~J}$ for gold-water-gold system. ${ }^{24}$ The van der Waals force for the sphere-plate geometry is described as:

$$
\frac{F}{R}=-\frac{A}{6 H^{2}}
$$

where $H$ is the closest distance separation the two microscopic surfaces. The inset shows the AFM force curve obtained for the immersion time of 45 minutes, and a calculated force curve using DLVO theory.

The parameters extracted from these calculations are shown in Table 4.1. Also included in Table 4.1 are the contact angles measured for different immersion times. As shown in Figure 4.6, the contact angle increased with time and reached a limited value of $87^{\circ}$. At the same time, the surface potential $\left(\psi_{0} \approx-45 \mathrm{mV}\right)$ of gold did not change much, while the Debye length $\left(\kappa^{-1}\right)$ increased from 25.4 to $38.7 \mathrm{~nm}$. As discussed before, for $1 \times 10^{-6} \mathrm{M}$ PAX, the xanthate ions cannot cover the entire gold surface. As the xanthate ions adsorbed onto the gold surfaces with time, the xanthate ions in the bulk solution were depleted, and the ionic strength decreased, increasing Debye length.

Table 4.1. The Contact Angles and Parameters (Surface Potentials, Debye Lengths) Obtained by Fitting the Surface Forces Measured in PAX Solutions after Different Immersion Times with DLVO Theory

\begin{tabular}{ccccc}
\hline$C(\mathrm{M})$ & $t(\mathrm{~min})$ & $\theta($ degree $)$ & $k^{-1}(\mathrm{~nm})$ & $\psi_{0}(\mathrm{mV})$ \\
\hline $1 \times 10^{-6}$ & 1 & 65 & 25.4 & -45.0 \\
& 45 & 81 & 28.6 & -44.0 \\
& 240 & 87 & 37.8 & -45.0 \\
& 1320 & 87 & 38.7 & -46.0 \\
$5 \times 10^{-6}$ & 5 & 81 & 24.5 & -48.0
\end{tabular}




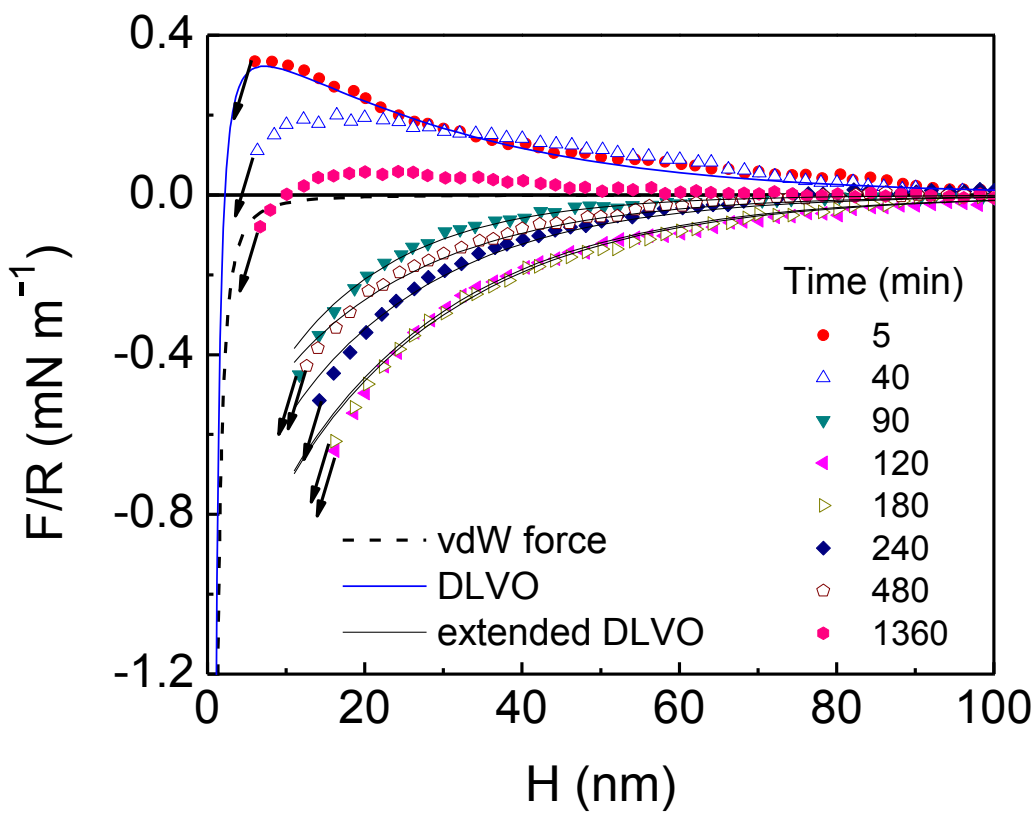

Figure 4.8. Surface forces measured between bare gold in a $5 \times 10^{-6} \mathrm{M}$ PAX aqueous solution at different immersion times.

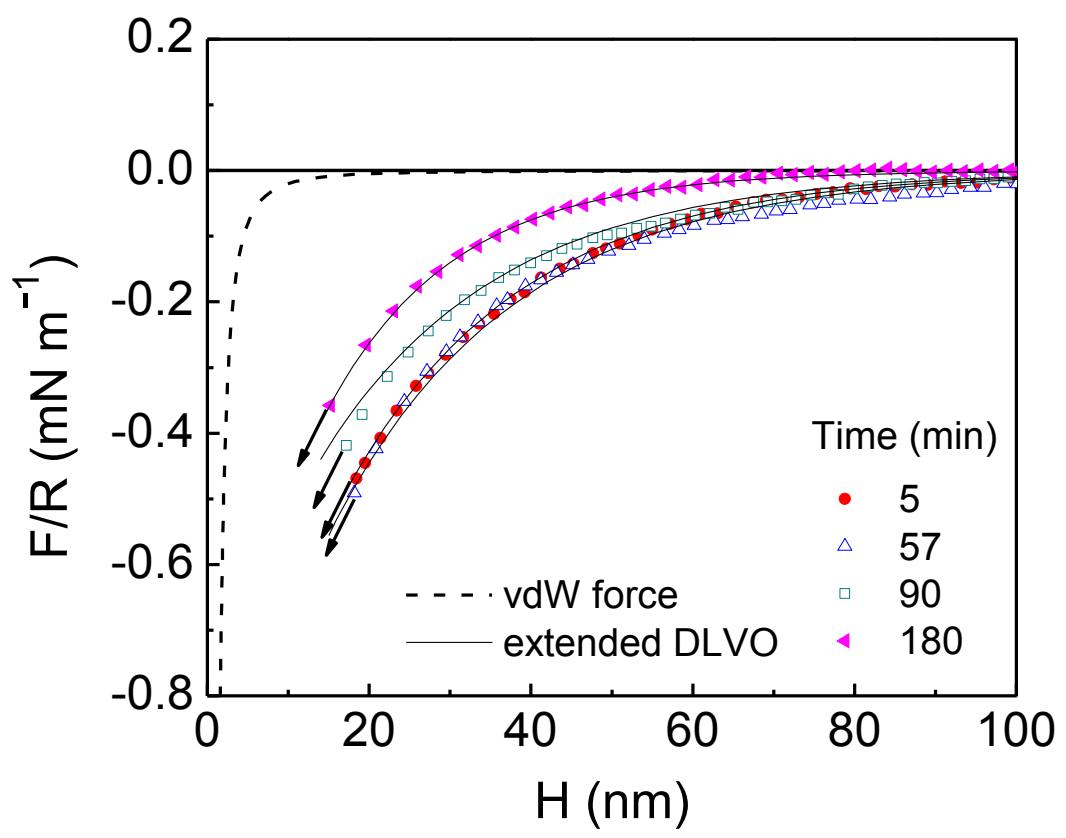

Figure 4.9. Surface forces measured between bare gold in a $1 \times 10^{-5} \mathrm{M}$ PAX aqueous solution at different immersion times. 
concentrations of $5 \times 10^{-6}$ and $1 \times 10^{-5} \mathrm{M}$ PAX. Figure 4.8 shows the surface forces measured at $5 \times 10^{-6} \mathrm{M}$ PAX. After the gold sphere and gold-coated glass plate were in contact with the surfactant solution for 5 minutes, the xanthate ions partially covered on gold, and a longrange repulsive force was observed. The repulsive force decreased with increased immersion time. At 90 minutes, a net-attractive force appeared which increased with further increase in immersion time. At 120 and 180 minutes, the measured force became most attractive. Presumably, the gold surfaces were completely covered by monolayers of xanthate. After a 240 minutes immersion time, the attractive force decreased, probably due to the inverse orientation of the physisorbed xanthate adlayer.

The results obtained in a $1 \times 10^{-5} \mathrm{M}$ PAX solution are presented in Figure 4.9. At this high concentration, the gold surfaces were completely covered by xanthate after 5 and 57 minutes of immersion times, and gave rise to the strongest attraction. After the 90 minutes of immersion time, the additional xanthate ions adsorbed on the underlying chemisorbed monolayer with reverse orientation, which gave rise to a weaker attractive force. The attractive force appeared sooner in $5 \times 10^{-6} \mathrm{M}$ than in $1 \times 10^{-5} \mathrm{M}$ PAX solution. According to the contact angle measurements, the xanthate adsorption process is a concentration dependent

\section{Table 4.2. Parameter $C$ and Decay Length $D$ Obtained by Fitting the Surface Forces Measured in PAX Solutions after Different Immersion Times with Extended DLVO Theory}

\begin{tabular}{ccccc}
\hline$C(\mathrm{M})$ & $t(\mathrm{~min})$ & $\theta($ degree $)$ & $C(\mathrm{mN} / \mathrm{m})$ & $D(\mathrm{~nm})$ \\
\hline \multirow{5}{*}{$5 \times 10^{-6}$} & 90 & 93 & -0.75 & 15.5 \\
& 120 & 94 & -1.1 & 22.5 \\
& 180 & 94 & -1.1 & 23 \\
& 240 & 93 & -0.9 & 20 \\
$1 \times 10^{-5}$ & 480 & 92 & -0.7 & 20 \\
& 5 & 91 & -1.05 & 22 \\
& 57 & 94 & -1.05 & 23 \\
& 90 & 92 & -0.8 & 22.5 \\
& 180 & 89 & -0.9 & 16
\end{tabular}

process. In summary, higher concentrations of PAX lead to faster adsorption rates on gold surfaces, creating a hydrophobic monolayer which in turn creates a long-range hydrophobic attractive force.

Previous studies ${ }^{25-28}$ on the hydrophobic force, using the surfaces hydrophobized by chemical reaction with hydrophobizing agents, suggest that the long-range interaction originates from the gas bridges. The gas bridges are formed by coalescence between nanosize bubbles, which are captured on the surfaces upon immersing them into an aqueous solution. In the present work, gold surfaces were hydrophobized by in-situ adsorption of xanthate. The substrates were never exposed to air at any stage. As can be seen from Figure 4.8 and 4.9, strong attractive forces with an exceptional range, up to $100 \mathrm{~nm}$, were observed. All of the force curves were smooth, without showing steps or kinks, indicating that the adsorption systems were relatively free of contamination or air bubbles. It further indicates that the 
hydrophobic forces measured in our study were not caused by bridging air bubbles as suggested by Parker et al., ${ }^{25}$ Higashitani et al., ${ }^{26,27,29}$ Attard et al., ${ }^{30-33}$ Ederth et al..${ }^{34-37}$ and Nguyen et al. ${ }^{28}$

Despite the extensive experimental evidence for the existence of the long-range hydrophobic force, no single theory can account for this interaction, and empirical equations were usually used to describe them. ${ }^{24,}{ }^{38-40}$ The long-range attractive force versus distance curves shown in Figure 4.8 and 4.9 were fitted to a single-exponential force law:

$$
\frac{F}{R}=C \exp \left(-\frac{H}{D}\right)
$$

where $F$ is the hydrophobic force, $R$ is the radius of the gold microsphere, $H$ is the surface separation distance of the gold microsphere and flat gold-coated glass plate, and $C$ and $D$ are the fitting parameters. $C$ represents the magnitude of the attractive force and $D$, usually called decay length, represents the range of this interaction. Table 4.2 gives the values of $C$ and decay length $(D)$ as well as the contact angle $(\theta)$. From Figure 4.8 and 4.9, it is clearly seen that the hydrophobic force increased with increasing contact angle, which is in agreement with previous studies. ${ }^{41}$ As shown in Table 4.2, the decay length $(D)$ increased with the contact angle, ranging from 15.5 to $23 \mathrm{~nm}$. For the same contact angle, the hydrophobic force was almost the same. The maximum contact angles measured in $5 \times 10^{-6} \mathrm{M}$ and $1 \times 10^{-5} \mathrm{M}$ PAX solutions were $94^{\circ}$. The fitting constants $C$ and $D$ were $-1.1 \mathrm{mN} / \mathrm{m}$ and $23 \mathrm{~nm}$ in $5 \times 10^{-6}$ M PAX and $-1.05 \mathrm{mN} / \mathrm{m}$ and $23 \mathrm{~nm}$ in $1 \times 10^{-5} \mathrm{M}$ PAX solution. In view of the results of contact angle and surface force measurements, it is suggested that hydrophobic force increased with the surface coverage of xanthate ions on gold. The hydrophobic force was

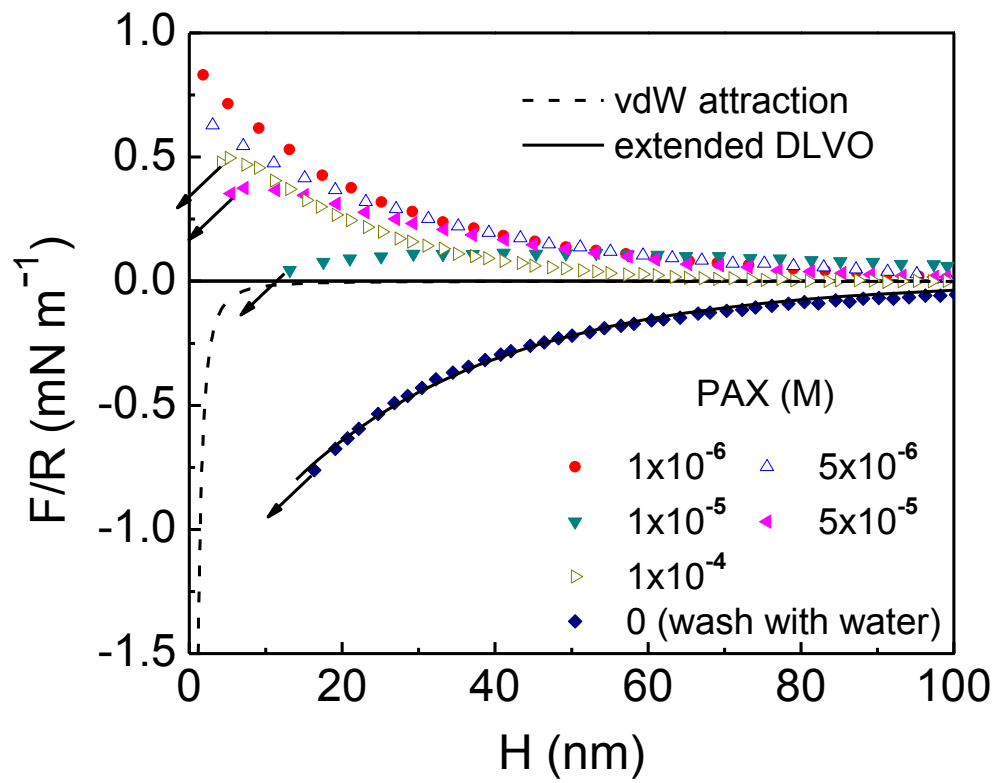

Figure 4.10. The surface forces measured between bare gold surfaces in PAX solutions as a function of concentration of PAX. 
most attractive when xanthate ions covered the entire gold surface by forming a selfassembled monolayer. The relation between the surface adsorption of xanthate ions and the hydrophobic force was also found for alkyl thiol on gold. ${ }^{24}$

Figure 4.10 shows the results of the surface force measurements conducted as a function of the PAX concentration. The PAX solutions were injected into an AFM liquid glass cell starting from the lowest concentration $\left(1 \times 10^{-6} \mathrm{M}\right)$ to the highest $\left(1 \times 10^{-4} \mathrm{M}\right)$. For each new concentration, the gold microsphere and gold-coated glass plate were exposed to the surfactant solution for a period of 35 minutes before the force measurements were taken.

Figure 4.10 shows the same trend as the surface forces measured between a glass sphere and a silica plate in $\mathrm{C}_{18} \mathrm{TACl}$ solution. ${ }^{39}$ At $1 \times 10^{-6} \mathrm{M}$, the xanthate ions were unable to cover the entire gold surfaces, allowing the surface charge on gold to give rise to a longrange electrostatic repulsive force. At $5 \times 10^{-6} \mathrm{M}$, more xanthate ions adsorbed on gold, reducing the electrostatic force. While relatively short-ranged, the attractive force was longer than the van der Waals force (represented as the dashed line), causing the gold sphere to jump to the gold-coated glass surface at a separation distance of $17 \mathrm{~nm}$. This attraction was actually the hydrophobic force, and the repulsive force was the electrostatic force caused by the physisorbed xanthate layer with reverse orientation. At $5 \times 10^{-5} \mathrm{M}$, the hydrophobic force decreased, represented by the decreased jump distance $(H=9 \mathrm{~nm})$. When the concentration of PAX was increased to $1 \times 10^{-4} \mathrm{M}$, an increase in surface charge and decrease in surface hydrophobicity were observed, caused by an adsorption of amyl xanthate ions on the chemisorbed monolayer. The repulsive electrostatic force increased and the hydrophobic force decreased with a jump distance of $5 \mathrm{~nm}$. After the force measurement in $1 \times 10^{-4} \mathrm{M}$ PAX, the AFM liquid cell was flushed with nanopure water, replacing the xanthate solution.

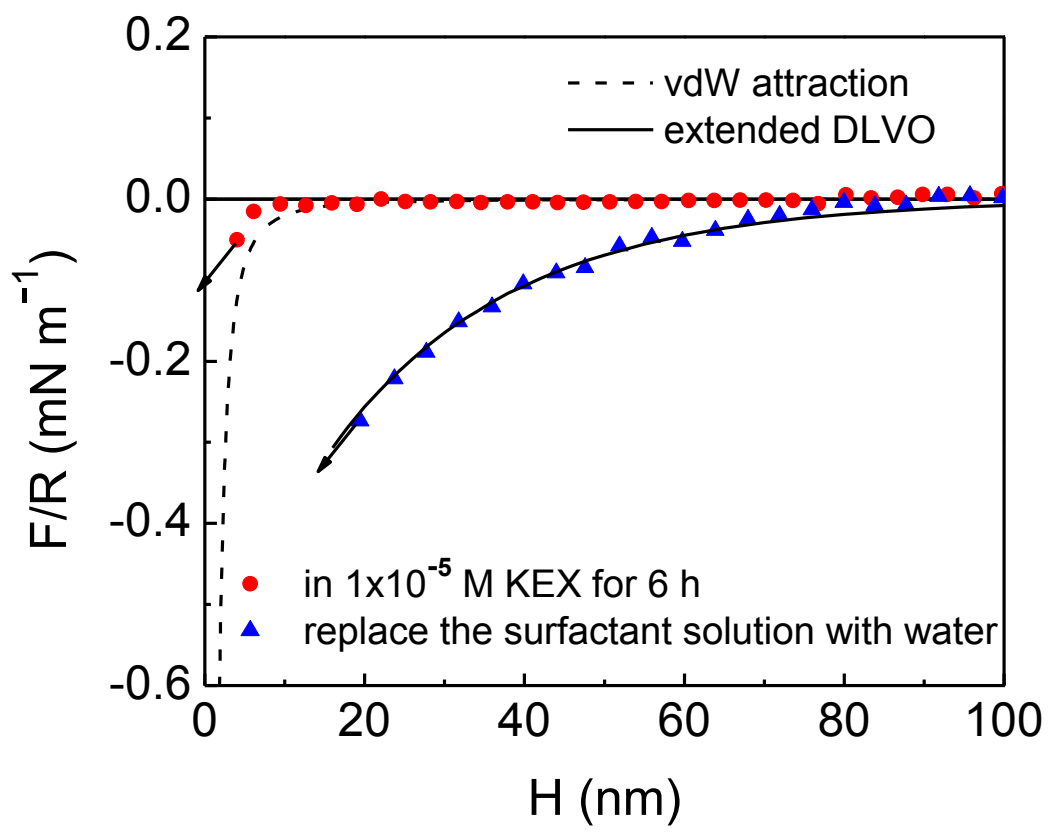

Figure 4.11. Surface forces measured between KEX adsorbed gold surfaces in $1 \times 10^{-5} \mathrm{M}$ $\mathrm{KEX}$ aqueous solution and in water. 
The force measurement revealed the existence of a strongly attractive hydrophobic force with a long range of up to $100 \mathrm{~nm}$. This means that after water washing, the physisorbed adlayer of xanthate was removed, leaving the chemisorbed monolayer intact and giving rise to the long-range hydrophobic force.

The force measurement was also conducted after the gold sphere and gold-coated glass plate were immersed in $1 \times 10^{-5} \mathrm{M}$ potassium ethyl xanthate $(\mathrm{KEX})$ solution for 6 hours. As shown in Figure 4.11, the red dotted line represents the force measured in KEX solution. The force was attractive and jumped at short separation distance. The thin dashed black line represents the van der Waals force calculated using an experimental Hamaker constant of $1.2 \times 10^{-20} \mathrm{~J}$. It is shown clearly that the measured attraction was smaller than the van der Waals force. It is speculated that the measured force was a combination of attractive hydrophobic force and a repulsive electrostatic force due to the physisorption of KEX. This physisorbed KEX layer can be washed away using water. As shown, a long-range hydrophobic force appeared when the KEX solution was replaced with water.

\section{Table 4.3. Effects of $\mathrm{NaCl}$ on Debye Length $\left(\kappa^{-1}\right), C$ and Decay Length $(D)$ for Gold Hydrophobized by in-situ Adsorption of PAX and KEX}

\begin{tabular}{ccccc}
\hline & $\mathrm{NaCl}(\mathrm{M})$ & $k^{-1}(\mathrm{~nm})$ & $C(\mathrm{mN} / \mathrm{m})$ & $D(\mathrm{~nm})$ \\
\hline PAX & 0 & 94.5 & -1.3 & 28 \\
& $1 \times 10^{-4}$ & 28.6 & -1.4 & 14 \\
& $5 \times 10^{-4}$ & 13.3 & -0.6 & 6 \\
KEX & 0 & 94.5 & -0.6 & 22
\end{tabular}

The hydrophobic force curves shown in Figure 4.10 and 4.11 were fitted to a single exponential law, as described in Equation 4.3. The Debye length $\left(\kappa^{-1}\right), C$ and decay length $(D)$ in water as well as in $\mathrm{NaCl}$ solutions were given in Table 4.3. In water, the $C$ and $D$ values were much larger for $\operatorname{PAX}(C=-1.3 ; D=28)$ than for $\operatorname{KEX}(C=-0.6 ; D=22)$. Compared to the most attractive hydrophobic forces measured in $5 \times 10^{-6} \mathrm{M}$ and $1 \times 10^{-5} \mathrm{M}$ of PAX, as shown in Figure 4.8 and 4.9, the hydrophobic force measured after water washing was larger and longer-ranged. According to the previous studies, the most attractive hydrophobic force appeared when the gold surfaces were covered by a monolayer of xanthate. In Figure 4.10, force measurements show that gold surfaces were fully covered in xanthate following an immersion in $1 \times 10^{-4}$ PAX solution for 35 minutes. Thus, the measured force when $1 \times 10^{-4} \mathrm{M}$ PAX was replaced by water should be the same in terms of magnitude and range as the most attractive forces shown in Figure 4.8 and 4.9. However, data analysis shows that the $C$ and decay length $D$ for forces $(C=-1.3 \mathrm{mN} / \mathrm{m} ; D=28 \mathrm{~nm})$ as shown in Figure 4.10 were much larger than that $(C=-1.1 \mathrm{mN} / \mathrm{m} ; D=23 \mathrm{~nm})$ shown in Figure 4.8 and 4.9. Zhang et al $^{40}$ had conducted surface force measurement using an AFM with a glass sphere and a silica plate immersed $\mathrm{C}_{n} \mathrm{TACl}$ solutions. The $\mathrm{C}_{n} \mathrm{TACl}$ represents the homologues of the surfactant with a carbon chain length from 12 to 18 . They found the presence of the hydrocarbon chain $\left(-\left(\mathrm{CH}_{2}\right)_{n} \mathrm{CH}_{3}\right)$ in water diminished the hydrophobic force. The decay length decreased linearly with the effective concentration of the $\mathrm{CH}_{2} / \mathrm{CH}_{3}$ groups of the $\mathrm{C}_{n} \mathrm{TACl}$ homologues in water. In view of the hydrophobic force model derived using a mean-field approach by Eriksson et ll $^{42}$, they concluded that hydrophobic chains in solution disrupted the surface-induced water structure and thus, caused the hydrophobic force to 
decrease. When $1 \times 10^{-4} \mathrm{M}$ PAX was replaced by water, the increase of the attractive force measured suggests a decreased repulsive force and an increased hydrophobic force. The repulsive electrostatic force disappeared when the physisorbed xanthate layer was removed by water flushing, and the hydrophobic force increased because water also dispelled the "water destroying" hydrocarbon chain.

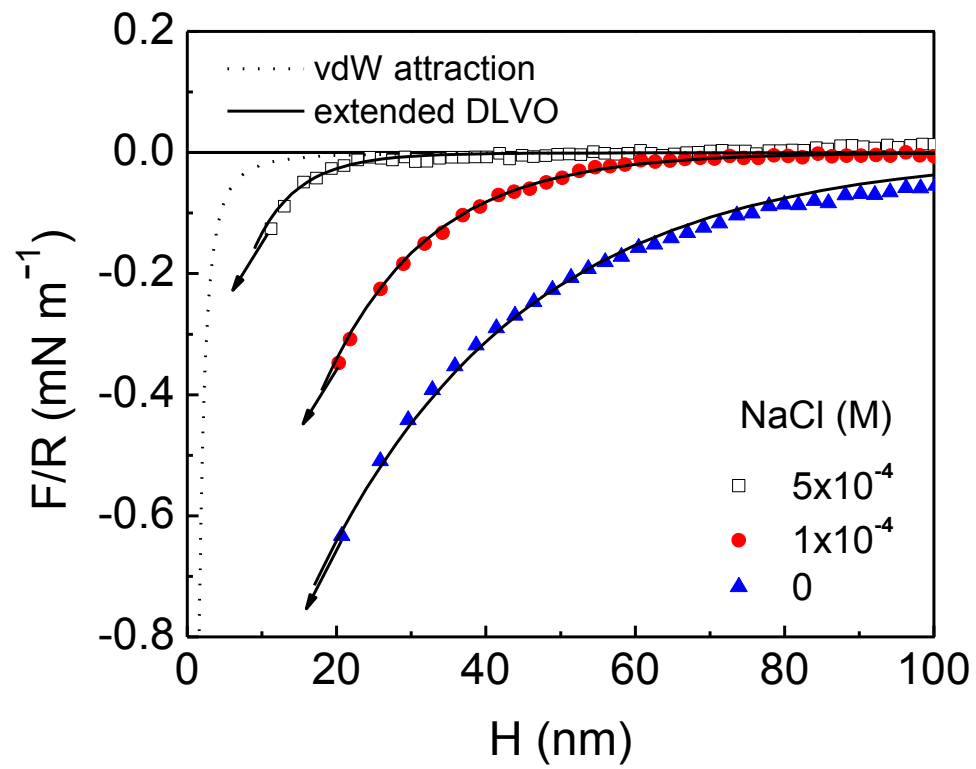

Figure 4.12. Surface force measured between PAX hydrophobic layers in different concentrations of $\mathrm{NaCl}$ aqueous solution.

After flushing with nanopure water, the liquid cell was filled by $\mathrm{NaCl}$ solutions with two different concentrations $\left(5 \times 10^{-4} \mathrm{M}\right.$ and $\left.1 \times 10^{-4} \mathrm{M}\right)$. The effect of added salt on the hydrophobic force was investigated, and the experimental data are shown in Fig. 4.12 and the fitted parameters are given in Table 4.3. It is shown that the addition of $\mathrm{NaCl}$ decreased the magnitude and range of the attractive hydrophobic force, which is consistent with previous investigations. ${ }^{39,}{ }^{43-46}$ Because of its salt dependent character, the hydrophobic force was regarded as the attraction due to electrostatic correlations in an early study. ${ }^{47} \mathrm{~A}$ chargedpatch model developed by Miklavic et al ${ }^{48}$ predict that the attraction should be exponentially decaying at a rate equal to one half the Debye length $\left(\kappa^{-1}\right)$, and data analysis shows that the decay lengths in $1 \times 10^{-4} \mathrm{M}$ and $5 \times 10^{-4} \mathrm{M}$ are actually equal to one half of the corresponding Debye length. However, Miklavic"s model presumes that the positive and negative surfactant patches on the two surfaces are free to migrate to lower the interaction. Because xanthate ions adsorbed on gold to form strong covalent bonding, it is not possible for them to migrate freely on gold. The "patchy bilayer" model developed by Meyer et al. ${ }^{49}$ suggests that the long-range hydrophobic force measured between two surfactant-coated surfaces is a long-range electrostatic attraction, which results from the natural alignment of oppositely charged surfactant domain as two such surfaces approach. Neither the "charged patch" nor the "patchy bilayer" model can explain the long-range hydrophobic force in the 
present work. It is believed that the addition of $\mathrm{NaCl}$ disturbs the water structure between the hydrophobic surfaces. ${ }^{40}$

\section{$\underline{4.5 \text { Conclusions }}$}

In the present work, cyclic voltammetry (CV), contact angle and surface force measurements were conducted to study the adsorption of amyl xanthate on gold and to measure the hydrophobic forces between xanthate-coated surfaces. The CV test verified the chemical adsorption of amyl xanthate ions on gold. The contact angle and surface force measurements revealed the reverse orientation of amyl xanthate on gold surfaces. According to the surface force measurement, the attractive hydrophobic force which was much greater in magnitude and range than expected for the van der Waals force has been detected at the high water contact angles. It has been found also that xanthate adsorption resulted in multilayer formation. The molecules adsorbed in the second-layer adsorbed with inverse orientation, which in turn caused a decrease in hydrophobic force. The molecules adsorbed in the second and subsequent layers can be readily washed by water, which gives rise to a strong hydrophobic force.

\section{References}

1. Yoon, R.-H.; Mao, L., J. Colloid Interface Sci. 1996, 181, 613-626.

2. $\quad$ Derjaguin, B. V.; Dukhin, S. S., Trans. Inst. Min. Metall. 1961, 70, 221-246.

3. Derjaguin, B. V.; Dukhin, S. S., In Proceedings 13th Int. Miner. Process. Cong., Warszawa, 1979; Vol. 2, pp 1261-1287.

4. Laskowski, J.; Kitchener, J. A., J. Colloid Interface Sci. 1969, 29, 670-679.

5. Blake, T. D.; Kitchener, J. A., J. Chem. Soc., Faraday Trans. 1 1972, 68, 1435-1442.

6. Israelachvili, J.; Pashley, R., Nature 1982, 300, 341-342.

7. Yoon, R.-H.; Aksoy, B. S., J. Colloid Interface Sci. 1999, 211, 1-10.

8. $\quad$ Wang, L.; Yoon, R.-H., Langmuir 2004, 20, 11457-11464.

9. Tzhayik, O.; Sawant, P.; Efrima, S.; Kovalev, E.; Klug, J. T., Langmuir 2002, 18, 3364-3369.

10. Rao, S. R., Xanthates and Related Compounds. In Marcel Dekker, Inc.: New York, 1971; pp 15-18.

11. Ducker, W. A.; Senden, T. J.; Pashley, R. M., Nature 1991, 353, 239-241.

12. Ducker, W. A.; Senden, T. J., Langmuir 1992, 8, 1831-1836.

13. Cleveland, J. P.; Manne, S.; Bocek, D.; Hansma, P. K., Rev. Sci. Instrum. 1993, 64, (2), 403-405.

14. Woods, R., J. Phys. Chem. 1971, 75, 354-362.

15. Leppinen, J. O.; Yoon, R.-H.; Mielczarski, J. A., Colloids Surf. 1991, 61, 189-203.

16. Talonen, P.; Sundholm, G.; Li, W.-H.; Floate, S.; Nichols, R. J., Phys. Chem. Chem. Phys. 1999, 1, 3661-3666.

17. Gothelf, K. V., J. Electroanal. Chem. 2000, 494, 147-150.

18. Sondag-Huethorst, J. A. M.; Fokkink, L. G. J., Langmuir 1992, 8, 2560-2566.

19. Woods, R.; Hope, G. A.; Brown, G. M., Colloids Surf., A 1998, 137, 339-344.

20. Persson, N. O.; Uvdal, K.; Liedberg, B.; Hellsten, M., Progr Colloid Polym Sci 1992, 88, 100-109. 
21. Ihs, A.; Uvdal, K.; Liedberg, B., Langmuir 1993, 9, 733-739.

22. Finklea, H. O.; Snider, D. A.; Fedyk, J., Langmuir 1993, 9, 3660-3667.

23. Bain, C. D.; Troughton, E. B.; Tao, Y.-T.; Evall, J.; Whitesides, G. M.; Nuzzo, R. G., J. Am. Chem. Soc. 1989, 111, 321-335.

24. Wang, J.; Yoon, R.-H., Langmuir 2008, 24, 7889-7896.

25. Parker, J. L.; Claesson, P. M.; Attard, P., J. Phys. Chem. 1994, 98, 8468-8480.

26. Ishida, N.; Sakamoto, M.; Miyahara, M.; Higashitani, K., Langmuir 2000, 16, (13), 5681-5687.

27. Ishida, N.; Sakamoto, M.; Miyahara, M.; Higashitani, K., J. Colloid Interface Sci. 2002, 253, 112-116.

28. Nguyen, A. V.; Nalaskowski, J.; Miller, J. D.; Butt, H.-J., Int. J. Miner. Process. 2003, 72, 215-225.

29. Sakamoto, M.; Kanda, Y.; Miyahara, M.; Higashitani, K., Langmuir 2002, 18, 57135719.

30. Carambassis, A.; Jonker, L. C.; Attard, P.; Rutland, M. W., Phys. Rev. Lett. 1998, 80, (24), 5357-5360.

31. Attard, P., Langmuir 2000, 16, 4455-4466.

32. Tyrrell, J. W. G.; Attard, P., Phys. Rev. Lett. 2001, 87, (17), 176104-1.

33. Attard, P.; Moody, M. P.; Tyrrell, J. W. G., Physica A 2002, 314, 696-705.

34. Ederth, T.; Claesson, P.; Liedberg, B., Langmuir 1998, 14, 4782-4789.

35. Ederth, T., J. Phys. Chem. B 2000, 104, 9704-9712.

36. Ederth, T.; Liedberg, B., Langmuir 2000, 16, $2177-2184$.

37. Ederth, T.; Tamada, K.; Claesson, P. M.; Valiokas, R.; Colorado, R.; Graupe, M.; Shmakova, O. E.; Lee, T. R., J. Colloid Interface Sci. 2001, 235, 391-397.

38. Rabinovich, Y. I.; Yoon, R.-H., Langmuir 1994, 10, 1903-1909.

39. Zhang, J.; Yoon, R.-H.; Mao, M.; Ducker, W. A., Langmuir 2005, 21, 5831-5841.

40. Zhang, J.; Yoon, R.-H.; Eriksson, J. C., Colloids Surf., A 2007, 300, 335-345.

41. Yoon, R.-H.; Ravishankar, S. A. R., J. Colloid Interface Sci. 1996, 179, 391-402.

42. Eriksson, J. C.; Ljunggren, S.; Claesson, P. M., J. Chem. Soc., Faraday Trans. 2 1989, 85, (3), 163-176.

43. Claesson, P. M.; Christenson, H. K., J. Phys. Chem 1988, 92, 1650-1655.

44. Christenson, H. K.; Claesson, P. M.; Berg, J.; Herder, P. C., J. Phys. Chem. 1989, 93, 1472-1478.

45. Kekicheff, P.; Spalla, O., Phys. Rev. Lett. 1995, 75, (9), 1851-1855.

46. Claesson, P. M.; Blom, C. E.; Herder, P. C.; Ninham, B. W., J. Colloid Interface Sci. 1986, 114, (1), 234-242.

47. Attard, P., J. Phys. Chem. 1989, 93, (17), 6441-6444.

48. Miklavic, S. J.; Chan, D. Y. C.; White, L. R.; Healy, T. W., J. Phys. Chem. 1994, 98, 9022-9032.

49. Meyer, E. E.; Lin, Q.; Hassenkam, T.; Oroudjev, E.; Israelachvili, J. N., Proc. Nat. Acad. Sci. U.S.A 2005, 102, 6839-6842. 


\section{TASK 4 SURFACE FORCES BETWEEN HYDROPHBIZED GOLD SURFACES SUBMERGED IN ALCOHOLS AND IN WATER-ETHANOL MIXTURES}

\section{$\underline{5.1 \text { Abstract }}$}

Hydrophobic surfaces prepared by the adsorption of alkanethiols of different chain lengths onto a gold microsphere and a gold-coated flat glass plate were used to study the long-range hydrophobic force. Direct surface force measurements were conducted in airequilibrated alcohols, water, and water/ethanol mixtures using an Atomic Force Microscope (AFM) at room temperature. The force measurements in alcohols showed that long range attractions existed in ethanol, 1-butanol and water between two $\mathrm{C}_{4} \mathrm{SH}$-coated gold surfaces. In methanol, the attractive force was slightly larger than the non-retarded van der Waals force.

The surface forces measured on $\mathrm{C}_{4} \mathrm{SH}-, \mathrm{C}_{12} \mathrm{SH}$ - and $\mathrm{C}_{16} \mathrm{SH}$-coated gold surfaces in water/ethanol mixtures were purely attractive, and the surface force curves were all smooth, exhibiting no kinks or steps. With all of the three hydrophobic surfaces, the attraction was strongest in pure water and pure ethanol. The attraction decreased in the ethanol-water mixtures, its range and strength reaching a minimum at mole fractions in the range of 0.1 to 0.9 , depending on the length of the hydrocarbon chain of the thiol used for hydrophobizing the gold surfaces. For all three cases, the decay length $(D)$ and pre-exponential constant $(C)$ passes through a minimum at the mole fraction of ethanol 0.2, indicating a transition from a water structure to an ethanol structure. Considerations of thin film thermodynamics suggests that at low ethanol concentration more of the ethanol is expelled from the film while more of the water is expelled from the film at high ethanol concentration.

\section{$\underline{5.2 \text { Background }}$}

For many years it has been recognized that both the water and ethanol surfaces exposed to air at room temperature are characterized by anomalously low surface entropies. Upon raising the temperature, however, the surface entropy increases. For a pure vaporliquid system, it passes through a pronounced maximum before vanishing at the critical temperature. ${ }^{1}$ This type of behavior is anticipated also for water or ethanol in contact with a (macroscopic) hydrophobic solid surface and can be explained, in a qualitative manner at least, by invoking the notion of a surface-induced, H-bond-dependent, dynamic structure that gradually becomes less extensive and less well-ordered at elevated temperatures.

Focusing on mixtures of water (1) and ethanol (2) in contact with air, we note that upon raising the ethanol concentration, the surface tension at first drops quite rapidly for alcohol mole fractions less than about 0.2 but only rather slowly, and almost linearly, in the range above $x_{2} \approx 0.2^{2,3}$. From the surface tension curves and available vapor pressure data, ${ }^{4}$ Butler and Wightman ${ }^{5}$ showed that the Gibbs surface excess of ethanol, $\Gamma_{2(1)}$, passes through a pronounced maximum at $x_{2}=0.17$ (Figure 5.1). This maximum can be rationalized by invoking that above $x_{2}=0.17$, the interfacial structure and composition vary at a considerably slower rate than the bulk composition, whereas the reverse holds true below this particular mole fraction. 

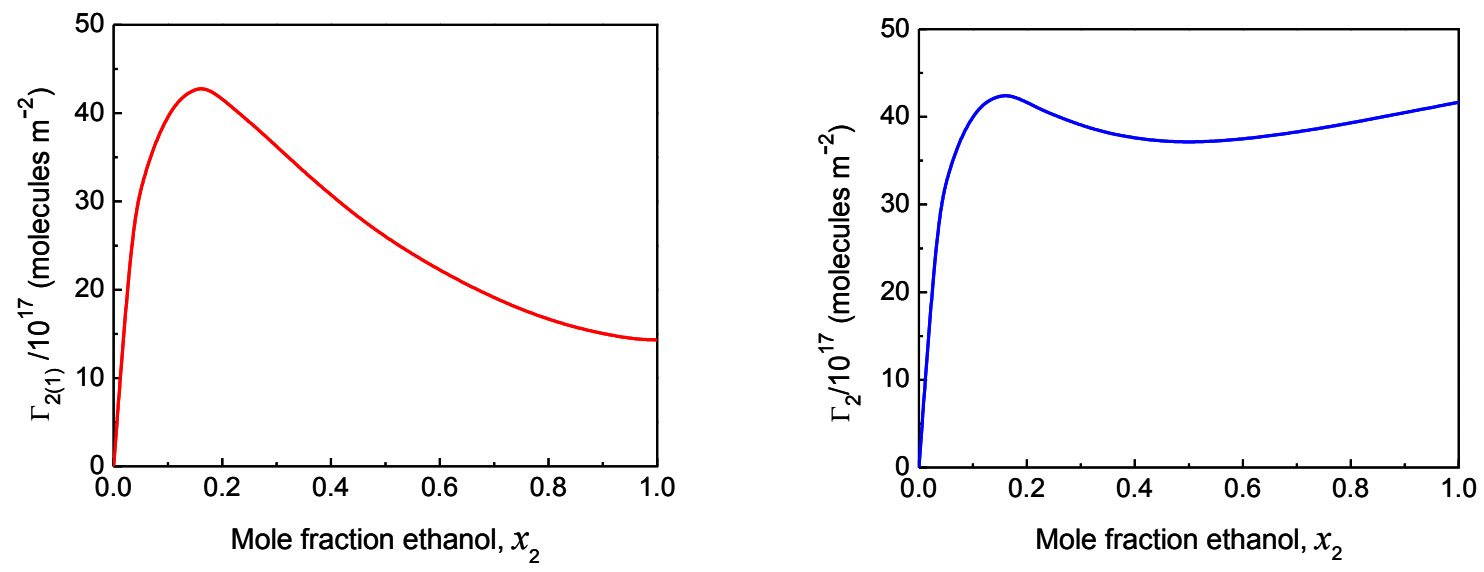

Figure 5.1. The Gibbs surface excess of ethanol, $\Gamma_{2(1)}$, in the water (1)-ethanol (2) mixture/air interface plotted versus the mole fraction of ethanol, $x_{2}$. Note the pronounced maximum for $x_{2} \approx 0.17$. For comparison, the superficial density of ethanol, $\Gamma_{2}$, as obtained on the basis of Equations 5.1 and 5.2 are also shown. Temperature is $25^{\circ} \mathrm{C}$.

A more transparent estimate of the ethanol adsorption is obtained by assuming a mixed monolayer surface phase and introducing the condition

$$
\Gamma_{1} a_{1}+\Gamma_{2} a_{2}=1
$$

to be used in conjunction with the expression for the Gibbs surface excess of ethanol, $\Gamma_{2(1)}$, viz.,

$$
\Gamma_{2(1)}=\Gamma_{2}-\Gamma_{1} x_{2} / x_{1}
$$

where $\Gamma_{1}$ and $\Gamma_{2}$ stand for the (monolayer) superficial densities of water and ethanol, respectively, and $a_{1}$ and $a_{2}$ denote the corresponding molecular areas that are assumed to be constant. As above, $x_{1}$ and $x_{2}$ are the bulk mole fractions. In Figure 5.1, we show the (room temperature) ethanol adsorption isotherm obtained in this manner by relying on the $\Gamma_{2(1)}$ data obtained from Butler and Wightman, ${ }^{5}$ and putting water molecular area $a_{1}$ equal to $10 \AA^{2}$ and ethanol molecular area $a_{2}$ equal to $24 \AA^{2}$ that are believed to be reasonable estimates of the molecular areas. ${ }^{6}$ This isotherm exhibits, however, the unexpected features of a maximum at $x_{2}=0.13$ and a faint minimum at $x_{2}=0.40$, indicating a rather complex, non-ideal interfacial behavior which the simplistic ansatz expressed by Equation 5.1 (in effect determining the surface phase considered) is unable to cover in a proper manner. Nevertheless, there is no question about the existence of a transition occurring between about $x_{2}=0.1$ and $x_{2}=0.3$, from a laterally mixed to a layer-wise arranged, alcohol-rich interface.

By the same token, the solubility of e.g., oxygen in water-ethanol mixtures, studied long ago by Shchukarev and Tolmacheva, ${ }^{7}$ was found to depend strongly on the structural features of water for mole fractions less than $x_{2} \approx 0.2$, whereas for higher mole fractions, the 
formation of clusters of aggregated ethyl groups appear to be decisive for the oxygen solubility. This is in line with more recent IR, x-ray and neutron diffraction results. ${ }^{8,9}$

From sum-frequency vibration spectroscopy (SFVS), studies of water-alcohol (methanol ${ }^{10}$ and ethanol ${ }^{11,12}$ ) mixtures, it has been clarified that in the low concentration range, the main event occurring is that dangling -OH groups (about one per $30 \AA^{2}$ ) which belong to the top layer of water molecules, successively become replaced by methyl (watermethanol) or ethyl (water-ethanol) groups. At high alcohol mole fractions, a hydrogenbonded bilayer of alcohol molecules is present as the uppermost layer which includes about twice as many alkyl groups as the number of dangling -OH groups originally present in the case of a pure water surface. The purpose of our investigation, to be presented below, was to investigate to what extent the structural changes occurring for alcohol-water mixtures are reflected in the surface forces operating between hydrophobic solid surfaces submerged in such mixtures. To this end, we have employed an AFM set-up and made use of the colloidal probe technique. $^{13,14}$

\section{$\underline{5.3 \text { Methods and Materials }}$}

\subsubsection{Surface Force Measurements by Means of AFM}

Surface force measurements were conducted at room temperature $\left(22 \pm 1^{\circ} \mathrm{C}\right)$ using a Digital Instruments Nanoscope III (Veeco Instruments, Inc., Santa Barbara, CA) atomic force microscope (AFM). The AFM instrument was equipped with a contact mode fluid cell and a scanner "E". All AFM force measurements were carried out in a manner described earlier by Zhang and Yoon. ${ }^{14}$ Triangular silicon nitride $\left(\mathrm{Si}_{3} \mathrm{~N}_{4}\right)$ cantilevers (Model: NP-20, Veeco probes) were used for force measurement. The spring constant was calibrated according to the Cleveland method. ${ }^{15}$ The force measurements were carried out without delay after thiol monolayers had been generated on the gold substrates.

\subsubsection{Reagents}

A Nanopure III (Barnstead, IA) water purification system was employed to obtain double-distilled, deionized water with a resistivity of $18.2 \mathrm{M} \Omega / \mathrm{cm}$ at $25^{\circ} \mathrm{C}$. To remove the particulates in water, a submicron postfilter $(0.2 \mu \mathrm{m}$ in pore size, Fisher Scientific) was integrated with the water purification unit. 1-butanethiol $\left(\mathrm{C}_{4} \mathrm{SH}, 97 \%\right.$, TCI America), 1 dodecanethiol $\left(\mathrm{C}_{12} \mathrm{SH}, 98 \%\right.$, Aldrich $)$ and 1-hexadecanethiol $\left(\mathrm{C}_{16} \mathrm{SH}, \geqq 97 \%\right.$, TCI America) were used to hydrophobize the gold substrates. Ethanol was obtained from AAPER alcohol, KY; methanol (99.8\%) from Fluka; and 1-butanol from Fisher Scientific. Other liquid reagents, such as $\mathrm{H}_{2} \mathrm{SO}_{4}\left(98 \%\right.$, VMR International) and $\mathrm{H}_{2} \mathrm{O}_{2}$ (29.0-32.0\%, Alfa Aesar), were used to clean the gold plates. All reagents listed were used as received.

\subsubsection{Gold Plates}

The surface force measurements were carried out using gold micro-spheres and goldcoated glass slides. Gold-coated glass slides were obtained by depositing gold onto glass using a vacuum evaporator. A $50 \AA$ chromium layer was first deposited, followed by coating 
a $500 \AA$ thick layer of gold. The chromium layer was necessary to ensure a strong adhesive bonding between gold and glass.

\subsubsection{Gold Probes}

Gold spheres with suitable diameters were produced following the procedure devised by Raiteri et al. ${ }^{16}$ A gold wire $(0.0127 \mathrm{~mm}$ dia, $99.9 \%$, Alfa Aesar) was connected to a power supply $(120 \mathrm{~V}, \mathrm{AC})$, briefly creating a short circuit. This was done in a glass tray. A small aerosol cloud of gold particles was produced in the spark. In this way, gold spheres with a wide size distribution in the micrometer range were produced. Spheres with diameters 10 20 $\mu \mathrm{m}$ were chosen for the experiments. In each experiment, a gold sphere was glued onto a cantilever with EPON 1004 resin (Shell Chemical Co) using a homemade three-dimensional micromanipulator under an Olympus BH-2 light microscope.

\subsubsection{Preparation of Hydrophobic Surfaces}

To generate high-quality self-assembled thiol monolayer coatings, the gold substrates were thoroughly cleaned prior to hydrophobizing them by thiol coating. ${ }^{17,} 18$ There are several cleaning procedures available for gold that are based on "piranha" solution, ${ }^{19-21}$ chromic acid ${ }^{22,23}$ or UV/ozone treatment. ${ }^{18,24}$ For the present investigation, a flat gold plate was first cleaned by immersion into a boiling "piranha solution" $\left(30: 70 \mathrm{H}_{2} \mathrm{O}_{2} / \mathrm{H}_{2} \mathrm{SO}_{4}\right)$ for 20 minutes. (WARNING: piranha solution reacts violently with organic matter, especially when hot, and is extremely corrosive). The surface was then flushed with nano-pure water for 1 minute to remove the residual acid, rinsed in pure ethanol for 2 minutes, and blow-dried with $\mathrm{N}_{2}$ gas. The cleaned gold substrates were immediately contacted by a thiol solution.

A hot $\mathrm{H}_{2} \mathrm{O}_{2}-\mathrm{H}_{2} \mathrm{SO}_{4}$ mixture is an extremely strong oxidizing agent. It is not only efficient in removing organic contaminants but also tends to oxidize the gold surface itself. ${ }^{17}$ The cleaning procedure results in a surface with zero water contact angle, which is due to formation of gold oxide $\left(\mathrm{Au}_{2} \mathrm{O}_{3}\right)$. The gold oxide is thermodynamically unstable in the ambient and tends to decompose. It has been reported that ethanol reduces $\mathrm{Au}_{2} \mathrm{O}_{3}$ to gold. ${ }^{17}$,

${ }^{18}$ In the present work, a gold plate rinsed in ethanol for 2 minutes gave a contact angle of $65^{\circ}$, indicating that the solvent washing substantially removed the oxidation product.

As for cleaning the gold sphere, a given sphere was glued onto an AFM cantilever and then cleaned by UV irradiation rather than being cleaned in the corrosive piranha solution. This was adopted to avoid the possibility of destroying the glue holding the sphere onto the cantilever. Typically, a gold sphere was flushed with ethanol, illuminated by a UV light $(254 \mathrm{~nm})$ for 2 hours, and then rinsed again with ethanol before being contacted in a thiol solution for hydrophobization.

Hydrophobization of the gold surfaces was achieved by immersing the spheres and plates in i) a $1 \times 10^{-2} \mathrm{mM} \mathrm{C}_{4} \mathrm{SH}$-in-ethanol (absolute) solution for 5 hours, ii) a $1 \times 10^{-2} \mathrm{mM}$ $\mathrm{C}_{12} \mathrm{SH}$-in-ethanol solution for 2 hours, and iii) a $1 \times 10^{-2} \mathrm{mM} \mathrm{C}_{16} \mathrm{SH}$-in-ethanol solution for 10 minutes. The strong chemical bonding between the - $\mathrm{SH}$ group and the gold surface coupled with assembly causes the thiols adsorb spontaneously from solution. ${ }^{19}$ The adsorption rate depends on critically the thiol concentration. ${ }^{25}$ Dilution to $1 \times 10^{-2} \mathrm{mM}$ or less can be applied to form smooth monolayers, given sufficient time for the adsorption to reach a completion. ${ }^{19}$ After the hydrophobization, the gold substrates were washed with ethanol and 
dried under a nitrogen gas stream. In a given force measurement, we used the same immersion time for gold plate and sphere. It was very important that the gold sphere was glued onto the cantilever prior to the hydrophobization procedure due to the poor adhesion between the glue and a hydrophobic gold sphere.

\section{$\underline{5.4 \text { Results and Discussion }}$}

Figure 5.2 shows the surface force curves recorded for the $\mathrm{C}_{4} \mathrm{SH}$-coated gold surfaces at room temperature in pure water, methanol, ethanol and 1-butanol, respectively. As shown, the strongest attraction was observed in pure water, but long-range attractions were also observed in ethanol and 1-butanol. With methanol, however, the attraction was almost as weak as the non-retarded van der Waals force. A commonality of these liquids is that all of them are H-bonding liquids.

Boinovich and Emelyanenko ${ }^{26}$ conducted the FTIR studies for the thin films of ethanol, butanol and pentanol sandwiched between fluorite $\left(\mathrm{CaF}_{2}\right)$ surfaces, and observed changes in the stretching vibrations of the - $\mathrm{OH}$ and $-\mathrm{CH}$ groups at thicknesses below about 5 $\mathrm{nm}$. This finding suggests that the structure of these H-bonded liquids in the vicinity of the solids is different from that of the bulk liquid. Although fluorite is hydrophilic, structural changes may also be expected when an H-bonded liquid is confined between hydrophobic surfaces such as thiol-coated gold. In this regard, the results presented in Figure 5.3 suggest that long-range attractions originate from increased structuring of liquids in the vicinity of hydrophobic surfaces.

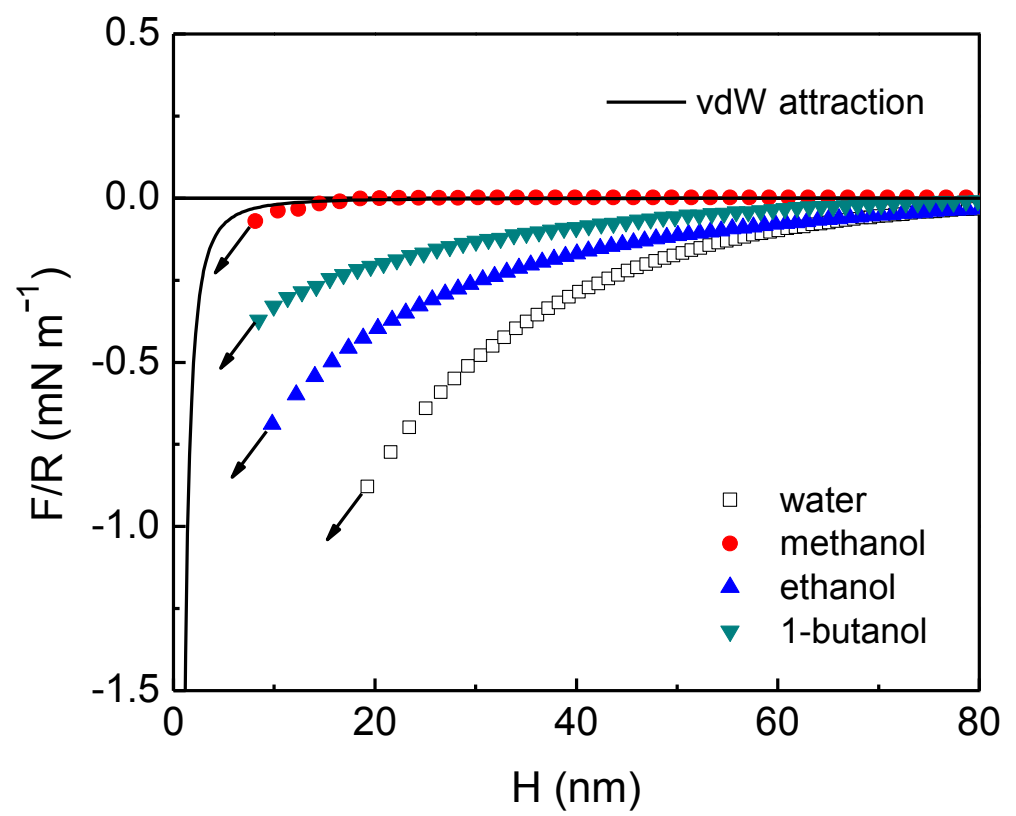

Figure 5.2. Surface force curves obtained for pure water, methanol, ethanol and 1-butanol at room temperature $\left(22 \pm 1{ }^{\circ} \mathrm{C}\right)$ using $\mathrm{C}_{4} \mathrm{SH}$-coated gold surfaces, which were prepared by immersing gold surfaces in a $1 \times 10^{-2} \mathrm{mM} \mathrm{C}_{4} \mathrm{SH}$-in-ethanol (absolute) solution for 5 hours. 


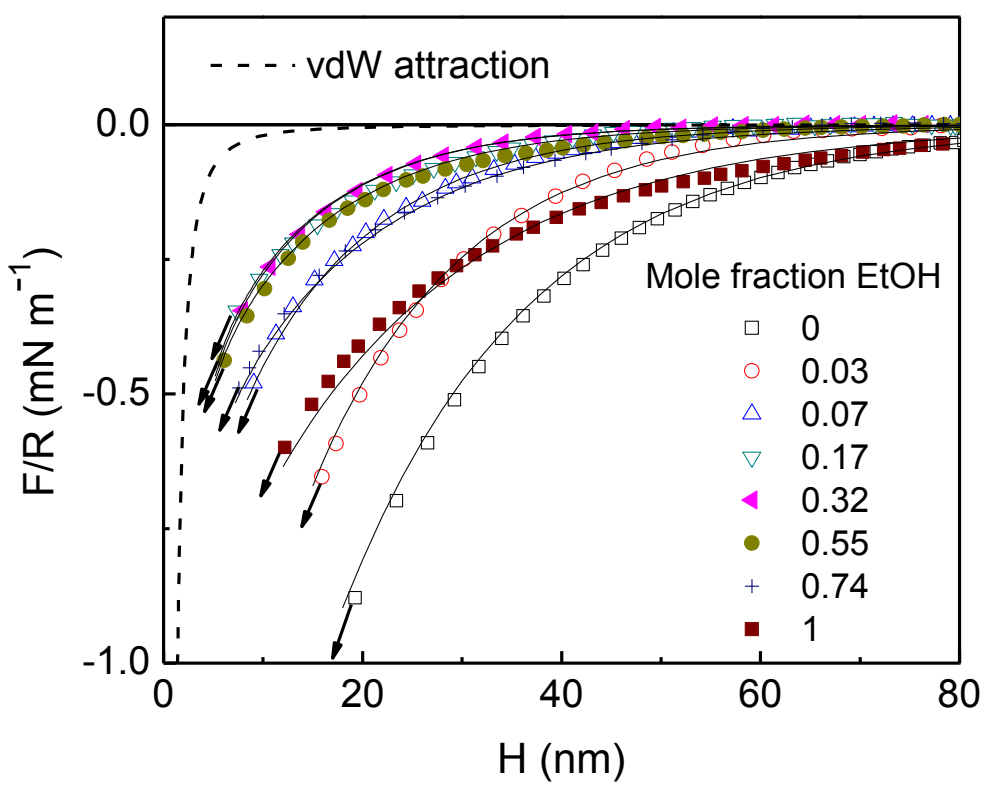

Figure 5.3. Surface force curves obtained at room temperature $\left(22 \pm 1{ }^{\circ} \mathrm{C}\right)$ for waterethanol mixtures using $\mathrm{C}_{4} \mathrm{SH}$-coated gold surfaces, which were prepared by immersing gold surfaces in a $1 \times 10^{-2} \mathrm{mM} \mathrm{C}_{4} \mathrm{SH}$-in-ethanol (absolute) solution for 5 hours.

Figure 5.3 shows the results of the surface force measurements conducted with $\mathrm{C}_{4} \mathrm{SH}$ coated gold substrates in varying concentrations of ethanol solutions. The water contact angle of the thiol-coated gold was $95^{\circ}$. As shown, the long-range attraction was the strongest in pure water, and diminished with increasing ethanol concentrations. It reached a minimum at mole fractions in the range of $0.17-0.55$, and began to increase as the ethanol concentration was further increased. A strong long-range attraction was observed in pure ethanol, but it was still weaker than in pure water.

Figure 5.4 shows a similar set of measurements conducted with $\mathrm{C}_{12} \mathrm{SH}$-coated gold substrates in varying mole fractions of ethanol. The water contact angle of the thiolated gold was $105^{\circ}$. The trend was the same as obtained with the $\mathrm{C}_{4} \mathrm{SH}$-coated gold in that the longrange attraction decreased with increasing ethanol concentration reaching a minimum at mole fractions of $0.17-0.32$, which was narrower in range than with the $\mathrm{C}_{4} \mathrm{SH}$-coated gold substrates. Also, the long-range attraction observed in pure ethanol was about the same as with pure water. 


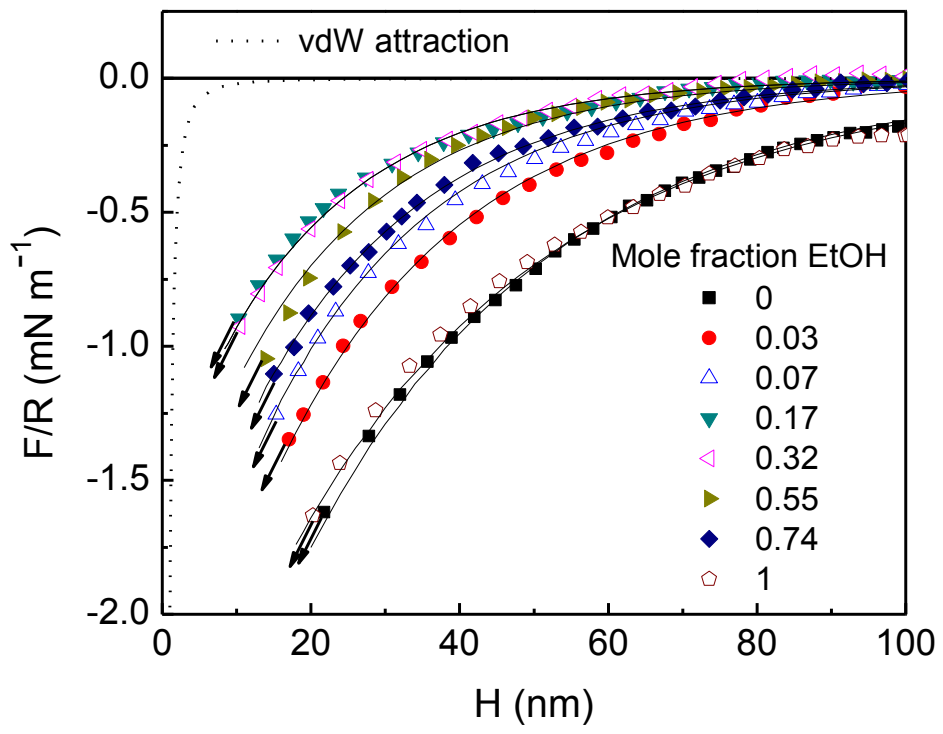

Figure 5.4. Surface force curves obtained at room temperature $\left(22 \pm 1{ }^{\circ} \mathrm{C}\right)$ for water-ethanol mixtures using $\mathrm{C}_{12} \mathrm{SH}$-coated gold surfaces, which were prepared by immersing gold surfaces in a $1 \times 10^{-2} \mathrm{mM} \mathrm{C}_{12} \mathrm{SH}$-in-ethanol (absolute) solution for 2 hours.

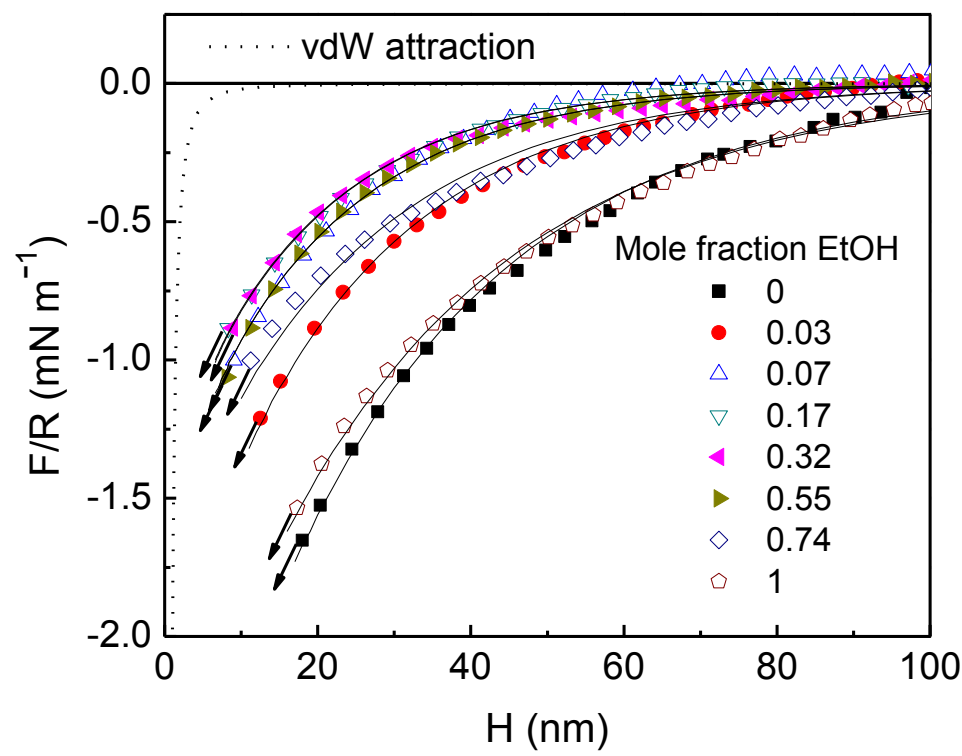

Figure 5.5. Surface force curves obtained at room temperature $\left(22 \pm 1{ }^{\circ} \mathrm{C}\right)$ for water-ethanol mixtures using $\mathrm{C}_{16} \mathrm{SH}$-coated gold surfaces, which were prepared by immersing gold surfaces in a $1 \times 10^{-2} \mathrm{mM} \mathrm{C}_{16} \mathrm{SH}$-in-ethanol (absolute) solution for 10 minutes. 


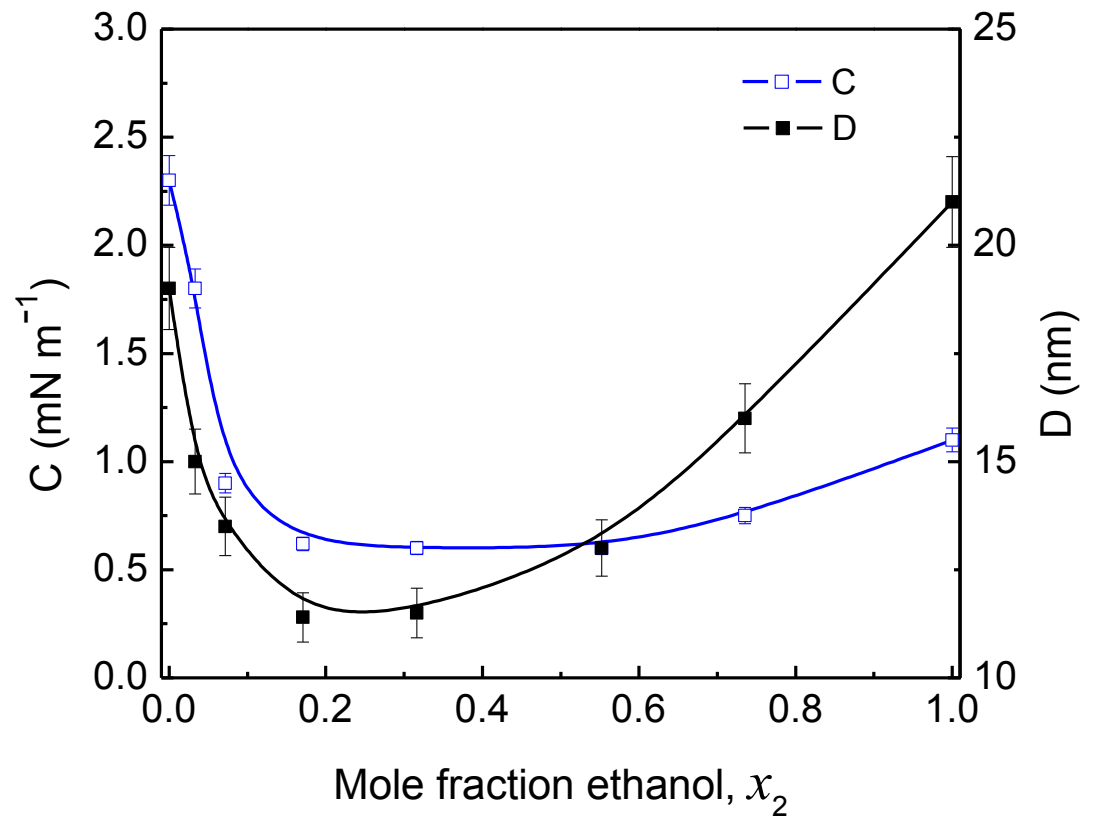

Figure 5.6. The parameters $C$ and $D$ in Equation 5.3 plotted versus the mole fraction of ethanol $\left(x_{2}\right)$ for the case of $\mathrm{C}_{4} \mathrm{SH}$-coated gold surfaces.

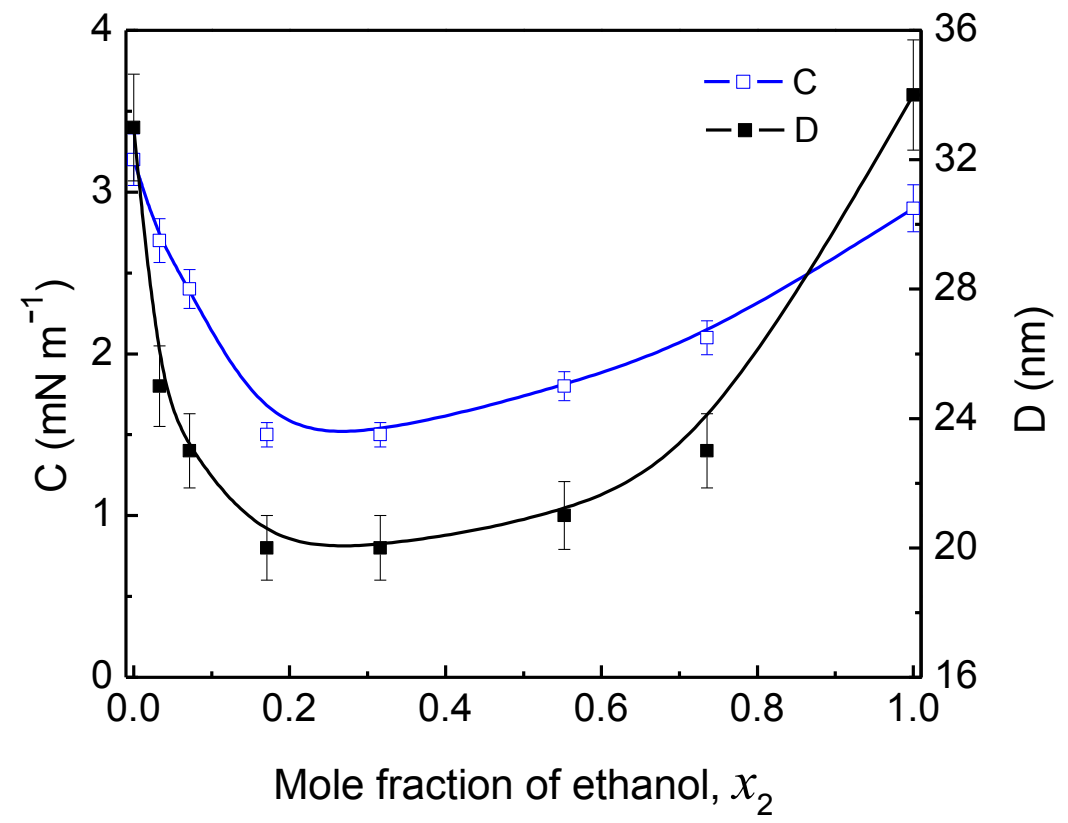

Figure 5.7. The parameters $C$ and $D$ in Equation 5.3 plotted versus the mole fraction of ethanol $\left(x_{2}\right)$ for the case of $\mathrm{C}_{12} \mathrm{SH}$-coated gold surfaces. 
A similar set of experiments was also conducted with $\mathrm{C}_{16} \mathrm{SH}$-coated gold substrates, and the results are presented in Figure 5.5. The weakest attractions were obtained in the mole fractions of $0.17-0.55$, and the result obtained in pure ethanol was about the same as in pure water.

The attractive surface forces for ethanol-water mixtures were first seen by Ederth et $a l .^{27}$ who, however, made just a couple of runs on solutions of ethanol in water (12.5 and $20 \%$ by weight). The forces he recorded are about an order of magnitude weaker than those we have obtained. However, the trend toward a rapidly diminishing surface force as a result of ethanol addition was also evident in his measurements.

It is noteworthy that all the surface forces recorded were purely attractive, and the force curves were all smooth without kinks or steps. Thus, these results do not support the charged-patch ${ }^{28}$ or bridging nano-bubble ${ }^{29}$ mechanism.

The results presented in Figures 5.3-5.6 have been fitted to single-exponential force law:

$$
\frac{F}{R}=C \exp \left(-\frac{H}{D}\right)
$$

where $F$ denotes the measured surface force, $R$ the radius of the gold sphere, $C$ a preexponential constant, and $D$ is the decay length. The $C$ and $D$ parameters obtained from the curve-fitting exercise are plotted in Figures 5.6-5.8 versus the ethanol mole fraction $\left(x_{2}\right)$. In all three cases, both $C$ and $D$ parameters pass through minima at $x_{2}=0.2$. As discussed above, earlier investigations showed that a transition from a water-structure related to an ethanol-cluster related behavior takes place at this mole fraction ${ }^{2,3,7}$. At the same time, the top layer of ethanol molecules become more or less fully developed by changing from a truly mixed water-ethanol monolayer to an ethanol surface layer devoid of dangling -OH groups.

The exponential force law obtained (Equation 5.3) from the experimental data obtained with the $\mathrm{C}_{4} \mathrm{SH}$-coated gold (Figure 5.3) were employed to derive the $\Delta \gamma^{\mathrm{f}}$-functions:

$$
F / 2 \pi R=\gamma^{f}-\gamma^{f, \infty}=\Delta \gamma^{f}
$$

where $\gamma^{f}$ represents the tension of the planar film of water with a thickness $H$, and $\gamma^{f, \infty}$ is the same at an infinite separation. Thus, $\Delta \gamma^{f}$ represents the change in film tension (or Gibbs free energy) as two surfaces approach each other from an infinitely large distance to $H$ and can be related to the surface force measured at a given film thickness. Figure 5.9 shows the changes in $\Delta \gamma^{f}$ as a function of the mole fraction of ethanol at different separations, $H$. Invoking the ethanol partial vapor pressure determined by Dobson ${ }^{4}$ (which was critically examined by Butler and Wightman ${ }^{5}$ ), it is then an easy matter to make use of the Gibbs surface tension equation analogue for a thin liquid film, viz.,

$$
\left(\frac{\partial \Delta \gamma^{f}}{\partial \ln p_{2}}\right)_{T, H}=-\Delta \Gamma_{2}^{f, e x}
$$




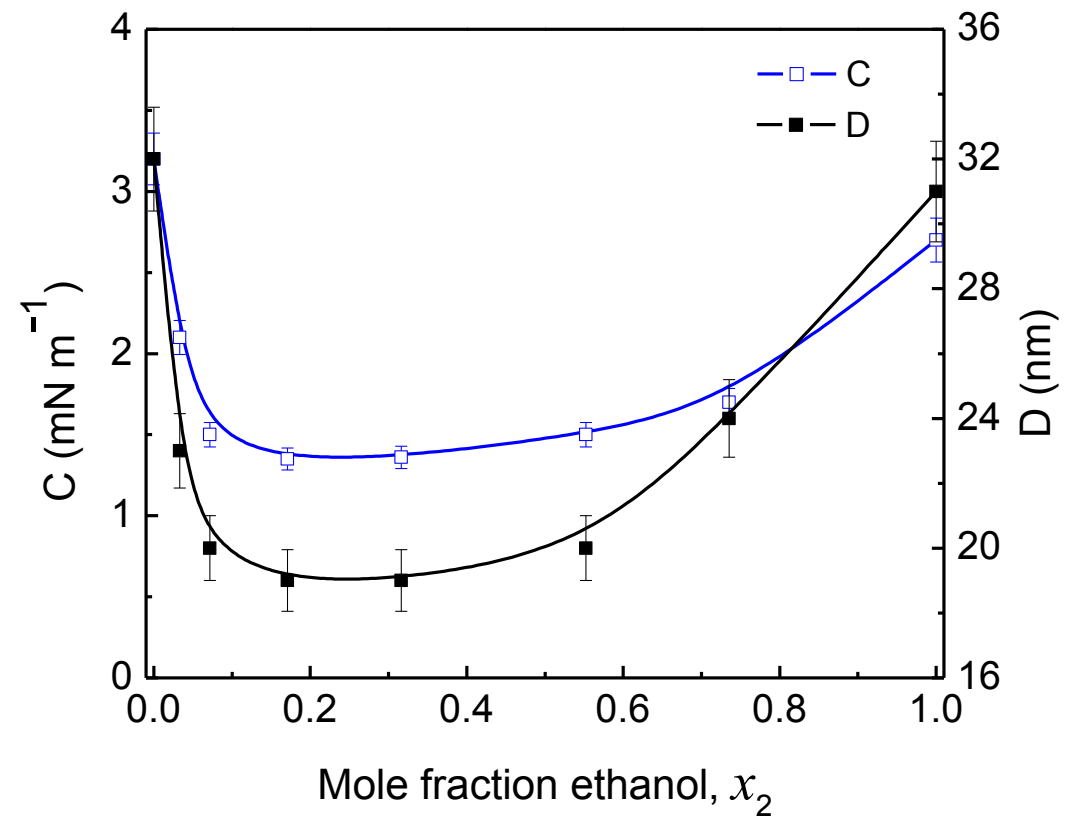

Figure 5.8. The parameters $C$ and $D$ in Equation 5.3 plotted versus the mole fraction of ethanol $\left(x_{2}\right)$ for the case of $\mathrm{C}_{16} \mathrm{SH}$-coated gold surfaces.

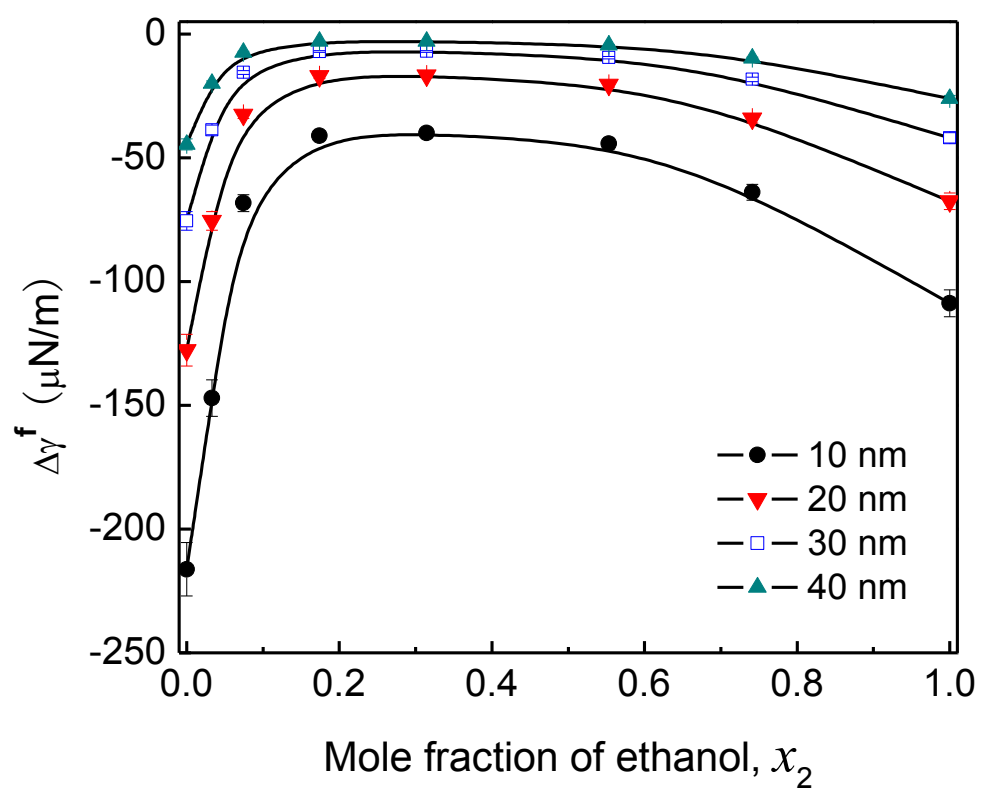

Figure 5.9. The $\Delta \gamma^{\mathrm{f}}$ versus $x_{2}$ functions derived for the film thickness $H=10,20,30$ and $40 \mathrm{~nm}$ by using the surface force data obtained for $\mathrm{C}_{4} \mathrm{SH}$-coated gold surfaces (Figure $5.3)$. 


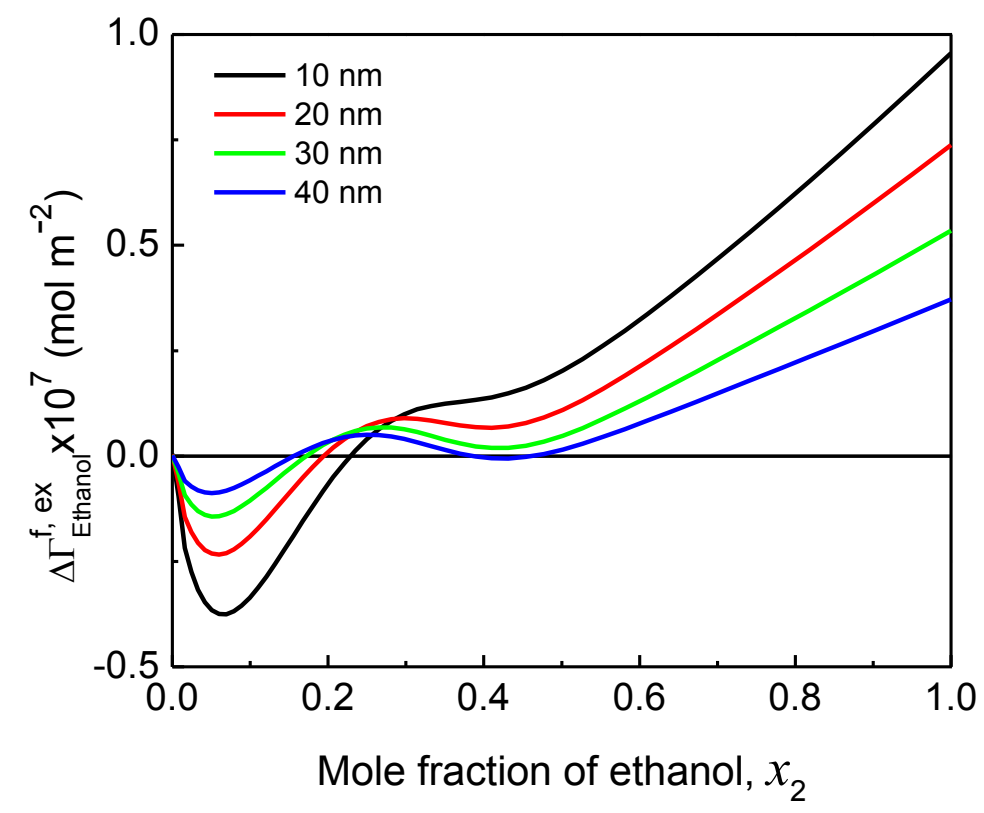

Figure 5.10. The film excess of ethanol, $\Delta \Gamma_{2}^{\mathrm{f}}$,ex , derived from the $\Delta \gamma^{\mathrm{f}}$-functions in Figure 5.9 by applying Equation 5.4.

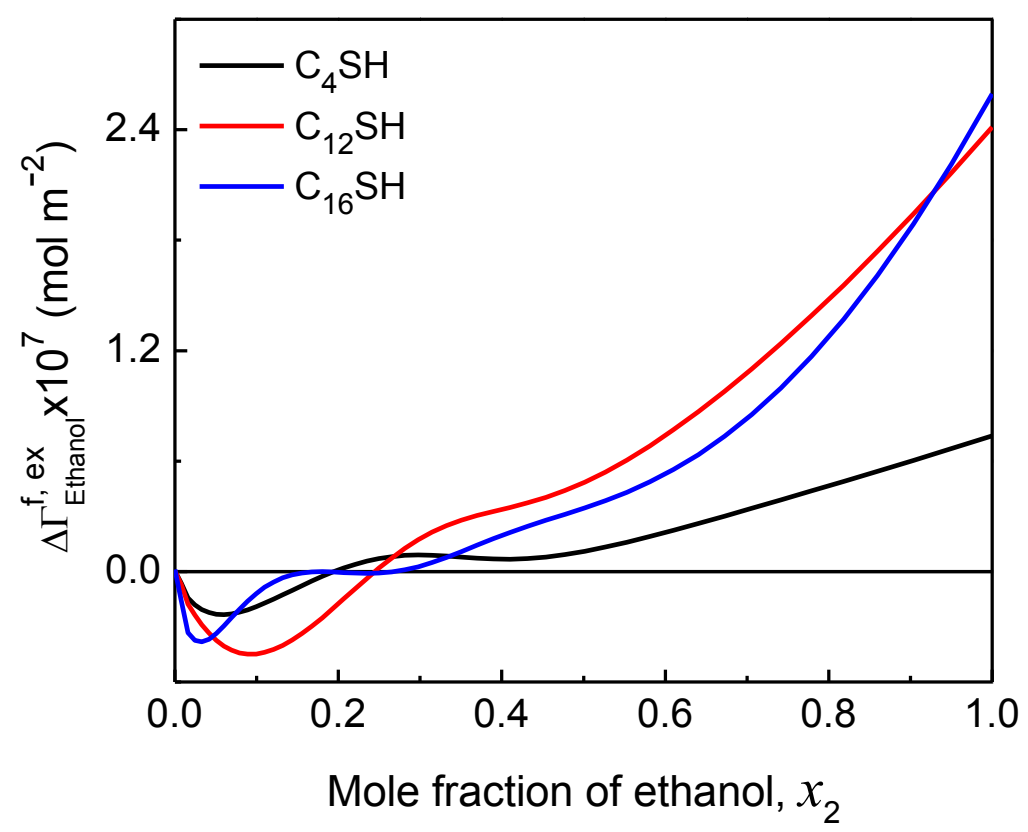

Figure 5.11. The film excess of ethanol, $\Delta \Gamma_{2}^{\text {fex }}$ for $H=20 \mathrm{~nm}$ obtained for $\mathrm{C}_{4} \mathrm{SH}-$, $\mathrm{C}_{12} \mathrm{SH}-$ and $\mathrm{C}_{16} \mathrm{SH}$-coated gold surfaces. 
to derive the changes arising in the thin film of its ethanol content as a result of the attractive interaction between the two hydrophobic surfaces.

The results of such exercises are shown in Figure 5.10. It appears that at low ethanol concentrations in the bulk, the ethanol excess of a thin film is less than for a very thick film. In other words, upon letting the two hydrophobic surfaces approach each other, more ethanol than might be expected on a regular basis, is being expelled from the film. Conversely, for mole fractions approaching unity, upon thinning the water component is preferably being expelled from the film and we are left with a positive excess of ethanol. Recall that diminishing the absolute value of the hydrophobic attraction force means that the film tension increases. As a consequence, according to Equation $5.5, \Delta \Gamma_{2}^{\mathrm{f}}$,ex must be negative.

Figure 5.11 shows the ethanol film excess functions, $\Delta \Gamma_{2}^{\text {f,ex }}\left(x_{2}\right)$ for $H=20 \mathrm{~nm}$ generated by employing the surface force data recorded for $\mathrm{C}_{4} \mathrm{SH}-, \mathrm{C}_{12} \mathrm{SH}-$ and $\mathrm{C}_{16} \mathrm{SH}-$ coated gold surfaces. A semi-quantitative agreement is noted. For all three cases $\Delta \Gamma_{2}{ }^{\text {fex }}$ is negative for low and positive for high ethanol mole fractions.

\section{$\underline{5.5 \text { Model Considerations }}$}

In terms of the recently presented bridging-cluster theory ${ }^{30}$ of hydrophobic attraction that might apply for dilute solutions of alcohol in water, the pre-exponential constant $C$ is given by the expression:

$$
C=\left(\frac{2 \pi k_{B} T}{a_{2}}\right) \times \exp \left(-\frac{2 a_{2} \Delta \gamma_{\text {eff }}}{k_{B} T}\right)
$$

where $a_{2}$ is the (mean) cross-sectional area of a cluster and $\Delta \gamma_{\text {eff }}$ stands for the effective change in interfacial free energy per unit area, arising due to attaching the cluster ends to the hydrophobic surface. Assuming the latter to be a positive quantity, approximately independent of the cluster cross section, one can anticipate $C$ to diminish when the cross section area $a_{2}$ becomes larger. Moreover, the inverse of the decay length $D$ is simply to be regarded as the work per unit length to form the middle part of a long bridging cluster. It should increase with $a_{2}$, and upon raising the ethanol concentration. In this way, we can tentatively account for the rapidly decreasing decay length $D$ upon adding alcohol to water, at least in a preliminary way.

As to the other end of the concentration scale, i.e., $x_{2}$ is high, the ethyl groups and the $\mathrm{H}$-bonded $-\mathrm{OH}$ groups plus water molecules may form alternating layers that are parallel to the hydrophobic surface. The structure may gradually become less and less well ordered with distance away from the surface. In this concentration range, our original order-parameterbased theory ${ }^{31}$ might apply, in which case the decay length would be given by the simple expression:

$$
D=\left(c_{3} / 2 c_{2}\right)^{1 / 2}
$$

where the cooperative constant $c_{3}$ represents a tendency to avoid gradients in the order parameter, $s$, inside the thin film. A comparatively large value of $c_{3}$ means that the layered structure arrangement will prevail for some distance toward the core of the thin film, giving 
rise to an attractive surface force. Adding more water should cause a reduced cooperatively (i.e., a smaller $c_{3}$ ) and increase the free energy of forming an ordered alcohol-water (i.e., a larger $c_{2}$ ), both contributing to decreasing the decay length $D$.

\subsection{Conclusions}

AFM surface force measurements were conducted between hydrophobized gold surfaces in water, $\mathrm{C}-1$ to $\mathrm{C}-4$ alcohols, and ethanol-water mixtures. Long-range attractions were observed in all of these H-bonding liquids, the range and strength of the attractions decreasing in the order of water, ethanol, butanol, and methanol. In the ethanol-water mixtures, the attractions were the maximum when the ethanol mole fraction was zero and unity, and the strength and range of the attractions varied with composition. At the higher mole fractions, the long-range attraction increased, most probably due to the increased structuring of ethanol. Since each ethanol molecule forms two H-bonds, it is likely that layered, laterally homogenous ethanol structures are formed at higher mole fractions. At lower mole fractions, three-dimensional water structure may be formed in view of the fact that each water molecule forms four H-bonds of equal strength.

The results obtained in the present work may thus indicate that the long-range attraction (or hydrophobic force) originates from the liquid structure. The structure of water or ethanol is disturbed in the presence of the other, causing a decrease in the hydrophobic force. That the hydrophobic force increases with decreasing separation between hydrophobic surfaces suggests that the structuring is induced by the hydrophobic surface.

\section{References}

1. $\quad$ Eriksson, J. C., Arkiv Kemi 1966, 26, (2), 49-72.

2. $\quad$ Butler, J. A. V.; Wightman, A., J. Chem. Soc. (London) 1932, 2089-2097.

3. Teitelbaum, B. Y.; Gortalova, T. A.; Sidorova, E. E., Doklady Acad. Nauk S. S. S. R 1951, (25), 911.

4. $\quad$ Dobson, H. J. E., J. Chem. Soc., Trans., 1925, 127, 2866-2873.

5. $\quad$ Butler, J. A. V.; Wightman, A., J. Chem. Soc. 1932, 2089-2097.

6. Li, Z. X.; Lu, J. R.; Styrkas, D. A.; Thomas, R. K.; Rennie, A. R.; Penfold, J., Mol. Phys. 1993, 80, (4), 925-939.

7. Shchukarev, S. A.; Tolmacheva, T. A., ZhurnalStrukt. Khim 1968, 9, 21-28.

8. Nishi, N.; Takahashi, S.; Matsumoto, M.; Tanaka, A.; Muraya, K.; Takamuku, T.; Yamaguchi, T., J. Phys. Chem. 1995, 99, 462-468.

9. Dixit, S.; Crain, J.; Poon, W. C. K.; Finney, J. L.; Soper, A. K., Nature 2002, 416, 829-832.

10. Liu, W.-T.; Zhang, L.; Shen, Y. R., J. Chem. Phys. 2006, 125, 144711-1 -6.

11. Sung, J.; Park, K.; Kim, D., J. Korean. Phys. Soc. 1989, 44, 1394.

12. Sung, J.; Park, K.; Kim, D., J. Am. Chem. Soc 1989, 111, 321-335.

13. Ducker, W. A.; Senden, T. J.; Pashley, R. M., Nature 1991, (353), 239-241.

14. Zhang, J.; Yoon, R.-H.; Mao, M.; Ducker, W. A., Langmuir 2005, 21, 5831-5841.

15. Cleveland, J. P.; Manne, S.; Bocek, D.; Hansma, P. K., Rev. Sci. Instrum. 1993, 64, (2), 403-405. 
16. Raiteri, R.; Preuss, M.; Grattarola, M.; Butt, H.-J., Colloids Surf., A 1998, 136, 191197.

17. Ron, H.; Rubinstein, I., Langmuir 1994, 10, 4566-4573.

18. Ron, H.; Rubinstein, I., J. Am. Chem. Soc. 1998, 120, 13444-13452.

19. Bain, C. D.; Troughton, E. B.; Tao, Y.-T.; Evall, J.; Whitesides, G. M.; Nuzzo, R. G., J. Am. Chem. Soc 1989, 111, 321-335.

20. Kane, V.; Mulvaney, P., Langmuir 1998, 14, 3303-3311.

21. Giesbers, M.; Kleijn, J. M.; Stuart, M. A. C., J. Colloid Interface Sci. 2002, 252, 138148.

22. Hillier, A. C.; Kim, S.; Bard, A. J., J. Phys. Chem. 1996, 100, 18808-18817.

23. Biggs, S.; Mulvaney, P., J. Chem. Phys. 1994, 100, (11), 8501-8505.

24. Sondag-Huethorst, J. A. M.; Fokkink, L. G. J., Langmuir 1992, 8, 2560-2566.

25. Karpovich, D. S.; Blanchard, G. J., Langmuir 1994, 10, 3315-3322.

26. Boinovich, L. B.; Emelyanenko, A. M., Adv. Colloid Interface Sci. 2002, 96, 37-58.

27. Ederth, T., J. Phys. Chem. B 2000, 104, 9704-9712.

28. Miklavic, S. J.; Chan, D. Y. C.; White, L. R.; Healy, T. W., J. Phys. Chem. 1994, 98, 9022-9032.

29. Parker, J. L.; Claesson, P. M.; Attard, P., J. Phys. Chem. 1994, 98, 8468-8480.

30. Eriksson, J. C.; Henriksson, U., Langmuir 2007, 23, 10026-10033.

31. Eriksson, J. C.; Ljunggren, S.; Claesson, P. M., J. Chem. Soc. Faraday Trans. 2 1989, $85,(3), 163-176$. 


\section{TASK 5 HYDROPHOBIC ATTRACTION ORIGINATES FROM CHANGES IN WATER STRUCTURE: THERMODYNAMIC EVIDENCE}

\section{$\underline{6.1 \text { Abstract }}$}

In 1982, Israelachvili and Pashley ${ }^{1}$ reported the first measurements of a hitherto unknown attractive force between two mica surfaces hydrophobized in cetyltrimethylammonium bromide (CTAB) solutions. Follow-up experiments conducted by many investigators confirmed their results, while others suggested that the "hydrophobic force" is an artifact due to nanobubbles or cavities. ${ }^{2-4}$ Evidences for the latter included the discontinuities (or steps) in the force versus distance curves ${ }^{5}$ and the pancake-shaped nanobubbles seen in atomic force microscopic (AFM) images. ${ }^{3}$ Recent measurements ${ }^{6-8}$ conducted in degassed water showed, however, smooth force versus distance curves, indicating that the hydrophobic force is not an artifact due to nanobubbles. In the present work, we have conducted AFM force measurements with gold substrates hydrophobized by self-assembly of alkanethiols with different chain lengths. The measurements carried out at $10-40{ }^{\circ} \mathrm{C}$ show that the hydrophobic force decreases with temperature for all cases. Thermodynamically, these results imply that as two hydrophobic surfaces approach each other the excess film entropy per unit area $\left(\mathrm{S}^{\mathrm{f}}\right)$ decreases, which suggests that the hydrophobic force is due to the structuring of water in the thin film confined by two hydrophobic surfaces rather than from an artifact. Our analysis also shows that the hydrophobic interaction entails a reduction in the excess film enthalpy per unit area $\left(\mathrm{H}^{\mathrm{f}}\right)$, with the change in excess film enthalpy $\left(\Delta \mathrm{H}^{f}\right)$ being slightly more negative than the corresponding entropy term $\left(T \Delta S^{f}\right)$. The difference between these two quantities gives the change in film tension $\left(\Delta \gamma^{\mathrm{f}}\right)$ or in Gibbs (free) energy per unit area. By multiplying this difference with $2 \pi$, in accordance with the Derjaguin approximation, ${ }^{9}$ one obtains the surface force $(F)$ normalized by the radius $(R)$ of the gold sphere used in the force measurement. The nearly parallel enthalpy and entropy changes observed may be associated with the formation of clathrate cages at the hydrophobic surface/water interfaces of the thin water film by virtue of cooperative hydrogen bonding. The presence of the clathrate cages at the hydrocarbonwater interface is supported by the decrease in water density observed by recent neutron reflectivity measurements. ${ }^{10}$ Surface force measurements conducted in ethanol-water mixtures also show that the long-range attractions are due to the structuring of the liquids in the thin liquid films between hydrophobic surfaces.

\section{$\underline{6.2 \text { Introduction }}$}

There have been intense debates in the scientific community as to the existence of a long-range hydrophobic force between hydrophobic solid surfaces in water and its possible origin. After the observations of such a non-DLVO force firstly made by Israelachvili and Pashley ${ }^{1}$ for in situ hydrophobized mica using a surface force apparatus (SFA) in the early 1980s, the scientific community had been divided into different camps. One argues that the long-range hydrophobic force is actually the capillary force in view of the "discontinuities" (or steps) characteristic, attributed to nano-bubble bridging, for the force curves recorded ${ }^{2,11}$, 12. Another one claims that the additional attractive force is related to the structuring of confined water induced by the hydrophobic solid surface. ${ }^{13,14}$ And the third one insists that, 
however, the hydrophobic force is an electrostatic correlation force of charged patches on the hydrophobic surfaces. ${ }^{15,16}$ Addressing the controversy should help us better understand a variety of important issues ranging from self-assembly, protein folding and stability, molecular origin of life, enzyme-coenzyme interactions, recovery of energy minerals (oil, coal, bitumen, kerogen, methane hydrates), etc.

A large part of the debate is due to the difficulty in preparing molecularly smooth and stable hydrophobic surfaces for surface force measurements. For unstable hydrophobic surfaces, indicated by contact angle hysteresis, the long-range attractions measured are usually believed to be ascribed to significant molecular rearrangement, ${ }^{17}$ local charge fluctuations ${ }^{18}$ when two hydrophobic surfaces come into contact in water, or patchy bilayer formation when the surfaces were immersed in water. ${ }^{16}$ Preparation of ideal hydrophobic surfaces is thus of importance to decide whether there is actually a true long-range hydrophobic force arising from thin water film between hydrophobic solid surfaces. Some of the supposedly most reliable surface force data were produced by Claesson and Christenson ${ }^{19}$ and, more recently, by Lin et $a .^{20}$ using Langmuir-Blodgett deposited monolayers of a double chain surfactant dioctadecyldimethylammonium bromide (DODAB) on mica. Unfortunately, these surfaces lack full stability, especially when exposed to salt solutions. More recently, self-assembly of long single-chain thiols on gold from alcohol solution has been used to generate robust hydrophobic surfaces. Ederth et $a l^{21}$ hydrophobized gold substrates in $1 \mathrm{mM} \mathrm{C}_{16} \mathrm{SH}$-in-ethanol solutions and used them for surface force measurements in pure water. However, their force curves exhibited steps, indicating coalescence of pre-existing gas bubbles on the two hydrophobic surfaces during the measurements. Under the conditions employed by these investigators, we obtained essentially the same results. ${ }^{22}$ But it was found that the steps disappeared when the thiolcoated gold surfaces were rinsed with appropriate organic solvents after hydrophobizing them in $1 \mathrm{mM}$ solutions for long periods of time ( $>6$ hours), or when they were hydrophobized in a dilute $\left(1 \times 10^{-2} \mathrm{mM}\right)$ solution for short periods of time $(<10$ minutes $)$. Thus, one has to apply appropriate treatment times and concentrations to ensure that the hydrophobic surfaces generated by chemisorbing an alkanethiol entail little else but full monolayers of thiols firmly bonded by their -SH end groups to the gold substrate. Otherwise, too small surface forces may be measured or nano bubbles will nucleate on surfaces causing steps in the force curves.

In the present work, gold spheres and gold-coated glass plates were hydrophobized using appropriate treating procedure. The gold surfaces, treated with alkanethiols with different chain lengths $(n=2-16)$, were used to measure the surface forces using the AFM colloidal probe technique. ${ }^{23,24}$ A Veeco Multimode AFM equipped with a temperature controller was used to record the forces in pure water at different temperatures.

\section{$\underline{6.3 \text { Materials and Methods }}$}

\subsubsection{Chemicals}

The alkanethiols used to hydrophobize the gold surfaces in this work are $\mathrm{H}-\left(\mathrm{CH}_{2}\right)_{\mathrm{n}^{-}}$$\mathrm{SH}$, denoted briefly as $\mathrm{C}_{\mathrm{n}} \mathrm{SH}$, where $\mathrm{n}=2,4,12,16$. They are all liquid at room temperature. Ethanethiol $\left(\mathrm{C}_{2} \mathrm{SH} ; 98 \%\right)$, 1-butanethiol $\left(\mathrm{C}_{4} \mathrm{SH} ; 97 \%\right)$ and 1-hexadecanethiol $\left(\mathrm{C}_{16} \mathrm{SH} ; \geq\right.$ $97 \%$ ) were purchased from TCI America (Portland, USA). 1-dodecanethiol $\left(\mathrm{C}_{12} \mathrm{SH} ; 98 \%\right)$ 
was obtained from Aldrich. They were stored in the refrigerator and used without further purification. The alkanethiol solutions were prepared in pure ethanol (Decon Laboratories, Inc). $\mathrm{H}_{2} \mathrm{SO}_{4}\left(98 \%\right.$, VMR International) and $\mathrm{H}_{2} \mathrm{O}_{2}(29.0-32.0 \%$, Alfa Aesar) were used as received to clean the gold plates. Ultrapure water $\left(18.2 \mathrm{M} \Omega \cdot \mathrm{cm}^{-1}, 25^{\circ} \mathrm{C}\right)$ was obtained using a Millipore direct-Q3 ultrapure (Millipore, MA) water system.

\subsubsection{Gold Surfaces Preparation}

The flat gold surfaces $(0.5 \times 0.5 \mathrm{sq}$ inch $)$ were produced by coating smooth glass substrates with a thermally evaporated 500 angstrom thick $\mathrm{Au}$ (99.9\%) in a vacuum evaporator. A 50 angstrom $\mathrm{Cr}$ interlayer was used to promote $\mathrm{Au}$ adhesion. The maximum peak-to-valley distance was $3.3 \mathrm{~nm}$ and a typical root mean square (rms) roughness of the gold-coated glass over a surface area of $1 \times 1 \mu \mathrm{m}^{2}$ was $0.8 \mathrm{~nm}$, as measured by AFM.

Gold spheres with suitable size were produced following the procedure devised by Raiteri et al. $^{25}$ Gold wire $(0.0127 \mathrm{~mm}$ dia, 99.9\%), purchased from Alfa Aesar, was connected to a power supply $(120 \mathrm{~V}, \mathrm{AC})$, briefly creating a short circuit. This was done in a glass tray. A small aerosol cloud of gold micro particles was produced in the electric spark. In this way, gold spheres with a wide size distribution in the micrometer range were produced. Spheres with radius of $3 \sim 7 \mu \mathrm{m}$ were chosen for the surface force measurements.

\subsubsection{Colloidal Probe Preparation}

In order to measure the force between surfaces of a gold microsphere and gold-coated flat glass, a gold probe was made by gluing a gold sphere onto to the end of a AFM cantilever with Epon 1004 (Shell Chemical Co) using a homemade 3-dimensional (X, Y, Z) translation stage under a high-resolution optical microscope (BH2, Olympus). The polymer glue which melts at about $105^{\circ} \mathrm{C}$, is insoluble in water. The translation stage is equipped with a hot plate, which sits under the microscope and was used to melt the polymer glue. A clean glass plate with gold microspheres and tiny particles of glue spreading on it was placed on the hot plate. An AFM cantilever, gripped by a clump, was attached to the 3 -dimensional translation stage. The AFM cantilever was cleaned by soaking in pure ethanol and followed by irradiating using a UV lamp (Model ENF-240 C, Spectronics Corporation; $\lambda=254 \mathrm{~nm}$ ) for 10 minutes before use. Firstly, the cantilever approached to a tiny glue drop and the cantilever tip dipped into it. Then the glue-laden tip came close to a desired gold sphere. The radius of sphere $R$ was determined under the microscope. When the sphere was touched by the glue, it spontaneously transfered to the cantilever tip due the capillary force.

\subsubsection{Hydrophobization of Gold}

To obtain high-quality thiol monolayers, the carbonaceous impurities on gold have to be removed first. The gold plates were cleaned by soaking in a boiling piranha etch solution (a mixture of $10 \mathrm{ml} \mathrm{H}_{2} \mathrm{O}_{2}$ and $20 \mathrm{ml} \mathrm{H}_{2} \mathrm{SO}_{4}$ ) for 20 minutes and then washed by rinsing with nanopure water for 1 minute, followed by an ethanol wash for 2 minutes. This was done in a fume hood. (Caution: piranha solution reacts violently with many organic materials and should be used with extreme care). For the cases of gold spheres, they were cleaned after they had been glued onto AFM cantilever springs. To prevent the glue from being destroyed 
by the piranha solution, each gold sphere was flushed with ethanol, exposed in a short-wave UV light $(\lambda=254 \mathrm{~nm})$ for 2 hours, and then rinsed with ethanol again. The high energy UV radiation can decompose and remove the organic compounds on gold.

All glassware such as volumetric flasks and pyrex dishes, used to prepare and contain thiol solutions, were left overnight in sulfuric acid bath and cleaned by rinsing in ultrapure water. Gold surfaces were hydrophobized with alkanethiols by soaking in ethanolic solution of the alkanethiol for a desired period of time. After self-assembly, the surfaces were thoroughly rinsed with pure ethanol and water and then dried in a nitrogen gas stream. For a given force measurement, a gold sphere and a gold-coated glass were soaked in the same solution at the same time, so that the hydrophobicity of the two microscopic surfaces would be the same.

In the present study, gold surfaces were hydrophobized using short and long-chain alkanethiols. The monolayers with different hydrocarbon chain lengths were obtained by placing gold substrates in $1 \mathrm{mM}$ solution of ethanethiol in ethanol for 6 hours, $1 \times 10^{-2} \mathrm{mM}$ ethanolic solution of butanethiol for 5 hours, $1 \times 10^{-2} \mathrm{mM}$ dodecanethiol for 3 hours and in 1 $\times 10^{-2} \mathrm{mM}$ hexadecanethiol for 10 minutes, respectively. Previous contact angle and force measurement studies established that these procedures produce monolayers that can cover the entire gold surface. To ensure the accuracy of the experiment, for every set of experiment, fresh thiol surfactant solutions were used to prepare the hydrophobic coatings, because the ethanolic thiol solutions degrade with time.

\subsubsection{AFM Force Measurement}

Surface force measurements with temperature control were carried out using a Nanoscope IVa (Digital Instruments, Inc.) in Nanoscale Characterization and Fabrication Laboratory (NCFL) at Virginia Tech. The MultiMode AFM was equipped with a heater/cooler accessory, which enables force measurement at both reduced and elevated temperatures. Primary components of the equipment include a heater/cooler Peltier element, a specialized scanner "J", a scanner cooling system which is comprised of a peristaltic pump and an ice bucket, and a Digital Instruments Thermal Applications Controller (TAC) which set and control the liquid temperature by regulating the Peltier element. The Peltier element was plugged into the connector on top of the scanner. The coated flat gold sample was mounted onto a sample puck and placed under the glass fluid cell sealed with a silicone Oring. The metallic sample puck was placed on top of the Peltier element. Triangular silicon nitride cantilevers (NP-20, Veeco Probes, Inc.) bearing the gold microspheres were used for the force measurements. The spring constant $(k)$ were determined using the resonant frequency technique. ${ }^{26}$ The unloaded and loaded resonant frequencies were obtained using Tapping mode AFM. In each experiment, the liquid cell, used to hold the sphere probe, was cleaned in an ultrasonic water bath. Ultrapure water was injected into liquid cell for force measurement using a Norm-Ject syringe. All of the measurements were conducted in airequilibrated solution. The separation distance $(H)$ between the gold sphere and the flat-gold coated glass plate was measured by monitoring the deflection of the cantilever on which the gold sphere was attached. Measured forces $(F)$ were normalized with respect to the radii $(R)$ of the gold spheres.

The initial measurements were conducted in water at $10{ }^{\circ} \mathrm{C}$ and subsequently at 20 , 30 , and $40{ }^{\circ} \mathrm{C}$. It usually takes 15 minutes for water temperature to increase $10{ }^{\circ} \mathrm{C}$. Because 
the reflective index of water changes with temperature, the deflection voltage signal of AFM changes with temperature as well. The stabilization of deflection voltage signal indicates the water temperature reaches the target temperature. After the measurement at $40{ }^{\circ} \mathrm{C}$, the temperature was tentatively brought back to $20{ }^{\circ} \mathrm{C}$ and the experiment repeated to check the reproducibility and the stability of the hydrophobic surfaces at the higher temperatures. The force measurements were reproducible, and the data presented here represent the results of 23 repeat experiments at a given temperature.

The force measurements were also carried out using a Nanoscope III (Digital Instruments, Inc.) atomic force microscope in ethanol-water mixtures at the room temperature $\left(22 \pm 1^{\circ} \mathrm{C}\right)$ in our lab. The MultiMode AFM was equipped with a standard fluid cell and a scanner "E". The water used in the present work was purified using the Millipore Direct Q-3 water purification system. No efforts were made to conduct the measurements in degassed water or ethanol-water mixtures.

\section{$\underline{6.4 \text { Results and Discussion }}$}

\subsubsection{Effect of Temperature}

The interaction forces $(F)$ measured between two hydrophobized gold surfaces as a function of separation distance $(H)$ in air-equilibrated water over a range of temperatures are presented here. Figure 6.1, 6.2, 6.3 and 6.4 shows the force versus distance curves obtained at $10,20,30$ and $40{ }^{\circ} \mathrm{C}$ for gold surfaces coated with $\mathrm{C}_{2} \mathrm{SH}, \mathrm{C}_{4} \mathrm{SH}, \mathrm{C}_{12} \mathrm{SH}$ and $\mathrm{C}_{16} \mathrm{SH}$, respectively. All of the curves are smooth without steps as shown, indicating that the surface forces measured are not due to the coalescence of pre-existing gas bubbles during the measurements. The isotherms recorded can be represented by a single-exponential expression:

$$
F / R=-C \exp (-H / D)
$$

where $\mathrm{F}$ denotes the measured surface force, normalized by the radius of the gold sphere, $R$, and $H$ is the closest separation distance between the gold sphere and plate. The solid lines in Figure 6.1, 6.2, 6.3 and 6.4 represent Equation 6.1 with appropriate $C$ and $D$ values that best fit the experimental data. Figure 6.5 shows the same data as shown in Figure 6.4 for $\mathrm{C}_{16} \mathrm{SH}$ coated gold which was plotted on a log-linear scale. The measured forces can be converted to film tension changes by means of the Derjaguin approximation: ${ }^{9,13}$

$$
F / 2 \pi R=\gamma^{f}-\gamma^{f, \infty}=\Delta \gamma^{f}=\Delta G^{f}
$$

where $\gamma^{\mathrm{f}}$ represents the tension of the planar film of water with a thickness $H$, and $\gamma^{\mathrm{f}, \infty}$ is the same at an infinite separation. Thus, $\Delta \gamma^{\mathrm{f}}$ represents the change in film tension (or Gibbs free energy $\Delta G^{f}$ ) as two surfaces approach each other from an infinitely large distance to a given separation distance $H$. The value of $2 \pi \Delta \gamma^{\mathrm{f}}$ is the surface force actually measured for sphere-plate geometry. 


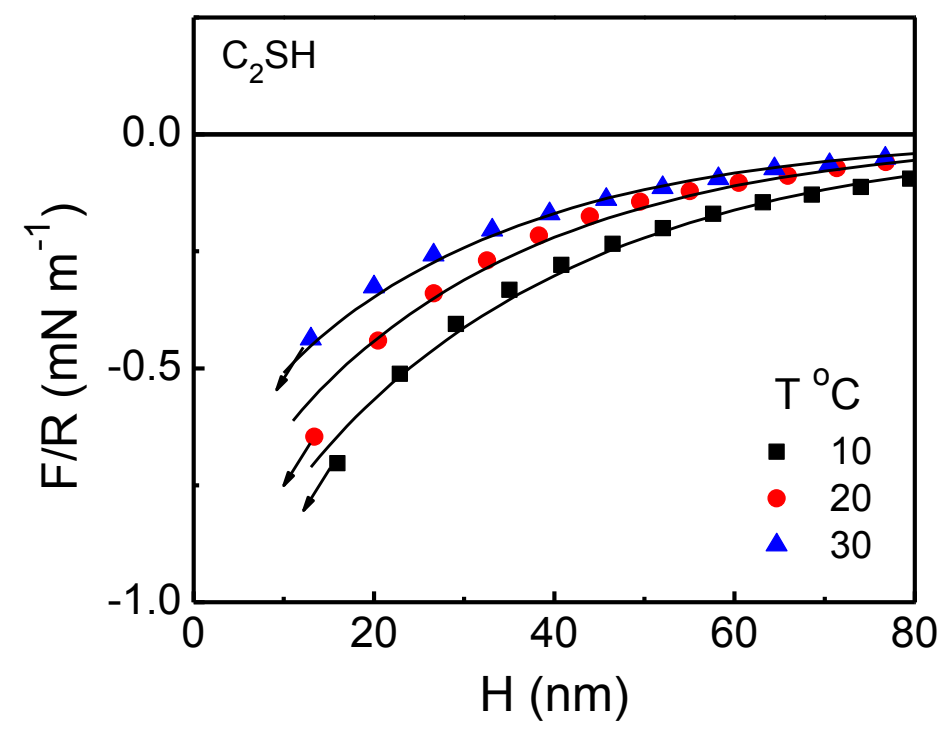

Figure 6.1. The long-range attractive forces between $\mathrm{C}_{2} \mathrm{SH}$-hydrophobized gold sphere and gold-coated glass plates as measured in air-equilibrated water at different temperatures. The solid lines represent fits of the data using a single-exponential force function of Equation 6.1, with $D=32,29,28 \mathrm{~nm}$ and $C=1.05,0.87$ and $0.7 \mathrm{mN} / \mathrm{m}$ at 10 , 20 , and 30 , respectively.

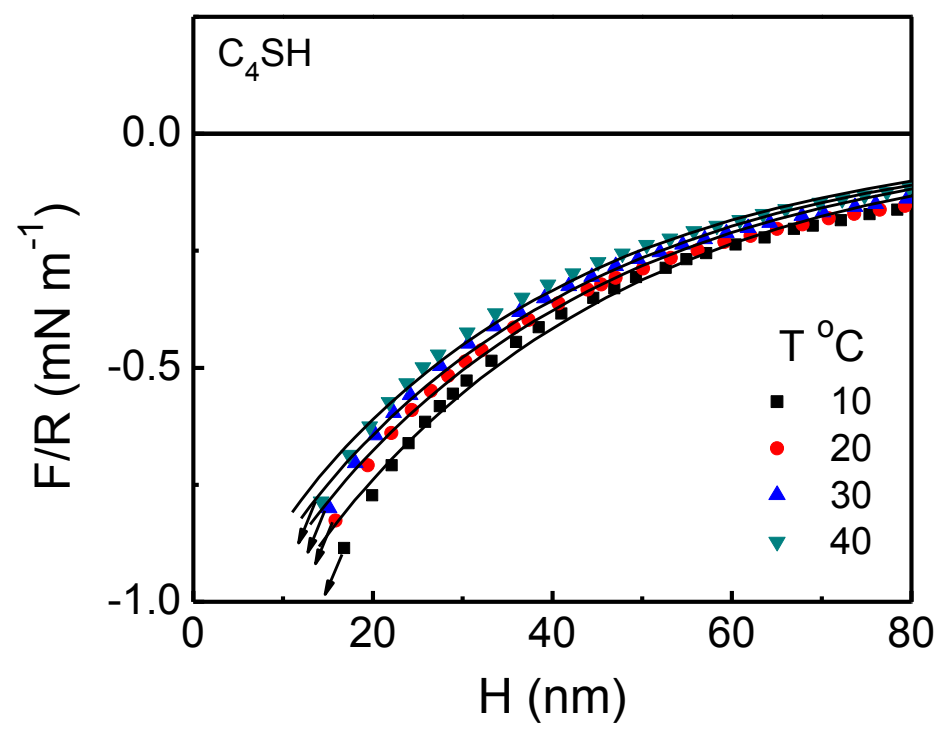

Figure 6.2. The long-range attractive forces between $\mathrm{C}_{4} \mathrm{SH}$-hydrophobized gold sphere and gold-coated glass plates as measured in air-equilibrated water at different temperatures. The solid lines represent fits of the data using a single-exponential force function of Equation 6.1, with $D=35,34.5,34.0,33.5 \mathrm{~nm}$ and $C=1.3,1.2,1.15$, and 1.1 $\mathrm{mN} / \mathrm{m}$ at $10,20,30$, and $40^{\circ} \mathrm{C}$, respectively. 


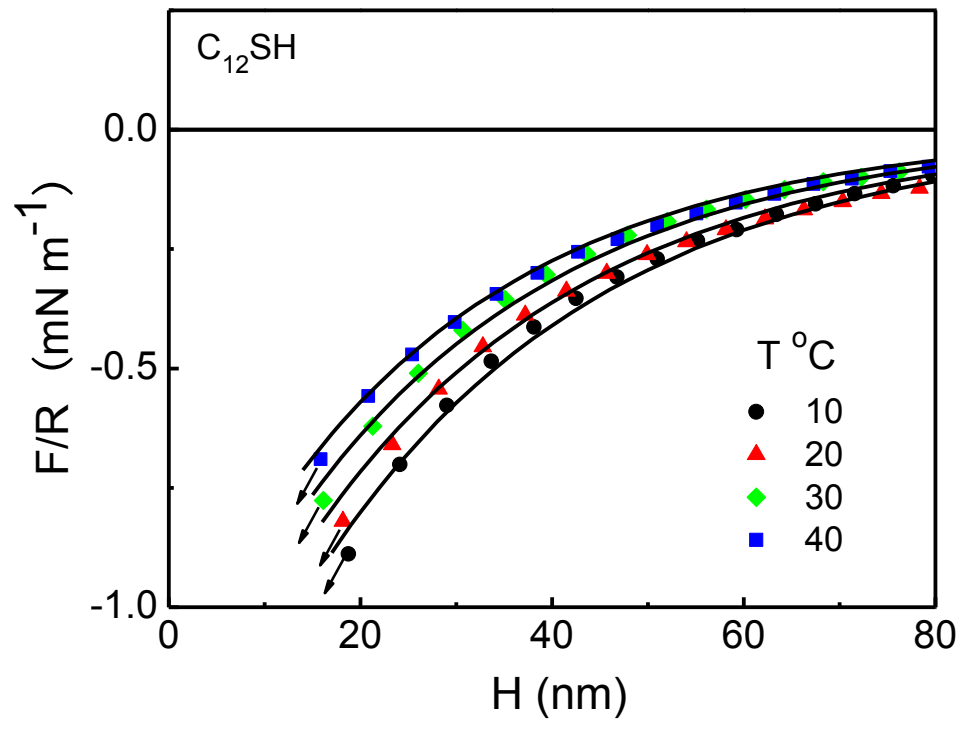

Figure 6.3. The long-range attractive forces between $\mathrm{C}_{12} \mathrm{SH}$-hydrophobized gold sphere and gold-coated glass plates as measured in air-equilibrated water at different temperatures. The solid lines represent fits of the data using a single-exponential force function of Equation 6.1, with $D=30,29.5,28.5,27.5 \mathrm{~nm}$ and $C=1.55,1.4,1.28$, and $1.17 \mathrm{mN} / \mathrm{m}$ at $10,20,30$, and $40^{\circ} \mathrm{C}$, respectively.

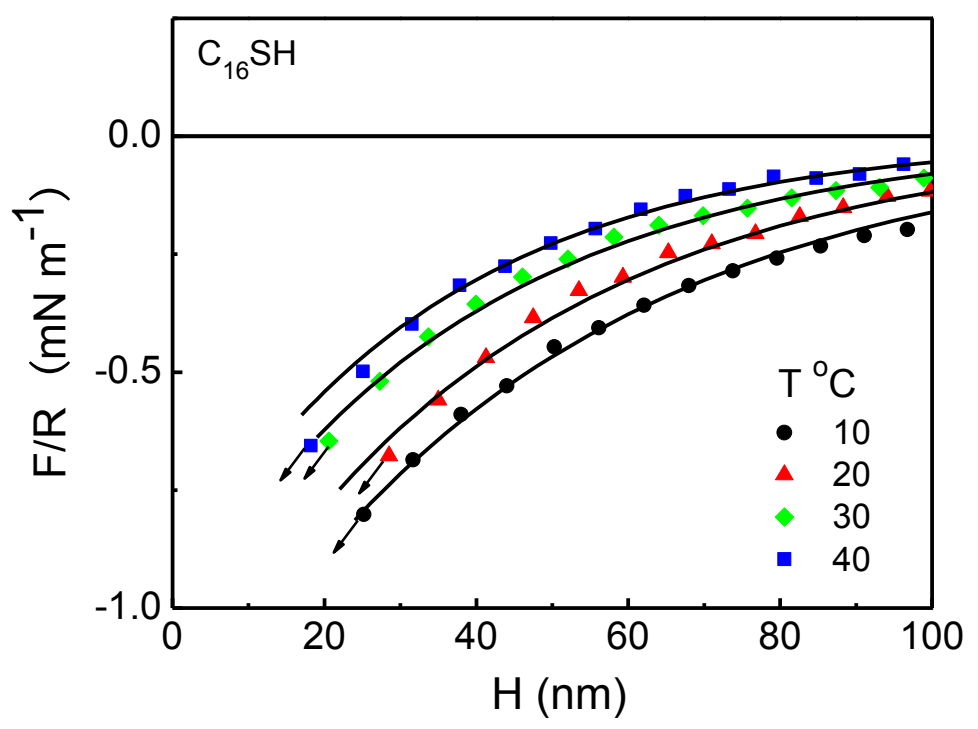

Figure 6.4. The long-range attractive forces between $\mathrm{C}_{16} \mathrm{SH}$-hydrophobized gold sphere and gold-coated glass plates as measured in air-equilibrated water at different temperatures. The solid lines represent fits of the data using a single-exponential force function of Equation 6.1, with $D=47.7,42.4,39.0,35.0 \mathrm{~nm}$ and $C=1.37,1.25,1.03$, and $0.95 \mathrm{mN} / \mathrm{m}$ at $10,20,30$, and $40^{\circ} \mathrm{C}$, respectively. 


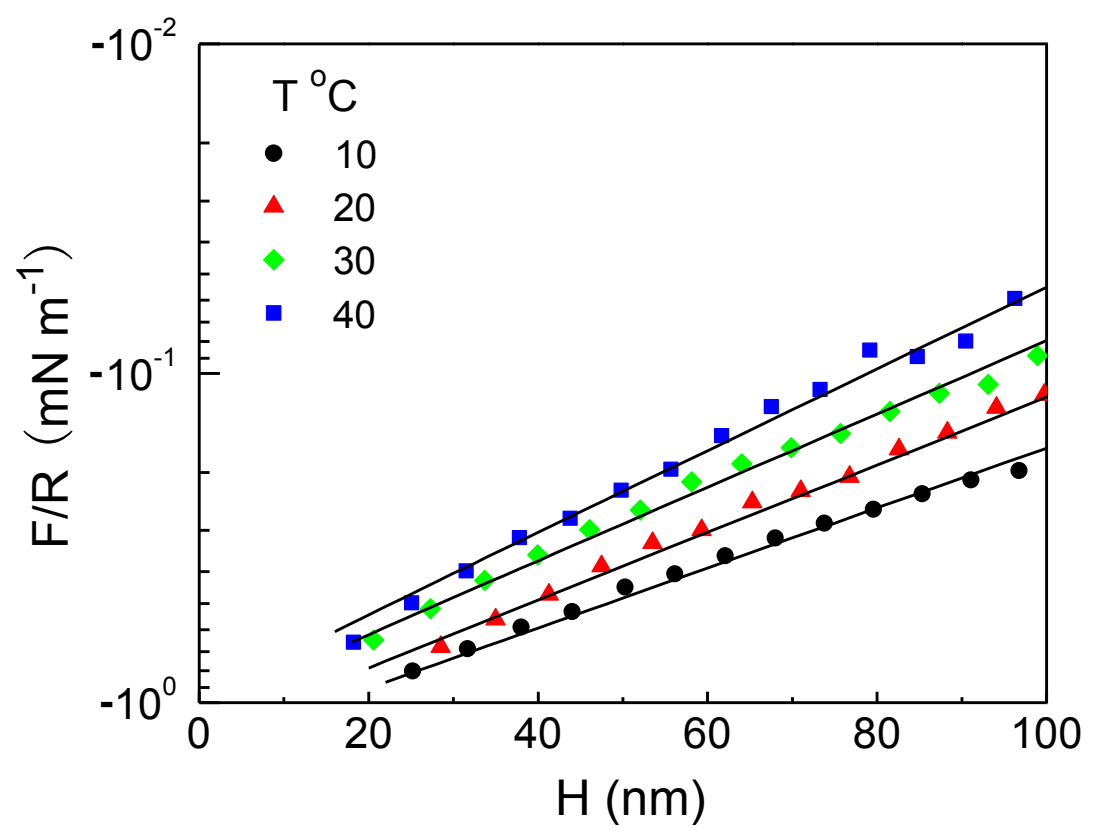

Figure 6.5. Same data as shown in Figure 6.4 for $\mathrm{C}_{16} \mathrm{SH}$-coated gold was plotted on a log-linear scale.

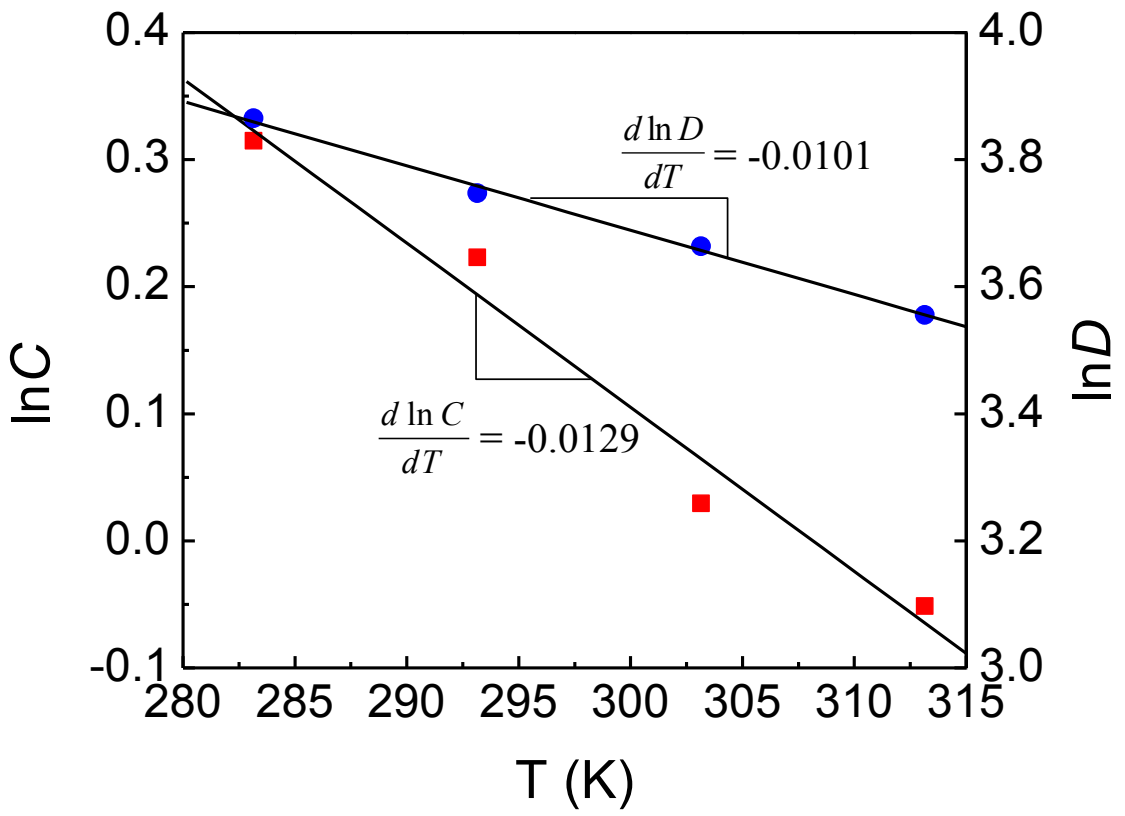

Figure 6.6. $\ln C$ and $\ln D$ obtained for $\mathrm{C}_{16} \mathrm{SH}$-coated gold as functions of absolute temperature. The temperature derivatives of $\ln C$ and $\ln D$ are -0.0129 , and -0.0101 , respectively. 


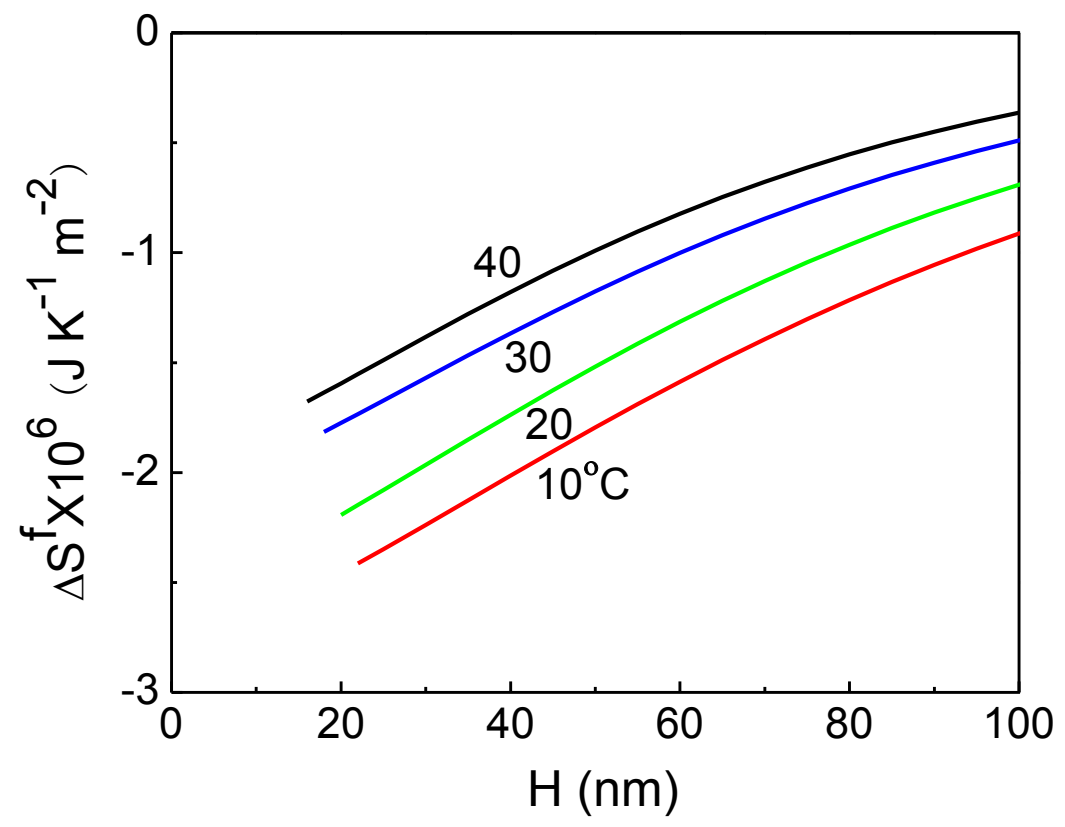

Figure 6.7. The changes in excess film entropy $\left(\Delta \mathrm{S}^{\mathrm{f}}\right)$ per $\mathrm{m}^{2}$ in the thin films of water between two $\mathrm{C}_{16} \mathrm{SH}$-coated gold surfaces as the film thickness $(H)$ decreases, or as the temperature increases.

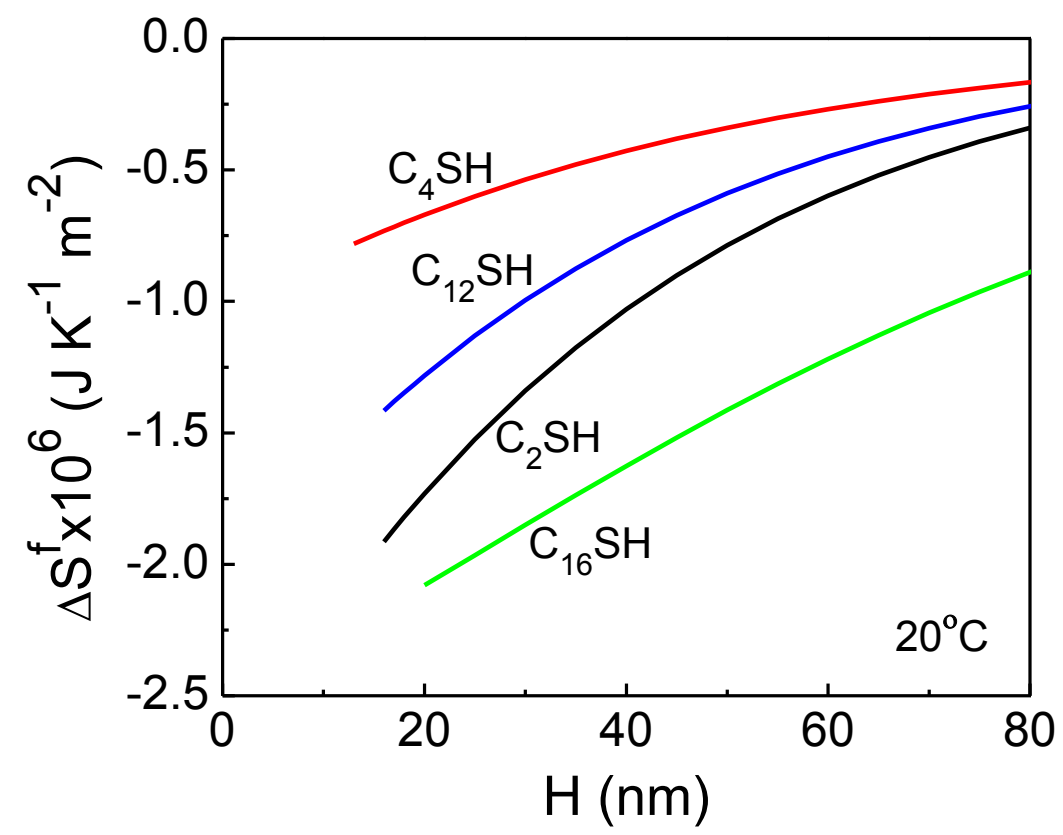

Figure 6.8. Changes in excess film entropy $\left(\Delta \mathrm{S}^{\mathrm{f}}\right)$ per $\mathrm{m}^{2}$ in the thin films of water between two gold surfaces hydrophobized by alkanethiols with different chain lengths at $20^{\circ} \mathrm{C}$. 
From the force versus distance curves recorded for $\mathrm{C}_{2}, \mathrm{C}_{4}, \mathrm{C}_{12}$ and $\mathrm{C}_{16} \mathrm{SH}$-coated gold at different temperature, we find that in all cases the temperature coefficients of the hydrophobic surface force for a fixed surface separation $H$ are positive. In other word, upon raising the temperature, the magnitudes of the hydrophobic attraction diminish. In principle, this temperature effect was first documented by Tsao et $a .^{27}$ on mica surfaces hydrophobized by the adsorption of cationic surfactants of different chain lengths from cyclohexane solutions. From film thermodynamics, we have the following relationship for a planar (pure) water film at constant pressure $P$ and separation distance $H$ :

$$
\left(\frac{\partial \Delta \gamma^{f}}{\partial T}\right)_{p, H}=-\Delta S^{f}
$$

in which $\Delta S^{f}\left(\equiv S^{f}-S^{f, \infty}\right)$ is the change in excess film entropy per unit area, and $T$ is the absolute temperature. From Equations 6.1-6.3, one obtains the following relation:

$$
\Delta \mathrm{S}^{\mathrm{f}}=-\Delta \gamma^{\mathrm{f}}\left(\frac{\mathrm{d} \ln \mathrm{C}}{\mathrm{dT}}+\frac{\mathrm{H}}{\mathrm{D}} \frac{\mathrm{d} \ln \mathrm{D}}{\mathrm{dT}}\right)
$$

which was used to determine the entropy changes from the values of $\Delta \gamma^{f}$ and the temperature coefficients of the $C$ and $D$ parameters. Since $\Delta \gamma^{\mathrm{f}}$ is a negative quantity and the temperature derivatives of $\ln C$ and $\ln D$ are negative (Figure 6.6), the excess entropy $\Delta \mathrm{S}^{\mathrm{f}}$ of a thin water film between thiol-coated gold surfaces is thus a negative quantity. The excess entropy of the film is equal to the entropy per $\mathrm{m}^{2}$ of the actual thin film minus the entropy of a hypothetical thin film per $\mathrm{m}^{2}$ that entails the same number of water molecules per $\mathrm{m}^{2}$ but is lacking face-to face interactions. Accordingly, the real film has somewhat lower entropy than the corresponding hypothetical film.

As shown in Figure 6.7 and 6.8, the change in excess film entropy $\left(\Delta \mathrm{S}^{\mathrm{f}}\right)$ becomes more negative when the thickness of the film $(H)$ confined between two hydrophobic surfaces is reduced. A typical value of $\Delta \mathrm{S}^{\mathrm{f}}$ for a plane-parallel film of thickness $20 \mathrm{~nm}$ between two $\mathrm{C}_{16} \mathrm{SH}$-coated surfaces turns out to be $-0.0022 \mathrm{mJm}^{-2} \mathrm{~K}^{-1}$, which is about $0.008 \%$ of the entropy change associated with the liquid water-to-ice phase transition. Although the effect dealt with here is an exceedingly minute one, the decrease in excess film entropy indicates that the thin film of water becomes increasingly structured as two hydrophobic surfaces approach each other. Note also that at a given film thickness $H, \Delta \mathrm{S}^{\mathrm{f}}$ becomes more negative at lower temperatures, which is akin to the anomalous behavior of supercooled water. As is well known, the density of water decreases below its maximum at $4^{\circ} \mathrm{C}$, which has invoked the presence of various low-density species (e.g., "ice-like" species, ${ }^{28}$ pentagonal dodecahedra, ${ }^{29}$ clathrate cages, ${ }^{30}$ etc.) in pure water. All of these species represent highly ordered structure of water; therefore, their formation in colder water entails entropy decrease. Likewise, the results presented in Figure 6.7 may indicate the formation of lowdensity species at the hydrophobic surface/water interface. Figure 6.8 shows changes in excess film entropy $\left(\Delta S^{f}\right)$ per $\mathrm{m}^{2}$ in the thin films of water as functions of separation distance 
between two gold surfaces hydrophobized by $\mathrm{C}_{2} \mathrm{SH}, \mathrm{C}_{4} \mathrm{SH}, \mathrm{C}_{12} \mathrm{SH}$ and $\mathrm{C}_{16} \mathrm{SH}$ at $20^{\circ} \mathrm{C}$. As the chain length decreases from 16 to 4, the change in excess film entropy decreases. The difference between changes in excess film entropy obtained from different surfaces may due to different states of the hydrophobic carbon chains on the surface (e.g., crystalline order of hydrocarbon chain $)^{27}$. For $\mathrm{C}_{2} \mathrm{SH}$, the obtained change in excess film entropy is comparable to that for $\mathrm{C}_{16} \mathrm{SH}$, and larger than that for $\mathrm{C}_{4} \mathrm{SH}$ and $\mathrm{C}_{12} \mathrm{SH}$, probably due to the experimental uncertainty.

Thermodynamically, the change in film tension $\left(\Delta \gamma^{f}\right)$ is composed of enthalpic $\left(\Delta \mathrm{H}^{\mathrm{f}}\right)$ and entropic $\left(\mathrm{T} \Delta \mathrm{S}^{\mathrm{f}}\right)$ parts. Using Equation 6.5, one can obtain the excess enthalpy per unit area $\left(\Delta \mathrm{H}^{\mathrm{f}}\right)$ for fixed surface separation distance $H$ :

$$
\Delta H^{f}=\Delta \gamma^{f}+T \Delta S^{f}
$$

As shown in Figure 6.9, the slope of a $\Delta \mathrm{H}^{\mathrm{f}}$ versus $\mathrm{T}$ plot at a given separation distance is positive, meaning that the constant-pressure heat capacity $\left(\mathrm{C}_{\mathrm{p}}\right)$ of the thin film between two hydrophobic surfaces is a little larger than that of the corresponding hypothetical water film without film face-face interactions, which is another indication that the film of water is more structured.

Figure 6.10 shows the changes in these thermodynamic functions (i.e., $\Delta \gamma^{f}, \Delta S^{f}$ and $\Delta \mathrm{H}^{\mathrm{f}}$ ) for the interaction between the $\mathrm{C}_{16} \mathrm{SH}$-coated gold surfaces at $20^{\circ} \mathrm{C}$. It is seen that the enthalpic part is a little larger than the entropic part. This finding contradicts the general perception that the hydrophobic interaction is entropic in nature, ${ }^{31}$ that is, the interaction is driven by a positive entropy change, in which case the attractive force at a fixed film

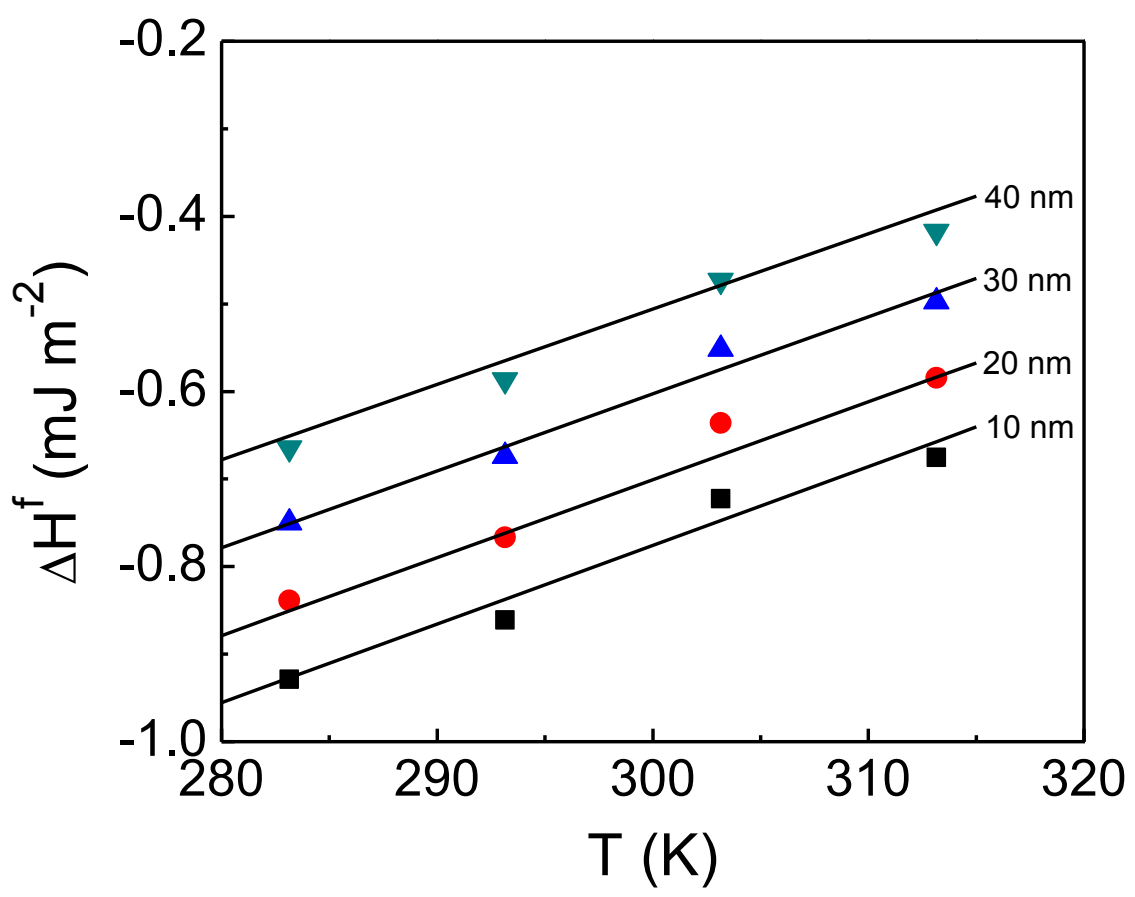

Figure 6.9. A plot of $\Delta \mathrm{H}$ versus $\mathrm{T}$ at surface separation distance of 10, 20, 30 and 40 $\mathrm{nm}$ for $\mathrm{C}_{16} \mathrm{SH}$-coated gold surfaces. 
thickness should increase as temperature rises. However, our results show the opposite; $\Delta \mathrm{S}^{\mathrm{f}}$ is negative and the hydrophobic force decreases with temperature, as shown in Figures 6.1-6.5, 6.7 and 6.8, respectively. Israelachvili and Pashley ${ }^{32}$ and Tsao et al. $^{27}$ also found that the hydrophobic force decreases with temperature. Since both $\Delta \mathrm{H}^{\mathrm{f}}$ and $\Delta \mathrm{S}^{\mathrm{f}}$ are negative, the Gibbs free energy change $\left(\Delta \gamma^{\mathrm{f}}\right)$ becomes negative or an attractive hydrophobic force appears when $\left|\Delta \mathrm{H}^{\mathrm{f}}\right|>\left|\mathrm{T} \Delta \mathrm{S}^{\mathrm{f}}\right|$, as shown in Figure 6.10. Thus, hydrophobic force originates from the thermodynamic properties of water rather than an artifact created by nanobubbles or cavitation ${ }^{2,3}$ The negative enthalpy change may be due to the formation of the low-density species such as clathrate cages ${ }^{30}$ at the hydrocarbon/water interfaces. The negative enthalpy change associated with the clathrate formation exceeds the corresponding entropy cost.

Recent investigations have clarified that the nature of a hydrophobic interaction depends critically on the length scale of the hydrophobic species involved ${ }^{33,34}$. For small hydrophobic solutes, such as noble gases and hydrocarbons (e.g., methane), water molecules can go around the hydrophobic species and form H-bonded network without compromising the number and strengths of the H-bonds. Each water molecule can "straddle" the small hydrophobic species due to its high curvature, ${ }^{30}$ which will allow them to maintain four, highly directional $\mathrm{H}$-bonds. As $\mathrm{H}$-bonding is cooperative, ${ }^{35}$ i.e., a pair of adjacent $\mathrm{H}$-bonds

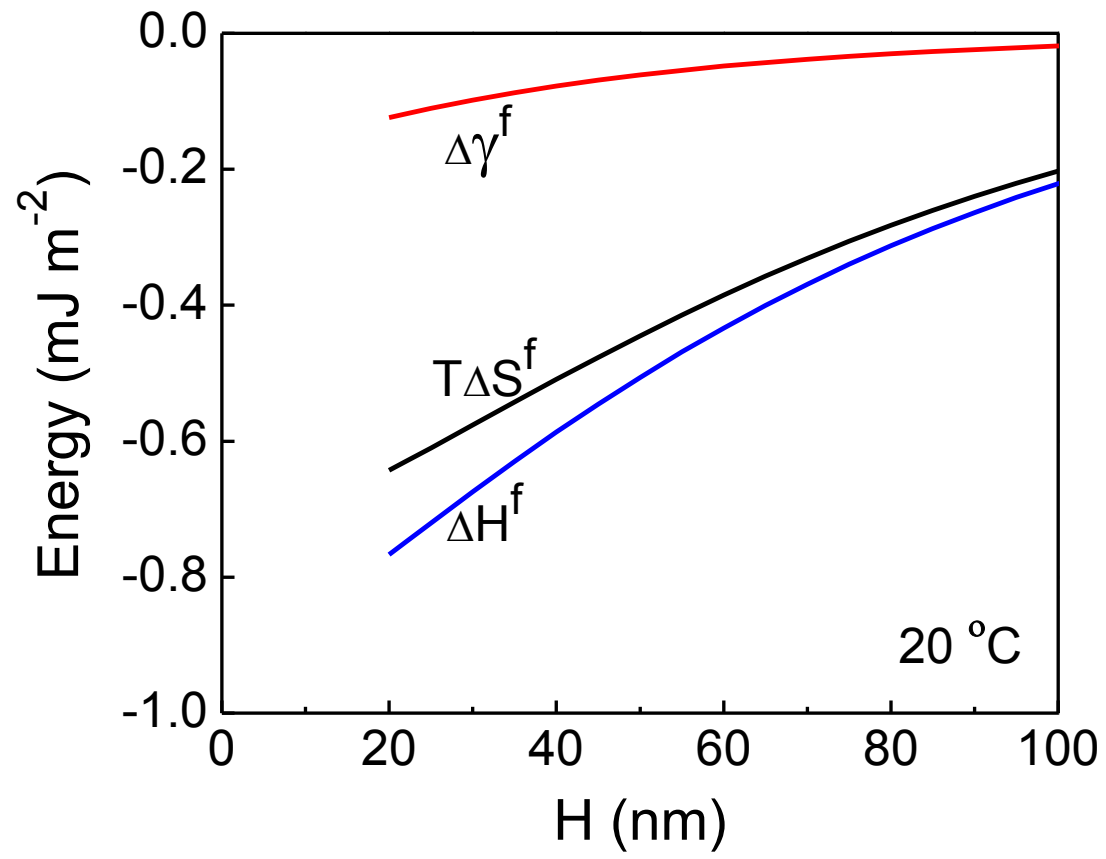

Figure 6.10. Changes in the excess thermodynamic functions for the hydrophobic interaction between $\mathrm{C}_{16} \mathrm{SH}$-coated gold macroscopic surfaces in air-equilibrated water at $20^{\circ} \mathrm{C}$. The enthalpy $\left(\Delta \mathrm{H}^{\mathrm{f}}\right)$ of interaction is slightly more negative than the corresponding entropic term $\left(\mathrm{T} \Delta \mathrm{S}^{\mathrm{f}}\right)$, and the difference between the two represents the change in the Gibbs free energy $\left(\Delta \gamma^{f}\right)$. Since both $\Delta \mathrm{H}^{\mathrm{f}}$ and $\Delta \mathrm{S}^{\mathrm{f}}$ are negative, attractive hydrophobic force is observed only when $\left|\Delta \mathrm{H}^{\mathrm{f}}\right|>\left|\mathrm{T} \Delta \mathrm{S}^{\mathrm{f}}\right|$. 
are more stable than two isolated bonds, the water molecules surrounding a hydrophobic solute can reinforce each other and form a concave clathrate structure with the mean bond energy stronger than that of a simple dimer. ${ }^{30}$ Monte Carlo simulations showed indeed that the mean H-bond length of water around an apolar solute is shorter, while they are longer around a polar group. ${ }^{36}$ Further, the extended x-ray absorption fine structure (EXAFS) spectrum of the solid krypton $(\mathrm{Kr})$ clathrate is the same as that of $\mathrm{Kr}$ in cold water, which confirmed the formation of clathrate (or "iceberg") structures around hydrophobic solutes. 37 The thermodynamic cost of forming the clathrate structure will for the most part be a decrease in entropy. However, the entropy cost is less than the cost of breaking some Hbonds to accommodate a hydrophobic solute in water and not forming the clathrate structure around it.

When a larger hydrophobic species is placed in water, the water molecules in the immediate vicinity will lose some of the H-bonds as a consequence of the low curvature of the extended surface, resulting in a significant enthalpy increase. According to Chandler ${ }^{34}$, the crossover from an entropic to an enthalpic hydration occurs at about $1 \mathrm{~nm}$. For $n$-alkanes of 20 or fewer carbons, entropic hydration is still possible due to the high curvatures of the $\mathrm{CH}_{2}$ and $\mathrm{CH}_{3}$ groups, which serves as the basis for the well-known entropic self-assembly of $n$-alkanes in water at room temperature. ${ }^{31}$

Recognizing the importance of the length scale in hydrophobic interaction, Lum et $a l .{ }^{38}$ suggested that the water molecules in the vicinity of the extended hydrophobic surfaces tend to move away from the surface, leading to drying and large forces of attraction. Many investigators followed this theory and determined the density of the vicinal water by the neutron reflectivity (NR) measurements. The results showed that preexisting nanobubbles are excluded $^{10,39,40}$ and the density of the vicinal water is lower than in the bulk, which has led to a suggestion that hydrophobic force is a "depletion force" 10 caused by the drying effect. The NR studies showed also that the depletion length (D), a measure of density decrease, increases with increasing temperature ${ }^{41,42}$ and electrolyte concentration ${ }^{42}$. Maccarini et al. ${ }^{42}$ suggested that these results corroborate with the AFM surface force measurements reported in the literature. ${ }^{43}$ The results presented in Figure 6.1-6.5 show, on the contrary, that hydrophobic forces decrease with increasing temperature. Moreover, it is well documented that hydrophobic forces decrease with increasing electrolyte concentration ${ }^{7,44}$. In addition, high-resolution in situ x-ray study shows that the hydrophobic water gas was not affected by dissolving gases ${ }^{39}$ (e.g., $\mathrm{Ar}, \mathrm{Xe}, \mathrm{Kr}, \mathrm{N}_{2}, \mathrm{O}_{2}, \mathrm{CO}$, and $\mathrm{CO}_{2}$ ), which should enhance the hydrophobic force ${ }^{8}$.

According to the clathrate cage model of Stillinger ${ }^{30}$, the clathrates formed in water can join together by sharing the edges and faces of polyhedrons, which entails less overall order than when they are dispersed. Clumping (or clustering) of clathrates is, therefore, a way to minimize the entropy cost associated with structuring water. Since clathrates represent the low-density species present in water, the results of the NR measurements ${ }^{10}$ may be considered to support the presence of clathrates in the thin films bounded by hydrophobic surfaces. The number and size of the clathrates may increase with decreasing film thickness, which may account for the corresponding decrease in water density, decrease in excess entropy $\left(\mathrm{S}^{\mathrm{f}}\right)$, and hence increase in hydrophobic force. Eriksson et al. ${ }^{14}$ assumed that the clusters are of quasi-cylindrical shape, and derived a surface-thermodynamic model for the long-range attractive forces observed between charge-free hydrophobic surfaces. ${ }^{14}$ The possibility of forming linear clusters is to some extent supported by the recent spectroscopic 
evidence that a water molecule is bonded to its neighbors by two strong and two weak Hbonds rather than four bonds of equal strength. ${ }^{45}$

\subsubsection{Effect of Solutes}

It has been shown that the long-range attractions between hydrophobic surfaces decrease considerably in degassed solutions. ${ }^{6,8}$ This important observation can be analyzed by means of the Gibbs surface tension equation adapted for thin planar films at constant temperature, pressure, and film thickness:

$$
\left(\frac{\partial \Delta \gamma^{\mathrm{f}}}{\partial \mu_{\mathrm{S}}}\right)_{\mathrm{T}, \mathrm{p}, \mathrm{H}}=-\Delta \Gamma_{\mathrm{S}}^{\mathrm{f}}
$$

in which $\mu_{\mathrm{S}}$ is the chemical potential of a solute (dissolved air) and $\Delta \Gamma_{\mathrm{S}}^{\mathrm{f}}$ is its excess quantity per $\mathrm{m}^{2}$ in the thin film of thickness H. Since the attractive force increases (i.e., $\Delta \gamma^{\mathrm{f}}$ becomes more negative) in the presence of dissolved air, $\Delta \Gamma_{S}^{f}$ must be positive in the thin film, that is, the amount of a dissolved gas in a thin film between two hydrophobic surfaces is higher than in a thick film. The excess dissolved gas in a thin film should promote the clathrate structure by the hydrophobic hydration mechanism described above and, hence, should give rise to a stronger hydrophobic force. The van der Waals attraction between the guest (gas) and host (water) molecules should also contribute to the stabilization of clathrate structure. Meagher et al. ${ }^{6}$ and Meyer et al. ${ }^{8}$ showed that hydrophobic force becomes shorter ranged in degassed water, which may be attributed to the weakening of the clathrate structure and, hence, smaller number and size of the clathrates.

Thermodynamically, clathrate structures are more readily formed at colder temperatures and higher pressures. However, remnants of the clathrates may also be found at ambient conditions. Evidence for this is that the solubilities of gases ${ }^{46}$ (e.g., $\mathrm{O}_{2}, \mathrm{~N}_{2}$ and Ar) and hydrocarbons ${ }^{47}$ in water at ambient conditions are substantially higher than predicted by the ordinary solution theory based on entropy of mixing. Shinoda ${ }^{47}$ attributed the deviation to the "iceberg" formation.

We have also conducted surface force measurements in ethanol-water mixtures between two $\mathrm{C}_{16} \mathrm{SH}$-coated gold surfaces and the work was reported in Chapter 5 . The hydrophobic surfaces were prepared by immersing a gold microsphere and a gold-coated glass in $1 \times 10^{-5} \mathrm{M}$ thiol in ethanol for 10 minutes. It is found that the long-range attraction in water changes significantly with adding ethanol. Figure 5.8 shows the changes in the $C$ and $\mathrm{D}$ constants of Equation 6.1. The attraction is the lowest at about 0.2 mole fraction of ethanol and increases on either side, and both $\mathrm{C}$ and $\mathrm{D}$ have minimum values at about the ethanol mole fraction 0.2. This finding mirrors other properties of ethanol-water mixtures regarding oxygen solubility $^{48}$ and the surface excess of ethanol at the air-solution interface. ${ }^{49}$

The data presented in Figure 5.8 have been used to calculate the film excess of ethanol $\left(\Delta \Gamma_{\mathrm{S}}^{\mathrm{f}}\right)$ using Equation 6.5. By invoking vapor pressure data of Butler and Wightman $^{49}$ that yield the changes in ethanol with the chemical potential changes of ethanol, we can thus compute the excess of ethanol in the film as a function of composition for 
different film thickness. The results presented in Figure 6.11 show that at low ethanol concentrations $\Delta \Gamma_{\mathrm{S}}^{\mathrm{f}}$ is negative, indicating that some of the ethanol is excluded from the thin

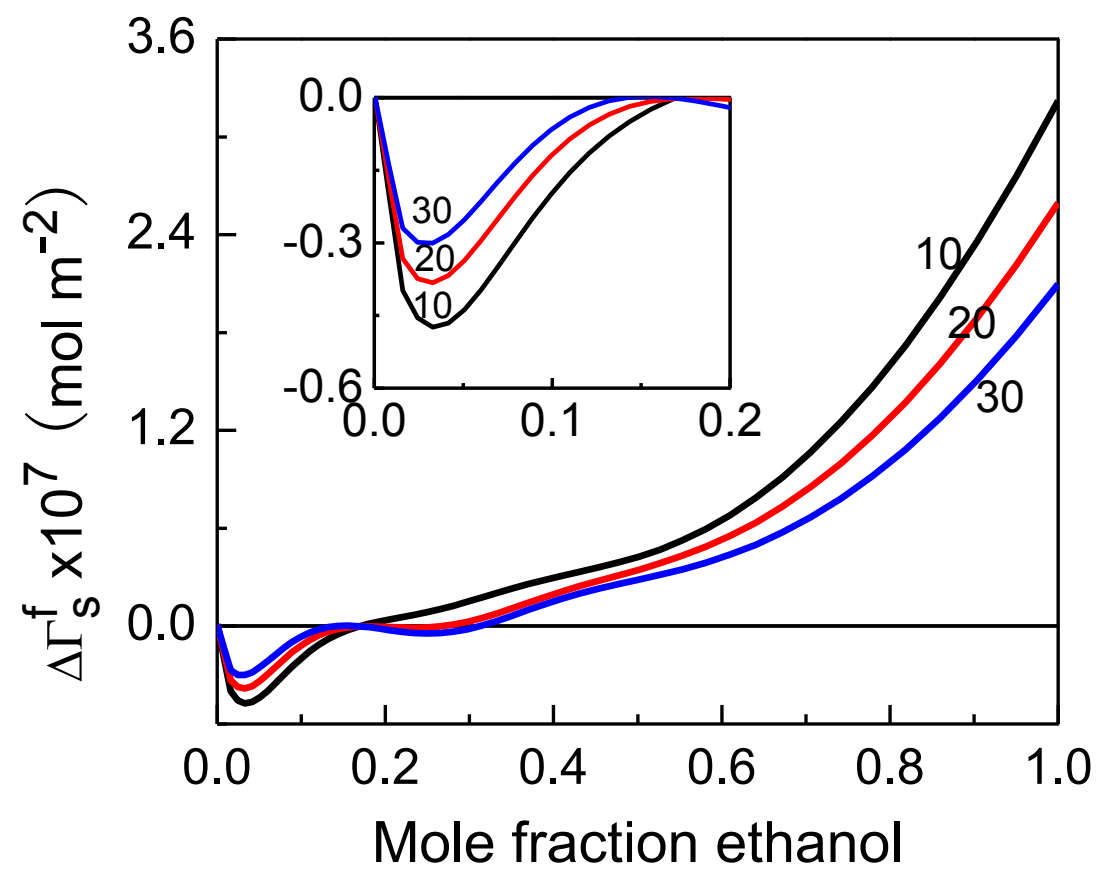

Figure 6.11. The excess quantities of ethanol $\left(\Delta \Gamma_{\mathrm{s}}{ }^{f}\right)$ per $\mathrm{m}^{2}$ in the thin films of water-ethanol mixtures between two $\mathrm{C}_{16} \mathrm{SH}$-hydrophobized surfaces plotted versus ethanol mole fraction.

film. This is most probably because ethanol disrupts the water structure in the vicinity of hydrophobic surfaces. As the ethanol concentration is increased, the strong, long-range attraction reappears, and $\Delta \Gamma_{\mathrm{S}}^{\mathrm{f}}$ becomes positive, which means that water molecules are to some extent expelled from the thin films between the hydrophobic surfaces. Both water and ethanol are H-bonding liquids. It appears, however, that the H-bonding of one becomes less extensive in the presence of the other, and causes the long-range attraction to diminish.

Neto $^{50}$ also observed strong long-range attractions between hydrophobic surfaces in pure ethanol, and Boinovich and Emelyanenko ${ }^{51}$ detected structural changes in the thin ethanol films $(<5 \mathrm{~nm})$ formed between fluorite surfaces using an infrared spectroscopic technique. In all likelihood, a layer-wise molecular arrangement that extends toward the core of the thin film is generated.

\subsection{Summary and Conclusions}

Our new thermodynamic data lend support for the hydrophobic force originating from the structural differences between water in the thin and thick films bounded by hydrophobic surfaces. This conclusion is the same as originally suggested by Rabinovich and Derjaguin ${ }^{52}$ 
and Eriksson et $a l^{13}$. The water molecules in the vicinity of hydrophobic surfaces reorganize themselves to form clathrates and clusters thereof, and cause hydrophobic attractions to appear. In the presence of dissolved gases, the clathrate structure becomes stronger, which gives rise to long-range hydrophobic forces. In degassed solutions, the clathrate structure becomes weaker, which in turn causes the hydrophobic force to become short-ranged ${ }^{8}$ and oil-in-water emulsions to become stabilized without surfactant ${ }^{53}$. Unlike the dissolved inert gases, ethanol disrupts the water structure and, hence, diminishes the long-range attractions. Further studies in the thermodynamics of hydrophobic interactions should lead to a better understanding of the origin of hydrophobic force, which plays an important role in a variety of scientific and technological fields.

\section{References}

1. Israelachvili, J.; Pashley, R., Nature 1982, 300, 341-342.

2. $\quad$ Parker, J. L.; Claesson, P. M.; Attard, P., J. Phys. Chem. 1994, 98, 8468-8480.

3. Tyrrell, J. W. G.; Attard, P., Phys. Rev. Lett. 2001, 87, (17), 176104-1.

4. $\quad$ Seo, H.; Yoo, M.; Jeon, S., Langmuir 2007, 23, 1623-1625.

5. Sakamoto, M.; Kanda, Y.; Miyahara, M.; Higashitani, K., Langmuir 2002, 18, 57135719.

6. $\quad$ Meagher, L.; Craig, V. S. J., Langmuir 1994, 10, 2736-2742.

7. $\quad$ Zhang, J.; Yoon, R.-H.; Mao, M.; Ducker, W. A., Langmuir 2005, 21, 5831-5841.

8. $\quad$ Meyer, E. E.; Lin, Q.; Israelachvili, J. N., Langmuir 2005, 21, 256-259.

9. Derjaguin, B. V., Kolloid Zeits 1934, 69, 155-164.

10. Doshi, D. A.; Watkins, E. B.; Israelachvili, J. N.; Majewski, J., Proc. Nat. Acad. Sci. U.S.A 2005, 102, 9458-9462.

11. Nguyen, A. V.; Nalaskowski, J.; Miller, J. D.; Butt, H.-J., Int. J. Miner. Process. 2003, 72, 215-225.

12. Ederth, T., J. Phys. Chem. B 2000, 104, 9704-9712.

13. Eriksson, J. C.; Ljunggren, S.; Claesson, P. M., J. Chem. Soc., Faraday Trans. 2 1989, 85, (3), 163-176.

14. Eriksson, J. C.; Henriksson, U., Langmuir 2007, 23, 10026-10033.

15. Miklavic, S. J.; Chan, D. Y. C.; White, L. R.; Healy, T. W., J. Phys. Chem. 1994, 98, 9022-9032.

16. Meyer, E. E.; Lin, Q.; Hassenkam, T.; Oroudjev, E.; Israelachvili, J., Proc. Nat. Acad. Sci. U.S.A 2005, 102, (19), 6839-6842.

17. Christenson, H. K.; Yaminsky, V. V., Colloids Surf., A 1997, 129-130, 67-74.

18. Podgornik, R., J. Chem. Phys 1989, 91, (9), 5840-5849.

19. Claesson, P. M.; Christenson, H. K., J. Phys. Chem 1988, 92, 1650-1655.

20. Lin, Q.; Meyer, E. E.; Tadmor, M.; Israelachvili, J. N.; Kuhl, T. L., Langmuir 2005, 21, 251-255.

21. Ederth, T.; Claesson, P.; Liedberg, B., Langmuir 1998, 14, 4782-4789.

22. Wang, J.; Yoon, R.-H., Langmuir 2008, 24, 7889-7896.

23. Ducker, W. A.; Senden, T. J.; Pashley, R. M., Nature 1991, 353, 239-241.

24. Rabinovich, Y. I.; Yoon, R.-H., Langmuir 1994, 10, 1903-1909.

25. Raiteri, R.; Preuss, M.; Grattarola, M.; Butt, H.-J., Colloids Surf., A 1998, 136, 191197. 
26. Cleveland, J. P.; Manne, S.; Bocek, D.; Hansma, P. K., Rev. Sci. Instrum. 1993, 64, (2), 403-405.

27. Tsao, Y.-H.; Yang, S. X.; Evans, D. F.; Wennerstrom, H., Langmuir 1991, 7, 31543159.

28. Roentgen, W. C., Ann. Phys. 1892, 281, 91-97.

29. Pauling, L., Science 1961, 134, 15-21.

30. Stillinger, F. H., Science 1980, 209, 451-457.

31. Tanford, C., The Hydrophobic Effect. 2 ed.; Wiley: New York, 1980.

32. Israelachvili, J. N.; Pashley, R. M., J. Colloid Interface Sci. 1984, 98, (2), 500-514.

33. Chandler, D., Nature 2002, 417, 491.

34. Chandler, D., Nature 2005, 437, 640-647.

35. Frank, H. S.; Wen, W.-Y., Discuss. Faraday Soc. 1957, 24, 133-140.

36. Sharp, K. A.; Madan, B., J. Phys. Chem. B 1997, 101, 4343-4348.

37. Kauzmann, W., Nature 1987, 325, 763-764.

38. Lum, K.; Chandler, D.; Weeks, J. D., J. Phys. Chem. B 1999, 103, 4570-4577.

39. Mezger, M.; Reichert, H.; Schoder, S.; Okasinski, J.; Schroder, H.; Dosch, H.; Palms, D.; Ralston, J.; Honkimaki, V., Proc. Nat. Acad. Sci. U.S.A 2006, 103, (49), 1840118404.

40. Poynor, A.; Hong, L.; Robinson, I. K.; Granick, S., Phys. Rev. Lett. 2006, 97, 266101.

41. Jensen, T. R.; Jensen, M. Q.; Reitzel, N.; Balashev, K.; Peters, G. H.; Kjaer, K.; Bjqrnholm, T., Phys. Rev. Lett. 2003, 90, (8), 086101-1.

42. Maccarini, M.; Steitz, R.; Himmelhaus, M.; Fick, J.; Tatur, S.; Wolff, M.; Grunze, M.; Janecek, J.; Netz, R. R., Langmuir 2007, 23, 598-608.

43. Kokkoli, E.; Zukoski, C. F., Langmuir 1998, 14, 1189-1195.

44. Christenson, H. K.; Claesson, P. M.; Berg, J.; Herder, P. C., J. Phys. Chem. 1989, 93, 1472-1478.

45. Wernet, P.; Nordlund, D.; Bergmann, U.; Cavalleri, M.; Odelius, M.; Ogasawara, H.; Naslund, L. A.; Kirsch, T. K.; Ojamae, L.; Glatzel, P.; Pettersson, L. G. M.; Nilsson, A., Science 2004, 304, 995-999.

46. Battino, R.; Clever, H. L., Chem. Rev. 1966, 66, (4), 395-463.

47. Shinoda, K., J. Phys. Chem. 1977, 81, (13), 1300-1302.

48. Shchukarev, S. A.; Tolmacheva, T. A., ZhurnalStrukt. Khim 1968, 9, 21-28.

49. Butler, J. A. V.; Wightman, A., J. Chem. Soc. (London) 1932, 2089-2097.

50. Neto, C. Universita degli Studi di Firenze, Firenze, 2001.

51. Boinovich, L. B.; Emelyanenko, A. M., Adv. Colloid Interface Sci. 2002, 96, 37-58.

52. Rabinovich, Y. I.; Derjaguin, B. V., Colloids Surf. 1988, 30, 243-251.

53. Maeda, N.; Rosenberg, K. J.; Israelachvili, J. N.; Pashley, R. M., Langmuir 2004, 20, 3129-3137. 


\section{CONCLUSIONS AND RECOMMENDATIONS}

\subsection{Conclusions}

In this work, the force measurements were conducted with gold surfaces pretreated by ex-situ adsorption of alkanethiols and in-situ adsorption of water-soluble xanthates of different chain lengths, concentrations, and immersion times. Further, the force measurements were conducted in the presence of various solutes such as inorganic electrolytes, ionic surfactants, and alcohols that can affect the properties (e.g., structure) of the thin water films between two hydrophobic surfaces. To gain an understanding of the water structure, the changes in the excess film entropies and enthalpies across the film thickness were determined by measuring the surface forces at different temperatures.

Based on the findings of the present study, conclusions can be drawn as follows:

1. I observed long-range attractions between gold surfaces pretreated by ex-situ adsorption of alkanethiols $(n=2-16)$ and in-situ adsorption of water-soluble xanthate surfactants (i.e., PAX, KEX). The long-range attractive forces decayed exponentially, and were detected at the separation distance of up to $120 \mathrm{~nm}$. The force curves exhibited no steps, indicating that the long-range attraction was not due to bridging bubbles.

2. The surface forces measured between hydrophobized-gold surfaces depend on surface treatment conditions such as surfactant concentration and immersion time. When the surfaces were hydrophobized in $1 \mathrm{mM}$ 1-hexadecanethiol of ethanol solution longer than 6 hours, the measured forces were net-attractive and long-ranged. However, the force curves exhibited steps, indicating that the measured forces were due to bridging bubbles. It is possible that the thiol monolayer can have defects (or pits) in which air bubbles can nucleate. When the gold substrates were hydrophobized in a dilute $\left(1 \times 10^{-2} \mathrm{mM}\right)$ thiol solution at relatively short contact time (10 minutes), a long-range attractive force which decayed exponentially with a decay length of $35 \mathrm{~nm}$ was obtained, and the force curve exhibited no steps. It is possible that at lower concentrations and shorter contact times smoother coatings are formed, which are less likely to trap nanobubbles and hence give rise to smooth force curves.

When the gold substrates were immersed in the $1 \times 10^{-2} \mathrm{mM} \mathrm{C}_{16} \mathrm{SH}-$ and $\mathrm{C}_{12} \mathrm{SH}-$ in-ethanol solutions for different periods of time, the attractive forces increased with immersion time, and then decreased. All the forces measured were stronger and longer-ranged than the van der Waals force. The contact angles on these surfaces were greater than $90^{\circ}$. When the force measurements were conducted after washing the substrates with appropriate solvents, strong long-range hydrophobic forces appeared. The same behavior was also found for gold in PAX solutions after different immersion times. It is suggested that the decrease of hydrophobic force is caused by the formation of the bilayers of thiol or xanthate, which can be washed away by using appropriate solvents.

3. The long-range attractions were observed between thiol-coated gold surfaces regardless of whether the contact angles were greater or smaller than $90^{\circ}$. When gold surfaces were hydrophobized with $\mathrm{C}_{2} \mathrm{SH}$, the contact angle was less than $90^{\circ}$. In this case, no cavitation is possible. Yet, the AFM force measurement gave 
a long-range attraction. It is suggested that hydrophobic force is not due to the bubbles present on hydrophobic surfaces or to the cavitation (or drying).

4. The long-range hydrophobic attraction increases with increasing chain length of alkanethiol, decreases in the presence of salt (e.g., $\mathrm{NaCl})$ and surfactant (e.g., $\left.\mathrm{C}_{12} \mathrm{TACl}, \mathrm{PAX}\right)$, and changes with ethanol concentration. The strongest hydrophobic attraction measured on the $\mathrm{C}_{4} \mathrm{SH}$-coated gold was weaker than those measured on $\mathrm{C}_{12} \mathrm{SH}$ - and $\mathrm{C}_{16} \mathrm{SH}$-coated gold surfaces. The latter two surfaces had the same water contact angles and were indistinguishable with respect to the hydrophobic attractions measured. It was also found that the hydrophobic force measured with PAX was much larger than that with KEX.

The attractive forces measured on $\mathrm{C}_{12} \mathrm{SH}-, \mathrm{C}_{16} \mathrm{SH}$ - and PAX-coated gold surfaces were effectively reduced by electrolyte $(\mathrm{NaCl})$, indicating that they may be of electrostatic origin. For this mechanism to work, it is necessary that the charged patches be mobile when two surfaces approach each other to maximize the correlation and give rise to a long-range attraction. However, the strong covalent $\mathrm{Au}-\mathrm{S}$ bonding provides robust and immobile hydrophobic monolayers, which precludes the possibility of charged patches being mobile. Further, there is no reason that thiol or xanthate adsorption produced charged patches as they chemisorbs on gold. For the case of xanthate adsorption, both the adsorbate and adsorbent are negatively charged. Our work leaves the possibility that the longrange attraction is caused by the changes in water structure near hydrophobic surfaces. It is possible that the electrolyte $(\mathrm{NaCl})$, ionic surfactant $\left(\mathrm{C}_{12} \mathrm{TACl}\right)$ and residual xanthate (PAX) in solution break the H-bonded water structure and, hence, reduce the hydrophobic force.

The surface force data obtained in water-ethanol mixtures showed that the attractions were strong and long-ranged in pure water and pure ethanol, whereas they were weaker and of shorter range in water-ethanol mixtures with mole fraction of ethanol from 0.1 to 0.9 . The data have been analyzed by using thin film thermodynamics. The results mirror the well-known, rather special bulk properties of water-ethanol mixtures. At mole fractions below about 0.2, a breakdown of the water structure due to introducing ethanol molecules was a predominant feature, explaining the reduction of hydrophobic attraction in both strength and range. For high ethanol mole fractions, we can assume that a layered, laterally homogenous surface structure was formed that stretches some distance away from the hydrophobic surfaces, likewise giving rise to a longrange attraction that was readily disturbed, however, by adding more water.

5. Hydrophobic force may be a structural force. The measurements carried out at $10-40^{\circ} \mathrm{C}$ show that the hydrophobic force decreased with increasing temperature. Thermodynamically, these results imply that as two hydrophobic surfaces approach each other the changes in excess film entropy $\left(\mathrm{S}^{\mathrm{f}}\right)$ and enthalpy $\left(\mathrm{H}^{\mathrm{f}}\right)$ per unit area decrease, which suggests that the hydrophobic force is due to the structuring of water in the thin film confined by two hydrophobic surfaces rather than from an artifact.

\subsection{Recommendations for Future Work}


Based on the results obtained in this work, the following areas of research are recommended:

1. It has been suggested that the hydrophobic force may be related to the structure of water confined between hydrophobic surfaces. Direct evidence for the structure change of water is needed. Therefore, systematic neutron reflectivity (NR) measurement, in-situ x-ray or nuclear magnetic resonance (NMR) studies on water between two hydrophobic surfaces which are close to each other are highly recommended.

2. Based on the surface force measurements at different temperatures, it was speculated that water molecules form partial or full clathrates in the vicinity of hydrophobic surfaces and the presence of gases facilitates the clathrate hydrate formation. In the future work, the AFM force measurements are recommended to be conducted in the presence of different gases (e.g., $\mathrm{CO}_{2}, \mathrm{CH}_{4}, \mathrm{H}_{2}, \mathrm{He}, \mathrm{N}_{2}$ ) dissolved in water to investigate the gas effect. Molecular dynamic simulation of water in the vicinity of hydrophobic surfaces is recommended to pursue the molecular origin of the hydrophobic force. 


\section{ACKNOWLEDGEMENT}

The authors would like to express their sincere appreciation to Professor Jan Christer Eriksson for his encouragement and helpful discussions. They would also like to acknowledge the financial support from the National Energy Technology Laboratory, U.S. Department of Energy (DE-FC26-02NT41607). 


\section{PUBLICATIONS}

1. Jialin Wang and Roe-Hoan Yoon, "Surface Forces Measured between XanthateCoated Gold Surfaces," ECS Trans. 28, 3 (2010)

2. Jialin Wang and Roe-Hoan Yoon, "Surface Force Measurements between Gold Surfaces in Xanthate Solutions," $48^{\text {th }}$ Annual Conference for Metallurgists, Ontario, Canada, August 23, 2009

3. Roe-Hoan Yoon, Amadeu K. Sum, Jialin Wang and Jan Christer Eriksson, "The Role of Hydrophobic Interactions for the Formation of Gas Hydrates," Proceedings of the $6^{\text {th }}$ International Conference on Gas Hydrates (ICGH 2008), Vancouver, British Columbia, Canada, July 6-10, 2008

4. Jialin Wang and Roe-Hoan Yoon, "AFM Forces Measured between Gold Surfaces Coated with Self-Assembled Monolayers of 1-Hexadecanethiol," Langmuir, 2008, 24(15), 7889-7896

\section{PRESENTATIONS}

1. Jialin Wang, Jan Christer Eriksson and Roe-Hoan Yoon, "Thermodynamics of Macroscopic Interactions," $13^{\text {rd }}$ ICSCS $/ 83^{\text {rd }}$ CSS Symposium, New York, USA, June 14-19, 2009

2. Jialin Wang, Roe-Hoan Yoon, Atte Kumpulainen and Jan Christer Eriksson, "Surface Forces Measured between Hydrophobic Gold Surfaces in n-Alcohols and in WaterEthanol Mixtures," $13^{\text {rd }}$ ICSCI $/ 83^{\text {rd }}$ CSS Symposium, New York, USA, June 14-19, 2009

3. Jialin Wang and Roe-Hoan Yoon, "Surface Forces Measured between Gold Surfaces," Zing Surface Chemistry Conference 2008, Bolans Village, Antigua and Barbuda, January 7-10, 2008

4. Jialin Wang, Jinhong Zhang, Monica Ma and Roe-Hoan Yoon, "Force Measurement between Hydrophobic Surfaces," CAST Workshop, July 24-26, 2007, Blacksburg, Viginia

5. Jialin Wang, Roe-Hoan Yoon and Jan Christer Eriksson, "Surface Forces Measured between Gold Surfaces Coated with Self-Assembled Monolayers of Thiols and Xanthates in Water," The $81^{\text {st }}$ ACS Colloid \& Surface Science Symposium, June 2427, 2007, Newark, DE, USA

6. Jinhong Zhang, Jialin Wang, Jan Christer Eriksson and Roe-Hoan Yoon, "Effect of Chain Length on Hydrophobic Force," XIII International Conference on Surface Forces, June 28-July 4, 2006, Moscow, Russia

\section{APPENDICES}

No appendices are included in this report. 
APPENDIX 3 - Alternative Materials for Dense Medium Separations (KY004) 
FINAL TECHNICAL REPORT

Contract Title and Number: Continuation of Croscutting Technology Development at CAST (DE-FC26-05NT42457)
Period of Performance:

Starting Date: $10 / 1 / 05$

Ending Date: 9/30/10 $\underline{\text { Sub-Recipient Project Title: }}$

Alternative Materials for Dense Medium Separation

Principal Investigators:

Rick Q. Honaker

Contact Address:

University of Kentucky

234B Mining and Mineral Resources

Lexington KY 40506

Subcontractor Address:
Report Information:

Type: Final

Number:

Period: 10/01/05-9/30/09

Date: 10/25/09

Code: $\quad$ KY004-FINAL

Contact Information:

Phone: (859) 257-1108

Fax: (858) 323-1962

E-Mail: rhonaker@engr.uky.edu

Subcontractor Information:

Phone:

Fax:

E-Mail:

\section{ABSTRACT}

In response to concerns regarding cost and future supply of magnetite, a study has been performed to evaluate the potential of alternative materials that can be used to generate a dense medium for coal cleaning applications. Alternative materials included waste steel slag, fine sand, high-density material existing in run-of-mine feed and magnetic material from coal combustion bottom ash. Under certain conditions, each of the alternative materials provided separation efficiencies that meet industrial standards including probable error values ranging from 0.03 to 0.05 . Dense medium derived from the non-magnetite sources and magnetic bottom ash particles achieved organic efficiency values exceeding the $95 \%$ level over a medium density range from 1.3RD to $1.6 \mathrm{RD}$ with lower than normal density offsets. Coarse magnetite, which has a lower commercial value than conventional magnetite, provided excellent separation efficiencies when evaluating medium density values greater than 1.6RD. From the assessment of recoverability after the coal cleaning process, the magnetic bottom ash material was easily recovered by a standard low intensity magnetic separator which is the current method used to recover magnetite in operating coal cleaning facilities. 


\section{DISCLAIMER}

This report was prepared as an account of work sponsored by an agency of the United States Government. Neither the United States Government nor any agency thereof, nor any of their employees, make any warranty, express or implied, nor assume any legal liability or responsibility for the accuracy, completeness, or usefulness of any information, apparatus, product, or process disclosed, or represents that its use would not infringe privately owned rights. Reference herein to any specific commercial product, process, or service by trade name, trademark, manufacturer, or otherwise does not necessarily constitute or imply endorsement, recommendation, or favoring by the United States Government or any agency thereof. The views and opinions of authors expressed herein do not necessarily state or reflect those of the United States Government or agency thereof. 


\section{Table of Contents}

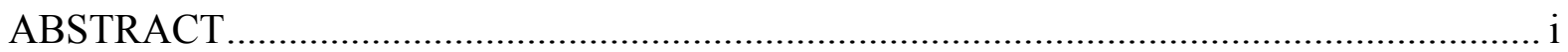

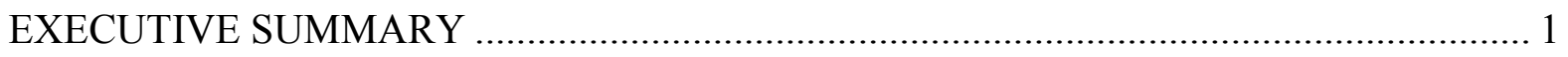

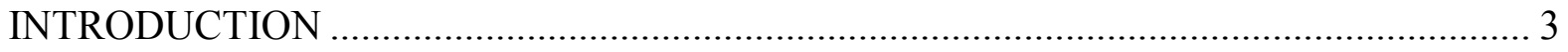

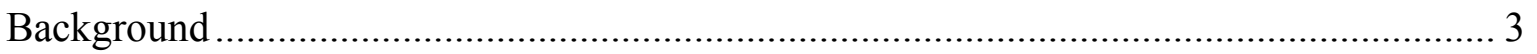

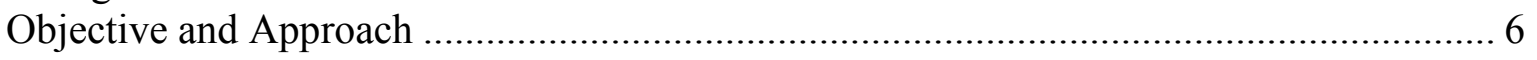

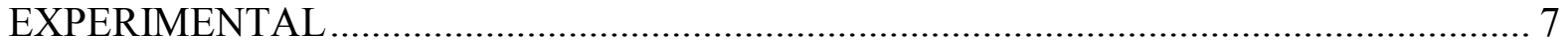

Dense Medium Cyclone Circuit................................................................................. 7

Coal Sample

Alternative Medium Materials ....................................................................................... 10

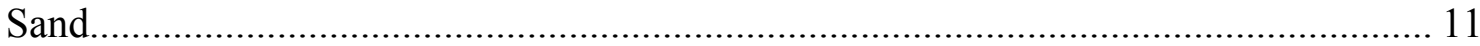

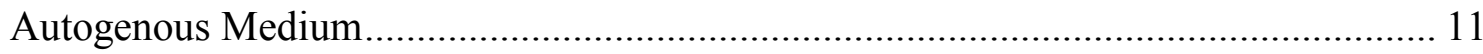

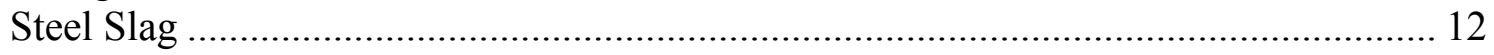

Magnetic Coal Combustion Byproducts ................................................................... 12

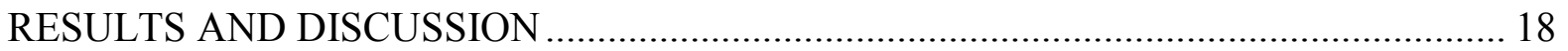

Reference Magnetite Performance............................................................................... 18

Alternative Medium Performance................................................................................ 20

Sand, Autogenous and Coarse Magnetite Medium.......................................................... 20

Magnetic Coal Combustion Byproduct ..................................................................... 24

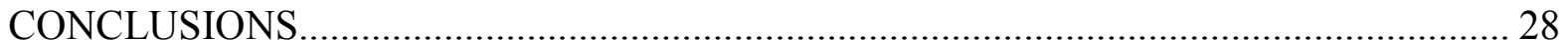

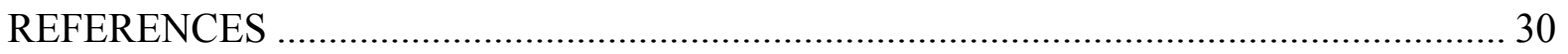

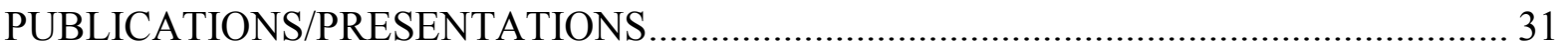




\section{EXECUTIVE SUMMARY}

Dense medium separation processes are currently used to clean over $50 \%$ of the 640 million tons of coal that is processed through preparation plants in the U.S. annually. They provide the most efficient separations for particle sizes greater than $1 \mathrm{~mm}$. To develop the dense medium, magnetite $\left(\mathrm{Fe}_{3} \mathrm{O}_{4}\right)$ particles finer than about 44 micrometer are dispersed in water at a concentration that provides a medium density between the solid densities of coal and rock. After the separation, the medium is passed through a low-intensity magnetic drum separator to recover the magnetite. Magnetite losses are typically within the range of 0.5 to $1.0 \mathrm{lbs} / \mathrm{ton}$ of raw coal processed.

In 2002, most of the magnetite used in the U.S. coal industry was obtained from domestic sources and worldwide production was adequate to meet the needs for coal cleaning and steel production as well as other needs. The cost for a ton of magnetite was $\$ 56$. However, closure of a mine that supplied magnetite to the coal industry containing very low amounts of silica particles resulted in the need to seek international sources of magnetite which resulted in imports mainly from South America and elevated costs. Economic and construction growth in developing countries brought a high demand for iron ore worldwide and, as a result, the concern for adequate magnetite supply for the coal industry. Prices rose to around $\$ 300$ per ton. Concerns over cost and supply resulted in worldwide interest in identifying alternative materials that could potentially replace magnetite is dense medium separation processes.

The project funded through the Center for Advanced Separation Technologies investigated several magnetite alternatives including a source of coarse magnetite that is sold internationally at a lower cost than conventional magnetite. Other materials included magnetic particles recovered from coal combustion bottom ash, fine sand and fine waste generated from steel production. A unique source of alternative material is the high-ash content, high density particles that exist in the fine reject streams in coal preparation plants. The autogenous particles have a density less than half of magnetite which requires the use of twice the amount of autogneous material to achieve a given medium density. The high particle population raises concerns over negative viscosity effects but separations at low medium density values may avoid these concerns.

The characteristics of a medium used to clean coal are that i) the particles forming the medium are relatively stable over a range of medium densities and ii) the medium has low viscosity. Medium stability is achieved by minimizing the settling velocity of the particles forming the medium. Elevating medium density improves stability since the increased volumetric concentration of particles creates a high level of hindered settling which decreases particle settling velocity. However, high particle populations result in viscosity issues that typically reduce the separation efficiency achieved when applied to coal cleaning. Decreasing particle size and sphericity also reduces particle settling rates and thus enhances stability.

The magnetic bottom ash particles collected from the Mill Creek power station, a pulverized coal fired power plant, operated by Louisville Gas and Electric (LG\&E), was comprised of mostly hollow spheres. The Mill Creek plant was selected as a source for the material due to the relatively high pyrite content of the feed coal which results in elevated concentrations of 
iron oxide material in the combustion byproduct streams, particularly in the bottom ash stream due to the high particle density of the iron oxide particles. The iron oxide particles were recovered using a spiral concentrator and a high intensity magnetic separator. The -100 mesh material recovered from the bottom ash stream represented $5.7 \%$ of the total feed. However, the sphericity of the iron oxide particles provided a relatively unstable medium. As such, the material was ground in a ball mill to reduce particle sphericity which resulted in a particle size distribution in which $90 \%$ of the particles were finer than 50 micrometers. Grinding caused liberation of the magnetic material which resulted in only $54 \%$ of the originally recovered magnetic material to be recoverable by a magnetic separator. A positive outcome is that $98 \%$ of the remaining magnetic particles can be recovered by a low intensity magnetic separator with a field strength of 1000 gauss.

The conventional magnetite alternatives were evaluated for their application in dense medium separators using a 15-cm diameter Krebs cyclone operated in a closed-looped circuit. The overflow and underflow streams of the cyclone were returned to a common feed sump from which feed to the cyclone was pumped under pressures ranging from 17.3 to 68.7 $\mathrm{kPa}$ (2.5 to $7.5 \mathrm{psi}$ ). Medium stability was assessed by measuring the difference in the pulp density values of the underflow and overflow streams. Differences less than 0.4 units are indicative of acceptable medium stability. With the exception of the coarse magnetite, each of the alternative materials provided a more stable medium than the conventional magnetite over the entire medium density range evaluated. This outcome was somewhat expected due to the lower solid density values of the alternative materials which means that higher volumetric solid concentrations relative to the conventional magnetite are needed to achieve a given medium density. Medium stability formed from coarse magnetite approached acceptable levels when the relative medium density exceeded 1.6.

The separation performance achievable using medium from each of the alternative sources was assessed using a relatively difficult to clean Coalburg coal having a top particle size of $6 \mathrm{~mm}$ and a bottom size of $0.6 \mathrm{~mm}$. Medium density was varied from $1.4 \mathrm{RD}$ to a high of 1.9RD. At medium density values of $1.4 \mathrm{RD}$ and $1.5 \mathrm{RD}$, the separation efficiency values achieved when using steel slag, fine sand, autogenous material, and magnetic bottom ash were nearly equal to the values obtained using the conventional magnetite (e.g., $\mathrm{E}_{\mathrm{p}}=0.03$ and organic efficiency $>95 \%$ ). However, when the medium density was raised to $1.6 \mathrm{RD}$, only the magnetic bottom ash material achieved separation efficiency values near but slighter lower than that realized from conventional magnetite. The inferior performances at 1.6RD are reflective of the elevated medium viscosity caused by particle populations far exceeding that of the conventional magnetite.

On the other hand, the lower cost coarse magnetite provided poor medium stability for all medium density values tested at or below 1.6RD. As a result, the separation efficiencies achieved while cleaning coal were significantly inferior. However, as the medium density was increased above 1.6RD, medium stability and thus separation efficiency improved significantly. In fact, the separation efficiency achieved with the coarse magnetite was superior to conventional magnetite at medium density values of 1.8RD and 1.9RD. The findings support previously reported conclusions that an optimum medium particle size distribution exists for a separation at a given medium density. 


\section{INTRODUCTION}

\section{Background}

Dense-medium separators are the most widely applied gravity concentration units in coal preparation. In the process, the separation of coal from mineral matter is achieved by creating a medium having a density between the solid densities of the two materials being separated. The medium is formed by suspending ultrafine particles in a medium of water. The materials that have been used to form the suspension in commercial applications include sand, clay, quartz, slate, galena, magnetite and ferrosilicon [1]. In the early development of dense medium separators, sand (Chance sand process) was used as medium. However, sand was difficult to recover. When magnetic separators were developed at acceptable capacities, sand was replaced with magnetite [2]. Since then, magnetite has become the predominate material used to form a dense medium when relative separation densities below 2.0 are desired.

Recently, worldwide demand for magnetite has increased significantly due to steel production requirements in developing countries. This has led to a growing interest in seeking alternate materials for dense medium separation. Material that is acceptable for producing a dense medium should provide the following characteristics: good medium stability; capability of achieving a broad range of separation densities, low viscosity and easy recoverability for reuse. Medium stability is typically monitored by the density difference between the overflow and the underflow media streams. Acceptable medium stability is indicated by medium density differences in the discharge streams of less than 0.4 density units. Particle size, shape and density are parameters that play vital roles in the stability and rheological properties of a medium. For example, spherical particles create unstable mediums at low medium densities but may become stable as the medium density is increased.

The control of density, viscosity, and settling rate of a medium is necessary for the efficient separation of coal and impurities. The following equation determines the density of a suspension, $\rho_{m}\left(\mathrm{~g} / \mathrm{cm}^{3}\right)$,

$$
\rho_{m}=\frac{100}{\left(100-\phi_{W}\right)+\frac{\phi_{W}}{\rho_{s}}},
$$

where $\rho_{s}$ is the density of the solid $\left(\mathrm{g} / \mathrm{cm}^{3}\right)$ used to form the medium and $\phi_{W}$ the solid concentration by weight (\%). From Eq. (1), it can be seen that the density of a suspension can be varied by adjusting the proportion of medium solids present in the suspension. However, there is a natural limit to the proportion of solids as imposed by the negative effects of medium viscosity. Relatively low viscosity is needed to permit the free travel of coal and impurities in it. This limiting volumetric concentration is similar for all suspensions with particles of similar size range but can be modified to a limited extent by a change in the size distribution of the medium solids. Eq. (1) also shows that the density of the suspension is a 
function of the relative density (RD) of the medium solids and it follows that media composed of higher specific gravity solids will have a higher density at the limiting volume concentration than suspensions composed of lower specific gravity solids.

Increasing the medium density above the density of pure water by the addition of magnetite or alternative material initially has a positive effect on separation performance due to improved medium stability and the positive impacts on differential density-based particle movement. Medium stabilization is a result of an elevated particle population which decreases the settling rate of the magnetite particles due to hindered settling as quantified by the expression:

$v_{H}=\frac{d^{2} g\left(\rho_{s}-\rho_{f}\right)}{18 \mu_{A}}\left(1-\phi_{V}\right)^{3.65}$

where $v_{H}$ is the hindered settling velocity $(\mathrm{cm} / \mathrm{s}), d$ particle size $(\mathrm{cm}), g$ acceleration due to gravity $\left(\mathrm{cm} / \mathrm{s}^{2}\right), \rho_{s}$ and $\rho_{f}$ the densities of particle and fluid, respectively, $\phi_{V}$ the solids concentration by volume and $\mu_{A}$ the actual suspension viscosity (poise). Equation (2) is applicable for ultrafine particle suspensions and clearly shows that increasing the solid concentration by volume reduces particle settling rates which leads to stabilization of the medium. However, as many studies have shown, a continued increase in the solids concentration above a critical value leads to a viscous suspension having non-Newtonian fluid characteristics and depressed separation efficiencies when cleaning coarse and fine coal $[3-4]$.

A semi-empirical model was developed by Shi and Napier-Munn [1996] that allows the prediction of slurry viscosity as a function of particle size and volumetric solids concentration of the suspension, i.e.,

$\mu_{A}=\mu_{W}\left[C_{1}(H-1)+C_{2} H \Omega+C_{3} \frac{\phi_{V}}{P_{80}} \Omega^{2}+C_{4} \Omega^{3}\right]$,

in which

$H=\exp \left[\frac{\phi_{V}}{1-\phi_{V}\left(P_{20}\right)^{C_{5}} \exp \left(C_{6} T\right)}\right]$. 
The variable $T$ is the medium temperature $\left({ }^{\circ} \mathrm{C}\right), \Omega$ the bobbin angular velocity of the viscometer ( $\mathrm{rad} / \mathrm{s}), P_{20}$ and $P_{80}$ the $20 \%$ and $80 \%$ passing sizes of the suspended solids (mm), respectively, $\mu_{W}$ the viscosity of water at $20^{\circ} \mathrm{C}(0.01$ Poise $)$ and $C_{1}-C_{6}$ model parameters that are determined experimentally for particular suspension type. Predictions using Eqs. (3) and (4) were found to accurately predict the output readings obtained from a rotational viscometer at various bobbin speeds.

An evaluation of three different medium solid materials conducted using Eqs. (2) - (4) shows a window of medium density values in which medium stability is realized and viscosity remains low (Figure 1). One of the materials (MAG) resembles conventional magnetite with a relative solids density of 5.2 and a $P_{80}$ of 36 microns. The characteristics of the other two cases represent those associated with materials that could potentially be used as an alternative to magnetite (FAM: $\rho_{\mathrm{s}}=3.6$ and $P_{80}=45$ microns; SR: $\rho_{\mathrm{s}}=2.45$ and $P_{80}=170$ microns). In all three cases, hindered settling velocity decreases with an elevation in medium density which stabilizes the medium. Simultaneously, medium viscosity increases and the rate of increase becomes significantly greater as medium density is raised above a relative value of 1.60. The case of using a low density solid (SR) requires the use of a relative coarse particle size distribution to reduce particle surface area and minimize particle suspension viscosity. The result is a relatively fast hindered settling velocity at low medium density values and thus medium instability. At a medium density of about $1.5 \mathrm{RD}$, the 'SR' material becomes stable due to the low settling velocity. However, at a medium density of 1.6RD, viscosity increases rapidly and provides undesirable separation conditions. Thus, an operating window exists for each medium type which provides the appropriate medium stability through hindered settling with relatively low viscosity effects.

The results from several recent studies have identified potential low cost materials that could be used as a substitute for magnetite in dense medium separators. Studies with magnetic fly ash particles have shown that the material could be an acceptable substitute for magnetite with some changes in the magnetite recovery circuit [ $6-7]$. The research revealed that the raw state of magnetic fly ash has spherical shape of particle sizes between $0.1 \mathrm{~mm}$ and $0.2 \mathrm{~mm}$ which creates unstable suspension conditions. Recent research found that the wet grinding of fly ash liberates the magnetic components and changes the shape of the material. As a result, the medium suspension was found to be relatively stable over a broad range of medium density values. This result was confirmed by pilot scale experiments and in-plant tests conducted at two different coal preparation plants. The recovery of $99.1 \%$ of the magnetic fly ash material was obtained in in-plant test [8]. Another investigation focused on the magnetic fraction from flue dust (MFFD) which achieved satisfactory results in a coal cleaning application [9].

A number of materials have potential for use as a substitute for magnetite in dense medium separations. However, they must provide the medium characteristics that have been previously discussed. A detailed research program has been conducted to evaluate the potential use of a relatively coarse magnetite as well as steel slag waste, fine sand and autogenous medium. The details of the study and the associated results are the subject of this publication. 


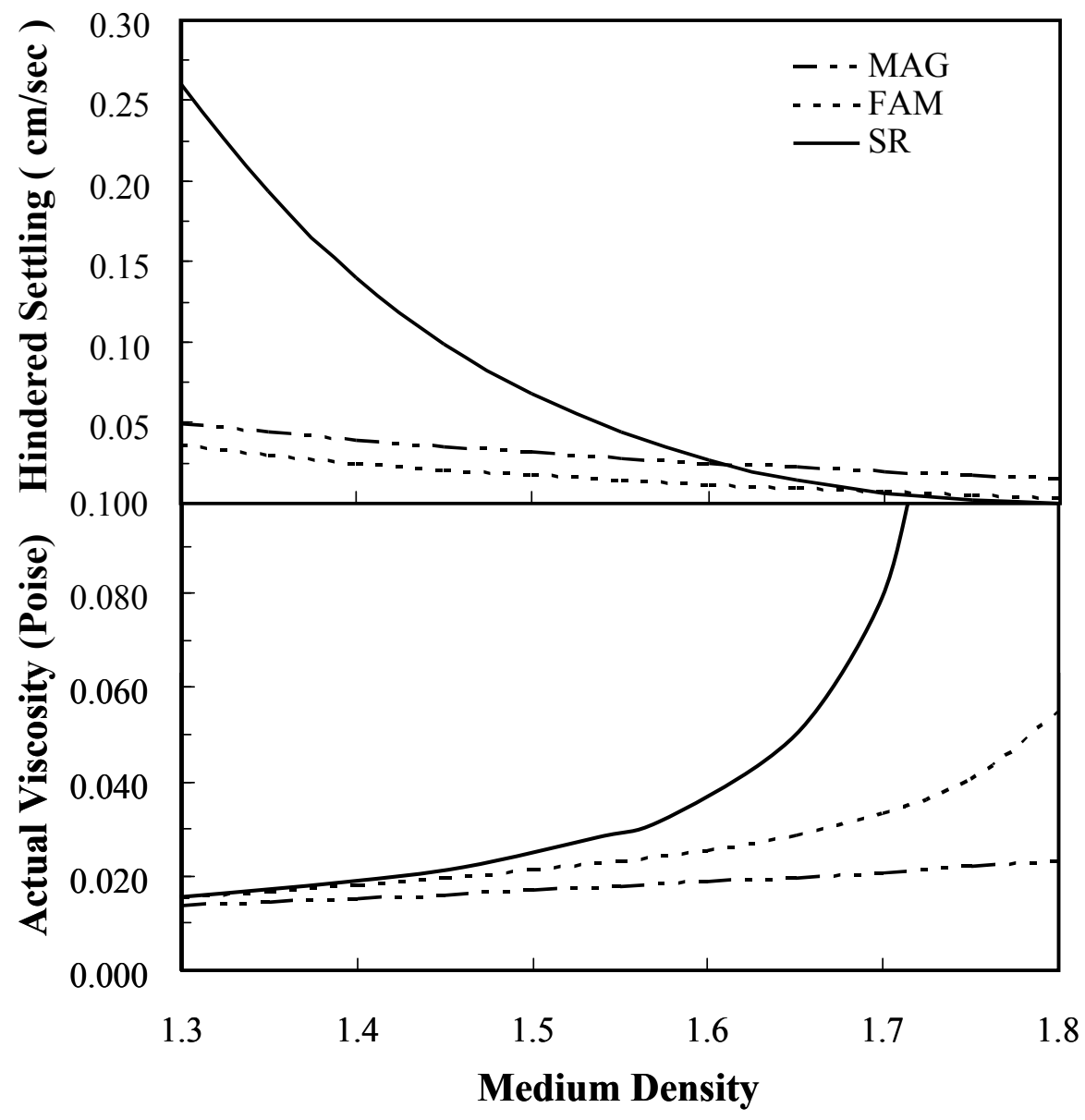

Figure 1. Predicted impact of the type of material used to develop a dense medium on medium stability (hindered settling rate) and medium viscosity; MAG: $\rho_{\mathrm{s}}=5.2$ and $P_{80}=36$ microns; FAM: $\rho_{\mathrm{s}}=3.6$ and $P_{80}=45$ microns; SR: $\rho_{\mathrm{s}}=2.45$ and $P_{80}$ $=170$ microns.

\section{$\underline{\text { Objective and Approach }}$}

The objectives of the proposal were i) to evaluate alternative materials that could be economically used as a substitute for the traditional magnetite in dense medium processes, ii) to develop and evaluate a process in which fine waste material may be used as whole or part of the material needed for adjusting medium density and iii) to provide an excellent educational experience for a graduate student that can be easily translated to industrial application. 


\section{EXPERIMENTAL}

\section{$\underline{\text { Dense Medium Cyclone Circuit }}$}

The tests were conducted using a $150 \mathrm{~mm}$ diameter Krebs dense medium cyclone (DMC) with a $63 \mathrm{~mm}$ diameter vortex and a 20-degree cone angle. The angle of inclination of the set up was 10 degrees from horizontal with inlet pressures operated between 17 to $69 \mathrm{kPa}$ ( 2.5 to $10 \mathrm{psi}$ ). The apex diameter was $4.5 \mathrm{~cm}$ and was maintained constant throughout the entire study.

The dense medium cyclone was set to operate in closed loop. Figure 2 shows the closed loop circuit set up of the dense medium cyclone. The feed to the cyclone is pumped from the feed sump with the overflow and underflow streams reporting back to the sump. A feed bypass also reported to the sump, which was used to collect samples of feed to cyclone. The feed pump was used to control the inlet feed pressure to the cyclone between 17 and $69 \mathrm{kPa}(2.5$ and $10 \mathrm{psi}$.

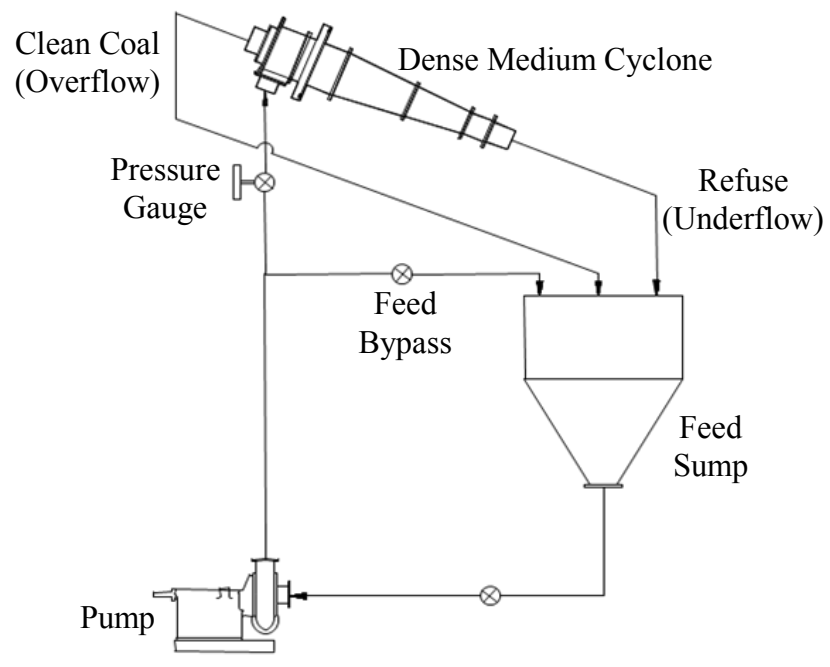

Figure 2. Schematic diagram of the dense medium cyclone circuit.

The evaluation of the material was conducted in two phases. The objective of the first phase was to evaluate the stability of the medium. The second phase evaluated the performances achieved using the medium when treating $6 \times 0.6 \mathrm{~mm}$ Coalburg coal, which has relatively difficult cleaning characteristics.

At the beginning of each stability test, the relative density of medium, which is a suspension of water and the high density magnetic material, was adjusted to the desired value in the circuit feed sump. The density was measured using a Marcy density scale. The medium was pumped to the cyclone at a pre-selected feed inlet pressure. A time interval of 10 minutes was allowed for the medium to stabilize. Samples were taken from the underflow and 
overflow streams to calculate the density differential between the two streams. The stability of the material was tested over a range of relative medium density values from 1.3 to 1.7 RD.

In the second phase of the experimental program, the medium was adjusted to the desired value and then the coal sample was added to the medium with the amount equivalent to achieve a medium-to-coal ratio of about 4:1. Again, a time interval of 10 minutes was allowed to ensure adequate mixing and steady state conditions. Samples from the overflow (clean coal and medium) and underflow (reject and medium) streams were taken simultaneously. Also, a feed sample was obtained from the bypass feed stream. The samples collected were wet screened using a $419 \mu \mathrm{m}$ (40 mesh) screen to separate the medium from the coal. Each coal sample was split into two factions for washability analysis and ash content. These results were used for data analysis and performance assessment of the medium

Previous research has shown the significant effect of centrifugal force on the separation performance achieved by DMCs. Near-gravity particles move slowly due to small density differences between the particle and the medium. As the centrifugal force is increased, the velocities of the near-density density particles are enhanced which improves the sharpness of the separation. However, elevated centrifugal forces can destabilize the medium suspension which leads to a deterioration in performance. Thus, an optimum centrifugal force or inlet pressure may exist for a given medium type having a characteristic particle size distribution. According to Bradley (1965) and later Zanker (1977), the centrifugal force applied within a DMC unit having standard dimensions varies with cyclone diameter $\left(d_{c}\right)$ as described by the expression:

$$
N_{g}=2 \alpha^{2} \frac{V_{i}^{2}}{D_{C} g}\left(\frac{D_{C}}{d_{C}}\right)^{(2 n+1)}
$$

where $\alpha$ is a modifying factor for inlet losses approximated by $3.7\left(D_{i} / D_{c}\right), D_{i}$ the cyclone inlet diameter, $D_{c}$ the cyclone diameter, $g$ the gravitational acceleration, $V_{i}$ the inlet velocity and $n$ a constant that normally varies between 0.5 and 0.8 . Equation (2) clearly shows that the centrifugal force in the $150 \mathrm{~mm}$ DMC used in this study is significantly greater than the typical DMC units in industry at the same inlet pressure.

To determine the inlet pressure needed to realize a centrifugal force in the $150 \mathrm{~mm}$ DMC unit that is nearly equal to the larger industrial units, a standard inlet pressure equivalent to 9 cyclone diameters was assumed which equates to about $41 \mathrm{kPa}$ for a $700 \mathrm{~mm}$ diameter cyclone. According to data generated from Eq. (5) and those reported by Mengelers (1982) the centrifugal force at this inlet pressure is approximately 36.9g's. Using Eq. (5), the centrifugal force over a range a range of inlet pressures was determined for two common DMC diameters (i.e., $700 \mathrm{~mm}$ and $1000 \mathrm{~mm}$ ) and a $150 \mathrm{~mm}$ diameter DMC (Figure 3). The inlet pressure providing an equivalent centrifugal force in the $150 \mathrm{~mm}$ cyclone is $31 \mathrm{kPa}(4.5$ psi). As such, most of the tests were conducted with an inlet pressure of $34.5 \mathrm{kPa}$ (5 psi) which provides a centrifugal force of $37.5 \mathrm{~g}$ 's while the remaining tests were performed over an inlet pressure range of $17.4 \mathrm{kPa}$ to $68.9 \mathrm{kPa}$. 


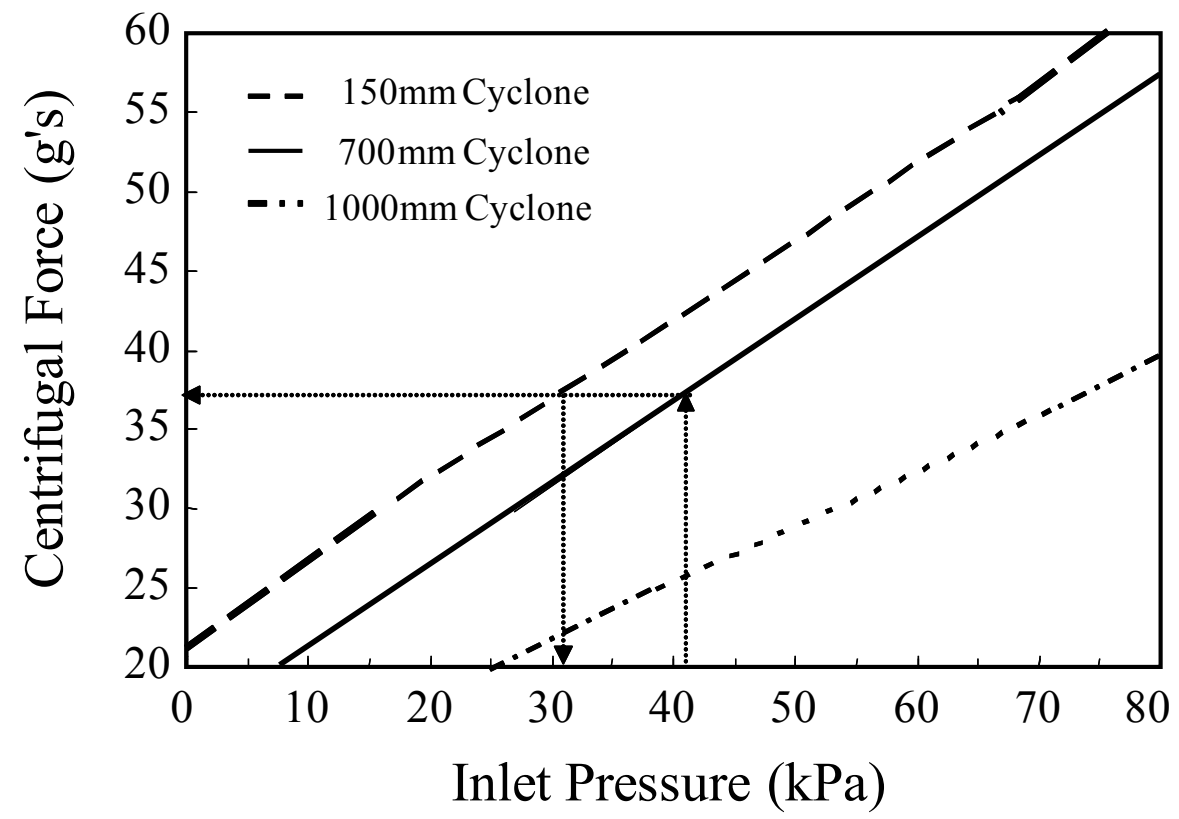

\begin{tabular}{|c|c|}
\hline \multicolumn{2}{|c|}{$\begin{array}{c}\text { Feed Pressure of Cyclone } \\
=9 \times 1 \\
\text { Mengelers }(1982)\end{array}$} \\
\hline $\begin{array}{l}\text { Cyclone } \\
\text { Diameter } \\
(\mathrm{mm})\end{array}$ & $\begin{array}{l}\text { Centrifugal } \\
\text { Force }\end{array}$ \\
\hline 250 & $54.9 \times \mathrm{g}$ \\
\hline 350 & $50.6 \times g$ \\
\hline 400 & $48.8 \times \mathrm{g}$ \\
\hline 500 & $42 . .2 \times g$ \\
\hline 600 & $39.4 \times \mathrm{g}$ \\
\hline 700 & $36.2 \times g$ \\
\hline 800 & $33.8 \times \mathrm{g}$ \\
\hline 1000 & $31.0 \times \mathrm{g}$ \\
\hline
\end{tabular}

Figure 3. Centrifugal force as a function of feed inlet pressure and cyclone diameter.

\section{$\underline{\text { Coal Sample }}$}

The bituminous coal sample was obtained from an operating coal processing plant treating coal extracted from the Coalburg seam located in southern West Virginia, USA. The samples were collected into four 208-liter drums. Upon arrival in the test facility, the samples were dry screened using $6 \mathrm{~mm}(1 / 4$-in) and $0.6 \mathrm{~mm}(28 \mathrm{mesh})$ screens. The $6 \times 0.6 \mathrm{~mm}$ fraction was retained while the material having a particle size greater than $6 \mathrm{~mm}$ was crushed using a laboratory hammer mill. The mill product was re-screened and the $6 \times 0.6 \mathrm{~mm}$ fraction combined with the previously obtained material. Coal particles that were smaller than $0.6 \mathrm{~mm}$ were discarded. The coal sample was mixed thoroughly and then used as the dense-medium cyclone feed material.

Representative samples were obtained to determine the particle size and density distribution of the $6 \times 0.6 \mathrm{~mm}$ coal. The wet screening results shown in Table 1 indicate that the majority of the total mass existed in the fractions greater than $1 \mathrm{~mm}$ in which the mass is equally distributed. The total ash content of the sample was $33.82 \%$ and the mineral matter was well distributed throughout the size fraction. The Coalburg seam coal typical has a low Hardgrove Index (i.e., 48) which means that the coal is difficult to break. This fact is important since the dense-medium circuit recycled slurry to maintain good suspension. The resistant to break limited particle size reduction as a result of recycling through a pump. 
Table 1. Characteristics of the Coalburg seam coal.

\begin{tabular}{||c|c|c|c|c|c||}
\hline \multicolumn{3}{|c|}{ Particle Size Distribution } & \multicolumn{2}{c|}{ Particle Density Distribution } \\
\hline $\begin{array}{c}\text { Particle Size } \\
\text { (mm) }\end{array}$ & $\begin{array}{c}\text { Weight } \\
(\%)\end{array}$ & $\begin{array}{c}\text { Ash } \\
(\%)\end{array}$ & $\begin{array}{c}\text { Specific } \\
\text { Gravity } \\
\text { Fraction }\end{array}$ & $\begin{array}{c}\text { Weight } \\
(\%)\end{array}$ & $\begin{array}{c}\text { Ash } \\
(\%)\end{array}$ \\
\hline+4.8 & 16.42 & 36.74 & 1.3 float & 27.96 & 3.56 \\
$4.8 \times 3.4$ & 18.76 & 34.70 & $1.3 \times 1.40$ & 22.33 & 11.68 \\
$3.4 \times 2.4$ & 22.26 & 34.45 & $1.40 \times 1.50$ & 10.24 & 25.98 \\
$2.4 \times 1.7$ & 18.26 & 30.97 & $1.50 \times 1.60$ & 6.45 & 37.72 \\
$1.7 \times 1.2$ & 15.46 & 27.98 & $1.6 \times 1.75$ & 4.81 & 45.56 \\
$1.2 \times 0.8$ & 4.54 & 34.73 & $1.75 \times 1.90$ & 4.85 & 54.73 \\
$0.8 \times 0.6$ & 1.71 & 36.72 & $1.90 \times 2.0$ & 2.35 & 63.07 \\
-0.6 & 2.26 & 57.84 & 2.0 sink & 21.02 & 84.53 \\
\hline Total & 100.00 & 33.82 & Total & 100.00 & 32.79 \\
\hline
\end{tabular}

The Coalburg coal seam is known to be a moderately difficult coal to clean based on relatively high quantities of mass existing in the $1.5 \times 2.0$ density fractions. Based on the washability data in Table 1 , the cleaning index (C.I. $=1.3$ cumulative weight $\%$ float $/ 1.6$ cumulative weight \% float) was determined to be less than 0.5 which indicates a very difficult-to-clean coal using differential densities. Over $15 \%$ of the feed mass is within \pm 0.1 relative density units for density values in the range of 1.4 and 1.9. The benefit to having a large amount of material in the middle density fractions is better data for partition curve development.

\section{$\underline{\text { Alternative Medium Materials }}$}

The tests evaluated five different dense medium materials, i.e., fine sand, steel slag, coarse magnetite, autogenous reject material and magnetic combustion residue. The magnetic combustion residue material was evaluated separately from the other four sources due to the unique recovery processes needed to recover the combustion residue and the time needed in the project to develop the recovery circuit. As such, the characterization and separation performance data and discussions are presented separately in this report.

Conventional grade B magnetite was used as the reference magnetite. The coarse magnetite was explored as an option when density separations greater than 1.6RD are targeted. The particle size distributions and specific gravities of each dense medium material including the conventional source are provided in Table 2. The particle size distributions of the sand, steel slag and autogenous material were made significantly coarser than the conventional magnetite to compensate for the lower solid densities. Low density materials require the addition of a greater volumetric amount of solid to achieve a given medium density, which elevates particle population and thus negative medium viscosity effects. Since viscosity is 
directly related to the amount of exposed particle surface area, increasing the particle size of the medium material can reduce viscosity, thereby allowing a high performance level.

Table 2. Particle size distribution and specific gravity of the alternative dense medium materials.

\begin{tabular}{|c|c|c|c|c|c||}
\hline \multirow{2}{*}{$\begin{array}{c}\text { Particle } \\
\text { Size } \\
\text { (micron) }\end{array}$} & \multicolumn{5}{|c||}{ Weight Distribution (\%) } \\
\cline { 2 - 6 } & Fine Sand & $\begin{array}{c}\text { Coarse } \\
\text { Magnetite }\end{array}$ & $\begin{array}{c}\text { Conventional } \\
\text { Magnetite }\end{array}$ & Steel Slag & $\begin{array}{c}\text { Autogenous } \\
\text { Material }\end{array}$ \\
\hline+150 & 0.26 & 17.91 & 0 & 0.54 & 52.56 \\
$150 \times 74$ & 48.85 & 32.23 & 0.5 & 46.92 & 36.98 \\
$74 \times 44$ & 29.48 & 14.45 & 4.95 & 22.49 & 6.6 \\
$44 \times 37$ & 6.34 & 6.44 & 7.04 & 6.70 & 2.74 \\
$37 \times 25$ & 6.83 & 5.88 & 13.83 & 6.08 & 1.05 \\
-25 & 8.24 & 23.09 & 73.68 & 17.25 & 0.07 \\
\hline Relative & & 5.2 & 4.9 & 3.8 & 2.2 \\
Particle & 2.6 & & & & \\
Density & & & & & \\
\hline
\end{tabular}

\section{Sand}

Fine sand was obtained from Belleview Sand and Gravel Inc., a local sand producer in Petersburg, Kentucky, U.S. The sand was used to assess the potential of using fine rock existing in the plant feed as an alternative dense medium. It is known in the early development of dense medium separations that sand was a popular dense medium material used for coal cleaning [2]. Upon arriving at the laboratory, the sand was wet screened using $150 \mu \mathrm{m}$ and $44 \mu \mathrm{m}$ Sweco screens. The $150 \times 44 \mu \mathrm{m}$ (100 x 325 mesh) fraction was retained as dense medium material while the $+150 \mu \mathrm{m}$ and $-44 \mu \mathrm{m}$ material from each screening step was discarded.

\section{Autogenous Medium}

Fine spiral reject was obtained from an eastern coal preparation plant located in eastern Kentucky. To obtain this material, the overflow of the high frequency screen currently treating the spiral reject was screened at $210 \mu \mathrm{m}(65-\mathrm{mesh})$ using a Sweco wet screen. Similar to the sand preparation, the underflow material was further screened at $44 \mu \mathrm{m}$. The $210 \mathrm{x}$ $44 \mu \mathrm{m}(65 \times 325 \mathrm{mesh})$ fraction was retained while the +210 and $-44 \mu \mathrm{m}$ material from each screening step was discarded. 


\section{Steel Slag}

The steel slag by-product was obtained from a steel production company, the Stein, Inc., Ashland, Kentucky, USA. Steel slag is used for cement manufacturing, concrete aggregates, fill, and glass manufacturing and in agriculture as a soil amendment. Steel slag is hard, dense material and contains significant amounts of free iron which provides its high density, hardness and magnetic susceptibility. Depending on the cooling method, three types of slag are produced, namely air-cooled, expanded, and granulated. When the slag is formed under controlled rapid cooling, the slag tends to be hard and dense, making it suitable for the dense medium application. This type of steel slag is called 'C-type', which has a higher iron content and solid density than the other slag types. Several million tons of C-type steel slag is produced each year in the U.S. and available at a relative low cost. The steel slag material used in the DMC tests was ground in a cage mill and subsequently screened to reach a top size of $150 \mu \mathrm{m}$. According to a Davis Tube test, $64.1 \%$ of the steel slag was magnetic and recoverable using a magnetic field strength of 700 gauss.

\section{Magnetic Coal Combustion Byproducts}

Research conducted by Baird et al. (1998) in Australia suggested the fly ash derived magnetite (FAM) to have potential advantages in terms of lower cost to the end user, greater medium stability at low separation densities, and utilization of waste by-product of coal fired power plant. Similar research by Sripriya et al. (2003) using magnetic fraction of flue dust (MFFD) as a substitute for magnetite showed satisfactory results.

Problems associated with magnetic fly ash are that the particles are unstable in a suspension due to their sphericity and the particles require stronger magnetic strengths to recover. However, Baird et al. (1998) found that grinding the fly ash reduces the sphericity sufficiently to allow a stable medium within a dense medium cyclone. Additionally, the magnetic portions were selectively liberated and thus the magnetic field strength needed for recovery was significantly reduced. However, high-density magnetic particles in fly ash are already extremely fine and grinding creates a significantly high surface area and thus a suspension that may be stable in part due to elevated viscosity.

A potentially better source of magnetic particles is the bottom ash material produced from a pulverized coal boiler. The pneumatic transport of particles through the boiler naturally causes the higher-density particles to concentrate in the boiler ash stream. The composition of bottom ash indicates the presence of Fe-rich magnetic material in the form of spheres mostly fused with Si and Al. The spherical magnetic particles in the bottom ash are coarser than those in the fly ash and thus can be ground to decrease sphericity. Since the initial particle size is coarser than those contained in fly ash, medium viscosity should be lower for the ground bottom ash material.

The initial efforts involved identifying a bottom ash source in which magnetic material can be economically recovered and suitably used in a dense medium suspension .Similar attempts had been made in the past on commercial scale to recover magnetic material but were unsuccessful due to economic constraints and stability issues. There is insufficient amounts 
of magnetic material in the combustion ash to economically justify the recovery of this material as a single product. The economic constraints led to the alternative of incorporating magnetite recovery into a process flowsheet in which several products of combustion ash were recovered. A process flowsheet referred to as FastFloat process was developed at the University of Kentucky Center for Applied Energy Research (CAER), which utilized physical beneficiation for recovering various marketable products from combustion ash.

It has been noted in the past that high iron content in combustion ash is associated with utilities that burn high sulfur coal in turn leading to higher magnetite production. The site selection for magnetite recovery was limited to utilities that burned high sulfur coal. The criteria for site selection was firstly to use a substrate that contained sufficient amounts of magnetic material, and secondly to select a site which either practiced ash beneficiation to recover marketable products in some form or planned to do so in future.

The evaluation suggested the use of material from the Mill Creek utility. The reasons for the selection are the existence of a commercial scale beneficiation process and the high magnetite yield relative to the other test sites. A bottom ash processing facility is in operation at Mill Creek which produces 80,000 tpy of construction grade fill sand from bottom ash and discharges $-49 \mu \mathrm{m}$ ( -100 mesh) fines as a reject stream. The addition of magnetite recovery into the process circuitry would enhance the overall economics of the beneficiation facility.

Mill Creek Station, a pulverized coal fired power plant, operated by Louisville Gas and Electric (LG\&E), began its commercial operation in 1972. It burns about 3.7 million tons of coal per year and is rated to produce $1717 \mathrm{MW}$ of power. All the units are fully scrubbed so as to meet the Clean Air Act's Phase II standards for sulfur-dioxide emissions. Bottom ash produced at this facility has historically been stored in an on-site storage pond along with hard coal and pyrite rejected from coal pulverisers. The bottom ash produced at this facility has higher density than that of other power plants, primarily attributed to the exclusive use of high sulfur coal fuel at this site. The high density of material combined with presence of pyrite and coal precluded the use of this ash as a lightweight aggregate, a product that has been produced at several other utility stations.

Market research by Charah Environmental Inc, an ash management company based in Louisville and LG\&E, identified that the material has a significant potential to be utilized as graded backfill for sanitary sewer construction projects in Louisville area. Bottom Ash is currently being processed at Mill Creek by Charah Environmental Inc., utilizing a process which was jointly developed with CAER. This facility has been in operation since 2002 and has successfully marketed about 500,000 tons of graded fill sand produced from the bottom ash at Mill Creek.

The boilers of the plant produced bottom ash which was sluiced into a trench that flowed to the ash pond. The trench was excavated periodically with a track hoe and the excavated material was stockpiled and allowed to drain. The material in the trench near the sluice entry point contained pyrite which is not suitable for beneficiation. So the other material was transported by front end loaders to a feeder hopper and conveyed to the process facility where the oversize $(+3 / 8$ inch) material was rejected. The $-3 / 8$ inch ash was dropped into a 
sump containing screw classifier to dewater the ash. The finished fill sand was conveyed to a stockpile. The dilute slurry which came out from the sump as overflow contained $-150 \mu \mathrm{m}$ (100 mesh) solids, which flow to a separate reject containment area. The material was periodically excavated from this area again using a track hoe and stockpiled so that the fine solids drained out. These stockpiled fines were being used as kiln feed by a nearby cement manufacturing plant named Cemex's Cosmos Plant. This material was used as substrate for magnetite recovery.

The stockpiled fines, which were used as substrate, contained high density magnetic material. The magnetic material was recovered using the process flowsheet as shown in Figure 4. A Bobcat loader was used to excavate the stockpiled fines and dump the material into a feeder hopper. The feeder hopper conveyed it into an agitated mix tank where water was added to make the pulp density $10 \%$ solids by weight. The slurry was fed to a single bank of concentrating spirals at a rate of 35 gpm using a feed pump.

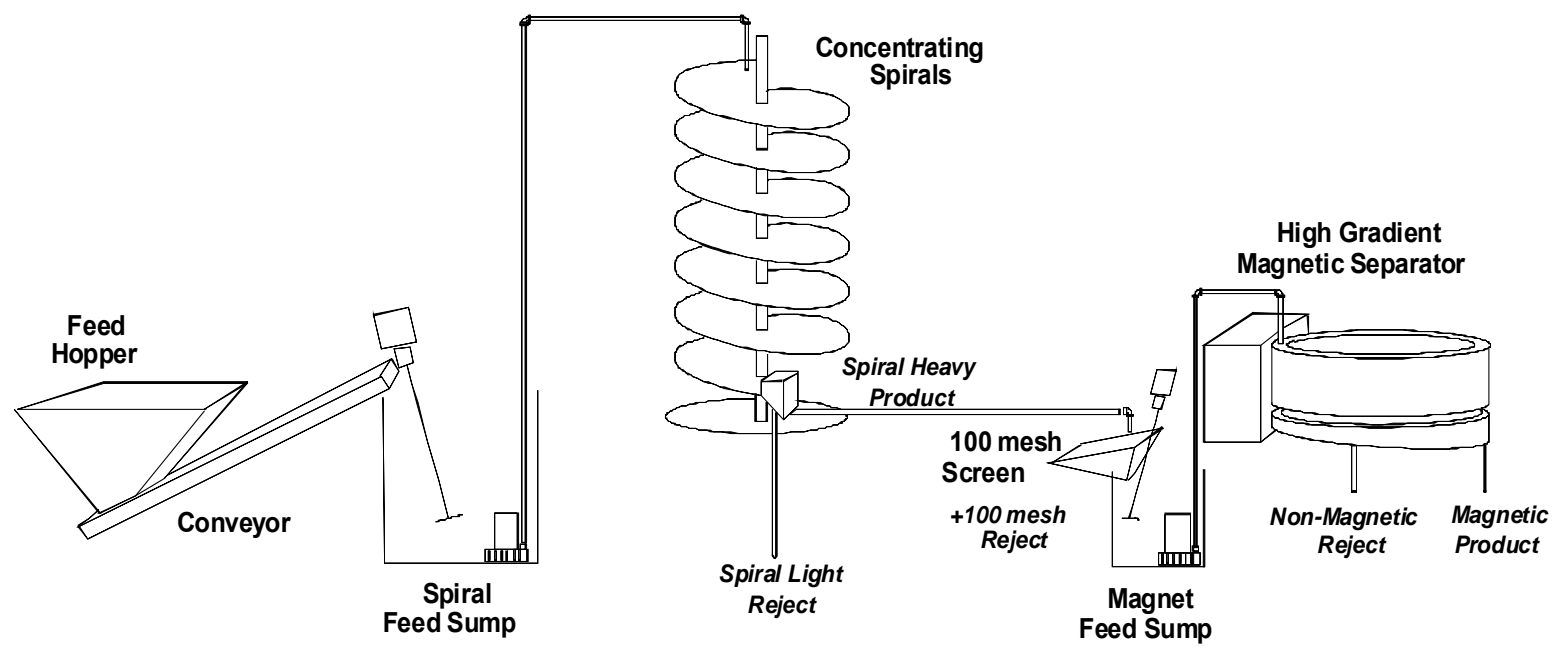

Figure 4. Magnetite recovery process flowsheet.

As the slurry flowed down the spiral, dense magnetite concentrated on the innermost section of race and was collected using a splitter at the bottom of the spiral. The light weight ash and water reported to the outer section of the race which was diverted back to the ash pond as reject. A $150 \mu \mathrm{m}$ (100 mesh) vibrating screen was used to remove the coarser particles from the spiral product. The screened magnetic material was fed to a continuous High Gradient Magnetic Seperator (HGMS) to remove the entrained non-magnetic material.

Samples were collected at each process stage to evaluate the incremental change in the magnetic content of the material. The results obtained during the recovery process are summarized in Figure 5. All the collected samples were dried and weighed, and a representative sample was subjected to Davis Tube Magnetic Separation (DTMS). The weight of material retained in the Davis Tube after two passes was defined to be magnetic. Figure 5 shows the grade of each stream, the incremental yield of each separation process and the overall yield as a weight percent of feed to the spirals. 


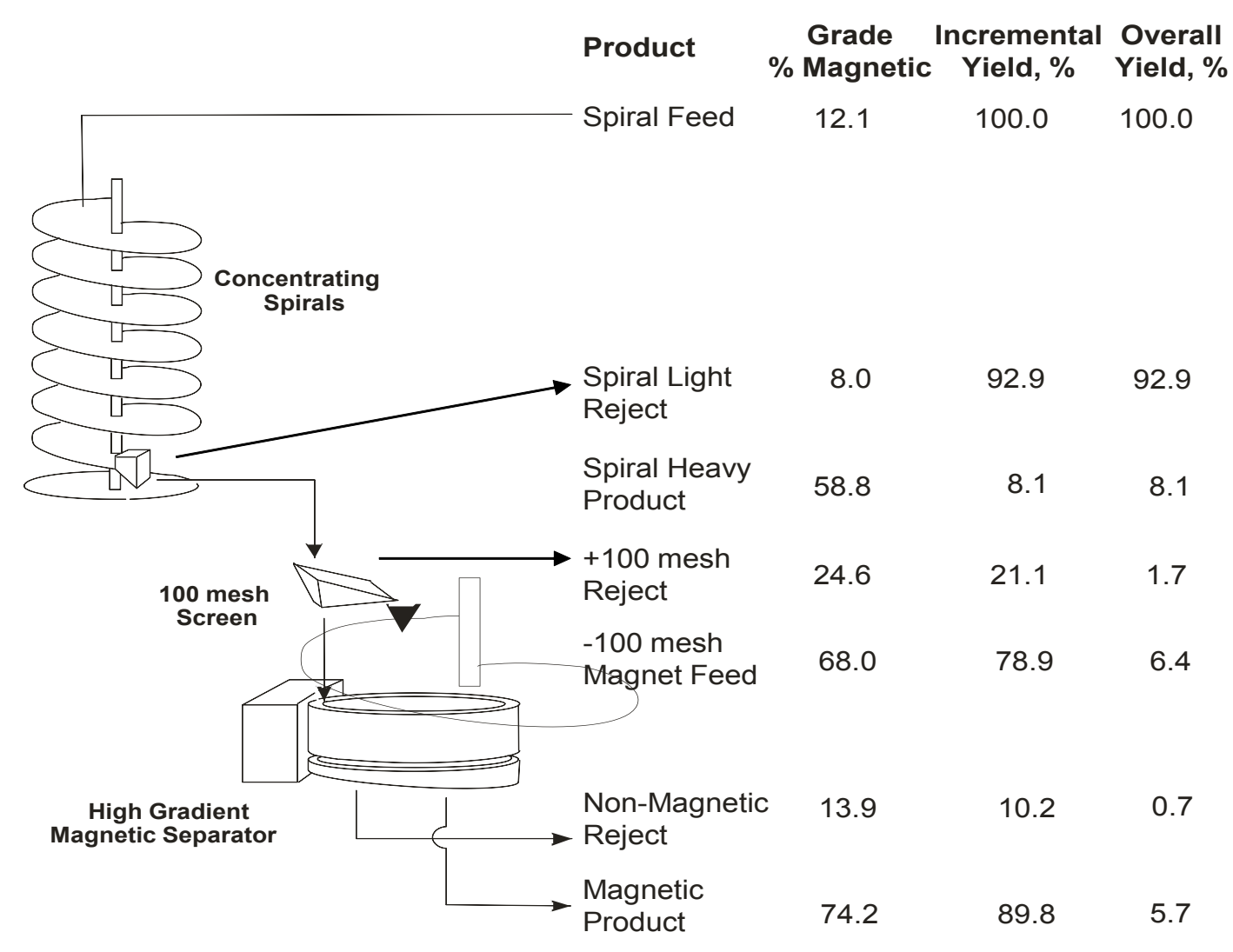

Figure 5. Material balance and magnetic content of the products produced by the magnetic recovery circuit.

The concentrating spiral upgraded the magnetic content from $12.1 \%$ to $58.8 \%$ and rejected a stream of low density ash and water with a magnetic content of about $8 \%$. The overall yield of classification process was $8.1 \%$. Further removal of $+150 \mu \mathrm{m}$ (100 mesh) particles led to the up-gradation of magnetics to $68 \%$ with an incremental yield of $78.9 \%$, resulting in an overall yield of $6.4 \%$. The final stage of magnetic upgrading using a HGMS gave a product which contained $74.2 \%$ magnetic in the recovered material. The overall yield of the magnetic bottom ash recovery process was $5.7 \%$ or $114 \mathrm{lbs}$ per ton of process feed.

Bottom Ash Characterization: Three representative bottom ash samples were used to determine the average solid density. The samples were weighed and mixed into a measured volume of water. The mass of each sample divided by the incremental volume increase when added to the water was used to quantify solid density. The average density from three measurements was $3.44 \mathrm{~g} / \mathrm{ml}$.

Particle size analysis was performed on a representative sample of the HGMS product using a CILAS Particle Size Analyzer. As shown in Table 3, about 87\% of the recovered HGMS product had a particle size less than $74 \mu \mathrm{m}$. This is significantly coarser than conventional magnetite in which $90 \%$ of the particles are less than $44 \mu \mathrm{m}$. As a result of its relatively 
coarse particle size and its spherical shape, particle size reduction was required to obtain a stable medium.

Table 3. Particle size distribution of the magnetic HGMS product.

\begin{tabular}{|c|c|}
\hline $\begin{array}{c}\text { Particle Size } \\
\text { (micron) }\end{array}$ & $\begin{array}{c}\text { Weight } \\
(\%)\end{array}$ \\
\hline+150 & 4.59 \\
$150 \times 74$ & 50.16 \\
$74 \times 44$ & 32.71 \\
$44 \times 37$ & 5.61 \\
$37 \times 25$ & 5.07 \\
-25 & 1.86 \\
\hline
\end{tabular}

Ball Mill Grinding: The preliminary analysis indicated that the particle size distribution of the HGMS product needed to be altered to lower size fractions before its use for evaluation in dense medium separation. A ball mill circuit as shown in the Figure 6 was set up to grind the material. The material was dry fed using a belt feeder and mixed with calculated amounts of water to maintain the pulp density at $33 \%$ solids by weight. The active grind volume in the ball mill was calculated to be $12 \%$ of the total volume. The retention time of material in the ball mill was controlled by controlling the feed rate. A representative sample was collected to analyze the change in the particle size distribution as a result of grinding.

Retention time in the ball mill was defined as the time taken by a particle to travel across the ball mill from feed end to the product end. The ball mill circuit set up was operated in open circuit with no recycle. The retention time was directly controlled by the feed rate to the ball mill. Lower feed rates increased the retention time which in turn increased the chances of the material to be broken, and thus reduce the particle size. 


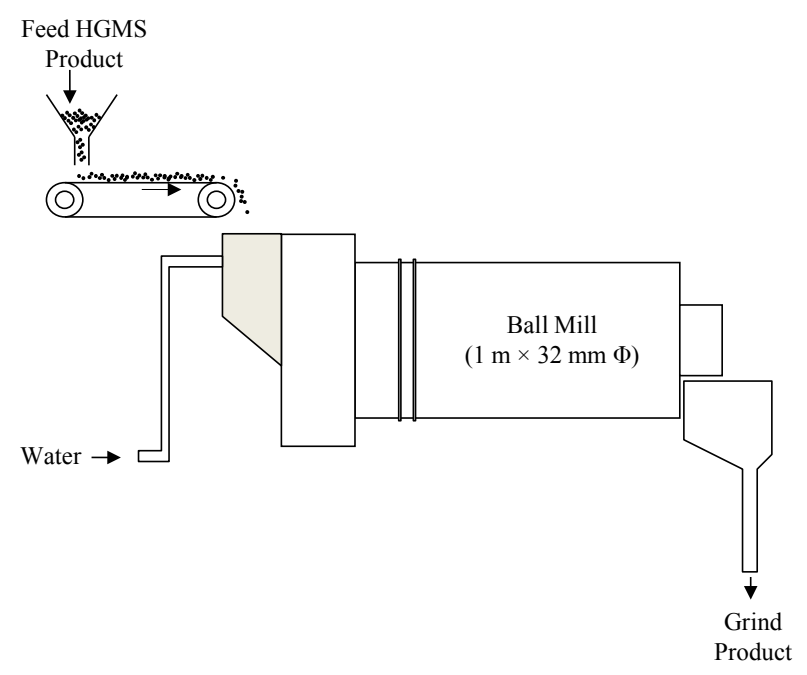

Figure 6. Schematic diagram of the ball mill circuit set up.

Figure 7 shows the cumulative passing of material as a function of particle size fraction for different grind variants produced as a result of grinding in the ball mill. Each grind variant represents a certain cumulative retention time in ball mill. Suspension stability was qualitatively assessed during the grinding trials. A residence time of 52 minutes was required to achieve good medium stability, which provided a mean particle size of about $22 \mu \mathrm{m}$ and a $\mathrm{d}_{90}$ of about $48 \mu \mathrm{m}$.

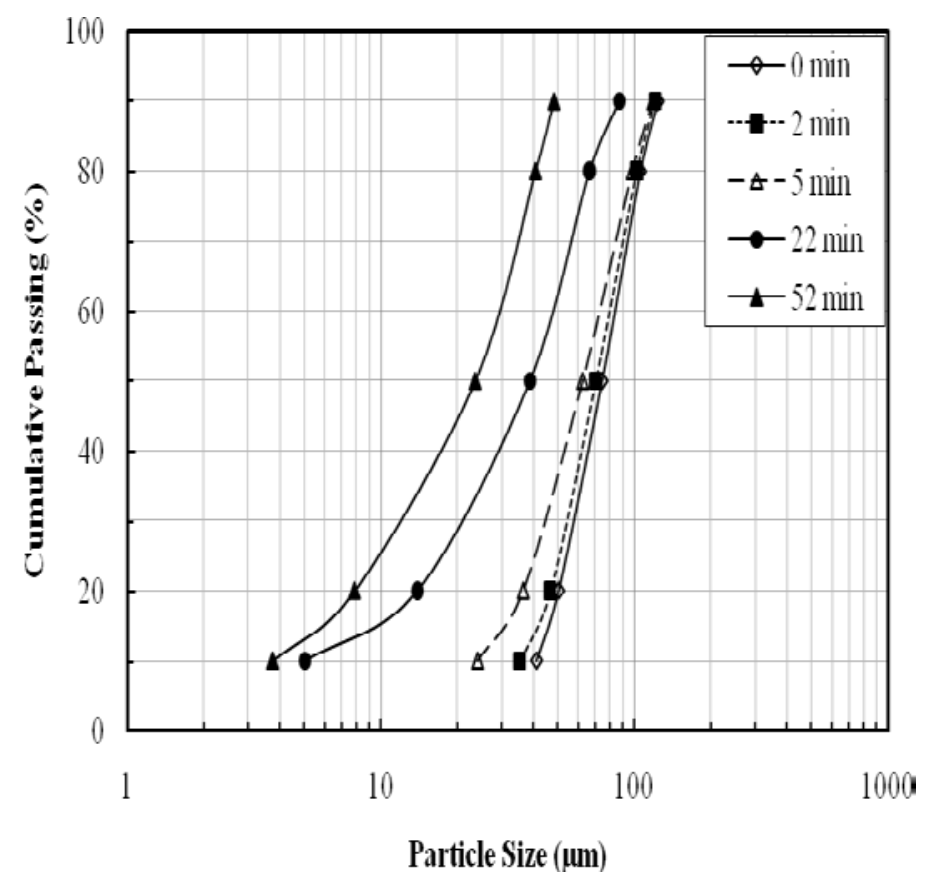

Figure 7. Change in particle size distribution with increased retention time in ball mill. 
The change in particle shape due to grinding is significant as indicated by the micrographs in Figure 8. As shown, the sphericity of the particles was significantly reduced.

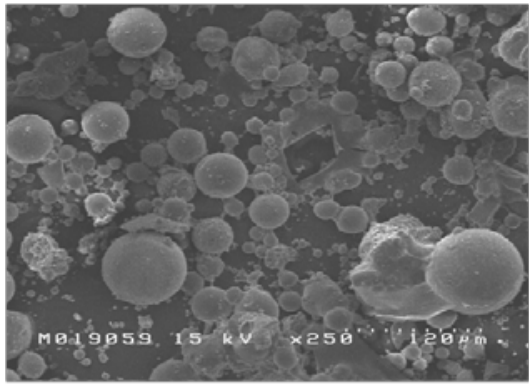

Original

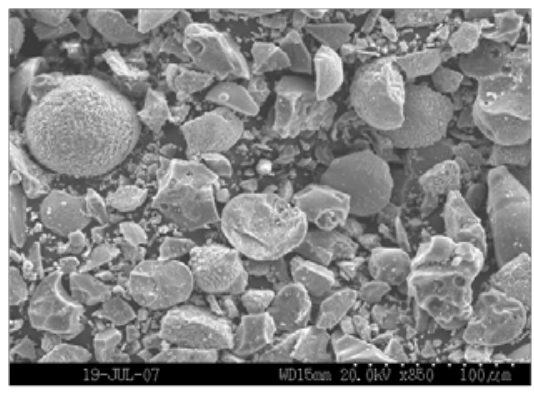

10 min grind

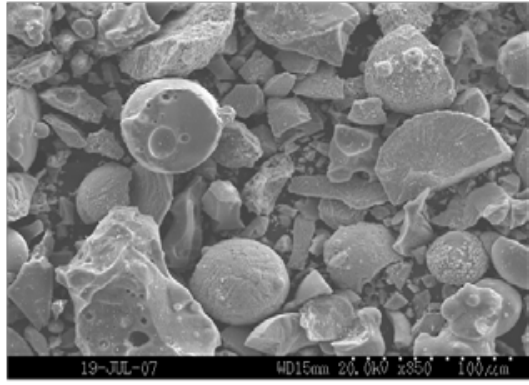

5 min grind

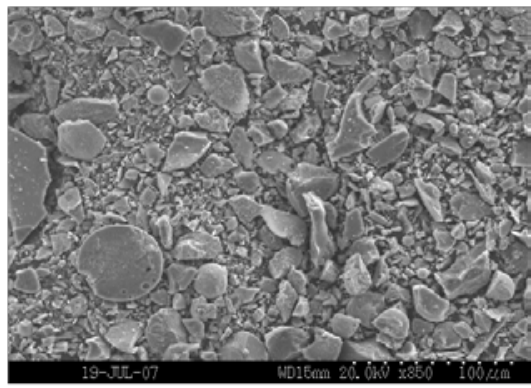

15 min grind

Figure 8. Effect of grinding on particle shape of the magnetic coal combustion byproduct..

\section{RESULTS AND DISCUSSION}

\section{$\underline{\text { Reference Magnetite Performance }}$}

Prior to the evaluation of each alternative material, tests were performed using the grade B magnetite. Medium stability was evaluated in the absence of coal by measuring the difference in the pulp density of the overflow and underflow streams of the DMC under inlet pressures ranging from 34.5 to $68.9 \mathrm{kPa}$ (2.5 to $10.0 \mathrm{psi}$ ). Generally, optimum separation performance is achieved when the relative density difference is maintained at or below 0.40 density units. As shown in Figure 9, stability of the conventional grade B magnetite suspension improved significantly with an increase in medium density and a decrease in inlet pressure. The trend associated with medium density is due to an increase in particle population, which reduces the hindered settling rates of the magnetite particles. Although hindered settling is beneficial for medium stability, another effect realized at high medium densities is an elevation in medium viscosity, which has negative impacts on separation performance. 


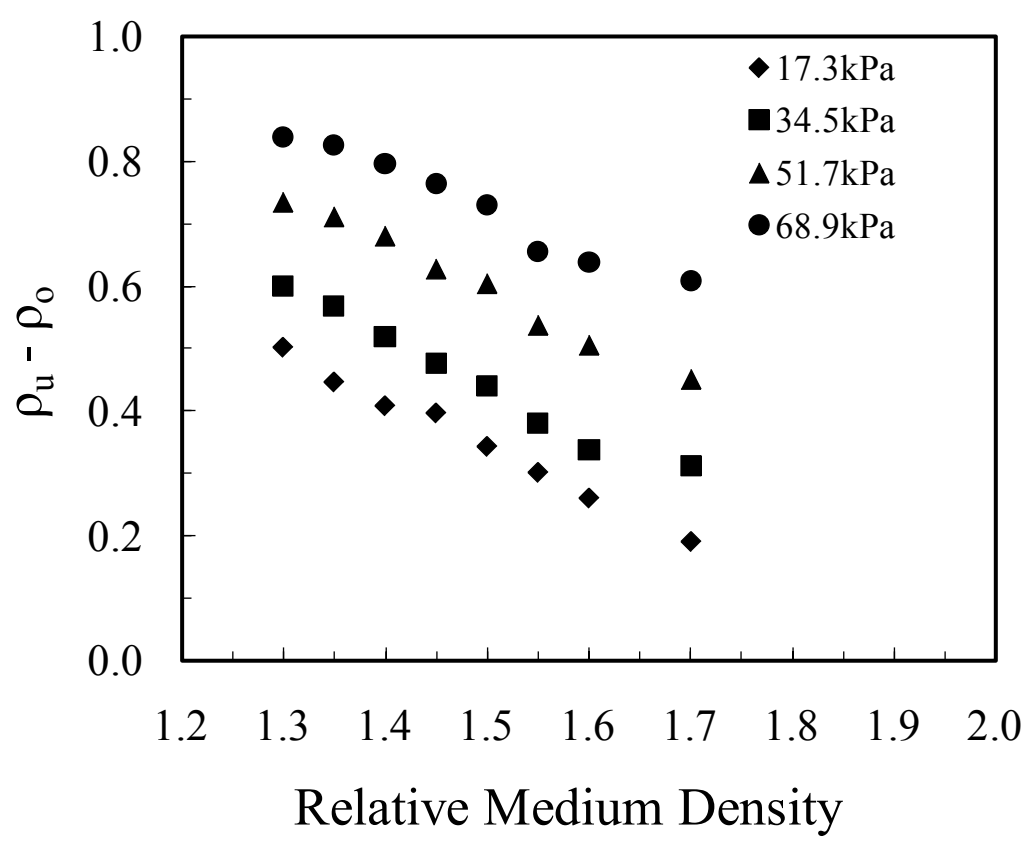

Figure 9. Medium stability achieved by grade B magnetite.

The tests involving the cleaning of the Coalburg coal were conducted over a range of medium density values and inlet pressures. The separation performances achieved using four different medium density values at an inlet pressure of $34.5 \mathrm{kPa}$ are represented by partition curves in Figure 10. The sharpest curves and thus highest separation efficiencies were obtained when using medium densities of 1.5RD and 1.6RD which correspond to improved medium stability as indicated in Figure 9.

The stability of the magnetite suspension when using a medium density of 1.4RD does not meet industrial standards $\left(0.4<\rho_{\mathrm{u}}-\rho_{\mathrm{o}}\right)$ which is likely due to slightly elevated centrifugal forces in the $150 \mathrm{~mm}$ cyclone used in the study. As such, the partition curve is less sharp. Likewise, a lower efficiency is indicated by the $1.7 \mathrm{RD}$ data in Figure 10. In this case, the reduced efficiency is most likely due to elevated medium viscosity which provides a resistance to particle movement. The level of coal cleaning achieved at each medium density is shown in Table 4 . The product ash values achieved when using the reference magnetite ranged from $9.36 \%$ to $14.89 \%$. The large range in mass yield and recovery values is reflective of the difficult cleaning characteristics of the Coalburg coal. 


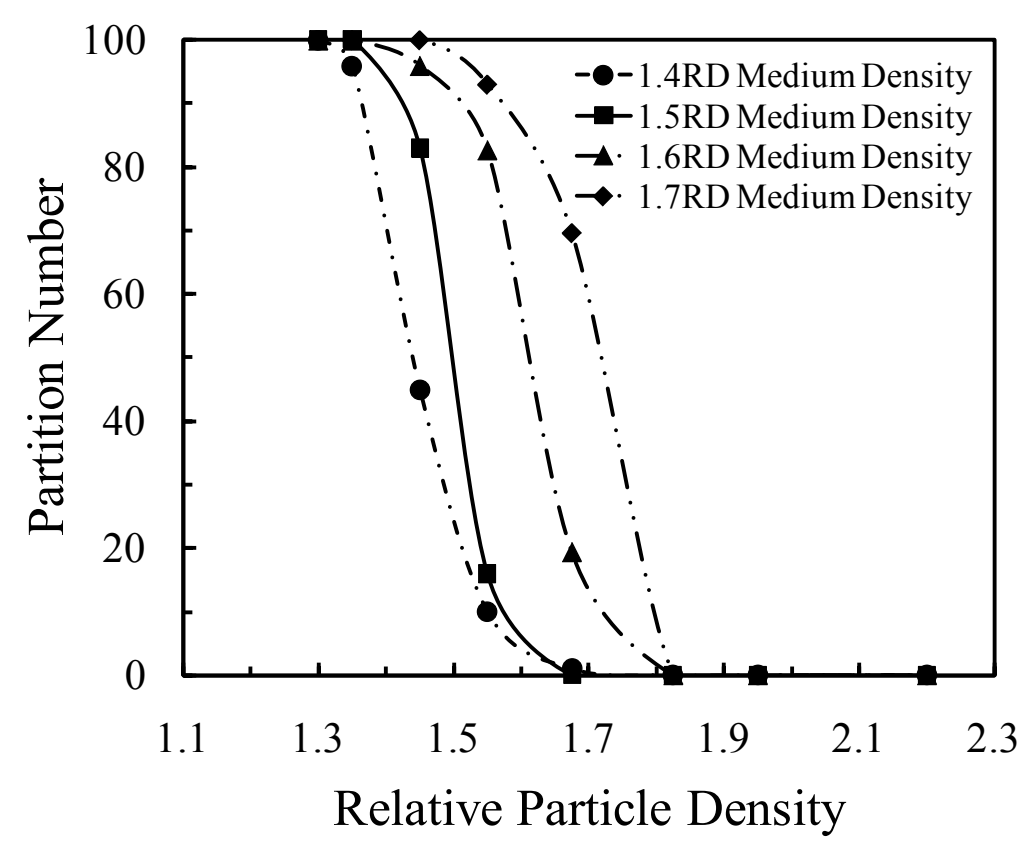

Figure 10. Partition curves achieved using the reference magnetite.

Table 4. Overall separation performances achieved with reference magnetite.

\begin{tabular}{|c|c|c|c|c|}
\hline \multirow{2}{*}{ Variable } & \multicolumn{4}{|c|}{ Relative Medium Density } \\
\cline { 2 - 5 } & 1.4 & 1.5 & 1.6 & 1.7 \\
\hline Feed Ash (\%) & 33.89 & 35.05 & 36.73 & 33.81 \\
Product Ash (\%) & 9.36 & 11.06 & 13.81 & 14.89 \\
Tailings Ash (\%) & 61.41 & 65.71 & 75.29 & 76.41 \\
\hline Yield (\%) & 52.9 & 56.1 & 66.0 & 69.3 \\
Recovery (\%) & 72.5 & 76.9 & 87.5 & 89.0 \\
Organic Efficiency (\%) & 91.3 & 92.9 & 95.7 & 96.7 \\
\hline$\rho_{50}$ & 1.440 & 1.525 & 1.615 & 1.73 \\
$\mathrm{E}_{\mathrm{p}}$ & 0.041 & 0.045 & 0.061 & 0.62 \\
$\rho_{50}-\rho_{\text {medium }}$ & 0.040 & 0.020 & 0.015 & 0.03 \\
\hline
\end{tabular}

\section{$\underline{\text { Alternative Medium Performance }}$}

\section{Sand, Autogenous and Coarse Magnetite Medium}

Stability and rheology are the two principal medium properties affecting DMC performance and both are strongly dependent on the physical characteristics of the solids comprising the medium. As expected, the coarse magnetite provided very unstable medium with a highly concentrated underflow and a much diluted overflow. However, acceptable stability standards were achieved as the medium density was increased beyond $1.7 \mathrm{RD}$. The 
alternative materials (sand, steel slag and autogenous mediums) provided better medium stability than the reference magnetite. As shown in Figure 11, the density differentials at an inlet pressure of $34.5 \mathrm{kPa}$ ( $5 \mathrm{psi}$ ) were much lower than that of the reference magnetite over the entire medium density range studied. This was mainly due to the lower particle density of the alternative materials which required the addition of a greater volume of material to achieve the same medium density. High volumetric solid concentrations induce hindered settling which aid stability but eventually initiates viscosity effects that negatively impact separation performance.

The higher level of medium stability appeared to be the key in reducing the density offset. Under most conditions, the separation densities provided by the alternative material mediums were nearly equal to the medium density. On the other hand, the density offsets achieved by the reference magnetite-based medium were similar to the typical industrial standards for dense medium cyclones.

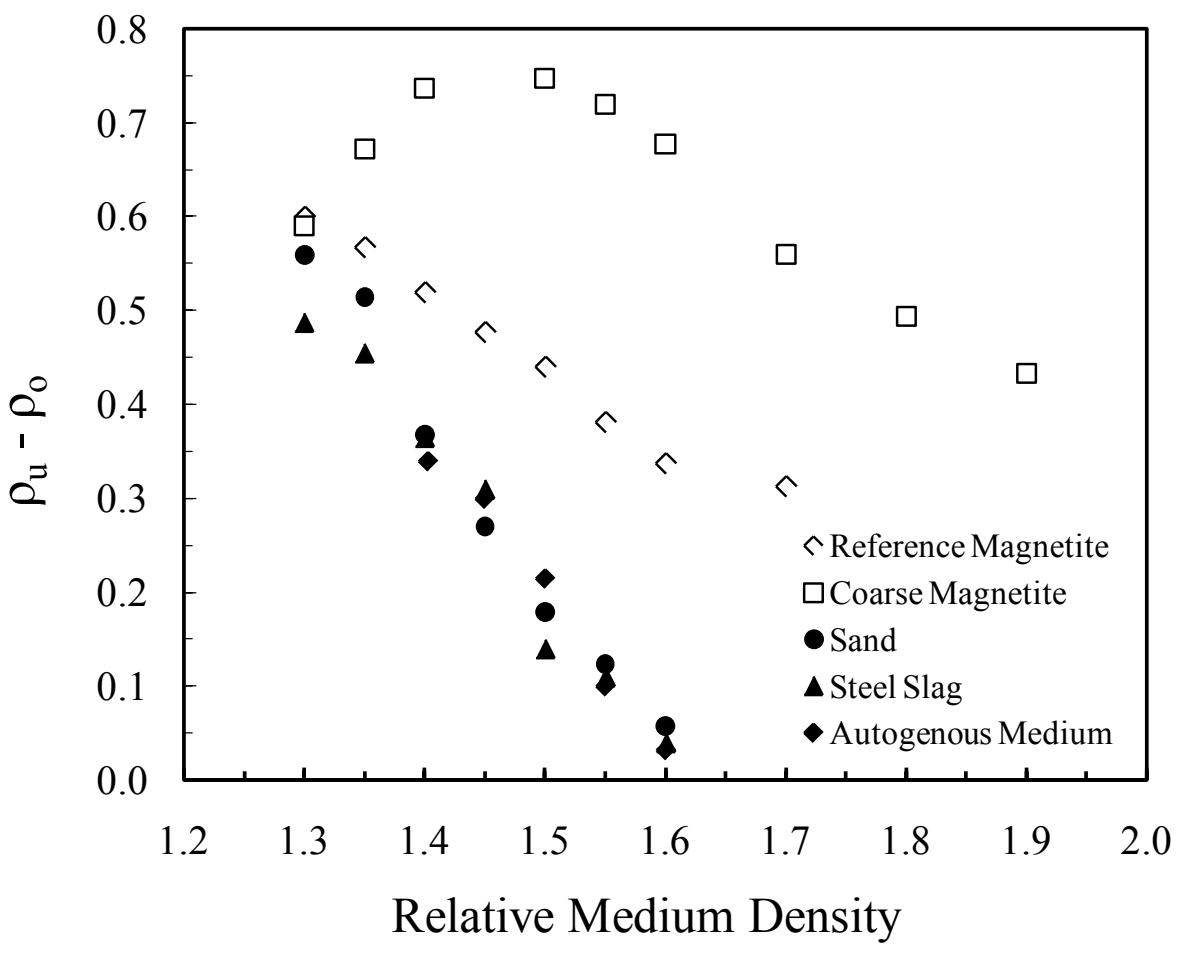

Figure 11. Medium stability as a function of alternative material and medium density at $34.5 \mathrm{kPa}$ (5 psi) feed inlet pressure.

Typical medium densities used in the U.S. coal industry to produce steam coal products are between 1.5 RD and 1.6 RD. As shown in Figures 12 and 13, the separation performances achieved by the alternative materials at 1.5 and 1.6 medium densities were comparable to the reference magnetite with $E_{p}$ values of 0.02 to 0.05 . The partition curves generated by the alternative materials at 1.5 medium density were much sharper than the 1.6 medium density. This suggested the impact of negative viscosity issues at the higher medium densities with the exception of coarse magnetite. 


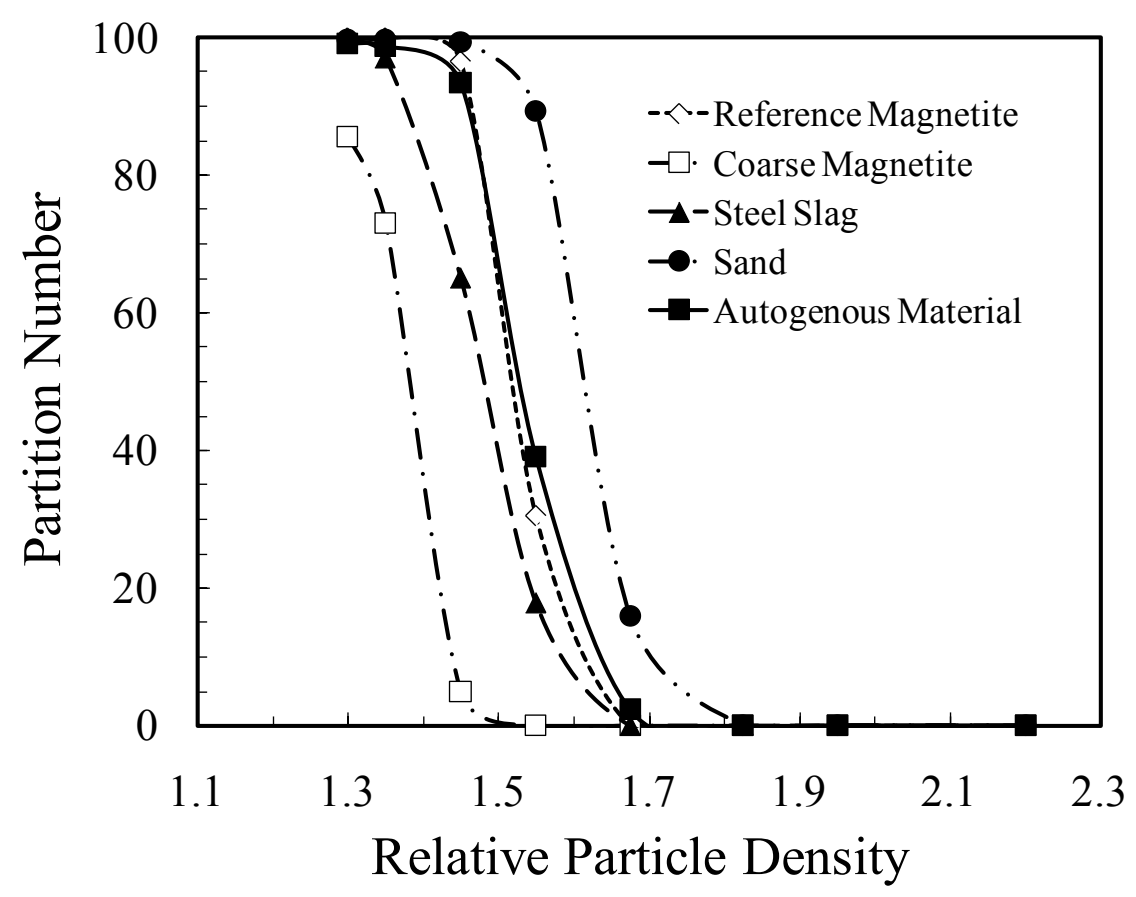

Figure 12. Comparison of separation performance achieved by alternative materials using a medium density of $1.5 \mathrm{RD}$ and feed inlet pressure of $34.5 \mathrm{kPa}$ (5 psi).

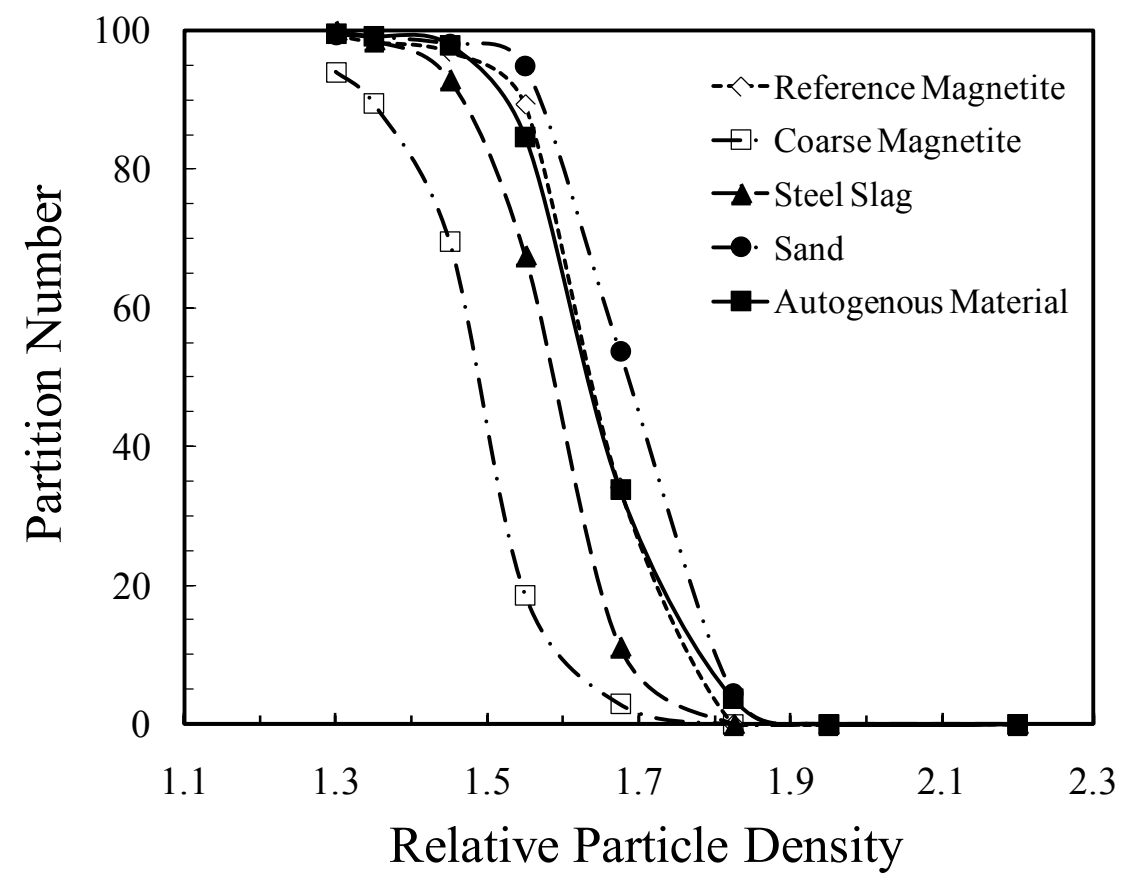

Figure 13. Comparison of separation performance achieved by alternative materials using a medium density of $1.6 \mathrm{RD}$ and an inlet pressure of $34.5 \mathrm{kPa}(5 \mathrm{psi})$. 
With dense media consisting of sand, steel slag, autogenous medium and reference magnetite, the Ep values tend to increase with medium density as expected due to viscosity effects (Figure 14). Surprisingly, the separation efficiency achieved by sand, steel slag and autogenous dense mediums provided $E_{p}$ values similar to the reference magnetite despite their significantly larger particle size distribution. It is believed, that at low medium densities, the relative coarseness of the sand, steel slag and autogenous mediums minimizes the viscosity effects and provides a stable medium due to hindered settling. At high medium density values, separation performance deteriorated due to medium viscosity and yield stress issues. For medium density values greater than 1.6RD, the coarse magnetite seems to become a more attractive option due to a stable, non-viscous medium.

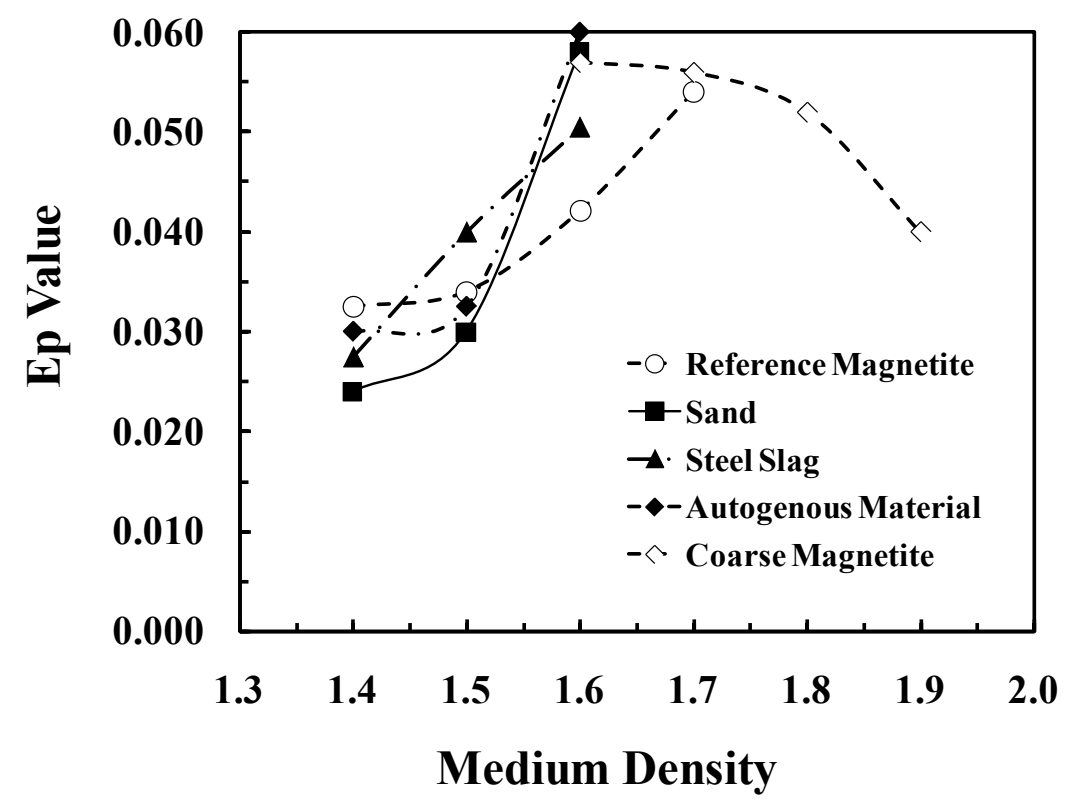

Figure 14. Comparison of the probable error values achieved by the various medium types using an inlet pressure of $34.5 \mathrm{kPa}$ (5 psi).

A summary of selected optimum performances achieved by the alternative materials is provided in Table 4. These performances were near perfect as indicated by the organic efficiency values (= actual yield/theoretical yield*100). One may question the performance achieved by the reference magnetite since the organic efficiency and $E_{p}$ values fall below standards that are commonly achieved in industrial practice. However, the performances obtained using the non-magnetite sources are consistent and would be acceptable in an operating preparation plant. Using coarse magnetite below 1.6RD is not acceptable due to medium stability issues. However, if a plant is often required to vary the medium density from low to high values, there may be a scenario where coarse magnetite provides an optimum solution. As shown in Table 4, the coarse magnetite provides a very good separation performance at a medium density of 1.9RD. 
Table 5. Optimized dense medium separation performances achieved by the alternative materials.

\begin{tabular}{||c|c|c|c|c|c||}
\hline \multirow{2}{*}{ Variable } & \multicolumn{4}{|c|}{$1.5 \mathrm{RD}$} & 1.9 RD \\
\cline { 2 - 6 } & Ref. Mag. & Sand & Steel Slag & $\begin{array}{c}\text { Autogenous } \\
\text { Medium }\end{array}$ & $\begin{array}{c}\text { Coarse } \\
\text { Magnetite }\end{array}$ \\
\hline Feed Ash (\%) & 35.03 & 33.64 & 33.54 & 36.65 & 39.09 \\
Product Ash (\%) & 11.06 & 10.59 & 8.57 & 9.64 & 18.45 \\
Tailings Ash (\%) & 65.71 & 66.32 & 62.35 & 67.88 & 87.15 \\
\hline Yield (\%) & 56.1 & 60.1 & 53.6 & 53.62 & 70.0 \\
Recovery (\%) & 76.9 & 78.2 & 73.7 & 76.49 & 93.7 \\
Organic Efficiency (\%) & 92.9 & 96.3 & 97.4 & 97.2 & 98.5 \\
\hline$\rho_{50}$ & 1.525 & 1.530 & 1.495 & 1.518 & 1.91 \\
$\mathrm{E}_{\mathrm{p}}$ & 0.045 & 0.042 & 0.040 & 0.033 & 0.040 \\
$\rho_{50}-\rho_{\text {medium }}$ & 0.02 & 0.03 & -0.005 & 0.018 & 0.01 \\
\hline
\end{tabular}

\section{Magnetic Coal Combustion Byproduct}

Stability: The medium formed by the original recovered material was unstable with density differential values being over 0.8 density units. As shown in Figure 15, medium stability deteriorated with an increase in medium density. The shape of the bottom ash particles was observed to be spheroidal with a relatively coarse particle size distribution when compared to

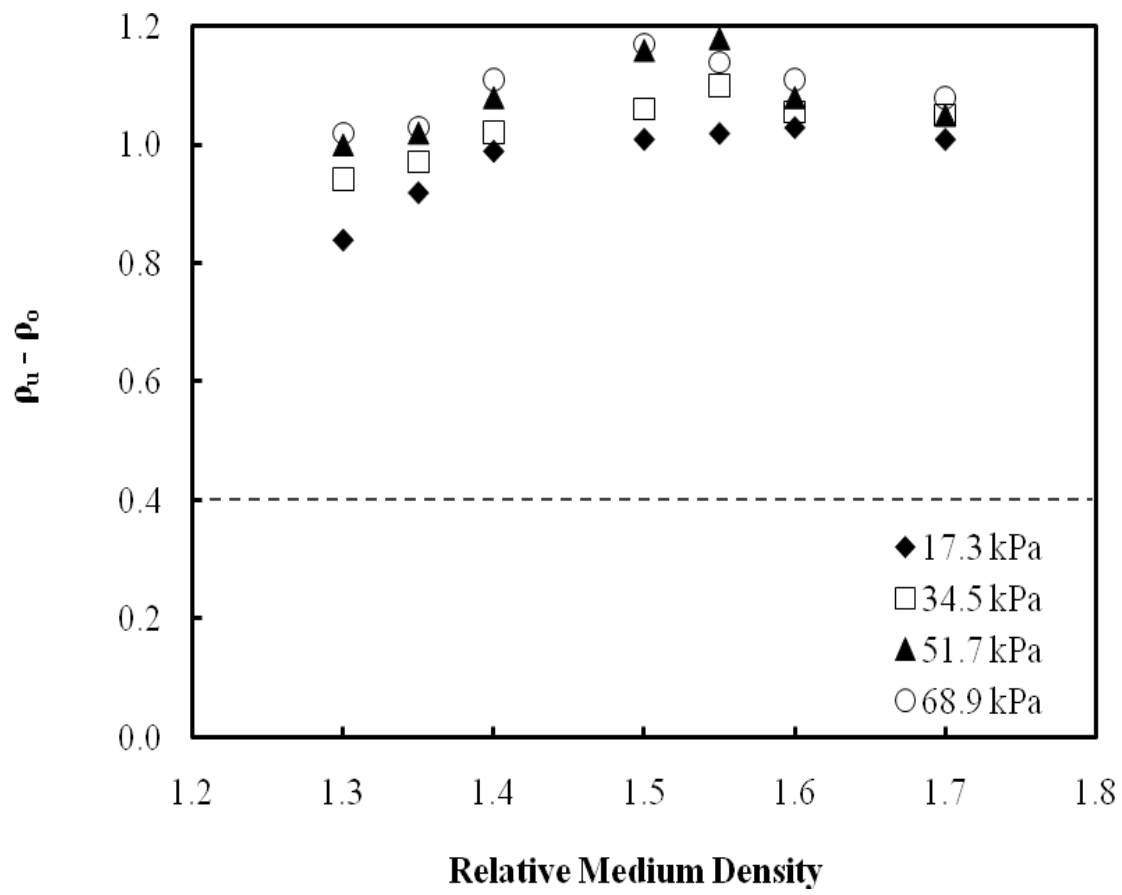

Figure 15. Medium stability achieved using the un-ground magnetic bottom ash. 
conventional magnetite used for coal cleaning. Spheroidal particles tend to exhibit lower drag forces and viscosities for a given particle density and size than irregularly shaped material (Collins et al., 1974). Another effect observed was that an increase in the inlet pressure to the cyclone had a negative impact on the stability at medium density less than 1.6 RD. The increase in inlet pressure elevated the centrifugal forces in the cyclone resulting in medium classification. At a relative medium density over $1.6 \mathrm{RD}$, pressure change had almost no effect on stability of the medium. This can be attributed to the combined role of increase in medium viscosity due to the high particle concentration and elevated hindered settling effects.

The medium formed using the ground bottom ash material was observed to be significantly more stable with relative density differences being within the industrial acceptable levels of around 0.4 density units and less (Figure 16). With increase in relative medium density, the suspension provided for improved stability. The particle size distribution of the ground material is nearly equal to that provided by conventional grade B magnetite used for coal cleaning. Enhanced stability with an increase in medium density occurs due to the development of hindered settling conditions as a result of an increased particle population. Also, elevated viscosity also helps to stabilize the suspension.

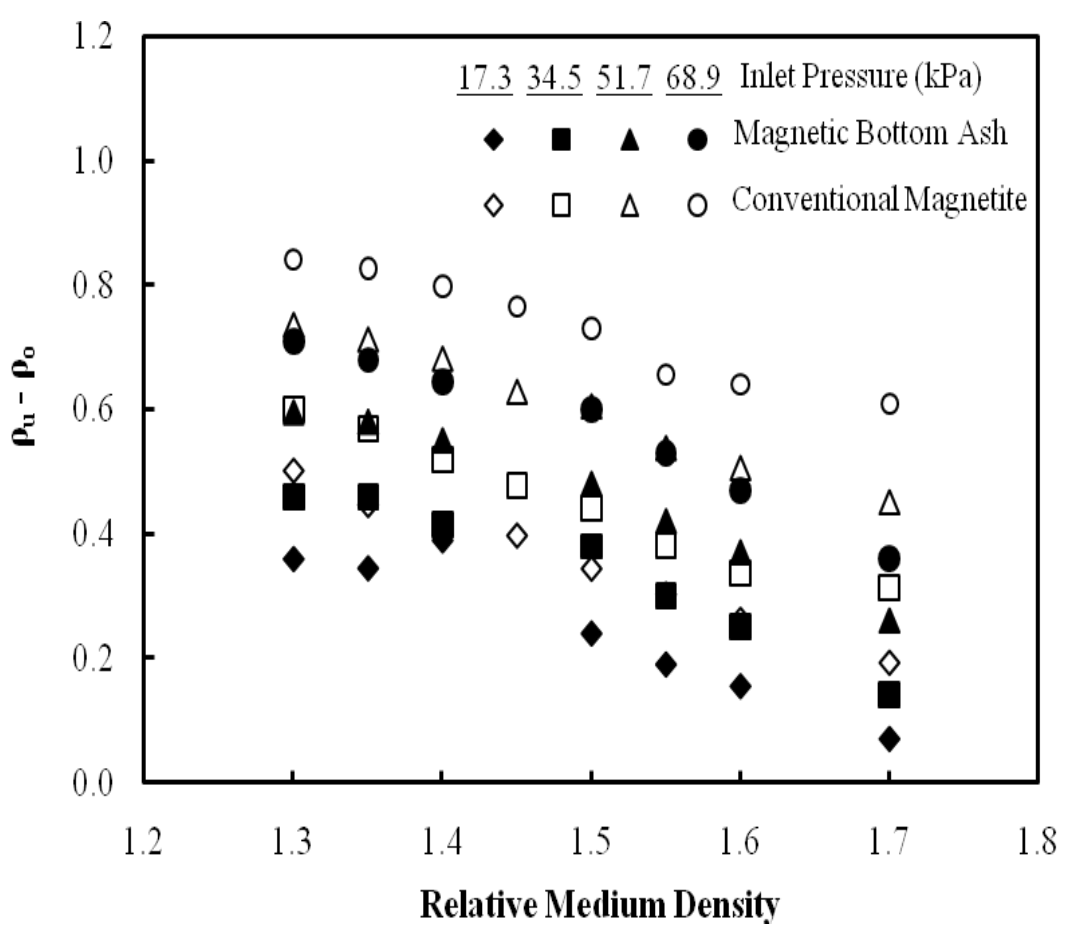

Figure 16. Comparison of medium stability achieved by ground bottom ash and grade 'B' magnetite. 
Dense medium cyclone tests were conducted to assess the separation efficiency achievable when using the magnetic bottom ash. Washability analyses were performed on samples collected from the clean coal, reject and feed process streams. The data was used to develop the partition curves shown in Figure 17 over a range in medium density values from 1.4RD to $1.7 \mathrm{RD}$.

A sign of medium stability is a low density offset, which is the difference between the separation density $\left(\rho_{50}\right)$ and the medium density. At medium density values of $1.4 \mathrm{RD}$ and $1.5 \mathrm{RD}$, the density offset was relatively high with values around 0.08 density units. At 1.6RD, the offset drops to 0.04 density units indicating medium stabilization.

Separation efficiency is measured by the slope of the partition curve which is typically quantified by the probable error value (i.e., $\mathrm{Ep}=\left[\rho_{25}-\rho_{75}\right] / 2$ ). Bypass of low-density particles to the reject stream and vice versa are also a sign of inefficiency. A parameter that combines the efficiency associated with the probable error and bypass is the organic efficiency. Organic efficiency is the ratio of the actual energy recovery to the theoretical energy recovery at a given product quality or product ash content. A summary of the separation performances represented in Figure 17 is shown in Table 6.

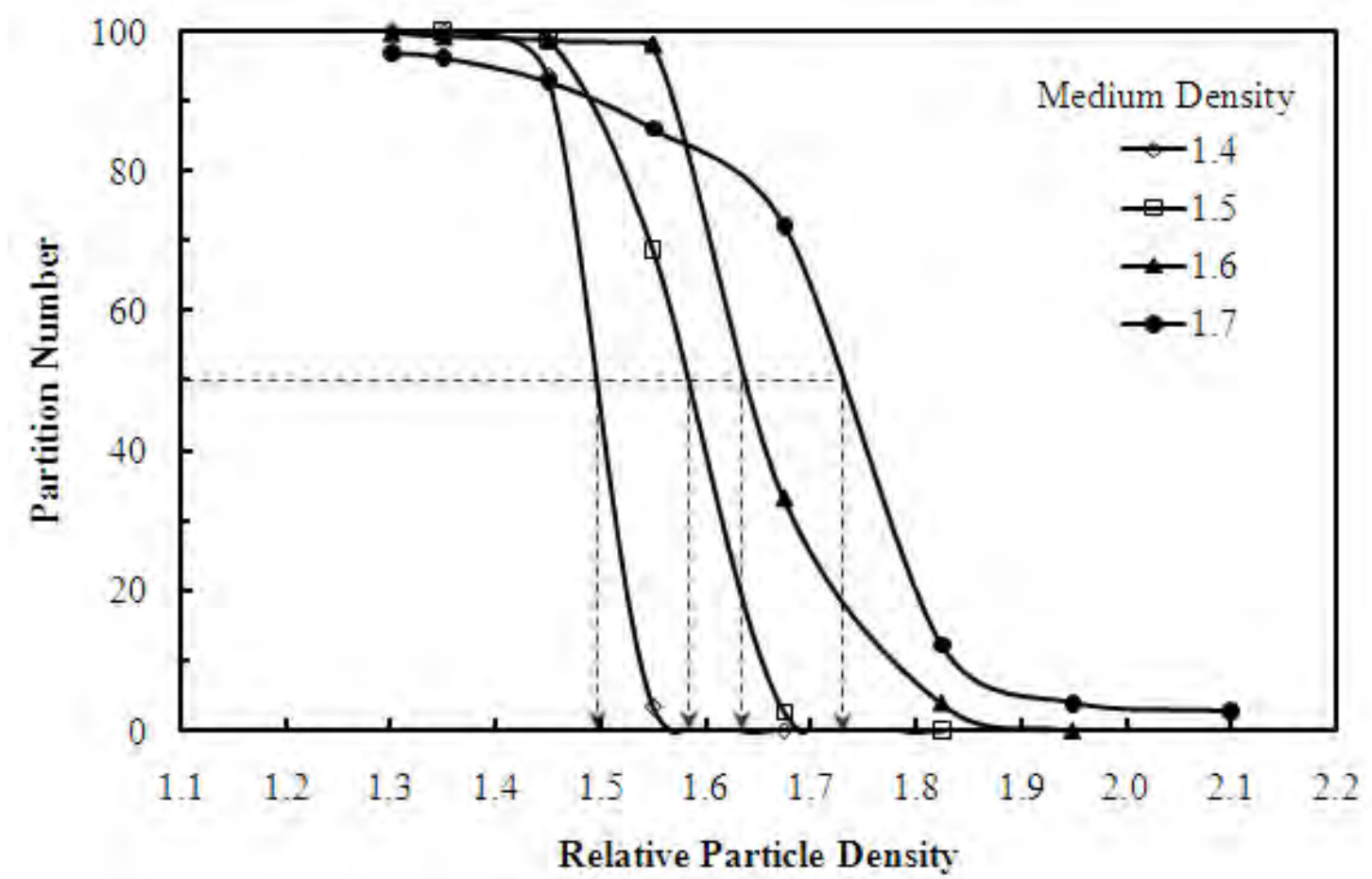

Figure 17 Partition curves generated over a range of medium density values using magnetic bottom ash. 
Table 6. Summary of separation performances achieved using the magnetic bottom ash at an inlet pressure of $34.5 \mathrm{kPa}$.

\begin{tabular}{||c|c|c|c|c||}
\hline \multirow{2}{*}{ Variable } & \multicolumn{4}{|c|}{ Relative Medium Density } \\
\cline { 2 - 5 } & 1.4 & 1.5 & 1.6 & 1.7 \\
\hline Feed Ash (\%) & 40.84 & 40.30 & 33.82 & 32.58 \\
Product Ash (\%) & 6.11 & 8.82 & 11.7 & 15.43 \\
Tailings Ash (\%) & 56.44 & 58.28 & 57.82 & 56.29 \\
\hline Yield (\%) & 31.00 & 36.36 & 52.04 & 58.03 \\
Recovery (\%) & 49.19 & 55.53 & 69.43 & 72.79 \\
Org. Efficiency (\%) & 96.81 & 98.92 & 95.49 & 89.94 \\
\hline$\rho_{50}$ & 1.495 & 1.583 & 1.640 & 1.733 \\
$\mathrm{E}_{\mathrm{p}}$ & 0.023 & 0.044 & 0.051 & 0.063 \\
$\rho_{50} \rho_{\text {medium }}$ & 0.095 & 0.083 & 0.040 & 0.033 \\
\hline \multicolumn{4}{|l|}{} \\
\hline
\end{tabular}

The separation efficiencies measured for the medium densities of $1.4 \mathrm{RD}$ and $1.5 \mathrm{RD}$ are excellent with organic efficiency values around $97 \%$ and probable error values of 0.04 and less. As the case with conventional magnetite, the probable error increases with an increase in medium density which represents a decline in efficiency due to medium viscosity impacts. The probable error values are comparable to those achieved when using conventional magnetite.

A trend that is atypical is the decline in organic efficiency with an elevation in medium density. In most cases, the amount of near gravity material in the feed decreases with an increase in medium density. As a result, organic efficiency generally improves with an increase in medium despite the declining efficiency caused by medium viscosity. For the coal in this study, a peak in middlings content occurs at a density around 1.75 which causes the inefficiencies quantified by the probable error values to by magnified in the determination of organic efficiency.

Magnetic Bottom Ash Recovery: In addition to DMC separation performance, magnetic recovery is another important issue in development of recovered magnetic material as an alternative to magnetite. Magnetic content of the initial recovered material was $74 \%$. After grinding the bottom ash material, the magnetic components were selectively liberated. This action reduced the amount of magnetic particles in the original feed to $54 \%$. However, the magnetic strength needed to recover the material was significantly reduced as shown in Figure 18. The magnetic strength needed is about 1000 gauss to recover $98 \%$ of the material. The typical magnetic field strength provided by the low-intensity wet drum commonly employed in the coal industry is 750 gauss. 


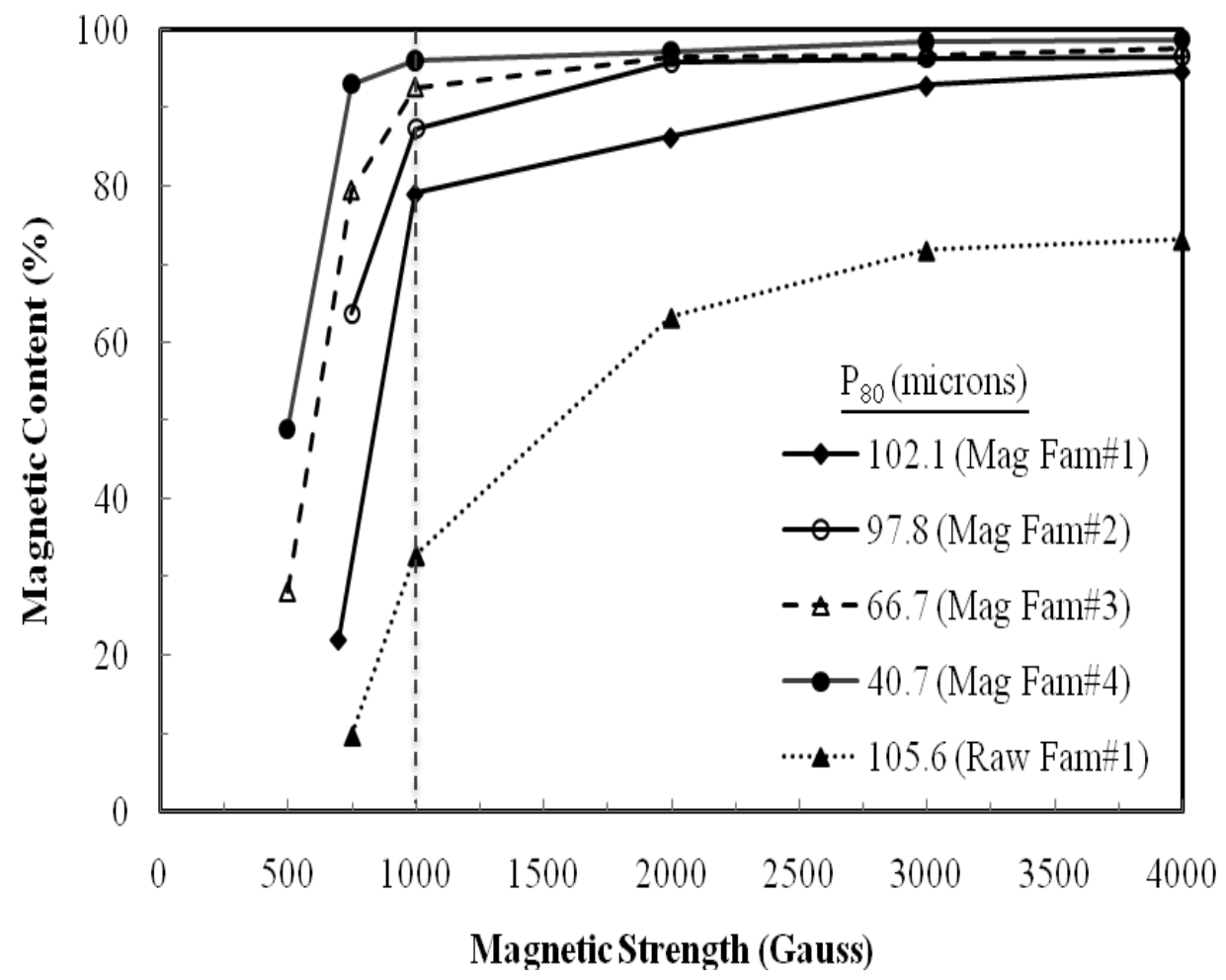

Figure 18. Magnetic intensity needed to recover the magnetic particles over a range of particle sizes.

\section{CONCLUSIONS}

Due to the global demand for iron ore over the last decade, concerns have developed regarding the cost and stable supply of magnetite for use in dense medium separations. In response, a study has been conducted to evaluate the feasibility of using alternative materials to replace magnetite in dense medium processes. Alternative materials included a lower cost, coarse magnetite, steel slag waste, fine sand, autogenous reject material and magnetic bottom ash. The autogenous material was obtained by recovering the finest size fractions from the spiral tailings stream. The evaluations were performed using a $150 \mathrm{~mm}$ diameter dense medium cyclone treating $6 \times 0.6 \mathrm{~mm}$ Coalburg coal over a range of medium densities and feed inlet pressures.

The magnetic bottom ash material was recovered from an electric utility that utilized a feed coal containing a relatively high amount of pyritic sulfur. As a result, the use of a spiral concentrator and a high gradient magnetic separator collected $5.7 \%$ of the total bottom ash having some degree of magnetic susceptibility. However, the sphericity of the -100 mesh material created a very unstable medium that required grinding to alter the particle shape. The result was $90 \%$ of magnetic bottom ash having a particle size less than 50 micrometer. In addition, the magnetic components were selectively liberated which resulted in $54 \%$ of the material being magnetic. The magnetic susceptibility of the magnetic portion was 
significantly improved as indicated by the ability to recover $98 \%$ of the magnetic particles using a relatively weak field strength of 1000 gauss.

With the exception of the coarse magnetite, the dense medium generated using the alternative materials provided superior medium stability when compared to the medium produced from conventional magnetite. This finding was a result of the lower solid density values of the alternative materials which resulted in the need to use greater amounts of the alternative materials to obtain a desired medium density. The higher mass and, more importantly, volume concentrations led to elevated particle populations and thus reduced particle settling velocities due to hindered effects. However, the positive impacts of improved medium stability were negated by a rise in medium viscosity as the medium density is increased. For coarse magnetite, a sufficiently stable medium was achieved only at relative medium density values above 1.7RD due to a need to reach a critical particle population for the development of hindered settling conditions.

The use of fine sand was investigated as an initial assessment of the potential to use the high density reject particles from a spiral concentrator as the material to form a dense medium. As a result of the stable medium formed when using sand, the density offset values (= separation density minus medium density) were positive and comparable to those realized using the conventional grade B magnetite. Organic efficiencies values achieved were greater than $95 \%$ at medium densities of $1.4 \mathrm{RD}$ and $1.5 \mathrm{RD}$ and decreased to $93 \%$ at $1.6 \mathrm{RD}$, which was likely due to the impact of medium viscosity. The performances were slightly better than those achieved by the magnetite-based medium at separation densities of $1.4 \mathrm{RD}$ and $1.5 \mathrm{RD}$.

The solid density of the spiral reject material was lower than sand density which resulted in a larger amount needed to reach a desired medium density. As result, a coarser particle size distribution was utilized in an effort to minimize the negative impacts of medium viscosity. The autogenous medium provided low density offsets which resulted in lower product ash values than those realized from the magnetite-based medium. The probable error values achieved using the autogenous medium were lower throughout the medium density range of 1.4RD to 1.6RD indicating superior performance. However, a significant elevation in the probable error value occurred at a 1.6RD medium density which is indicative of medium viscosity issues.

Compared to all medium types, steel slag provided the most stable suspension. As a result, the separation density achieved using the steel slag was approximately equal to the medium. The separation efficiency achieved was also superior to all other medium types. The organic efficiency increased from $96.3 \%$ at a medium density of $1.40 \mathrm{RD}$ to $98.9 \%$ at a density of 1.6RD. However, the steel slag medium did experience the typical trend of a rise in the probable error value from 0.025 to 0.051 as the medium density was increased. The partition curves were notably sharp even at the tail ends of the curves. Laboratory Davis Tube tests found that $64 \%$ of the steel slag was magnetic. Of the magnetic portion of the steel slag, nearly $100 \%$ was recovered using a magnetic field strength of around 700 gauss. As such, current magnetite recovery circuits used in the coal industry could be used to recovery the steel slag if applied to dense medium separations. 
The separations achieved using the magnetic bottom ash particles are comparable to those achieved through the use of conventional magnetite. Probable error values ranged from 0.02 at a medium density of $1.4 \mathrm{RD}$ to 0.06 at $1.7 \mathrm{RD}$. For the bottom ash material used in this study, the upper medium density limit appears to be around 1.6RD. Above this value, medium viscosity appears to have a significant impact.

One of the key concerns with the use of coarse magnetite is the ability to maintain a stable suspension. However, an acceptable stable suspension was achieved in a dense medium cyclone at medium densities of 1.7RD and higher at an inlet pressure of $34.5 \mathrm{kPa}$. The stability trend agreed well with data reported by He and Laskowski [1995]. Relatively efficient separations were achieved throughout the medium density range of 1.4RD to 1.9RD despite the instability of the medium at the low density values. Organic efficiencies increased and the probable error values decreased as the medium density was elevated from 1.4RD to 1.9RD, which is the opposite trend observed for the conventional magnetite. This tendency is due to improved stability with little or no viscosity effects. At a $1.9 \mathrm{RD}$, the Ep value was 0.04 and the organic efficiency was $98.5 \%$.

\section{REFERENCES}

Baird, G. A., Hornsby D. T. and Lief, H., 1998, "Development of fly ash derived magnetite for coal cleaning", 13th Proceedings, International Coal Preparation Conference, Vol. 1, pp. 314-321 (1998).

Birlingmar, D., Murtha, M., and Killmeyer, R., Characterization and Performance of Fly Ash-Derived Heavy Medium Material, DOE Report, IS-4839 (1983).

Bradley, D., The Hydrocyclone, Pergamon Press, Oxford (1965).

Burt, R. O., The Theory of Heavy Medium Separation in Gravity Concentration Technology, Developments in Mineral Processing, Elsevier, Amsterdam. vol. 5, pp.66-68 (1984a).

Davis, J. J., Cleaning Coarse and Small Coal - Dense Medium Processes, in Advanced Coal Preparation Monograph Series Part VIII, Australian Coal Preparation Society, vol. 3, pp. 138 (1994).

He, Y. B. and Laskowski, J. S., Separation of Fine Particles in Dense Medium Cyclone Effect of the Medium Yield Stress", in Proceedings of the 12th International Coal Preparation Congress, Krakow, Poland (1994).

He, Y. B. and Laskowski, J. S., "Dense Medium Cyclone Separation of Fine Particles: Part 2, The Effect of Medium Composition on Dense Medium Cyclone Performance", International Journal of Coal Preparation, vol. 16, pp. 27-49 (1995).

Leonard, J. W., III and Hardinge, B. C., Coal Preparation, 5th Edition, Society for Mining Metallurgy and Exploration, Inc., Littleton, Colorado, pp 272-300 (1991). 
Luttrell, G.H., Barbee, C.J., Bethell, P.J. and Wood, C.J., "Dense medium optimization", U.S. Department of Energy Technical Report (2005).

Mengelers, J., "The Influence of Cyclone Diameter on Separating Performance and Economy," in Proceedings of the 9th International Coal Preparation Congress, New Delhi, India (1982).

Palowitch, R. R., Deurbrock, A. W., Tork, E., and Akers, D. J., 1991, Part 1, Wet Coarse Particle Concentration, Section 1: Dense Media, in Coal Preparation, 5th ed. (J.W. Leonard and B. C. Hardinge) Society for Mining, Metallurgy and Exploration, Littleton, Colorado, pp. 271- 273 (1991).

Shi, F. N. and Napier-Munn, T. J., A Model for Slurry Rheology, International Journal of Mineral Processing, vol. 47, pp. 103-123 (1996).

Sripriya, R., P. Rao, V. T., Bapat, J. P., Singh, N. P. and Das, P., Development of an Alternative to Magnetite for Use as Heavy Media in Coal Washeries, International Journal of Minerals Processing, vol. 71, pp. 55 - 71 (2003).

Zanker, A., Hydrocyclones: Dimensions and Performance, Chemical Engineering Journal, vol. 84, pp. 122-125 (1977).

\section{PUBLICATIONS/PRESENTATIONS}

Honaker, R. Q., Bimpong, C. and Bethell, P., "Alternative Materials for Dense Medium Separations," 136th SME Annual Meeting, Denver, Colorado, February 25 - 28, 2007. (presentation).

Honaker, R. Q. and Bimpong, C., "Alternative Materials for Dense Medium Separations," Proceedings, $25^{\text {th }}$ International Coal Preparation Conference, Lexington, Kentucky, pp. 19 36, April 28-May 1, 2008, pp. 63 - 79.

Honaker, R. Q., Akram, Z. and Groppo, "Recovery and Utilization of Bottom Ash Magnetics for Coal Cleaning Medium," Proceedings, Society of Mining, Metallurgy and Exploration Annual Meeting, February 2009, Preprint 09-02.

Honaker, R. Q., and C. Bimpong, "Alternative Materials for Dense Medium Separations," International Journal of Coal Preparation and Utilization, Vol. 29, No. 4, pp. 173 - 191, 2009. 
APPENDIX 4 - Novel Design of DM Cyclones to Improve Coal Cleaning Efficiency with Large Eddy Simulations (LES) (UT010) 


\section{FINAL TECHNICAL REPORT}

Contract Title and Number: $\quad$ Period of Performance:

Continuation of Cross Cutting Technology Development Starting Date: 10/1/2005

at CAST (DE-FC26-05NT42457)

Ending Date: $\quad$ 9/30/2010

Sub-Recipient Project Title:

Report Information:

Novel Design of DM cyclones to improve coal cleaning

Efficiency with large eddy simulations (LES)

Principal Investigators:

Raj K. Rajamani

Contact Address:

University of Utah

135 South 1460 E. Room 412

Salt Lake City UT 84112

Subcontractor Address:

"No subcontracts issued."
Type: Final

Number: 3

Period: $\quad$ 10/1/05-9/30/09

Date: $\quad 2 / 24 / 10$

Code: UT010-FINAL

Contact Information:

Phone: (801) 581-3107

Fax: $\quad$ (801) 581-8119

E-Mail: raj.rajamani@utah.edu

Subcontractor Information:

Phone:

Fax:

E-Mail: 


\section{Disclaimer}

This report was prepared as an account of work sponsored by an agency of the United States Government. Neither the United States Government nor any agency thereof, nor any of their employees, make any warranty, express or implied, nor assume any legal liability or responsibility for the accuracy, completeness, or usefulness of any information, apparatus, product, or process disclosed, or represents that its use would not infringe privately owned rights. Reference herein to any specific commercial product, process, or service by trade name, trademark, manufacturer, or otherwise does not necessarily constitute or imply endorsement, recommendation, or favoring by the United States Government or any agency thereof. The views and opinions of authors expressed herein do not necessarily state or reflect those of the United States Government or agency thereof. 


\section{$\underline{\text { ABSTRACT }}$}

A number of empirical models are in existence for the dense medium cyclone (DMC) and in recent years this subject has been broached with computational fluid dynamics (CFD). The dense medium presents a centrifugal field within the cyclone body. The coal particles separate in this field due to various forces acting on them. Hence, CFD is ideally suited for the modeling of the DMC. The Large Eddy Simulation (LES) method for resolving the turbulence was used in the CFD simulation of a $76 \mathrm{~mm}$ dense medium cyclone. The study is oriented toward developing a methodology for predicting the separation characteristics of DMC for cleaning coal. The methodology includes developing primary flow field using multi-phase simulation of water-air-magnetite mixture followed by generating the distribution characteristics for coal particles of different density and size using discrete phase modeling. In particular, the magnetite was modeled as three granular fluids. The selection of the size of magnetite particles was decided from the simulated cut size values of water only phase simulation followed by magnetite particles injection. Coal particles of different sizes and densities are injected into the established mixture phase using Lagrangian particle tracking method and the corresponding distribution points of the particles are generated. In the simulation the diameter of the vortex finder and spigot are varied to compare with the experimental data of Verghese, P. A. and Rao, T.C. (Coal Preparation, 1994). Simulations using three fluid phases and Reynolds Stress Method (RSM) for turbulence were attempted towards reducing the computational efforts. The Large Eddy Simulation (LES) method for resolving the turbulence, with 5 fluid phases, was more comprehensive. Among the simulation studies carried out by using 3 magnetite size particles, fairly matching results are obtained adopting multiphase simulation with RSM model for turbulence. However, the results obtained using LES turbulence model is found to be more accurate in terms of the cut density and the slope of the distribution curves. Hence, LES turbulence closure was used in all of the simulations.

The discussions are also made on the system physics like fractional volume concentration of individual phases due to phase segregation, density profiles, velocity profiles at different radial and axial positions, for change in the magnetite particle size in the simulation. 


\section{Table of contents}

1. $\quad$ LIST OF GRAPHIC MATERIALS ................ 5

2. INTRODUCTION $\quad \ldots \ldots \ldots \ldots \ldots \ldots \ldots$

3. EXECUTIVE SUMMARY $\quad \ldots \ldots \ldots \ldots \ldots \ldots . . . . .7$

4. METHODS AND MODEL $\quad \ldots \ldots \ldots \ldots \ldots . . . . .7$

5. RESULTS AND DISCUSSIONS $\ldots \ldots \ldots \ldots \ldots . . . . .10$

6. CONCLUSIONS $\quad \ldots \ldots \ldots \ldots \ldots \ldots . \ldots$

7. REFERENCES $\quad \ldots \ldots \ldots \ldots \ldots \ldots . . . .25$

8. APPENDIX $\quad \ldots \ldots \ldots \ldots \ldots \ldots . . . . .27$ 


\section{LIST(S) OF GRAPHICAL MATERIALS}

\section{List of figures:}

Figure 1 Dense medium cyclone design used in the CFD simulation $\ldots \ldots \ldots \ldots \ldots \ldots \ldots \ldots 10$

Figure 2 CFD Simulation results on the distribution points of magnetite particles in the $76 \mathrm{~mm}$ Heavy medium cyclone using water-only as fluid and particle injection technique...11

Figure 3a Experimental and CFD simulated results distribution points (1673 $\mu \mathrm{m}$ coal particles)

Figure 3b Experimental and CFD simulated results distribution points (1285 $\mu \mathrm{m}$ coal particles).

Figure 3c Experimental and CFD simulated results distribution points (1086 $\mu \mathrm{m}$ coal particles).

Figure 3d Experimental and CFD simulated results distribution points (550 $\mu \mathrm{m}$ coal particles).

Figure 4 Volume concentration of air in the central core of the $76 \mathrm{~mm}$ DMC...............14

Figure 5 Mixture density distribution in the hydrocyclone body $\ldots \ldots \ldots \ldots \ldots \ldots \ldots \ldots \ldots \ldots . \ldots \ldots$

Figure 6 Volume fraction distribution of magnetite granular fluids $\ldots \ldots \ldots \ldots \ldots \ldots \ldots \ldots \ldots$

Figure 7 Predicted partition curve compared with experimental data for case 22, 19, and 6 (Percentage of feed to overflow versus relative density, SG/SG ${ }_{50}$ )..................17-21

Figure 8 Axial (a) and tangential (b) velocity predictions with LES in a $250 \mathrm{~mm}$ hydrocyc-

lone (Delgadillo and Rajamani, 2005)

\section{List of tables:}

Table 1 Experimental conditions. 


\section{$\underline{\text { INTRODUCTION }}$}

In principle it is widely known that magnetite suspension in water generates an intermediate density between the coal and the ash forming minerals and researchers have identified that a density gradient naturally occurs within the separation chamber of heavy medium cyclone. The gradient is due to the centrifugal forces on the magnetite particles and their balance with the drag force at different radial and axial positions in the cyclone body (Verghese and Rao, 1994; Davis, 1994; He and Laskowski, 1994). There exists a density differential in the overflow and the underflow products of the cyclone, both of which are different from that of the density maintained in the feed. However, very limited information is available on the segregation of magnetite and hence the slurry density distribution at different radial and axial positions in the cyclone body. Studies on such behavior of medium will be useful both in terms of engineering principles and in the design modifications for specific applications.

The simulation of DMC with CFD is a leap forward based on the enormous success of CFD in hydrocyclones. Recently, developments in computational systems enabled the simulation of hydrocyclone performance using CFD techniques (Hsieh and Rajamani, 1988; Monredon et al., 1992; Rajamani and Milin, 1992; Devulapalli and Rajamani, 1996; Griffths and Boysan, 1996; Slack and Wraith, 1997; Slack and Boysan, 1998; Stovin and Saul, 1998; Suasnabar and Fletcher, 1999; Slack et al., 2000, 2003; Ma et al., 2000; Nowakowski et al.,2000, 2004; Grady et al., 2002, 2003; Nowakowski and Dyakowski, 2003; Schuetz et al., 2004; Cullivan et al., 2003, 2004; Delgadillo and Rajamani, 2005, 2007; Narasimha et al., 2005, 2006, 2007; Udaya Bhaskar et al., 2005, 2007, 2007). However, applying the CFD technique learned in hydrocyclones to DMC may not produce correct results for obvious reasons.

CFD simulation of a heavy medium cyclones is a complex task and numerically intensive. The direct Eulerian simulation for different medium sizes, and size and density based coal classification is extremely difficult to achieve in years to come. Toward this objective, Narasimha et al. (2007) presented the results of LES and Reynolds Stress Model (RSM) and compared it with density distribution measured in a $350 \mathrm{~mm}$ DMC. These authors presented the discrepancies between the computations and experimental results as well as the means to remedy these discrepancies. Though the simulated results agree with values of density distribution within the cyclone body, the computational effort is enormous and further the unsteady state simulation needs good expertise and judgment for solution convergence. It is apparent that high concentration of magnetite in water would exhibit non-Newtonian rheology. Hence, Suasnabar and Fletcher (1999) studied non-Newtonian rheology and turbulence of DMC using CFD. They concluded that the turbulence effect is more important than the rheology effect. The multiphase approach by Brennan (2003) produced nearly matching results in terms of density distribution and coal separation characteristics, that too with a reasonable computational effort. 
However, medium segregation was found to be over predicted by CFD, and it was concluded that it might be caused by the fact that turbulent mixing of the dispersed phase was more significant for sizes typical to DMC $(\sim 30 \mu \mathrm{m})$.

Chu et al. took the DMC simulation one level above what is possible with CFD. They combined the discrete element method for particle behavior with CFD. With this approach they proved the existence of surging phenomena in DMC. While this approach is a leap forward, the computations would be so prohibitive for reasonable use.

The understanding of the effect of magnetite particles on the changes in fluid characteristics in the centrifugal field via fluid dynamics is the key aspect of LES simulations. The simulation methodologies which are relatively less computationally expensive and yet produce reasonable results would be attractive for the analysis and design of DMC. The present study is an attempt to develop such a CFD methodology.

\section{EXECUTIVE SUMMARY}

In the present study, validation of the CFD results and experimental results is shown for a $75 \mathrm{~mm}$ dense medium hydrocyclone. The experimental results of Verghese and Rao, 1994 and the corresponding CFD simulated results of the distribution of coal particles of different size and density are presented. The RSM and LES turbulent closure models were used in the Fluent computations. The three phase CFD simulations with RSM predicted cut sizes slightly lower than the experimental values. Also, the slopes of the distribution curves were steeper in comparison with the experimental values. Of all the predictions made with CFD methodologies, the distribution curves predicted using LES model provided the closest match with the experimental values. Although computationally time consuming, the LES model was chosen for all subsequent DMC simulations. A variety of experimental results (different sizes of vortex finders and spigots) were successfully predicted with the LES turbulence closure model. The details of the multiphase model and the computational results are discussed in this report. Such computational model paves the way for understanding and designing dense medium cyclones of larger sizes. It was felt that computing cost is prohibitively high, which however can be overcome via parallelized computations with multiple processors.

\section{METHODS AND MODEL}

Simulation was carried out using a 3-D double precision, unsteady state, and segregated solver under Cartesian coordinate systems. In order to enhance the capture of flow features, at critical regions such as cyclone walls, around the air-core, in the vicinity of the vortex finder and at the spigot, methods like boundary layer meshing, block-structured meshing at the air-core, and larger number of meshes near the spigot were adopted. 
The computational domain is divided into about 150,000 elements, a reasonably optimum mesh domain for such CFD problems. In all the three groups, the size and density based distribution characteristics of coal particles are evaluated using particle injection through discrete phase modeling technique.

In a dense medium cyclone, volumetric concentration of coal in proportion to the mixture of water and magnetite can be presumed to be reasonably dilute to simulate the path of coal particles using the Lagrangian tracking approach. This assumption though is far off from the actual condition, would reduce the computational effort to a great extent. It is also reported that using this method partition curves can be generated with reasonable accuracy in both sharpness and cut density (Suasnabar, 2000, Brenan, 2003 and Narasimha et al., 2006). The distribution characteristics of different size and density coal particles are generated using particle injection technique. In this method, at the converged flow conditions of the primary and secondary phases, constant number of coal particles of different size and density were injected into the fluid body and the percentage reporting into the overflow and underflow products were noted. The distribution data of different size and density coal particles was used for validation with the published experimental and model results (Verghese and Rao, 1994).

In the first phase, three phase simulations i.e., water, air and specific size magnetite particles were carried out. Effects of variations in the magnetite particle size on the particle dispersion and hence the slurry density distribution in the cyclone body is evaluated. It is found that the cut density values obtained are slightly lower than the cut densities from the reported experimental results though higher cut densities are achieved with decrease in the particle size.

Thus in the second phase of simulation works, attempts were made to simulate the separation characteristics using pure magnetite fluid of density around $1400 \mathrm{~kg} / \mathrm{m}^{3}$. The results under these conditions are found to be of lower cut-density and the distribution curve has a higher steepness than the published results. Attempts in this second phase of simulations also included combination of high density fluids (water) $1200 \mathrm{~kg} / \mathrm{m}^{3}$ and the $5100 \mathrm{~kg} / \mathrm{m}^{3}$ (magnetite). Though the results indicated a reasonable match with the density of the cut, there is not much improvement in the steepness of the distribution curve.

Finally in the third phase of the research work, simulations were carried out using five phases i.e., three magnetite phases with different granular sizes and with air and water, and by using LES method for resolving the turbulence. The particle distribution characteristics indicated that there is a very good match between the experimental and the simulated results both in terms of the density of cut and also in terms of the slope of the distribution curve.

In LES approach for turbulence closure modeling, eddies larger than the mesh size are resolved and eddies of smaller scales are modeled. Thus the equations of motion are filtered which results in an additional stress tensor term in the filtered Navier Stokes equations which account for the transfer of momentum by subgrid scales of turbulence. The subgrid scale (SGS) stresses are usually modeled using a simple eddy viscosity model known as Smagorinsky -Lilly SGS model. The SGS model presumes that the eddy viscosity is related 
to the local average grid spacing and the mean strain rate. In the mixture model with granular flow, the granular viscosity, the granular temperature and the drag law formulations are the important parameters. Granular viscosity is comprised of collisional viscosity and kinetic viscosity. In the present work granular viscosity was set as a constant in the calculations. The drag law formulations incorporated in phase interactions, as per available (Fluent) model library, were respectively Schiller - Nauman model for air - water, and Syamlal - Obrien model for magnetite - water. The slip velocity for air was modeled with Manninen et al. law. In the present study, the use of 5 fluids required a reasonable computational time. A typical value of mass flow inlet of water was set at $0.71 \mathrm{~kg} / \mathrm{s}$. The magnetite fluid inlet flow was set at $0.41 \mathrm{~kg} / \mathrm{s}$ with $33.5,33.9$, and 32.6 weight percents apportioned to the three granular fluids i.e., $7.0 \mu \mathrm{m}, 19.4 \mu \mathrm{m}$, and $38 \mu \mathrm{m}$ sizes. Specific experimental values were taken from Table 3 of Verghese and Rao, 1994.

The typical computation for five fluids takes over 20 to 40 days on $2.6 \mathrm{GHz}$ single CPU processor. Upon convergence of flow conditions of the primary and secondary phases, constant number of coal particles of different size and density were injected into the fluid body and the percentage reporting to the overflow and underflow products were generated.

\section{Geometry}

The $76 \mathrm{~mm}$ diameter hydrocyclone geometry used in the experimental studies of Verghese and Rao (1994) was simulated in the present study. The dimensions of the DMC are shown in figure 1. In particular four different spigots were used in this study. These authors report 25 experiments conducted with magnetite as the heavy medium. The experiments were performed with the aim of understanding the effects of design variables and operating variables. These authors presented detailed size and specific gravity for all the streams in each of the 25 experiments. Hence, the data set is valuable for model verification.

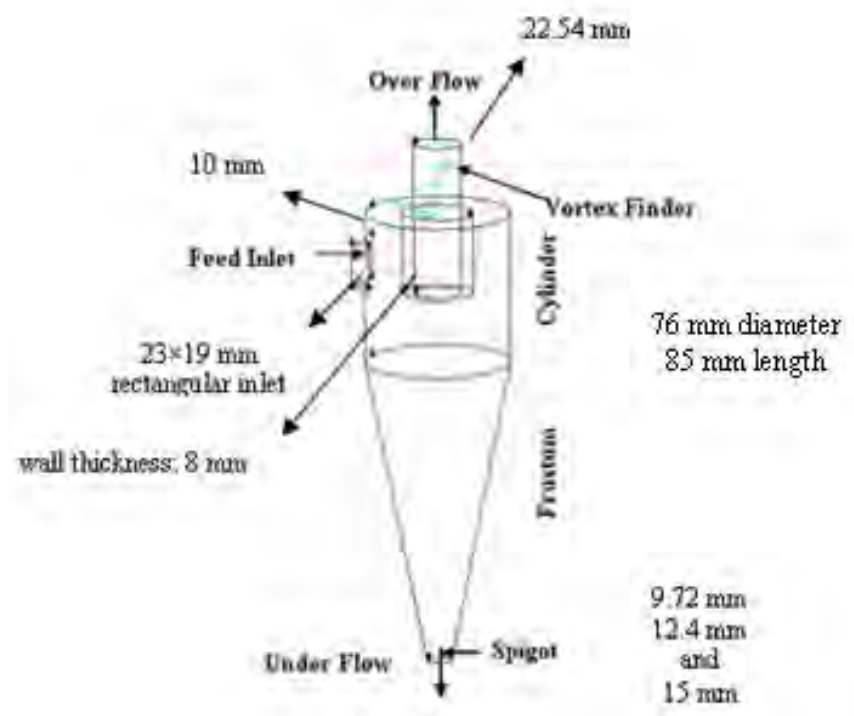

Figure 1. Dense medium cyclone design used in the CFD simulation. 


\section{$\underline{\text { Boundary and initial conditions }}$}

Water, air and magnetite particles were defined as fluids with density values corresponding to 1000,1 , and $5100 \mathrm{~kg} / \mathrm{m}^{3}$ respectively. Magnetite is defined as a granular phase with a suitable particle size. Water was the primary phase for simulation, air and three different phases of magnetite granular fluids were used as secondary phases. Feed inlet was designated as mass flow inlet. The necessary values of mass flow rates (taken from Verghese and Rao, 1994) were assigned for the water and magnetite phases at the feed inlet. The vortex finder and spigot outlets were designated as pressure outlets. At both the outlets, the backflow volume fraction of air was assigned as unity for air entry into the cyclone body.

\section{$\underline{\text { RESULTS AND DISCUSSION }}$}

The efficiency measurement of coal processing in dense medium cyclone is very much similar to efficiency measurement of classification cyclones. Both are represented in terms of distribution curves which essentially reveal information on the probability of each size or density fraction to report into the product under consideration.

Verghese and Rao present the distribution of coal particles as a function of reduced specific gravity. Furthermore, they present a functional form for the mean value of the experimentally obtained data points. In other words, the experimental data points are nearly clustered around this mean distribution curve. Therefore, in the present study, the mean curve is taken as the experimental response and the CFD simulations are compared with it.

The simulation results are obtained from the water-only phase simulation followed by magnetite particle injection between sizes 1 and $100 \mu \mathrm{m}$ is presented in Fig. 2. For the design and process conditions used in the present study, a cut density $\left(\mathrm{d}_{50}\right)$ which is obtained through plotting the distribution points to underflow is observed to be about of $10 \mu \mathrm{m}$. The results also indicate that the $\mathrm{d}_{20}$ and $\mathrm{d}_{80}$ sizes of separation of the DMC correspond to about 5 and $15 \mu \mathrm{m}$. 


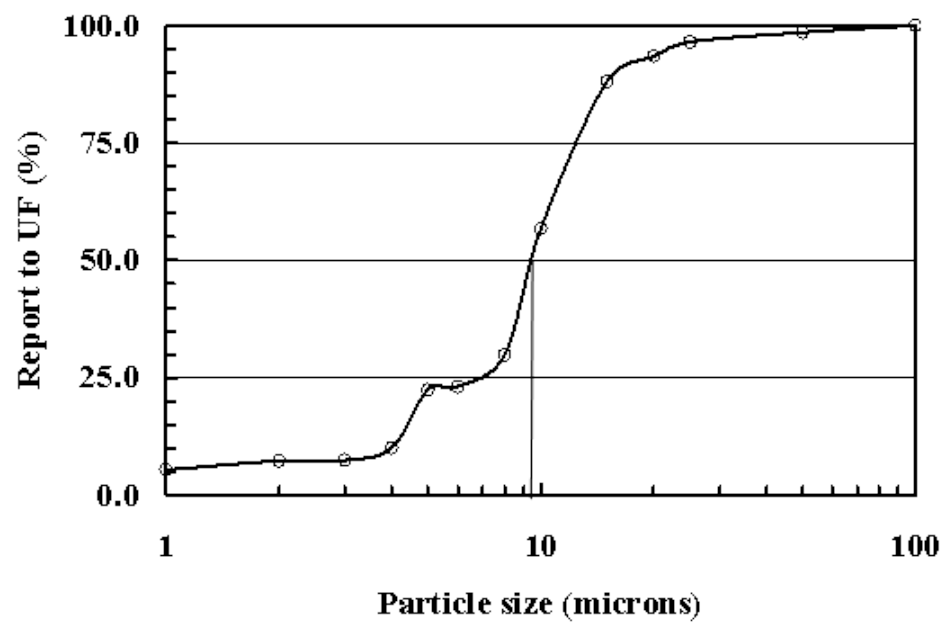

Figure 2. CFD Simulation results on the distribution points of magnetite particles in the $76 \mathrm{~mm}$ Heavy medium cyclone using water-only as fluid and particle injection technique.

Therefore the size of the single granular phase was varied from $5 \mu \mathrm{m}$ to $10 \mu \mathrm{m}$ to $15 \mu \mathrm{m}$. In figures $3 \mathrm{a}-3 \mathrm{~d}$ the experimental values of the published results (Verghese and Rao, 1994) and the CFD simulated results (using LES turbulence model) of the distribution points for different size and density of coal particles are presented.

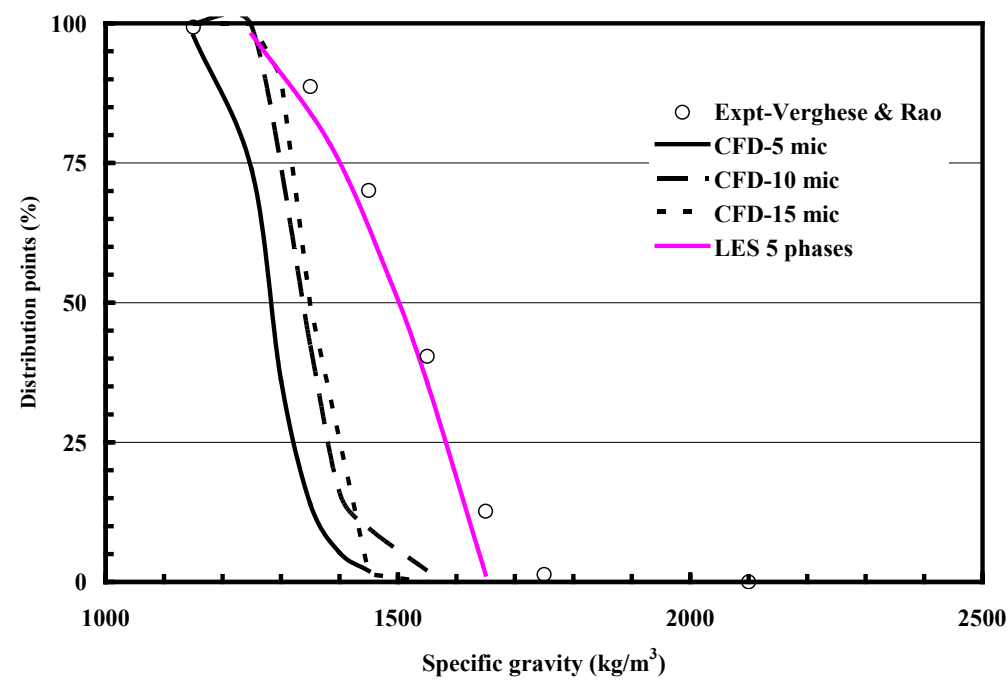

Figure 3a. Experimental and CFD simulated results distribution points (1673 $\mu$ m coal particles). 


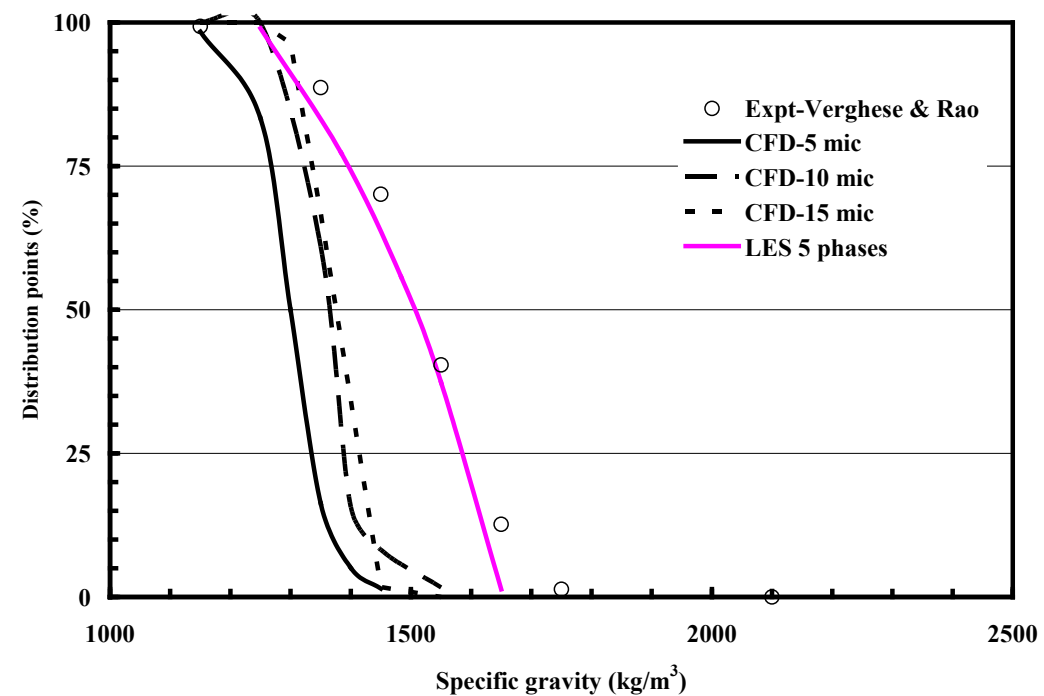

Figure 3b. Experimental and CFD simulated results distribution points (1285 $\mu \mathrm{m}$ coal particles).

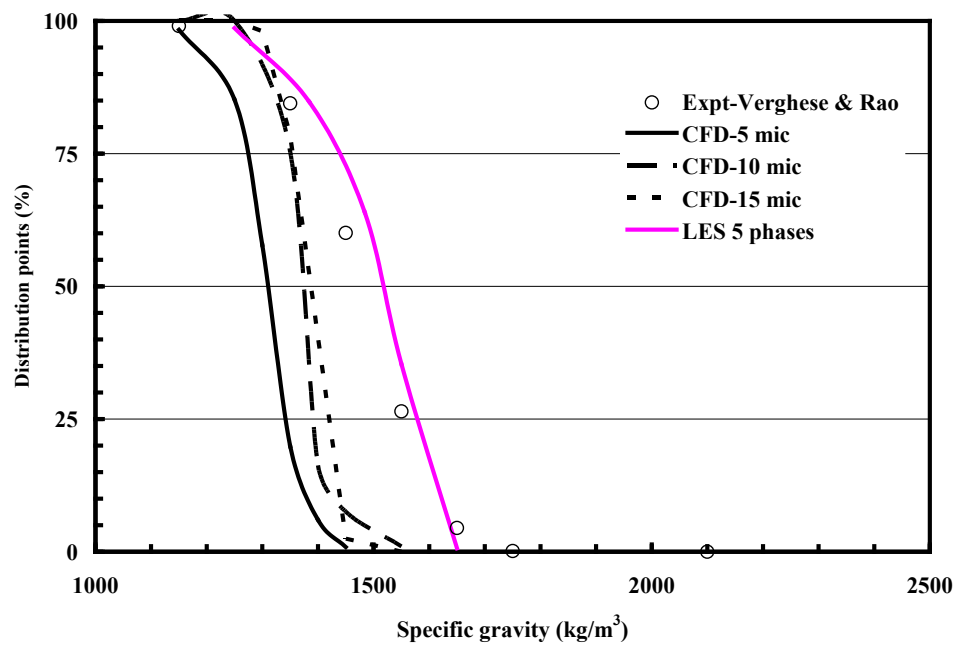

Figure 3c. Experimental and CFD simulated results distribution points (1086 $\mu \mathrm{m}$ coal particles). 


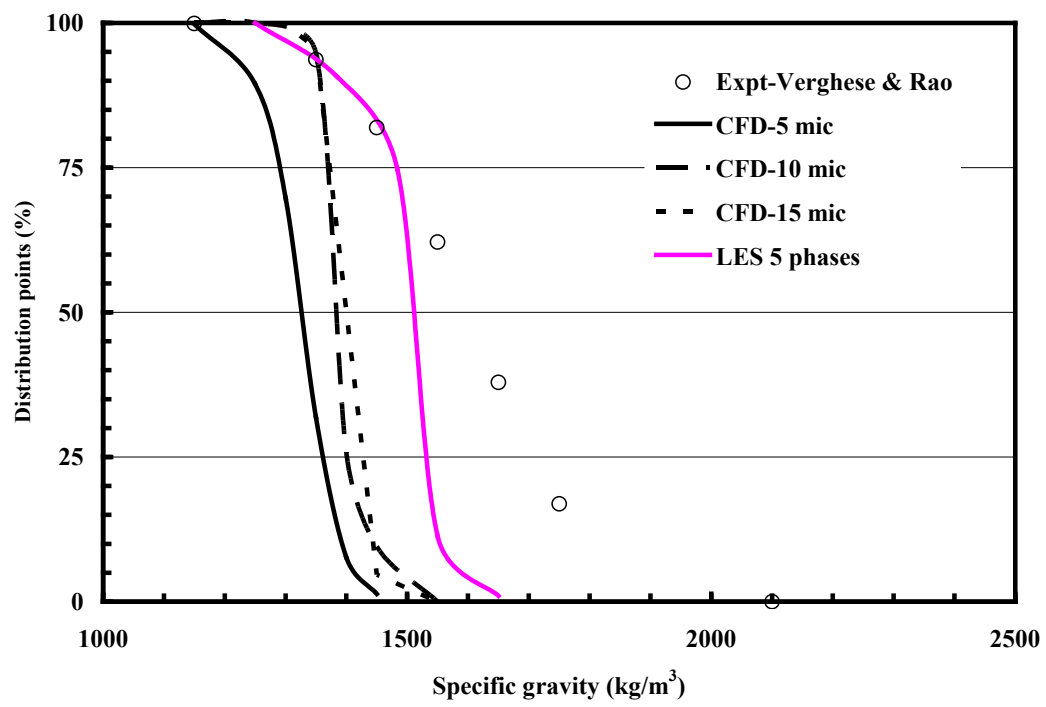

Figure 3d. Experimental and CFD simulated results distribution points (550 $\mu \mathrm{m}$ coal particles).

As shown in figures the results of single granular fluid systematically fall short of experimental results. Therefore, in the rest of this manuscript the discussions are about the five-fluid LES model predictions. The CFD predicted values of cut densities of coal particles with LES simulation are $1510 \mathrm{~kg} / \mathrm{m}^{3}, 1520 \mathrm{~kg} / \mathrm{m}^{3}, 1530 \mathrm{~kg} / \mathrm{m}^{3}$, and $1520 \mathrm{~kg} / \mathrm{m}^{3}$ corresponding to coal particle sizes 1673, 1285, 1086, and $550 \mu \mathrm{m}$ respectively.

\section{Air core:}

The air core was nearly $10 \mathrm{~mm}$ in diameter at or near the spigot opening. With increasing vertical heights from the spigot opening, the diameter of the air core broadens and the fractional volume of air in this region decreases. Figure 4 shows the volume fraction of air profiles throughout the $76 \mathrm{~mm}$ DMC. 


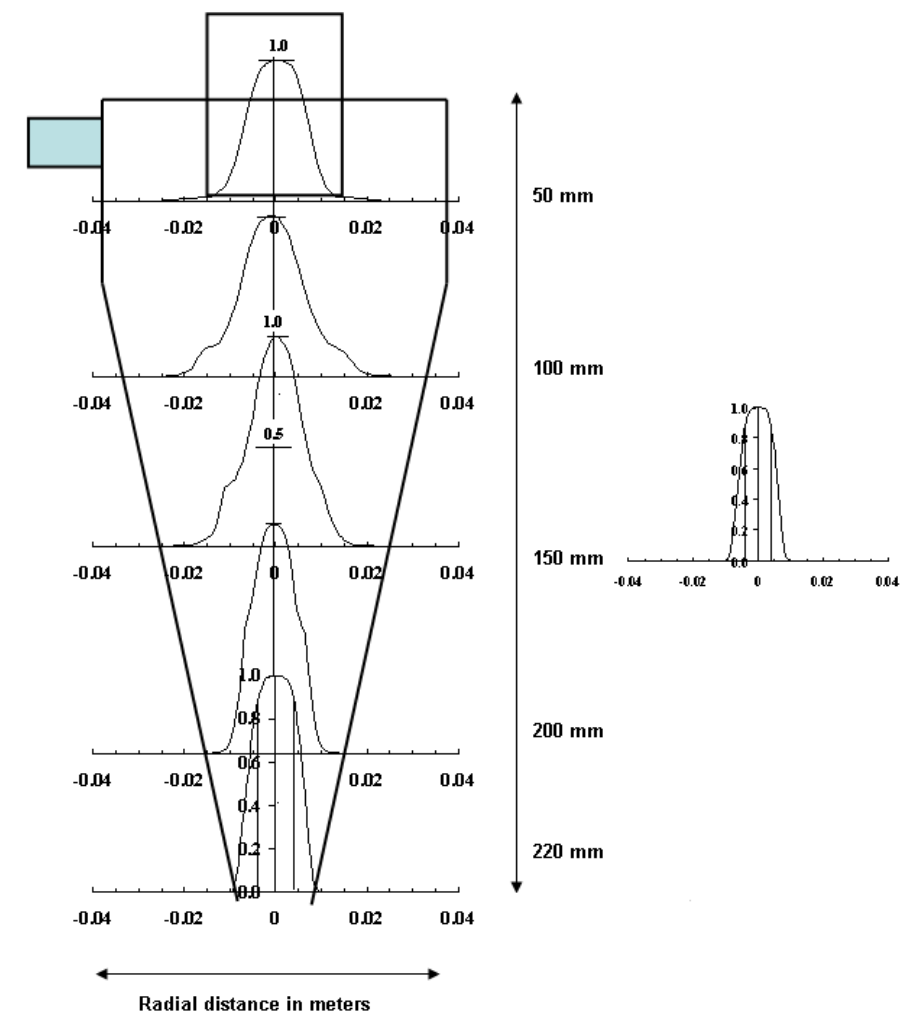

Figure 4. Volume concentration of air in the central core of the $76 \mathrm{~mm}$ DMC.

The central air-core has a parabolic contour shape. However, the air-core in the vortex finder is nearly cylindrical. The decrease in the volume fraction of air at increased vertical heights from the spigot opening is due to entrapment of water and the magnetite particles reporting to the overflow product through the vortex finder.

\section{Mixture density distribution in the cyclone body:}

The slurry density is the most important variable which decides the separation efficiency in side a DMC. Because of the range of particle sizes of the media used in the feed slurry, a natural segregation of the media within the cyclone occurs. The coarser size particles tend to report towards the walls while layers of relatively finer particles are found at smaller radial distance from the cyclone axis. Ideally, the fines below the cut size of the cyclone mostly report into the overflow product while the size coarser than the cut size mostly report to the underflow product. Therefore, the density which is maintained in the feed slurry to the cyclone will be always lower than the cut density. It is widely reported that most of the magnetite pumped to the cyclone reports into the underflow product. Realizing the importance of density distribution due to media segregation on the separation behavior, attempts were made to understand the mixture density at different radial and axial positions in the cyclone body. The density distribution characteristics are presented in Figure 5. 


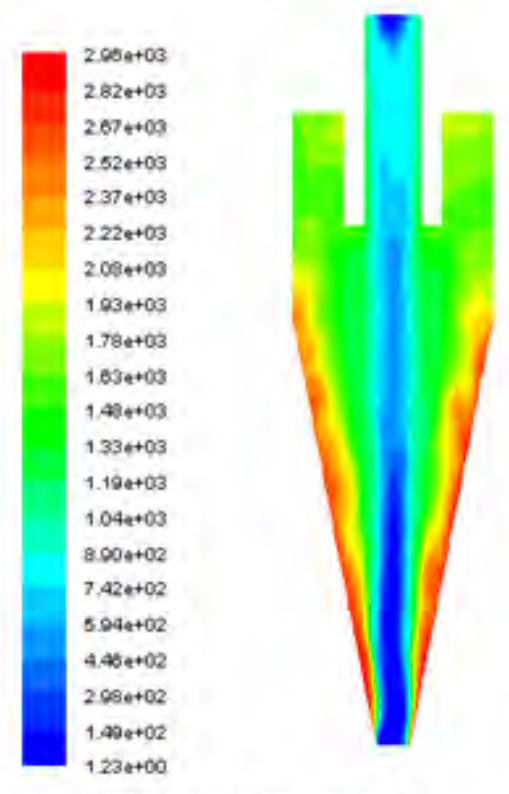

Slurry density $\left(\mathrm{kgm}^{3}\right)$

Figure 5. Mixture density distribution in the hydrocyclone body.

The maximum value of mixture densities are observed more at the lower conical portions of the cyclone body. For example, at a distance of $50 \mathrm{~mm}$ from the cyclone roof, the maximum value of mixture density observed at the walls is $1787 \mathrm{~kg} / \mathrm{m}^{3}$ while the maximum value of mixture density at $225 \mathrm{~mm}$ axial distance is $2820 \mathrm{~kg} / \mathrm{m}^{3}$. Within the central core region, the density is predominantly that of air.

\section{Media segregation behavior in the cyclone body:}

The behavior of the three different sizes of the magnetite granular fluid is helpful in understanding how the overall density gradation is established within the cyclone. The distribution behavior of these fluids in terms of the volume fraction contours is presented in Figure 6. 

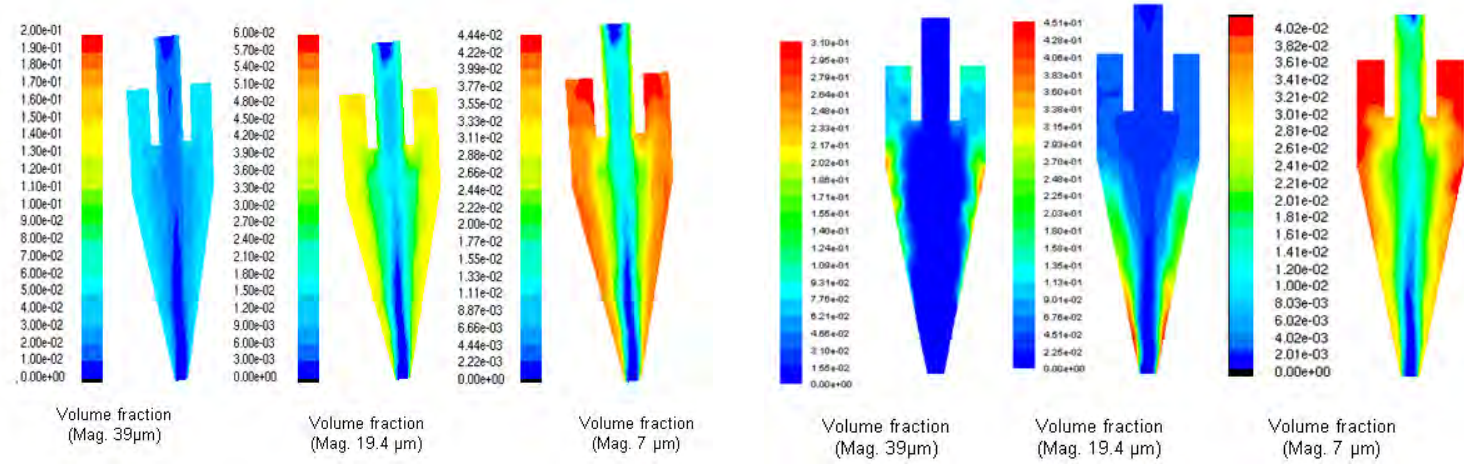

Figure 6. Volume fraction distribution of magnetite granular fluids.

The magnetite granular fluid of size of $38 \mu \mathrm{m}$ is mostly segregated along the walls of the cyclone body, preferentially in the cylindrical and upper conical regions. The fluid of a grain size of $19.4 \mu \mathrm{m}$ also segregates along the walls of the cyclone body. However, this fluid segregates in the lower conical portions of the cyclone body indicating preferential discharge of this fluid into the underflow. The fluid of grain size of $7.0 \mu \mathrm{m}$ is mostly distributed around the cylindrical and upper conical portions of the cyclone body. Lower values of the fractional volume concentration of this phase can be observed at lower conical heights indicating that this fraction of the fluid essentially decides the density distribution in the cylindrical portion of the cyclone body, where most of the classification effects are anticipated to occur.

Figure 7 compares the LES predictions for each coal particle size class with the experimental data. The experimental conditions used in three cases are shown in Table 1.

\begin{tabular}{|l|l|l|l|}
\hline \multicolumn{4}{|l|}{ Table 1. Experimental conditions } \\
\hline Case no. & Spigot (mm) & $\begin{array}{l}\text { Medium } \\
\text { density }\end{array}$ & $\begin{array}{l}\text { Feed slurry flowrate } \\
(\mathrm{l} / \mathrm{min})\end{array}$ \\
\hline 22 & 12.4 & 1.42 & 40.0 \\
19 & 12.4 & 1.39 & 52.5 \\
23 & 12.40 & 1.47 & 33.3 \\
6 & 9.7 & 1.39 & 55.1 \\
4 & 9.7 & 1.32 & 57.5 \\
\hline
\end{tabular}


CASE 22

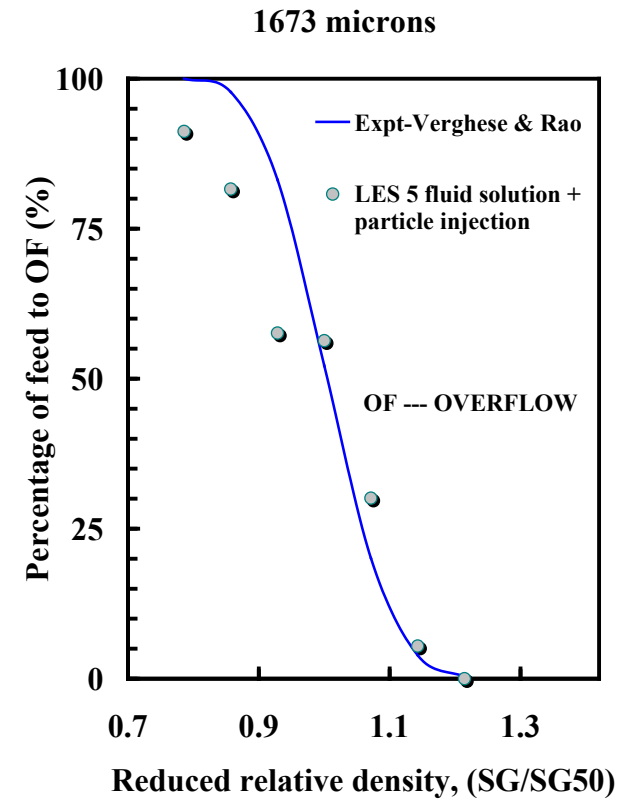

$1673 \mu \mathrm{m}$

1086 microns

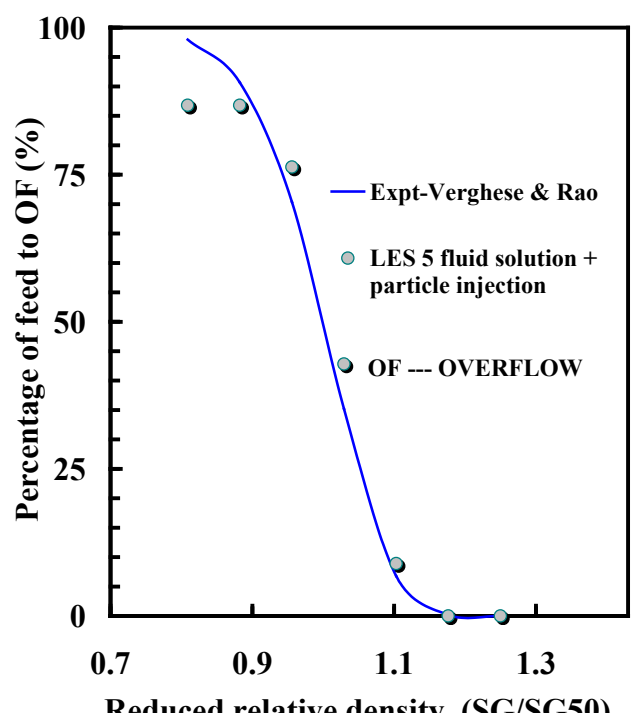

$1086 \mu \mathrm{m}$
1285 microns

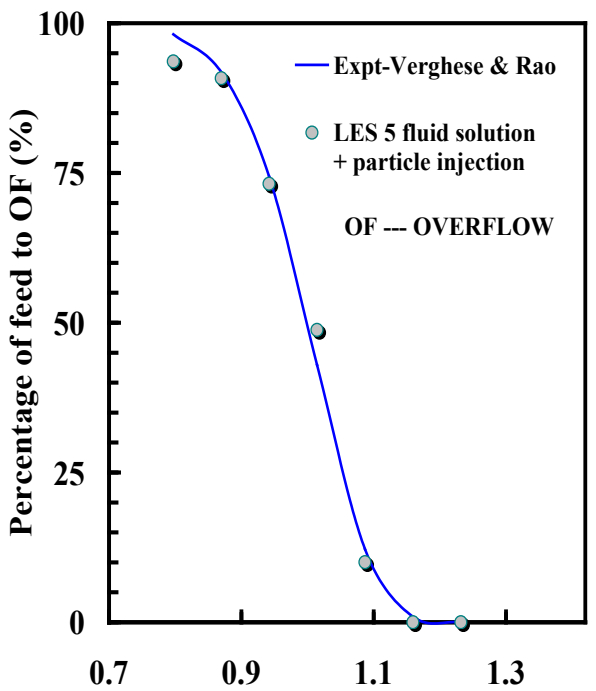

Reduced relative density, (SG/SG50)

$1285 \mu \mathrm{m}$

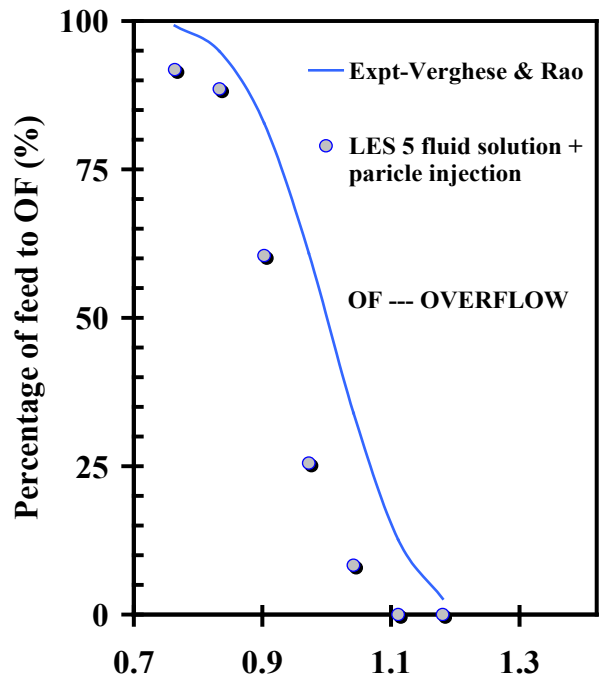

Reduced relative density, (SG/SG50)

$550 \mu \mathrm{m}$ 
CASE 19

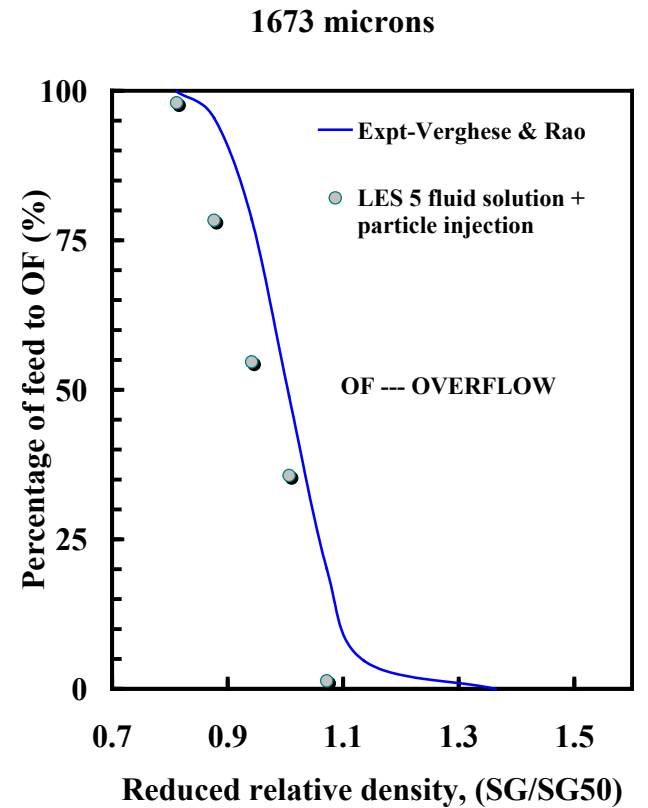

$1673 \mu \mathrm{m}$

1086 microns

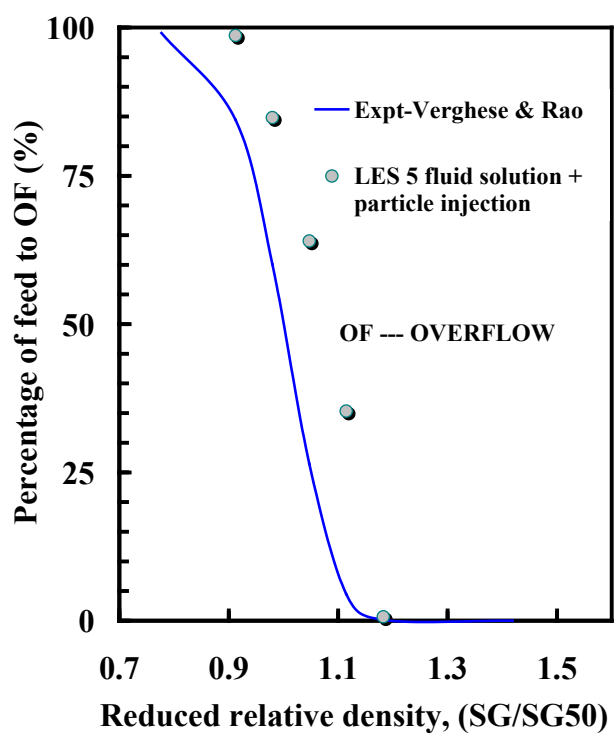

$1086 \mu \mathrm{m}$

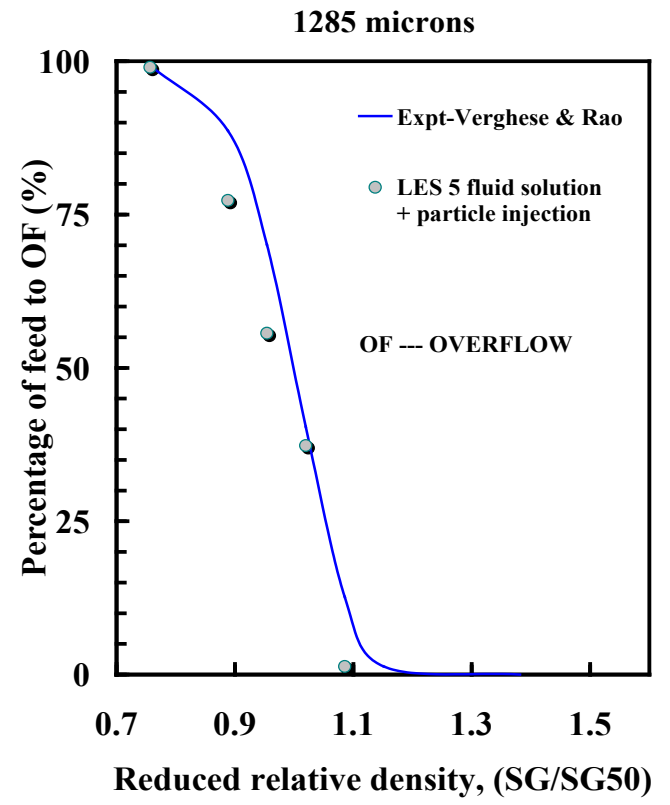

$1285 \mu \mathrm{m}$

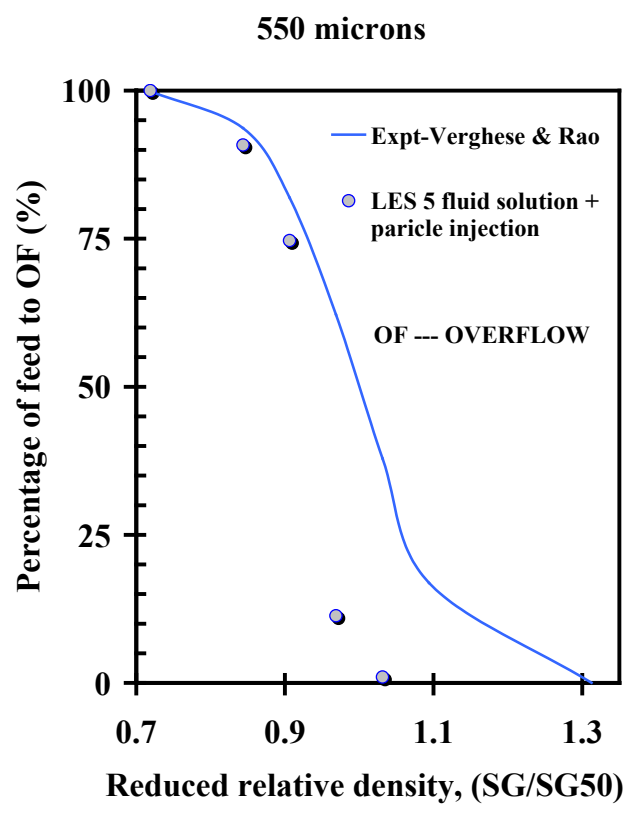

$550 \mu \mathrm{m}$ 


\section{CASE 4}

1673 microns

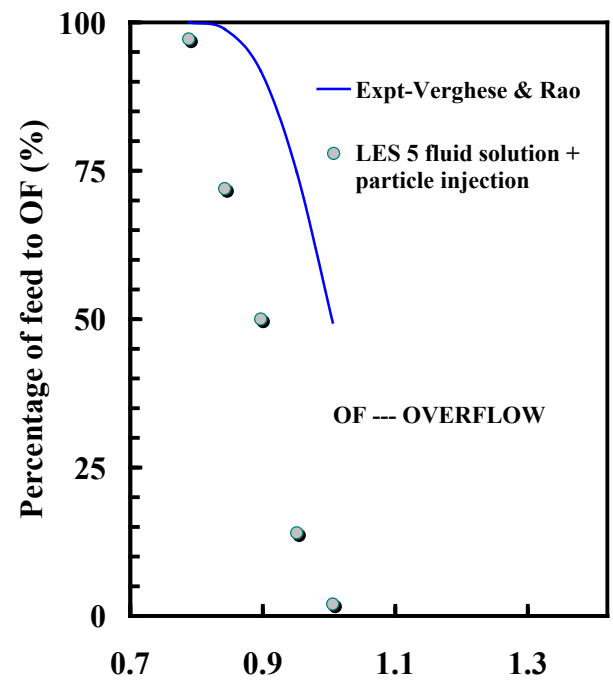

Reduced relative density, (SG/SG50)

$1673 \mu \mathrm{m}$

1086 microns

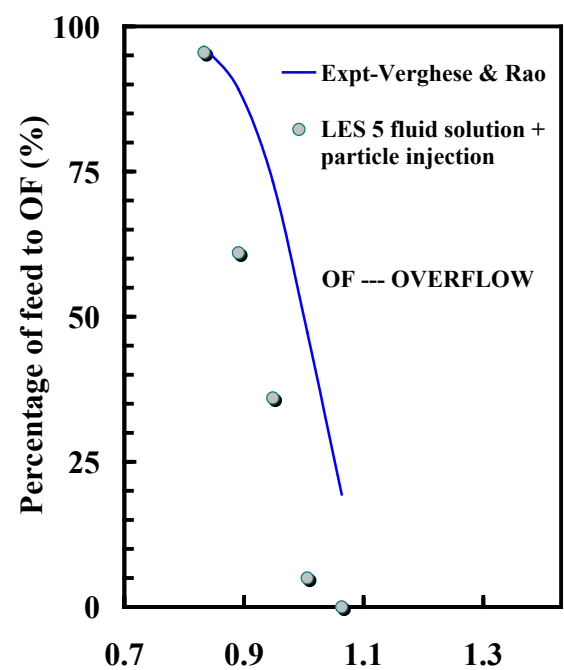

Reduced relative density, (SG/SG50)
1285 microns

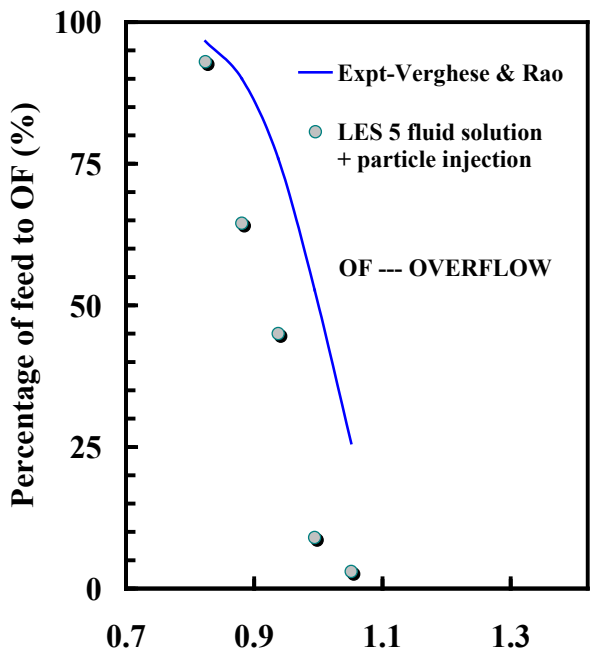

Reduced relative density, (SG/SG50)

$1285 \mu \mathrm{m}$

550 microns

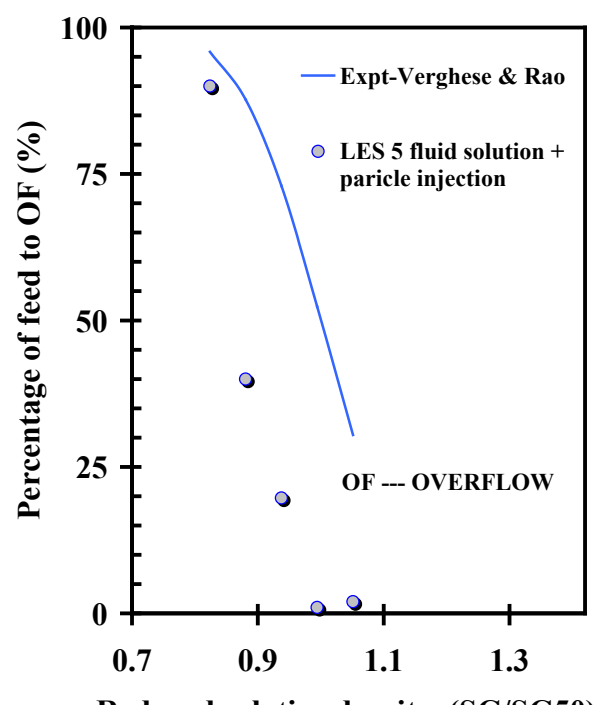

Reduced relative density, (SG/SG50)

$550 \mu \mathrm{m}$ 


\section{CASE 6}

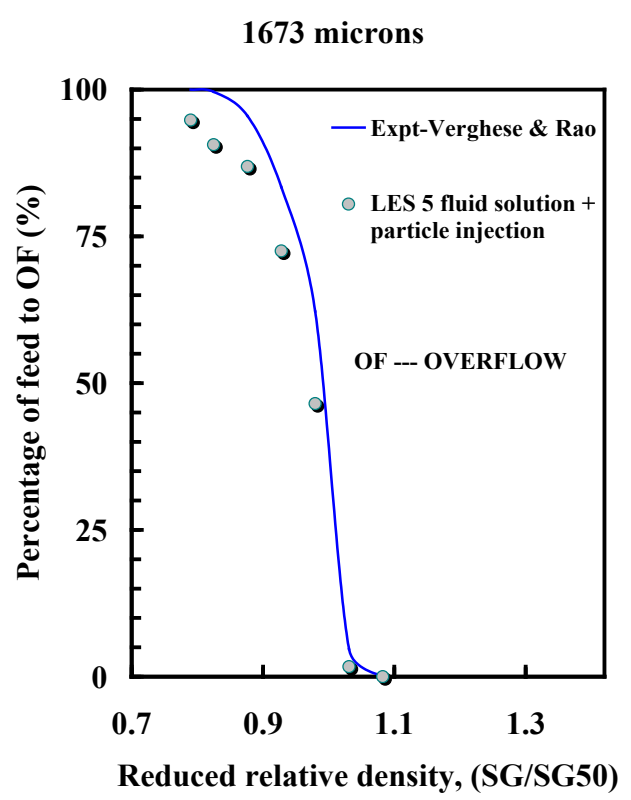

$1673 \mu \mathrm{m}$

1086 microns

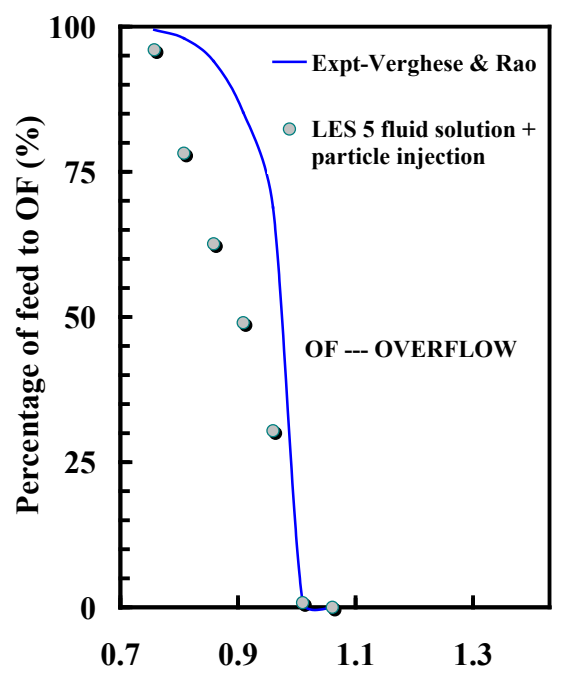

Reduced relative density, (SG/SG50)

$1086 \mu \mathrm{m}$
1285 microns

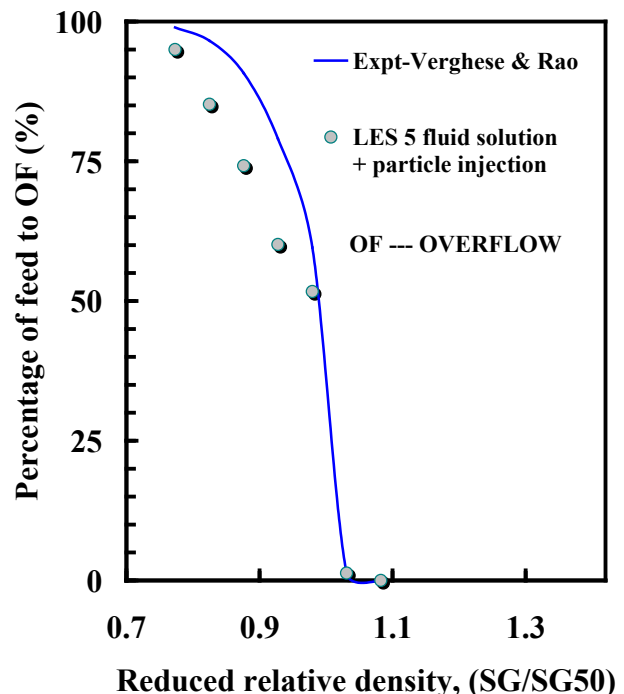

$1285 \mu \mathrm{m}$

550 microns

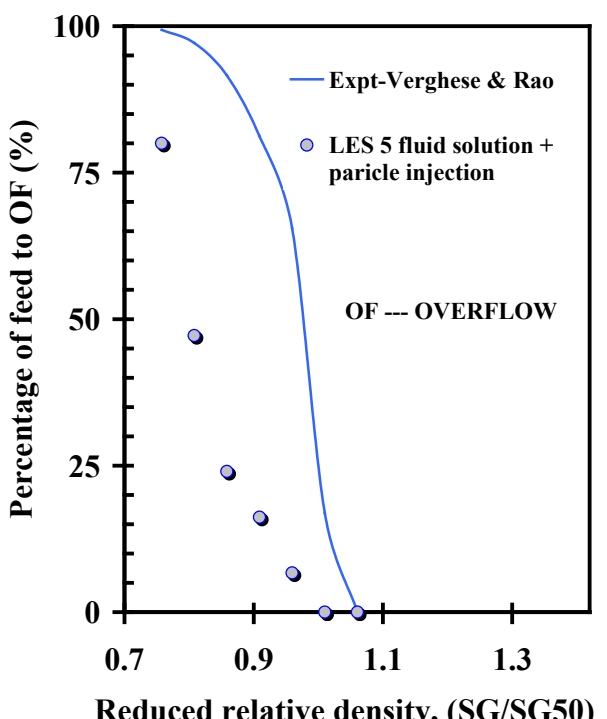

$550 \mu \mathrm{m}$ 
CASE 23

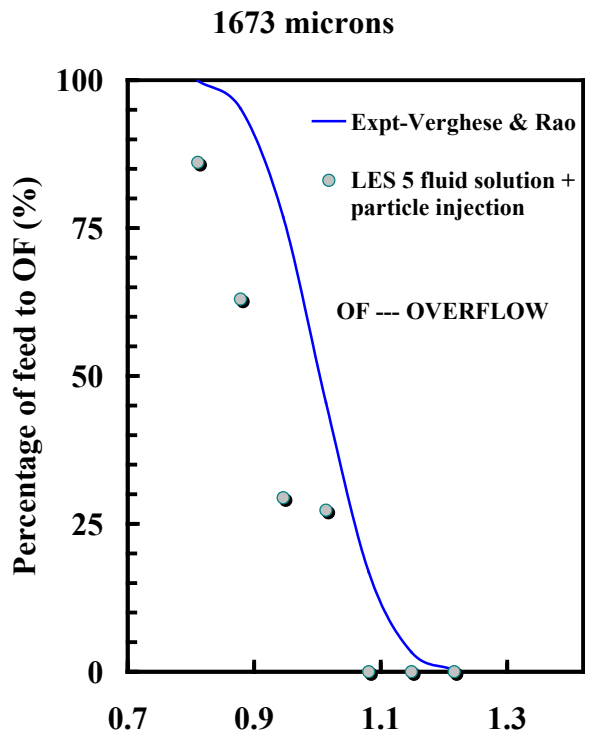

Reduced relative density, (SG/SG50)

$1673 \mu \mathrm{m}$

1086 microns

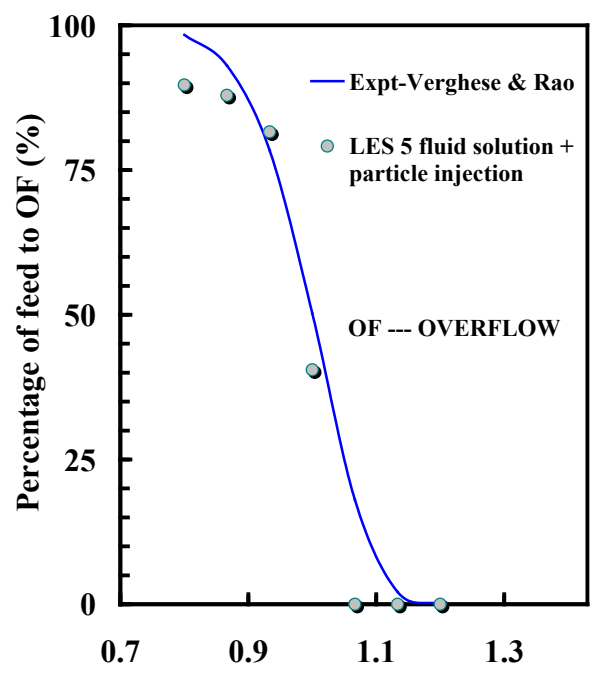

Reduced relative density, (SG/SG50)

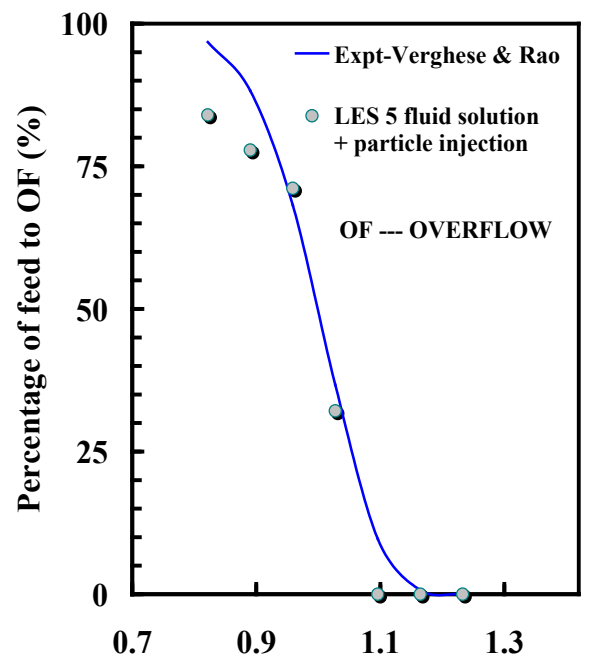

Reduced relative density, (SG/SG50)

$1285 \mu \mathrm{m}$

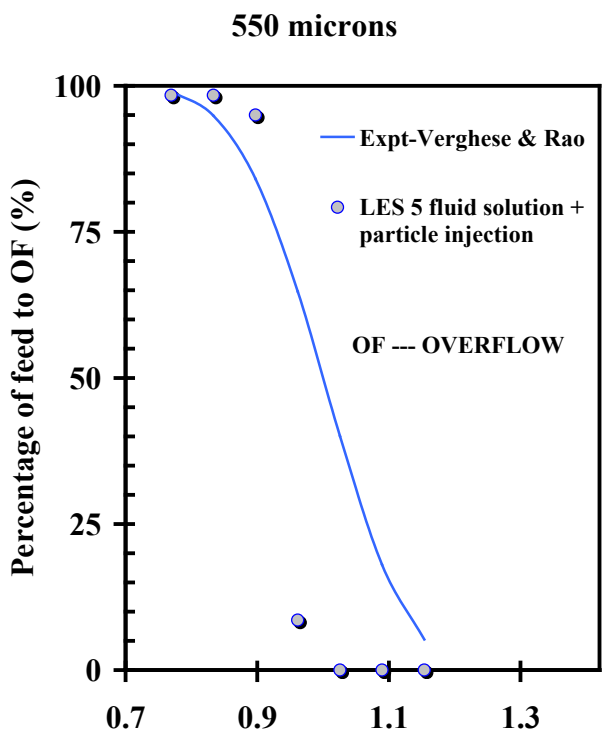

Reduced relative density, (SG/SG50)

\section{$1086 \mu \mathrm{m}$}

$550 \mu \mathrm{m}$

Figure 7. Predicted partition curve compared with experimental data for case 22, 19, 4, 6 and 23 (Percentage of feed to overflow versus relative density, SG/SG $\mathrm{S}_{50}$ ). 
The solid line in the figure is the fitted line to the large volume of experimental data, and the results from CFD simulation are marked by small circles. It is readily apparent that CFD predictions compare well with experiments. Both the slope and the cut density is well predicted for 550 to $1673 \mu \mathrm{m}$ sizes. However, it was noted that the predictive quality diminished at smaller particle sizes. It is believed that the force balance done by the Lagrangian tracking method may not accurately describe the drag forces acting on the coal particle especially in very small size range. While much is known in dilute fluid systems, accurate descriptions of the flow characteristics of highly concentrated fluid-particle systems are not yet available.

Velocity profile prediction is the most ubiquitous method of verification, although predicting Reynolds's stresses is more advanced in characterizing modeled turbulence if such measurement is available. The velocity field can be resolved into three components, tangential, radial, and axial. The tangential velocity determines the distribution of centrifugal force that constitutes the major contribution to the total pressure drop. The tangential velocity increases rapidly in the radial direction in a free vortex zone. In the central part of the hydrocyclone, the vortex and the air-core forces the tangential velocity to decrease creating a force vortex zone. The axial velocity sets the boundary between the inner spiral, which is moving upward, and outer spiral, which is moving downward. The accurate prediction of this component is the key to predicting particle size classification.

Delgadillo and Rajamani (2005) compared the LES model with RSM and $\kappa-\varepsilon$ model for the prediction of 75-mm and 250-mm hydrocyclones. Figure 8 shows the comparison between computed axial and tangential velocity profiles against experimental values in a 250 $\mathrm{mm}$ hydrocyclone. The trends in the experimental data which are obtained by Laser Doppler Velocity (LDV) measurements are extremely well predicted. Likewise, the velocity predictions were made at various heights within the body of the hydrocyclone compared well with experimental data. Since the velocity profiles along the axial and tangential direction are well described by LES, the conclusion is that the anisotropy of turbulence is well captured. 


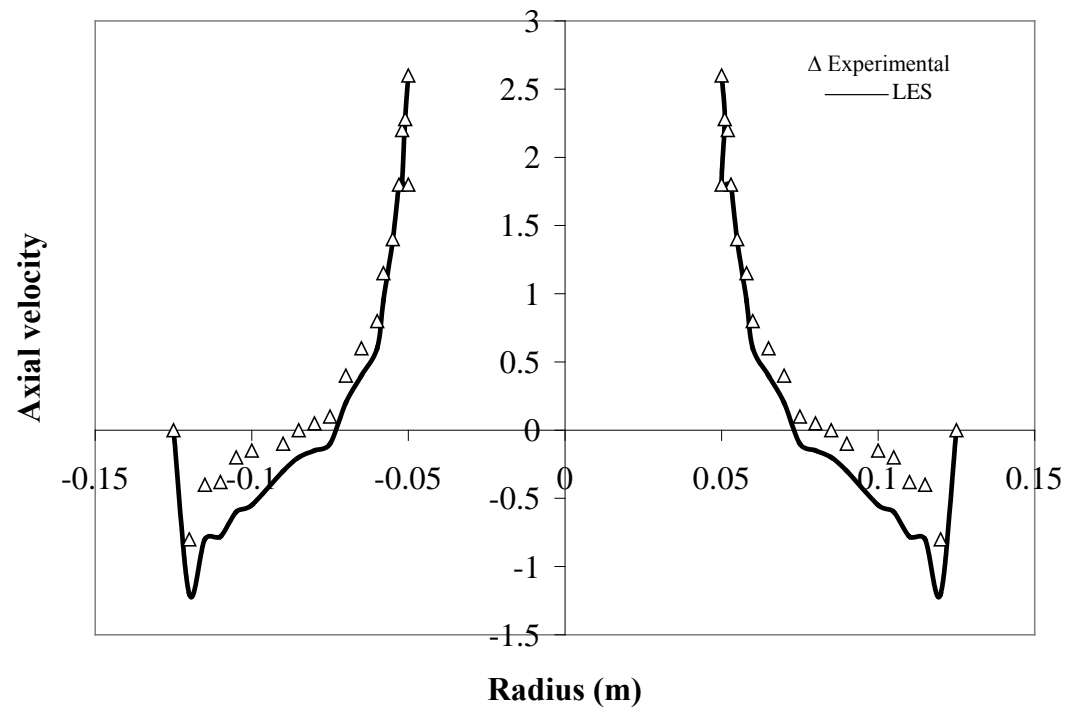

(a)

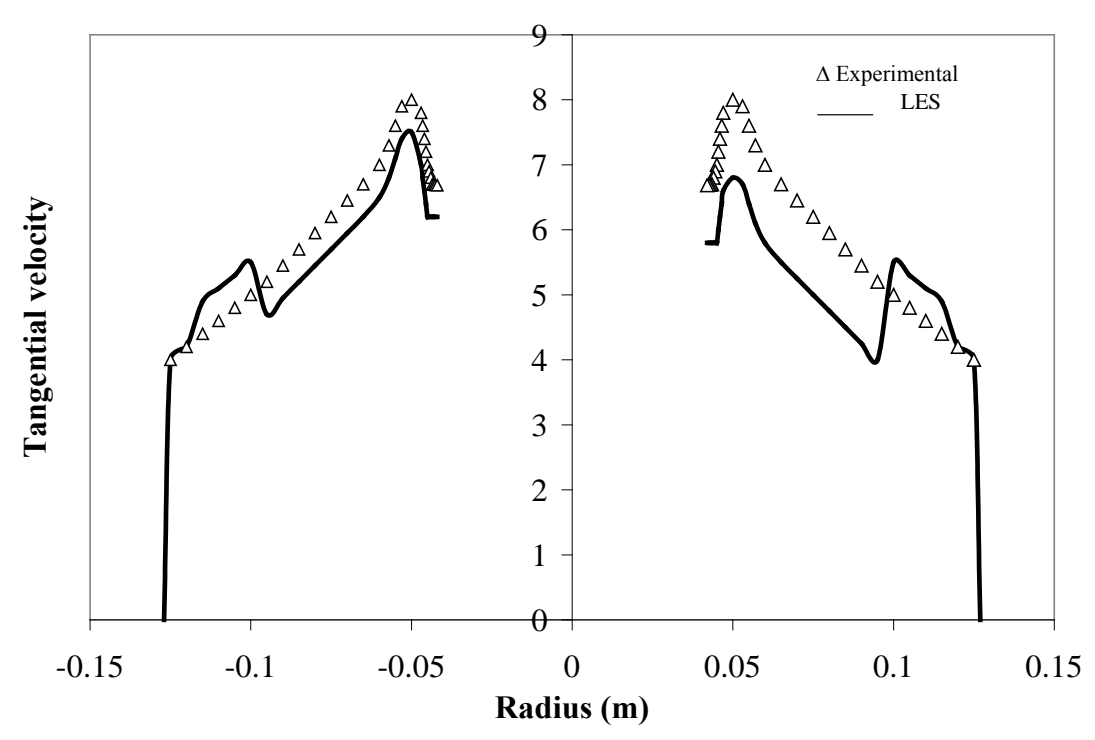

(b)

Figure 8. Axial (a) and tangential (b) velocity predictions with LES in a $250 \mathrm{~mm}$ hydrocyclone (Delgadillo and Rajamani, 2005). 
In the same study (Delgadillo and Rajamani, 2005) it was demonstrated that once the velocity profiles are accurately described, the particle size classification can also be predicted by following the path of each size class of particles in the computed velocity field. The particle path is traced by what is known as Lagrangian tracking method. Indeed such success with the LES method shows its promise for application in the dense medium hydrocyclone. More fundamental verification of fluid dynamics in the DMC field was shown by Narasimha et al. (2007) in their computer tomography verification of predicted density profiles.

\section{CONCLUSION}

A CFD simulation methodology is described for simulating the classification of dense medium cyclone. The methodology included multi-phase mixture modeling using five different fluids. Water being the primary fluid, air and three different granular magnetite fluid particles are used as the secondary phases. The turbulence is resolved using LES method. The classification behavior of $76 \mathrm{~mm}$ dense medium cyclone is evaluated. The distribution characteristics were generated using particle injection using the discrete particle modeling technique. The results show that the cut density of different size coal particles and the slopes of the distribution curves match with the published results. The simulation method using LES turbulence and five phase simulation is found to have improved matching characteristics than the multi-phase simulations generated using just three phases (water, air and single granular magnetite fluids), and two phases (pure high density water $1400 \mathrm{~kg} / \mathrm{m}^{3}$ density, and air) with RSM method for resolving the turbulence.

The study was about finding the minimum fluid configuration in the FLUENT solver code that would accurately describe the DMC performance. The five-fluid configuration was adequate for this purpose, yet it should be mentioned that for solution convergence CPU time was in the range of 20 to 40 days. 


\section{REFERENCES}

Brennan, M. S., 2003. Multiphase CFD simulations of dense medium and classifying hydrocyclones. Third International Conference on CFD in the Minerals and Process Industries: CSIRO, Melbourne, Australia.

Brennan, M. S., Narasimha, M., Holtham, P. N., 2007. Multiphase modeling of drocyclones - prediction of cut size. Minerals Engineering 20, 395 - 406.

Chu, K. W., Wang, B., Vince, A., and Yu, A. B., 2009. CFD - DEM modeling of multiphase flow in dense medium cyclones. Powder Technology 193, 235-247.

Cullivan, J.C., Williams, R.A., Dyakowski, T., Cross, C.R., 2004. New understanding of a hydrocyclone flow field and separation mechanism from computational fluid dynamics. Minerals Engineering 17, 651- 660.

Davis, J.J., 1994. A study on coal washing dense medium cyclone, Ph.D. Thesis, JKMRC, University of Queensland.

Delgadillo, A.J., Rajamani, R.K., 2005. A comparative study of three turbulence-closure models for the hydrocyclone problem. International Journal of Mineral Processing 77, 217-230.

Delgadillo, A.J., Rajamani, R.K., 2007. Large-Eddy Simulation (LES) of large hydrocyclones, Particulate Science and Technology, 25: 227-245.

Devulapalli, B., Rajamani, R.K., 1996. A comprehensive CFD model for particle-size classification in industrial cyclones. In: Hydrocyclones '96. Mechanical Engineering Publications, Ltd., London, UK, 83-104.

Griffths, W.D., Boysan, F., 1996. Computational fluid dynamics (CFD) and empirical modeling of the performance of a number of cyclone samples. Journal of Aerosol Science 27 (2), 281-304.

He, Y.b., Laskowski, J.S., 1994. Effect of dense medium properties on the separation performance of a dense medium cyclone. Minerals engineering, 7, 209-221.

Hsieh, K.T., 1988, A Phenomenological model of hydrocyclone, Ph.D. Thesis, University of Utah.

Hsieh, K.T., Rajamani, K., 1988. Phenomenological model of the hydrocyclone: model development and verification for single-phase flow. International Journal of Mineral Processing 22 (1-4), 223-237.

King, R. P., and Juckes A. H.., 1984. Cleaning of fine coals by dense-medium hydrocyclone. Powder Technology 40, $147-160$.

Manninen, M. , Taivassalo, V. and Kallio, S., 1996. On the mixture model for multiphase flow. VTT Publications 288, Technical Research Centre of Finland.

Monredon, T.C., Hsieh, K.T., Rajamani, R.K., 1992. Fluid flow model of the hydrocyclone: an investigation of device dimensions. International Journal Mineral Processing 35 (1-2), 65-83.

Narasimha, M., Brennan, M. S., Holtham, P. N., Napier - Munn T. J., 2007.A Comprehensive CFD model of dense medium cyclone peformance. Minerals Engineering 20, 414 426.

Narasimha, M., Sripriya, R., Banerjee, P.K., 2005. CFD modeling of hydrocyclone-prediction of cut size. International Journal Mineral Processing 75 (1-2), 53-68. 
Nowakowski, A.F., Dyakowski, T., 2003. Investigation of swirling flow structure in hydrocyclones. Transactions of the Institution of Chemical Engineers, Chemical Engineering Research and Design 81 (A), 862- 873.

Rajamani, R.K., and Milin, L., 1992. Fluid flow model of the hydrocyclone for concentrated slurry classification. Hydrocyclones: Analysis and Application: 4th Intentional Conference, vol. 12, 95-108.

Rajamani, R.K, Delgadillo, A.J, Udaya Bhaskar, K., and Alkac D., CFD modeling of dense medium cyclone, XVI. International Coal Preparation Congress 2010, Lexington, Kentucky.

Schiller, L. and Naumann.Z. ,1935. Z. Ver. Deutsch. Ing., 77:318.

Schuetz, S., Mayor, G., Bierdel, M., Piesche, M., 2004. Investigations on the flow and separation behavior of hydrocyclones using computational fluid dynamics. International Journal of Mineral Processing 73 (2-4), 229-237.

Slack, M.D., Wraith, A.E., 1997. Modelling the velocity distribution in a hydrocyclone, 4th International Colloquium on Process Simulation, 65-83.

Smagorinsky. J, 1963. General circulation experiments with the primitive equations. I. The BasicExperiment. Month. Wea. Rev., 91:99-164.

Suasnabar D. J., Fletcher C. A., 1999. A CFD model for dense medium cyclones. Second International Conference on CFD in the Minerals and Process Industries: CSIRO, Melbourne, Australia

Syamlal M., 1987. The Particle-Particle drag term in a multiparticle model of fluidization. National Technical Information Service, Springfield, VA, DOE/MC/21353-2373, NTIS/DE87006500.

Udaya Bhaskar, K., Rama Murthy, Ramakrishnan, N., Srivastava, J.K., Supriya Sarkar., Vimal Kumar., 2007, CFD validation for flyash classification in hydrocyclones, Minerals Engineering 20, 290-302.

Udaya Bhaskar K., Rama Murthy, Y., Ravi Raju, M., Sumit Tiwari, Srivastava, J.K., Ramakrishnan, N., 2007. CFD simulation and experimental validation studies on hydrocyclone, Minerals Engineering 20 , 60-71.

Verghese, P.A., Rao, T.C., 1994. Modelling of a 76mm dense medium cyclone, Coal preparation, Vol.15, 71-91. 


\section{APPENDIX}

The model used for flow simulation solves the conservation equations for mass (or continuity) and momentum. The turbulence in the system is solved through transport equations. Navier-Stokes equations for incompressible flows along with appropriate turbulence model are adopted for flow predictions. Under steady state conditions, the equations for mass and momentum in a general form are as follows:

$\nabla \cdot \rho v=0$

$\nabla \cdot(\rho \vec{v} \vec{v})=\nabla p+\nabla \cdot(\vec{\tau})+\rho \vec{g}$

where, $\mathrm{p}$ is a static pressure, $\rho \vec{g}$ is the gravitational body force $\vec{\tau}$ is the stress tensor given by

$$
\begin{array}{r}
\vec{\tau}=\mu_{\text {effective }}\left[(\nabla v)-\frac{2}{3} \nabla \cdot \rho \vec{v}^{2}\right] \\
\text { where } \mu_{\text {effective }}=\mu+\mu_{t}
\end{array}
$$

\section{Reynolds stress model (RSM)}

For steady-state, the Reynolds-Stress Model (RSM) adopted, uses the following transport equations for the Reynolds stresses:

$$
\begin{aligned}
& \frac{\partial}{\partial t}\left(\rho u_{k}^{\prime} u_{i}^{\prime} u_{j}^{\prime}\right)=P_{i j}+F_{i j}+D_{T_{i j}}+\phi_{i j}-\varepsilon_{i j} \\
& P_{i j} \text { (Stress production) }=-\rho\left(\overline{u_{i}^{\prime} u_{k}^{\prime}} \frac{\partial u_{j}}{\partial x_{k}}+\overline{u_{j}^{\prime} u_{k}^{\prime}} \frac{\partial u_{i}}{\partial x_{k}}\right) \\
& F_{i j} \text { (Rotation production) }=-2 \rho \Omega\left(\overline{u_{j}^{\prime} u_{m}^{\prime} \varepsilon_{i k m}}+\overline{u_{i}^{\prime} u_{m}^{\prime}} \varepsilon_{j k m}\right) \\
& \left.D_{T i j} \text { (Turbulent diffusion) }=-\frac{\partial}{\partial x_{k}}\left[\overline{\rho u_{i}^{\prime} u_{j}^{\prime} u_{k}^{\prime}}+\overline{P\left(\delta_{k j} u_{i}^{\prime}+\delta_{i k} u_{j}^{\prime}\right.}\right)\right] \\
& \phi_{i j} \text { (Pressure strain) }=+\left[\frac{\partial u_{i}^{\prime}}{\partial x_{j}}+\frac{\partial u_{j}^{\prime}}{\partial x_{i}}\right] \\
& \varepsilon_{i j} \text { (Dissipation) }=-2 \mu \frac{\partial u_{i}^{\prime}}{\partial x_{k}} \frac{\partial u_{j}^{\prime}}{\partial x_{k}}
\end{aligned}
$$




\section{Large Eddy Simulation model (LES)}

In LES modeling, larger energy carrying eddies are resolved and the smaller eddies are filtered and modelled using subgrid scale model. The following governing equations represent the resolving the turbulence using LES technique.

Equation of continuity $\frac{\partial \rho}{\partial t}+\rho \frac{\partial \bar{u}_{i}}{\partial x_{i}}=0$

Equation of motion $\frac{\partial \bar{u}_{i}}{\partial t}+\frac{\partial\left(\bar{u}_{i} \bar{u}_{j}\right)}{\partial x_{j}}=-\frac{1}{\rho} \frac{\partial \bar{p}}{\partial x_{i}}+\frac{\partial}{\partial x_{j}}\left(\mu \frac{\partial \bar{u}_{i}}{\partial x_{j}}\right)-\frac{\partial \tau_{i j}^{s g s}}{\partial x_{j}}+g_{i}$

Residual stress tensor $\quad \tau_{i j}^{s g s} \equiv \overline{u_{i} u_{j}}-\bar{u}_{i} \bar{u}_{j} \quad \tau_{i j}^{s g s}=-\mu_{t}\left(\frac{\partial \bar{u}_{i}}{\partial x_{j}}+\frac{\partial \bar{u}_{j}}{\partial x_{i}}\right)$

Turbulent viscosity $\quad \underbrace{\mu_{t}}=\mu_{\text {eff }}-\mu$

Effective viscosity $\quad \underbrace{\mu_{\text {eff }}}=\mu[1+H(x)]^{1 / 3}$

Heaviside function

$$
\underbrace{H(x)}=\left\{\begin{array}{l}
x, x \geq 0 \\
0, x \leq 0
\end{array} \quad x=\frac{\mu_{s}^{2} \mu_{\text {eff }}}{\mu^{3}}-100\right.
$$

Turbulent viscosity in sub grid scale $\underbrace{\mu_{s}}=\left(C_{R N G} V^{1 / 3}\right)^{2} \sqrt{2 \bar{S}_{i j} \bar{S}_{i j}}$

\section{Multiphase Mixture model}

In the dense medium cyclone modeling, the multi-phase mixture model used solves transport equations for the volume fraction for different secondary phases $\mathrm{p}$ (magnetite and air), which are dispersed in the primary phase water.

$\frac{\partial}{\partial t} \alpha_{p}+\frac{\partial}{\partial x i}\left(\alpha_{p} u_{i}\right)+\frac{\partial}{\partial x i}\left(\alpha_{p} u_{\rho m, i}\right)=0$

$u_{\rho m, i}=u_{p i}-u_{i}$

$u_{\rho m, i}$ is the drift velocity of phase p relative to the mixture phase m. 


\section{Equations of motion for particles}

In cyclone systems operating at dilute concentration of solids (typically below a value of $10 \%$ solids by weight), discrete phase modeling technique can be adopted for identifying the particle positions inside the system. The particles introduced into the system are simulated in a Lagrangian frame of reference using the definitions for material parameters like particle size, pecific gravity, and initial position etc. assuming solids spherical particles.

The trajectory of the discrete phase particle is obtained by integrating the force balance on the particle. This force balance equates the particle inertia with the other forces acting on the particle, and can be written (for the $\mathrm{x}$ direction in Cartesian coordinates) as

$\frac{d u_{p}}{d t}=F_{D}\left(u-u_{p}\right)+\left(g_{x}\left(\rho_{p}-\rho_{l}\right) / \rho_{p}\right)+F_{x}$

where $F_{D}\left(u-u_{p}\right)$ is the drag force per unit particle mass and

$F_{D}=\left(\frac{18 \mu_{m} d_{d}{ }^{2}}{\rho d}\right) *\left(\frac{C_{D} R_{e d}}{24}\right)$

Here $u$ is the fluid phase velocity, ${ }^{u_{p}}$ is the particle velocity, ${ }^{\mu}$ is the molecular viscosity of the fluid, $\rho_{l}$ is the fluid density, $\rho_{p}$ is the density of the particle and ${ }^{D_{p}}$ is the particle diameter, $R_{e}$ is the relative Reynolds number which is defined as

$$
R_{e}=\rho \times D_{p}\left(u_{p}-u\right) / \mu
$$


APPENDIX 5-Improving Coal Flotation Recovery Using Cavitation Concepts (KY007) 


\section{FINAL TECHNICAL REPORT}

Contract Title and Number:

Continuation in Crosscutting Technology Development

at CAST. (DE-FC26-05NT42457)
Period of Performance:

Starting Date: $10 / 1 / 05$

Ending Date: 9/30/2011
Sub-Recipient Project Title: Improving Coal Flotation Recovery Using Cavitation Concepts

Principal Investigators:

Tao, Honaker

Contact Address:

University of Kentucky

234 MMRB

Lexington KY 40506

Subcontractor Address:

No subcontracts issued.
Report Information:

Type: Final

Number: KY007-Final

Period: $\quad 10 / 1 / 07-9 / 30 / 11$

Date: $\quad 11 / 30 / 2011$

Code: KY007-FINAL

Contact Information:

Phone: (859) 257-2953

Fax: (859) 323-1962

E-Mail: dtao@engr.uky.edu

Subcontractor Information:

Phone:

Fax:

E-Mail:

\section{Disclaimer}

The Disclaimer must follow the Title Page and must contain the following paragraph:

"This report was prepared as an account of work sponsored by an agency of the United States Government. Neither the United States Government nor any agency thereof, nor any of their employees, make any warranty, express or implied, nor assume any legal liability or responsibility for the accuracy, completeness, or usefulness of any information, apparatus, product, or process disclosed, or represents that its use would not infringe privately owned rights. Reference herein to any specific commercial product, process, or service by trade name, trademark, manufacturer, or otherwise does not necessarily constitute or imply endorsement, recommendation, or favoring by the United States Government or any agency thereof. The views and opinions of authors expressed herein do not necessarily state or reflect those of the United States Government or agency thereof." 


\begin{abstract}
Froth flotation is the most widely used solid-solid separation process for coal and minerals beneficiation. However, its high process efficiency is limited to a narrow particle size range, which is usually 10-100 $\mu \mathrm{m}$ beyond which its efficiency decreases sharply, especially for oxidized coal that is more difficult to float due to weak hydrophobicity

The particle-bubble collision, attachment and detachment are the most critical steps in the flotation process. The low flotation recovery of fine particles is mainly due to the low probability of bubble-particle collision while the main reason for poor flotation recovery of coarse particles is the high probability of detachment of particles from bubble surface. Fundamental analysis indicated that use of smaller bubbles is the most effective approach to increase the probability of collision and reduce the probability of detachment.

The main goal of the proposed project was to enhance recovery of difficult-to-float coal particles $(-0.15 \mathrm{~mm}$ or $-100 \mathrm{mesh})$, particularly ultrafine $(<44$ or -325 mesh $\mu \mathrm{m})$ particles using a flotation column and a mechanical flotation cell featured with a hydrodynamic picobubble generator or an air eductor for feed preconditioning. Picobubbles (usually less than $1 \mu \mathrm{m}$ ) selectively attach to the hydrophobic coal particles. They are characterized by an inherently high probability of collision with particles and high probability of attachment and low probability of detachment due to their tiny size, low ascending velocity and rebound velocity from the surface, and high surface free energy to be satisfied, and therefore are very effective for enhancing flotation recovery of fine and coarse particles. Other major advantages of the proposed technique include lower collector dosage and air consumption since picobubbles are produced from air naturally dissolved in water and they act as the secondary collector on particle surfaces thereby resulting in considerably lower operating costs.
\end{abstract}




\section{TABLE OF CONTENTS}

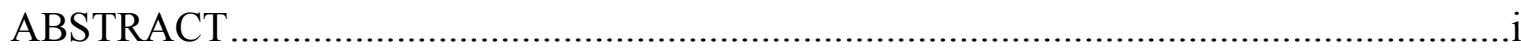

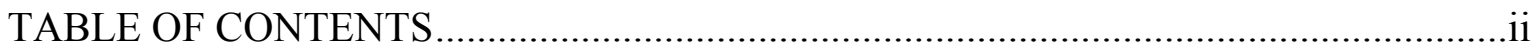

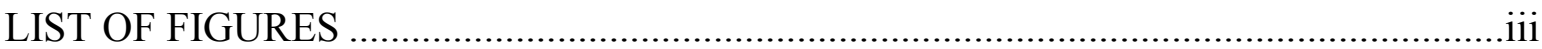

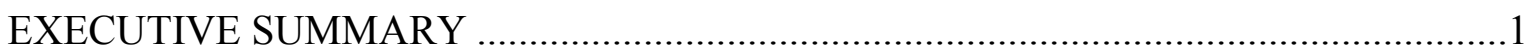

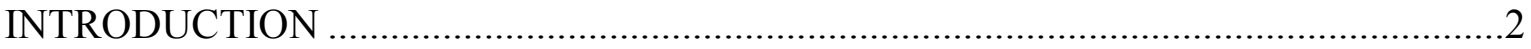

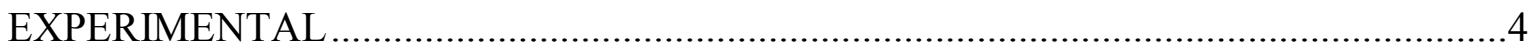

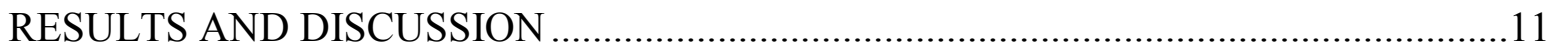

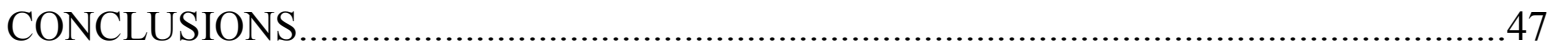

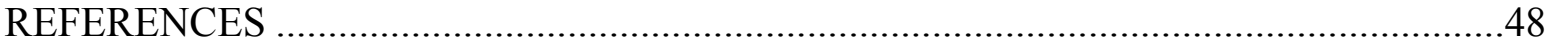

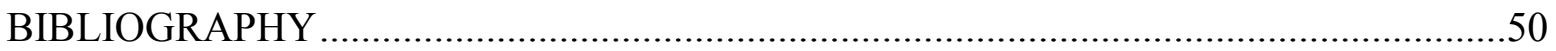




\section{LIST OF FIGURES}

Figure 1 Schematic diagram of bubble size measurement system using laser particle size analyzer.

Figure 2 Specially designed vibration cell for studying the effect of picobubble on bubble/particle detachment probability at varying vibration frequency and vibration amplitude.

Figure 3 Modified Hallimond tube for flotation efficiency experiments 8

Figure 4 Laboratory flotation column with picobubble generator and the conventionalsized bubble generator

Figure 5 A bank of three 10-liter mechanical cells ....................................................... 11

Figure 6 Flotation feed size distribution data ……………....................................... 12

Figure 7 Flotation feed ash content in different size fractions......................................... 13

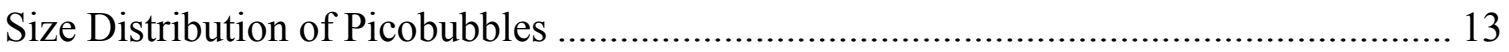

Figure 8 Picobubble generated from air naturally dissolved in water at varying concentrations of surfactant .................................................................................. 13

Figure 9 Bubbles generated by cavitation tube …………………............................. 14

Figure 10 Picobubble generated in water and on hydrophobic/hydrophilic particle surface.

Figure 11 Bubbles generated by the cavitation tube and the static mixer ........................ 15

Figure 12 Bubbles generated by the static mixer only ................................................... 15

Figure 13 The size distribution curve for bubbles in water solution with $10 \mathrm{ppm}$ MIBC and F507.

Figure 14 The size distribution curve for bubbles in water solution as a function of time.

Figure 15 Zeta potential of coal and picobubble as a function of $\mathrm{pH}$.............................. 17

Figure 16 Vibration and oscillation of particle-bubble aggregate in a vibration cell...... 17

Figure 17 Attachment and detachment fores and detachment probability as a function of phosphate (a) and coal (b) particle size.................................................................... 19 
Figure 18 Effect of vibration frequency on predicted detachment probability of $1.18 \mathrm{~mm}$, $0.85 \mathrm{~mm}, 0.60 \mathrm{~mm}, 0.425 \mathrm{~mm}, 0.30 \mathrm{~mm}$, and $0.075 \mathrm{~mm}$ particles (a) and experimental detachment probability on $1.18 \mathrm{~mm}$ amd $0.425 \mathrm{~mm}$ particle (b)....... 20

Figure 19 Effect of picobubble on experimental detachment probability of $1.18 \mathrm{~mm}$ and $0.425 \mathrm{~mm}$ phosphate (a) and coal (b) particles at varying vibration frequency. ...... 20

Figure 20 Effect of vibration amplitude and picobubble on detachment probability of 1.18 $\mathrm{mm}, 0.425 \mathrm{~mm}$ and $0.075 \mathrm{~mm}$ phosphate and coal particles.

Figure 21 Effect of bubble size on collection efficiency of $0.038-0.075 \mathrm{~mm}, 0.075-$ $0.212 \mathrm{~mm}$ and $0.212-0.425 \mathrm{~mm}$ phosphate particles with single bubble.

Figure 22 Effect of bubble size on flotation efficiency of $0.038-0.075 \mathrm{~mm}, 0.075-$ $0.212 \mathrm{~mm}$ and $0.212-0.425 \mathrm{~mm}$ phosphate particles at a given air flow rate.

Figure 23 Phosphate and coal particle flotation efficiency versus bubble size at a given air flow rate with or without picobubbles 23

Figure 24 Effect of picobubble on the flotation probability of varying coal particle sizes at collector dosage of $0.3 \mathrm{~kg} / \mathrm{ton}$.

Figure 25 Combustible recovery and product ash as a function of superficial air velocity with and w/o picobubbles 25

Figure 26 Combustible recovery vs. product ash at different superficial air velocity with and w/o picobubbles 25

Figure 27 Combustible recovery and product ash as a function of tailing recirculating flow rate ratio between static mixer and picobubble generator.

Figure 28 Combustible recovery and product ash as a function of collector dosage with and w/o picobubbles

Figure 29 Combustible recovery vs. product ash at different collector dosage with and w/o picobubbles

Figure 30 Combustible recovery and product ash as a function of frother dosage with and w/o picobubbles 28

Figure 31 Combustible recovery vs. product ash at different frother dosage with and w/o picobubbles 28

Figure 32 Combustible recovery and product ash as a function of superficial wash water velocity with and w/o picobubbles 29

Figure 33 Combustible recovery vs. product ash at different superficial wash water velocity with and w/o picobubbles 
Figure 34 Combustible recovery and product ash as a function of superficial feed rate with and w/o picobubbles

Figure 35 Combustible recovery vs. product ash at different superficial feed rate with and

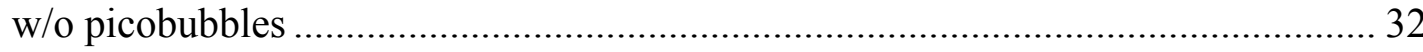

Figure 36 Combustible recovery and product ash as a function of particle size. ............. 33

Figure 37 Combustible recovery vs. product ash with and w/o picobubbles ................. 34

Figure 38 Separation efficiency as a function of particle size with and w/o picobubbles 35

Figure 39 Effect of picobubbles on the combustible recovery, product ash and flotation selectivity index at varying feed slurry solids concentration............................... 37

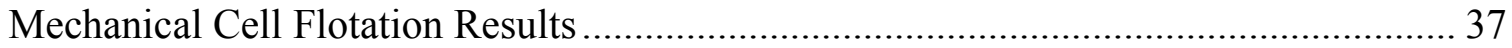

Figure 40 Effect of picobubbles on coal flotation recovery and separation density......... 38

Figure 41 Effect of picobubbles on yield and combustible recovery .......................... 40

Figure 42 Incremental and cumulative particle size distribution of flotation product with

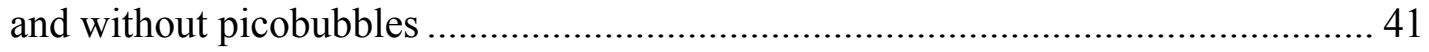

Figure 43 Cumulative size distribution of each flotation cell product with and without

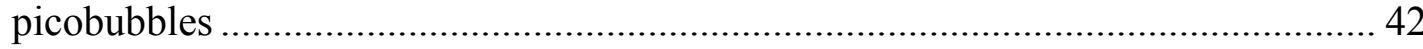

Figure 44 Effects of picobubbles on the flotation kinetics of various size coal particles. 44

Figure 45 Effects of picobubbles on the flotation rate constant .................................. 45 


\section{EXECUTIVE SUMMARY}

Froth flotation is the most widely used separation process for coal and minerals beneficiation. In this process hydrophobic particles captured by air bubbles ascend to the top of the pulp zone and eventually report to the froth product whereas hydrophilic particles remain in the pulp and are discharged as tailings. However, high flotation process efficiency is limited to a narrow particle size range, which is usually $10-100 \mu \mathrm{m}$. It is not very effective for cleaning ultrafine oxidized coal particles. Flotation of micron or sub-micron ultrafine particles is well known to be a difficult task. Recent studies have shown that the low flotation efficiency of ultrafine particles is mainly due to the low probability of bubble-particle collision. Oxidized coal is more difficult to clean by froth flotation due to its poor surface hydrophobicity. Enhanced flotation of fine oxidized coal can be achieved by increasing collision and attachment probabilities and decreasing the detachment probability.

The overall objective of the proposed research program was to develop an innovative flotation process for enhanced recovery of fine particles $(-0.15 \mathrm{~mm}$ or $-100 \mathrm{mesh})$ of oxidized coal, by enhancing bubble particle collision/attachment and minimizing detachment. Picobubble technology based on a Venturi tube was integrated into mechanical and column flotation cells to achieve the goal by feed preconditioning and/or tailing scavenging. Major tasks performed during this study include: 1) Sample acquisition and characterization; 2) Microflotation experiments on flotation efficiency; 3) Column flotation experiments; 4) Mechanical flotation experiments. Microflotation experiments were conducted to to investigate the effect of bubble size on the flotation rate of particles of different size and with different contact angles. Column and mechanical flotation experiments were performed to understand how primary operating parameters such as superficial gas velocity, superficial feed flow rate, wash water flow rate, collector dosage, and frother dosage affect cavitation picobubble enhanced flotation performance. A size-by-size analysis of flotation concentrate and tailings was conducted to determine separation efficiency for each size fraction under some conditions.

Tiny bubbles or gas nuclei of usually less than $1 \mu \mathrm{m}$ are referred to as picobubbles. They naturally exist in liquids such as seawater and distilled water. Picobubbles attach more readily to particles than large bubbles due to their lower ascending velocity and rebound velocity from the surface and higher surface free energy to be satisfied. More efficient attachment of particles and improved flotation rate have been observed when tiny bubbles co-exist with air bubbles commonly used in flotation cells. It has been shown that the combined flotation by gas nuclei from air supersaturation and by mechanically generated bubbles produced higher flotation recovery than by either of them alone. Gas nuclei or picobubbles on particle surface activate flotation by promoting the attachment of larger bubbles since attachment between gas nuclei or picobubbles and large bubbles is more favored than bubble/solid attachment. In other words, picobubbles act as a secondary collector for particles, reducing flotation collector dosage, enhancing particle attachment probability and reducing the detachment probability. This leads to substantially improved flotation recovery of poorly floating oxidized coal particles and reduced 
reagent cost, which is often the largest single operating cost in commercial mineral flotation plants.

Hydrodynamic cavitation is the process of creating picobubbles in a liquid due to the rupture of a liquid-liquid or a liquid-solid interface under the influence of external forces. The bubbles generated on a particle surface by cavitation naturally attach to the particle, eliminating the collision and attachment process which is often the rate determining step for flotation. Cavitation also improves the flotation efficiency of coarse particles by reducing the detachment probability during the rise of particle-bubble aggregate.

The experimental results have shown that hydrodynamically produced picobubbles are about two orders of magnitude smaller in size than the conventional sized bubbles. The use of picobubbles increased the probabilities of collision and attachment and decreased the probability of detachment during the flotation process. The application of the picobubble flotation process to coal flotation resulted in an increase in flotation recovery up to $20 \mathrm{wt} \%$ or more, a frother dose reduction of one third to one half and a collector dose reduction of up to two thirds. It also greatly expanded the particle size range for effective flotation.

\section{INTRODUCTION}

Froth flotation is the most widely used separation process for coal and minerals beneficiation. In this process hydrophobic particles captured by air bubbles ascend to the top of the pulp zone and eventually report to the froth product whereas hydrophilic particles remain in the pulp and are discharged as tailings. However, high flotation process efficiency is limited to a narrow particle size range, which is usually $10-100 \mu \mathrm{m}$. It is not very effective for cleaning ultrafine oxidized coal particles. Flotation of micron or sub-micron ultrafine particles is well known to be a difficult task. Recent studies have shown that the low flotation efficiency of ultrafine particles is mainly due to the low probability of bubble-particle collision. Oxidized coal is more difficult to clean by froth flotation due to its poor surface hydrophobicity. Enhanced flotation of fine oxidized coal can be achieved by increasing collision and attachment probabilities and decreasing the detachment probability.

The key to the success of effective particle separation by flotation is the efficient capture of hydrophobic particles by air bubbles, which is accomplished in three distinct processes: collision, adhesion, and detachment.

The probability of collision $\left(\mathrm{P}_{\mathrm{c}}\right)$ can be calculated from stream functions for quiescent conditions (Weber and Paddock, 1983; Yoon and Luttrell, 1989) and microturbulence models for well mixed conditions (Schubert and Bischofberger, 1979; Yoon, 2000). One of the mathematical models for $P_{c}$ is shown in equation (1):

$P_{c}=\left[\frac{3}{2}+\frac{4 R e^{0.72}}{15}\right]\left(\frac{D_{p}}{D_{b}}\right)^{2}$,

where $D_{b}$ is the bubble size, $D_{p}$ the particle size and Re is the Renolds number. Equation (1) shows that $P_{c}$ increases with increasing particle size and decreasing bubble size. Fine 
particles have low probability of collision with bubbles and are thus difficult to catch by bubbles, particularly by large size bubbles. This is the main reason for low flotation rate of fine particles.

The probability of attachment $\left(\mathrm{P}_{\mathrm{a}}\right)$ is related to the energy barrier for the bubble-particle adhesion $E_{1}$ and the kinetic energy of collision $E_{k}$ in Equation (2) (Yoon and Luttrell, 1989; Mao and Yoon, 1997):

$P_{a}=\exp \left(-\frac{E_{1}}{E_{k}}\right)$

$\mathrm{P}_{\mathrm{a}}$ can be calculated using Equation (3) (Yoon and Luttrell, 1989):

$P_{a}=\sin ^{2}\left[2 \tan ^{-1} \exp \left(-\frac{45+8 R e^{0.72} u_{b} t_{i}}{15 D_{b}\left(D_{b} / D_{p}+1\right)}\right)\right]$

where $t_{i}$ is the induction time and $u_{b}$ is the bubble rise velocity. Equation (3) indicates that $\mathrm{P}_{\mathrm{a}}$ decreases with increasing $\mathrm{D}_{\mathrm{p}}$, suggesting that coarse particles are more difficult to attach to air bubbles. $\mathrm{P}_{\mathrm{a}}$ increases with increasing particle hydrophobicity or decreasing $\mathrm{t}_{\mathrm{i}} ; \mathrm{P}_{\mathrm{a}}$ also increases with decreasing bubble size until the bubble size becomes too small.

All particles attached to air bubbles do not report to the froth phase. Some of them detach from bubble surface and drop back into the pulp phase. Particle detachment occurs when detachment forces exceed the maximum adhesive forces. The adhesive forces include the capillary force $F_{p}$ and the excess force $F_{e}$ while detachment forces consist of real weight of particle in the liquid medium $F_{w}$ and the hydrodynamic drag force $F_{d}$. These forces are typically represented by the following equations (Drzymala, 1994; Ralston and Dukhin, 1999; Ralston et al., 1999a; Ralston et al., 1999b):

$F_{p}=\pi D_{p} \gamma\left(1-\cos \theta_{d}\right) / 2$

$F_{e}=\frac{1}{4} \pi D_{p}^{2}\left(1-\cos \theta_{d}\right)\left(2 \gamma / D_{b}-\rho_{w} g D_{b} / 2\right)$

$F_{w}=\frac{1}{6} \pi D_{p}^{3} \rho_{p} g-\frac{1}{8} \pi D_{p}^{3} \rho_{w} g\left[2 / 3+\cos \left(\theta_{d} / 2\right)-(1 / 3) \cos ^{3}\left(\theta_{d} / 2\right)\right]$

$F_{d}=3 \pi D_{p} \eta u$

where $\gamma$ is the liquid surface tension, $\rho_{\mathrm{p}}$ and $\rho_{\mathrm{w}}$ the densities of the particle and water respectively, $\eta$ dynamic viscosity of fluid, $u$ particle rising velocity, and $\theta_{d}$ is the critical value of three phase contact angle right before detachment. It is very interesting to point out that the excess force increases with decreasing bubble size $D_{b}$, which means that smaller bubbles can be used to reduce coarse particle detachment. Therefore, flotation recovery of coarse particles can be enhanced using smaller rather than larger bubbles.

The probability of detachment $\left(\mathrm{P}_{\mathrm{d}}\right)$ may be described by Equation (8):

$P_{d}=\frac{1}{1+F_{a t} / F_{d e}}$

where $F_{\text {at }}$ represents the total attachment force and $F_{d e}$ the total detachment force. Using equations (4) - (6) and neglecting the drag force, one obtains: 
$\frac{F_{a t}}{F_{d e}} \approx \frac{3\left(1-\cos \theta_{d}\right) \gamma}{g\left(\rho_{p}-\rho_{w}\left(1 / 2+3 / 4 \times \cos \left(\theta_{d} / 2\right)\right)\right)} \cdot \frac{1+D_{p} / D_{b}}{D_{p}^{2}}$

It can be readily seen from Equations (8) and (9) that $F_{a t} / F_{d e}$ decreases and $P_{d}$ increases with increasing $D_{p}$ and increasing $D_{b}$. This conclusion is in agreement with the empirical correlation that shows that the detachment rate constant for flotation increases with increasing $\mathrm{D}_{\mathrm{b}}$ and $\mathrm{D}_{\mathrm{p}}$ (Gorain et al., 1995; Gorain et al., 1997; Deglon et al., 1999). Therefore, coarse particles are more likely to detach from air bubbles and use of small bubbles will increase flotation recovery of coarse particles.

The first order flotation rate constant (k) is determined by (Yoon et al., 1989):

$k=\frac{3 V_{g}}{2 D_{b}} P=\frac{1}{4} S_{b} P=\frac{1}{4} S_{b} P_{c} P_{a}\left(1-P_{d}\right)$

where $V_{g}$ is the superficial gas rate, $P$ the probability of collection, and $S_{b}$ is the bubble surface area flux. Since $P_{c}, P_{a}$, and $P_{d}$ are all dependent on $D_{b}$ and $D_{p}$, as discussed earlier, Equation (10) indicates that $\mathrm{k}$ is strongly dependent on $\mathrm{D}_{\mathrm{b}}$ and $\mathrm{D}_{\mathrm{p}}$. It has been shown that $\mathrm{k}$ varies as $D_{p}^{2} / D_{b}^{3}$ under quiescent conditions in flotation columns. Obviously, the flotation recovery of particles can be enhanced effectively by use of smaller bubbles.

\section{EXPERIMENTAL}

\section{Sample Acquisition and Characterization}

Two 55-gallon drums of fine coal sample (characterized with high ash and low hydrophobicity) were acquired from a major coal Company in West Virginia and the ash analysis and size distribution analysis were conducted. Table 1 shows the size distribution data of the coal sample. Obviously, almost all coal particles (about 98\%) were smaller than $1.18 \mathrm{~mm}$ or 16 mesh. A small portion of coal particles $(6.83 \%)$ were smaller than $43 \mu \mathrm{m}$ or 325 mesh. The feed ash content was $30.28 \%$ and the solids content was $15.6 \%$.

Table 1. Particle Size and Ash Distribution of Coal Sample

\begin{tabular}{|c|c|c|c|c|}
\hline $\begin{array}{c}\text { Particle Size } \\
\text { Fraction (mm) }\end{array}$ & Wt (\%) & $\begin{array}{c}\text { Cumulative } \\
\text { Wt (\%) }\end{array}$ & Ash (\%) & $\begin{array}{c}\text { Cumulative } \\
\text { Ash (\%) }\end{array}$ \\
\hline Minus 0.043 & 6.83 & 6.83 & 76.21 & 76.21 \\
\hline $0.075-0.043$ & 11.47 & 18.30 & 51.26 & 60.57 \\
\hline $0.15-0.075$ & 17.66 & 35.96 & 37.32 & 49.15 \\
\hline $0.212-0.15$ & 17.61 & 53.57 & 29.54 & 42.71 \\
\hline $0.30-0.212$ & 15.65 & 69.22 & 21.17 & 37.84 \\
\hline $0.425-0.30$ & 11.07 & 80.29 & 15.32 & 34.73 \\
\hline $0.85-0.425$ & 13.70 & 93.99 & 12.29 & 31.46 \\
\hline $1.18 \sim 0.85$ & 3.86 & 97.85 & 11.93 & 30.69 \\
\hline Plus 1.18 & 2.15 & 100.00 & 11.71 & 30.28 \\
\hline
\end{tabular}




\section{$\underline{\text { Picobubbles Size Measurements }}$}

Figure 1 schematically shows the picobubble generating and sampling system for measuring the bubble size. Cillas 1064 laser particle size analyzer was used to measure the size distribution of bubbles generated by Venturi tube and static mixer. It uses the principle of laser diffraction to analyze particles from 0.04 to 500 micrometers in size in a time span of several minutes.

The column was made of plexiglass with $50.8 \mathrm{~mm}$ in diameter and $356 \mathrm{~mm}$ in height. The bottom of column is $152.4 \mathrm{~mm}$ in diameter and $152.4 \mathrm{~mm}$ in height. The larger diameter of the bottom of the column compared to the top of the column was designed to achieve a stable flow. The specially designed cavitation tube and the static mixer that were installed in the parallel configuration were used to generate picobubbles and conventional sized bubbles, respectively. The picobubbles were generated from dissolved gas in water by pumping water and frother solution through the cavitation tube. In the tests, the Frother MIBC (Methyl Isobutyl Carbinol) and F507 (a kind of glycol) were used as surfactants. $<50 \mathrm{~nm}$ titanium oxide $\left(\mathrm{TiO}_{2}\right)$ particles coated with $\mathrm{Al}_{2} \mathrm{O}_{3}$ and fatty acid salt, and $<50 \mathrm{~nm}$ titanium oxide $\left(\mathrm{TiO}_{2}\right)$ particles coated with $\mathrm{SiO}_{2}$ were used as the hydrophobic particle and the hydrophilic particles, respectively.

The cavitation tube generated picobubbles and the static mixer generated conventional sized bubbles in the column were pumped into and out of the Cillas 1064 laser particle size analyzer by two peristaltic pumps. The bubble sample, which was pumped into the laser size analyzer, is internally dispersed and circulated through the path of the laser light beam. As the bubbles pass through the laser beam, the light scatters at angles inversely proportional to bubble size and with intensity directly proportional to bubble size. A $45^{\circ}$ rotational charge-coupled device collects the scattered light intensity, which is converted to electrical signals and bubble size distribution, analyzed in a microprocessor. 


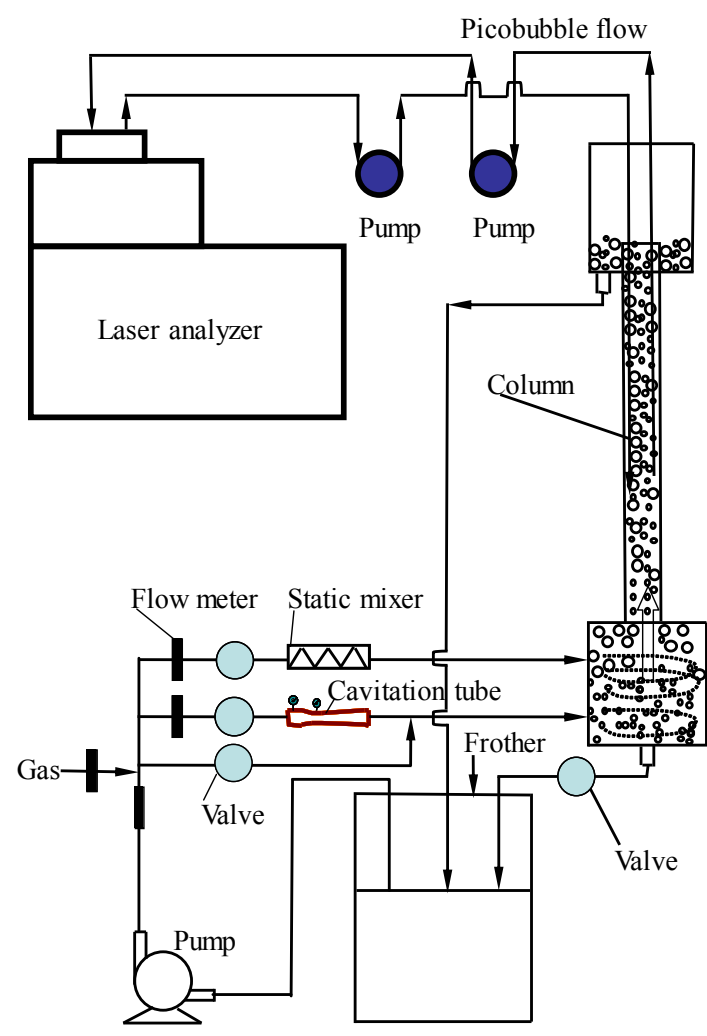

Figure 1 Schematic diagram of bubble size measurement system using laser particle size analyzer.

\section{$\underline{\text { Zeta Potential of Picobubbles }}$}

Zeta potential is a measure of the magnitude of the electrostatic repulsion or attraction between particles and bubbles. The data on Zeta potential offers insights into the interaction mechanisms between bubbles and particles. The Zeta potential of picobubbles generated by the cavitation tube was measured using ZetaPlus (Brookhaven Instruments Co., Holtsville, NY) to quantify the surface charge of the bubbles.

\section{$\underline{\text { Vibration Cell for Detachment Tests }}$}

Figure 2 shows a specially designed vibration cell that was used to study the effect of picobubble on bubble/particle detachment probability at varying vibration frequency and vibration amplitude. The frequency and amplitude were adjusted through electromagnetic vibrator. A cylindrical glass cell $(\phi 50 \times 15 \mathrm{~mm})$ containing a single bubble with attached particles was fixed to the above mentioned vibrator and vibrated at the given amplitude and frequency for a fixed time period and the number of particles detached was determined by counting. The particles used in the experiments were in three size fractions of $1.18-0.85 \mathrm{~mm}, 0.85-0.60 \mathrm{~mm}$, and $0.60-0.425 \mathrm{~mm}$. Each size fraction was further upgraded by hand-picking under a microscope. 


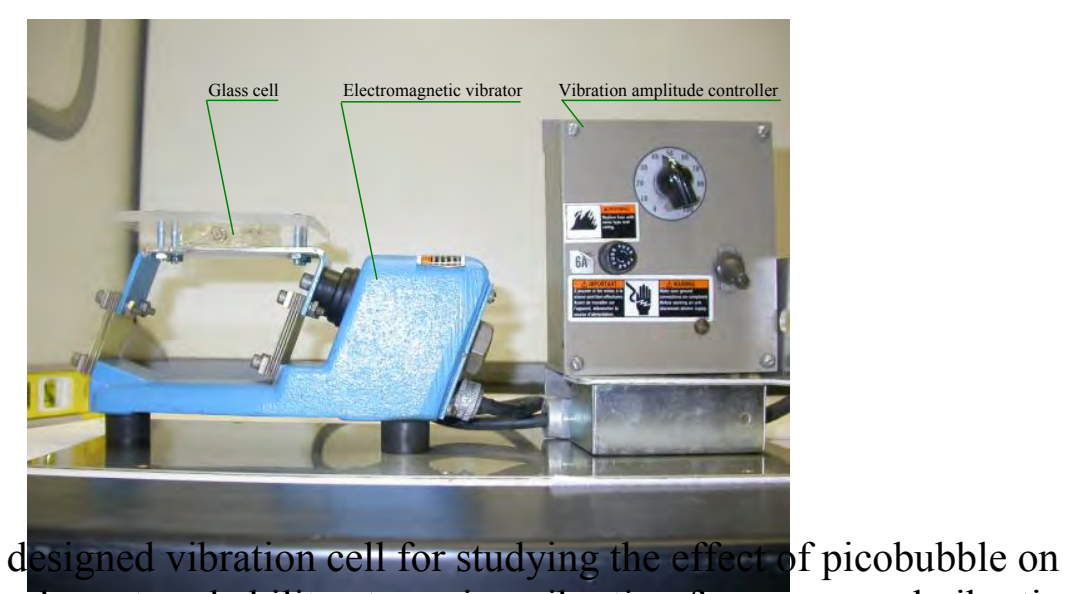

Figure 2 Specially designed vibration cell for studying the effect-of picobubble on bubble/particle detachment probability at varying vibration frequency and vibration amplitude.

The glass cell was filled with the dilute collector solution. The cell was carefully sealed, ensuring that no air bubbles were trapped inside, and a single air bubble was then introduced through a syringe. Bubbles of $3.0 \mathrm{~mm}$ in diameter were used. The cell and its contents were then agitated by hand causing a number of the particles to become attached to the bubble, the number being determined by manual counting. The experiments were performed in the absence and presence of picobubbles in the solution. Diesel was used as collector for coal particle detachment tests.

The cell was then securely attached to the motor driven vibrator. By increasing the motor speed or frequency, the particle-bubble aggregate could be exposed to increasing levels of vibrational amplitude or frequency. After reaching the desired frequency level, the motor was turned off. The number of particle detached was determined by manual counting as before. At each frequency level, the number of particles detached from each aggregate was counted to get an average value. This procedure was repeated at increasing values of frequency or amplitude output until a level was reached at which all the particles were detached from the bubble in absence and presence of picobubbles in the solution.

\section{$\underline{\text { Monobubble Flotation }}$}

The flotation collection efficiency is primarily related to bubble size, particle size and contact angle of particles. Microflotation experiments were conducted to provide a better understanding of effects of bubble size and particle size on flotation efficiency and thus foster the development of the innovative process to enhance flotation recovery. The pure fine and coarse phosphate mineral sample used for the single bubble flotation tests was prepared by multiple-pass tribo electrostatic separation (Tao, et al., 2009; Tao, et al., 2008) and hand picking under microscope, respectively. The pure phosphate analyzed $32.43 \% \mathrm{P}_{2} \mathrm{O}_{5}$ and $4.69 \%$ insol. To make accurate measurements, the suspension in the Hallimond tube should be kept at constant solids concentration. However, when slurry was pushed into the Hallimond tube, all particles began to settle down and the solid concentration decreased. To maintain the concentration in a certain range, the testing time should be maintained within a certain limit. In our experiments, approximately 60 
bubbles were generated in 30 seconds for each test. For the relatively large size particles that settled fast, the experiments were performed in two batches, each with 30 bubbles in 15 seconds. For particles larger than $0.425 \mathrm{~mm}$, a specially designed monobubble flotation column was fabricated to carry out the tests, as described below.

Modified Monobubble Hallimond Tube Flotation

A modified monobubble Hallimond tube shown in Figure 3 was used to perform microflotation experiments. Because the number of the particles floated was very small compared with the total number of particles in the feed suspension, it was very important to minimize any upward mixing of particles from the suspension into the concentrate. The key to the success of such experiments was the formation of a particle-free water column in the upper part of the flotation tube before the individual single bubbles were introduced into the bottom of the column. The suspension was kept in the cone flask and stirred with a magnetic stirrer to keep the slurry homogeneous. The compressed air was introduced into the flask and pushed the homogeneous suspension to the Hallimond

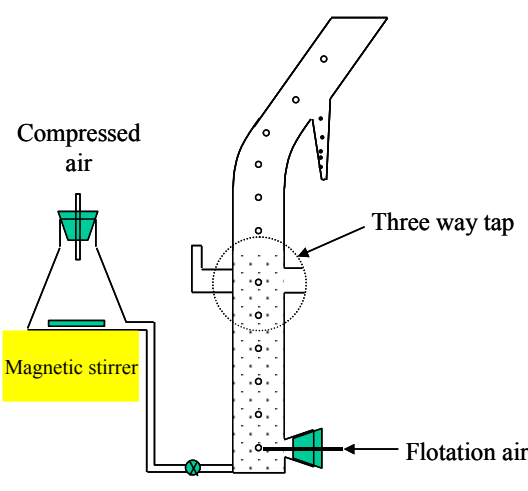

Figure 3 Modified Hallimond tube for flotation efficiency experiments tube. A three-way tap was used to divide the Hallimond tube into the upper part and the lower part. The lower part was full of the suspension while the upper part was filled with the clear water. Flotation air bubbles were injected through the opening at the bottom of the tube. A container was utilized to collect the slurry overflow.

The experimental procedure is described as follows:

1) Diluted water suspensions (about $0.3 \%$ by mass) of slid particles with or without picobubble solutions were stirred in a conditioning cell for 5 min with a magnetic stirrer.

2) The three-way tap was closed and the section of the flotation column below the tap was open to air through the overflow weir.

3) Clean water was added into the column from the top to the desired level.

4) At the end of the conditioning period, compressed air was introduced to force the suspension into the column. This upward flow prevented particle settling prior to flotation.

5) When the suspension was about to overflow from the overflow weir which was at the same height as the three-way tap, the suspension flow was stopped and the three-way tap was turned so that the suspension came into contact with the clear water above. This caused little turbulence and no particles were entrained into the clear water.

6) Immediately after step 5, single bubbles were generated and timed flotation started.

7) Flotation lasted 30 seconds with a total of 60 bubbles.

8) The number of particles floated by each bubble was calculated by dividing the total floated particles by the number of bubbles. 
9) Division of this value by the number of particles in the suspension column yielded the collection efficiency.

The number of particles floated by each bubble was calculated by dividing the total floated particles by the number of bubbles. Division of the value by the number of particles in the suspension column yielded the collection efficiency. The flotation efficiency was defined as the product of the collection efficiency multiplied by the number of bubbles. Bubbles of different sizes were produced using syringes or microsyringe of different specifications.

\section{$\underline{\text { Flotation Column }}$}

A cylindrical flotation column of $5 \mathrm{~cm}$ (2 inch) in diameter and $1.8 \mathrm{~m}$ (6 feet) in height shown in Figure 4 was utilized for coal flotation tests. The column was made of Plexiglas and featured a Venturi cavitation tube to produce picobubbles and a static mixer to produce conventional-sized bubbles. The length of collection and froth zones typically used in the test program were $210 \mathrm{~cm}$ and $30 \mathrm{~cm}$, respectively. With a diameter of $5.1 \mathrm{~cm}$, the length-to-diameter ratio was around 51:1, which provided near plug-flow conditions. Wash water was added in the froth zone at a

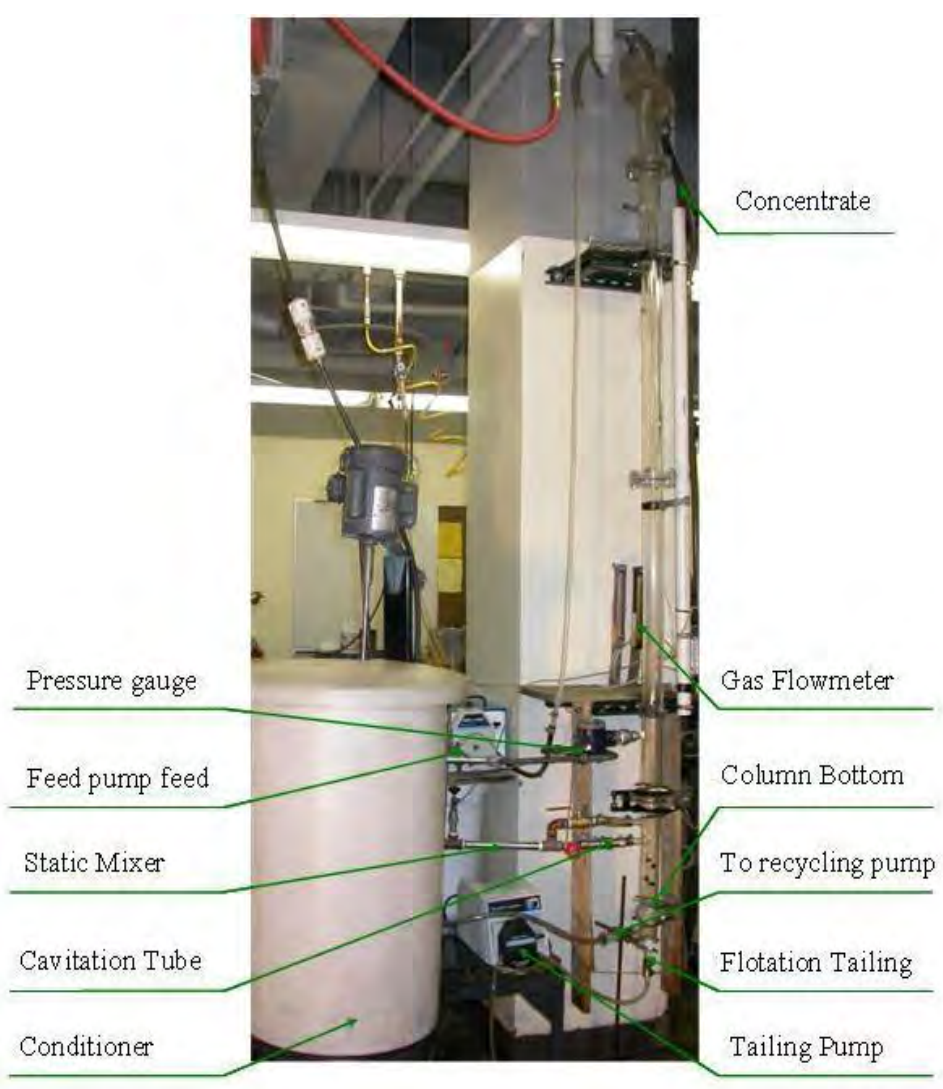
depth that was $1 / 3$ of the froth zone height below the overflow lip.

The cavitation tube and the static mixer were used to generate picobubbles and conventional-sized bubbles, respectively. Both are compact and have no moving parts. The pipe diameter of the cavitation tube is $12 \mathrm{~mm}$ and the neck diameter is $3.2 \mathrm{~mm}$. Frother was injected into the feed stream while air was injected into the stream prior to the static mixer. Feed slurry entered the column in the upper pulp zone, $45 \mathrm{~cm}$ below the overflow lip. After being fed into the column, coal particles collected by rising bubbles ascend to the top. Those that settle to the bottom of the column are pumped through the static mixer and the cavitation tube to have more chances for recovery. 
The slurry jet comes out of the neck of the Venturi cavitation tube at a speed of 6 to 10 $\mathrm{m} / \mathrm{s}$ causing hydrodynamic cavitation in the stream with picobubbles formed preferentially on coal particle surfaces. Picobubbles formed on hydrophobic coal particle surfaces remain attached while those on hydrophilic particle detached, which was a selective process that enhanced flotation separation efficiency. Hydrophobic particles had higher collision probability with picobubbles, higher attachment probability, and lower detachment probability, as explained earlier, resulting in a greater flotation rate constant and flotation recovery. The slurry jet enters the column tangentially at the bottom. The total recycling flow rate through the static mixer is $11 \mathrm{~L} / \mathrm{min}$, which splits at a two-way connector into the cavitation tube and a pipe. As a result, the flow rate distribution (flow rate in cavitation tube/total flow rate in static mixer) can be adjusted to be $6.6 \mathrm{~L} / 11.0 \mathrm{~L}=$ $60 \%$ (with picobubble) or $0 \mathrm{~L} / 11.0 \mathrm{~L}=0 \%$ (without picobubble). A microprocessor series 2600 Love Controls receives signals from a pressure transducer located at the bottom of the column. The signal adjusts the Miniflex pinch valve that controls the underflow flow rate and the desired froth level.

Prior to each test, feed slurry was conditioned for five minutes with fuel oil, which was used to enhance the hydrophobicity of coal particle surfaces. Conditioning was conducted in a sump that was equipped with a mixer and four baffles placed vertically and separated by an equal distance along the circumference of the sump. The feed slurry was pumped to a feed tank, which utilized a recirculating line to ensure suspension of all solids. A peristaltic pump was used to draw a pre-determined amount of feed into the flotation column. Unless otherwise specified, all column flotation tests were performed under the following conditions: froth depth of $30 \mathrm{~cm}$; superficial gas flow rate of $0.5 \mathrm{~cm} / \mathrm{s}$; fuel oil collector dosage of $1.0 \mathrm{lb} /$ ton; MIBC frother concentration of $30 \mathrm{ppm}$; superficial wash water flow rate of $0.12 \mathrm{~cm} / \mathrm{s}$; superficial feed slurry flow rate of $0.5 \mathrm{~cm} / \mathrm{s}$; feed slurry solids concentration of $7 \%$. A period of time equivalent to three particle retention times was allowed to achieve steady-state conditions. After reaching the steady-state, samples of feed, product, and tailing streams were collected simultaneously. These samples were filtered, dried, weighed, and analyzed for ash content.

Major process parameters were examined individually to investigate their effects on flotation recovery and concentrate ash content with and without the cavitation tube. They include superficial gas velocity, frother dosage, collector dosage, superficial wash water flow rate, and superficial feed flow rate. A size-by-size analysis of the flotation concentrate and tailings was conducted to determine flotation recovery and separation efficiency for each size fraction. Data from this analysis demonstrate how picobubbles affect flotation recovery and efficiency of different particle sizes, which can be further used for process simulation and performance prediction for different coal samples.

\section{Mechanical Cells Flotation Test}

To assess the impact of using picobubbles on coal separation performance, experiments were conducted with a bank of three 10-liter mechanical cells shown in Figure 5. During tests with picobubbles, part of the slurry in the third cell was pumped through a picobubble generator with $3.2 \mathrm{~mm}$ inner neck diameter and $12 \mathrm{~mm}$ inner pipe diameter, 
and then fed back to the first cell. In this configuration, picobubbles formed preferentially on hydrophobic coal particle surfaces remained attached while those produced on hydrophilic particle surfaces detached, which was a selective process that enhanced flotation separation efficiency. Hydrophobic particles had higher collision probability with picobubbles, higher attachment probability, and lower detachment probability, as explained earlier,

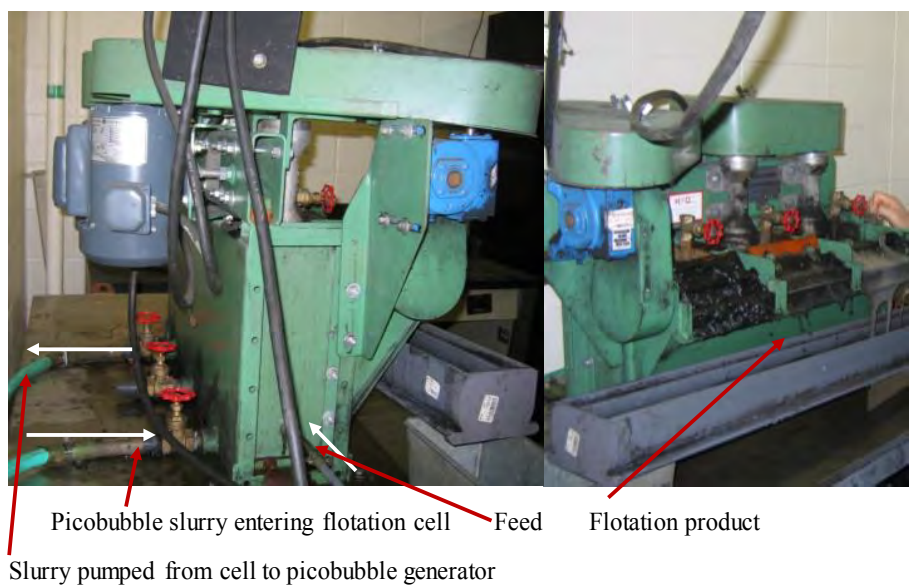

Figure 5 A bank of three 10-liter mechanical cells resulting in a greater flotation rate constant and higher flotation recovery.

Operating variables examined include flow rate to the cavitation tube, collector dosage, frother concentration, feed solids percentage, and feed slurry flow rate. Unless otherwise specified, all conventional flotation tests were performed under the following conditions: MIBC froth concentration of $20 \mathrm{ppm}$; fuel oil collector dosage of $0.49 \mathrm{~kg} / \mathrm{ton}$; flow rate to cavitation tube of $6000 \mathrm{ml} / \mathrm{min}$; feed slurry solids concentration of $23 \%$, and feed slurry flow rate of $2100 \mathrm{ml} / \mathrm{min}$.

Kinetic flotation tests were also conducted to show the effect of picobubbles on flotation rate constants. Flotation performance data from these tests were compared to the release analysis data for a better understanding of effects of picobubbles.

\section{RESULTS AND DISCUSSION}

Figure 6 shows the size distribution data for the fine coal flotation feed obtained from a major coal Company in West Virginia. Obviously, $\mathrm{d}_{50}$ and $\mathrm{d}_{90}$ of the coal sample were approximately $0.18 \mathrm{~mm}$ and $0.5 \mathrm{~mm}$, respectively. Figure 7 shows the ash content as a function of particle size in the feed. The ash content increased with decreasing particle size. Higher ash content in the smaller size fraction is at least partially caused by clay particles that are characterized by tiny sizes. Particles larger than $0.5 \mathrm{~mm}$ had an ash content of lower than $12 \%$. 


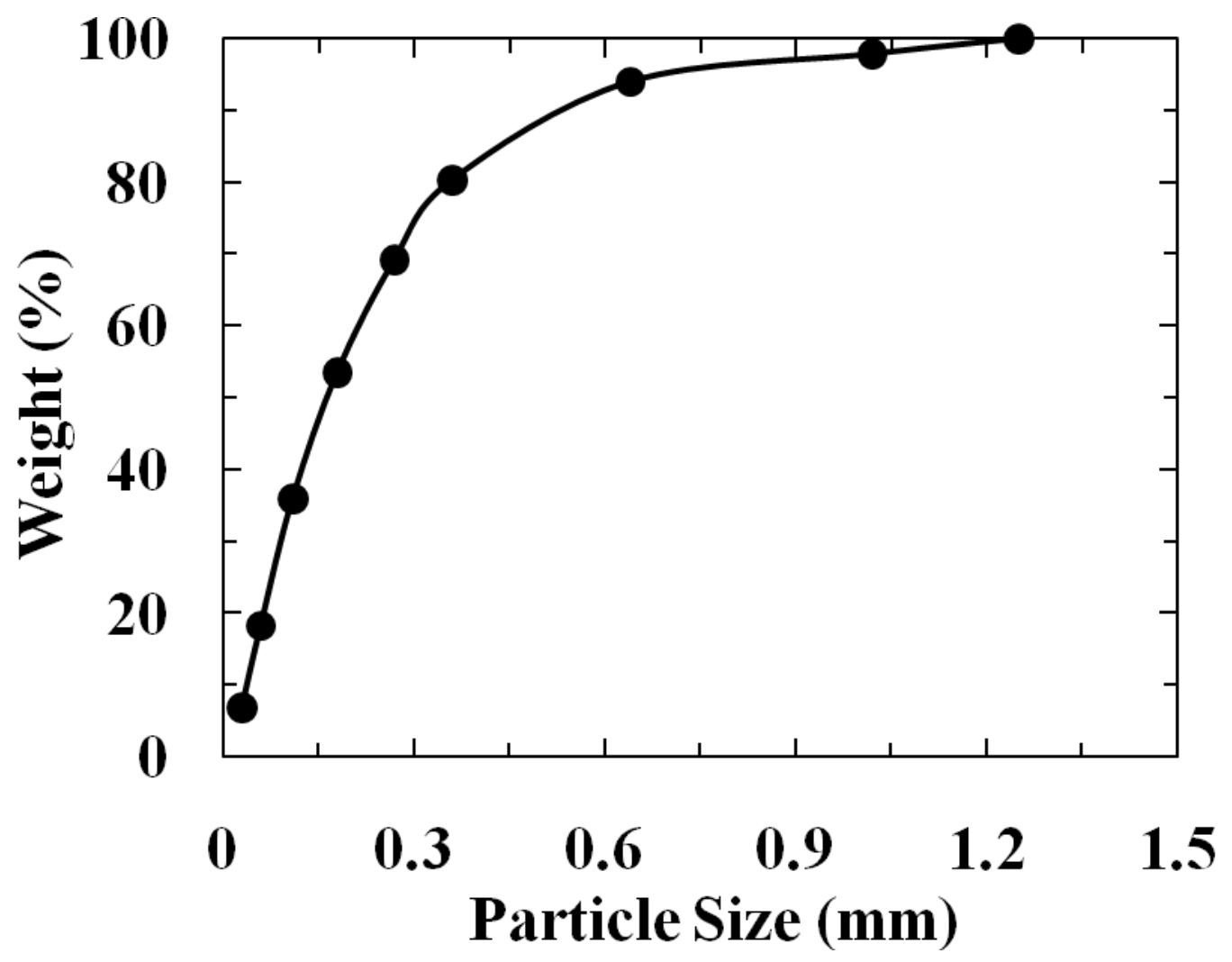

Figure 6 Flotation feed size distribution data 


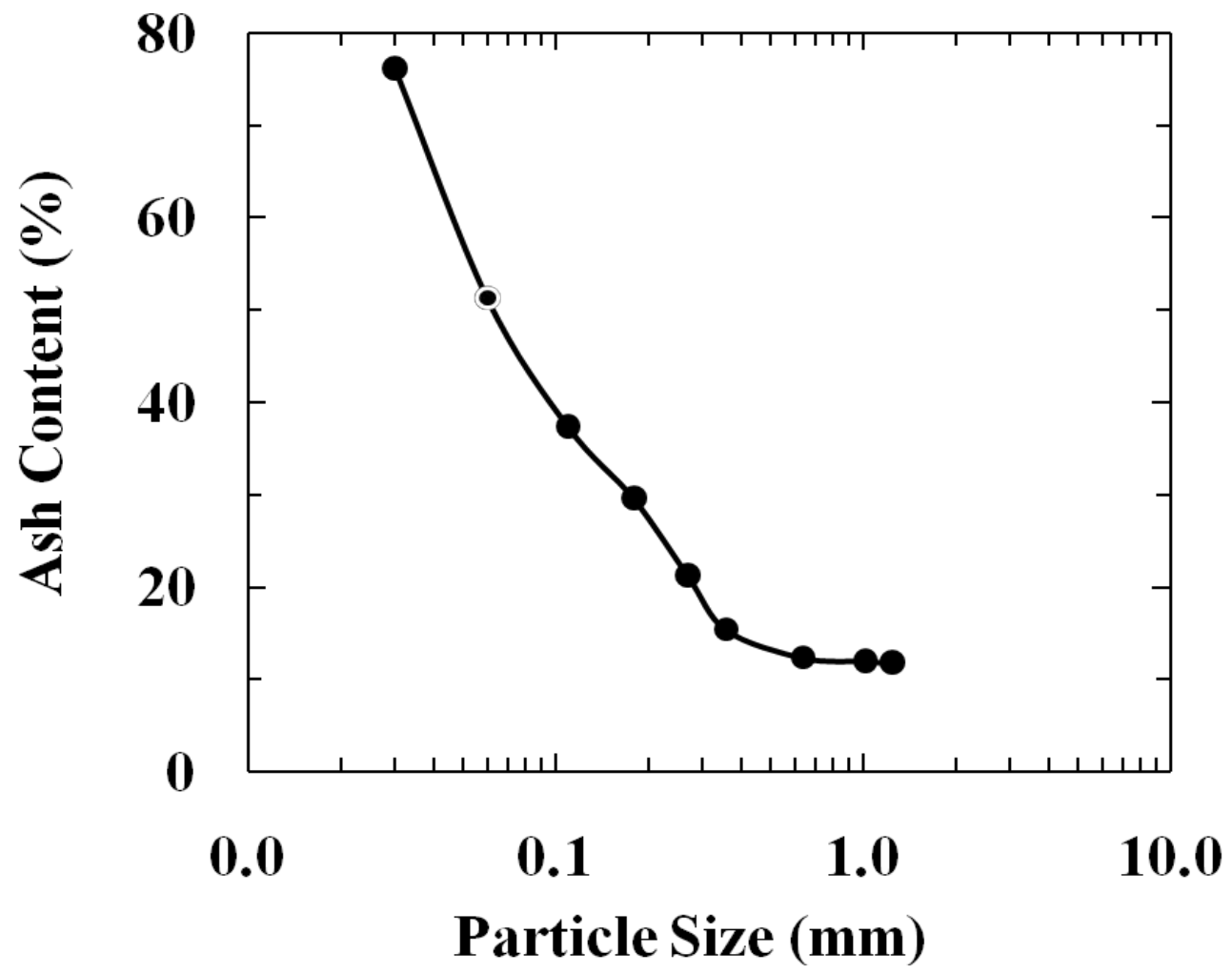

Figure 7 Flotation feed ash content in different size fractions. $\underline{\text { Size Distribution of Picobubbles }}$

Figure 8 shows the size distribution of picobubbles generated from air naturally dissolved in water with varying surfactant (F507, a kind glycol) concentration. It can be clearly seen from the figure that the bubble size decreases with increasing surfactant concentration from $10 \mathrm{ppm}$ to $100 \mathrm{ppm}$. The peaks are located within the region from 300 $\mathrm{nm}$ to $800 \mathrm{~nm}$

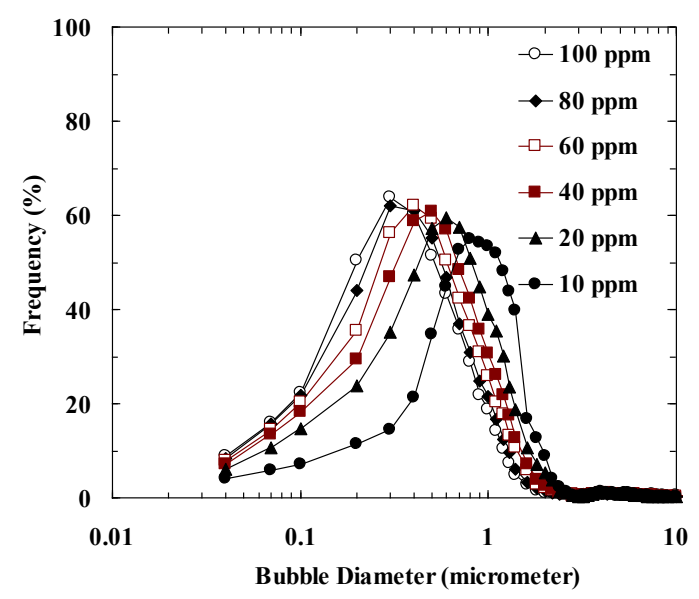

Figure 8 Picobubble generated from air naturally dissolved in water at varying concentrations of surfactant 
Figure 9 shows a size distribution of picobubbles measured at 10 minutes after the final generation from air naturally dissolved in water. The picobubble size increased with increasing time. During the tests, the picobubble solution was recycled slowly without passing through cavitation tube to reduce the effect of bubble rising on measurement.

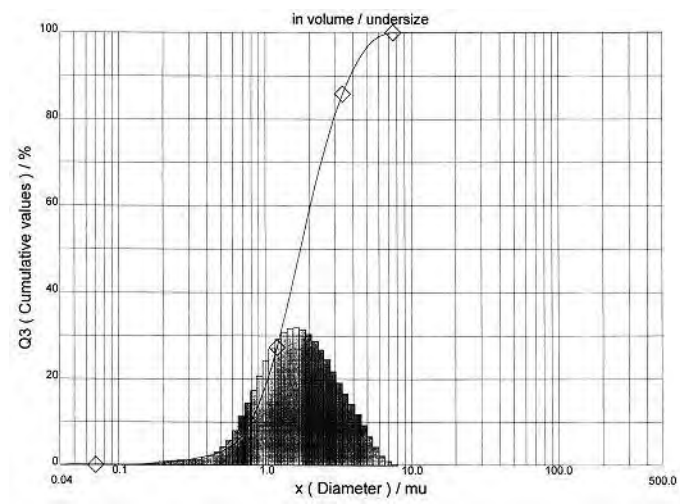

Figure 9 Bubbles generated by cavitation tube

Figure 10 shows the picobubbles generated by pressure drop in the water solution and on the hydrophobic and hydrophilic solid/water interfaces. It can be seen from the Figure 10 that picobubbles were more preferably formed on the hydrophobic particle surface than in the water solution. The creation of picobubbles is due to the rupture of a liquid-liquid or a liquid-solid interface under the influence of external forces. Because the work of adhesion $W_{a}=\gamma_{l}(1+\cos \theta)$ between a solid particle and water is always smaller than the work of cohesion of water $W_{c}=2 \gamma_{l}$, picobubbles preferentially nucleate on the surface of solid particles, especially hydrophobic particle with higher contact angle $(\theta)$.
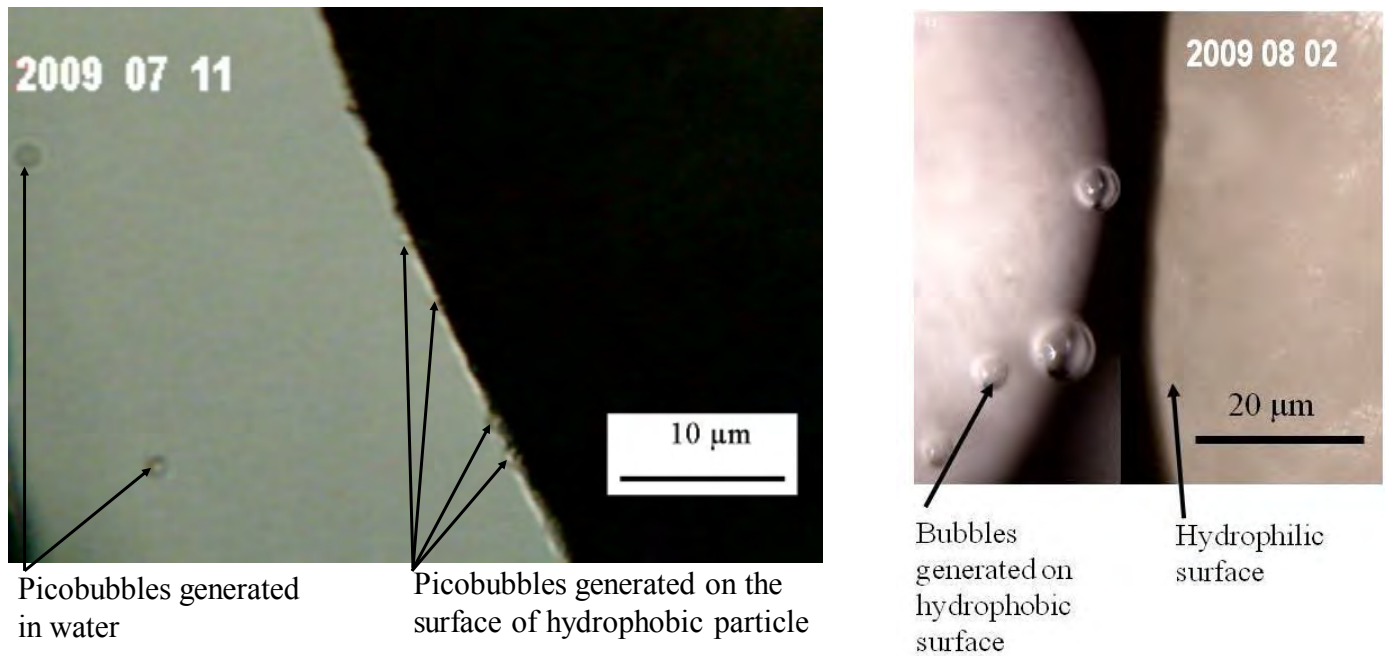

Figure 10 Picobubble generated in water and on hydrophobic/hydrophilic particle surface. 
$\underline{\text { Size Distribution of Co-existing Picobubbles and Microbubbles }}$

Figure 11 shows the picobubbles and microbubbles generated by the cavitation tube and the static mixer, respectively. There are two distinct peaks observed on each population density curve. Figure 12 shows that the bubbles produced by the static mixer only, which mostly consist of microbubbles.

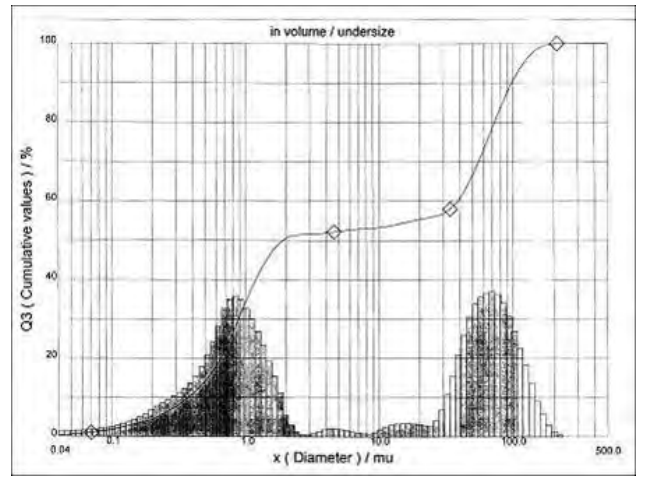

Figure 11 Bubbles generated by the cavitation tube and the static mixer

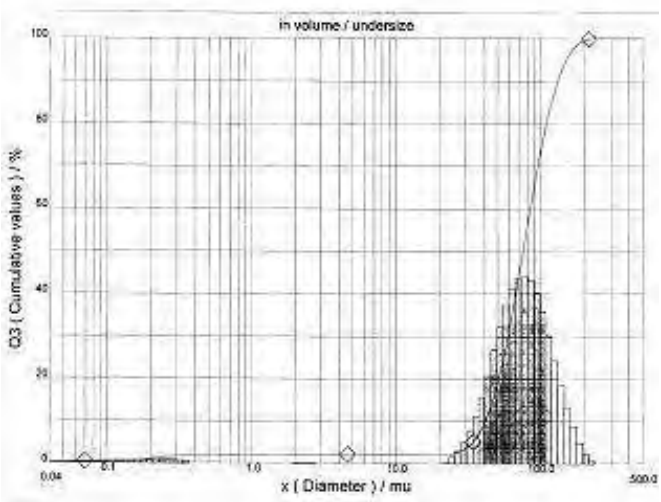

Figure 12 Bubbles generated by the static mixer only

Figure 13 gives the size distribution curve for bubbles in water solution inside the testing column with 10 ppm MIBC (Methyl Isobutyl Carbinol) or F507 (a kind of glycol). It can be seen that both curves have two peaks. On the population density curve with frother F507, two peaks are located at $0.7 \mu \mathrm{m}$ and $63 \mu \mathrm{m}$, representing picobubbles generated by the cavitation tube and microbubbles produced by the static mixer, respectively. With frother MIBC, the modes of the picobubble and microbubble size distribution are $0.9 \mu \mathrm{m}$ and $70 \mu \mathrm{m}$, respectively. The picobubble size is smaller than the microbubble size by about two orders of magnitude. Figure 13 also shows that the picobubbles and microbubbles produced with F507 are finer than those formed with MIBC. This is because frother F507 is stronger than MIBC and the surface tension reduction by F507 is more significant than by MIBC. 


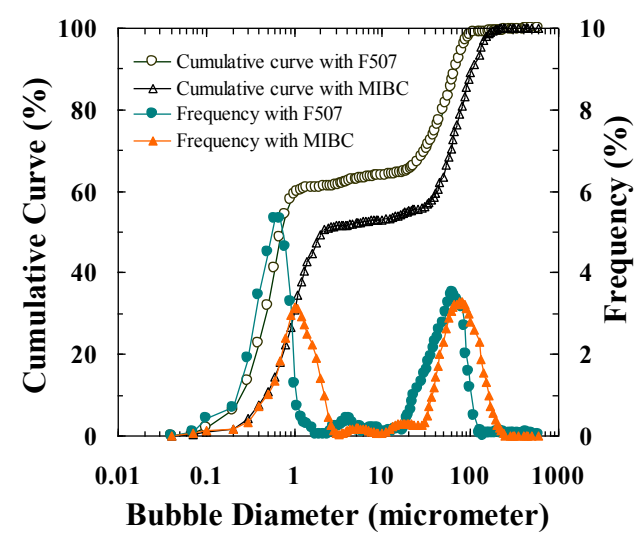

Figure 13 The size distribution curve for bubbles in water solution with 10 ppm MIBC and F507.

Figure 14 depicts the bubble size of picobubble and microbubble increases with time. In order to reduce the effect of bubble rising on measurement during these tests, the bubble slurry was recycled slowly bypassing the cavitation tube and the static mixer.

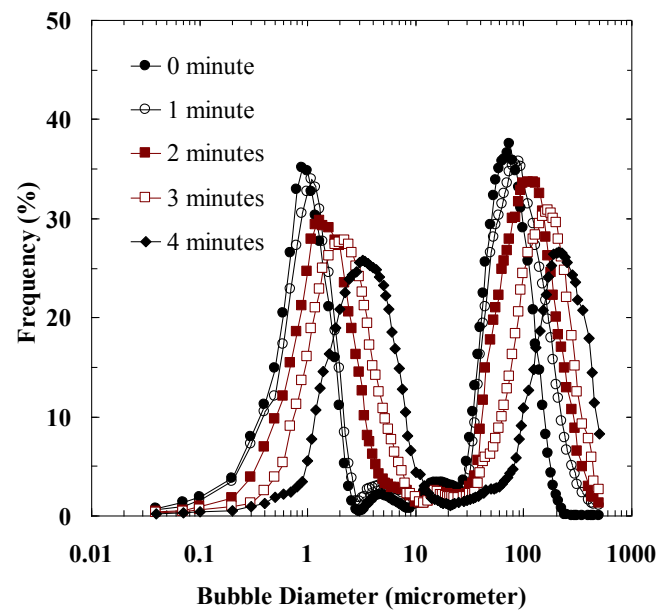

Figure 14 The size distribution curve for bubbles in water solution as a function of time.

\section{Zeta Potential Measurements}

Figure 15 depicts the zeta potentials of picobubbles, bituminous, anthracite, subminous and oxided coal as a function of $\mathrm{pH}$ value at the range of 2 to 12. The isoelectric point for picobubbles was nearly at the neutral $\mathrm{pH}$ at which all coal particles are negatively charged. 


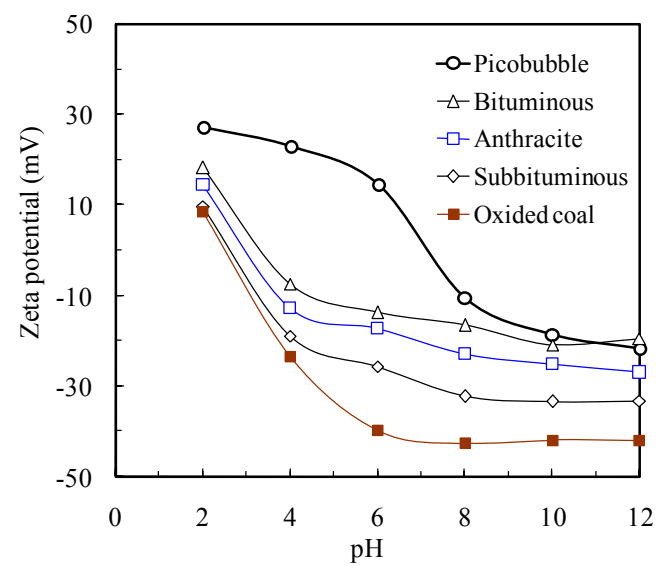

Figure 15 Zeta potential of coal and picobubble as a function of $\mathrm{pH}$.

\section{Detachment Studies with Vibration Cell}

Figure 16 shows the motion of attached solid particle on bubble in the vibrated cell. The air bubble was excited by external vibrating forces. It was found that the external vibrating force resulted in two types of particle motions: the vertical vibration and horizontal oscillation. The velocity of the particle decreased as it moved away from the equilibrium position, which is similar to a spring-mass system.

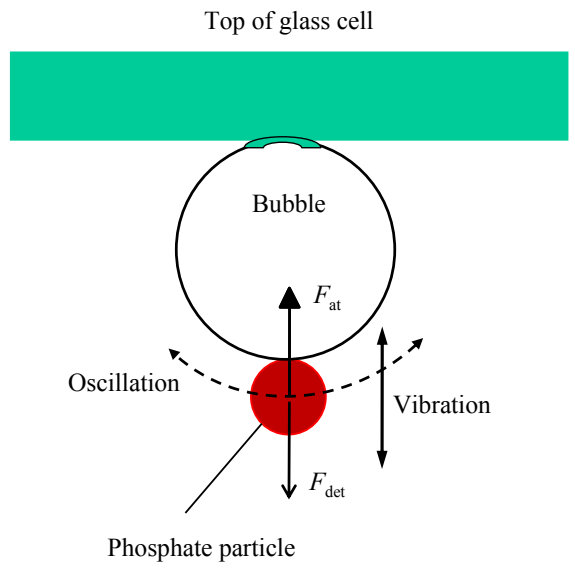

Figure 16 Vibration and oscillation of particle-bubble aggregate in a vibration cell.

The vibration acceleration was directly proportional to the displacement from the equilibrium position. During a vibration cycle, the particle's vibrating force acted as the attachment force as the particle moved from the equilibrium to the top position and from the bottom position to the equilibrium. But the particle's vibrating force acted as the detachment force as the particle moved from the top position to the equilibrium and from the equilibrium to the bottom position. The maximum detachment force from the particle's vibration occurred when it moved to the bottom position, which can be calculated by the following equation (Cheng and Holtham, 1995): 
$F_{V, \max }=\frac{1}{6} \pi D_{p}^{3}\left(\rho_{p}-\rho_{f}\right)(2 \pi f)^{2} A$

where $D_{p}$ is particle diameter, $\rho_{p}$ particle density, $\rho_{f}$ water solution density, $t$ the vibration frequency $(\mathrm{Hz})$ and $A$ the vibration amplitude (m).

For the oscillation motion, the centrifugal force acting on the particle was always the detachment force as the particle moved back and forth. The maximum detachment force caused by the oscillation when it approached to the equilibrium position, can be calculated by the following equation:

$F_{c, \text { max }}=\frac{1}{6} \pi D_{p}^{3}\left(\rho_{p}-\rho_{f}\right) \frac{v^{2}}{R}$

where $v$ is the phosphate particle velocity and $R$ is the radius of motion. $R$ is given by the following equation:

$R=D_{b}+\frac{1}{2} D_{p}$

where $D_{b}$ is bubble diameter. $v$ is given by

$v=\frac{2 \pi R}{\frac{1}{f}}=2 \pi R f=2 \pi\left(D_{b}+\frac{1}{2} D_{p}\right) f$

So, substituting Eqs. (13) and (14) into Eq. (12) yields the maximum detachment centrifugal force acting on the phosphate particle:

$$
\begin{aligned}
& F_{c, \text { max }}=\frac{1}{6} \pi D_{p}{ }^{3}\left(\rho_{p}-\rho_{f}\right) \frac{\left(2 \pi\left(D_{b}+\frac{1}{2} D_{p}\right) f\right)^{2}}{D_{b}+\frac{1}{2} D_{p}} \\
& F_{c, \text { max }}=\frac{1}{6} \pi D_{p}{ }^{3}\left(\rho_{p}-\rho_{f}\right)(2 \pi f)^{2}\left(D_{b}+\frac{1}{2} D_{p}\right)
\end{aligned}
$$

Figure 17(a) depicts that the maximum vibration force $F_{v, \text { max }}$, the maximum centrifugal force $F_{c \text {,max }}$, the major adhesion capillary force $F_{p}$, the excess force $F_{e}$, the weight of particle in the liquid medium $F_{w}$, the hydrodynamic drag force $F_{d}$, the sum of the attachment forces $F_{a t}=F_{p}+F_{e}$, the sum of the detachment forces $F_{\text {det }}=$ $F_{v, \text { max }}+F_{c \text {, max }}+F_{w}+F_{d}$, and the net force of $F_{\text {det }}-F_{a t}$, assuming the vibration frequency $t=60 \mathrm{~Hz}$, the vibration amplitude $A=3 \mathrm{~mm}, D_{b}=3 \mathrm{~mm}, \theta_{d}=90^{\circ}, \gamma=72.94 \mathrm{mN} / \mathrm{m}$, $\rho_{p}=3200 \mathrm{~kg} / \mathrm{m}^{3}, \square \rho_{w}=1000 \mathrm{~kg} / \mathrm{m}^{3}, g=9.80 \mathrm{~m} / \mathrm{s}^{2}, \eta=0.001 \mathrm{~N} . \mathrm{s} / \mathrm{m}^{2}$. While all the forces increased with particle size, the phosphate particle size had more significant effect on the detachment forces $F_{v, \text { max }}, F_{c, \text { max }}$ and $F_{w}$ than on the detachment force $F_{d}$ and on the attachment forces $F_{p}$ and $F_{e}$. Therefore, the net force of $F_{\text {det }}-F_{a t}$ increased with particle size, suggesting detachment is more likely with coarse particles. Indeed, the 
probability of detachment $P_{d}$ defined by Eq. (16) that derived from Eq. (12) increased significantly with increasing phosphate particle size, as shown in Figure 17(a). The probability of detachment $P_{d}$ for phosphate particles larger than $0.425 \mathrm{~mm}$ was higher than $65 \%$.

Figure 17(b) shows that the forces between a coal particle and a $3 \mathrm{~mm}$ diameter attached bubble: $F_{v, \text { max }}, F_{c, \text { max }}, F_{p}, F_{e}, F_{w}, F_{d}, F_{w}+F_{d}, F_{a t}=F_{p}+F_{e}, \quad F_{\text {det }}=$ $F_{v, \text { max }}+F_{c, \text { max }}+F_{w}+F_{d}$, and $F_{\text {det }}-F_{a t}$, when the vibration frequency $f=60 \mathrm{~Hz}$, the vibration amplitude $A=3 \mathrm{~mm} \theta_{d}=90^{\circ}, \gamma=72.94 \mathrm{mN} / \mathrm{m}, \rho_{p}=1500 \mathrm{~kg} / \mathrm{m}^{3}, \square \rho_{w}=1000$ $\mathrm{kg} / \mathrm{m}^{3}, g=9.80 \mathrm{~m} / \mathrm{s}^{2}, \eta=0.001 \mathrm{~N} . \mathrm{s} / \mathrm{m}^{2}$. Similar to the phosphate particle discussed above, all the forces increased with particle size, the coal particle size had more significant effect on the detachment forces $F_{v, \text { max }}, F_{c, \text { max }}$ and $F_{w}$ than on the detachment force $F_{d}$ and on the attachment forces $F_{p}$ and $F_{e}$. So the net force of $F_{\text {det }}-F_{a t}$ increased with particle size, which means that coarser coal particles has higher detachment probability.

$$
\begin{aligned}
& P_{d}=\frac{1}{1+\frac{F_{a t}}{F_{\mathrm{det}}}}=\frac{F_{\mathrm{det}}}{F_{\mathrm{det}}+F_{a t}} \\
& =\frac{F_{v, \text { max }}+F_{c, \text { max }}+F_{w}+F_{d}}{F_{v, \text { max }}+F_{c, \text { max }}+F_{w}+F_{d}+F_{p}+F_{e}}
\end{aligned}
$$

where $F_{p}, F_{e}, F_{w}, F_{d}, F_{v, \text { max }}$ and $F_{c, \text { max }}$ are expressed by Eqs. (4), (5), (6), (7), (12), and (15), respectively.

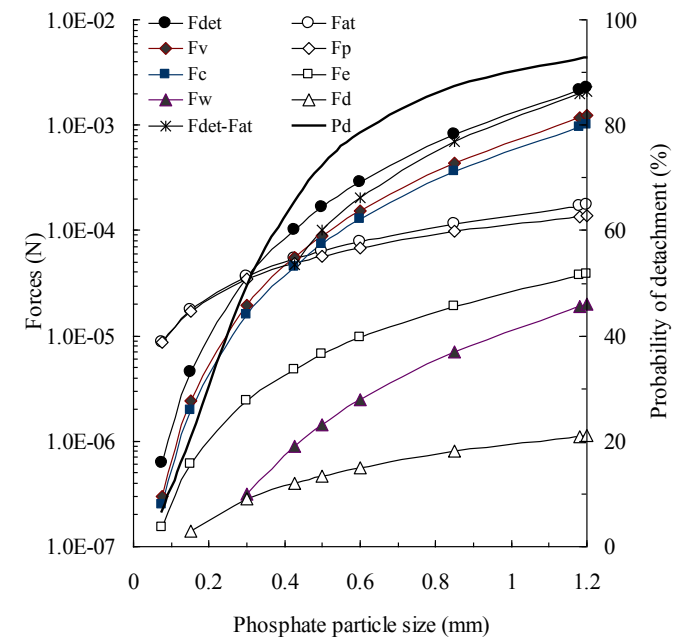

(a)Phosphate

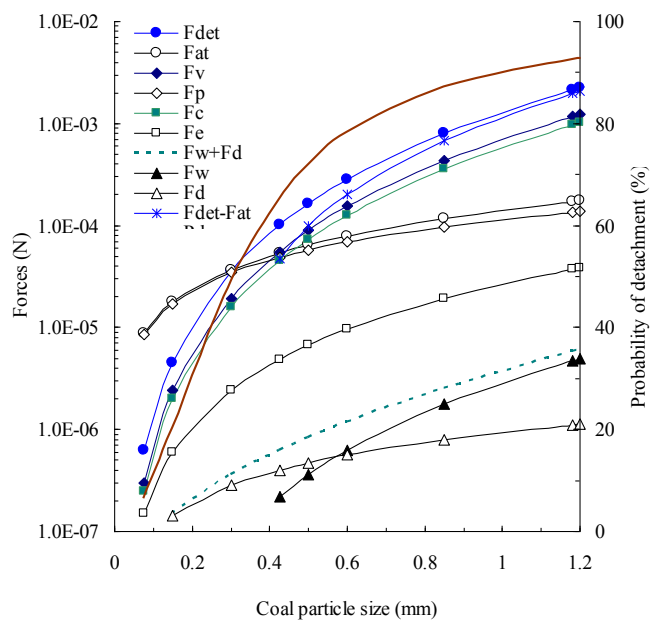

(b) Coal

Figure 17 Attachment and detachment fores and detachment probability as a function of phosphate (a) and coal (b) particle size. 
Figure 18 (a) shows the theoretical $P_{d}$ results predicted by Eq. (16), which clearly indicates that the probability of detachment increased with increasing vibration frequency from 0 to $120 \mathrm{~Hz}$ at vibration amplitude of $3 \mathrm{~mm}$. At lower vibration frequency, the influence of vibration frequency on the probability of detachment is more significant for the coarse particles than for the fine particles. Figure 18 (b) shows the experimental
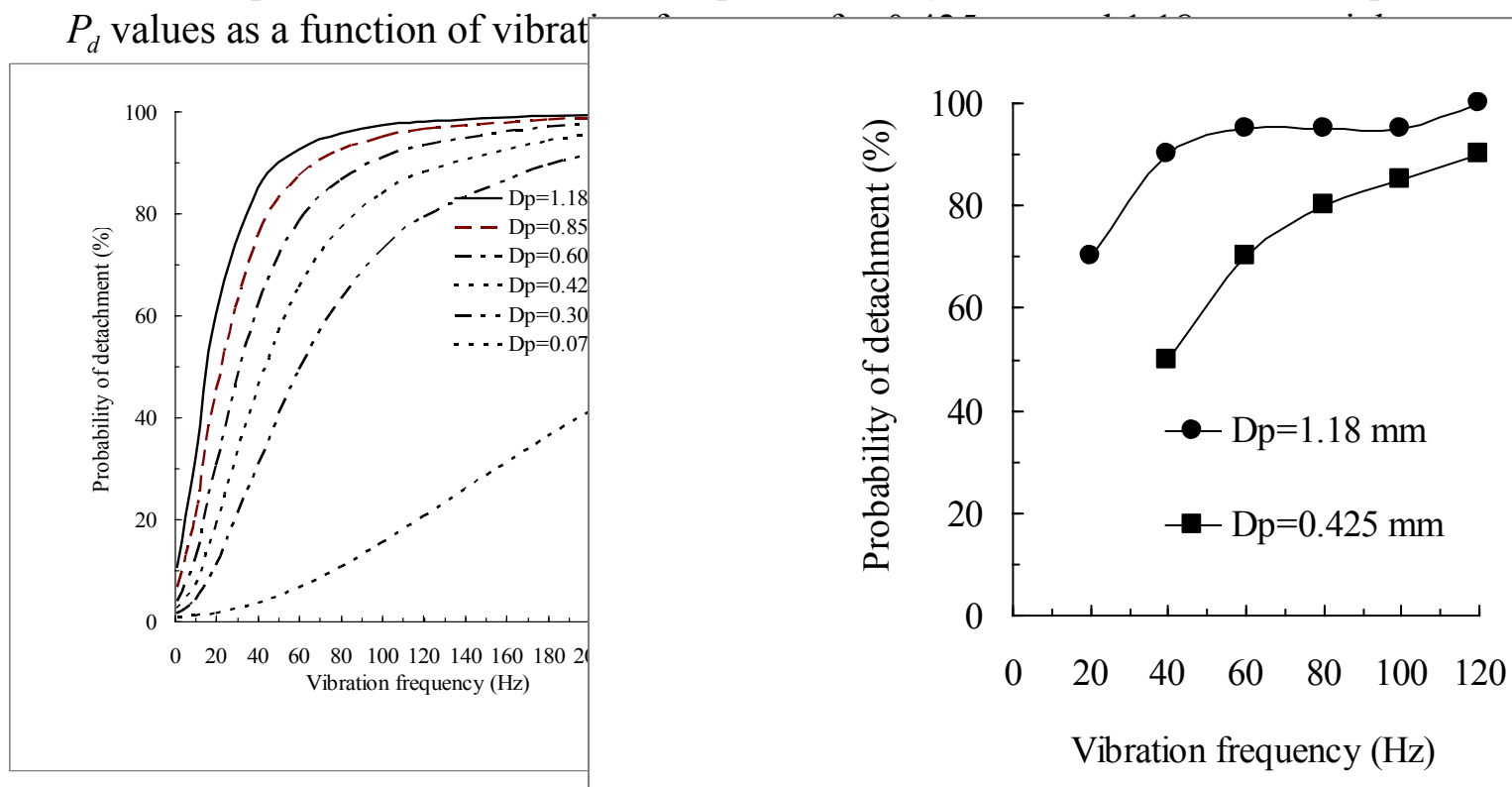

Figure 18 Effect of vibration fre $0.85 \mathrm{~mm}, 0.60 \mathrm{~mm}, 0.425 \mathrm{~mm}, 0.30 \mathrm{~mm}$, and $0.0 / 3 \mathrm{~mm}$ particies (a) and expermmental detachment probability on $1.18 \mathrm{~mm}$ amd $0.425 \mathrm{~mm}$ particle (b).

Figs. 19 (a) and (b) shows effects of picobubbles on experimental $P_{d}$ values for 0.425 $\mathrm{mm}$ and $1.18 \mathrm{~mm}$ coal and phosphate particles. Obviously, the presence of nanobbubbles reduced the probability of detachment, thus favoring the flotation.

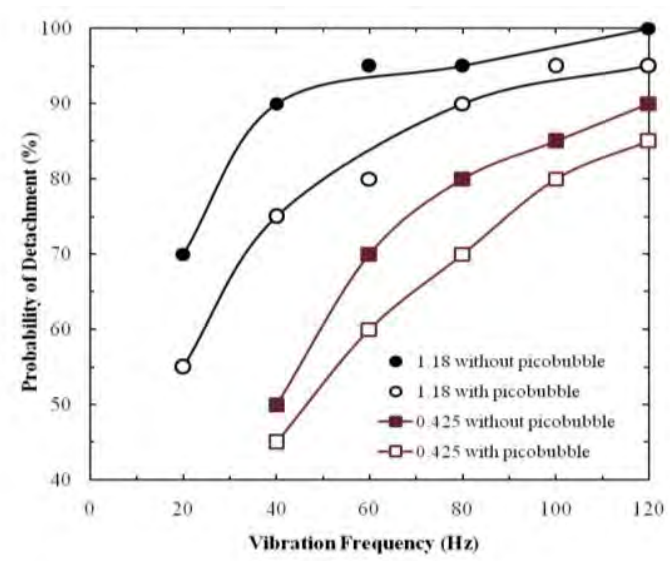

(a)

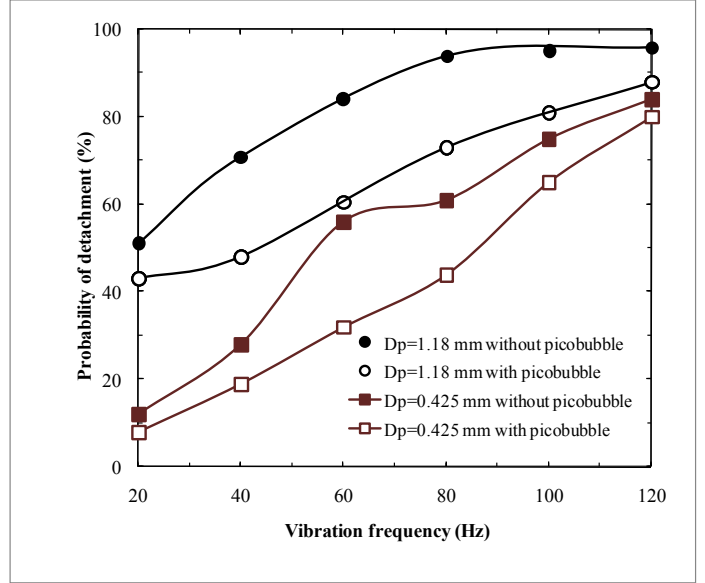

(b)

Figure 19 Effect of picobubble on experimental detachment probability of $1.18 \mathrm{~mm}$ and $0.425 \mathrm{~mm}$ phosphate (a) and coal (b) particles at varying vibration frequency. 
Figure 20 (a) shows the experimental detachment probability data as a function of vibration amplitude for different particle sizes with or without picobubbles at the vibration frequency of $60 \mathrm{~Hz}$. For the purpose of comparison, the theoretical detachment probability value from Eq. (16) is also shown in Figure 20 (a). The probability of detachment slightly increased with increasing vibration amplitude from $0.1 \mathrm{~mm}$ to 3.0 $\mathrm{mm}$. The curves show that particle size had significant effects on the detachment probability. For $0.075 \mathrm{~mm}$ phosphate particles, the detachment probability was less than $7 \%$ at varying vibration amplitude. The probabilities of detachment were about $47 \%-65 \%$ and $86 \%-93 \%$ for $0.425 \mathrm{~mm}$ and $1.18 \mathrm{~mm}$ phosphate particle, respectively. Figure 20 depicts that the effect of picobubbles on the detachment of coal particles. It can be seen from both Figs. 20 (a) and (b) that the existence of nanobbubble reduced the probability of detachment.

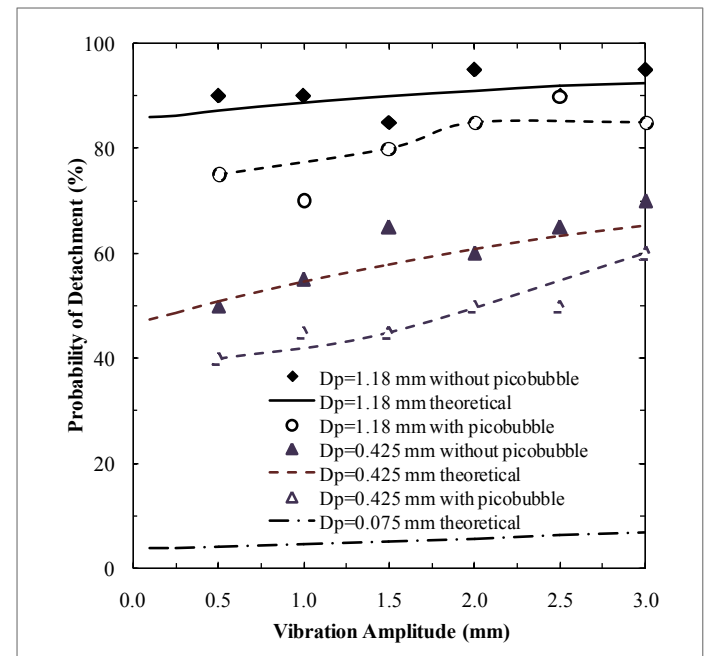

(a)Phoshapte

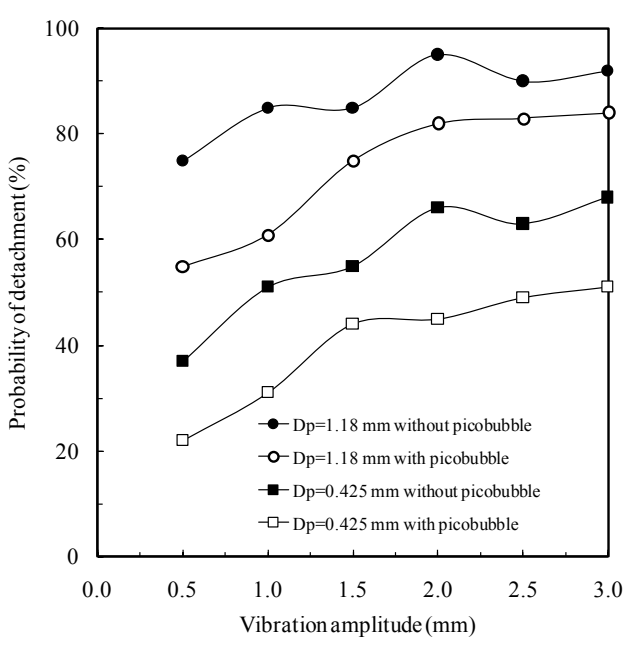

(b) Coal

Figure 20 Effect of vibration amplitude and picobubble on detachment probability of 1.18 $\mathrm{mm}, 0.425 \mathrm{~mm}$ and $0.075 \mathrm{~mm}$ phosphate and coal particles.

Modified Monobubble Hallimond Tube Flotation

Figure 21 shows that the collection efficiency by a single bubble decreased with reducing the bubble size due to reduced surface area. However, Figure 21 still suggests that the collection efficiency should increase with decreasing bubble size for a given gas flow rate since the number of bubbles is inversely proportional to the third power of bubble diameter. This is because as the bubble diameter decreased from $2.4 \mathrm{~mm}$ to 0.8 $\mathrm{mm}$, the number of bubbles increased $3^{3}$ or 27 times. But the collection efficiency for a single bubble decreases much less than 27 times. Therefore, the flotation efficiency, defined as the product of the collection efficiency multiplied by the number of bubbles was greater for smaller bubbles, as shown in Figure 22. For a given bubble size, phosphate particles in $0.075-0.212 \mathrm{~mm}$ size range showed higher flotation efficiency than that in $0.038-0.075 \mathrm{~mm}$ size range. This is because the solids concentration was maintained constant at $0.3 \%$ for all size particles and the relatively coarser particles had smaller number in the modified Hallimond tube. The detachment did not play a very important role in the flotation efficiency when the particle size is still relatively small compared to bubble size. 


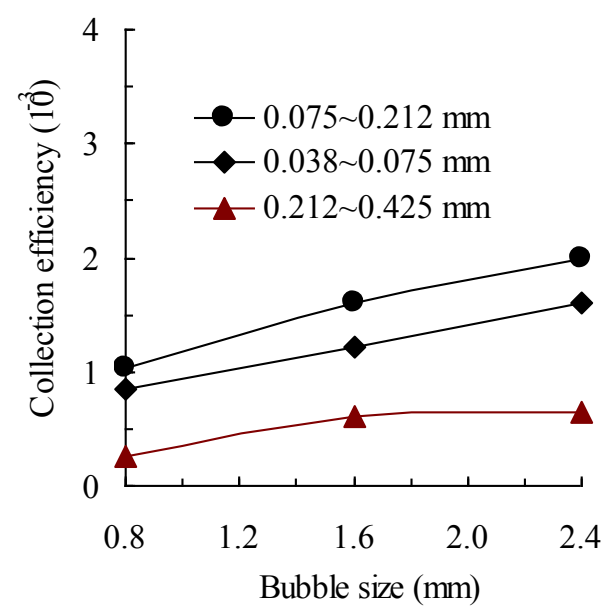

Figure 21 Effect of bubble size on collection efficiency of $0.038-0.075 \mathrm{~mm}, 0.075-$ $0.212 \mathrm{~mm}$ and $0.212-0.425 \mathrm{~mm}$ phosphate particles with single bubble.

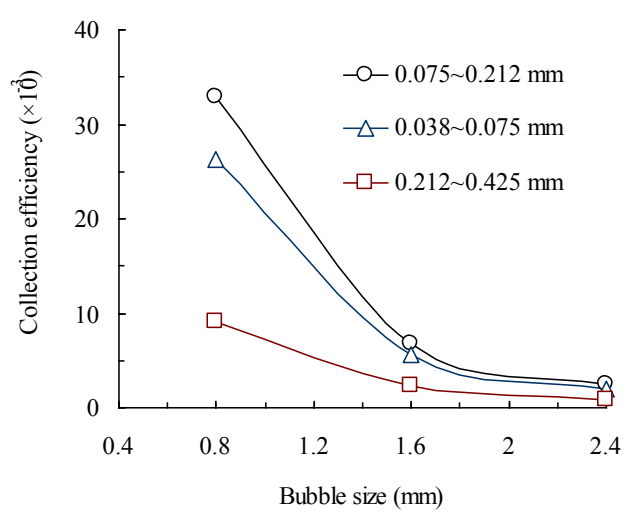

Figure 22 Effect of bubble size on flotation efficiency of $0.038-0.075 \mathrm{~mm}, 0.075-$ $0.212 \mathrm{~mm}$ and $0.212-0.425 \mathrm{~mm}$ phosphate particles at a given air flow rate.

Figs. 23 (a) and (b) depicts the effect of picobubbles on the flotation efficiency of different size phsophate and coal particles with varying bubble size at a given air flow rate. Both figures indicates that the addition of picobubble slurry increased flotation efficiency, especially for $0.038 \sim 0.075 \mathrm{~mm}$ and $0.212 \sim 0.425 \mathrm{~mm}$ particles. During the experiment, it was also observed that picobubbles induced some fine particle coagulation which helped flotation. This effect was more significant with smaller particles. 


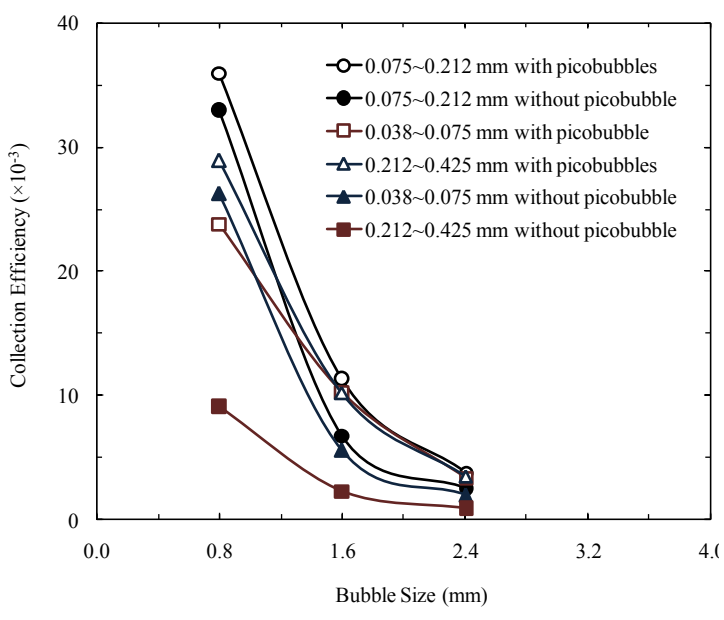

(a) Phosphate

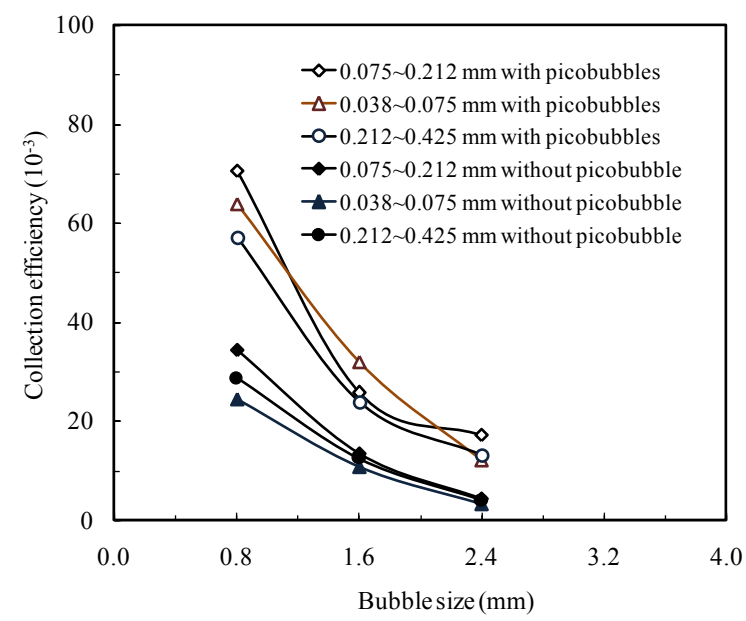

(b) Coal

Figure 23 Phosphate and coal particle flotation efficiency versus bubble size at a given air flow rate with or without picobubbles

It can be seen from Figs. 21, 22, and 23 that the bubble size, particle size and the presence of picobubbles have significant effect on the collection efficiency, especially on coarse and fine particles collection efficiency. For a given air flow rate, the reduction of bubble size will increase the flotation rate. Within a certain range of particle size, the collection efficiency can be increased by increasing particle size. However, when the particle size exceeds a certain limit, the collection efficiency decreased with increasing particle size as a result of higher detachment probability. For fine or coarse particles, use of picobubbles enhanced collection efficiency and flotation efficiency.

\section{Specially designed monobubble column flotation}

Figure 24 depicts the effect of picobubbles on the coarse coal particles flotation probability at varying particle size when particle condition collector dosage was at 0.3 $\mathrm{kg} /$ ton. Figure 24 indicates that the presence of picobubble increased the coarse coal flotation probability with more significant effect on coarser particles. The picobubble increased the flotation probability by up to $27 \%$.

The test results shown in Figs. 24 are in agreement with the study by Fan and Tao (2208) and Fan et al (2010b) that the existence of picobubbles in flotation slurry reduced the rinsing velocity of conventional-sized flotation bubble, thus increased the bubble-particle sliding time, decreased the tangential velocities of particle sliding on bubble surface, and therefore increased the bubble-particle attachment probability and decreased the detachment probability. The theoretical analysis and modified monobubble Hallimond tube flotation test results have demonstrated that the flotation recovery of coarse particles can be enhanced by using a combination of small and large bubbles (Wyslouzil et al., 2009). Specially designed monobubble column flotation test results proved that the presence of picobubble increased the coarse coal flotation probability. 


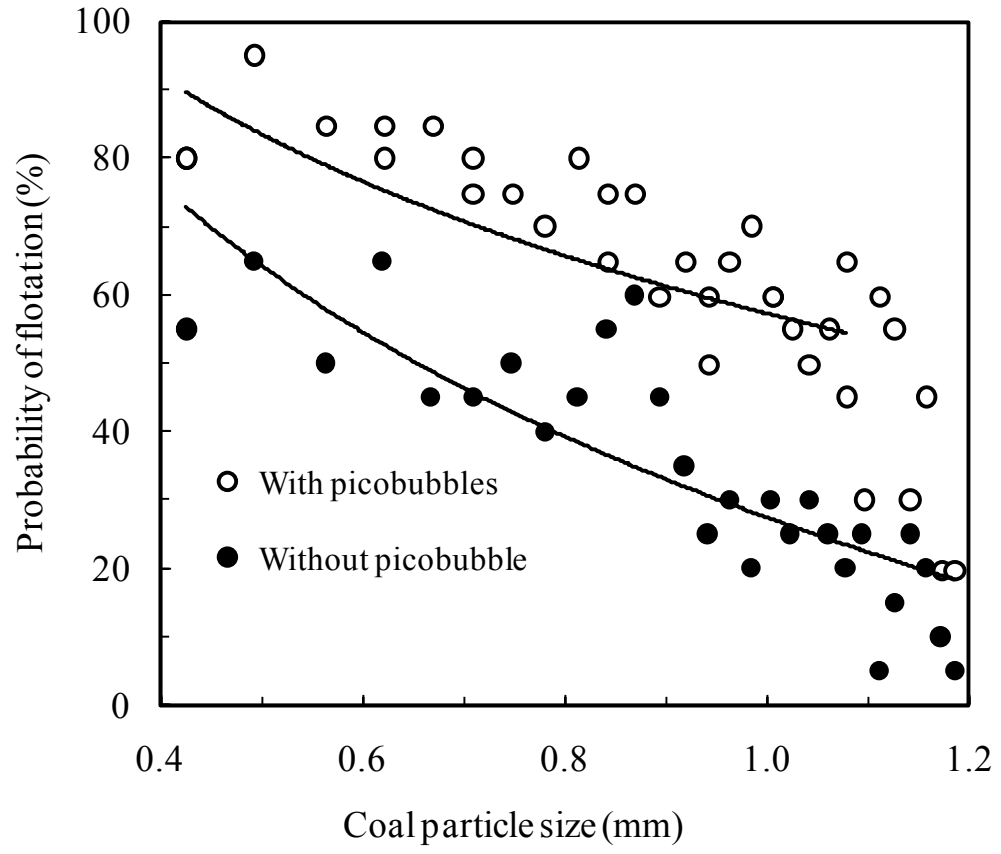

Figure 24 Effect of picobubble on the flotation probability of varying coal particle sizes at collector dosage of $0.3 \mathrm{~kg} / \mathrm{ton}$.

\section{$\underline{\text { Column Flotation Results }}$}

Figure 25 shows the combustible recovery and product ash as a function of superficial air velocity with and without picobubbles. Apparently the presence of picobubbles significantly increased combustible recovery, especially at lower air velocities. For example, at $0.5 \mathrm{~cm} / \mathrm{s}$ air velocity, the recovery was almost $30 \%$ higher in the presence of picobubbles. At the highest air velocity of $2.5 \mathrm{~cm} / \mathrm{s}$, the difference in recovery was about $8 \%$. It is noticed that the product ash was slightly higher when the picobubbles were present in the column. In order to evaluate the effect of picobubbles on the flotation separation efficiency, the separation curve, i.e., combustible recovery vs. product ash curve, is shown in Figure 26. The fact that the separation curve obtained with picobubbles is above the one without picobubble indicats that the use of picobubbles improved the separation efficiency. The improvement was more significant at lower product ashes. For example, the combustible recovery with picobubbles was more than $28 \%$ higher at a product ash of $6 \%$. The minimum improvement in combustible recovery created by picobubbles was about $8 \%$, which was still very significant for the industry.

Figure 27 shows the combustible recovery, product ash, and separation efficiency (defined as the difference between combustible recovery and ash recovery) as a function of the tailing re-circulating flow ratio between the static mixer that generated conventional size bubbles and the picobubble generator. A flow rate ratio of zero means there were no picobubbles in the column and a $100 \%$ flow ratio implies that all tailing slurry flow went to the picobubble generator. The separation efficiency curve clearly indicates that the best separation was achieved with about $60 \%$ tailing flow going through the picobubble generator under the given conditions employed for these tests. 


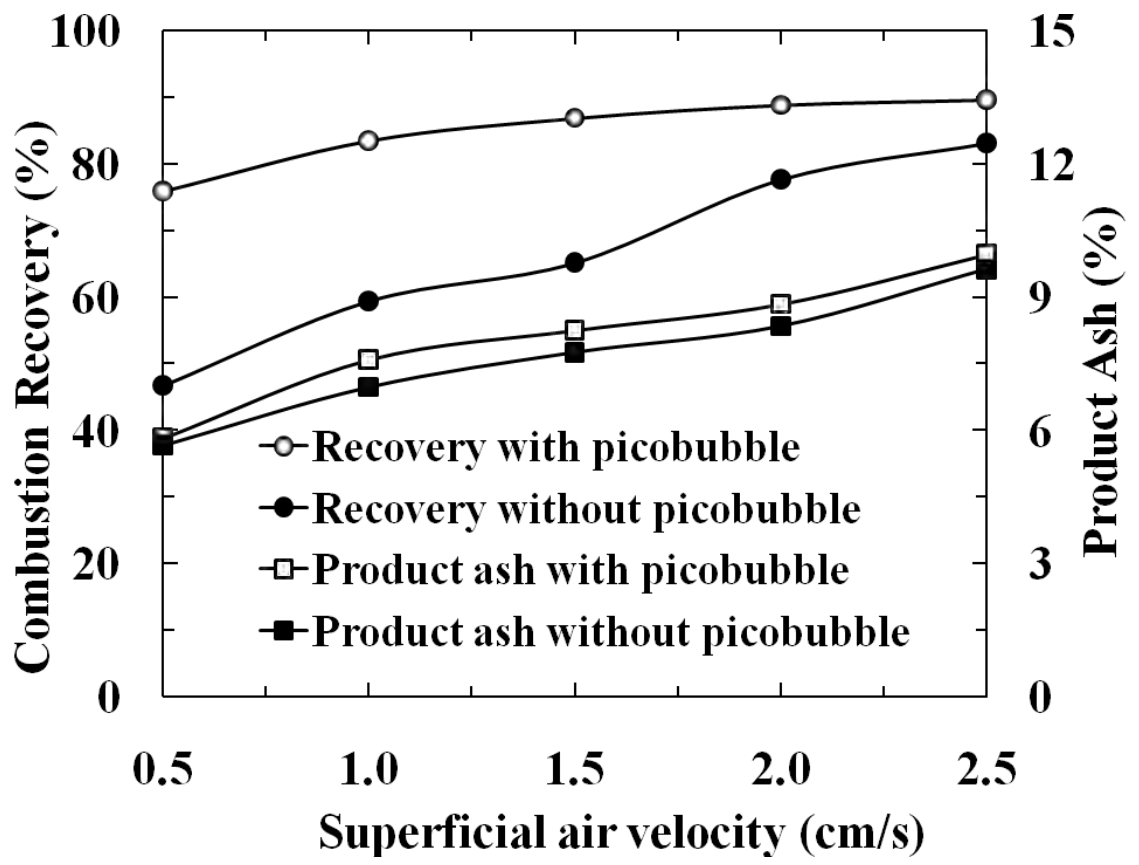

Figure 25 Combustible recovery and product ash as a function of superficial air velocity with and w/o picobubbles

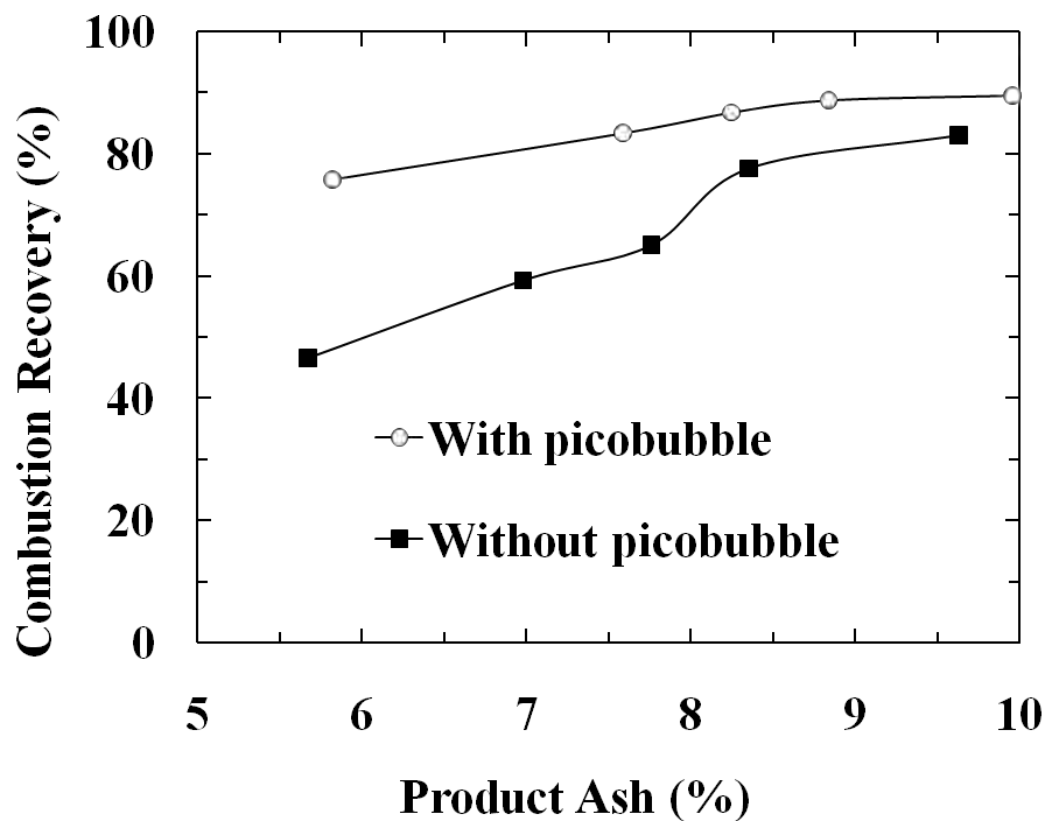

Figure 26 Combustible recovery vs. product ash at different superficial air velocity with and w/o picobubbles 


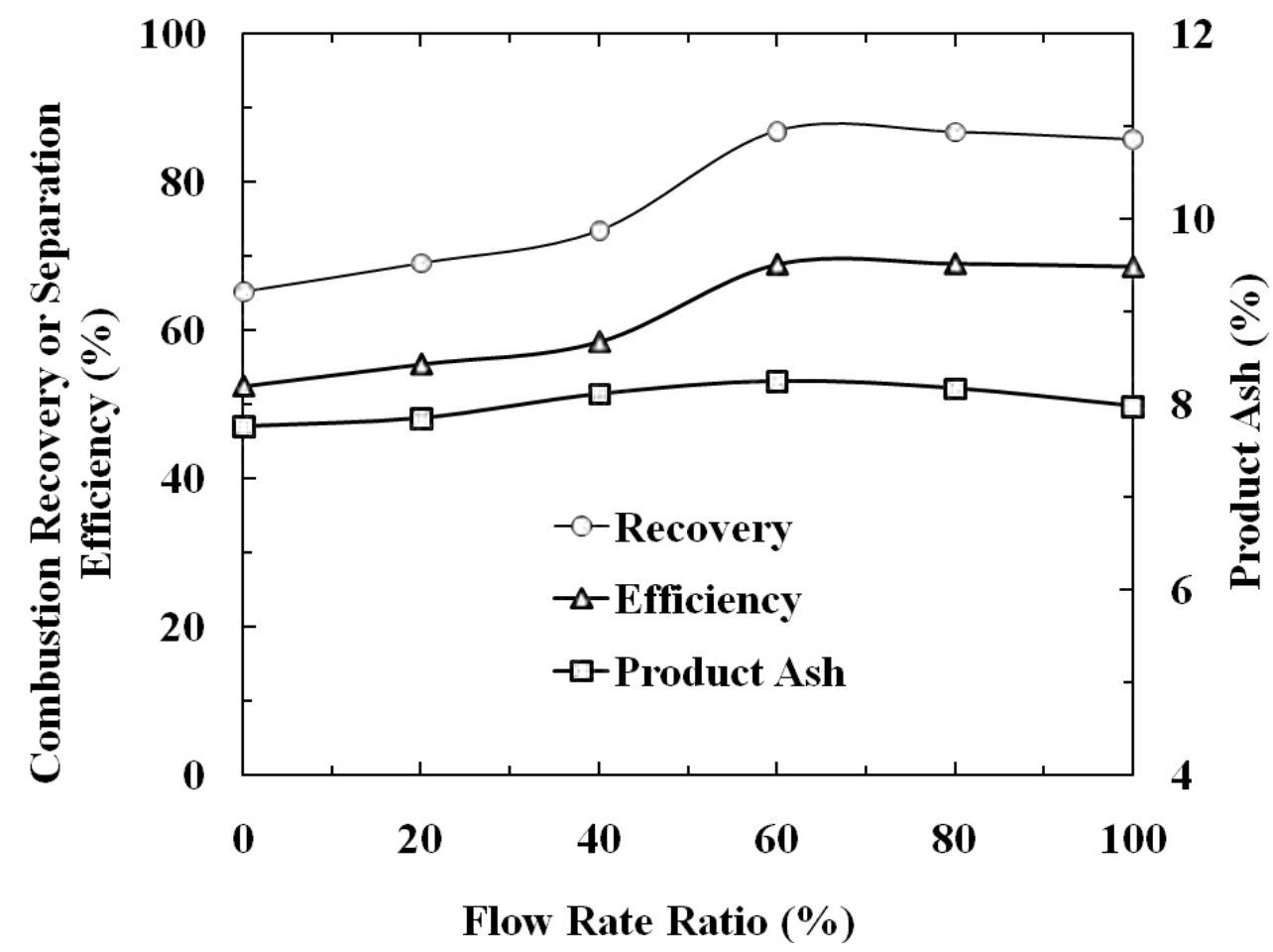

Figure 27 Combustible recovery and product ash as a function of tailing recirculating flow rate ratio between static mixer and picobubble generator.

Figure 28 shows the combustible recovery and product ash as a function of collector dosage with and without picobubbles Similar to Figure 25, the combustible recovery curve generated with picobubbles is always substantially above the one without picobubbles and the product ash was slightly higher when picobubbles were present. The use of picobubbles increased recovery by about $25-40 \%$ at a given collector dosage. The separation performance curve shown in Figure 29 suggests that the combustible recovery was $12-21 \%$ higher for a given product ash when picobubbles were employed.

The effects of picobubbles on flotation performance were also investigated at different frother disages and the results are shown in Figures 30 and 31. Similar to Figure 28 and Figure 29, a pronounced increase in combustible recovery was observed at a given frother dosage when picobubbles were introduced. A $28-32 \%$ increase in combustible recovery was generated at a given frother dosage and a $17-20 \%$ improvement in combustible recovery was observed for a given product ash. 


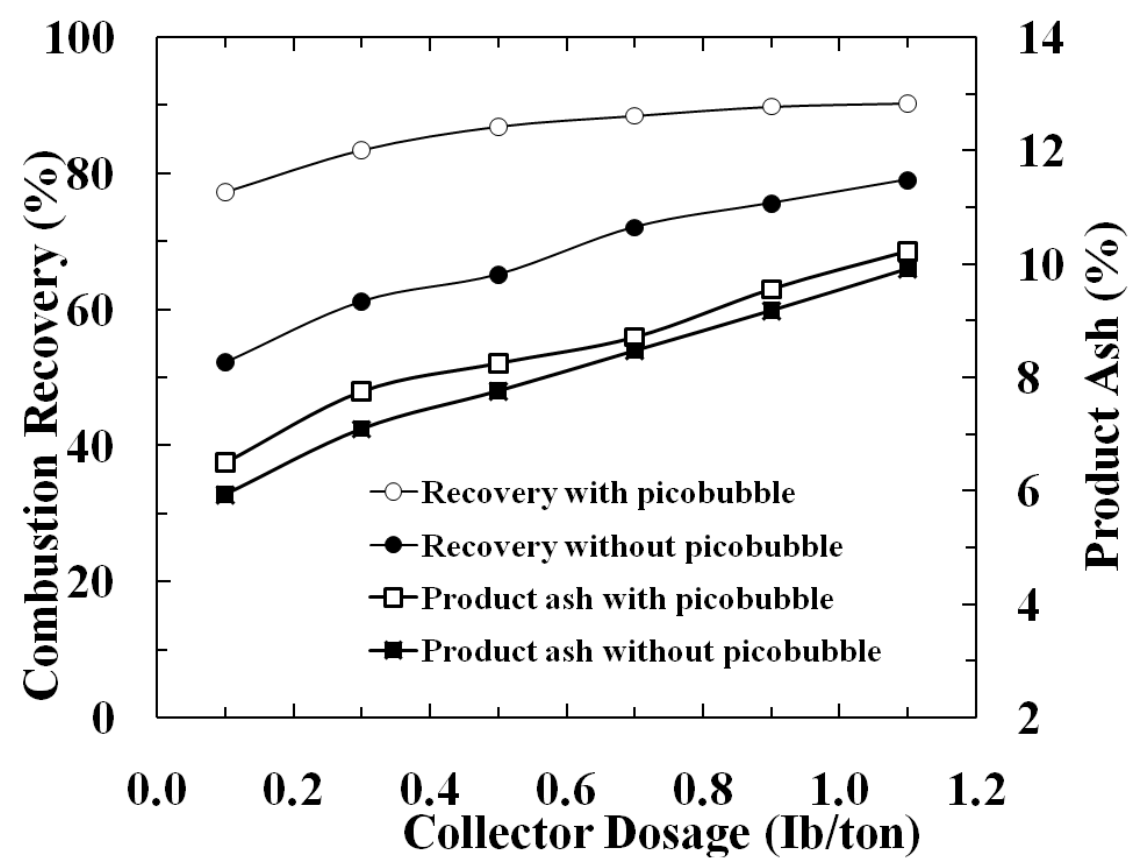

Figure 28 Combustible recovery and product ash as a function of collector dosage with and w/o picobubbles

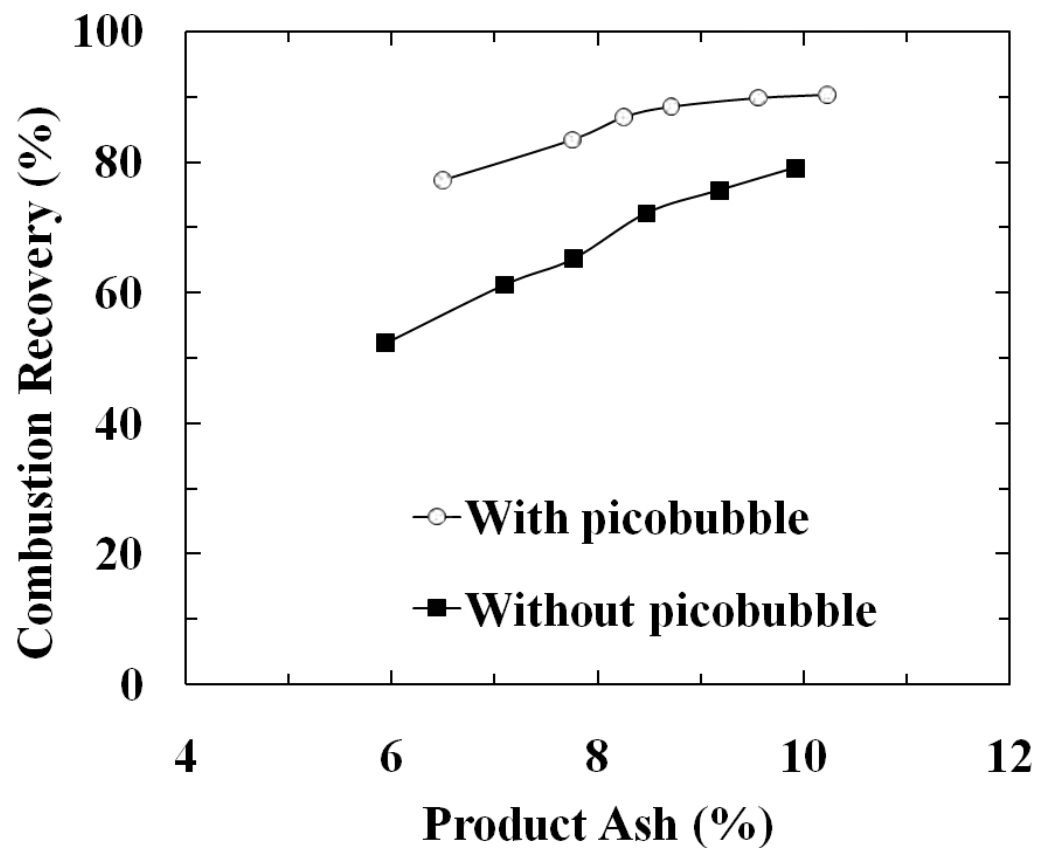

Figure 29 Combustible recovery vs. product ash at different collector dosage with and w/o picobubbles 


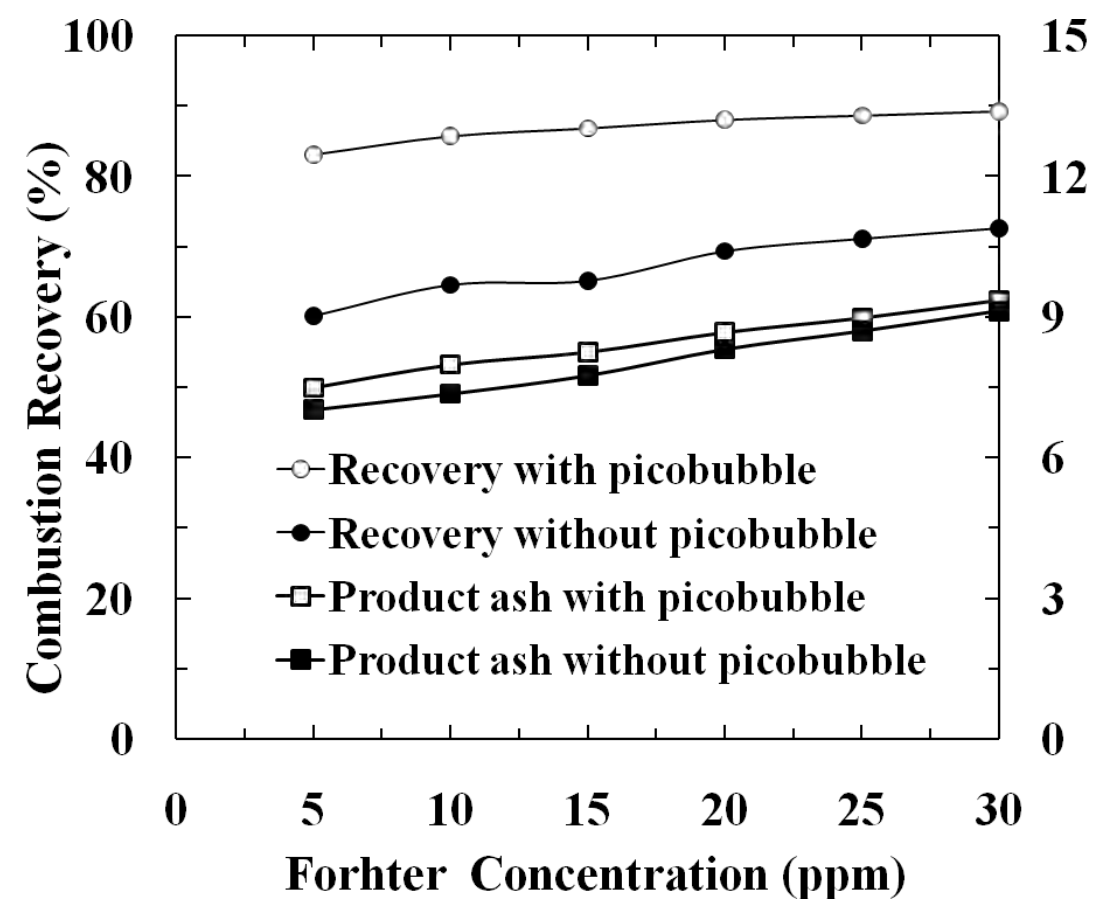

Figure 30 Combustible recovery and product ash as a function of frother dosage with and w/o picobubbles

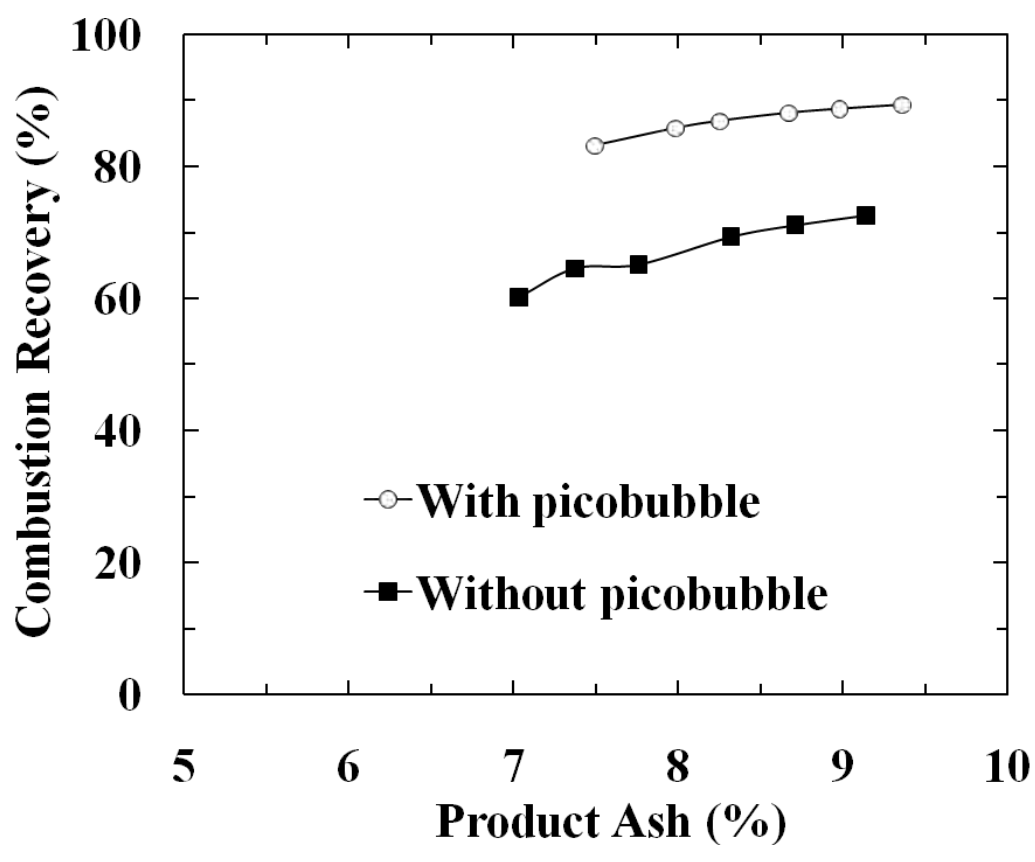

Figure 31 Combustible recovery vs. product ash at different frother dosage with and w/o picobubbles 
Figure 32 shows the combustible recovery and product ash as a function of superficial wash water velocity with and without picobubbles. Recovery and product ash decreased with increasing wash water velocity since more particles were washed back to the slurry phase in the column at higher wash water velocity and higher ash material was removed preferentially from the froth phase due to its weaker affinity to air bubbles. Comparison of data obtained with and without picobubbles indicates that the presence of picobubbles significantly increased combustible recovery. The most significant improvement in recovery of approximately $21 \%$ was observed at about $0.3 \mathrm{~cm} / \mathrm{s}$ and $0.4 \mathrm{~cm} / \mathrm{s}$ wash water velocity. On the other hand, use of picobubbles increased product ash to some extent as a result of more recovery of coal particles. In order to evaluate the effect of picobubbles on the flotation separation efficiency at different wash water velocity, the separation curve, i.e., combustible recovery vs. product ash curve, is shown in Figure 33. The fact that the separation curve obtained with picobubbles is above the one without picobubble indicates that the use of picobubbles improved the separation efficiency. The degree of improvement was nearly identical over the entire region, i.e., about $16 \%$.

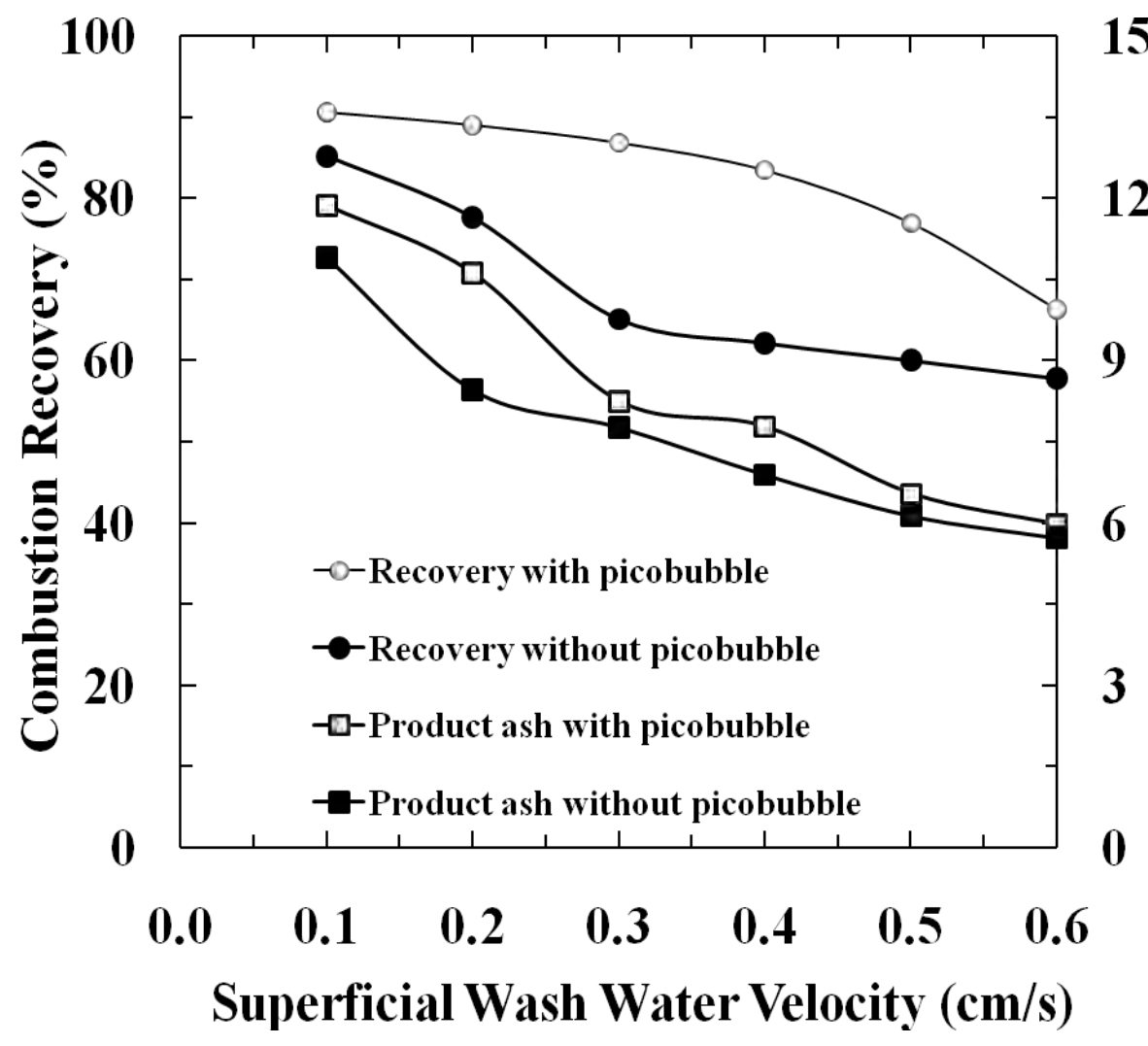

Figure 32 Combustible recovery and product ash as a function of superficial wash water velocity with and w/o picobubbles 


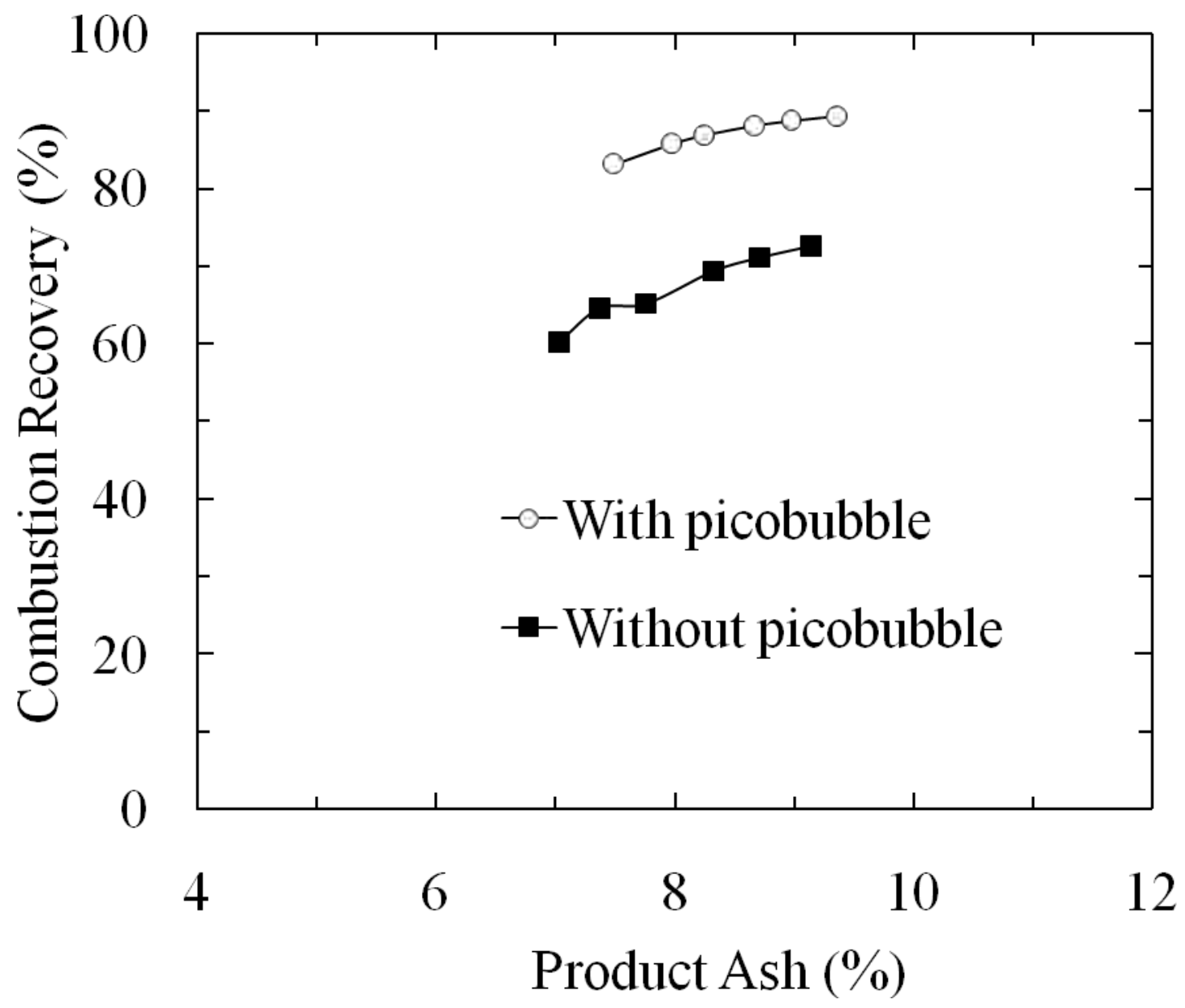

Figure 33 Combustible recovery vs. product ash at different superficial wash water velocity with and w/o picobubbles 


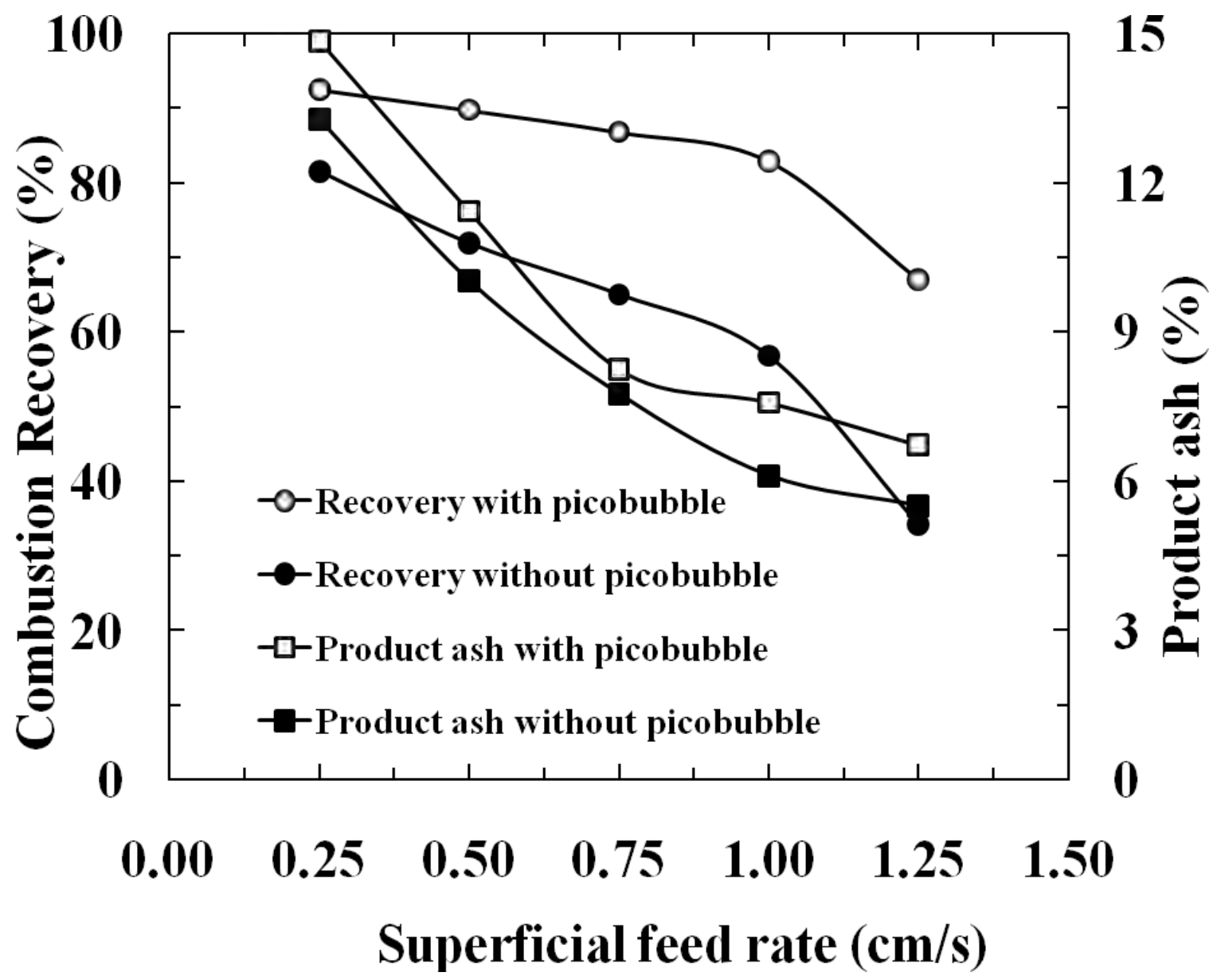

Figure 34 Combustible recovery and product ash as a function of superficial feed rate with and w/o picobubbles

Figure 34 shows the combustible recovery and product ash as a function of superficial feed rate with and without picobubbles. Recovery and product ash decreased with increasing feed rate as a result of reduced retention time. Comparison of data obtained with and without picobubbles indicates that the use of picobubbles considerably increased combustible recovery by $10-33 \%$, depending on the feed rate. More significant improvement was observed at higher feed rate. Product ash was also higher in the presence of picobubbles since more particles were recovered. Nevertheless the separation curve shown in Figure 35 clearly demonstrates that use of picobubbles increased the separation efficiency since the separation curve obtained with picobubbles is always above the one without picobubbles. 


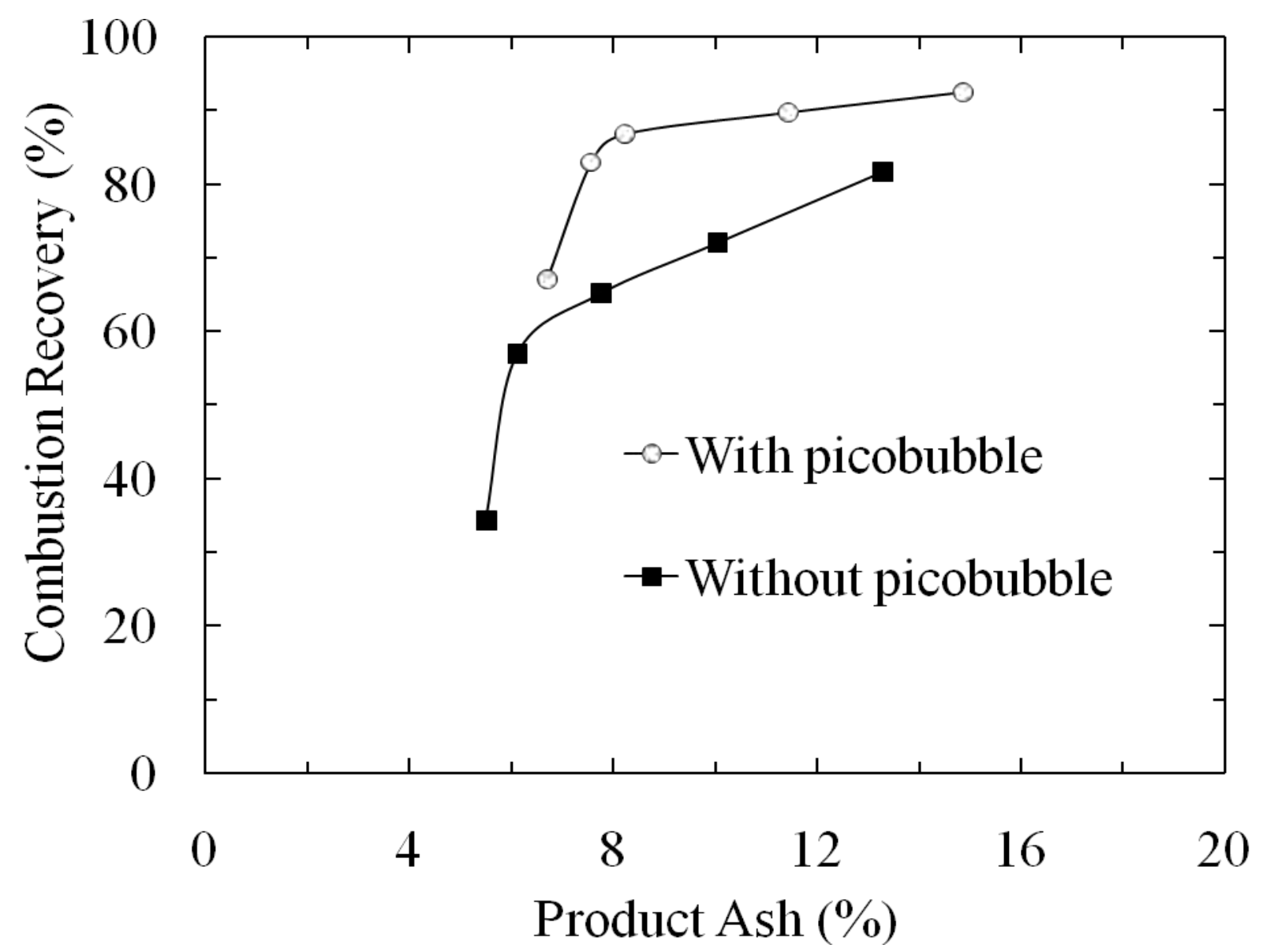

Figure 35 Combustible recovery vs. product ash at different superficial feed rate with and w/o picobubbles 


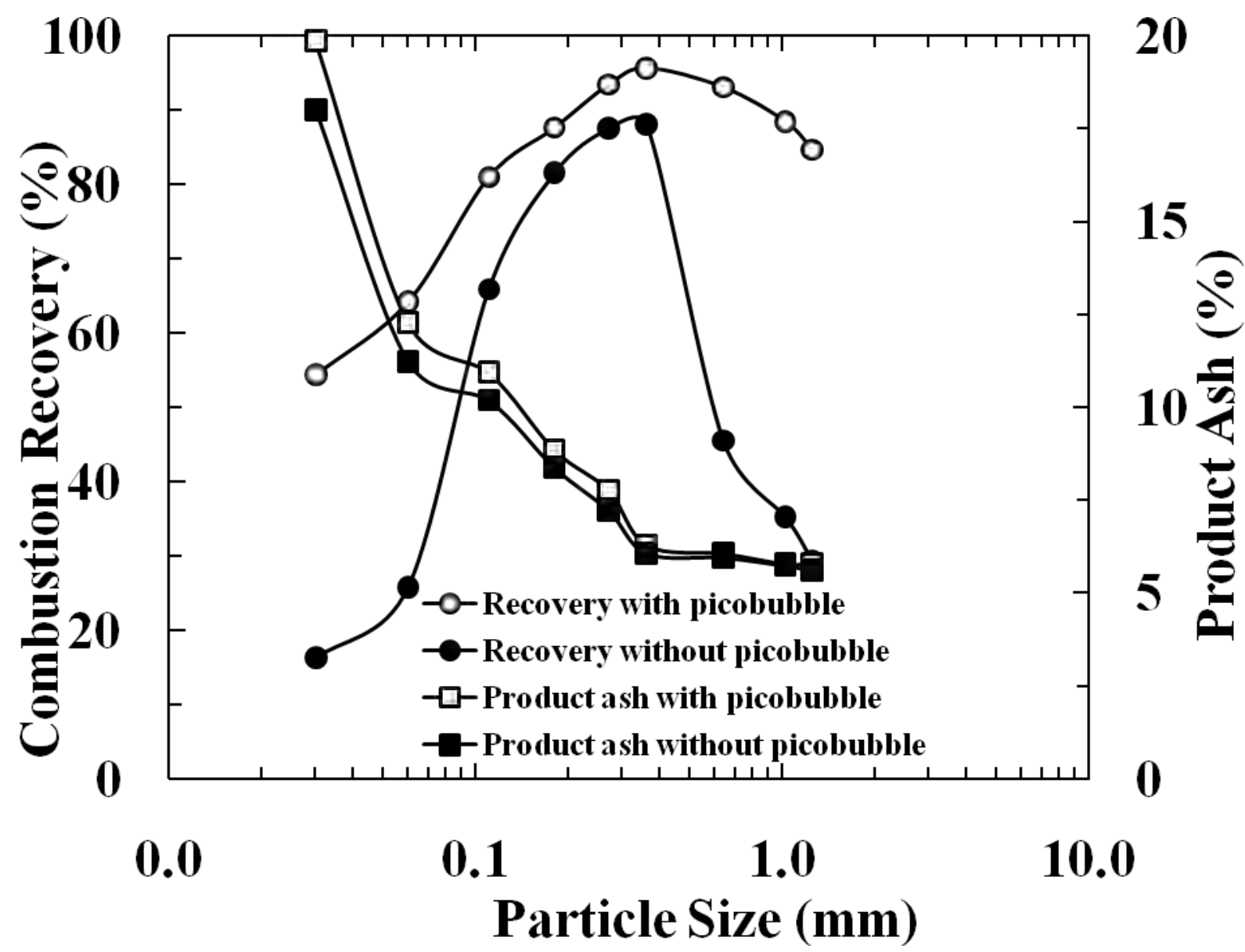

Figure 36 Combustible recovery and product ash as a function of particle size.

Figure 36 shows the combustible recovery and product ash as a function of particle size with and without picobubbles The purpose of this plot was to show the effect of picobubbles on different particles. The data was obtained by wet screening product and tailing samples after flotation tests. Figure 36 clearly shows that picobubbles showed different effects on different particles. More significant improvement in recovery was observed with relatively coarse $(+0.4 \mathrm{~mm})$ and very fine $(-0.1 \mathrm{~mm})$ particles. The most significant increase in recovery was achieved with the coarsest and finest particles. The recovery increase was $56 \%$ for $1.2 \mathrm{~mm}$ particles and $40 \%$ for $0.03 \mathrm{~mm}$ particles. It is interesting to note that use of picobubbles only increased the product ash marginally with most particle sizes. The data shown in Figure 36 suggests that use of picobubbles would improve the separation efficiency more effectively with very coarse and very fine particles that are difficult to float using the conventional flotation methods. 


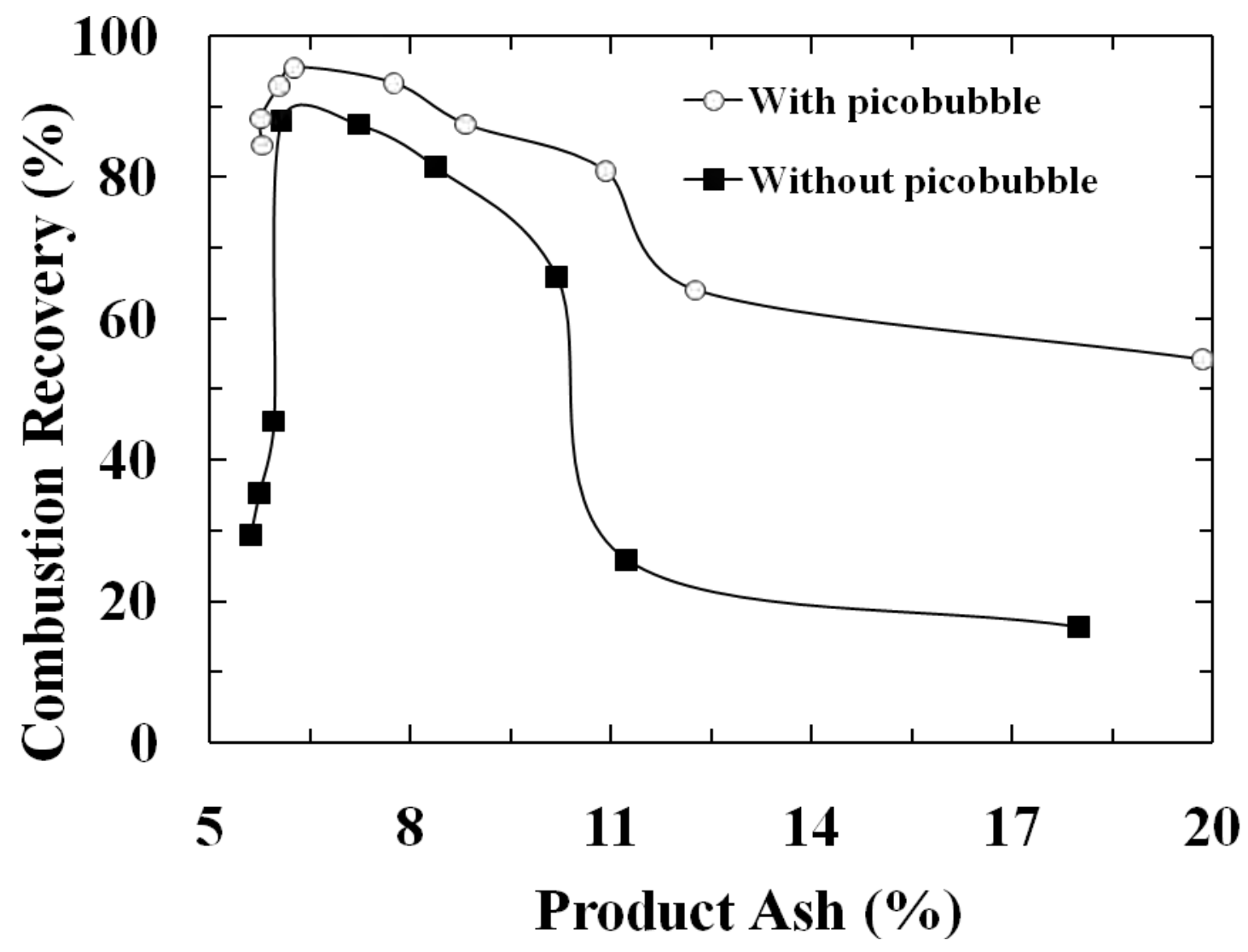

Figure 37 Combustible recovery vs. product ash with and w/o picobubbles

Figure 37 shows the combustible recovery vs. product ash relationship derived from Figure 36. The curve obtained in the presence of picobubbles is always above the one obtained without picobubbles. The difference in combustible recovery at a given product ash is the improvement that can be expected from use of picobubbles. Apparently, more significant improvement is observed on both ends of the curve that are associated with coarse and fine particles, as discussed above.

Figure 38 shows the separation efficiency as a function of particle size with and without picobubbles. When no picobubbles were used, the flotation window for effective separation with a separation efficiency of more than $50 \%$ was approximately in the range of $0.1 \mathrm{~mm}$ to $0.4 \mathrm{~mm}$. Use of picobubbles greatly expanded the window to the range of $0.02 \mathrm{~mm}$ to $0.7 \mathrm{~mm}$. In fact, even the coarsest particles showed a separation efficiency of more than $43 \%$. 


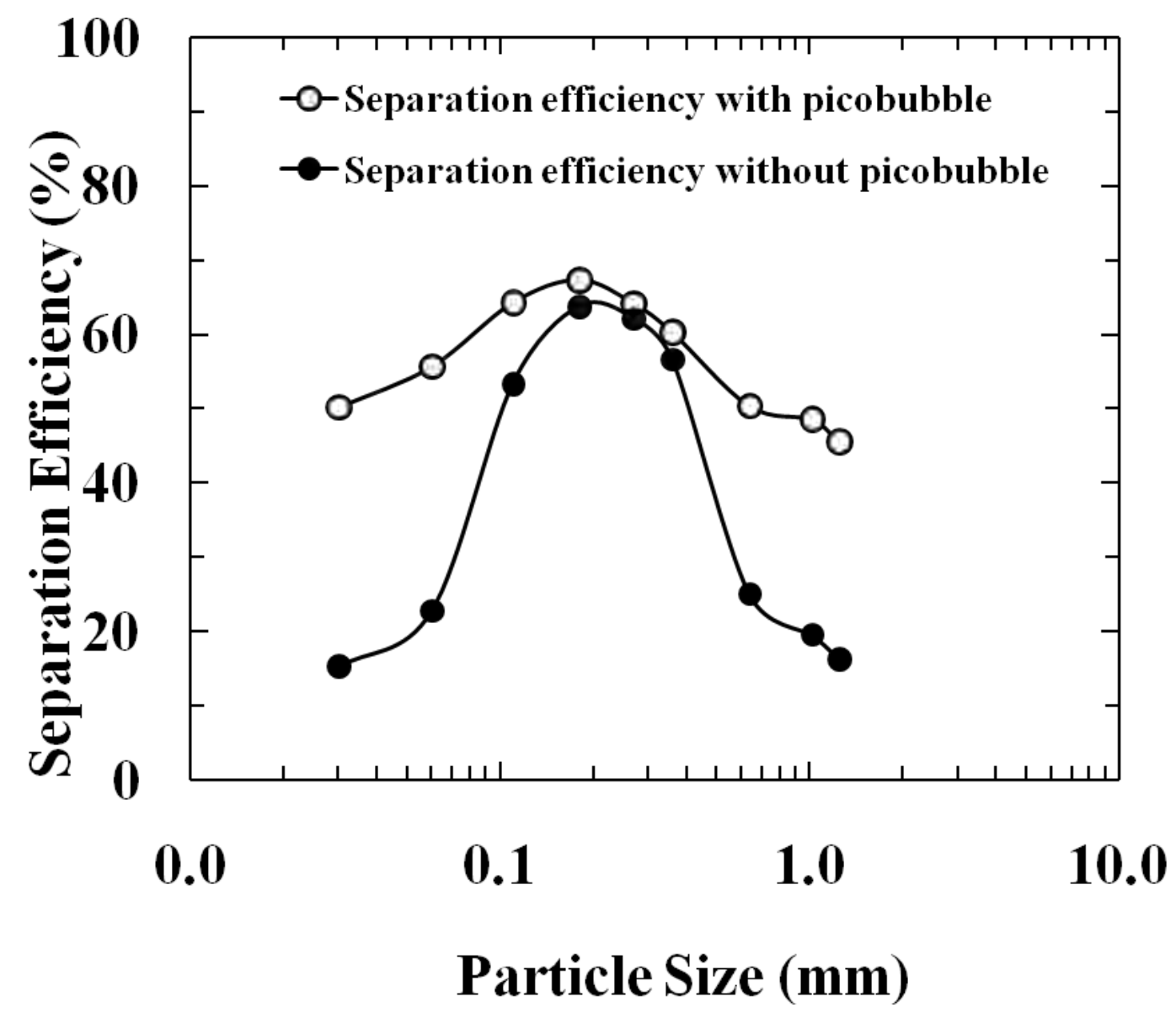

Figure 38 Separation efficiency as a function of particle size with and w/o picobubbles

Figure 39 indicates the impact of picobubbles on the flotation combustible recovery, product ash and flotation selectivity index at different feed slurry solids concentration. It can clearly be seen from the figure that the flotation combustible recovery decreased with increasing flotation feed solids concentration, due to the fact that less bubble surface was available for each particle at higher solids concentration. However, the flotation combustible recovery curve with picobubbles is always substantially above that without picobubbles, suggesting that use of picobubbles significantly increased the flotation combustible recovery. The flotation combustible recovery was $10 \%, 16 \%, 22 \%, 24 \%$, $25 \%$, and $26 \%$ higher with picobubbles than without picobubble at the flotation feed solids concentration of $5 \%, 7 \%, 9 \%, 11 \%, 13 \%$, and $15 \%$, respectively. The product ash with picobubbles was slightly higher than without picobubbles at varying tested solids concentration. Figure $39 \mathrm{~b}$ shows the flotation combustible recovery vs. product ash content curve that was drawn based on the data shown in Figure 39a. It can be observed from Figure $39 \mathrm{~b}$ that the flotation combustible recovery was $24 \%, 23 \%, 22 \%, 15 \%, 14 \%$, and $12 \%$ higher with picobubbles than without picobubble at the flotation product ash contents of $6 \%, 7 \%, 8 \%, 9 \%, 10 \%$, and $11 \%$, respectively. Figure $39 \mathrm{c}$ indicates that the use of picobubbles increased the flotation selectivity index by $19 \%, 18 \%, 17 \%, 12 \%$, $11 \%$, and $9 \%$ at the flotation product ash contents of $6 \%, 7 \%, 8 \%, 9 \%, 10 \%$, and $11 \%$, 
respectively. It can be concluded from Figure 39a and Figure 39c that picobubbles play the biggest role in the flotation combustible recovery and flotation selectivity at the highest flotation feed solids concentration. This is because picobubbles remarkably increased the bubble surface available for each particle at higher solids concentration. The better selectivity or separation sharpness observed with picobubbles at varying solids concentration may be attributed to the preference of picobubbles to coal particles. In other words, picobubbles not only increased flotation recovery, it also improved flotation selectivity. This is because picobubbles generated using the cavitation principle form preferentially on the surface of more hydrophobic particles that have lower work of adhesion $\mathrm{W}_{\mathrm{a}}$ or $\gamma_{1}(1+\operatorname{Cos} \theta)\left(\gamma_{1}\right.$ is water surface tension and $\theta$ is contact angle), as discussed earlier.

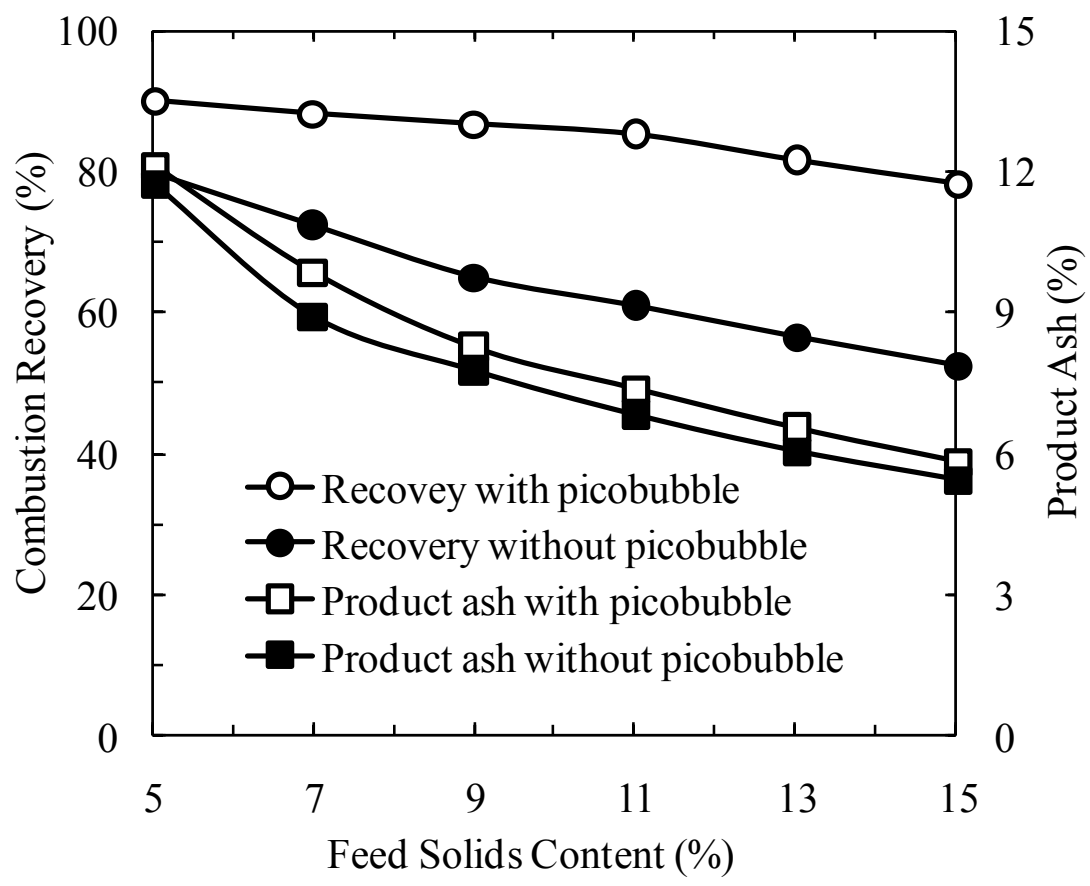

(a) 


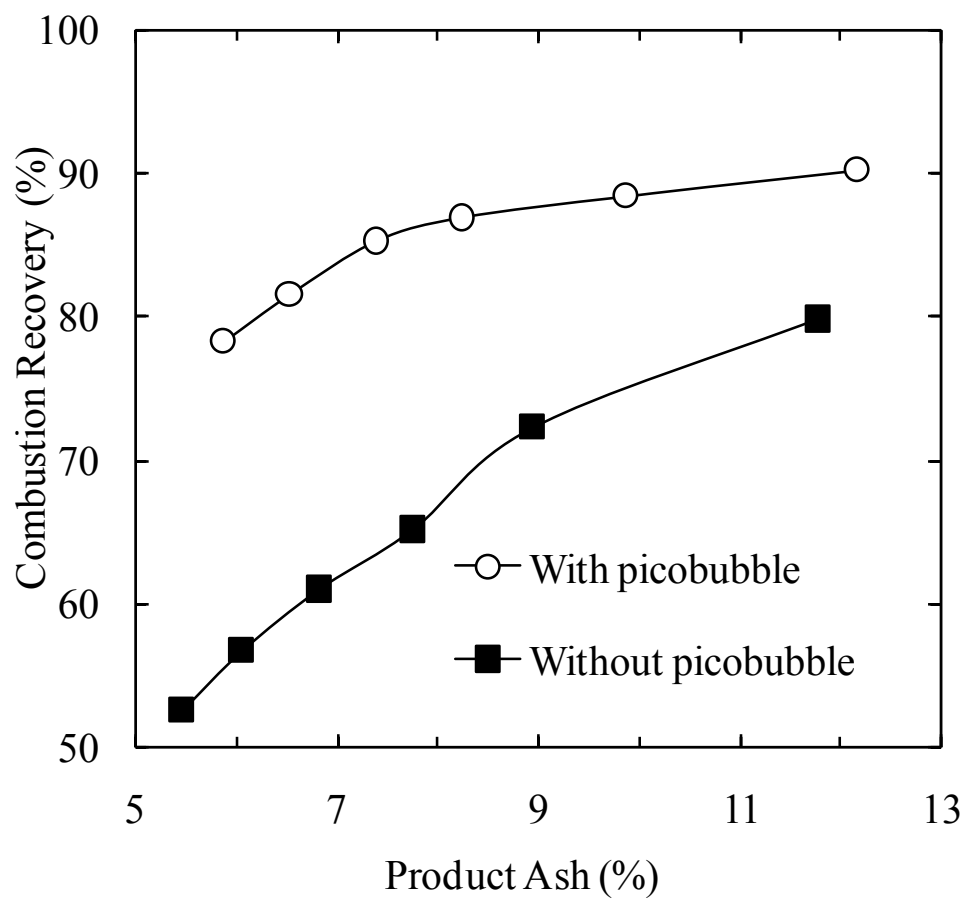

(b)

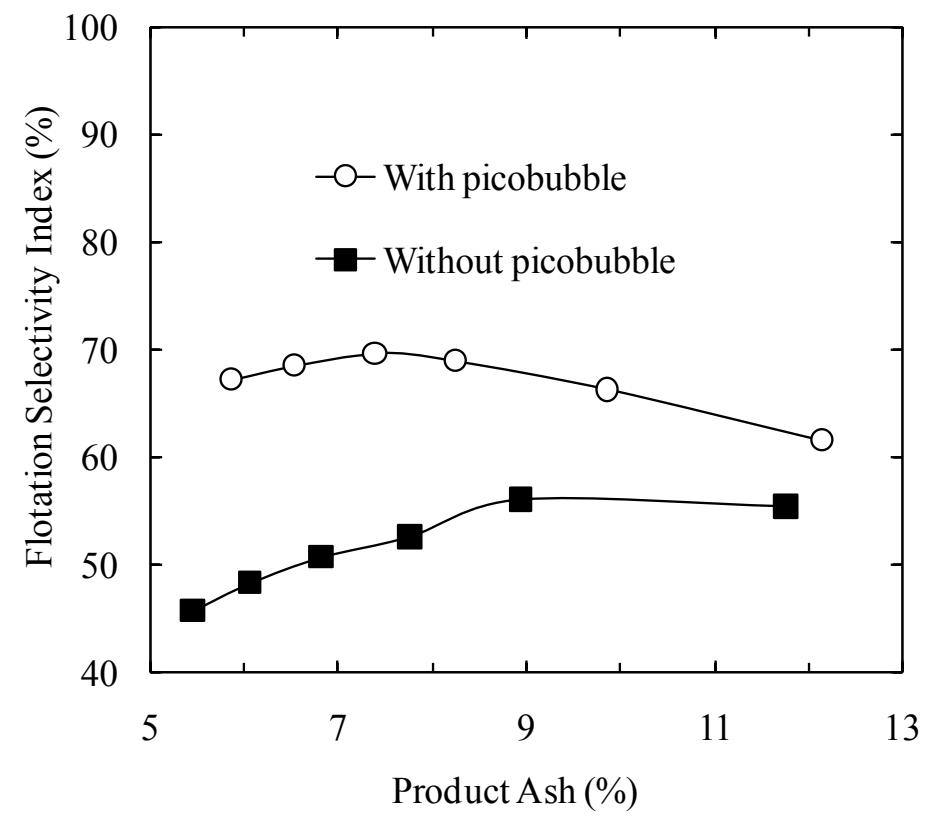

(c)

Figure 39 Effect of picobubbles on the combustible recovery, product ash and flotation selectivity index at varying feed slurry solids concentration

Mechanical Cell Flotation Results 
To assess the impact of picobubbles on mechanical cell coal flotation performance, experiments were conducted with a bank of three 10-liter mechanical cells as shown in Figure 5. During the tests with picobubbles, part of the slurry in the third cell was pumped through a picobubble generator with $3.2 \mathrm{~mm}$ inner neck diameter and $12 \mathrm{~mm}$ inner pipe diameter, and then fed back to the first cell. In this recycling, picobubbles took place preferentially on the hydrophobic coal particle surface. The picobubbles formed on coal particle surface remained attached while those on hydrophilic particle detached, which was a selective process that enhanced flotation separation efficiency. The hydrophobic particles had higher collision probability with picobubbles, higher attachment probability, and lower detachment probability, as explained earlier, resulting in greater flotation rate constant and flotation recovery.

Figure 40 illustrates how the coal particle density and picobubbles affect the flotation performance of 600 355 microns and 75 0 microns particle size, respectively. In these figures, the partition factor refers the ratio of the mass of a given density fraction in the flotation product to the mass of the corresponding density fraction in the flotation feed. The combustible recovery in Figure 40 was calculated by dividing the mass of combustible material recovered in a given density fraction by the mass of the combustible material in the flotation feed of the corresponding density fraction. It can be clearly seen from Figure 40 that the combustible recovery significantly decreases with increasing the particle density. The coal particle floatability of a given coal particle size range decreases with increasing the coal particle density. First, because the organic components those are most amenable to bubble attachment decrease and the inorganic mineral matter components which mostly resist bubble attachment increase as the coal particle density increases. Second, the bubble volume or the bubble number required for floating a lower density coal particle of a given particle size is smaller than that required for floating a higher density coal particle. In other words, the increase in coal particle density of a given particle size results in decrease of the amount coal surface area available for bubble attachment which reduces coal particle/bubble attachment probability, and increase coal particle mass which increases the coal particle detachment probability.

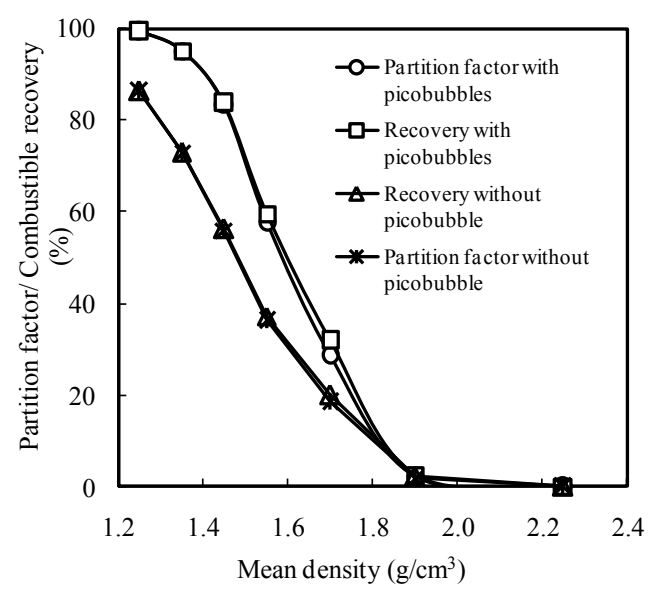

(a) 600 355 microns

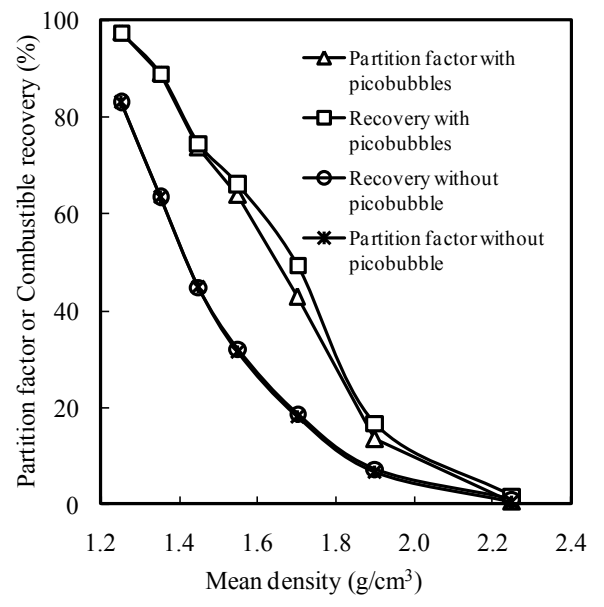

(b) 75 0 microns

Figure 40 Effect of picobubbles on coal flotation recovery and separation density 
Figure 40 shows that the use of picobubbles remarkably increase the various density coal flotation combustible recoveries of 600 355 microns and 75 0 microns particles. Figure $40 \mathrm{a}$ indicates that the presence of picobubbles increases the 600 355 microns coal particles combustible recoveries of the various density fractions: minus 1.3, 1.3-1.4, 1.4$1.5,1.5-1.6$, and $1.6-1.8 \mathrm{gram} / \mathrm{cm}^{3}$ by $13.1 \%, 22.2 \%, 27.6 \%, 22.5 \%$, and $11.9 \%$, respectively. The picobubbles increase the effective separation density when the partition factor is $50 \%$ from $1.44 \mathrm{gram} / \mathrm{cm}^{3}$ to $1.54 \mathrm{gram} / \mathrm{cm}^{3}$. That means picobubbles have more significant effect on the medium density fractions than on the low and high density fractions. Figure $40 \mathrm{~b}$ depicts that the presence of picobubbles increases the $75 \sim 0$ microns coal particles combustible recoveries of the various density fractions: minus 1.3, 1.3-1.4, $1.4-1.5,1.5-1.6,1.6-1.8$, and $1.8-2.0$ gram $/ \mathrm{cm}^{3}$ by $14.6 \%, 25.7 \%, 29.7 \%, 33.8 \%, 30.6 \%$ and $9.5 \%$, respectively. The picobubbles increase the effective separation density when the partition factor is $50 \%$ from $1.42 \mathrm{gram} / \mathrm{cm}^{3}$ to $1.61 \mathrm{gram} / \mathrm{cm}^{3}$. Similarly the picobubbles have more significant effect on the medium density fractions than on the low and high density fractions. It can be seen from Figure 40 that the picobubbles have more remarkable effect on the 75 0 microns finer particles than the 600 355 microns coarser particles. More high density fraction particles of the $75 \sim 0$ microns finer particles were floated than that of the $600 \sim 355$ microns coarser particles.

Figure 41 is the comparisons of the yield and the combustible recoveries with and without picobubbles for $600 \sim 355$ microns coal particles and $75 \sim 0$ microns coal particles, respectively. At a given product ash content, Figure 4la indicates that the presence of picobubbles increases the 600 355 microns coal particles flotation yield and the combustible recovery by up to $18 \%$ and $35 \%$, respectively. Figure $41 \mathrm{~b}$ shows that the presence of picobubbles increases the 75 0 microns coal particles flotation yield and combustible recovery by up to $10 \%$ and $38 \%$, respectively. The picobubbles have more significant effect on the combustible recoveries of 600 355 microns coarse coal particles and $75 \sim 0$ microns fine coal particles in the medium product ash range than in the lower or the higher prc

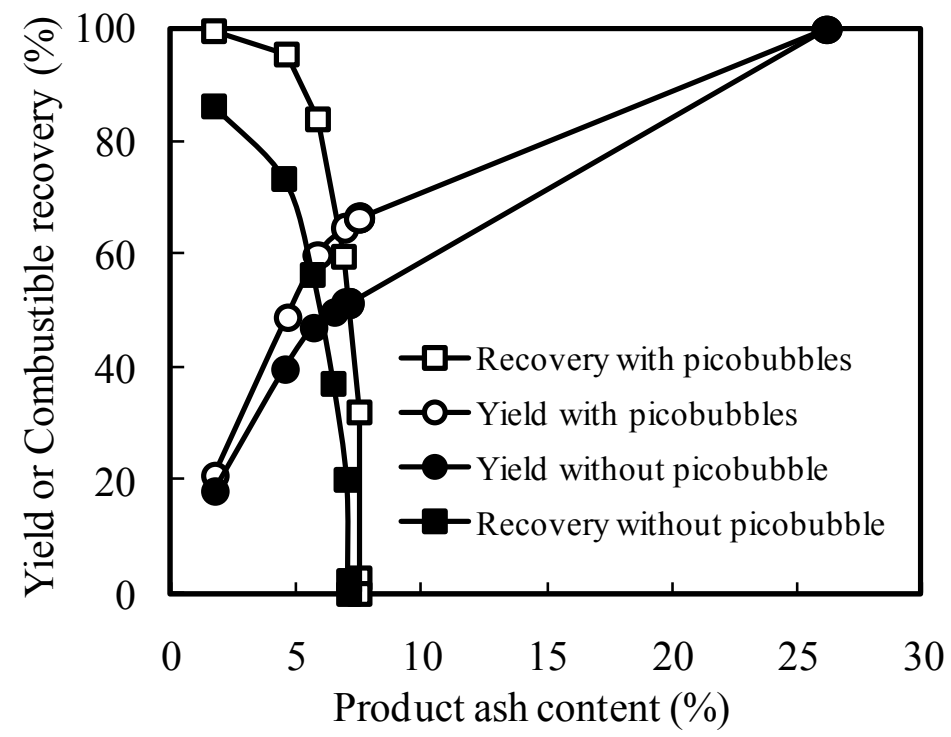

(a) 600 355 microns 


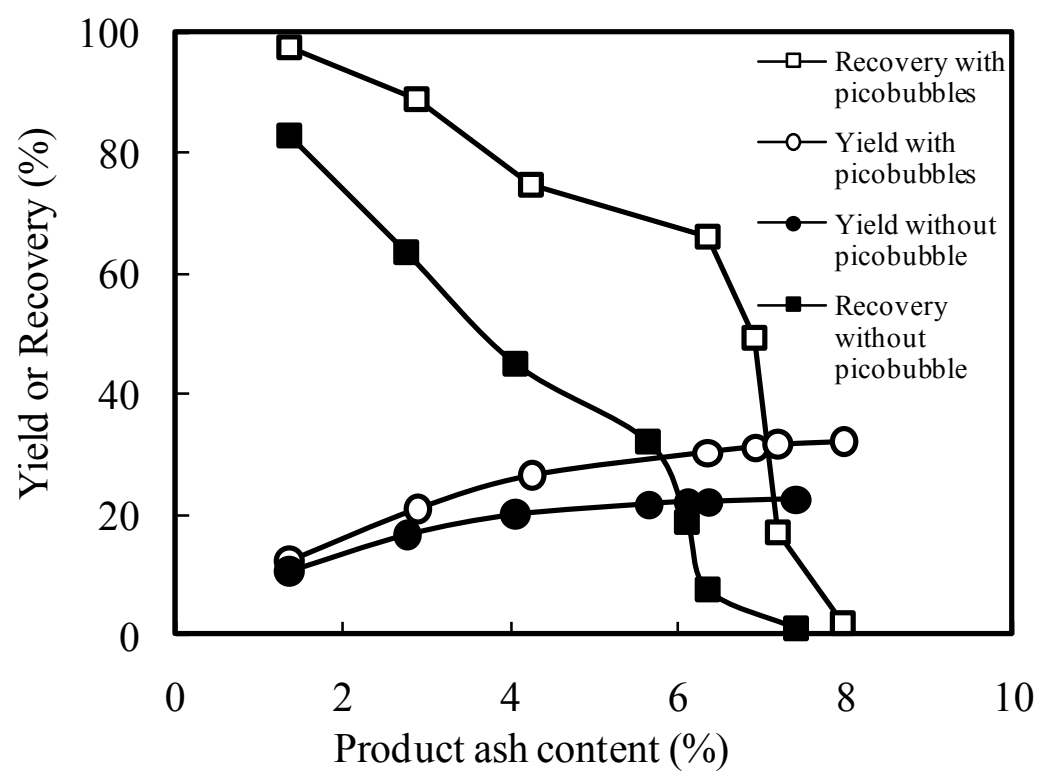

(b) $75 \sim 0$ microns

Figure 41 Effect of picobubbles on yield and combustible recovery

Figure 42 reveals the effect of picobubbles on the flotation product particle size distribution of 600 180 microns and 180 0 microns flotation feed in presence and in the absence of picobubbles. It can be clearly seen from cumulative particle size distribution curves in Figure 42a that the use of picobubbles increased the 600 180 microns coarse coal flotation product mean particle size from 250 microns to 325 microns, which means that picobubbles greatly improved the coarse coal particle flotation recovery. Figure $42 \mathrm{~b}$ depicts that the presence of picobubbles decreased the 180 0 microns fine/ultrafine coal flotation product mean particle size from 103 microns to 69 microns, significantly improved the fine/ultrafine coal particle flotation recovery.

Figure 43 shows the cumulative undersize mass distribution of flotation concentrate samples collected from each flotation cell as a function of particle size. Figure 43a indicates that the $600 \sim 180$ microns coarse coal flotation product mean particle size increases from cell 1 , cell 2 , to cell 3 . The use of picobubbles increases the mean particle size of cell 1, cell 2 and cell 3 flotation products by 65, 90 and 105 microns, respectively. Picobubbles remarkably improved the coarse coal particle flotation recovery. On the contrary, Figure $43 \mathrm{~b}$ reveals that the 180 0 microns fine/ultrafine coal flotation product mean particle size decreases from cell 1 , cell 2 , to cell 3 . The presence of picobubbles decreased the mean particle size of cell 1 , cell 2 and cell 3 flotation products from 108 to 80,96 to 64 and 90 to 62 , respectively. Picobubbles significantly improved the fine/ultrafine coal particle flotation recovery. Therefore, picobubbles can widen flotation size range. 


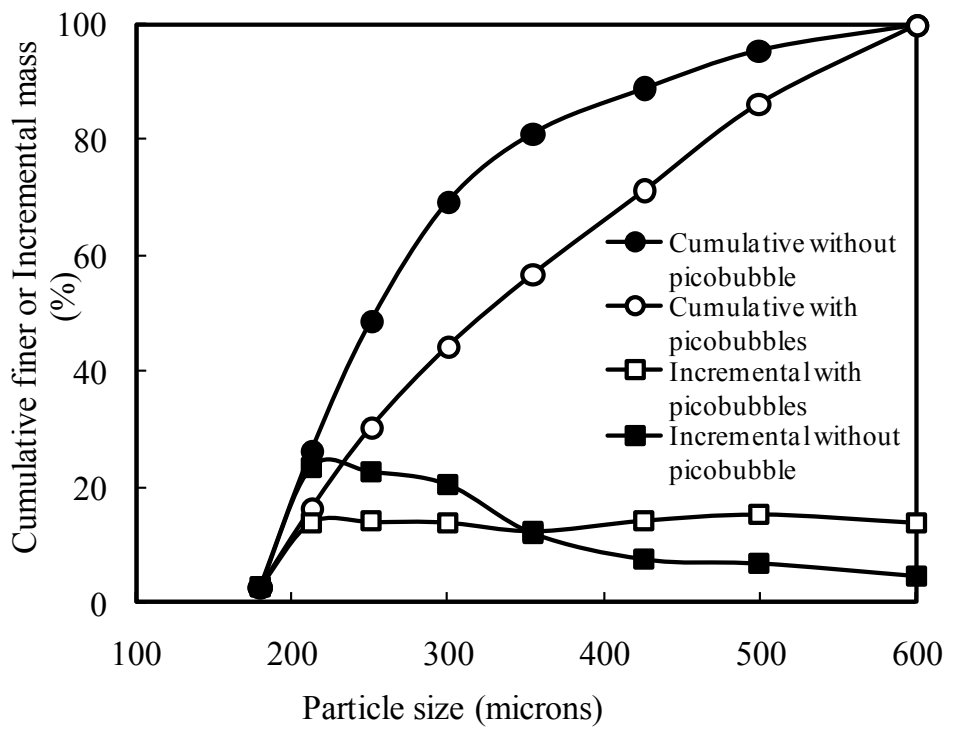

(a) Product of 600 180 microns flotation feed

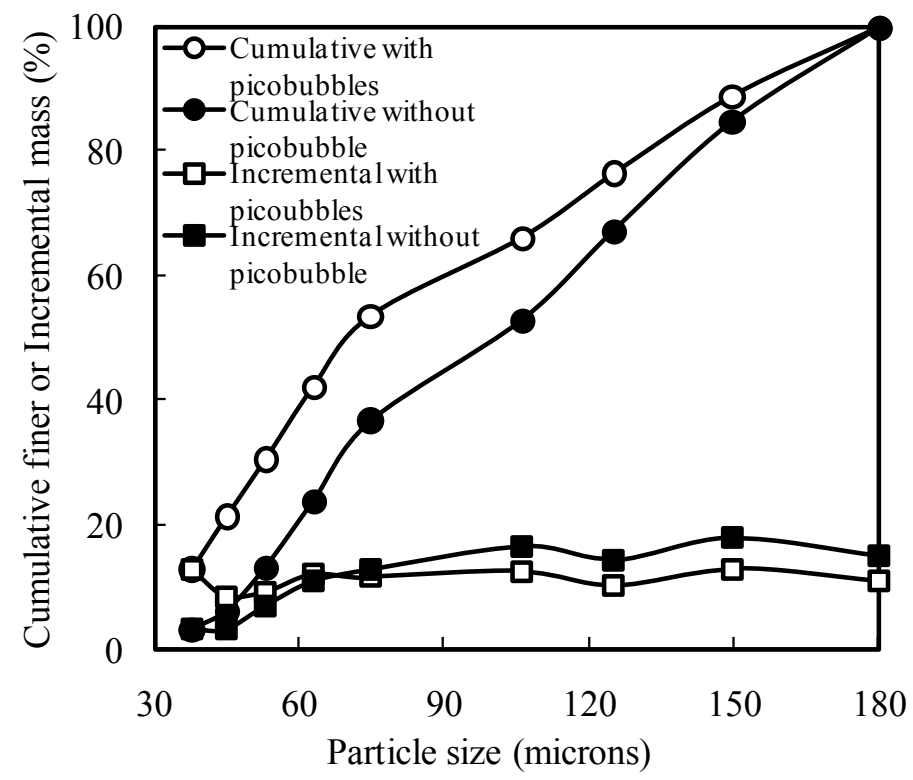

(b) Product of 180 0 microns flotation feed

Figure 42 Incremental and cumulative particle size distribution of flotation product with and without picobubbles 


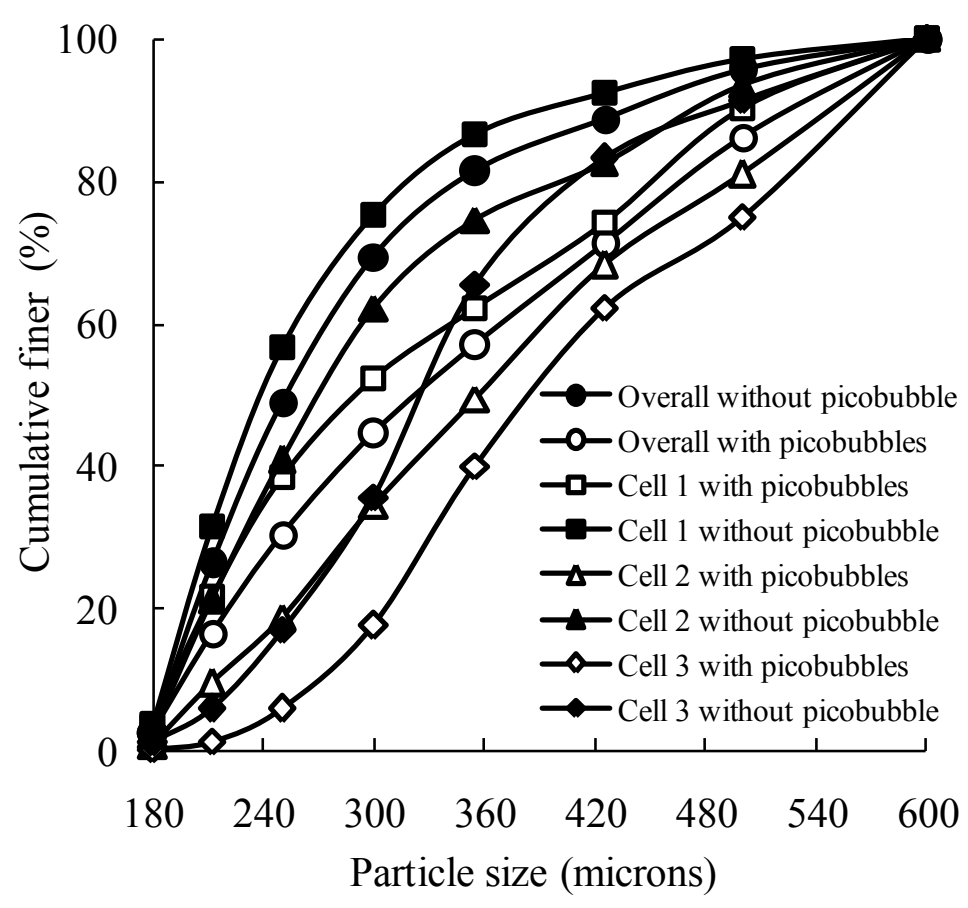

(a) Flotation product of 600 180 microns feed

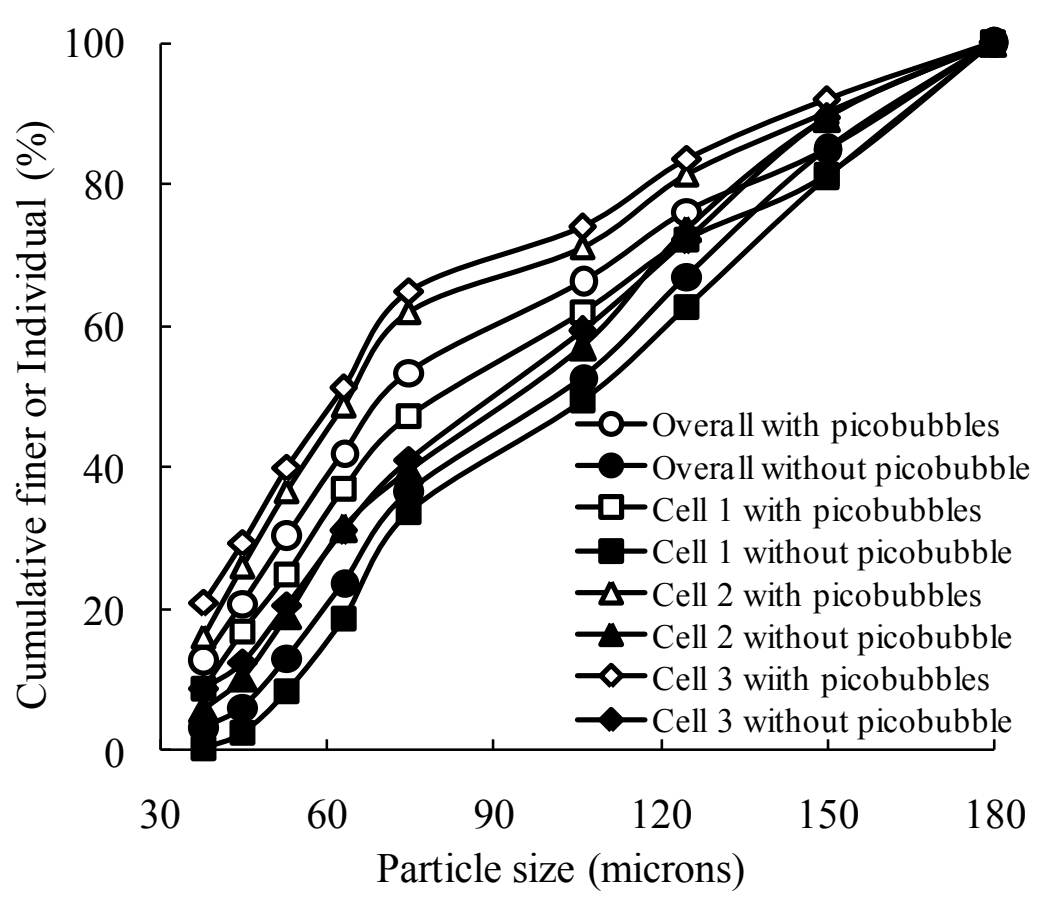

(b) Flotation product of 180 0 microns feed

Figure 43 Cumulative size distribution of each flotation cell product with and without picobubbles 
The investigation of picobubbles' effect on flotation rate constant was performed with four coal particle size fractions: $600 \sim 355,355 \sim 180,180 \sim 75$, and $75 \sim 0$ microns in the above described three 10-liter mechanical cells. Figure 44a shows the effect of picobubbles on the recovery-flotation time curves in which the combustible recoveries for $600 \sim 355,355 \sim 180,180 \sim 75$, and $75 \sim 0$ microns particle size fractions were plotted against the flotation time. The curves indicate that the combustible recovery in the presence of picobubbles was significantly higher than in the absence of picobubbles, which means the presence of picobubbles increased the flotation recovery of combustible matter. It can be clearly observed from Figure $44 \mathrm{~b}$ that picobubbles have more significant effect on the flotation combustible recovery before 1.5 minutes flotation time than that after 1.5 minutes flotation time. Picobubbles increased the combustible recovery of 75 0 microns particle size fraction by $20.5 \%, 18.3 \%, 10.4 \%, 8.8 \%, 6.8 \%$ and $4.8 \%$ at the flotation time of $0.5,1.0,1.5,2.0,3.0$, and 4.0 minutes, respectively. The difference of the combustible recovery decreased as the cumulative flotation time was increased from 0.5 minute to 4 minutes. Figure $44 \mathrm{~b}$ also depicts that picobubbles have more remarkable effect on the flotation combustible recovery of the coarse coal particle size fraction and the ultrafine coal particle size fraction than on that of the medium particle size fractions. For an example, when the cumulative flotation time is 1 minute, the combustible recovery in the presence of picobubbles was about $27.0 \%, 24.7 \%, 17.9 \%, 18.3 \%$ higher than in the absence of picobubbles for 600 355, 355 180, 180 75, and 75 0 microns particle size fractions, respectively.

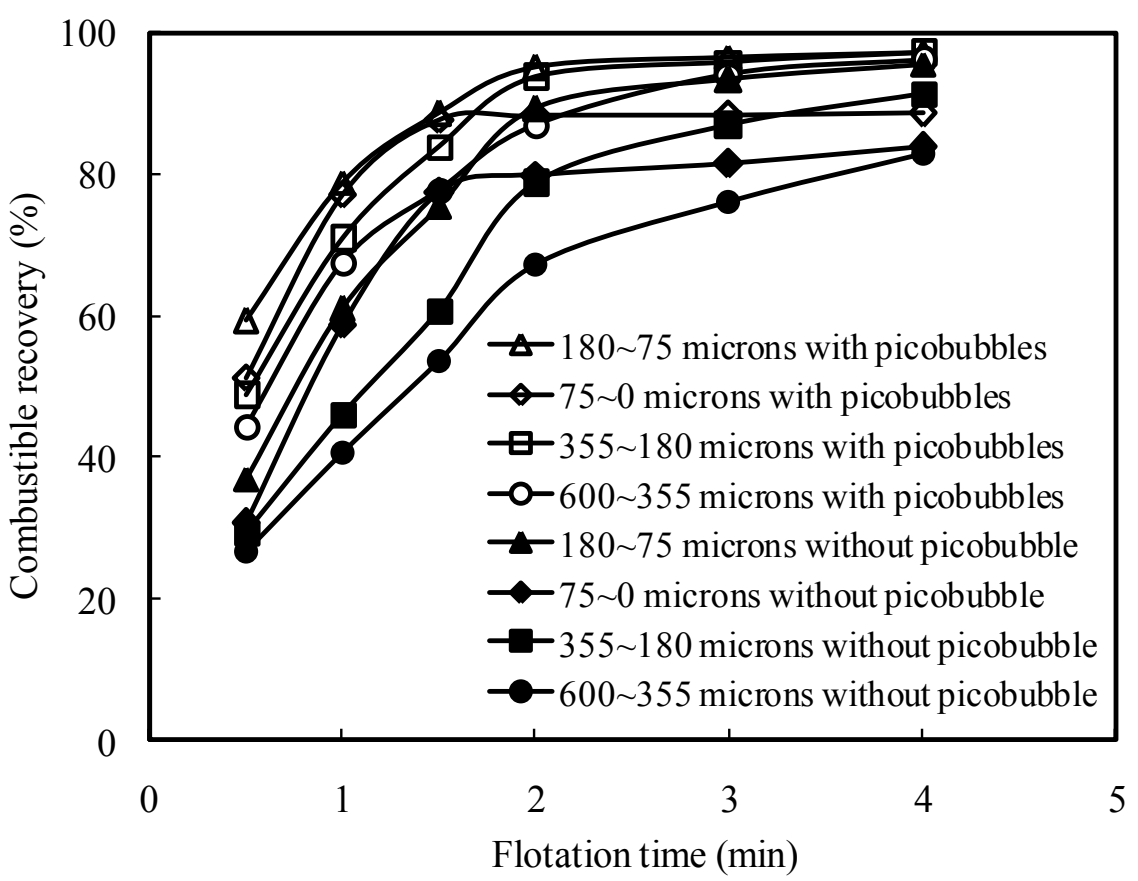

(a) 


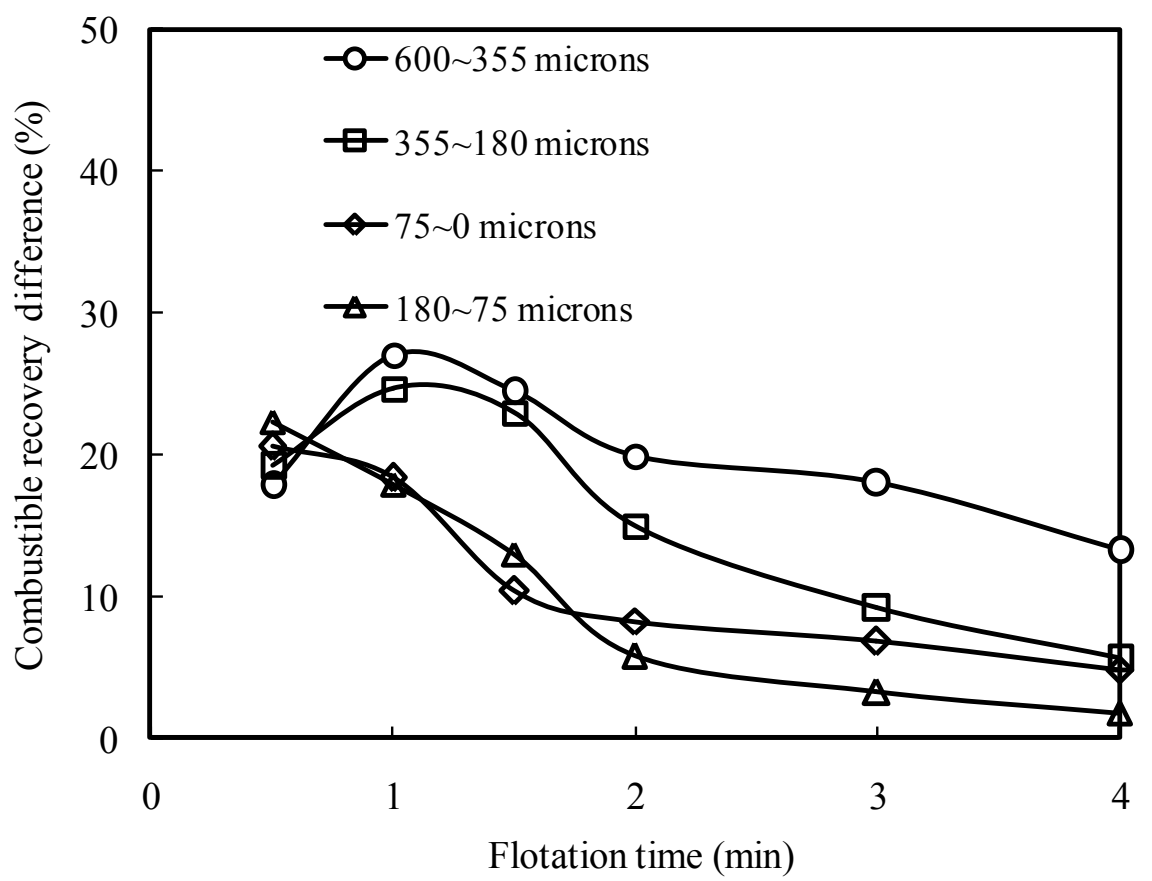

(b)

Figure 44 Effects of picobubbles on the flotation kinetics of various size coal particles

The fact that the curve with picobubbles is always above the other curve indicates that the presence of picobubbles improved the flotation kinetics. Assuming perfect mixing and first-order kinetics conditions, the flotation rate constants shown in Figure $45 \mathrm{~b}$ were determined from the data in Figure 45a, in which the flotation recovery $(R)$ for each particular size range is plotted against the flotation time. Figure 45a depicts the curves of $\ln (1-\mathrm{R})$ versus flotation time for four coal particle size ranges of $600 \sim 355,355 \sim 180$, $180 \sim 75$, and 75 0 microns. Figure $45 \mathrm{~b}$ shows the flotation rate constants, the slope of each plot shown in Figure 45a, are 0.50, 0.61, 0.94, and $1.01 \mathrm{~min}^{-1}$ for $600 \sim 355$, 355 180, 180 75, and 75 0 microns size range coal particles, respectively, when no picobubbles were present. In the presence of picobubbles, the flotation rate constants of coal particles in these four particle size fractions increased to $0.99,1.21,1.41$, and 1.43 $\min ^{-1}$, respectively. The flotation rate constants with picobubbles are $98.0 \%, 98.4 \%$, $50.0 \%$ and $41.6 \%$ higher than without picobubble for $600 \sim 355,355 \sim 180,180 \sim 75$, and 75 0 microns coal particles, respectively. This indicates that the use of picobubbles increased the various size coal particle flotation rate, which leads to the increased capacity of a given flotation machine. Figure 45 also shows that the flotation rate constant increases with decreasing coal particle size. The test results are in agreement with previous research results about the effects of very small bubbles on the fine mineral or coal flotation kinetics (Zhou et al., 1996; Feng and Aldrich, 1999). 


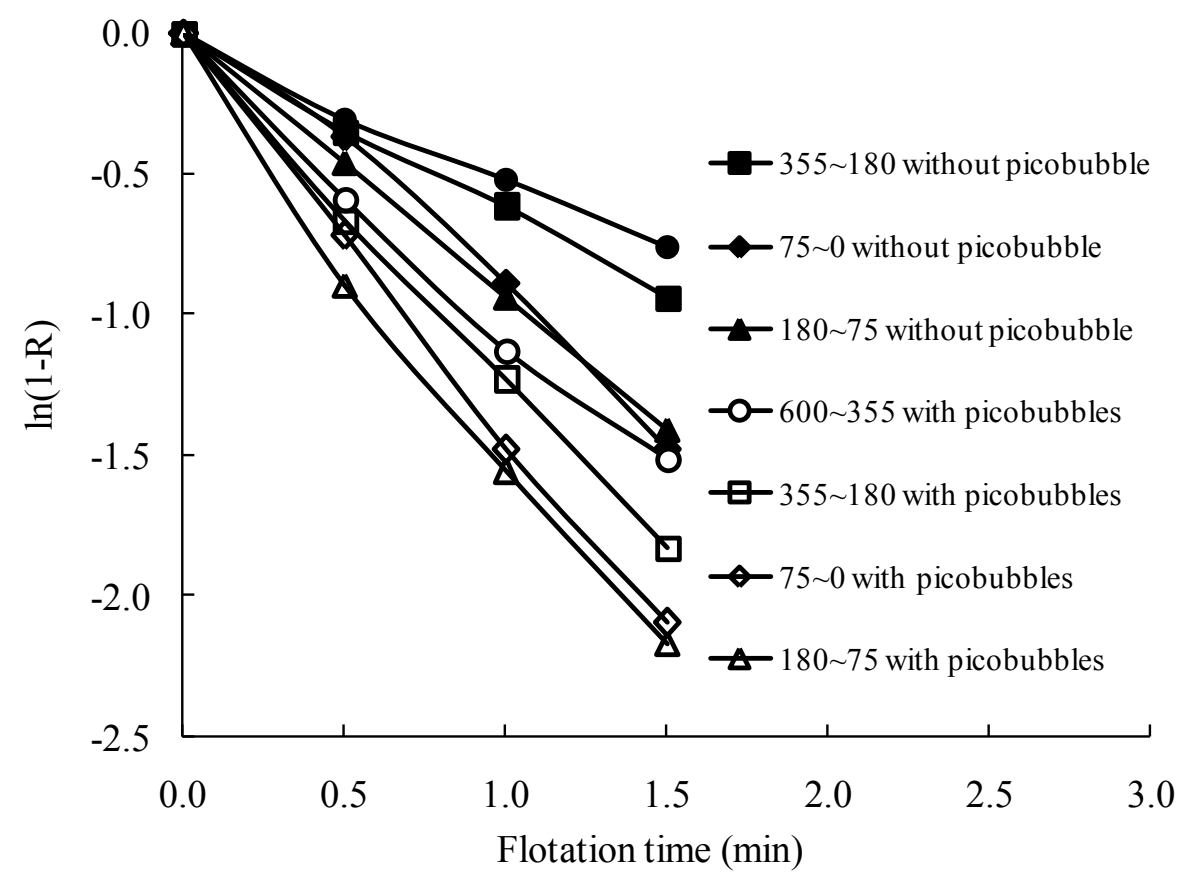

(a)

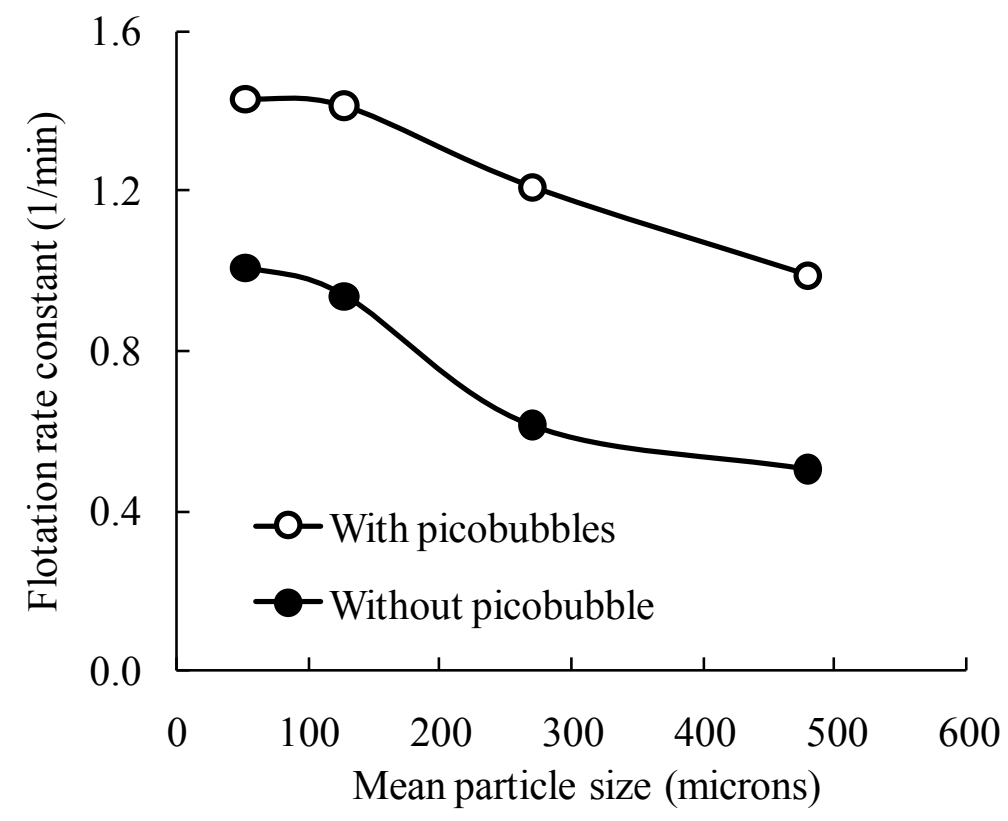

(b)

Figure 45 Effects of picobubbles on the flotation rate constant 


\section{CONCLUSIONS}

The following summarizing conclusions are made based on the test results described above:

1) Two distinct peaks can be observed on the bubble size distribution curve of the picobubbles generated by the cavitation tube and the microbubbles generated by the static mixer. Picobubbles are about two orders of magnitude smaller than microbubbles.

2) The presence of picobbubbles increased the probabilities of collision and adhesion, and reduced the probability of detachment. The modified Hallimond tube flotation tests show that the bubble size, particle size and the presence of picobubbles have significant effect on the collection efficiency, especially on fine particles collection efficiency.

3) The use of picobubbles significantly increased flotation recovery and separation efficiency. For example, at $0.5 \mathrm{~cm} / \mathrm{s}$ air velocity, the recovery was almost $30 \%$ higher in the presence of picobubbles. The use of picobubbles increased recovery by about $25-40 \%$ at a given collector dosage. The separation performance curve suggests that the combustible recovery was $12-21 \%$ higher for a given product ash when picobubbles were employed. A $28-32 \%$ increase in combustible recovery was produced at a given frother dosage and a 17-20\% improvement in combustible recovery was observed for a given product ash.

4) Most significant improvements in flotation recovery is observed with ultrafine (smaller than approximately $0.08 \mathrm{~mm}$ ) and coarse coal particles (larger than 0.4 or $0.5 \mathrm{~mm})$. The flotation recovery is increased by almost $40 \%$ and $55 \%$ with 30 microns and $1.5 \mathrm{~mm}$ coal particles, respectively. Use of picobubbles not only increases the flotation recovery but also improves the flotation selectivity.

5) The presence of picobubbles had more significant effect on the medium density coal particles than on the low and high density particles.

6) Picobubbles substantially improved the flotation recovery of coarse and ultrafine coal particle flotation recovery and greatly expanded the flotation particle size range. The use of picobubbles expanded the particle size range from $0.1 \mathrm{~mm}$ to $0.4 \mathrm{~mm}$ to the range from $0.02 \mathrm{~mm}$ to $0.7 \mathrm{~mm}$ for effective separation.

7) Picobubbles can reduce the required frother dosage by up to $50 \%$ and collector dosage by almost two thirds.

8) Picobubbles have more significant effects on the flotation combustible recovery before 1.5 minutes flotation time than that after 1.5 minutes flotation time. Picobubbles increased the combustible recovery of 75 0 microns particle size fraction by $20.5 \%, 18.3 \%, 10.4 \%, 8.8 \%, 6.8 \%$ and $4.8 \%$ at the flotation time of $0.5,1.0,1.5,2.0,3.0$, and 4.0 minutes, respectively..

9) The presence of picobubbles improves the flotation kinetics. Picobubbles increase the flotation rate constants of $600 \sim 355,355 \sim 180,180 \sim 75$, and 75 0 microns particles by $98.0 \%, 98.4 \%, 50.0 \%$, and $41.6 \%$, respectively, which leads to the increased capacity of a given flotation machine.

10) The use of picobubbles increases the flotation combustible recovery at the "elbow point" on the curves of flotation ash rejection vs. flotation combustible recovery of varying size particles by $5 \sim 10 \%$. The ash rejection of $600 \sim 355$ microns size 
coal particles is about $11 \%, 7.5 \%, 6 \%$, and $5 \%$ higher with picobubbles than without picobubble at the flotation combustible recoveries of $83 \%, 75 \%, 70 \%$, and $60 \%$, respectively. At the flotation combustible recovery of $90 \%$, picobubbles increased the flotation ash rejections by $13.5 \%$ and $22 \%$ for $355 \sim 180$ and $180 \sim 75$ microns size fraction coal particles, respectively.

\section{REFERENCES}

Cheng T, Holtham P N, 1995. "The particle detachment process in flotation," Minerals Engineering, 8(8):883-891.

Deglon, D.A., Sawyerr, F., and O'Connor, C.T., 1999. "A model to relate the flotation rate constant and the bubble surface area flux in mechanical flotation cells," Mineral Engineering, 12(6):599-608.

Drzymala, J., 1994. "Characterization of materials by Hallimond tube flotation. Part 2: maximum size of floating particles and contact angle," Int. J. Miner. Process., 42:153167.

Fan M, Honaker R, Tao D., 2010a. "An experimental study on cavitation enhanced fine coal column flotation," in: Processings of 2010 SME Annual meeting, Phoenix. Society for mining, metallurgy, and exploration, Inc., 2010.

Fan M, Tao D, Honaker R, Luo Z F., 2010b. "Nanobubble generation and its application in froth flotation(Part I): nanobubble generation and its effects on the properties of microbubble and millimeter scale bubble solutions," Mining Science and Technology, 2010, 20(1): 1-19.

Fan M, Tao D, Honaker R, Luo Z F., 2010c. "Nanobubble generation and its application in froth flotation(Part II): fundamental study and theoretical analysis," Mining Science and Technology, 20(2): 159-177.

Fan M, Tao D, Honaker R, Luo Z F., 2010d. "Nanobubble generation and its application in froth flotation(Part IV): mechanical cells and specially designed column flotation of coal" Mining Science and Technology, 20(5): 641-671.

Fan M, Tao D., 2008. "A study on nanobubble enhanced coarse phosphate froth flotation," Separation Science and Technology, 43(1):1-10.

Feng D, Aldrich., G, 1999. "Effect of particle size on flotation performance of complex sulfide ores, Minerals Engineering," 12(7):721-731.

Gorain, B.K., Franzidis, J.P., and Manlapig, E.V., 1995. "Studies on impeller type, impeller speed and air flow rate in an industrial scale flotation cell. Part 1. Effect of bubble size distribution," Minerals Engineering, 8(6):615-635. 
Gorain, B.K., Franzidis, J.P., and Manlapig, E.V., 1997. "Studies on impeller type, impeller speed and air flow rate in an industrial scale flotation cell. Part 4. Effect of bubble surface area flux on floattion performance," Minerals Engineering, 10(4):367-379.

Mao, L. and Yoon, R.-H., 1997. "Predicting flotation rates using a rate equation derived from first principles," Int. J. Miner. Process., 51:171-181.

Ralston, J. and Dukhin, S.S., 1999. "The interaction between particles and bubbles", Colloids and Surfaces, 151:3-14.

Ralston, J., Fornasiero, D., and Hayes, R., 1999a. "Bubble -particle attachment and detachment in flotation,” Int. J. Miner. Process., 56:133-164.

Ralston J, Fornasiero D, Hayes R, 1999b. "Bubble-particle attachment and detachment in flotation," International Journal of Mineral Processing, 56:133-164.

bert, H. and Bischofberger, C., 1979. "On the optimization of hydrodynamics in flotation processes," Proceedings of $13^{\text {th }}$ Int. Miner. Process. Cong., warszawa, 2:1261-1287.

Tao D, Fan M, Jiang X, 2008. "A novel rotary triboelectrostatic separator and its applications in waste fine particle separation," XXIV International Mineral Processing Congress, Beijing, China.

Tao, Daniel, Fan, Maoming, Jiang, Xinkai, 2009. "Dry coal fly ash cleaning using rotary triboelectrostatic separator," Mining Science and Technology, 19 0642-0647

Weber, M.E. and Paddock, D., 1983. "Interceptional and gravitational collision efficiencies for single collectors at intermediate Reynolds numbers," J. Colloid Interface Sci., 94:328:335.

WyslouzilH E, Kohmeunch J, Christodoulou L, Fan M, 2009. Coarse and fine particle flotation. In: Proceedings of the 48th Conference of metallurgists. Sudbury, Metallurgical Society of CIM.

Yoon, R.-H. and Luttrell, G.H., 1989. "The effect of bubble size on fine particle flotation,” Miner. Process. Extr. Metall. Rev., 5:101-122.

Yoon, R.-H., 2000. "The role of hydrodynamic and surface forces in bubble-particle interaction," Inter. J. Miner. Proces., 58:128-143.

Yoon, R.-H., Luttrell, G.G., Adel, G.T., and Mankosa, M.J., 1989. "Recent advances in fine coal flotation," in: Chander, S. (ed.), Advances in Coal and Mineral Processing Using Flotation, Chap 23. Society of Mining Engineers, Littleton, CO, pp. 211-218. bubble interaction in flotation", International Journal of Mineral Processing, 56:207-256.

Zhou Z A, Xu Z H, Finch J A. Effect of gas nuclei on hydrophobic coagulation. Journal of Colloid Interface Science, 1996(79): 311-314. 


\section{BIBLIOGRAPHY}

Dr. Daniel Tao

Professor

Department of Mining Engineering, University of Kentucky

234E MMRB, Lexington, KY 40504-0107

(859)-257-2953; (859)-323-1962(Fax); dtao@engr.uky.edu

\section{Research Interest}

Fine particle processing; froth flotation; dewatering; surface chemistry of metals and minerals; coal cleaning and pelletization; waste recycling and utilization; corrosion prevention.

\section{Education}

Ph.D. (Mining and Minerals Engineering), Virginia Polytechnic Institute and State University, 1994

M.Sc. (Minerals Processing Engineering), China University of Mining and Technology, 1986

B.Sc. (Minerals Processing Engineering), Beijing University of Science and Technology, 1983

\section{Professional Experience}

2008-present Professor, Department of Mining Engineering, University of Kentucky (UK), Lexington, Kentucky.

2004-08 Associate Professor, the Department of Mining Engineering, UK.

1998-04 Assistant Professor, the Department of Mining Engineering, University of Kentucky, Lexington, Kentucky.

1996-98 Research Engineer, Center for Applied Energy Research, University of Kentucky, Lexington, Kentucky

1994-96 Senior Research Associate, Center for Coal and Minerals Processing, Blacksburg, VA.

1990-94 Research Assistant, Department of Mining and Minerals Engineering, Virginia Polytechnic Institute and State University

1989-90 Visiting Scholar, Center for Coal and Minerals Processing, Virginia Polytechnic Institute and State University, Blacksburg, Virginia

\section{Professional Activities}

Member, Society of Mining, Metallurgy, and Exploration (SME)

Member, American Chemical Society (ACS)

Member, American Institute of Chemical Engineers (AIChE)

Member, American Filtration Society (AFS)

\section{$\underline{\text { Awards }}$}

SME Robert Stefanko Best Paper Award, 2009.

Mining Engineering Foundation Distinguished Professor, 2008.

Wethington Award, University of Kentucky, 2004, 05, 06, 07, 08, 09, 10.

Senior Scientist Award, American Filtration Society, 2005

Outstanding Professor, Mining Engineering Department, 2004. 


\section{Relevant Recent Journal Publications (*: corresponding author)}

1. Tao*, D., Zhou, X. Dopico, P., Hines, J., and Kennedy, D., 2010. "Evaluation of Novel GP Clay Binders in Iron Ore Flotation," J. of Mine.s and Metal. Processing, 27(1):42-46.

2. Tao, D. Zhou, X., Kennedy, D., Dopico P., and Hines J., 2010. "Improved Phosphate Flotation Using Clay Binder", Separation Science and Technology, 45(5):604-609.

3. Tao*, D., Fan ${ }^{+}$, M., and Jiang ${ }^{+}$, X., 2009. "Dry coal fly ash cleaning using rotary triboelectrostatic separator", Mining Science and Technology, 19:642-647.

4. Tao", D., Yu ${ }^{+}$, S., Zhou ${ }^{+}$, X., and Honaker, R., Parekh, B.K., 2008. "Picobubble Column Flotation of Fine Coal," Coal Preparation, 28(1):1-14.

5. Fan, M. and Tao, D.*, 2008. "A study on picobubble enhanced coarse phosphate froth flotation," Separation Science and Technology, 43(1):1-10.

6. Youjun Tao, Jiongtian Liu, Samuel Yu and Daniel Tao", 2007. "Picobubble Enhanced Fine Coal Flotation," Separation Science and Technology, 41:3597-3607.

7. Tao*, D., Chen, G.L., Fan, M.M., Zhou, X.H., Zhao, C., Aron, M., and Wright, J. , 2007. "Coal and Potash Flotation Enhancement by Using Clay Binders", Canadian Metallurgical Quarterly, 46(3):243-250.

8. Tao*, D., Chen, G.L. and Parekh, B.K., 2007. "An Electrochemical Study of Corrosive Wear of Phosphate Grinding Mill", Journal of Applied Electrochemistry, 37(2):187-194.

9. Allen, B.L. and Tao*, D., 2006. "Asphalt Emulsion Enhanced Fine Coal Dewatering," Filtration, 6(3):235-241.

10. $\mathrm{Fan}^{+}$, M., Chen, Q., Zhao, Y., Tao, D., Luo, Z., Zhang, X., Yang, G., 2006. "Fine Coal Dry Classification and Separation", Minerals \& Metallurgical Processing Journal, 23(1):17-21.

11. Chen G. and Tao*, D., 2005. "An Experimental Study of Stability of Oil/Water Emulsion for Preparation of Oil-water-coal Slurry," Fuel Processing Technology, 86:499-508.

12. Chen, G.L., Tao * D., Ren, H., Qiao J.K., 2005. "An Investigation of Niobite Flotation with Octyl Diphosphonic Acid as Collector", Inter. J. of Miner. Process, 76:111-122.

13. Chen, G. and Tao*, D., Parekh, B.K., 2005. "Corrosion Protection of Mild Carbon Steel Media in Phosphate Grinding Mill Using Impressed Current Technology," Minerals Engineering, 18:481-488.

14. Honaker, R. Q., Taulbee, D., Parekh, B. K., Tao, D., and Patil, D., 2004, "Premium Fuel Production from Coal and Timber Waste," Miner. \& Metal.l Process. J., 21(4):183-188.

15. D. Tao, G.L. Chen and B.K. Parekh, 2004. "Statistical Analysis of Wear Rate of Phosphate Grinding Mill," Corrosion, 60(11):1072-1081.

16. Chen, G. and Tao*, D., 2004. "Effect of Solution Chemistry on Flotability of Magnesite and Dolomite," International Journal of Mineral Processing, 74:343-357.

17. Ren, H., Ji, F., Chen, G., and Tao*, D., 2004. "An Investigation of Fersmite Flotation," International Journal of Mineral Processing, 74:271-279. 


\section{CURRICULUM VITAE OF RICKY Q. HONAKER}

\section{PERSONAL DATA}
A. Business Address:
234-B Mining \& Mineral Resources . Lexington, Kentucky 40506-0107
B. Phone/Fax:
(859) 257-1108/(859) 323-1962
C. E-mail Address:
D. Present University Position:
rhonaker@engr.uky.edu
Associate Professor

\section{EDUCATION}

Ph. D. Virginia Polytech. Inst. \& State Univ. - 1992 Mining \& Minerals Engineering

M. S. Virginia Polytech. Inst. \& State Univ. - 1988 - Mining \& Minerals Engineering

B. S. Virginia Polytech. Inst. \& State Univ. - 1986 - Mining \& Minerals Engineering

A. S. Southwest Virginia Community College- 1984 - Engineering

\section{PROFESSIONAL EXPERIENCE}

$1 / 00-$ Present

$4 / 97-12 / 99$

University

University

$$
8 / 92-4 / 97
$$

$8 / 91-8 / 92$

$9 / 90-7 / 91$
Professor, University of Kentucky, Lexington, Kentucky

Associate Professor, Southern Illinois

Carbondale, Illinois

Assistant Professor, Southern Illinois

Carbondale, Illinois

Instructor, Southern Illinois University

Carbondale, Illinois

Research Associate, Virginia Tech

Blacksburg, Virginia

\section{TEACHING EXPERIENCE}

Principles of Mining Engineering; Underground Mine Systems Design; Minerals and Coal Processing; Mineral Processing Plant Design; Mine Ventilation Systems Design; Operations Research and Computers in Mine Design; Mine Health \& Safety; Physical Coal Processing; Fine Coal Beneficiation; Simulation of Mineral Processing Circuits

\section{RESEARCH}

Coal and Minerals Processing, Fine Particle Processing, Automation and Control, Optimization of Unit Processes, Applied Surface Chemistry. 


\section{RELEVANT RECENT PUBLICATIONS}

Honaker, R. Q., Luttrell, G. H. and Lineberry, G. T., "Improved Coal Mining Economics Using Near-Face Deshaling, Minerals and Metallurgical Process. J., Vol. 23. No. 2, pp. $73-79,2006$.

Honaker, R. Q., Ozsever, A. V. and Parekh, B. K., "Selective Detachment Process in Column Flotation Froth,” Minerals Engineering, Vol. 19, pp. 687-695, 2006.

Honaker, R. Q. and Patwardhan, A., "In-Plant Evaluation of Dense Medium Process Performances," Coal Preparation: An International Journal, Vol. 26, pp. 1 - 16, 2006.

Honaker, R. Q., Boaten, F., Luttrell, G. H., Moorhead, R. and Coker, T., "Ultrafine Coal Classification using 6-inch gMax Cyclone Circuits," Proceedings, $22^{\text {nd }}$ International Coal Preparation Conference, Lexington, Kentucky, pp. 19 - 36, May 1-4, 2006.

Honaker, R. Q., Luttrell, G. H., Bratton, R and. Patil, D., "Improving Mine Profitability Using Dry Deshaling Technologies," CPSA Journal, Volume 5, Number 2, pp. 21 - 25, 2006.

Snoby, R., Honaker, R. Q. and Weinstein, R., "Dry Jigging Coal: Advantages and Limitations," Proceedings, XV International Coal Preparation Congress, China University of Mining and Technology Press, Beijing, China, Vol. 2, pp. 448 - 456, 2006

Honaker, R. Q., Jain, M., Parrekh, B. K. and Saracoglu, M., "Optimized Spiral Separation Performance for Ultrafine Coal Cleaning," Proceedings, XV International Coal Preparation Congress, China University of Mining and Technology Press, Beijing, China, Vol. 2, pp. $502-508,2006$.

Honaker, R. Q. and Patwardhan, A., "In-Plant Evaluation of Dense Medium Process Performances," Coal Preparation: An International Journal, Vol. 26, pp. 149 - 164, 2006.

Kohmuench, J. N., Mankosa, M. J., Honaker, R. Q. and Bratton, R. C., "Applications of the Crossflow Teeter-Bed Separator in the U.S. Coal Industry," Minerals and Metallurgical Processing Journal, Vol. 23. No. 4, pp. 187 - 195, 2006.

Honaker, R. Q., Das, A. and Boaten, F., "Slurry Viscosity Modification Effects on Classifying Cyclone Performance," Canadian Metallurgical Quarterly, Vol. 46, No. 3, pp. $341-348,2007$.

Honaker, R. Q., Boaten, F. and Luttrell, G. H., "Ultrafine Coal Classification using 150mm gMax Cyclone Circuits, Minerals Engineering, Vol. 20, pp. 1218 - 1226, 2007.

Honaker, R. Q., Jain, M. and Saracoglu, M., "Ultrafine Coal Cleaning using Spiral Concentrators," Minerals Engineering, Vol. 20, No. 14, pp. 1315 - 1319, 2007. 
APPENDIX 6 - Surface Force Measurement Between Hydrophic Surfaces (VA0020) 
FINAL TECHNICAL REPORT

Contract Title and Number:

Continuation in Crosscutting Technology Development

at CAST. (DE-FC26-05NT42457)

Sub-Recipient Project Title:

Surface Force Measurement Between Hydrophobic

Surfaces

Principal Investigators:

Roe-Hoan Yoon

Contact Address:

CAST

146 Holden Hall

Blacksburg VA 24061

Subcontractor Address:

No subcontracts issued.
Period of Performance:

Starting Date: 10/1/05

Ending Date: 3/31/12

\section{Disclaimer}

"This report was prepared as an account of work sponsored by an agency of the United States Government. Neither the United States Government nor any agency thereof, nor any of their employees, make any warranty, express or implied, nor assume any legal liability or responsibility for the accuracy, completeness, or usefulness of any information, apparatus, product, or process disclosed, or represents that its use would not infringe privately owned rights. Reference herein to any specific commercial product, process, or service by trade name, trademark, manufacturer, or otherwise does not necessarily constitute or imply endorsement, recommendation, or favoring by the United States Government or any agency thereof. The views and opinions of authors expressed herein do not necessarily state or reflect those of the United States Government or agency thereof. ” 


\begin{abstract}
Surface force measurements conducted with thiolated gold surfaces showed previously that hydrophobic interaction entails a decrease in excess film entropy, suggesting that hydrophobic force originates from changes in the structure of the medium (water) confined between hydrophobic surfaces [1]. As a follow-up work, surface force measurements have been conducted in the present work using an atomic force microscope (AFM) with silica surfaces coated with octadecyltrichlorosilane (OTS) at temperatures in the range of 10 to $40^{\circ} \mathrm{C}$. A thermodynamic analysis of the results show that both the excess film entropy $\left(\Delta S^{\mathrm{f}}\right)$ and excess film enthalpy $\left(\Delta H^{\mathrm{f}}\right)$ decrease with decreasing thickness of the water films between the hydrophobic surfaces. It has been found also that $\left|\Delta H^{\mathrm{f}}\right|>\left|T \Delta S^{\mathrm{f}}\right|$, which represents a necessary condition for the excess free energy change $\left(\Delta G^{\mathrm{f}}\right)$ to be negative and hence the hydrophobic interaction be attractive. Thus, the results obtained with both the thiolated and silylated surfaces show that hydrophobic forces originate from the structural changes in the medium. It is believed that the water molecules in the thin liquid films (TLFs) of water form clusters as a means to reduce the free energy when they cannot form $H$-bonds to neighboring hydrophobic surfaces.
\end{abstract}

Keywords: hydrophobic force, excess thermodynamic properties, Derjaguin approximation, structural force, atomic force microscope (AFM), octadecyltrichlorosilane (OTS). 


\section{INTRODUCTION}

Hydrophobic particles placed in water are attracted to each other much more readily than predicted by the DLVO theory due to the presence of the hydrophobic force [2, 3], which is longer-ranged and stronger than the van der Waals force. Israelachvili and Pashley [4] reported the first direct measurement of hydrophobic force using the surface force apparatus (SFA). Many investigators [5-10] confirmed its existence and discussed possible origins, while others suggested that the hydrophobic force is an artifact due to bubbles or cavities [11-13]. Still others suggested that hydrophobic forces, particularly those of longer range, may originate from mechanisms that are unrelated to surface hydrophobicity [14, 15].

In an effort to better understand the origin(s) of hydrophobic force, one of us determined the thermodynamic functions of the hydrophobic interactions by conducting surface force measurements at several different temperatures [1]. The results showed that macroscopic hydrophobic interactions entail decreases in both the excess entropy $\left(S^{f}\right)$ and the excess enthalpy $\left(H^{f}\right)$ of the thin liquid films (TLF) of water confined between hydrophobic surfaces. Here, the term "excess" refers to the thermodynamic indicator of the thin film in question relative to that of the infinitely thick film (or bulk water). It was found also that the changes in excess film enthalpy $\left(\Delta H^{f}\right)$ are slightly larger than the absolute temperature $(T)$ times the changes in excess film entropy $\left(\Delta S^{f}\right)$. Based on these results, it was suggested that macroscopic hydrophobic interactions may involve building structures of water in the vicinity of hydrophobic surfaces. In effect, $\Delta H^{f}$ represents the energy gained in building the structures, while $T \Delta S^{\mathrm{f}}$ represents the thermodynamic cost of building the structure. In this previous work, the surface force measurements were conducted using an atomic force microscope (AFM) with gold-coated silica surfaces hydrophobized with $n$-ethanethiol $\left(\mathrm{C}_{2}\right.$ $\mathrm{SH})$ and $n$-hexadecane thiol $\left(\mathrm{C}_{16}\right.$-SH).

The thermodynamic studies described above suggested that hydrophobic force is a structural force, a term first used by Derjaguin and Kusakov [16] to describe the repulsive hydration force present in wetting films. In general, the term structural force refers to the non-DLVO force created when two boundary layers, whose structures are different from that of the bulk solution, overlap [17]. The non-DLVO forces observed between two hydrophilic surfaces are referred to as positive structural force, while those observed between hydrophobic surfaces are referred to as negative structural force [18]. Eriksson et al. [19] suggested that the long-range attractive forces observed between hydrophobic surfaces may be due to the surface-induced changes in water structure.

In the present work, we have conducted AFM force measurements using silica surfaces hydrophobized with octadecyltrichlorosilane (OTS) at temperatures in the range of 10 to $40^{\circ} \mathrm{C}$. The results have been converted to the excess Gibbs free energies $\left(\Delta G^{f}\right)$ of the TLFs using the Derjaguin approximation [20], which were then used to determine $\Delta S^{\mathrm{f}}$ and $\Delta H^{\mathrm{f}}$ in the same manner as described previously [1].

Silylated silica may provide a more stable and robust hydrophobic surface than thiolated gold, as the latter is susceptible to oxidation. It has been shown that upon exposure to air 
under ambient conditions, self-assembled monolayers (SAMs) of alkane thiolates oxidize to alkane sulfonates [21, 22]. In the presence of halide, the thiol groups oxidize to disulfide and subsequently to sulfonate, with the substrate $(\mathrm{Au})$ being oxidized to $\mathrm{Au}^{3+}$ species [23]. Stability of hydrophobic surfaces using thiolated gold may thus become an issue particularly when conducting force measurements at elevated temperatures. On the other hand, OTScoated silica surfaces are stable at temperatures up to $175^{\circ} \mathrm{C}$ [24].

Surface force data obtained with silylated surfaces and reported in the literature are controversial. Some investigators showed strong hydrophobic forces [25, 26], while others showed capillary forces related to bubbles or cavities [11, 27, 28]. The experimental data obtained in the present work showed the presence of long-range hydrophobic forces with no evidences for bubbles or cavitation causing them. Thermodynamic analysis of the results led to the same conclusion as obtained previously using thiolated gold surfaces: hydrophobic forces originate from changes in the water structure [1].

\section{EXPERIMENTAL}

\section{a) Materials}

All experiments were conducted using ultrapure water obtained using a Direct-Q3 water purification system from Millipore. The water had a resistivity of $18.2 \mathrm{M} \Omega \cdot \mathrm{cm}$ and $<10 \mathrm{ppb}$ of total organic carbon. Silica spheres (Whitehouse Scientific) of $\sim 10 \mu \mathrm{m}$ radius and fusedquartz plates (Technical Glass Products Inc.) were used as macroscopic surfaces for surface force measurements. The spheres and plates were hydrophobized by immersion in octadecyltrichlorosilane (OTS, 95\% purity, Alfa Aesar)-in-toluene (99.9\% purity, Fisher Chemical, dried with a molecular sieve) solutions. The hydrophobized surfaces were rinsed with chloroform (99.9\% purity, Fisher Chemical), acetone (99.9+\% HPLC grade, Aldrich) and ultrapure water sequentially. Silicon wafers (Sumco, Oregon) were used for imaging the OTS-coated surfaces. Sulfuric acid $\left(\mathrm{H}_{2} \mathrm{SO}_{4}, 98 \%\right.$ purity, VMR international) and hydrogen peroxide $\left(\mathrm{H}_{2} \mathrm{O}_{2}, 29.0-32.0 \%\right.$ purity, Alfa Aesar) were used to clean the macroscopic surfaces before hydrophobization with OTS.

\section{b) Sample Preparation}

The macroscopic surfaces used in the present work were cleaned in piranha solutions $\left(\mathrm{H}_{2} \mathrm{O}_{2} / \mathrm{H}_{2} \mathrm{SO}_{4}, 3: 7\right.$ by volume) at $\sim 80^{\circ} \mathrm{C}$ for 1 hour to remove organic contaminants. The plates were then rinsed thoroughly with ultrapure water in an ultrasonic bath for 10 minutes and blow-dried in a pure nitrogen stream.

The cleaned surfaces were hydrophobized by immersing them in freshly-prepared OTSin-toluene solutions. Special care was taken to remove water from the solvent. The hydrophobicity of the silylated surfaces, as measured by water contact angles, was controlled by varying the immersion time in a $5 \times 10^{-5}$ or $3 \times 10^{-3} \mathrm{M}$ OTS-in-toluene solutions contained in TFPE beakers. The excess OTS present on silylated surfaces was removed by subjecting the plates and spheres to ultrasonic vibration sequentially in chloroform, acetone, and pure 
water for a few minutes. The silylated surfaces free of excess OTS were then blow-dried with pure nitrogen gas and stored.

\section{c) AFM Imaging}

A Nanoscope V atomic force microscope (AFM), Veeco Instruments Inc., was used to capture the images of the silylated surfaces under contact mode in air. A silicon nitride AFM cantilever (Nano world Innovative Technologies) with a spring constant of $\sim 0.48 \mathrm{~N} / \mathrm{m}$ was used.

\section{d) Surface Force Measurement}

The Nanoscope V AFM was also used to measure the surface forces acting between silylated silica sphere and silica plate in ultrapure water. The AFM was equipped with a temperature control system, which allowed the measurement of surface forces at different temperatures. The measurement was conducted in the range of 10 to $40^{\circ} \mathrm{C}$ in the same manner as described previously [1]. For a given series of measurements, a silylated silica sphere was glued onto the tip of a cantilever (AI silicon probe, Budget Sensors) with a spring constant (k) of $\sim 7.4 \mathrm{~N} / \mathrm{m}$ using a polymer resin (EPON-1004F, Shell Chemical Co.). The resonant frequency technique[29] was used to accurately determine the spring constant for each cantilever.

\section{e) Contact Angle Measurement}

Droplets of ultrapure water were placed on the silylated fused-silica and silicon wafer surfaces to determine the contact angles using the sessile drop technique. A Ramé-Hart goniometer was used for the measurements. Advancing and receding angles were measured by changing the volume of the ultrapure water drops by means of a Microliter syringe. For a given surface, five different measurements were conducted and averaged.

\section{RESULTS AND DISCUSSION}

\section{a) OTS Monolayer on Silica}

The AFM images of the fused-silica plates used in the present work showed grains of $\sim 100 \mathrm{~nm}$ in dimension, and the distinct boundaries between them made it difficult to see the surfactant molecules adsorbed on the surface [30]. Further, some of the images showed lines representing scratches created during polishing. Therefore, we used silicon wafers for imaging OTS-coated surfaces. Silicon wafers readily oxidize to form silicon dioxide [31]; therefore, the information derived using the wafer may be relevant to the surface force data obtained using the silylated silica surfaces. 


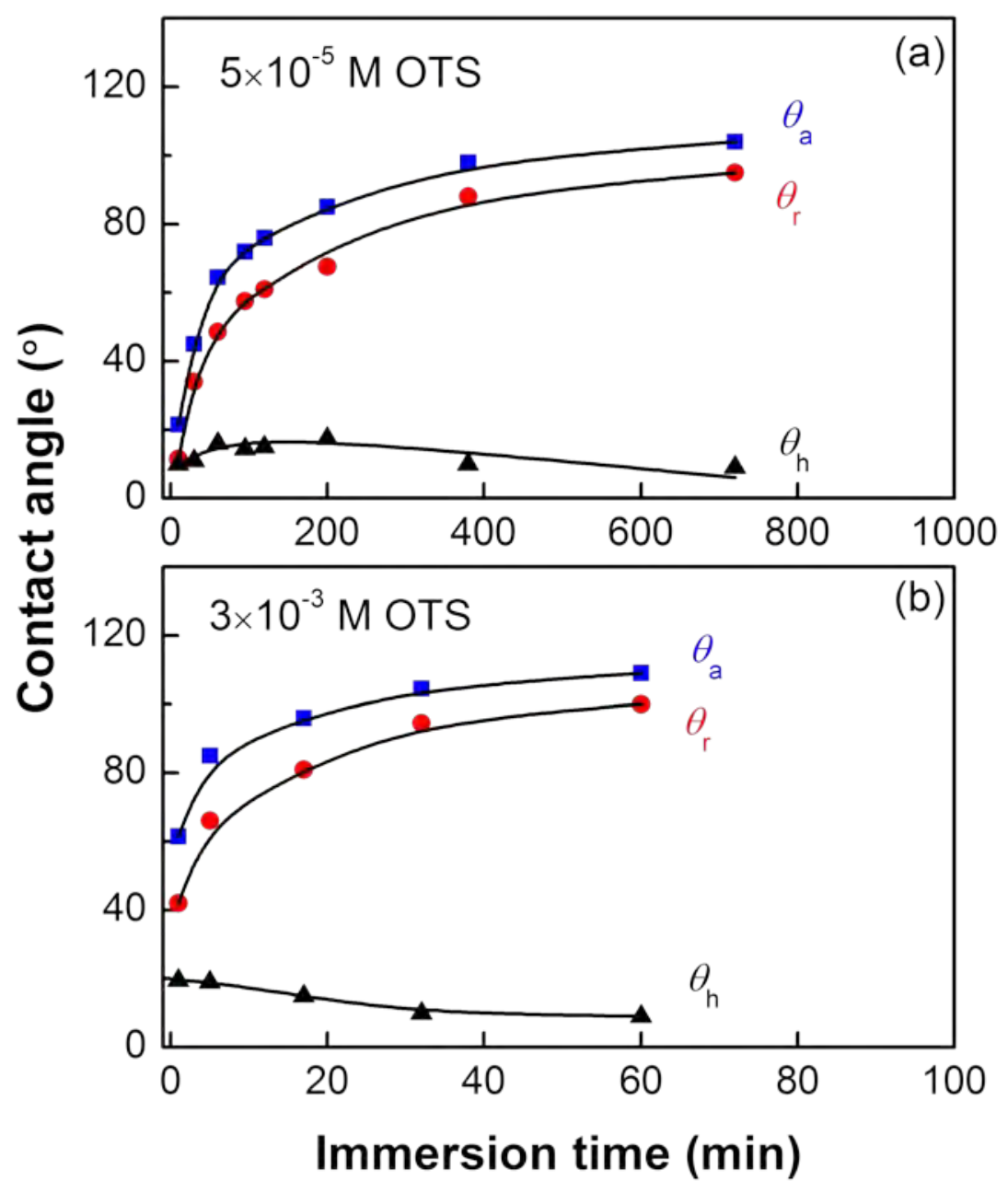

Fig. 1. Effects of immersion time on the water contact angles of silicon wafer in a) $5 \times 10^{-5} \mathrm{M}$ and b) $3 \times 10^{-3}$ M OTS-in-toluene solutions. $\theta_{\mathrm{a}}$ and $\theta_{\mathrm{r}}$ represent advancing and receding angles. The contact angle hysteresis $\left(\theta_{\mathrm{h}}\right)$ decreases with time indicating formation of smoother OTS monolayers. OTS adsorbs much faster at the higher concentration.

Figure 1 shows the water contact angles of ultrapure water placed on the surfaces of silicon wafers treated with OTS-in-toluene solutions at $5 \times 10^{-5}$ and $3 \times 10^{-3} \mathrm{M}$. Table 1 shows the data obtained at the lower concentration. As anticipated, both the advancing $\left(\theta_{\mathrm{a}}\right)$ and receding angles $\left(\theta_{\mathrm{r}}\right)$ increased with contact time, and the kinetics of adsorption was much faster at the higher concentration. Also shown is the contact angle hysteresis at the two different concentrations. In both cases, the hysteresis decreased with increasing contact time, indicating that as the adsorption density of OTS increased, the adsorption layers became smoother. 
Table 1. Water Contact Angles and Surface Roughness of the Silicon Wafer Surfaces Treated in a $5 \times 10^{-5}$ M OTS Solution at Different Contact Times

\begin{tabular}{|c|c|c|c|c|c|}
\hline \multirow{2}{*}{$\begin{array}{c}\text { Contact Time } \\
(\mathrm{min})\end{array}$} & \multicolumn{3}{|c|}{ Contact Angle $\left(^{\circ}\right)$} & \multirow{2}{*}{$\begin{array}{c}\text { Contact Angle } \\
\text { Hysteresis }\left(^{\circ}\right)\end{array}$} & $\begin{array}{c}\text { Surface } \\
\text { Roughness } \\
(\mathrm{nm})\end{array}$ \\
\cline { 2 - 4 } & $\theta_{\mathrm{e}}$ & $\theta_{\mathrm{a}}$ & $\theta_{\mathrm{r}}$ & 10.0 & 0.18 \\
\hline 20 & 30 & 35 & 25 & 17.5 & 0.46 \\
\hline 200 & 80 & 85 & 67.5 & 10.0 & 0.22 \\
\hline 380 & 93 & 98 & 88 & 9.0 & 0.11 \\
\hline 720 & 101 & 104 & 95 & & \\
\hline
\end{tabular}

Figure 2 shows the AFM images of the silicon wafers contacted with $5 \times 10^{-5}$ M OTS-intoluene solutions for different time periods to obtain equilibrium contact angles $\left(\theta_{\mathrm{e}}\right)$ of $30^{\circ}$, $80^{\circ}, 93^{\circ}$ and $101^{\circ}$. Untreated surface exhibited an equilibrium contact angle of $4^{\circ}$. The images were captured in air with a scan area of $1 \times 1 \mu \mathrm{m}$ and a vertical color scale of $3 \mathrm{~nm}$. The vertical profiles shown under the images represent the changes in height along the horizontal lines drawn in the images. The bare silicon surface with $\theta_{\mathrm{e}}=4^{\circ}$ showed RMS roughness of $0.11 \mathrm{~nm}$, demonstrating that the silicon wafer surface was smooth (Figure 2a).

After a short contact time of $20 \mathrm{~min}, \theta_{\mathrm{e}}$ became $30^{\circ}$ (Figure $2 \mathrm{~b}$ ) and small spots of 20 $\mu \mathrm{m}$ diameter appeared. These spots indicated that OTS adsorbs in clusters due to lateral interactions. As $\theta_{\mathrm{e}}$ increased to $80^{\circ}$ (Figure 2c), the clusters became more abundant, larger (40-100 $\mathrm{nm})$, and taller as shown in the vertical profiles. It appears that as $\theta_{\mathrm{e}}$ increased to $93^{\circ}$ (Figure $2 \mathrm{~d}$ ) and then to $101^{\circ}$ (Figure $2 \mathrm{e}$ ) with increasing contact time, the clusters coalesced and became larger, eventually creating a smooth monolayer.
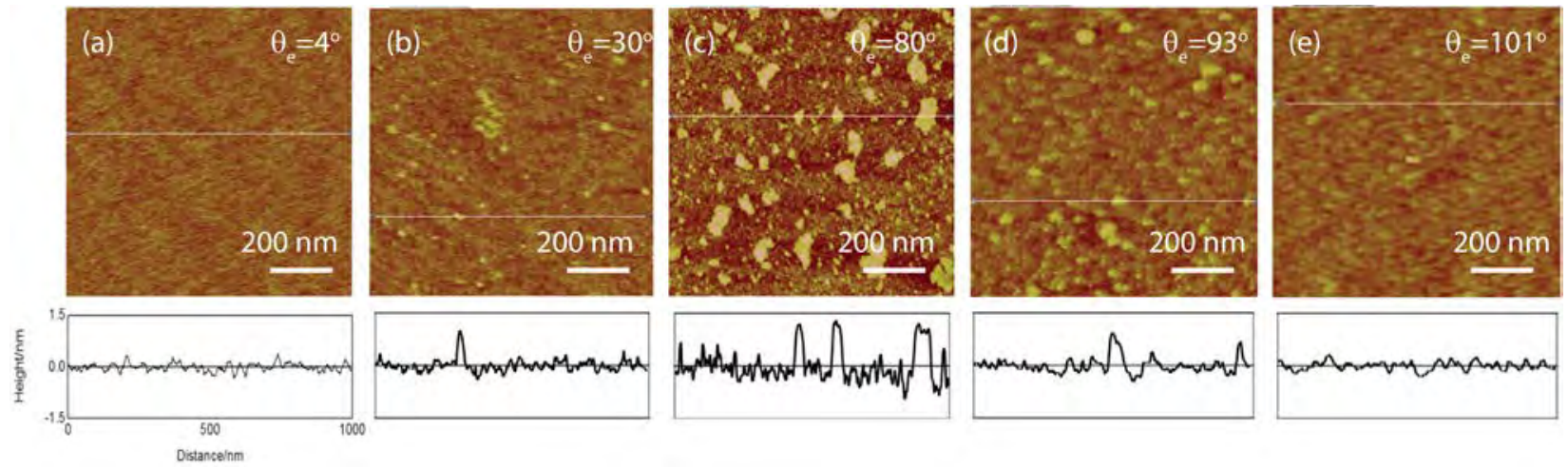

Fig. 2. Contact mode $1 \times 1 \mu \mathrm{m}$ AFM images of the silicon wafers of different equilibrium contact angles. a) bare surface with $\theta_{\mathrm{e}}=4^{\circ}$, b-e) surfaces with different $\theta_{\mathrm{e}}$ values as obtained by varying the immersion times in a $5 \times 10^{-5} \mathrm{M}$ OTS solution. Height profiles given below each image were obtained along the white line across the image. OTS adsorbs in patches, which coalesce to obtain a smooth monolayer. 
The series of AFM images presented in Figure 2 shows that the monolayer coating has a thickness of approximately $2.5 \mathrm{~nm}$, as was also reported by other investigators [32, 33]. This value is close to the length of 18 carbon alkane chain, indicating that the OTS coatings obtained in the present work were monomolecular layers. It is believed that the key to forming a smooth monolayer was to ensure that the solvent (toluene) was completely dehydrated before use. The adsorption layers formed in the presence of water produced much thicker ( 200 nm) layers [34], possibly indicating the formation of polymeric silanes.

\section{b) Surface Forces and Contact Angle}

In Figure 3, surface forces $(F)$ measured between silylated silica plates and spheres normalized by the radii $(R)$ of the spheres are plotted $v s$. thickness $(h)$ of the TLF of water formed between the two macroscopic surfaces. In each experiment, a pair of silica plate and sphere was hydrophobized in an OTS solution $\left(5 \times 10^{-5} \mathrm{M}\right.$ in toluene) simultaneously, so that the two surfaces have identical contact angles. Figure 3 shows the results obtained with silica

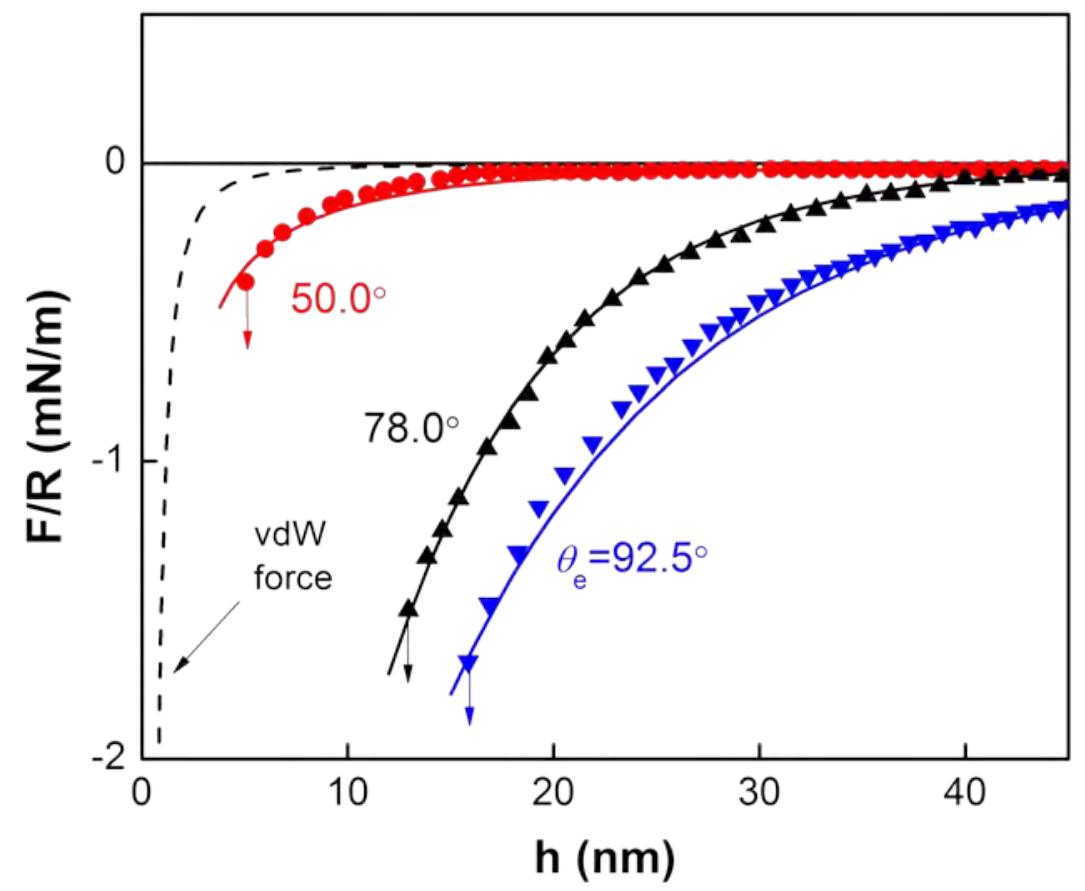

Fig. 3. Surface forces (F) normalized by the radius (R) of spheres $v s$. the closest separation distance (h). The surfaces were treated in a $5 \times 10^{-5} \mathrm{M}$ OTS solution for different times to obtain different contact angles. Solid lines represent the extended DLVO plots with $\psi_{1}=-40 \mathrm{mV}$ and $A_{131}=0.8 \times 10^{-20} \mathrm{~J}$. The double-layer and van der Waals forces are negligibly small particularly at longer separations. Thus, the measured forces practically represent the hydrophobic forces, which are shown to increase with $\theta_{\mathrm{e}}$. The arrows represent the separations at which two surfaces jumped into contact. 
surfaces of $\theta_{\mathrm{e}}=50.0,78$ and $92.5^{\circ}$. The contact angles were varied by controlling the immersion time in the OTS solution. As shown, all of the force curves exhibited net attractive forces stronger than the van der Waals force with a Hamaker constant $\left(A_{131}\right)$ of $0.8 \times 10^{-20} \mathrm{~J}$ [35]. Note here that the force curve obtained at $\theta_{\mathrm{e}}=92.5^{\circ}$ were smooth, showing no evidence for bubbles or vapor cavities affecting the measurement. Cavitation is thermodynamically possible at contact angles above $90^{\circ}$. If bubbles and/or cavities were the causes for the non-DLVO attractive forces [11], the forces measured at $\theta_{\mathrm{e}}<90^{\circ}$ should be equal to the van der Waals force. The experimental results were contrary as shown in Figure 3. Further, the attractive forces increased with increasing contact angle, indicating that the non-DLVO forces shown in Figure 3 are related to the hydrophobicity of the surfaces used in the measurements.

The arrow on each curve represents the distance at which a sphere jumps into contact with a plate due to the presence of the attractive hydrophobic force in the TLF confined between the macroscopic hydrophobic surfaces. The solid lines in Figure 3 represent the surface forces calculated using the extended DLVO theory, which includes contributions from the van der Waals force, electrical double-layer force, and the hydrophobic force. The hydrophobic force is represented by the single-exponential form,

$$
\frac{F}{R}=-C \exp \left(-\frac{h}{D}\right)
$$

where $C$ and $D$ are fitting parameters. The double-layer force was calculated using the surface potential of $-40 \mathrm{mV}$ for the OTS-coated silica surfaces, which was assumed to be the same as the $\zeta$-potential measured in the present work using the Zetasizer Nano-ZS, Malvern. We found that the $\zeta$-potentials did not change significantly with the OTS concentrations used in the present work for hydrophobization. It was found that contributions from van der Waals and double-layer forces were minimal as compared to those from hydrophobic forces.

\section{c) Effect of Temperature on Hydrophobic Force}

Figures 4 and 5 show the surface forces measured with OTS-coated silica surfaces in ultrapure water at temperatures in the range of 10 to $40^{\circ} \mathrm{C}$. The objective of these measurements was to determine the thermodynamic functions of the macroscopic hydrophobic interactions, which may help better understand the origin(s) of the hydrophobic force. It has been shown previously that hydrophobic interactions entail decrease in the excess entropy of the TLFs, which lead to a conclusion that the water structure becomes more ordered in thin films than in thick films.

Parker et al. [11] reported the measurement of attractive forces between silica surfaces hydrophobized with (tridecafluoro,1,1,2,2,-tetrahydroxyoctyl)dimethylchorosilane at $41^{\circ} \mathrm{C}$ and room temperature $\left(22^{\circ} \mathrm{C}\right)$. They found that the forces became stronger at the higher temperature, which was contrary to what was observed in the present work. The fluorinated surfaces exhibited advancing and receding contact angles of $110^{\circ}$ and $90^{\circ}$, respectively, and the surfaces showed no crystallinity. The force curve obtained at the room temperature showed significant steps, which became stronger at the higher temperature. Therefore, the 


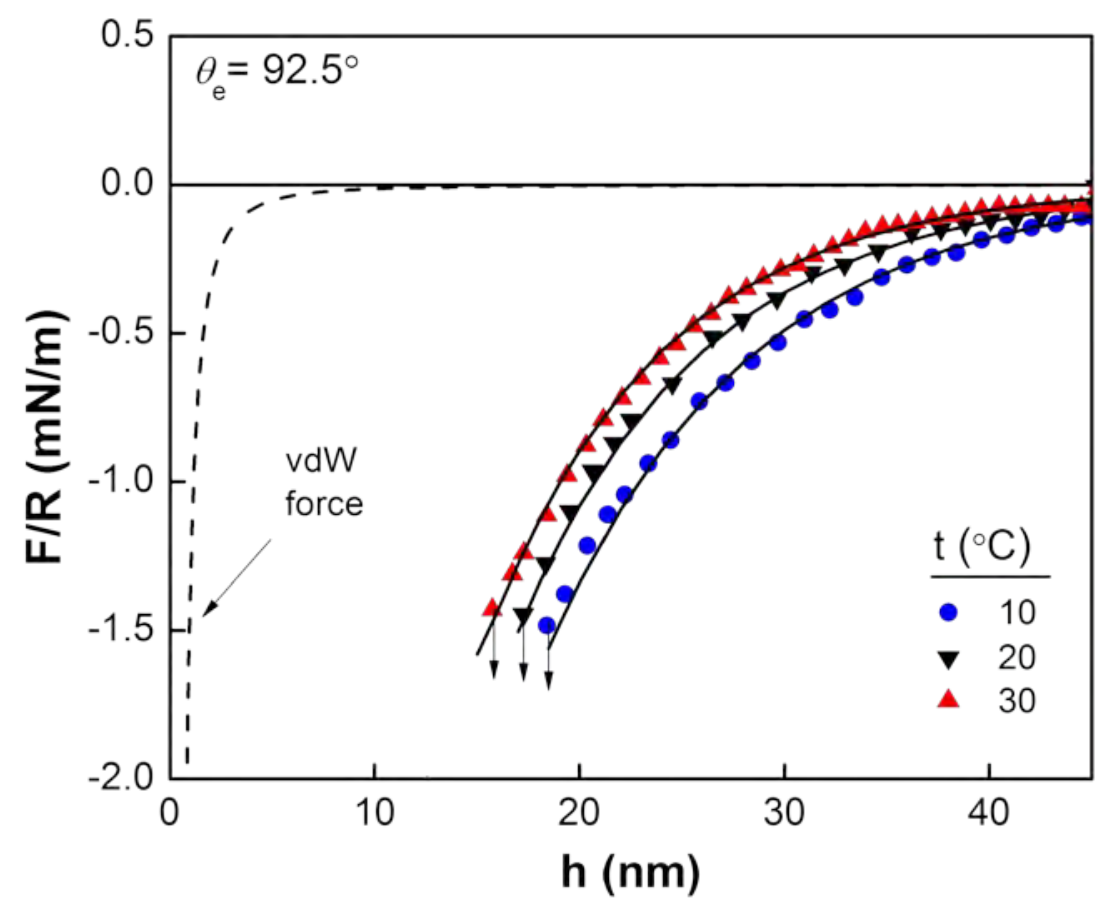

Fig. 4. Surface forces (F) measured at different temperatures in the range of 10 to $30^{\circ} \mathrm{C}$ and normalized by the radius of sphere (R) $v s$. separations (h). The silica surfaces were treated in a $5 \times 10^{-5}$ M OTS solution to obtain $\theta_{\mathrm{e}}=92.5^{\circ}$. The measured forces became stronger and longer-ranged with decreasing temperature of the medium.

authors attributed the increase in attractive force with temperature to the growth of bubble size, which should promote the bridging mechanism.

In the present work, two sets of force measurements were conducted, one with OTScoated silica surfaces of $\theta_{\mathrm{e}}=92.5^{\circ}$ and $\theta_{\mathrm{e}}=78.0^{\circ}$. If the bridging mechanism played a role, the result obtained at the higher contact angle would have been similar to what has been reported by Parker et al. [11]. The results were diametrically opposite. First, the force curves obtained at $\theta_{\mathrm{e}}=92.5^{\circ}$ were smooth, as shown in Figure 4, providing no evidence for bridging nano-bubbles affecting the force measurement. Second, the measured forces decreased with increasing temperature. Further, the hydrophobic forces increased with increasing surface hydrophobicity. It appears, therefore, that the long-range attractions observed in the present work were due to hydrophobic interactions rather than to bridging bubbles.

The results obtained with surfaces with $\theta_{\mathrm{e}}=78.0^{\circ}$ are presented in Figure 5. Again, the force curves were smooth without visible steps, and yet the forces were substantially stronger than the van der Waals force with a Hamaker constant of $0.8 \times 10^{-20} \mathrm{~J}$. It may be of interest to note here that the surface force measurements began at $10^{\circ} \mathrm{C}$, and then stepped up to $20^{\circ} \mathrm{C}$, $30^{\circ} \mathrm{C}$, and $40^{\circ} \mathrm{C}$. After the last set of measurements, the temperature was brought down to 
$20^{\circ} \mathrm{C}$ and the measurements were repeated. The results were reproducible indicating that the OTS-coated surface was stable in the range of temperatures where the measurements were conducted. It has actually been reported that OTS-coated surfaces are stable up to $175^{\circ} \mathrm{C}$ as measured by water contact angle [24].

Note that Figure 4 shows the data obtain at temperatures at 10,20 and $30^{\circ} \mathrm{C}$ only. At $40^{\circ} \mathrm{C}$, we saw some bubbles nucleating on the OTS-coated silica plate with $\theta_{\mathrm{e}}=92.5^{\circ}$, and the results were not very reproducible. Nevertheless, the force curves were smooth and showed no discontinuities or steps. In view of the difficulties with reproducibility, we decided not to include the results obtained at $40^{\circ} \mathrm{C}$ in the data set presented in Figure 4. We did not have this problem with the OTS-coated silica surfaces with $\theta_{\mathrm{e}}=78.0^{\circ}$ as shown in Figure 5. Obviously, bubbles can more readily nucleate on the more hydrophobic surface, and the surface force measurements become irreproducible in the presence of bubbles.

The solid lines of Figures 4 and 5 represent the extended DLVO theory, with the hydrophobic forces represented by Eq. [1]. The $C$ and $D$ parameters used to fit the experimental data are plotted in Figure 6. As shown, both of these parameters decrease

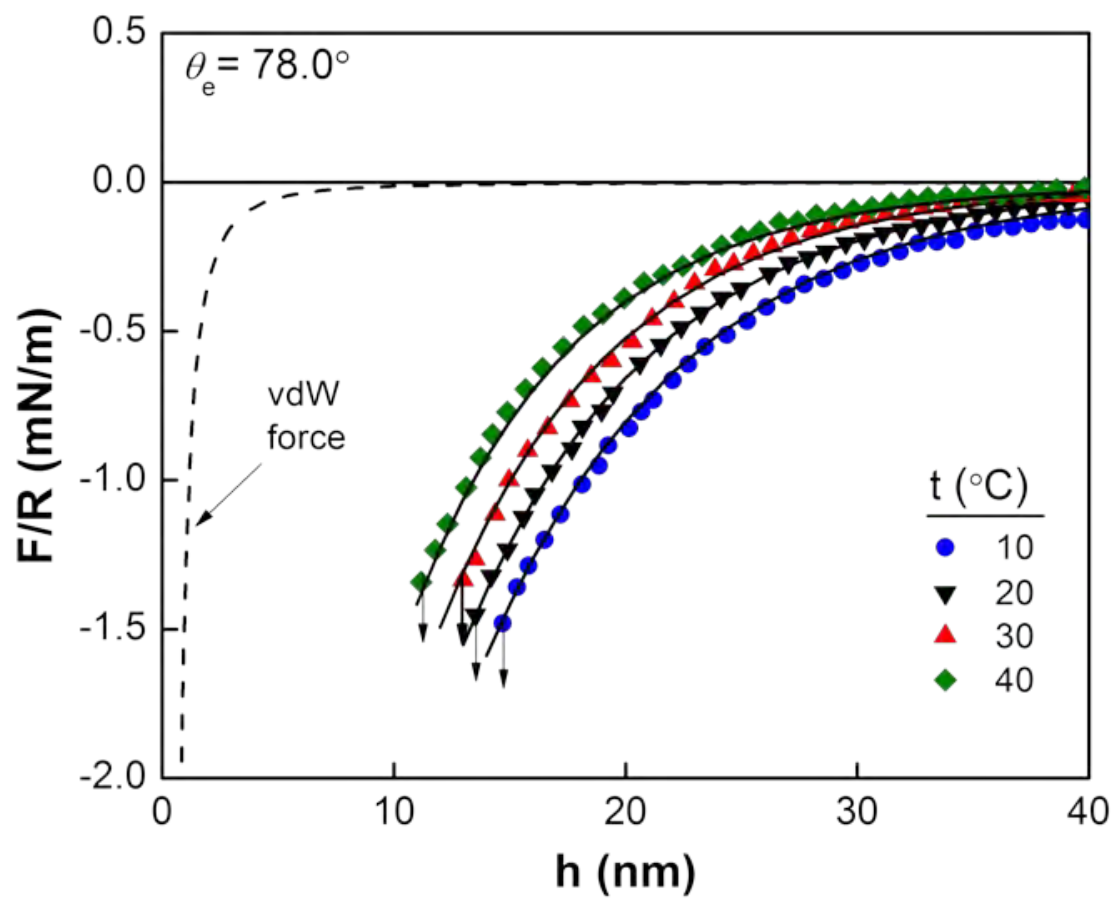

Fig. 5. Surface forces (F) measured at different temperatures in the range of 10 to $40^{\circ} \mathrm{C}$ and normalized by the radius of sphere (R) $v s$. separations (h). The silica surfaces were treated in a $5 \times 10^{-5} \mathrm{M}$ OTS solution to obtain $\theta_{\mathrm{e}}=78^{\circ}$. The measured forces became stronger and longer-ranged with decreasing temperature of the medium. 

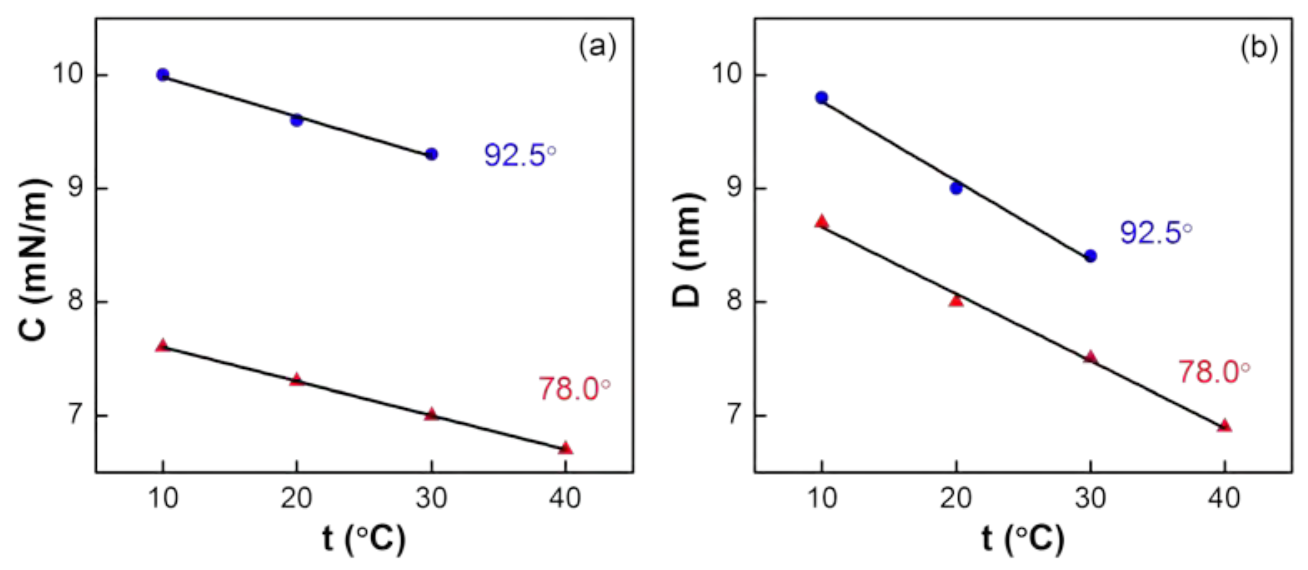

Fig. 6. a) $C v s . \mathrm{t}$ and b) $D v s$, $\mathrm{t}$ plots. The $\mathrm{C}$ and $\mathrm{D}$ parameters of the hydrophobic force represented by Eq. [1] were obtained by fitting the experimental data presented in Figure 4 and 5 to the extended DLVO theory with $\psi_{1}=-40 \mathrm{mV}$ and $A_{131}=$ $0.8 \times 10^{-20} \mathrm{~J}$.

linearly with increasing temperatures $(T)$, indicating that hydrophobic forces decrease with increasing temperature. The slopes of the $C v s$. $T$ plots obtained at $\theta_{\mathrm{e}}=92.5^{\circ}$ and $78.0^{\circ}$ are about the same. The slopes of the $D$ vs. $T$ plots are also about the same, although the slope obtained at $\theta_{\mathrm{e}}=92.5^{\circ}$ tends to be slightly steeper than that obtained at $\theta_{\mathrm{e}}=78.0^{\circ}$. The results presented in Figure 6 are similar to those reported by Wang et al. [1] with gold surfaces thiolated with $\mathrm{C}_{2} \mathrm{SH}$ and $\mathrm{C}_{16} \mathrm{SH}$. These results are consistent with those reported by other investigators. Israelachvili and Pashley [36] showed that the hydrophobic forces measured between mica surfaces coated with CTAB by self-assembly decreased as the temperature was increased from 21 to $50^{\circ} \mathrm{C}$. Tsao et al. [37] also showed that the attractive forces measured between mica surfaces hydrophobized by L-B deposited double-chain cationic surfactants decreased as the temperature was raised from 25 to $50^{\circ} \mathrm{C}$.

Using the Derjaguin approximation [20], one can relate the surface force $(F / R)$ measured at a separation distance $h$ to the Gibbs free energy change per unit area $\left(\Delta G^{\mathrm{f}}\right)$ as follows,

$$
\frac{F}{R}=2 \pi \Delta G^{\mathrm{f}}
$$

where

$$
\Delta G^{\mathrm{f}}=-\int_{\infty}^{h} \Pi(h) d h
$$

As shown in Eq. [3], the free energy change is the same in magnitude as the work done in thinning an infinitely-thick plane-parallel film to a finite thickness $h$ against the disjoining pressure $\Pi(h)$, which in turn is a function of film thickness. For a water film confined between two hydrophobic surfaces, the disjoining pressure should include contributions from the hydrophobic force as discussed above. 
The partial differential of $\Delta G^{\mathrm{f}}$ with respect to $T$ at a constant pressure and film thickness allows us to calculate the change in the excess film entropy for moving a planeparallel film from an infinite thickness to an $h$ as follows,

$$
\left(\frac{\partial \Delta G^{\mathrm{f}}}{\partial T}\right)_{\mathrm{p}, \mathrm{h}}=\left(\frac{\partial(F / 2 \pi R)}{\partial T}\right)_{\mathrm{p}, \mathrm{h}}=-\Delta S^{\mathrm{f}}
$$

in which $\Delta S^{\mathrm{f}}$ is the change in excess entropy per unit area of the liquid film confined between two hydrophobic surfaces.

From Eqs. [1], [2] and [4], one obtains,

$$
\Delta S^{\mathrm{f}}=-\frac{F}{2 \pi R}\left(\frac{d \ln C}{d T}+\frac{h}{D} \frac{d \ln D}{d T}\right)
$$

which can be used to calculate the changes in excess film entropy $\left(\Delta S^{\mathrm{f}}\right)$ at different film thicknesses using the surface force data, such as $F / R, D$, and the temperature coefficients for the $C$ and $D$ parameters given in Figure 6.

Figure 7 gives the values of $\Delta S^{\mathrm{f}}$ in this manner from the surface force data obtained with OTS-coated silica surfaces with $\theta_{\mathrm{e}}=78.0^{\circ}$ and $92.5^{\circ}$. The entropy change becomes increasingly negative with decreasing $h$, indicating that the entropy $\left(S^{f}\right)$ of the TLF confined between two hydrophobic surfaces decrease with decreasing film thickness. As has been suggested previously [1], the low entropy may be due to structuring of the water molecules in the vicinity of hydrophobic surfaces. The vicinal water molecules must be of high free energy state as they cannot form $H$-bonds to the hydrophobic surface. This is akin to the case for the non- $H$-bonded water molecules at the air/water and hydrocarbon-water interfaces being responsible for high interfacial tensions [38]. One way to expend the excess free energy would be to form structures, in which the 'frustrated' vicinal water molecules form stronger $H$-bonds with neighbors by taking advantage of the cooperative bonding mechanism first recognized by Frank and Wen [39] and used to explain the mechanism of hydrophobic hydration. It has been shown that the $\mathrm{O}-\mathrm{O}$ distance between water molecules decreases with increasing number of water molecules in a structure (or cluster) [40]. The structure can be coplanar or concave (or clathrate-like) depending upon the temperature, pressure, and dissolved species [41, 42]. It may be reasonable to assume that the clusters become larger and more abundant when water is confined in a TLF formed between very hydrophobic surfaces. Recent spectroscopic studies conducted in bulk water showed that water molecules can form low-density liquid (LDL) species more readily than previously thought to be possible[43-46]. These clusters may be collectively referred to as 'partial clathrates.' Clathrates are found in supercooled water or at high pressures and lower temperatures particularly in the presence of dissolved hydrophobic solutes [41, 42]. 


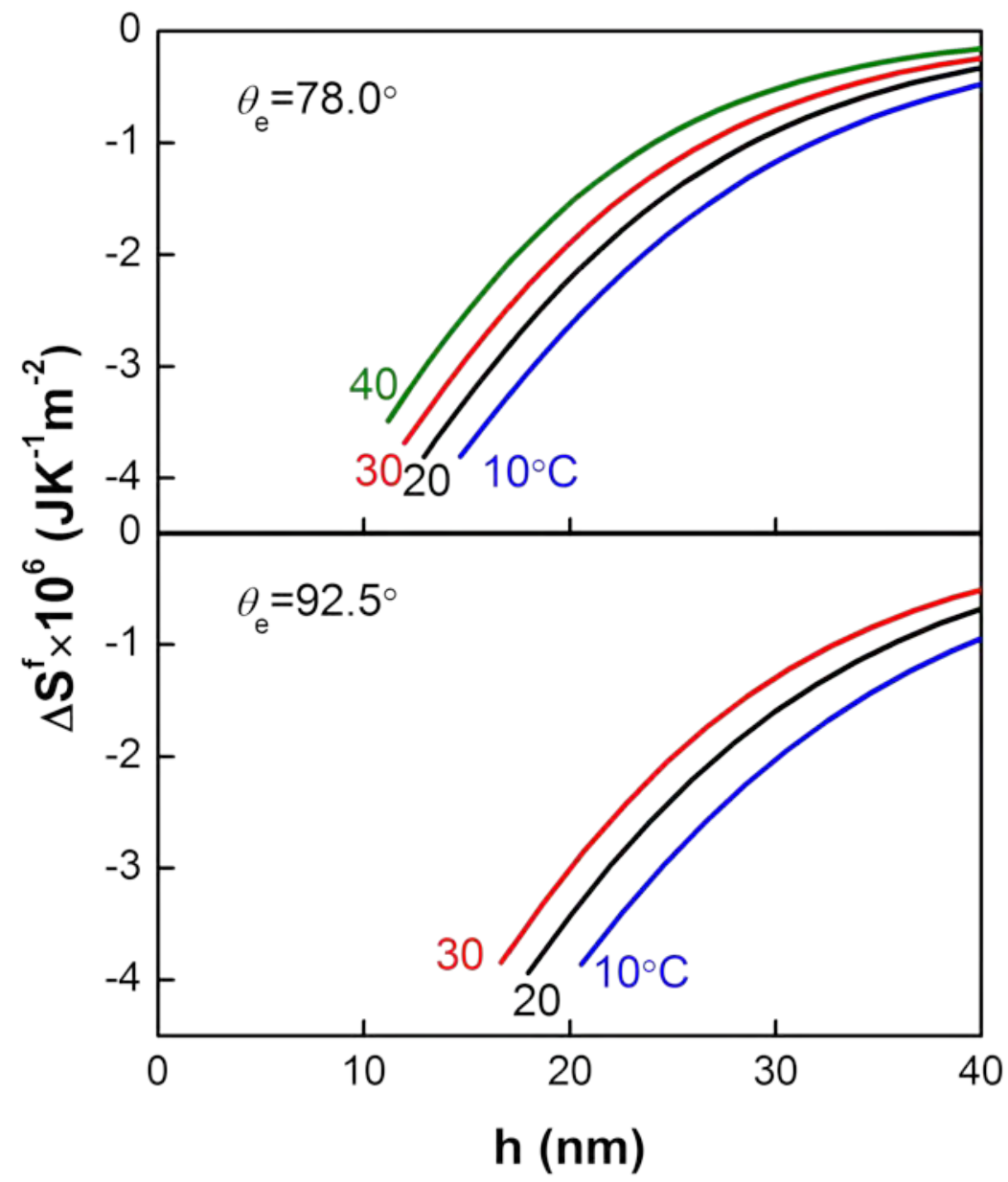

Fig. 7. Changes in excess film entropy $\left(\Delta S^{f}\right)$ in the thin films of water between OTScoated silica surfaces with contact angles of $78.0^{\circ}$ and $92.5^{\circ}$ at different medium temperatures.

Figure 7 shows also that $\Delta S^{\mathrm{f}}$ becomes more negative with increasing hydrophobicity and decreasing temperature. The former may be due to the likelihood that the number of non- $H$ bonded water molecules increases with increasing surface hydrophobicity, while the latter can be attributed to the low kinetic energies of the water molecules at a low temperature and hence increased propensity to form clusters.

One can readily obtain $\Delta G^{\mathrm{f}}$ from the surface force data using the Derjaguin approximation (Eq. [2]). From the values of $\Delta G^{\mathrm{f}}$ and $\Delta S^{\mathrm{f}}$, the excess film enthalpy per unit area $\left(\Delta H^{f}\right)$ can be determined as follows,

$$
\Delta H^{\mathrm{f}}=\Delta G^{\mathrm{f}}+T \Delta S^{\mathrm{f}}
$$


Figure 8 shows the changes in $\Delta H^{\mathrm{f}}$ determined in this manner. As shown, the enthalpy change associated in forming $H$-bonded structures becomes more negative with decreasing $h$ and $T$. This finding suggests that both the number and size of the clusters increase with decreasing film thickness. Further, the strength and life time of the clusters may also increase as film becomes thinner. Figure 8 shows also that the enthalpy change becomes more negative as the confining surfaces become more hydrophobic and the temperature is lowered. As has been the case with the entropy change, the former may be related to the increased number of non$H$-bonded water molecules, while the latter to decreased kinetic energies of water molecules.

The values of $\Delta G^{\mathrm{f}}, T \Delta S^{\mathrm{f}}$ and $\Delta H^{\mathrm{f}}$ determined in the manner described above are presented in Figure 9. These values represent the changes in the thermodynamic functions involved in

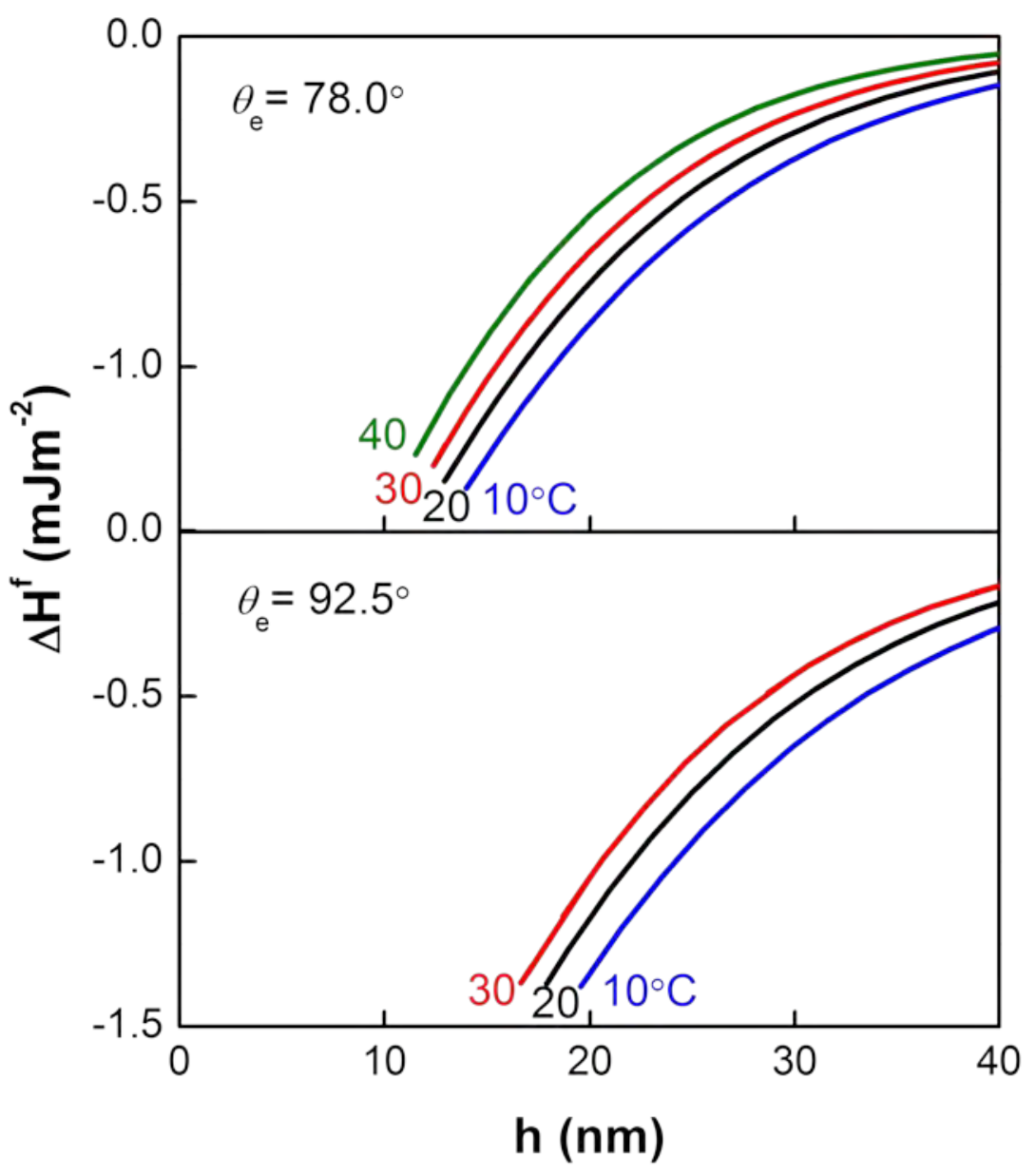

Fig. 8. Changes in excess film enthalpy $\left(\Delta H^{\mathrm{f}}\right)$ in the thin films of water between OTScoated silica surfaces with contact angles of $78.0^{\circ}$ and $92.5^{\circ}$ at different medium temperatures. 
the macroscopic hydrophobic interactions between the OTS-coated silica surfaces with $\theta_{\mathrm{e}}=$ $78.0^{\circ}$ and $92.5^{\circ} \mathrm{C}$ at $20^{\circ} \mathrm{C}$. Note that at both contact angles $\left|\Delta H^{\mathrm{f}}\right|>\left|T \Delta S^{\mathrm{f}}\right|$, which is a necessary condition for the hydrophobic force to be attractive when both enthalpy and entropy changes are negative. The enthalpy term represents the free energy gained in building the structures, while the entropy $\left(T \Delta S^{\mathrm{f}}\right)$ term representing the thermodynamic cost for building structures. As a result of the enthalpy-entropy tradeoff, the hydrophobic forces in general are weak and are difficult to be measured. As noted by Eriksson et al [19], the excess free energy per molecule related to the long-range attractions is in the range of $10^{-5}$ to $10^{-3}$ $\mathrm{kT}$, while the energy of a hydrogen bond is about $7 \mathrm{kT}$. Note also that $\Delta G^{\mathrm{f}}$ is more negative at the higher contact angle, which can be attributed to more negative $\Delta H^{\mathrm{f}}$.

The condition that $\left|\Delta H^{\mathrm{f}}\right|$ be larger than $\left|T \Delta S^{\mathrm{f}}\right|$ for macroscopic hydrophobic interactions

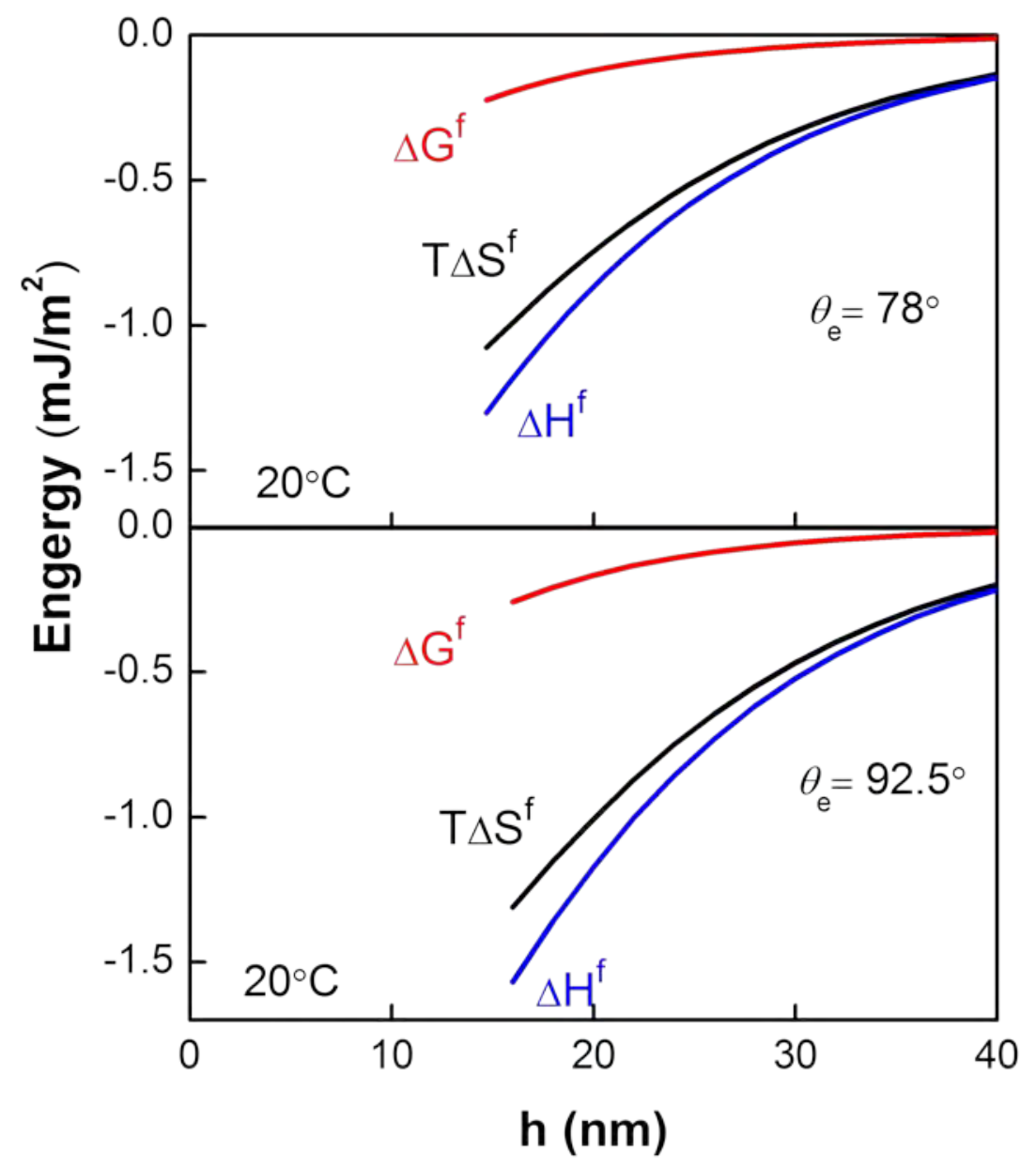

Fig. 9. Changes in the excess thermodynamic properties for the thin liquid films between OTS-coated silica surfaces with $\theta_{\mathrm{e}}=78.0^{\circ}$ and $92.5^{\circ}$ at $20^{\circ} \mathrm{C}$. 
is opposite to what is generally known for molecular-scale hydrophobic interactions or hydrophobic effect. For the latter, it is necessary that $\left|\Delta H^{\mathrm{f}}\right|<\left|T \Delta S^{\mathrm{f}}\right|$ for two hydrophobic molecules to attract each other as in self-assembly. It is generally accepted that the inequality arises from the entropy increase associated with the release of the water molecules surrounding hydrophobic moieties of high curvatures. The basic difference between the macroscopic- and molecular-scale hydrophobic interactions is due to the differences in curvatures of the surfaces involved, as discussed by Chandler [42].

The authors of this communication showed recently that long-range attractions are also observed in short-chain alcohols such as methanol, enthanol, and butabol-all well-known $\mathrm{H}$ bonding liquids [47]. It has been suggested that the origin of the long-range attractions observed in these short-chain alcohols is the same as the hydrophobic forces observed in water, i.e., structure formation. It was found also that the long-range attractions become stronger as the confining surfaces become more hydrophobic. The same has been observed in the present work with water as $H$-bonding liquid. It may be suggested, therefore, that hydrophobic force is the consequence of an $H$-bonding liquid striving to minimize free energy when it cannot form $H$-bonds to confining surfaces. Conversely, a key to increasing hydrophobic force is to eliminate $H$-bond sites from the confining surfaces.

There have been long-standing controversies concerning the origin of the hydrophobic force since Israelachvili and Pashley [4] published a seminal paper in 1982. It is hoped that the results presented in this communication have provided a basis for the hydrophobic force be recognized as a thermodynamic force rather than an artifact due to bubbles or cavities.

\section{ACKNOWLEDGEMENT}

The authors acknowledge the financial support from the U.S. Department of Energy (DE-FC26-05NT42457), and Professor Jan Christer Eriksson for helpful discussions and encouragement.

\section{REFERENCES}

[1] J. Wang, R.-H. Yoon, J.C. Eriksson, J. Colloid Interface Sci. 364 (2011) 257.

[2] Z. Xu, R.-H. Yoon, J. Colloid Interface Sci. 132 (1989) 532.

[3] Z. Xu, R.-H. Yoon, J. Colloid Interface Sci. 134 (1990) 427.

[4] J. Israelachvili, R. Pashley, Nature 300 (1982) 341.

[5] H.K. Christenson, P.M. Claesson, Adv. Colloid Interface Sci. 91 (2001) 391.

[6] E.E. Meyer, K.J. Rosenberg, J. Israelachvili, Proc. Natl. Acad. Sci. U. S. A. 103 (2006) 15739.

[7] R.H. Yoon, S.A. Ravishankar, J. Colloid Interface Sci. 166 (1994) 215.

[8] Y.I. Rabinovich, D.A. Guzonas, R.H. Yoon, J. Colloid Interface Sci. 155 (1993) 221.

[9] Y.I. Rabinovich, D.A. Guzonas, R.H. Yoon, Langmuir 9 (1993) 1168.

[10] R.H. Yoon, S.A. Ravishankar, J. Colloid Interface Sci. 179 (1996) 391.

[11] J.L. Parker, P.M. Claesson, P. Attard, J. Phys. Chem. 98 (1994) 8468.

[12] J.W.G. Tyrrell, P. Attard, Phys. Rev. Lett. 87 (2001) 176104.

[13] M. Sakamoto, Y. Kanda, M. Miyahara, K. Higashitani, Langmuir 18 (2002) 5713. 
[14] E.E. Meyer, Q. Lin, J.N. Israelachvili, Langmuir 21 (2005) 256.

[15] M.U. Hammer, T.H. Anderson, A. Chaimovich, M.S. Shell, J. Israelachvili, Faraday Discuss. 146 (2010) 299.

[16] B.V. Derjaguin, M.M. Kusakov, Izv. Akad. Nauk SSSR, Ser. Khim. 5 (1936) 741.

[17] B.V. Derjaguin, N.V. Churaev, Langmuir 3 (1987) 607.

[18] N.V. Churaev, B.V. Derjaguin, J. Colloid Interface Sci. 103 (1985) 542.

[19] J.C. Eriksson, S. Ljunggren, P.M. Claesson, J. Chem. Soc., Faraday Trans. II 85 (1989) 163.

[20] B.V. Derjaguin, Kolloid. Zeits. 69 (1934) 155.

[21] Y. Li, J. Huang, R.T. McIver, J.C. Hemminger, J. Am. Chem. Soc. 114 (1992) 2428.

[22] M.H. Schoenfisch, J.E. Pemberton, J. Am. Chem. Soc. 120 (1998) 4502.

[23] M. Dasog, R.W.J. Scott, Langmuir 23 (2007) 3381.

[24] W.R. Ashurst, C. Yau, C. Carraro, R. Maboudian, M.T. Dugger, J Microelectromech S 10 (2001) 41.

[25] Y.I. Rabinovich, R.H. Yoon, Langmuir 10 (1994) 1903.

[26] R.H. Yoon, D.H. Flinn, Y.I. Rabinovich, J. Colloid Interface Sci. 185 (1997) 363.

[27] N. Ishida, N. Kinoshita, M. Miyahara, K. Higashitani, J. Colloid Interface Sci. 216 (1999) 387.

[28] N. Ishida, M. Sakamoto, M. Miyahara, K. Higashitani, Langmuir 16 (2000) 5681.

[29] J.P. Cleveland, S. Manne, D. Bocek, P.K. Hansma, Rev. Sci. Instrum. 64 (1993) 403.

[30] D.H. Flinn, D.A. Guzonas, R.H. Yoon, Colloid Surf., A 87 (1994) 163.

[31] A.H. Carim, M.M. Dovek, C.F. Quate, R. Sinclair, C. Vorst, Science 237 (1987) 630.

[32] N. Tillman, A. Ulman, J.S. Schildkraut, T.L. Penner, J. Am. Chem. Soc. 110 (1988) 6136.

[33] M. Mao, J.H. Zhang, R.H. Yoon, W.A. Ducker, Langmuir 20 (2004) 4310.

[34] M.J. Wang, K.M. Liechti, Q. Wang, J.M. White, Langmuir 21 (2005) 1848.

[35] J.H. Zhang, R.H. Yoon, J.C. Eriksson, Colloid Surf., A 300 (2007) 335.

[36] J.N. Israelachvili, R.M. Pashley, J. Colloid Interface Sci. 98 (1984) 500.

[37] Y.H. Tsao, S.X. Yang, D.F. Evans, H. Wennerstrom, Langmuir 7 (1991) 3154.

[38] Q. Du, E. Freysz, Y.R. Shen, Science 264 (1994) 826.

[39] H.S. Frank, W.-Y. Wen, Discuss. Faraday Soc. 24 (1957) 133.

[40] K. Liu, J.D. Cruzan, R.J. Saykally, Science 271 (1996) 929.

[41] F.H. Stillinger, Science 209 (1980) 451.

[42] D. Chandler, Nature 437 (2005) 640.

[43] F. Mallamace, C. Branca, M. Broccio, C. Corsaro, C.Y. Mou, S.H. Chen, Proc. Natl. Acad. Sci. U. S. A. 104 (2007) 18387.

[44] F. Mallamace, C. Corsaro, M. Broccio, C. Branca, N. Gonzalez-Segredo, J. Spooren, S.H. Chen, H.E. Stanley, Proc. Natl. Acad. Sci. U. S. A. 105 (2008) 12725.

[45] F. Mallamace, Proc. Natl. Acad. Sci. U. S. A. 106 (2009) 15097.

[46] O. Mishima, H.E. Stanley, Nature 396 (1998) 329.

[47] J. Wang, Z. Li, R.-H. Yoon, J.C. Eriksson, J. Colloid Interface Sci. (2012). 


\section{Captions of Figures and Tables}

Table 1. Water Contact Angles and Surface Roughness of the Silicon Wafer Surfaces Treated in a $5 \times 10^{-5} \mathrm{M}$ OTS Solution at Different Contact Times

Fig. 1. Effects of immersion time on the water contact angles of silicon wafer in a) $5 \times 10^{-}$

${ }^{5} \mathrm{M}$ and b) $3 \times 10^{-3} \mathrm{M}$ OTS-in-toluene solutions. $\theta_{\mathrm{a}}$ and $\theta_{\mathrm{r}}$ represent advancing and receding angles. The contact angle hysteresis $\left(\theta_{\mathrm{h}}\right)$ decreases with time indicating formation of smoother OTS monolayers. OTS adsorbs much faster at the higher concentration.

Fig. 2. Contact mode $1 \times 1 \mu \mathrm{m}$ AFM images of the silicon wafers of different equilibrium contact angles. a) bare surface with $\theta_{\mathrm{e}}=4^{\circ}$, b-e) surfaces with different $\theta_{\mathrm{e}}$ values as obtained by varying the immersion times in a $5 \times 10^{-5} \mathrm{M}$ OTS solution. Height profiles given below each image were obtained along the white line across the image. OTS adsorbs in patches, which coalesce to obtain a smooth monolayer.

Fig. 3. Surface forces (F) normalized by the radius (R) of spheres $v s$. the closest separation distance (h). The surfaces were treated in a $5 \times 10^{-5} \mathrm{M}$ OTS solution for different times to obtain different contact angles. Solid lines represent the extended DLVO plots with $\psi_{1}=-40 \mathrm{mV}$ and $A_{131}=0.8 \times 10^{-20} \mathrm{~J}$. The double-layer and van der Waals forces are negligibly small particularly at longer separations. Thus, the measured forces practically represent the hydrophobic forces, which are shown to increase with $\theta_{\mathrm{e}}$. The arrows represent the separations at which two surfaces jumped into contact.

Fig. 4. Surface forces (F) measured at different temperatures in the range of 10 to $30^{\circ} \mathrm{C}$ and normalized by the radius of sphere (R) $v$ s. separations (h). The silica surfaces were treated in a $5 \times 10^{-5} \mathrm{M}$ OTS solution to obtain $\theta_{\mathrm{e}}=92.5^{\circ}$. The measured forces became stronger and longer-ranged with decreasing temperature of the medium.

Fig. 5. Surface forces (F) measured at different temperatures in the range of 10 to $40^{\circ} \mathrm{C}$ and normalized by the radius of sphere (R) vs. separations (h). The silica surfaces were treated in a $5 \times 10^{-5}$ M OTS solution to obtain $\theta_{\mathrm{e}}=78^{\circ}$. The measured forces became stronger and longer-ranged with decreasing temperature of the medium.

Fig. 6. a) $C v s$. $\mathrm{t}$ and b) $D v s$, t plots. The $\mathrm{C}$ and $\mathrm{D}$ parameters of the hydrophobic force represented by Eq. [1] were obtained by fitting the experimental data presented in Figure 4 and 5 to the extended DLVO theory with $\psi_{1}=-40 \mathrm{mV}$ and $A_{131}=$ $0.8 \times 10^{-20} \mathrm{~J}$.

Fig. 7. Changes in excess film entropy $\left(\Delta S^{\mathrm{f}}\right)$ in the thin films of water between OTScoated silica surfaces with contact angles of $78.0^{\circ}$ and $92.5^{\circ}$ at different medium temperatures. 
Fig. 8. Changes in excess film enthalpy $\left(\Delta H^{\mathrm{f}}\right)$ in the thin films of water between OTScoated silica surfaces with contact angles of $78.0^{\circ}$ and $92.5^{\circ}$ at different medium temperatures.

Fig. 9. Changes in the excess thermodynamic properties for the thin liquid films between OTS-coated silica surfaces with $\theta_{\mathrm{e}}=78.0^{\circ}$ and $92.5^{\circ}$ at $20^{\circ} \mathrm{C}$. 
APPENDIX 7 - On Improving the Hydrophobicity of Oxidized Coal (WV024) 
FINAL TECHNICAL REPORT

\section{Contract Title and Number:}

Continuation of Crosscutting Technology Development at the

Center for Advanced Separation Technologies

(DE-FC26-05NT42457)

Sub-Recipient Project Title:

On improving the hydrophobicity of oxidized coals

Principal Investigator: M. S. Seehra

Contact Address:

Physics Department

111 White Hall

West Virginia University

Morgantown, WV 26506-6315

Subcontractor Address:

No subcontracts issued.
Period of Performance:

Starting Date: $\quad 10 / 1 / 08$

Ending Date: $\quad$ 9/30/11
Report Information:

Type: Final

Number:

Period: $\quad 10 / 01 / 08$ to $09 / 30 / 11$

Date: $\quad 11 / 28 / 11$

Code: WV024-FINAL

Contact Information:

Phone: 304-293-5098

Fax: $\quad 304-293-5732$

E-Mail: mseehra@wvu.edu

Subcontractor Information: N/A

Phone:

Fax:

E-Mail:

\section{DISCLAIMER}

The Disclaimer must follow the Title Page and must contain the following paragraph: "This report was prepared as an account of work sponsored by an agency of the United States Government. Neither the United States Government nor any agency thereof, nor any of their employees, make any warranty, express or implied, nor assume any legal liability or responsibility for the accuracy, completeness, or usefulness of any information, apparatus, product, or process disclosed, or represents that its use would not infringe privately owned rights. Reference herein to any specific commercial product, process, or service by trade name, trademark, manufacturer, or otherwise does not necessarily constitute or imply endorsement, recommendation, or favoring by the United States Government or any agency thereof. The views and opinions of authors expressed herein do not necessarily state or reflect those of the United States Government or agency thereof.". 


\begin{abstract}
:
The question of why the hydrophobicity varies among different coals leading to poor flotation of some coals in coal preparation plants is investigated in this project. Various properties and compositional characteristics of ten different coals (eight Argonne Premium Coals and two coals from Consol Inc, one a 'poor' floater and the other a 'good floater) were investigated along with flotation characteristics. Techniques employed included proximate and ultimate analysis, $\mathrm{x}-$ ray diffraction/Rietveld analysis and thermogravimetric analysis to determine the percentages of the different chemical phases present in the ash of the coals, FTIR spectroscopy for determining the surface species, and zeta potential measurements for determining the surface charge and isoelectric point (IEP) in terms of the $\mathrm{pH}$. Comparison of the obtained results among good and poor floaters is then used to determine the conditions under which improved flotation behavior can be expected.

Since the air bubbles used in froth flotation are negatively charged, the effective charge on the coal particles and hence the zeta potential must be positive for the electrostatic attraction to play the dominant role for coal flotation unless coal particles are naturally hydrophobic. It is found that the presence of large amount of kaolin and silica in coal ash are detrimental for coal flotation since these minerals yield negative zeta potential. Generally an initial $\mathrm{pH}>6$ leads to good flotation whereas the reverse is true if the initial $\mathrm{pH}<6$. In general, the presence of large amounts of kaolin and silica and surface acidic groups make the zeta potential negative, leading to poor flotation. Finally, addition of $\mathrm{NaCl}$ and certain ionic surfactants make the zeta potential positive which in turn leads to considerable improvements in coal flotation for some coals.
\end{abstract}




\section{INTRODUCTION:}

The fact that certain coals are poor floaters in coal preparation plants is a long standing unsolved problem in the coal industry $[1,2]$. In this project, our objectives were to investigate the reasons behind the poor flotation characteristics of certain coals and possibly devise/suggest ways to improve the hydrophobicity of the poor floaters in order to recover the useful carbons from these coals.

Our approach in this project was to investigate the physical and chemical characteristics of about a dozen coals including both "good" and "poor" floaters to determine the reasons for poor flotation of certain coals. The coals investigated in this project included the eight Argonne Premium Coals (APC) and two coals, one "good" and the other "poor" floater, obtained from Consol Inc. Assisted by my associates Dr. Vivek Singh and Mohita Yalamanchi, these coals were subjected to the following analytical investigations:

- Ultimate analysis

- Chemical phases and their \% present in ash by x-ray diffraction

- FTIR spectroscopy

- Particle size analysis

- Surface area measurements

- Moisture content by thermogravimetric analysis (TGA)

- Zeta potential measurements

- Flotation behavior in water

- Flotation behavior in $0.5 \mathrm{M} \mathrm{NaCl}$ and in $1.0 \mathrm{M} \mathrm{NaCl}$.

A summary of the results and conclusions drawn from the results is present below.

\section{EXPERIMENTAL RESULTS ON ARGONNE PREMIUM COALS}

The Argonne Premium Coals (APC) represent a variety of commercial coals and these are listed in Table I along with their percentages of C, H, O and S (ash free basis) and \% of ash. These coals were purchased from the national coal bank maintained by the Argonne National Laboratory. The percentages of various minerals presented in the ash were determined in our laboratory using X-ray diffraction - Rietveld analysis (XRD-RA) and these are shown in Table II. A more detailed analysis of the ash components of APC samples is given in the paper by Ward et al [3]. The results given in Tables I and II form the basis for the other results presented here. 
Table I: List of the eight (8) Argonne Premium Coals investigated in this work. The percentages of $C, H, O$ and $S$ (ash free basis) and percentages of ash were provided by the Argonne National Lab. These samples represent a variety of coals available on the market.

\begin{tabular}{|c|c|c|c|c|c|c|c|c|}
\hline & Seam & State & Rank & C & H & O & S & Ash \\
\hline 1. & Upper Freeport & PA & Med. Vol. Bit. & 85.5 & 4.70 & 7.5 & 2.32 & 13.2 \\
\hline 2. & Wyodak-Anderson & WY & Sub-bituminous & 75.0 & 5.35 & 18.0 & 0.63 & 8.8 \\
\hline 3. & Illinois \#6 & IL & High Vol. Bit. & 77.7 & 5.00 & 13.5 & 4.83 & 15.5 \\
\hline 4. & Pittsburgh \#8 & PA & High Vol. Bit. & 83.2 & 5.32 & 8.8 & 2.19 & 9.2 \\
\hline 5. & Pocahontas \#3 & VA & Low Vol. Bit. & 91.1 & 4.44 & 2.5 & 0.66 & 4.8 \\
\hline 6. & Blind Canyon & UT & High Vol. Bit. & 80.7 & 5.76 & 11.6 & 0.62 & 4.7 \\
\hline 7. & Lewiston-Stockton & WV & High Vol. Bit. & 82.6 & 5.25 & 9.8 & 0.71 & 19.8 \\
\hline 8. & Beulah-Zap & ND & Lignite & 72.9 & 4.83 & 20.3 & 0.80 & 9.7 \\
\hline
\end{tabular}

Table II: Mineral Composition of Argonne Premium Coals determined by XRD/Rietveld Analysis is shown.

\begin{tabular}{|l|l|c|l|}
\hline \multicolumn{1}{|c|}{ Coal } & \multicolumn{1}{|c|}{ Composition (\%) } & Ash (\%) & \multicolumn{1}{|c|}{ Flotation properties } \\
\hline Upper Freeport & $\begin{array}{l}\text { Illite (2.9), FeS }(1.6), \text { Kaolinite (7.1), } \\
\text { Quartz (1.6) }\end{array}$ & 13.2 & $\begin{array}{l}\text { some settling in water, ok in } \\
1 \mathrm{M} \mathrm{NaCl}\end{array}$ \\
\hline $\begin{array}{l}\text { Wyodak } \\
\text { Anderson }\end{array}$ & Kaolinite (5.7), Quartz (3.1) & 8.8 & $\begin{array}{l}\text { floats well in water and in } \\
1 \mathrm{M} \mathrm{NaCl}\end{array}$ \\
\hline Illinois \#6 & $\mathrm{FeS}_{2}(1.9)$, Kaolinite (9.7), Quartz (3.9) & 15.5 & $\begin{array}{l}\text { poor flotation in water, and } \\
\text { in NaCl }\end{array}$ \\
\hline Pittsburgh \#8 & $\mathrm{FeS}_{2}(1.2)$, Kaolinite (5.6), Quartz (2.4) & 9.2 & $\begin{array}{l}\text { poor flotation in water, some } \\
\text { improvement in NaCl }\end{array}$ \\
\hline Pocahontas \#3 & Kaolinite (1.3), Calcite (3.5) & 4.8 & $\begin{array}{l}\text { poor flotation in water, } \\
\text { major improvement in NaCl }\end{array}$ \\
\hline Blind Canyon & Calcite (2.0), Quartz (2.7) & 4.7 & $\begin{array}{l}\text { floats well in water and in } \\
\text { NaCl }\end{array}$ \\
\hline $\begin{array}{l}\text { Lewiston- } \\
\text { Stockton }\end{array}$ & Kaolinite (16.7), Quartz (3.1) & 19.8 & $\begin{array}{l}\text { poor flotation in water, and } \\
\text { in NaCl }\end{array}$ \\
\hline Beulah Zap & Quartz (9.7) & 9.7 & $\begin{array}{l}\text { poor flotation in water, some } \\
\text { improvement in 1M NaCl }\end{array}$ \\
\hline
\end{tabular}




\subsection{Effect of $\mathrm{NaCl}$ on coal flotation:}

Previous studies have shown [3] that addition of $\mathrm{NaCl}$ to water affects the flotation behavior of coals. Encouraged by these results, we investigated and compared the flotation behavior of the eight APC samples in de-ionized water, $0.5 \mathrm{M} \mathrm{NaCl}$ and $1 \mathrm{M} \mathrm{NaCl}$. The results are shown as photographs in Fig. 1 and Fig. 2. We used deionized water for these experiments to eliminate any effects of the impurities in water, although it is understood that eventually ordinary available water has to be used for practical cases. We used about 0.5 grams of each coal in $100 \mathrm{ml}$ of deionized water in separate beakers. The coals were thoroughly mixed by hand-stirring with a glass rod. This was followed by allowing the coals to settle. After equilibrium was reached in a few minutes, photographs of each beaker were taken. These photographs of coals in deionized water are shown in Fig. 1 and in Fig. 2. Among the eight coals, Blind Canyon Coal is the best floater, followed by Wyodak and to a lesser extent Upper Freeport.

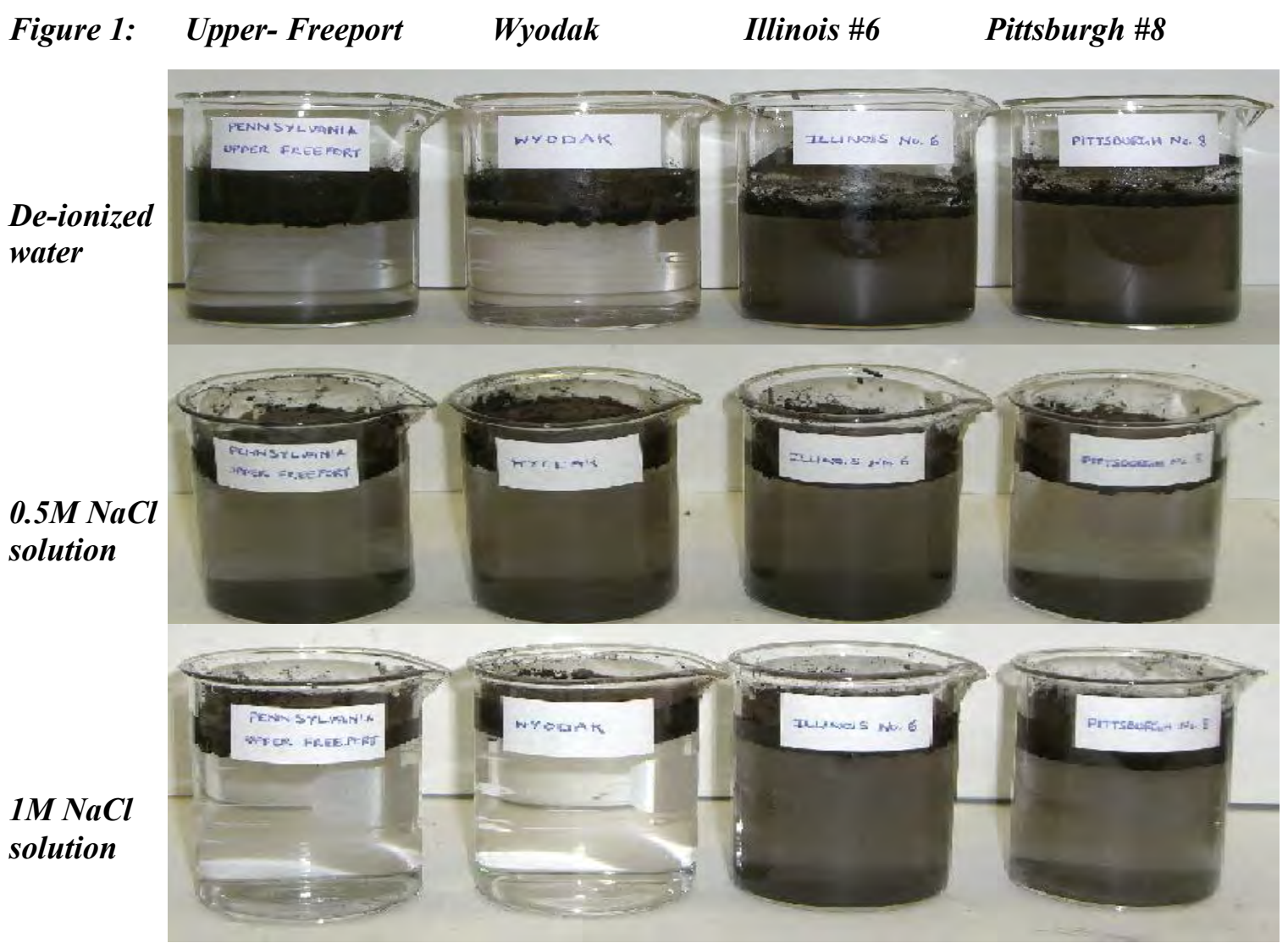


Figure 2: Pocahontas \#3 Blind Canyon Stockton Beulah-Zap

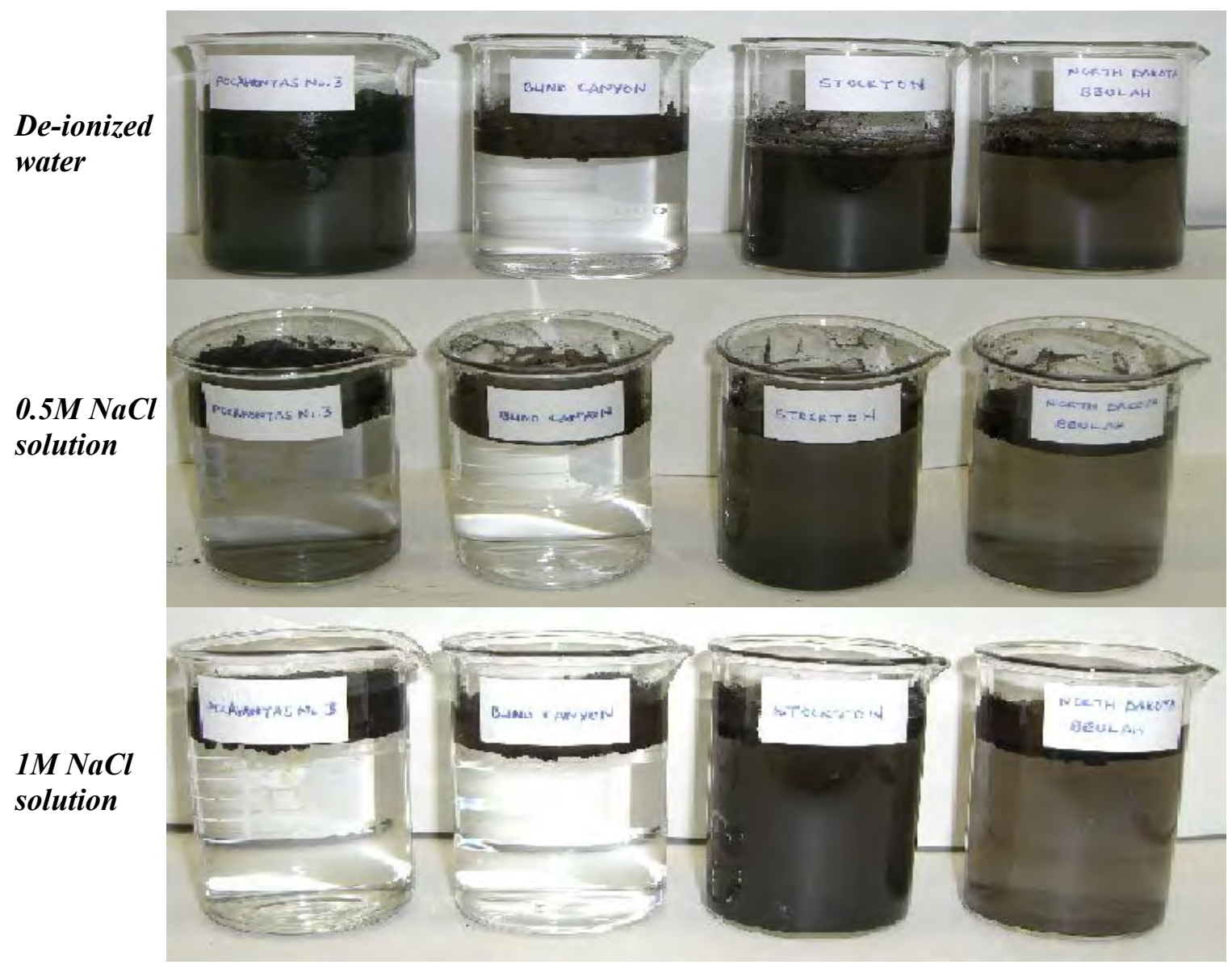

Our next tests were done with $0.5 \mathrm{M} \mathrm{NaCl}$ and $1 \mathrm{M} \mathrm{NaCl}$ solutions prepared in deionized water. The rationale for using $\mathrm{NaCl}$ is the work by $\mathrm{Li}$ et al [4] who showed that $\mathrm{NaCl}$ disrupts the air-water interface. There have been other follow-up studies on this matter [5]. Results from our experiments in $0.5 \mathrm{M} \mathrm{NaCl}$ and $1 \mathrm{M} \mathrm{NaCl}$ solutions for all eight coals are shown in Figs. 1 and 2. Excepts for four coals, namely Lewiston-Stockton, Illinois \# 6, Pittsburgh \# 8 and Beulah-Zap lignite, there is a considerable improvement on the flotation behavior of the coals in $1 \mathrm{M} \mathrm{NaCl}$. These simple experiments have provided some insight although these experiments do not quite simulate the actual flotation experiments. Also the use of $\mathrm{NaCl}$ may not be practical in industry because of the effect of $\mathrm{NaCl}$ on quality of the discharged water.

The Blind Canyon Coal with the best flotation characteristics has the lowest levels of ash (4.7\%) with calcite and quartz being the only minerals present. The other good floaters are Wyodak with $8.8 \%$ ash (5.7\% kaolinite, $3.1 \%$ quartz) and Pocahontas \# 3 with $4.8 \%$ ash (calcite $3.5 \%$, kaolinite $1.3 \%$ ) as they both showed major improvement in flotation in $\mathrm{NaCl}$ solution. 
Surprisingly Upper Freeport with a relatively large ash content of 13.2\% (kaolinite $7.1 \%$, Illite $2.9 \%$, quartz $1.6 \%$ and pyrite $1.6 \%$ ) also showed major improvement in flotation behavior in $1 \mathrm{M}$ $\mathrm{NaCl}$. Illinois \# 6 with ash content of $15.5 \%$ (pyrite $1.9 \%$, kaolinite $9.7 \%$ and quartz $3.9 \%$ ), Pittsburgh \# 8 with ash content of $9.2 \%$ (pyrite $1.2 \%$, kaolinite $5.6 \%$ and quartz $2.4 \%$ ) and Lewiston-Stockton with ash content of $19.8 \%$ (kaolinite $16.7 \%$, quartz $3.1 \%$ ) are poor floaters with only minor effects of the treatment with $1 \mathrm{M} \mathrm{NaCl}$. Beulah Zap coal, with quartz $(9.7 \%)$ being the only major impurity, does show some improvement in flotation with $1 \mathrm{M} \mathrm{NaCl}$ solution. Thus at first glance, high levels of ash with significant amount of kaolinite and the presence of pyrite may appear to be a signature of poor floaters. However, further work is essential to reach such a conclusion since hydrophobicity may be expected to be governed by several other factors such as porosity and surface structure of a coal [6].

How does the addition of $\mathrm{NaCl}$ improve the flotation behavior of some coals shown in Figs. 1 and 2? A thorough review of the literature and our own analysis leads to the following interpretation. Air bubbles used in coal preparation plants are negatively charged in the whole $\mathrm{pH}$ range of 2 to 12 . As shown later, coal particles are usually negatively charged for most coals if $\mathrm{pH}>6$ but they become positively charged for $\mathrm{pH}<6$. It is noted that the $\mathrm{pH}$ of deionized water equals 8.6 whereas $\mathrm{pH}=6.02$ for $1 \mathrm{M} \mathrm{NaCl}$ prepared in deionized water. Therefore $\mathrm{NaCl}$ lowers the $\mathrm{pH}$ which may impart positive charge to the coal particles. For negatively charged air bubbles to attach to coal particles, the electrostatic interaction requires that coal particles be positively charged. So this may be one reason for the observed improvement with $\mathrm{NaCl}$. Of course if the coal particles are naturally hydrophobic such as high rank coals, then flotation occurs naturally without the need to invoke electrostatic attraction.

As discussed later, for charged coal particles suspended in water, there is a double layer surrounding each particle making the electrostatic interaction less effective. It is now believed that for $\mathrm{NaCl}$ concentration $>0.1 \mathrm{M}$, this double layer is severely compressed which makes the electrostatic attraction between the air bubbles and coal particles more effective [4]. This may be the key to improving the hydrophobicity of the poor flotation of some coals. Later on, measurements on zeta potentials are presented which provide further insights into this phenomenon. The lowering of $\mathrm{pH}$ by $\mathrm{NaCl}$ combined with the suppression of the double layer is the likely source of observed hydrophobicity in $\mathrm{NaCl}$.

\subsection{Effects of ash components:}

In our investigations, both XRD and FTIR spectroscopy were used to determine the impurities in the APC samples. Results from the XRD analysis are shown in Table 1 whereas the results from FTIR spectroscopy in the $2700 \mathrm{~cm}^{-1}$ to $4000 \mathrm{~cm}^{-1}$ range are shown in Fig.3. 
Surface characteristics of coals are important since interaction of water and coals occurs at the surface. IR spectroscopy is a well known technique to determine the nature of surface species. Previous studies on the IR spectroscopy of the APC samples are by Solomon et al $[7,8]$. We use that knowledge along with the results from our work using both the XRD and IR spectroscopy.

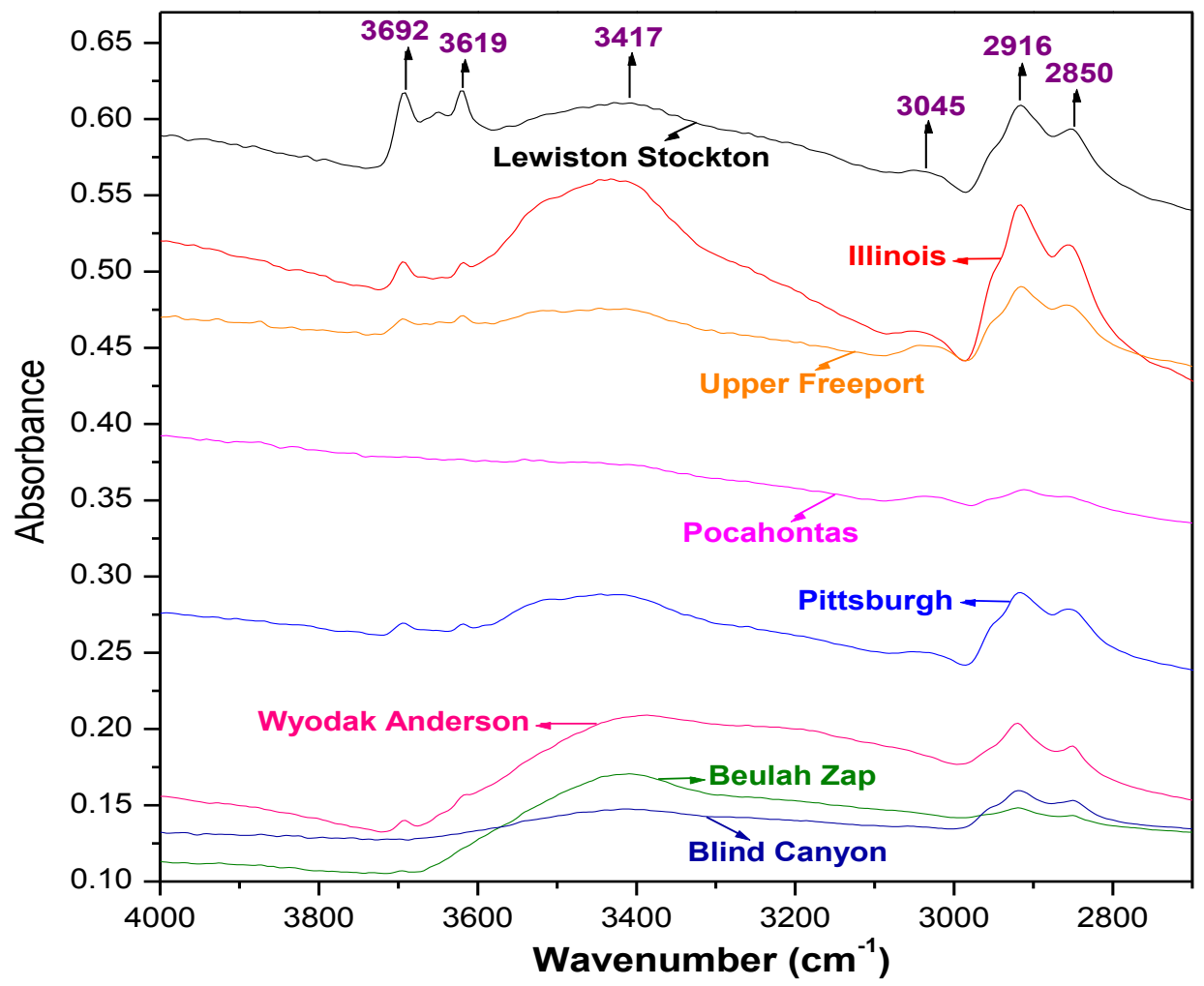

Figure 3: IR spectra of the eight AP coals over the $4000 \mathrm{~cm}^{-1}$ to $2700 \mathrm{~cm}^{-1}$ range

The IR spectra of the eight AP coals, covering the standard $400 \mathrm{~cm}^{-1}$ to $4000 \mathrm{~cm}^{-1}$ range are shown in Fig. 4. The IR spectrum of pure Kaolin which is a significant impurity in a large number of AP coals (Table II) is also included for comparison. The triplet in the $3600 \mathrm{~cm}^{-1}$ to $3700 \mathrm{~cm}^{-1}$ range is due to the vibration mode of kaolin with the chemical formula $\mathrm{Al}_{2} \mathrm{Si}_{2} \mathrm{O}_{5}(\mathrm{OH})_{4}$. Using the area under these peaks as a measure of the concentration of Kaolin in the AP coals, we have compared the concentration of Kaolin determined by XRD - Rietveld analysis and IR spectroscopy. In Fig. 5 and Fig. 6, this comparison shows near one-to-one correspondence between the concentrations determined by the two techniques. Coincidently, the coals with the highest Kaolin concentration viz. Lewiston-Stockton, Pittsburgh \#8, Illinois \#6 and Upper Freeport are among the poor floaters in our initial tests. Additional comments on this issue are given later in this report. 


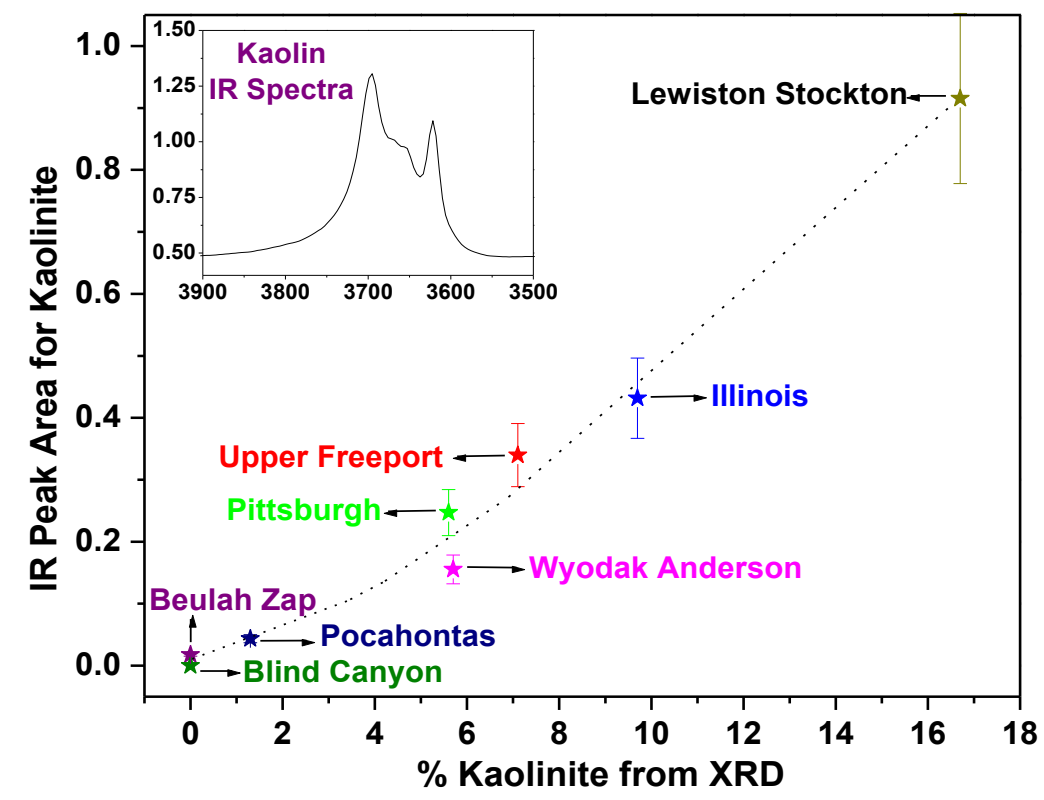

Figure 5: Comparison of the concentration of Kaolin determined by XRD-Rietveld analysis and IR spectroscopy.

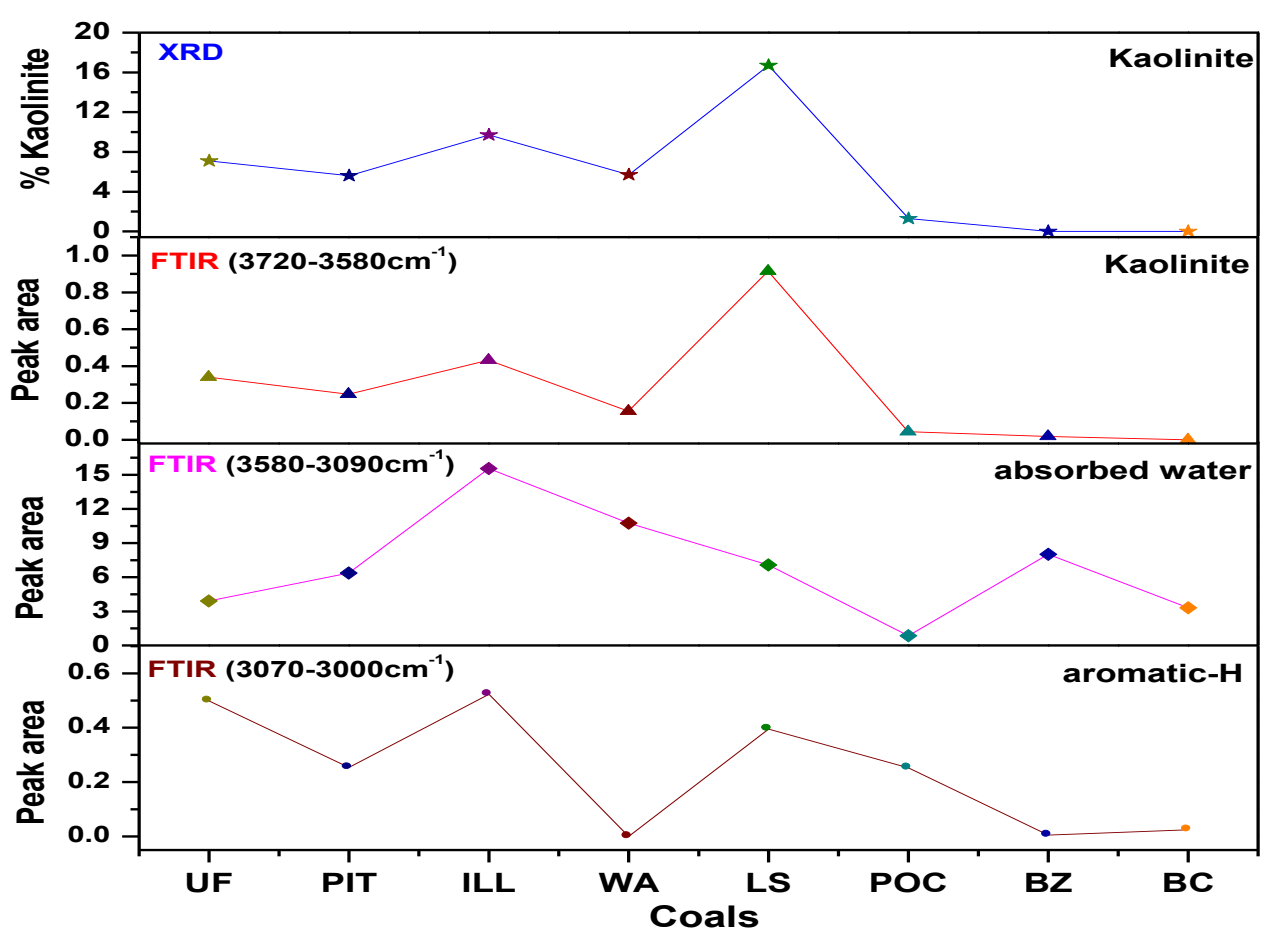

Figure 6: One-to-one correspondence between the concentrations of Kaolin determined by XRD and IR. Concentration of absorbed water and aromatic-H present is also shown. 
Previous studies [5] have indicated that the wetting behavior of carbonaceous matter of coal which is normally hydrophobic may change significantly as coals get oxidized. The chemisorption of oxygen during oxidation produces acidic surface carboxylic and phenolic groups viz. $-(\mathrm{COOH})$ and $-(\mathrm{OH})$ groups respectively. These groups make the coals more hydrophilic because they interact with water. These acidic surface functional groups produced by oxidation of coals lowers the $\mathrm{pH}$ of coals and make the zeta potential of the coals more negative.

In the expanded view of IR spectroscopy data of Fig. 3, the peak marked at $3045 \mathrm{~cm}^{-1}$ is usually associated with aromatic $\mathrm{C}-\mathrm{H}$ stretching where as the broad absorption in the $3100-3550$ $\mathrm{cm}^{-1}$ is associated with the $\mathrm{O}-\mathrm{H}$ stretch of adsorbed water and alcohol/phenol O-H stretch. As shown in Fig. 4, the peaks at 2850 and $2916 \mathrm{~cm}^{-1}$ are due to aliphatic $\mathrm{C}-\mathrm{H}$ stretching. The carboxyl acid $\mathrm{OH}$ stretch usually leads to broad absorption below $2800 \mathrm{~cm}^{-1}$. In Fig. 6, we have re-plotted the data of Fig. 3 keeping the ordinate scale to be the same for all the coals. The relative concentration of the surface $\mathrm{OH}$ groups which make the coals more hydrophilic can be gauged by the strength of IR absorption in the 3100 to $3550 \mathrm{~cm}^{-1}$. Based on this analysis, Lewiston-Stockton, Pittsburgh \#8, Illinois \#6, Upper Freeport, and Pocahontas coals are more hydrophilic (see Fig. 6). This is in general agreement with the results from the flotation behavior of these coals listed in Table II in that hydrophilicity leads to poor flotation. In Fig. 6, the area under the $3045 \mathrm{~cm}^{-1}$ representing aromatic-H groups for different coals is also plotted.

\subsection{Zeta potential measurements:}

When a negatively charged coal particle is placed in water, it is immediately surrounded by positive charges to neutralize the negative charge creating the Stern layer which in turn is surrounded by a more diffused layer of a positive charge. This creates an electrical double layer. Zeta potential $\mathrm{Z}$ is the potential measured at the slipping plane. Under an applied electric field, this compound particles moves which is affected by the viscosity $\eta$ and dielectric constant $\varepsilon$ of the medium. For dilute solutions with particles larger than $0.2 \mu \mathrm{m}$, the zeta potential $\mathrm{Z}=\eta \mathrm{U}_{\mathrm{E}} / \varepsilon$ where $\mathrm{U}_{\mathrm{E}}$ is the elctrophoretic mobility (particle velocity/electric field). Thus the sign of $\mathrm{Z}$ is determined by the sign of $U_{E}$ and it is affected by $\mathrm{pH}$ of the solution. Change in the sign of $\mathrm{Z}$ with respect to $\mathrm{pH}$ occurs at the isoelectric point (IEP) where $\mathrm{Z}=0$ at a specific $\mathrm{pH}$. As noted earlier, air bubbles are charged negatively. So for flotation of coal particles to occur, the coal particles must have a positive effective charge, producing a strong attraction between the air bubbles and the positively charged coal particles.

In our experiments we first measured the zeta potential of kaolinite and silica (quartz) particles. The results in Fig. 7 show that zeta potential of kaolinite and silica particles remains negative for all $\mathrm{pH}$ values. Thus isolated particles of silica and kaolinite are not expected to float 
with the air bubbles. Also for coals with large percentages of kaolin and silica, the measured zeta potential is likely affected by these impurities. For coal particles attached to kaolin and silica particles, the overall zeta potential is very likely to be negative.
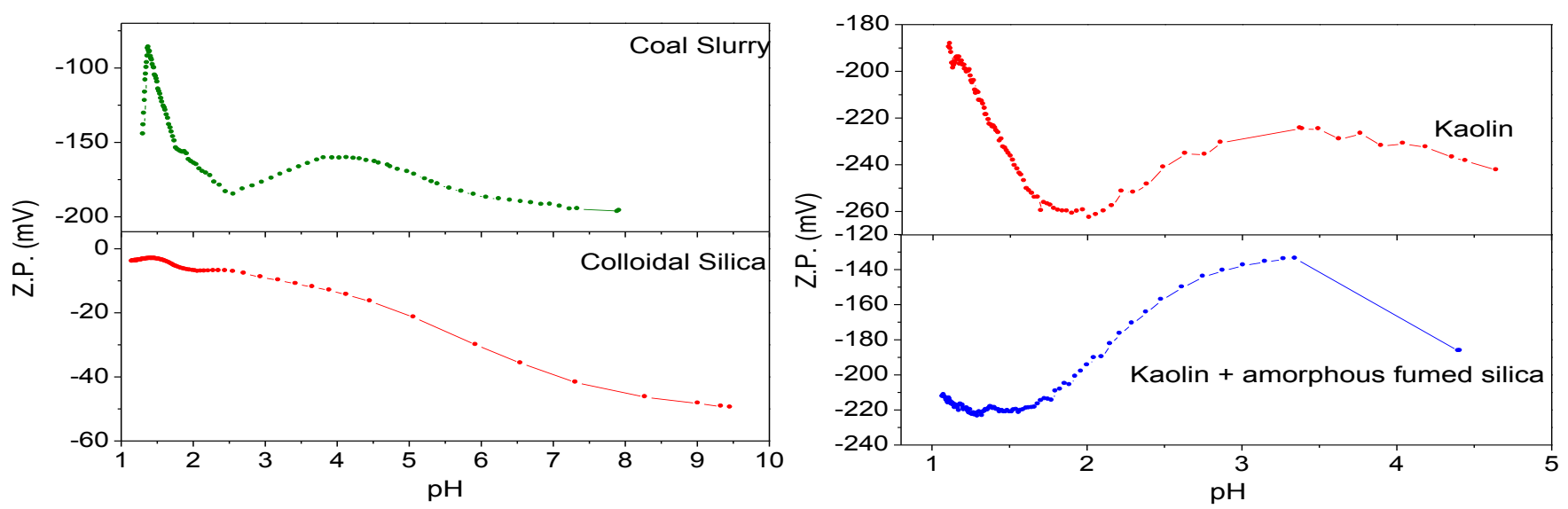

Figure 7: Zeta potential vs. pH for coal slurry, silica, kaolin, kaolin + silica.

There have been a number of studies on the measurement of zeta potentials of coals. Measurements reported by Fuerstenau et al used $0.1 \%$ concentration of coals of $-38 \mu \mathrm{m}$ size [9]. These studies showed that both the increase in the ash content and oxidation of coals made the zeta potential more negative and pushed the IEP point to lower $\mathrm{pH}$ values in agreement with the discussion presented above. Earlier studies by Wen and Sun [10] measured the effect of oxidation, surfactants and oils on the zeta potential of bituminous coals; these studies also showed that oxidation make the zeta potential more negative and shifts the IEP to lower $\mathrm{pH}$ values. An opposite effect was observed with the use of different ionic compounds $\left(\mathrm{Fe}^{2+}, \mathrm{Fe}^{3+} \ldots\right)$ and surfactants in that zeta potential becomes more positive and the IEP shifts to higher $\mathrm{pH}$ values $[10,11]$. On the other hand, non-ionic surfactants diacetone alcohol $\left(\mathrm{C}_{6} \mathrm{H}_{12} \mathrm{O}_{2}\right)$ and 2ETH $\left(\mathrm{C}_{8} \mathrm{H}_{18} \mathrm{O}\right)$ had no measurable effect on the zeta potential [12].

Measurements of the zeta potentials of the three Argonne Premium Coals are presented in Fig. 8. For the Blind Canyon coal which was found to have relatively small amount of ash (Table II and was found to be an excellent floater (Fig. 2), zeta potential remains positive till very low $\mathrm{pH}$ values. On the other hand, for the Illinios \# 6 and Pittsburgh \# 8 coals, the starting zeta potential is negative meaning the charge on the coal particles is negative. Only at lower pH values below the IEP point, does the zeta potential become positive. Note that both coals showed considerable flotation when $\mathrm{NaCl}$ is added (Fig. 1) and $\mathrm{NaCl}$ lowers the $\mathrm{pH}$. Therefore it is essential that for good flotation characteristics, the zeta potential and charge be positive which happens below IEP. 


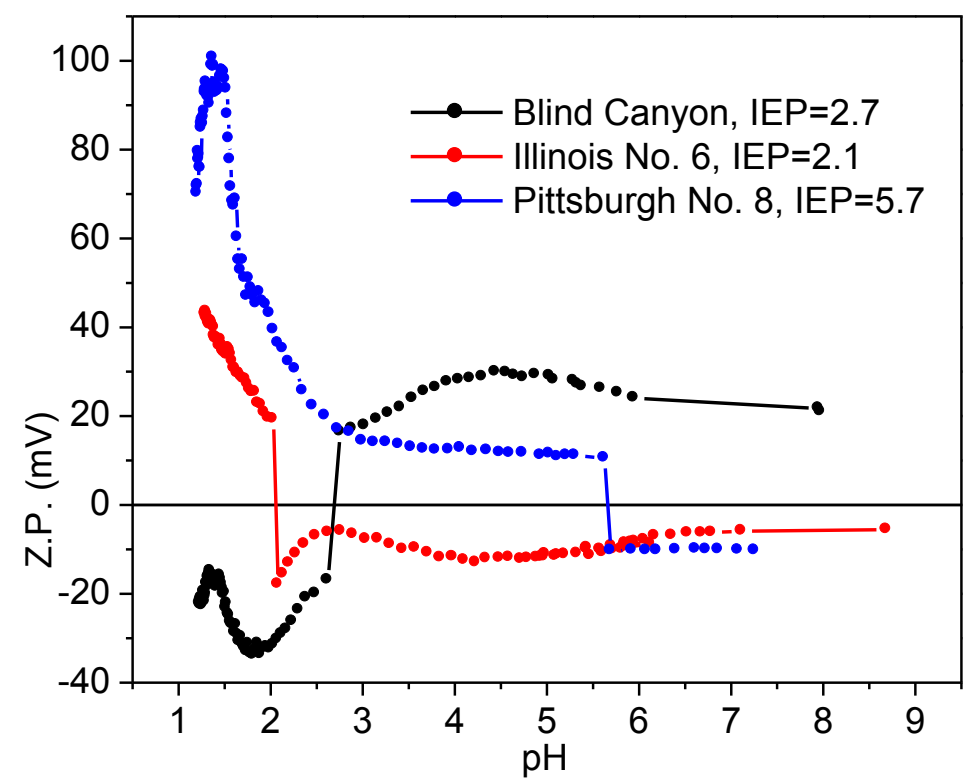

Figure 8: Zeta potential data on three Argonne Premium Coals

\section{EXPERIMENTAL RESULTS ON A "POOR FLOATER" AND A "GOOD FLOATER"}

At our request, Consol. Inc provided us two samples of coals from their preparation plant: a "poor floater" and a "good floater" as characterized by Consol engineers. The "poor floater" coal was collected in the preparation plant prior to any "conditioner frother" (Freedom Industries "shurflot" TF-944) being added. This sample was a mixture of several coals (coalburg$30 \%$, 5 block $-32 \%$, Middle Kittanning - 19\%, stockton $-9 \%$ and clarion $-7 \%$ ). The sample listed as a "good floater" was from the Pittsburg \# 8 seam. It was collected in the preparation plant after the conditioner frother (TF- 944) was added. For our experiments, we separated the solid samples of the two coals and dried them in an oven set at $80^{\circ} \mathrm{C}$. These dried coals were used for tests in our experiments.

First, $\mathrm{pH}$ was measured by adding $0.2 \mathrm{~g}$ of the coals in $200 \mathrm{~mL}$ of de-ionized water. For the "good floater" $\mathrm{pH}=8.2$ was obtained whereas for the "poor floater, $\mathrm{pH}=6.5$ was measured. So the acidic nature of the "poor floater" may be an important criterion.

\subsection{Composition:}

We carried out x-ray diffraction, ultimate analysis and thermogravimetric analysis (TGA) of the two coals to determine their mineral content, moisture and carbon content. Based on the results obtained from these experiments, the percentages of various components in these two coals are listed in Table III. 
Table III: Percentages of the components present in the "good" and the "poor" floater.

\begin{tabular}{|c|c|c|c|c|c|c|}
\hline Sample & $\mathrm{H}_{2} \mathrm{O}$ & $\mathrm{C}$ & $\mathrm{SiO}_{2}$ & $\mathrm{FeS}_{2}$ & Kaolin & Illite \\
\hline "Good floater" & 4 & 51 & 14 & 2 & 7 & 22 \\
\hline "Poor floater" & 3 & 51 & 11 & 0 & 14 & 21 \\
\hline
\end{tabular}

From the above table, it is evident that the "poor floater" contains more Kaolin than the "good floater". To verify this difference, we carried out FTIR spectroscopy of the two coals. The data of Fig. 9 clearly shows the presence of the strong triplet in the "poor floater" in the 3600$3700 \mathrm{~cm}^{-1}$ range which has been established to be due to Kaolin (See Fig.5). On the other hand, this triplet in the "good floater" is relatively weak. The importance of this difference in the \% of Kaolin in the two samples is discussed later.

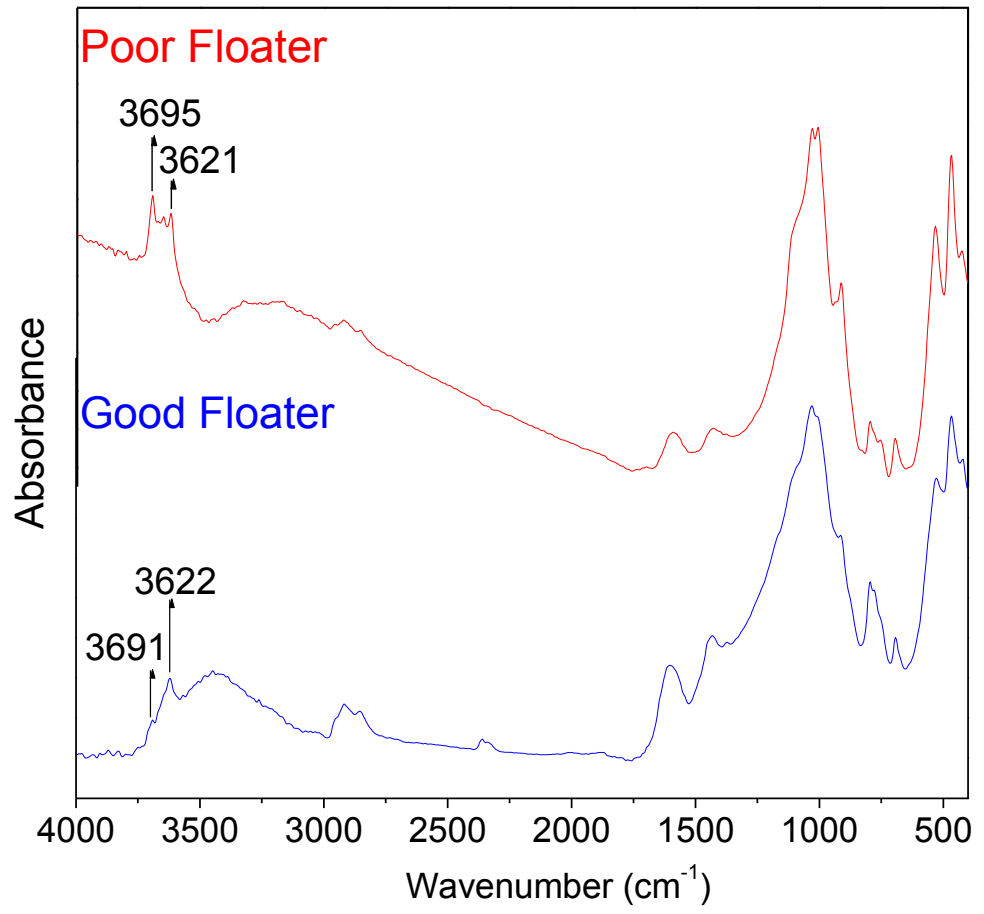

Figure 9: IR spectra of "poor" and "good" floater coals.

\subsection{Zeta Potential measurements:}

In Fig.10, measurements of the zeta potential vs. $\mathrm{pH}$ are shown for the "good" and "poor" floater coals. For the "good floater", zeta potential remains positive satisfying the criterion for good floatation characteristics as discussed earlier in connection with the good floatation behavior 
of the Blind Canyon Coal. Good flotation occurs if the charge on the coal particles and hence the zeta potential is positive leading to strong electrostatic attraction to the negatively charged air bubbles. For the "poor floater" coal, the zeta potential at the starting $\mathrm{pH}=6.5$ is negative. However, $\mathrm{pH}$ is lowered by adding $\mathrm{HCl}$, the zeta potential becomes positive below IEP $=4.3$ because the addition of the positively charged $\mathrm{H}^{+}$ions. So it would appear that a criterion for improving the floatation behavior of coals is to bring their $\mathrm{pH}$ to below their IEP.

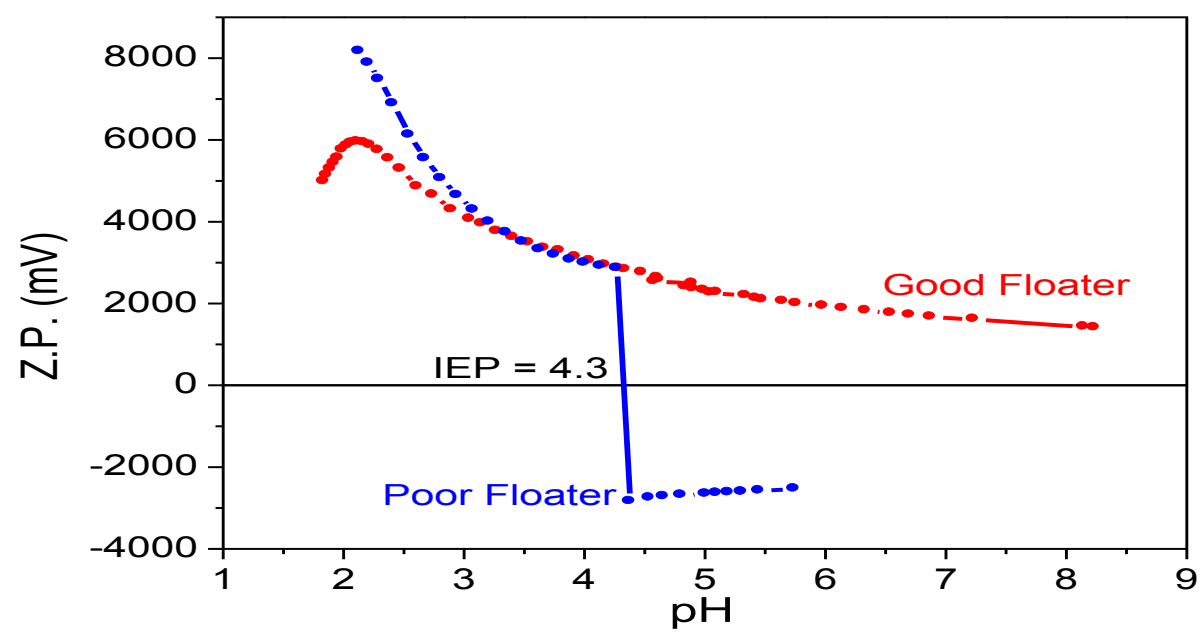

Figure 10: Zeta potential data for the good and poor floater Consol coals.

\section{DISCUSSION:}

Clay minerals which are often present in coals (See Table II and III) are hydrous aluminum silicates with layered structures and particle size less than $<2 \mu \mathrm{m}$. Due to this layered structure, (001) is the cleavage plane. Kaolin is the 1:1 layer type whereas other clays such as smectite, illite and muscovite are 2:1 layer types. The 1:1 layer type clays such as kaolin usually have no surface charge whereas the 2:1 layer types have surface charge between 0.2 and 0.6 per formula unit. Because of this surface charge, water binds to the 2:1 layer type clays and they swell in the presence of water. On the other hand, pure kaolin does not absorb any moisture. This is quite evident by comparing the IR spectra of "good" and "poor" floaters in Fig.9. The broad band near $3450 \mathrm{~cm}^{-1}$ due to adsorbed moisture is much stronger in the "good floater" than in the "poor floater", the latter containing more kaolin. The kaolin particles also have a large negative zeta potential for all $\mathrm{pH}$ value (Fig. 7). Thus the presence of a large amount of kaolin in a coal sample appears to be detrimental to its flotation in a coal preparation plant. Silica particles have a similar detrimental effect on coal flotation. On the other hand, coal flotation has been shown to 
improve considerably by the addition of $\mathrm{NaCl}$. A paper by Yoon and Sabey [13] has reviewed the effect of a variety of salts on coal flotation and reached a similar conclusion that salts improve the flotation by suppressing the electrical double layer around the coal particles. Effects of oilwater wetting for improving coal flotation has been reviewed by Capes and Jonasson [14] and chemistry of flotation is discussed by Fuerstenau et al [15]. Thus a number of approaches to improve flotation of coals are possible as shown in the experiments done in this project.

\section{SUMMARY OF RESULTS:}

- From the results and discussion presented in the above pages on the eight Argonne premium coals and the two Consol coals (good and poor floater), the following conclusions are drawn:

- Presence of significant amount of kaolin in coals is detrimental to their flotation in coal preparation plants. The other clays such as illite and muscovite absorb moisture and most likely settle down.

- The initial $\mathrm{pH}$ of a coal appears to affect its floatation behavior. Coals with $\mathrm{pH}>6$ have good flotation characteristics whereas the reverse is true if initial $\mathrm{pH}<6$. The presence of surface acidic groups resulting from coal oxidation and the presence of Kaolin and silica likely lowers the $\mathrm{pH}$.

- A negative zeta potential usually implies poor flotation characteristics. This negative zeta potential results from the negative surface charge on the coal particles. Since the air bubbles are also negatively charged, the electrostatic repulsion between the air bubbles and coal particles leads to poor flotation.

- Addition of $\mathrm{NaCl}$ and other ionic salts and surfactants suppress the electrical double layer, lower the $\mathrm{pH}$ below IEP and improve flotation characteristics.

- Since coals from different mines have different compositions and characteristics, each coal may require a specific approach to improve its hydrophobicity and flotation.

\section{PUBLICATIONS/PRESENTATIONS:}

1. "Electricity from coal combustion: Improving the hydrophobicity of oxidized coals"

Podium presentation \# V31-1 by M. S. Seehra (coauthor V. Singh) under the session entitled "Energy Production" made on March 24, 2011 at the Annual Meeting of the American Physical Society, Dallas, TX.

2. An invited podium presentation entitled" Microwave dewatering of coal slurries" made by M. S. Seehra at the International Coal Prep 2011 Conference held at Lexington, KY (May 3, 2011). 
3. A book chapter entitled " Use of microwave heating in coal research and in material synthesis" M. S. Seehra and Vivek Singh published in the book entitled " Microwave Heating" (edited by U. Chandra, Intech Publishers, 2011. ISBN: 979-953-307-020-8) pages 163-180.

4."Zeta potentials and flotation behavior of Argonne Premium Coals" by M. S. Seehra, V. Singh and M. Yalamanchi, Manuscript under preparation.

\section{PATENTS : None}

\section{REFERENCES:}

1. T. D. Wheelock, "Development and demonstration of selected fine coal beneficiation methods", in Physical Cleaning of Coals edited by Y. A. Liu (Marcel Dekker, NY, 1982) page 353-397.

2. T. D. Wheelock and R. Markuszewski, "Coal Preparation and Cleaning" in The Science and Technology of Coal and Coal Utilization edited by B. R. Cooper and W. A. Ellingson (Plenum Press, NY 1984).

3. C. R. Ward, J. C. Taylor, C. E. Matulis and L. S. Dale, Intern. J. Coal Geology, 40 (2001) 67-82.

4. C. Li and P. Somasundaran, Energy \& Fuels 7 (1993) 244-248.

5. D. S. Walker and G. L. Richmond, J. Phys. Chem. C 111(2007) 8321.

6. J. W. Larsen, P. Hall and P. C. Wernett, Energy \& Fuels 9 (1995) 324.

7. P. R. Solomon and R. M. Carangelo, Fuel 61(1982) 663.

8. E. L. Fuller, Jr. and N. R. Smyrl, Fuel 64 (1985) 1143.

9. D. W. Fuerstenau, J. M. Fosenbaum and Y. S. You, Energy \& Fuels 2 (1988) 241-245.

10. W. W. Wen and S. C. Sun, in Separation Science and Technology, 16 (1981) 1491-1521.

11. M. S. Celik and R. H. Yoon, Langmuir, 7 (1991) 1770-1774.

12. Z. Aktas, Turk. J. Chem. 24(2000) 117-129.

13. R. H. Yoon and J. B. Sabey, in Interfacial Phenomenon in Coal Technology, (edited by G. D. Botsaris and Y. M. Glazman, Marcel Dekker, Inc. New York, 1988) pages 87- 114.

14. C.E. Capes and K. A. Janasson, in Interfacial Phenomenon in Coal Technology,(edited by G. D. Botsaris and Y. M. Glazman, Marcel Dekker, Inc. New York, 1988) pages 115155.

15. Chemistry of Flotation by M. C. Fuerstenau, J. D. Miller and M. C. Kuhn (Society of Mining Engineers, New York 1985). 
APPENDIX 8 - Warm Air Drying of Fine Particles (WV021) 
Contract Title/Number:

Establishment of the Center for Advanced Separation

Technologies (DE-FC26-05NT424S7)

Sub-Recipient Project Title:

Warm Air Drying of Fine Particles

Principal Investigators:

Dr. Eric K Johnson

Dr. Bruce Kang

Contract Address:

Dept. of Mechanical and Aerospace Engineering

West Virginia University

PO Box 6106

Morgantown, WV
Period of Performance:

Starting Date: 7/26/2006

Ending Date: 3/31/2012

Report Information:

Date: $05 / 31 / 2012$

Report No.: 1

Report Period: 7/29/2007

$3 / 31 / 2012$

Contact Phone/e-mail:

Phone: (304) 293-3134

e-mail: eric.johnson@mail.wvu.edu

\section{DISCLAIMER}

This report was prepared as an account of work sponsored by an agency of the United States Government. Neither the United States Government nor any agency thereof, nor any of their employees, make any warranty, express or implied, nor assume any legal liability or responsibility for the accuracy, completeness, or usefulness of any information, apparatus, product, or process disclosed, or represents that its use would not infringe privately owned rights. Reference herein to any specific commercial product, process, or service by trade name, trademark, manufacturer, or otherwise does not necessarily constitute or imply endorsement, recommendation, or favoring by the United States Government or any agency thereof. The views and opinions of authors expressed herein do not necessarily state or reflect those of the United States Government or agency therof. 


\begin{abstract}
The small transparent riser system has provided the necessary data and operating experience for operating the large warm air drying of fine particles, WADFP, system. During the large amount of time required to rectify the air compressors problems, the development of the scaling processes between the hydrodynamics of the small riser and the larger WADFP system has been completed. This effort has been shown to be successful after experimental data was obtained for the two systems. Several preliminary coal drying runs have been completed. The results from these runs have led to recommendations for improving the performance of the dryer. The research completed prior to $11 / 15 / 2010$ is described in the attached PhD Dissertation written by Dr. Steve Rowan. The activities following that date are detailed below. These additional activities include modifying the WADFP system to address issues that Dr. Steve Rowan referred to in his dissertation. These include design and installation of a new coal feed system, replacing the heat exchanger used to pre-heat the fluidizing gas (air) with an electrical heating system, installation of a secondary cyclone and replacing the water filtration system (for exhaust gasses) with a HEPA filtration system.
\end{abstract}




\section{TABLE OF CONTENTS}

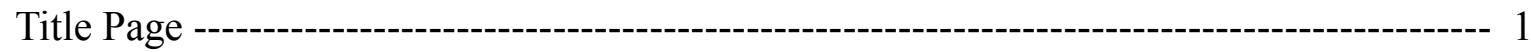

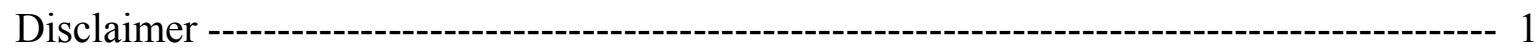

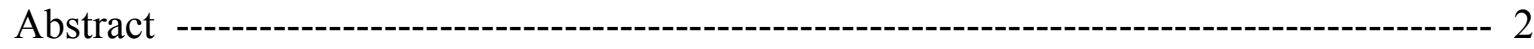

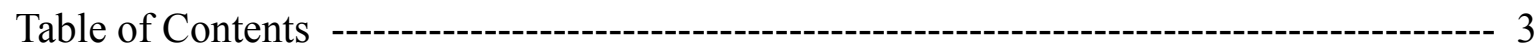

Introduction ------ 4

Background

Objective and History

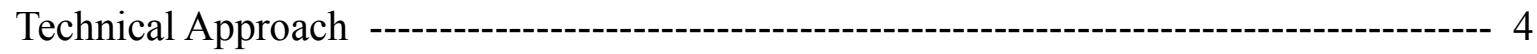

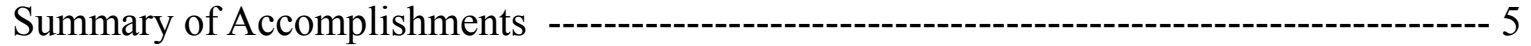

System Modifications --- 5

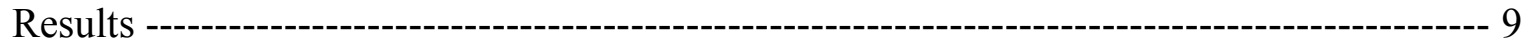

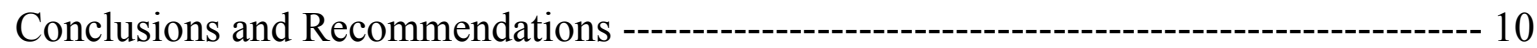

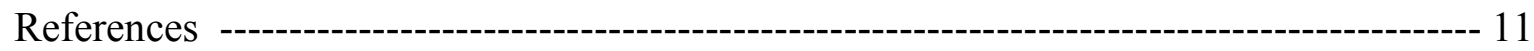




\section{INTRODUCTION:}

\section{Background:}

The goal of this research project is to evaluate the performance of a warm air dryer for fine particles, WADFP, system. Drying takes place in a two-stage circulating fluidized bed CFB riser. Warm dry air is supplied to each stage of the riser. The riser is enclosed in a steam jacket and the dry air is heated by condensing steam. The steam condenses at low pressures to avoid producing any hot spots in the dryer. As the wet fine particles are injected into the riser, they become lighter and are eventually blown out of the riser into the product bin. A "bare" WADFP system was fabricated and was purchased by WVU. Consequently, a considerable effort has been expended in designing the necessary auxiliary equipment and to make the research system operational, Instrumentation.

In order to better understand the fluidization process in a two-stage riser, a small transparent model of the riser has been designed and constructed. This model provides the necessary data to achieve a better understanding of the fluidization in a two-stage CFB system. This information was necessary to analyze the results from the large scale WADFP. This small scale model has no ability to dry particles.

Another major activity involved the development of a mathematical model for the drying process in the riser model. It is intended that this model will aid in correlating the experimental data and to lead to producing scaling parameters for the WADFP system.

\section{Objective and History:}

The objective of this research project is to evaluate the concept of a Warm Air Dryer of Fine Particles, WADFP. In order to accomplish this objective, several tasks must be completed. The original proposal for this project was for a one-year effort. This led to a rather optimistic work schedule. It was decided that this project should be reformulated into a two year project. A new work schedule was developed. A second development with the work schedule was that the actual work on the project could not begin until January 1, 2007. Initially, the project was to start September 2006, but all of the project members had to first complete their obligations for the Fall 2006 semester for WVU. Therefore, the work schedule reflects that the project activities started on January 1, 2007. The project was to be completed on 09/31/10. However, because of several significant delays, this project received additional funds and the ending date was extended to $03 / 31 / 12$. These significant delays were due to air compressor and electrical problems which have now been resolved.

\section{TECHNICAL APPROACH}

This section remains unchanged from the previous annual technical progress reports, [1,2]. A more detailed description of the technical approach may be found in Dr. Rowan's dissertation, [3]. 


\section{SUMMARY of ACCOMPLISHMENTS}

The following tasks provide a general outline of the work that has been completed performed. Each task was discussed in detail in [1]. The status of each task is indicated.

Task 1: Drawings of Warm Air Dryer and scale model. --Completed

Task 2: Selection of Instrumentation.-Completed

Task 3: Manufacturing, assembly and testing of scale model components.-Completed

Task 4: Manufacturing, assembly and testing of WADFP components.-Completed

Task 5: Flow visualization/verification tests with scale model.-Completed

Task 6: Coal Drying tests with WADFP.-Completed

Task 7: Data reduction and analysis-Completed

Task 8: Final report.-Completed

The following sections detail the work done between September 30, 2010 and March 31, 2012. It should be noted, however, that due to availability of project funding, the work detailed was not begun until June 2011.

As noted in the PhD dissertation of Dr. Steve Rowan [3], a number of design deficiencies within the WADFP system became apparent over the course of the initial testing. The original solids feed system, in which the solids material was fed pneumatically into the bottom of the WADFP riser via one of the lower air feed lines, proved to be problematic when feeding wet coal. The original feed system was restricted to very low feed rates and was prone to stoppage of feed due to clogging in the feed hopper. A second problem that was encountered was that the pressure drop across the heat exchanger used to pre-heat the fluidizing gas was large enough that it severely limited to flow rates of air into the WADFP unit, preventing the system from be operated at higher superficial gas velocities. A third problem that was encountered was that the cyclone located within the gas exhaust system failed to capture smaller diameter coal particles exiting the system $(<\sim 100 \mu \mathrm{m})$, leading the formation of a thin coal foam/slurry within the water filtration drum downstream of the cyclone. The final issue to be addressed was the previously mentioned water filtration system. In this system, the exhaust gasses leaving the cyclone where piped into a water-filled drum. Upon exiting the exhaust pipe, the gasses would be bubbled up through a column of water, where any remaining solid particles would be separated from the gas flow. Two problems were encountered with this system: the first was that of incomplete separation of solids from the exiting gas, leading to venting of coal dust into the air; the second issue was the formation of bubbles in the filtration system created pressure fluctuations that would propagate back into the riser system and affect the data collected by the pressure transducers located in the upper riser stage. The following section details the modifications made to the WADFP system to address these system deficiencies.

\section{SYSTEM MODIFICATIONS}

The current system and flow and instrumentation diagrams for the WADFP unit, incorporating all of the design modifications discussed below, is shown in Fig. 1. 


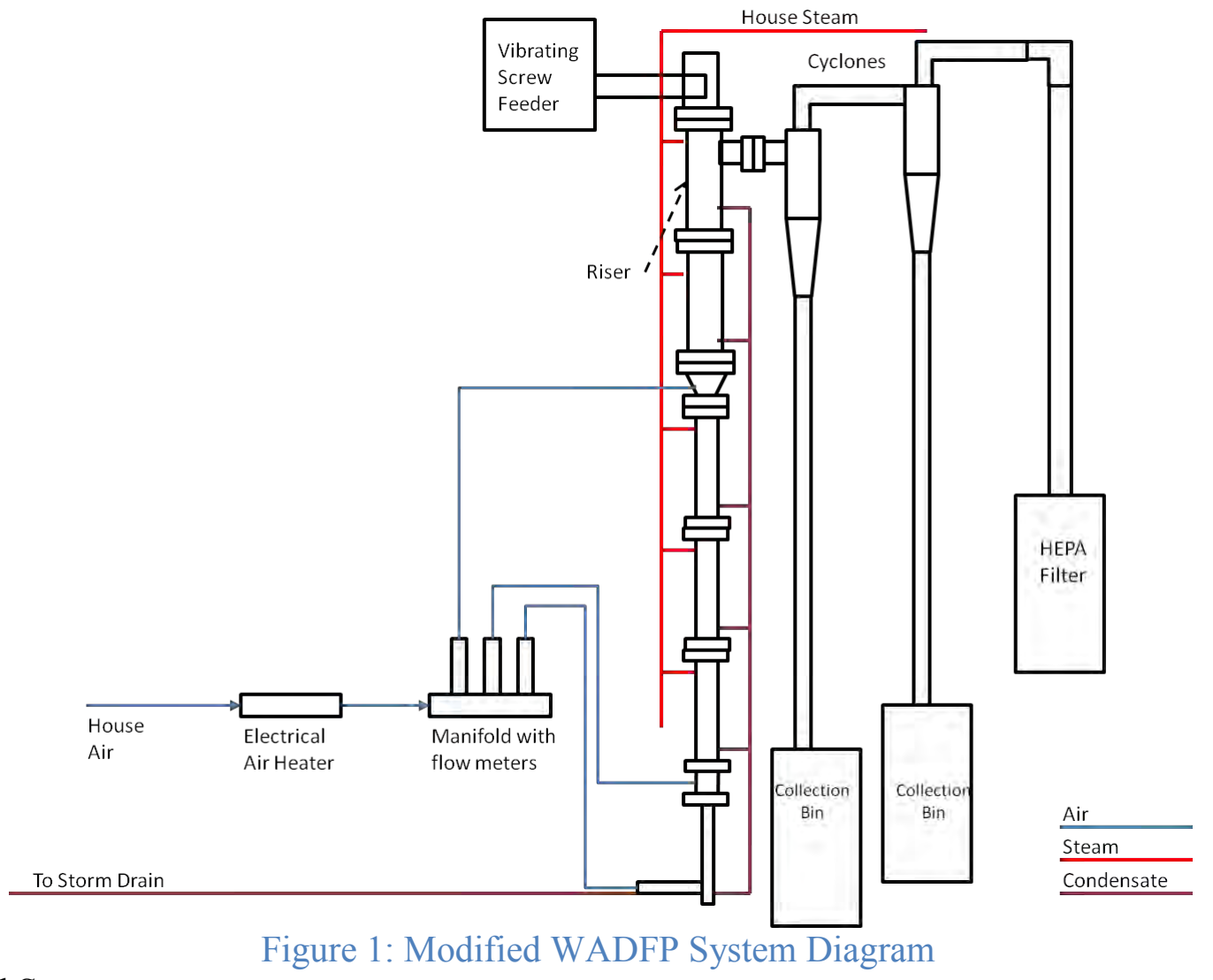

Solids Feed System

The original solids system has been replaced with an entirely new feed system that incorporates a custom-built vibrating feed hopper and motor-driven horizontal feed screw. (See Fig. 2) The shaker unit attached to the feed hopper is used to induce vibrations along the walls of the hopper, which prevents wet coal particles from adhering to the surface. This prevents the clumping together of coal (which led to clogs in the previous hopper system), and ensures a steady stream of solids material to the 3-inch diameter horizontal feed screw located below the hopper. The solids material is them transferred along the approximately 40 -inch long feed screw into the top of the fluidized bed riser, where it is then allowed to drop into the system along its central axis. As with the previous feed system, the screw is motor-driven and can be operated over a speed range of 0-60 rpm. A 20-ft tall masonry scaffold with a 5 -ft $\mathrm{x} 7$-ft platform was erected next to the riser system to provide structural support for the new feed hopper, as well as to provide a working area for system operators to fill and operate the new feed system. 


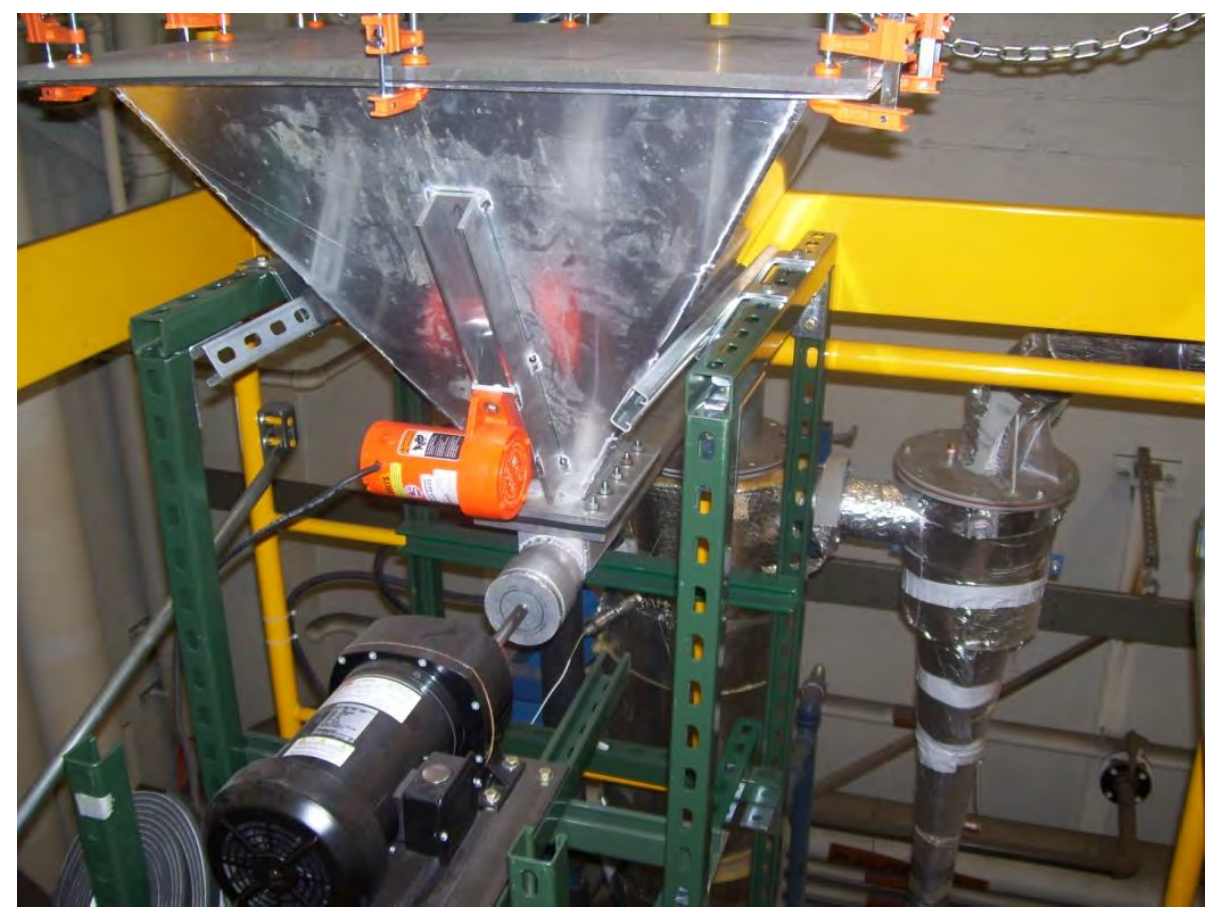

Figure 2: New vilbrating screw coal feed system

\section{Air Pre-Heating System}

As previously mentioned, the original heat exchanger used to pre-heat the fluidizing air prior to entering the WADFP unit allowed the operator little control over the resulting air temperature, and the high pressure losses across the heat exchanger severely restricted the maximum air flow rates. To address these issues, the heat exchanger was replaced with a threaded inline electrical resistance heater. In essence, this heater consists of an 18-inch long section of $2 \frac{1}{2}$-inch inside diameter pipe that is threaded on either end. Located inside this pipe section is a 480V, 3-phase, $22 \mathrm{amp}$ electrical heating element capable of generating $18.5 \mathrm{~kW}$ of thermal energy. This electrical heater is controlled by a temperature control system consisting of a SMART-3 temperature controller with a PID feedback control system and a Silicone-Controlled Rectifier (SCR). The temperature controller can be programmed to maintain a specified temperature for the air exiting the heater. A k-type thermocouple provides the controller with the actual air temperature. The controller then sends a 4-20 mA signal to the SCR to adjust the voltage applied to the heater. Over the course of the tests run with this new air heating system, it was found that the temperature control was able to maintain an air temperature within $+/-2^{\circ} \mathrm{F}$ of the desired set temperature. In addition, the maximum pressure drop across the heating element was only 7 psi, which had little to no effect on the maximum airflow rates into the system. At an operating air temperature of $220^{\circ} \mathrm{F}$, it was determined that a single heater could handle a volumetric air flow rate of $300 \mathrm{scfm}$. If higher temperatures and airflow rates would be desired, this could be addressed by use of multiple heating elements in series. 


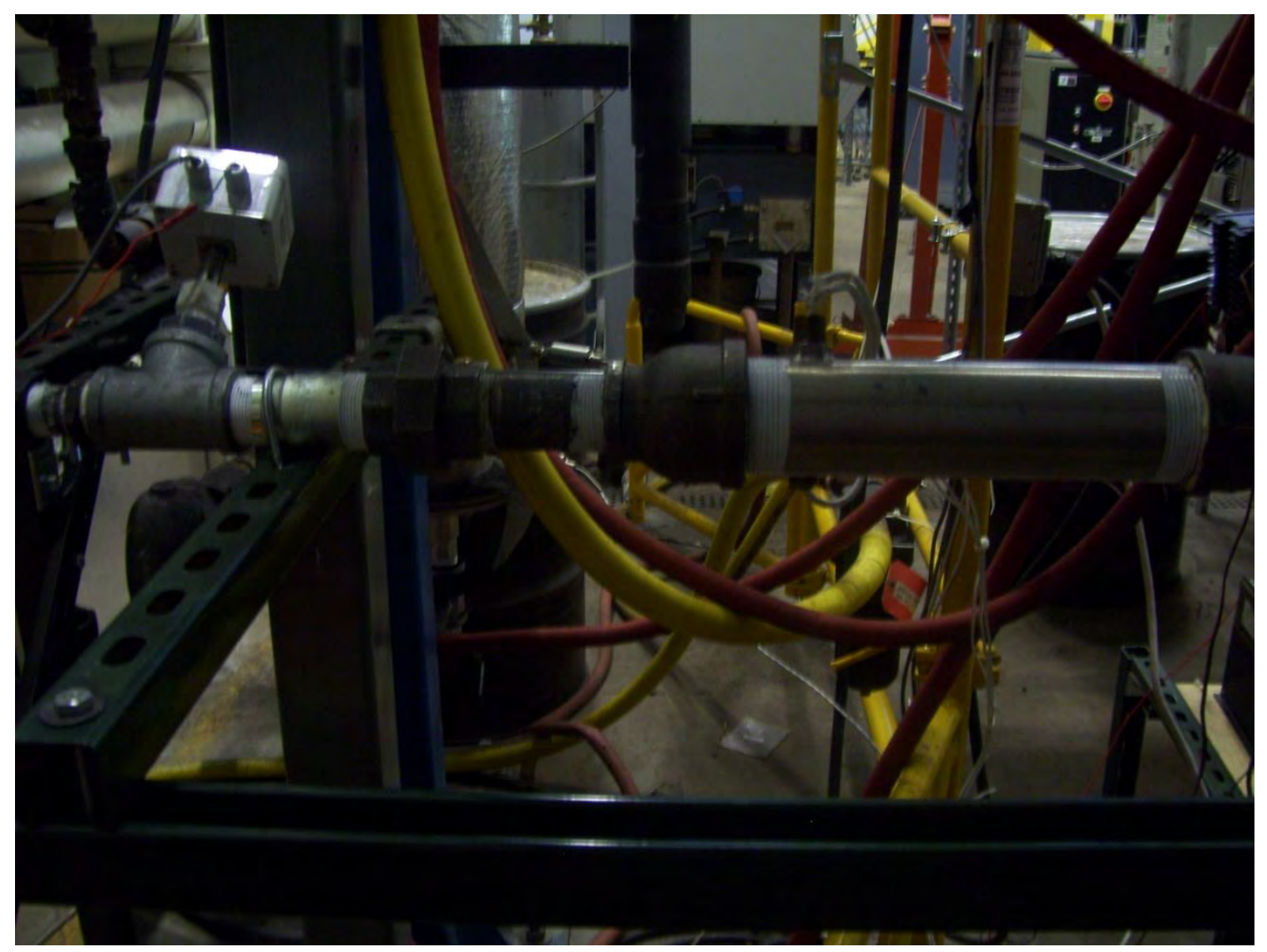

Figure 3: Electrical Air Heater

Secondary Cyclone

To improve the efficiency of capturing small particulate sizes $(<100 \mu \mathrm{m})$, a secondary cyclone was installed within the WADFP exhaust gas stream. The secondary cyclone has a smaller gas inlet and exit ports than the primary cyclone (2-inch diameter instead of 4-inch). This forces an acceleration of the exhaust entering the cyclone and increases the radial acceleration forces acting upon the entrained particles. This has the effect of forcing more particles to the inside wall of the cyclone, leading to higher collection rates of small particles. During experimental runs, it was found that the secondary cyclone was able to collect approximately an additional $5-7 \%$ of the dried coal particles over that collected with the original single cyclone configuration.

\section{HEPA Filtration System}

Finally, the original water filtration system was completely replaced with a filter box. A 2-ft x 2-ft, 3$\mathrm{ft}$ tall metal frame was fabricated. To the top, bottom and 3 sides of this frame were bolted clear acrylic sheeting. To the final side was bolted a 2-ft x 3-ft HEPA filter. This filter is listed as 99.97\% efficient at capturing particles as small as $0.3 \mu \mathrm{m}$ in diameter, and has a maximum air flow rate of 90 $1 \mathrm{fm}$ (linear feet per minute). The system is designed so that additional filters (up to 3 ) can be added if more airflow is necessary. 


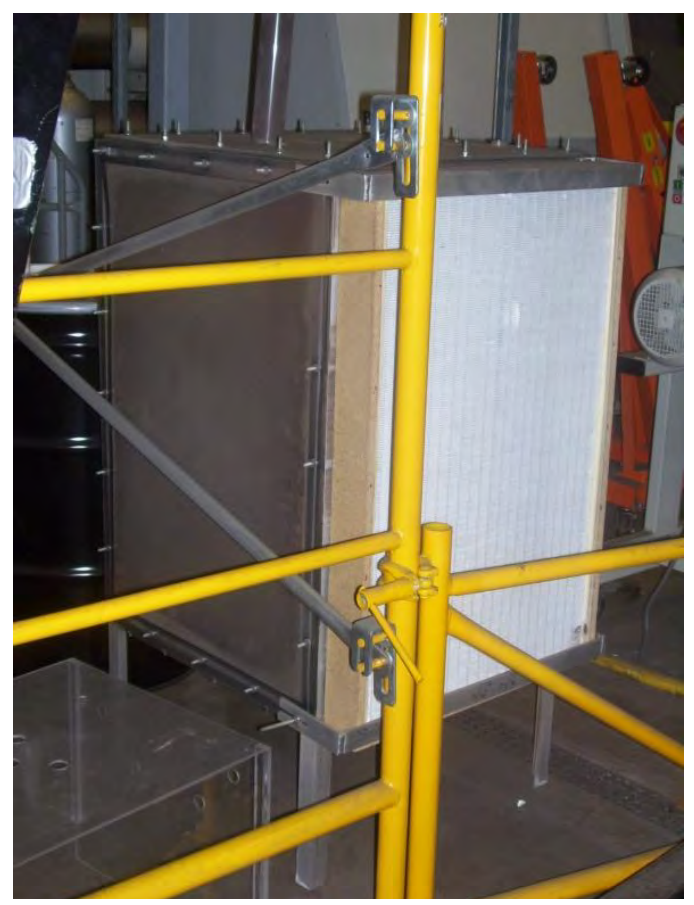

Figure 4: HEPA filtration system

\section{RESULTS}

In order to verify the effectiveness of the system modifications detailed in the previous section, a number of additional tests were completed. Table 1 below lists the feed rate of wet coal and the superficial gas velocity within the riser for each test condition. For each of the test conditions listed, the fluidization gas (air) was heated to $220^{\circ} \mathrm{F}$ prior to entering the WADFP riser system.

\begin{tabular}{|c|c|}
\hline Feed Rate of Wet Coal $(\mathrm{kg} / \mathrm{min})$ & Superficial Gas Velocity $(\mathrm{m} / \mathrm{s})$ \\
\hline $0.19(0.4125 \mathrm{lb} / \mathrm{min})$ & 1.38 \\
\hline$“$ & 1.66 \\
\hline “ & 1.93 \\
\hline $0.75(1.65 \mathrm{lb} / \mathrm{min})$ & 1.38 \\
\hline " & 1.69 \\
\hline " & 2.20 \\
\hline
\end{tabular}

\section{Table 1: Test Conditions}

For each of the test conditions listed above, samples of the feedstock and the dried product collected by the two cyclones were subjected to both proximate and calorimetry analysis. Table 2 provides data on the average moisture content and higher heating values of the feedstock and resulting dried product for each of the specified test conditions.

As can be seen from Table 2, there is a significant reduction of moisture, as well as a significant increase in the higher heating value (HHV) between the initial feedstock samples and the samples collected from the two cyclones for each test condition. Interestingly enough, upon further examination of these results, two distinctly different trends can be seen in this data. The first trend is that the final moisture content of the material collected from cyclone \#1 is greater than that of the material collected from cyclone \#2. However, even though there is more moisture remaining in the cyclone \#1 samples, those same samples also have greater HHV values. One possible explanation for this is that the smaller particles that are collected by the secondary cyclone contain a larger percentage 
of non-combustible, non-volatile material, which would lead to greater ash content. This is supported by the data presented in Table 3 .

\begin{tabular}{|c|c|c|c|c|c|c|c|}
\hline $\begin{array}{c}\text { Feed Rate } \\
(\mathrm{kg} / \mathrm{min})\end{array}$ & $\begin{array}{c}\text { ugas } \\
(\mathrm{m} / \mathrm{s})\end{array}$ & \multicolumn{3}{|c|}{$\begin{array}{c}\text { Moisture } \\
(\%)\end{array}$} & \multicolumn{3}{c|}{$\begin{array}{c}\text { HHV } \\
(\mathrm{btu} / \mathrm{lb})\end{array}$} \\
\hline & & initial & cyclone 1 & cyclone 2 & initial & $\begin{array}{c}\text { cyclone } \\
1\end{array}$ & cyclone 2 \\
\hline 0.19 & 1.38 & 28.3 & 11.9 & 7.3 & 7807.6 & 9614.7 & 8499.4 \\
\hline$"$ & 1.66 & 28.3 & 11.7 & 8.2 & 7807.6 & 9350.1 & 8583.4 \\
\hline$"$ & 1.93 & 28.3 & 13.3 & 9.0 & 7807.6 & 9460.3 & 8644.4 \\
\hline & & & & & & & \\
\hline 0.75 & 1.38 & 28.2 & 17.5 & 8.8 & 7839.1 & 9005.4 & 8736.9 \\
\hline$"$ & 1.69 & 28.2 & 17.9 & 8.6 & 7839.1 & 8734.0 & 8503.8 \\
\hline$"$ & 2.20 & 29.0 & 19.8 & 8.9 & 7719.4 & 8651.4 & 8375.1 \\
\hline
\end{tabular}

Table 2: Average Sample Moisture Percentages and Higher Heating Values

\begin{tabular}{|c|r|r|r|r|}
\hline $\begin{array}{c}\text { Feed Rate } \\
(\mathrm{kg} / \mathrm{min})\end{array}$ & \multicolumn{1}{|c|}{$\begin{array}{c}\text { ugas } \\
(\mathrm{m} / \mathrm{s})\end{array}$} & \multicolumn{3}{|c|}{$\begin{array}{c}\text { Ash Content } \\
(\%)\end{array}$} \\
\hline & & initial & cyclone 1 & cyclone 2 \\
\hline 0.19 & 1.38 & 9.81 & 12.76 & 23.00 \\
\hline$"$ & 1.66 & 9.81 & 15.25 & 20.96 \\
\hline$"$ & 1.93 & 9.81 & 11.02 & 20.34 \\
\hline & & & & \\
\hline 0.75 & 1.38 & 9.79 & 12.17 & 19.63 \\
\hline$"$ & 1.69 & 9.79 & 12.55 & 20.25 \\
\hline$"$ & 2.20 & 9.65 & 11.39 & 20.61 \\
\hline
\end{tabular}

Table 3: Average Sample Ash Contents

As can be seen in Table 3, on average, there is approximately an $8 \%$ difference in ash content between the material collected in the primary (cyclone 1) and the secondary (cyclone 2) cyclones. This seems to suggest that, for the coal used in this portion of the study (the Texas lignite), more of the ash producing material is concentrated at smaller particle sizes. Another possible explanation for this observed difference in ash content is that a larger amount of moisture was removed from the material in the secondary cyclone. While this is certainly a contributing factor, it seems to be a minor one given the fact that similar ash contents were found in the primary cyclone for the two feed rates, yet their moisture contents differed by approximately $6 \%$.

\section{CONCLUSIONS AND RECOMMENDATIONS}

The purpose of the work detailed in this report was to address the system deficiencies noted in the prior final report that was submitted in 2010. There were a total of 4 of these that were addressed. The original feed system which supplied solids material to the air feed line that supplied the air for the jet portion of the lower riser stage distributor was replaced by a vibrating hopper/horizontal feed screw system that introduces solids material into the top of the upper riser stage. The heat exchanger that was previously used to pre-heat the fluidizing air was replaced with an electrical resistance heating system in order to address flow rate restrictions due to pressure losses. A secondary cyclone was installed to improve the separation of fine particulate matter from the gas stream exiting the dryer system. Finally, the original water filtration system was replaced with a large HEPA filter that both eliminated release 
of particles into the laboratory environment, and eliminated the pressure fluctuations that were induced by the formation of bubbles in the water column.

Analysis of the data obtained from the experimental runs conducted with the new system configuration shows that significant levels of de-watering (or drying) of coal fines is possible in a warm air fluidized bed system. The reduction in moisture content in low rank "steam" coal has many advantages. The first of these advantages is a significant upgrade in the energy density of the coal, as shown by the increases in higher heating values (HHV) between the wet feedstock and the dried coal. The second advantage is a reduction in the thermal efficiency of combustion systems utilizing this coal for generation of energy. Less moisture in the feedstock means less energy lost to evaporation of moisture and more energy available for steam generation. A third and final advantage is that coal with lower moisture content has fewer inherent materials handling issues.

It was, however, noted during operation of the system that there was a significant decrease in the temperature of the air exiting the riser system once the feed of wet coal was started. The magnitude of the temperature decrease varied as a function of the feed rate of solids and mass flow rate of heated air entering the system. This decrease in temperature had the effect of decreasing the thermal energy available for the drying process. This can be seen when comparing the average final moisture contents of the two solids feed rates displayed in Table A-2. It is assumed that better control of the bed temperature within the WADFP riser can prevent this temperature loss and lead to enhanced drying results. With this in mind, it is recommended that any future work incorporates a temperature feedback control system that adjusts the temperature of the pre-heated air in order to maintain the desired internal bed temperature. This would have been addressed as part of this study if there had been additional time and funding to do so.

\section{PUBLICATIONS}

Rowan,S. "Analysis and Scaling of Two-Stage Fluidized Bed for Drying of Fine Coal Particles Using Shannon Entropy, Thermodynamic Exergy, and Statistical Methods" PhD. dissertation, WVU 2010.

Johnson, E. and Rowan, S. "Effect of Secondary Air Injection Upon the Fluidization Characteristics of the Lower Stage in a Two-Stage Variable -Area Fluidized Bed Riser", submitted to the International Conference on Circulation Fluidized Bed Technology CFB 10, Oregon, May 2011.

The following abstract has been submitted to the 2012 Pittsburgh Coal Conference for consideration:

Rowan, S. and Johnson, E. "Evaluation of the Performance of a Two-Stage, Warm Air Dryer for Fine Coal Particles".

\section{REFERENCES}

1. Johnson, E. and Kang, B. "Annual Technical Progress Report for CAST III, Warm Air Drying of Fine Particles, Report \#1, 9/29/07.

2. Johnson, E. and Kang, B. “Annual Technical Progress Report for CAST III, Warm Air Drying of Fine Particles, Report \#2, 11/15/10.

3. Rowan, Steve "Analysis and Scaling of a Two-Stage Fluidized Bed for Drying of Fine Coal Particles Using Shannon Entropy, Thermodynamic Exergy, and Statistical Methods" Ph. D Dissertation WVU,2010. A copy of this PhD. dissertation is attached. 


\title{
Analysis and Scaling of a Two-Stage Fluidized Bed for Drying of Fine Coal Particles Using Shannon Entropy, Thermodynamic Exergy and Statistical Methods
}

\author{
Steven Lee Rowan \\ Dissertation Submitted to the \\ College of Engineering and Mineral Resources \\ at \\ West Virginia University \\ In Partial Fulfillment of Requirements \\ For the Degree of
}

\author{
Doctor of Philosophy \\ In \\ Mechanical Engineering \\ Eric Johnson, Ph.D. Chair \\ Bruce Kang, Ph.D. John \\ Kuhlman, Ph.D. \\ Matthew Valenti, Ph.D. \\ Frank Saus, Ph.D. \\ Lawrence Shadle, Ph.D. \\ Department of Mechanical and Aerospace Engineering
}

Morgantown, West Virginia

2010

Keywords: Coal Drying, Fluidization, Shannon Entropy, Exergy

Copyright 2010 Steven L. Rowan 


\section{Acknowledgements}

I would like to express my appreciation and gratitude to my advisor, Dr. Eric Johnson. I Can't begin to express how fortunate I consider myself to be for his mentorship during my time studying under him. It would not be an exaggeration to say that his influences have transformed me into a much better-rounded engineer.

I am likewise thankful to both Dr. Bruce Kang and Dr. Frank Saus for being there to provide the proverbial boot to the rear when I needed extra motivation, as well as their financial support without which much of this research may never have taken place.

I would like to thank the remaining members of my dissertation committee: Dr. John Kuhlman, Dr. Lawrence Shadle and Dr. Matthew Valente.

I would also like to express my thanks to everyone at Wilson Works, Chuck Coleman and Clifford Judy from the MAE electrical and machine shops, as well as Liviu Magean and everyone in the chemical engineering department's analytical lab.

I would like to thank my fellow graduate students: Eric, Jackson, Rajiv and Femi. Without your assistance I do not know where I'd be today.

Most of all I'd like to thank my wife, Melissa. Without your support and understanding none of this would have been possible.

Finally, I would like to say thank you to all of the faculty, secretaries and other staff members in the Mechanical and Aerospace Engineering Department for their help and support over the years. 


\begin{abstract}
Liquid water (moisture) in coal causes a number of economic and environmental issues for the mining and electrical power generation industries. Coal preparation plants utilize large amounts of water for cleaning coal and removing unwanted materials such as clay, sulfur, pyrite and mercury. After the cleaning process, it is necessary to separate as much of the water from the coal as possible. Unfortunately, current dewatering techniques are not effective with particle sizes below $150 \mu \mathrm{m}$, which compromises $6-8 \%$ of the total energy found in mined coal. In most cases, these fine coal particles end up in slurry waste ponds. Additionally, coal-fired power plants typically purchase coal on a per btu of heating value basis. In many cases, coal can reabsorb moisture during transportation from mine to power plant, and some pulverized coal plants operate with moisture contents as high as $40 \%$. It has been shown previously that a $1 \%$ reduction in the moisture content of coal leads to approximately a $0.1 \%$ increase in the heating value of coal.
\end{abstract}

To address this issue, two two-stage, variable-area fluidized bed prototypes have been constructed. The first bed is a steam-jacketed warm-air dryer for fine particles (WADFP) with a lower riser stage bed diameter of 5" and an upper riser stage bed diameter of 8". The second is a half-scale transparent model. One of the primary objectives of this study is to utilize the scalemodel fluidized bed to study the unique fluidization characteristics of a large scale fluidized bed consisting of a lower small-diameter riser stage and an upper large-diameter riser stage with secondary air injection. The second objective of this study is to develop a simplified set of scaling relationships that allow for the scaling of fluidization regime transition velocities between different fluidized beds. The final objective of this study is to perform a thermodynamic exergy analysis on the fluidized bed drying process.

Preliminary test results show a similar trend of secondary air injection being the controlling factor of fluidization regime determination for both the large scale dryer and the small scale model riser. A proposed scaling method using riser area-normalized mass flow rates resulted in good matching between the two systems. Experiments with fluidizing wet coal also resulted in significant reductions in the moisture content of coal after drying. 


\section{Table of Contents}

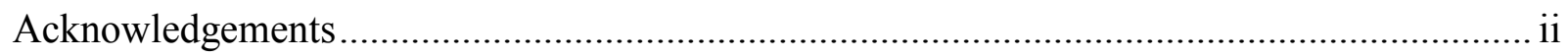

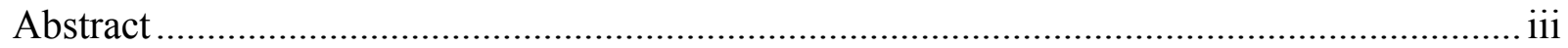

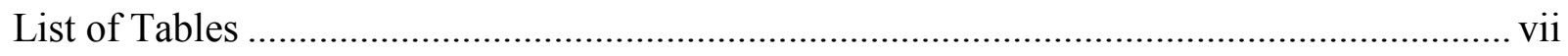

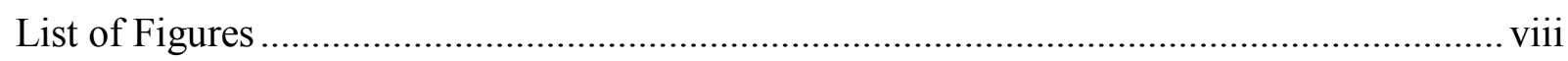

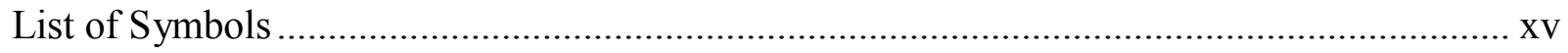

List of Video Files ......................................................................................................... xvii

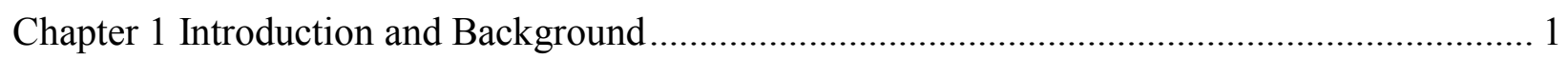

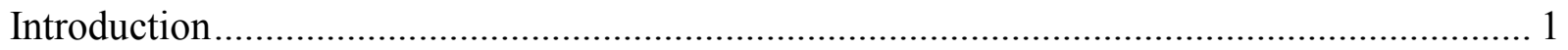

Statement of Objective:

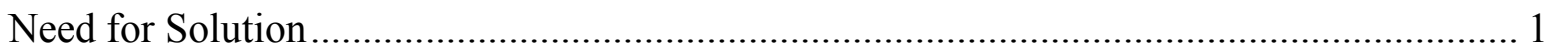

Experimental Approach ……………………………………………………………. 3

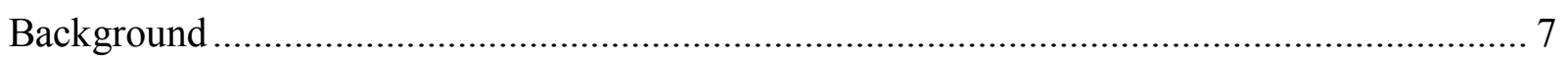

General types and applications of fluidized bed dryers .................................................. 7

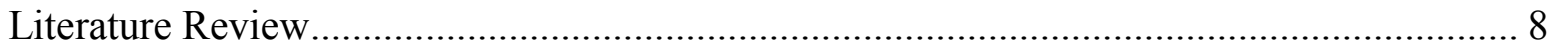

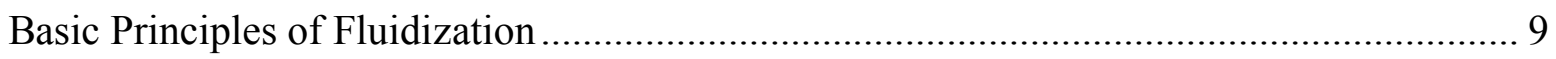

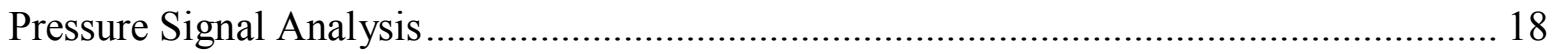

Chapter 2 Theoretical Approach for Fluidized Bed Drying ……………………….................. 28

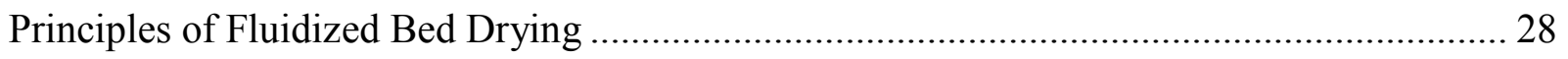

Droplet evaporation/film drying model ......................................................................... 30

Thermodynamic Analysis of Fluidized Bed Drying ................................................................ 35

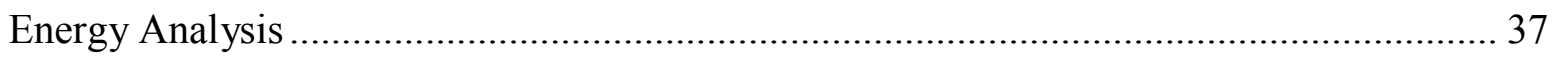

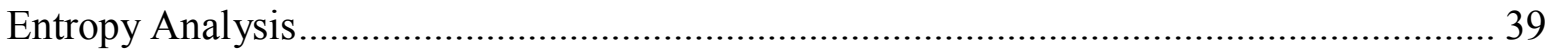

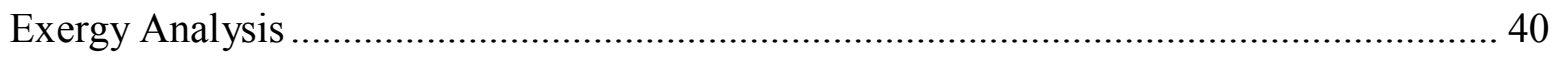

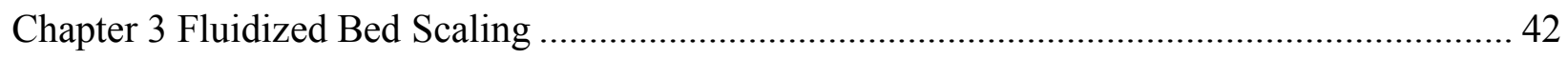

Hydrodynamic Scaling Relationships ............................................................................. 42

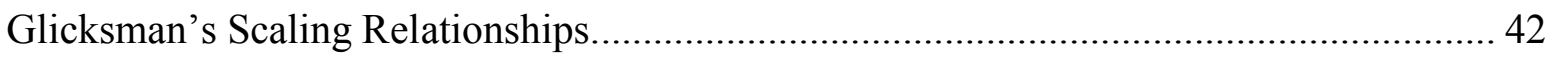

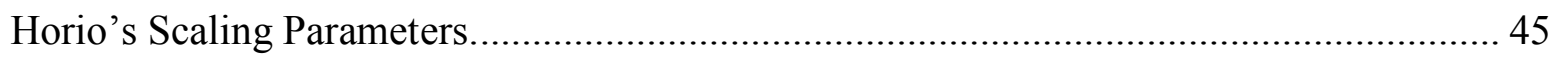

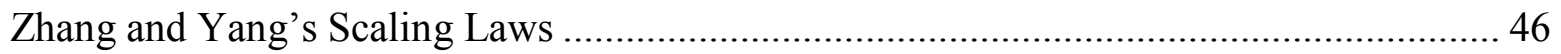

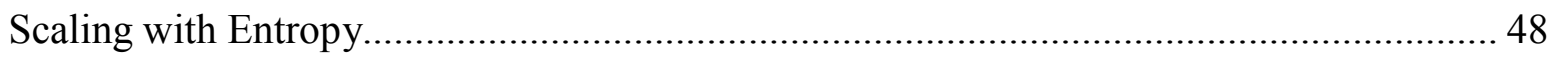

Experimental Verification Techniques of Scaling Laws ........................................................ 48 


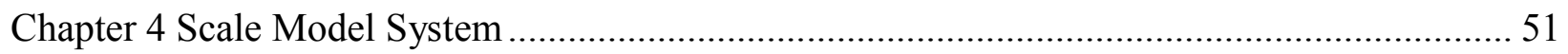

General Design Concepts................................................................................................ 51

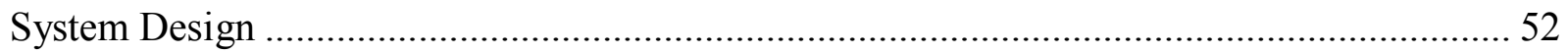

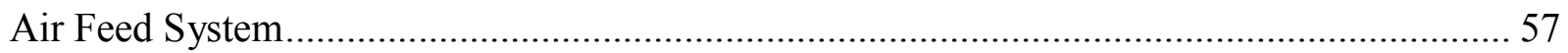

Data Acquisition System and Instrumentation ……………….......................................... 58

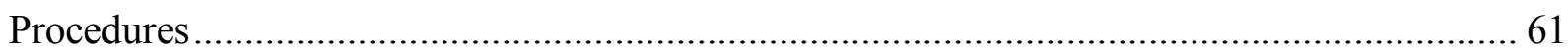

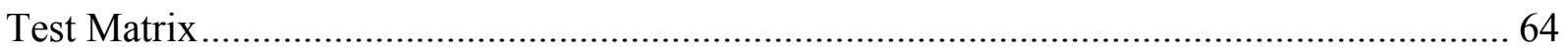

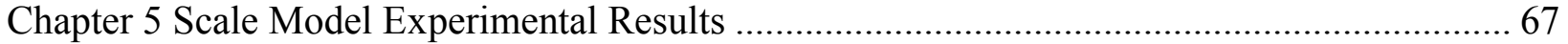

Lower Riser Stage Turbulent Fluidization Mapping and Characterization ............................... 67

Upper Riser Stage Fast Fluidization Mapping and Characterization....................................... 79

Effects of Secondary Air Injection on the Lower Riser Stage Fluidization .............................. 86

Comparison of Standard Deviation of Pressure with Riser Stage Pressure Drop..................... 97

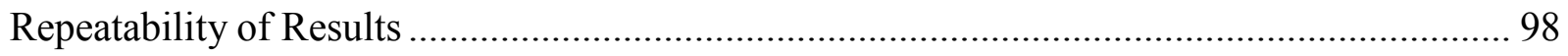

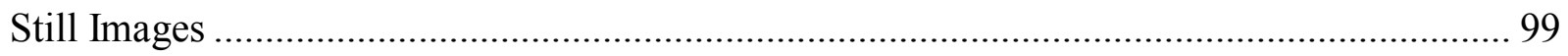

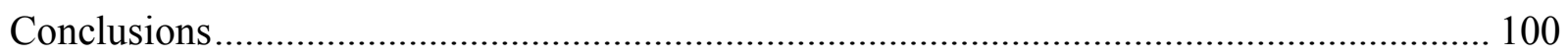

Chapter 6 Warm Air Dryer Experimental System .............................................................. 102

General Design Concepts............................................................................................... 102

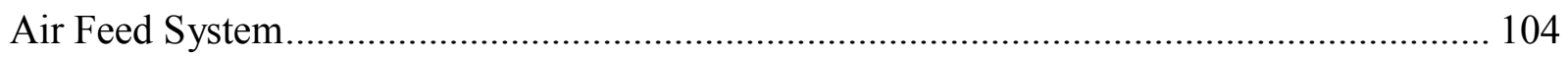

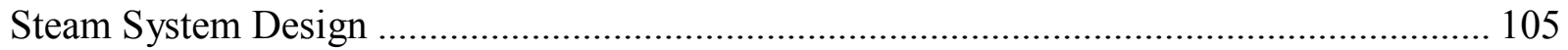

Data Acquisition System and Instrumentation .................................................................... 106

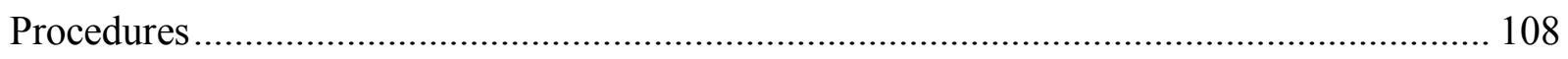

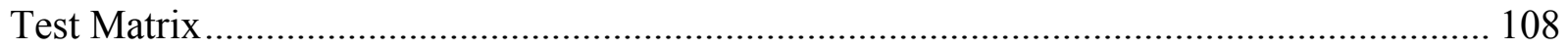

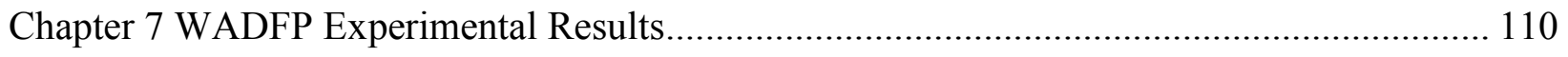

Lower Riser Stage Turbulent Fluidization Mapping and Characterization ............................ 110

Upper Riser Stage Fast Fluidization Mapping and Characterization...................................... 119

Effects of Secondary Air Injection on Lower Riser Stage Fluidization ................................ 124

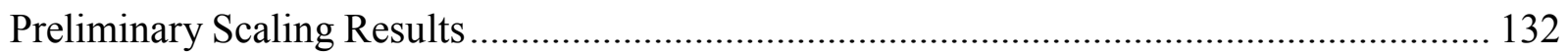

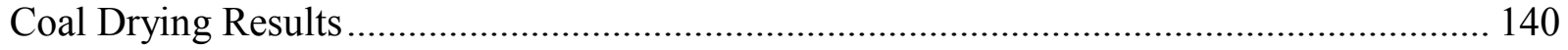

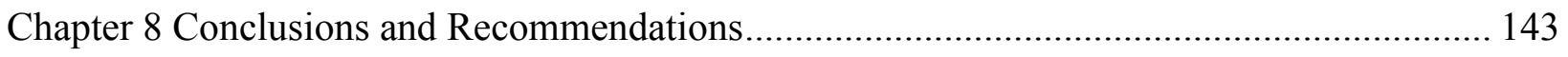

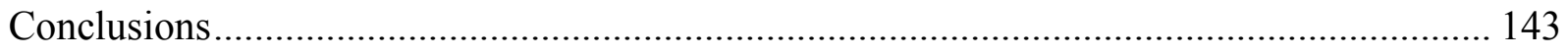

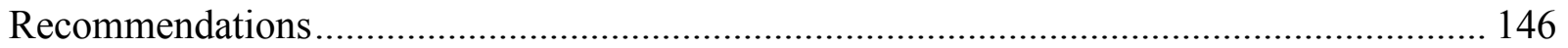


Chapter 9 Bibliography. 


\section{List of Tables}

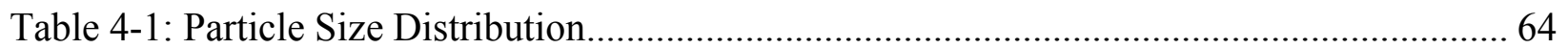

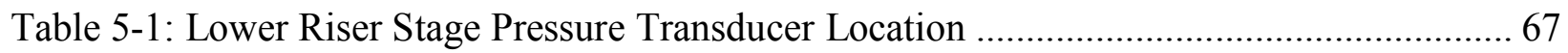

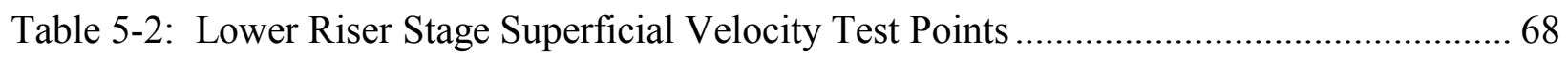

Table 5-3: Upper Riser Stage Superficial Velocity Test Points ……………………………..... 79

Table 5-4: Upper Riser Stage Pressure Transducer Location ..................................................... 80

Table 7-1: WADFP Lower Riser Stage Pressure Transducer Locations ................................... 110

Table 7-2: WADFP Lower Riser Stage Superficial Velocity Test Points ................................... 111

Table 7-3: WADFP Upper Riser Stage Pressure Transducer Location ......................................... 119

Table 7-4: WADFP Upper Riser Stage Superficial Velocity Test Points ................................... 119

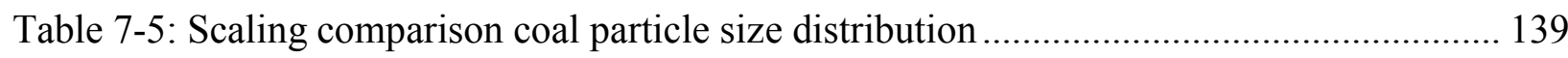

Table 7-6: Sub-bituminous Underflow Tailings Particle Size Distribution.................................. 141

Table 7-7: Coal Sample Proximate Analysis Moisture Content Results (* samples not ignitable at

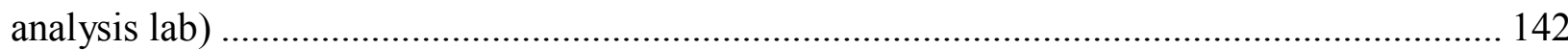

Table 7-8: WADFP Thermal and Exergy Efficiencies for Coal Drying Runs ........................... 142 


\section{List of Figures}

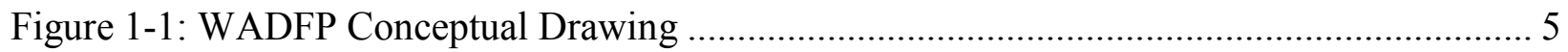

Figure 1-2: Effect of Secondary Air Injection on Specific Humidity.......................................... 7

Figure 1-3: Geldart's classification of fluidized particles [24] ................................................. 10

Figure 1-4: Dense fluidization regimes (a) Particulate; (b) Bubbling; (c) Turbulent; (d) Slugging;

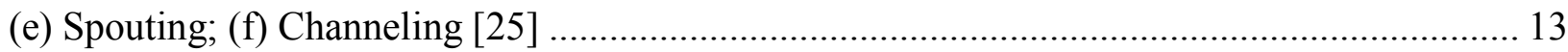

Figure 1-5: Variations of pressure drop per unit riser length with solids circulation rate and gas

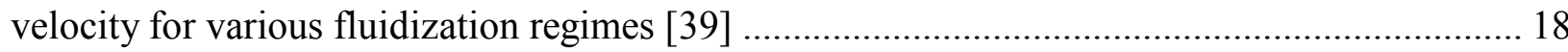

Figure 1-6: Variation of pressure fluctuations with velocity for dense-phase fluidization [40]... 19

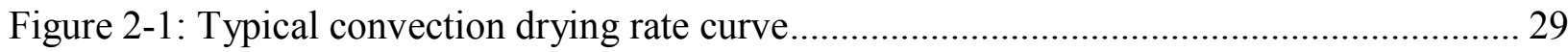

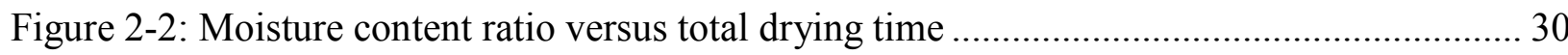

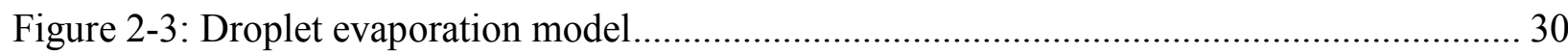

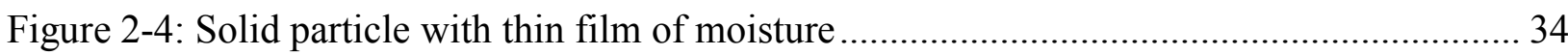

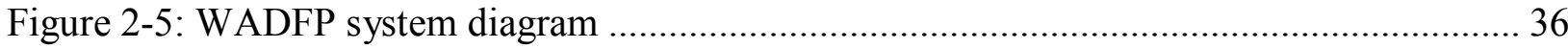

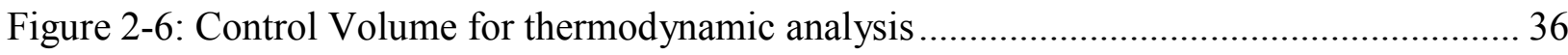

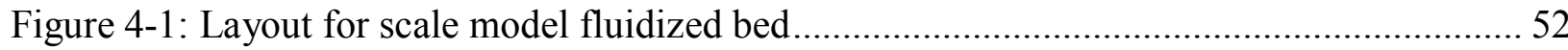

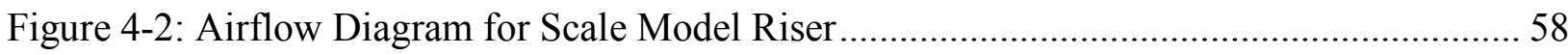

Figure 4-3: Instrumentation Diagram for Small Scale Riser .................................................................. 61

Figure 5-1 : Standard Deviation vs Superficial Velocity for Lower Riser Stage Pressures: (a) Lbottom (b) Lmid (c) Ltop; poly-dispersed sand, 3.5 psig feed hopper pressure, no secondary air

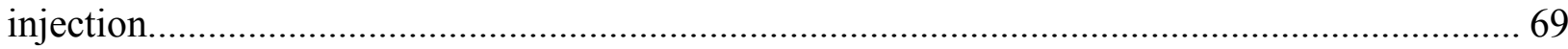

Figure 5-2: Skew vs Superficial Velocity for Lower Riser Stage Pressures: (a) Lbottom (b) Lmid (c) Ltop; poly-dispersed sand, 3.5 psig feed hopper pressure, no secondary air injection. .......... 70 Figure 5-3: Kurtosis vs Superficial Velocity for Lower Riser Stage Pressures: (a) Lbottom (b) Lmid (c) Ltop; poly-dispersed sand, 3.5 psig feed hopper pressure, no secondary air injection. 71 Figure 5-4: Shannon Entropy vs Superficial Velocity for Lower Riser Stage Pressures: (a) Lbottom (b) Lmid (c) Ltop; poly-dispersed sand, 3.5 psig feed hopper pressure, no secondary air

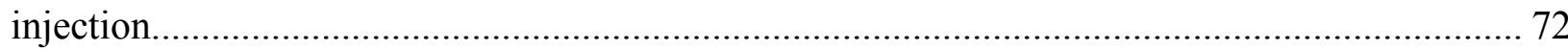

Figure 5-5: Autocorrelation of scale model Lbottom Pressure: poly-dispersed sand, 3.5 psig feed hopper pressure, no secondary air injection, $\mathrm{U}_{\mathrm{L}}=$ : (a) 0.57 (b) 0.76 (c) 1.14 (d) 1.52 (e) 1.90 (f) 2.29 (g) 2.67 (h) 3.05 (i) $3.43 \mathrm{~m} / \mathrm{s}$.

Figure 5-6: Autocorrelation of scale model Lmid Pressure: poly-dispersed sand, 3.5 psig feed hopper pressure, no secondary air injection, $\mathrm{U}_{\mathrm{L}}=$ : (a) 0.57 (b) 0.76 (c) 1.14 (d) 1.52 (e) 1.90 (f) 2.29 (g) 2.67 (h) 3.05 (i) $3.43 \mathrm{~m} / \mathrm{s}$. 75

Figure 5-7: Autocorrelation of scale model Ltop Pressure: poly-dispersed sand, $3.5 \mathrm{psig}$ feed hopper pressure, no secondary air injection, $\mathrm{U}_{\mathrm{L}}=$ : (a) 0.57 (b) 0.76 (c) 1.14 (d) 1.52 (e) 1.90 (f) 2.29 (g) 2.67 (h) 3.05 (i) $3.43 \mathrm{~m} / \mathrm{s}$. 76

Figure 5-8: Mutual Information of scale model Lbottom Pressure: poly-dispersed sand, $3.5 \mathrm{psig}$ feed hopper pressure, no secondary air injection, $U_{L}=$ : (a) 0.57 (b) 0.76 (c) 1.14 (d) 1.52 (e) 1.90 (f) 2.29 (g) 2.67 (h) 3.05 (i) $3.43 \mathrm{~m} / \mathrm{s}$. 
Figure 5-9: Mutual Information of scale model Lmid Pressure: poly-dispersed sand, 3.5 psig feed hopper pressure, no secondary air injection, $\mathrm{U}_{\mathrm{L}}=$ : (a) 0.57 (b) 0.76 (c) 1.14 (d) 1.52 (e) 1.90 (f) 2.29 (g) 2.67 (h) 3.05 (i) $3.43 \mathrm{~m} / \mathrm{s}$.

Figure 5-10: Mutual Information of scale model Ltop Pressure: poly-dispersed sand, 3.5 psig feed hopper pressure, no secondary air injection, $U_{L}=$ : (a) 0.57 (b) 0.76 (c) 1.14 (d) 1.52 (e) 1.90 (f) 2.29 (g) 2.67 (h) 3.05 (i) $3.43 \mathrm{~m} / \mathrm{s}$. 77

Figure 5-11: Power Spectral Density Plot of scale model Lbottom Pressure: poly-dispersed sand, 3.5 psig feed hopper pressure, no secondary air injection, $U_{L}=:$ (a) 0.57 (b) 0.76 (c) 1.14 (d) 1.52 (e) 1.90 (f) 2.29 (g) 2.67 (h) 3.05 (i) $3.43 \mathrm{~m} / \mathrm{s}$. 78

Figure 5-12: Power Spectral Density Plot of scale model Lmid Pressure: poly-dispersed sand, 3.5 psig feed hopper pressure, no secondary air injection, $U_{L}=$ : (a) 0.57 (b) 0.76 (c) 1.14 (d) 1.52

(e) 1.90 (f) 2.29 (g) 2.67 (h) 3.05 (i) $3.43 \mathrm{~m} / \mathrm{s}$. 78

Figure 5-13: Power Spectral Density Plot of scale model Ltop Pressure: poly-dispersed sand, 3.5 psig feed hopper pressure, no secondary air injection, $\mathrm{U}_{\mathrm{L}}=$ : (a) 0.57 (b) 0.76 (c) 1.14 (d) 1.52 (e) 1.90 (f) 2.29 (g) 2.67 (h) 3.05 (i) $3.43 \mathrm{~m} / \mathrm{s}$. 79

Figure 5-14: Standard Deviation of model upper riser stage pressures (a) Ubottom (b) Utop: poly-dispersed sand, feed hopper pressure $3.5 \mathrm{psig}, \mathrm{U}_{\mathrm{L}}=2.36 \mathrm{~m} / \mathrm{s}$..... 80

Figure 5-15: Skew of model upper riser stage pressures (a) Ubottom (b) Utop: poly-dispersed sand, feed hopper pressure $3.5 \mathrm{psig}, \mathrm{U}_{\mathrm{L}}=2.36 \mathrm{~m} / \mathrm{s}$.

Figure 5-16: Kurtosis of model upper riser stage pressures (a) Ubottom (b) Utop: poly-dispersed sand, feed hopper pressure $3.5 \mathrm{psig}, \mathrm{U}_{\mathrm{L}}=2.36 \mathrm{~m} / \mathrm{s}$. 81

Figure 5-17: Shannon Entropy of model upper riser stage pressures (a) Ubottom (b) Utop: polydispersed sand, feed hopper pressure $3.5 \mathrm{psig}, \mathrm{U}_{\mathrm{L}}=2.36 \mathrm{~m} / \mathrm{s}$...... 82 Figure 5-18: Autocorrelation of model Ubottom pressure: poly-dispersed sand, 3.5 psig feed hopper pressure, $\mathrm{U}_{\mathrm{L}}=2.36 \mathrm{~m} / \mathrm{s}, \mathrm{U}_{\mathrm{U}}=$ : (a) 1.42 (b) 1.74 (c) 2.06 (d) 2.38 (e) 2.70 (f) 3.02 (g) $3.35 \mathrm{~m} / \mathrm{s}$

Figure 5-19: Autocorrelation of model Utop pressure: poly-dispersed sand, 3.5 psig feed hopper pressure, $\mathrm{U}_{\mathrm{L}}=2.36 \mathrm{~m} / \mathrm{s}, \mathrm{U}_{\mathrm{U}}=$ : (a) 1.42 (b) 1.74 (c) 2.06 (d) 2.38 (e) 2.70 (f) 3.02 (g) $3.35 \mathrm{~m} / \mathrm{s}$.

Figure 5-20: Mutual Information Function of model Ubottom pressure: poly-dispersed sand, 3.5 psig feed hopper pressure, $\mathrm{U}_{\mathrm{L}}=2.36 \mathrm{~m} / \mathrm{s}, \mathrm{U}_{\mathrm{U}}=$ : (a) 1.42 (b) 1.74 (c) 2.06 (d) 2.38 (e) 2.70 (f) $3.02(\mathrm{~g}) 3.35 \mathrm{~m} / \mathrm{s}$.

Figure 5-21: Mutual Information Function of model Utop pressure: poly-dispersed sand, 3.5 psig feed hopper pressure, $\mathrm{U}_{\mathrm{L}}=2.36 \mathrm{~m} / \mathrm{s}, \mathrm{U}_{\mathrm{U}}=$ : (a) 1.42 (b) 1.74 (c) 2.06 (d) 2.38 (e) 2.70 (f) 3.02 (g) $3.35 \mathrm{~m} / \mathrm{s}$.

Figure 5-22: Spectral Density Plot for model Ubottom pressure: poly-dispersed sand, 3.5 psig feed hopper pressure, $\mathrm{U}_{\mathrm{L}}=2.36 \mathrm{~m} / \mathrm{s}, \mathrm{U}_{\mathrm{U}}=$ : (a) 1.42 (b) 1.74 (c) 2.06 (d) 2.38 (e) 2.70 (f) 3.02 (g) $3.35 \mathrm{~m} / \mathrm{s}$. 
Figure 5-23: Spectral Density Plot for model Utop pressure: poly-dispersed sand, 3.5 psig feed hopper pressure, $\mathrm{U}_{\mathrm{L}}=2.36 \mathrm{~m} / \mathrm{s}, \mathrm{U}_{\mathrm{U}}=$ : (a) 1.42 (b) 1.74 (c) 2.06 (d) 2.38 (e) 2.70 (f) 3.02 (g)

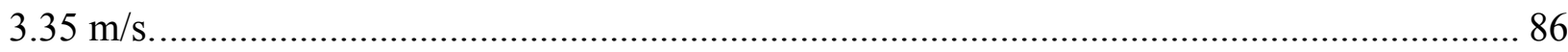
Figure 5-24: Effect of varying secondary air injection on lower riser stage average pressures (a) Lbottom (b) Lmid $\quad$ (c) Ltop: poly-dispersed sand, 3.5 psig feed hopper pressure, $\mathrm{U}_{\mathrm{L}}=2.36$ $\mathrm{m} / \mathrm{s}$.

Figure 5-25: Effect of secondary air injection on lower riser voidage profile: poly-dispersed sand, $3.5 \mathrm{psig}$ feed hopper pressure, $\mathrm{U}_{\mathrm{L}}=2.36 \mathrm{~m} / \mathrm{s}$. 88

Figure 5-26: Effects of varying secondary air injection on model lower riser stage standard deviation of pressures (a) Lbottom (b) Lmid (c) Ltop: poly-dispersed sand, 3.5 psig feed hopper pressure, $\mathrm{U}_{\mathrm{L}}=2.36 \mathrm{~m} / \mathrm{s}$. 89

Figure 5-27: Effects of varying secondary air injection on model lower riser stage skew of pressures (a) Lbottom (b) Lmid (c) Ltop: poly-dispersed sand, 3.5 psig feed hopper pressure, $\mathrm{U}_{\mathrm{L}}$ $=2.36 \mathrm{~m} / \mathrm{s}$. 90

Figure 5-28: Effects of varying secondary air injection on model lower riser stage kurtosis of pressures (a) Lbottom (b) Lmid (c) Ltop: poly-dispersed sand, 3.5 psig feed hopper pressure, $\mathrm{U}_{\mathrm{L}}$ $=2.36 \mathrm{~m} / \mathrm{s}$. 90

Figure 5-29: Effects of varying secondary air injection on model lower riser stage Shannon entropy of pressures (a) Lbottom (b) Lmid (c) Ltop: poly-dispersed sand, 3.5 psig feed hopper pressure, $\mathrm{U}_{\mathrm{L}}=2.36 \mathrm{~m} / \mathrm{s}$.

Figure 5-30: Autocorrelation plots for model Lbottom Pressure with Secondary Air Injection, poly-dispersed sand, 3.5 psig feed hopper pressure, $\mathrm{U}_{\mathrm{L}}=2.36 \mathrm{~m} / \mathrm{s}, \mathrm{U}_{\mathrm{U}}=$ : (a) 1.42 (b) 1.74 (c) 2.06 (d) 2.38 (e) 2.70 (f) 3.02 (g) $3.35 \mathrm{~m} / \mathrm{s}$. 93

Figure 5-31: Autocorrelation plots for model Lmid Pressure with Secondary Air Injection, polydispersed sand, 3.5 psig feed hopper pressure, $U_{\mathrm{L}}=2.36 \mathrm{~m} / \mathrm{s}, \mathrm{U}_{\mathrm{U}}=:$ (a) 1.42 (b) 1.74 (c) 2.06 (d) 2.38 (e) 2.70 (f) 3.02 (g) $3.35 \mathrm{~m} / \mathrm{s}$.

Figure 5-32: Autocorrelation plots for model Ltop Pressure with Secondary Air Injection, polydispersed sand, 3.5 psig feed hopper pressure, $U_{\mathrm{L}}=2.36 \mathrm{~m} / \mathrm{s}, \mathrm{U}_{\mathrm{U}}=:$ (a) 1.42 (b) 1.74 (c) 2.06 (d) 2.38 (e) 2.70 (f) 3.02 (g) $3.35 \mathrm{~m} / \mathrm{s}$.

Figure 5-33: Mutual Information plots for model Lbottom pressure with secondary air injection, poly-dispersed sand, 3.5 psig feed hopper pressure, $U_{\mathrm{L}}=2.36 \mathrm{~m} / \mathrm{s}, \mathrm{U}_{\mathrm{U}}=$ : (a) 1.42 (b) 1.74 (c) 2.06 (d) 2.38 (e) 2.70 (f) 3.02 (g) $3.35 \mathrm{~m} / \mathrm{s}$.

Figure 5-34: Mutual Information plots for model Lmid pressure with secondary air injection, poly-dispersed sand, 3.5 psig feed hopper pressure, $\mathrm{U}_{\mathrm{L}}=2.36 \mathrm{~m} / \mathrm{s}, \mathrm{U}_{\mathrm{U}}=$ : (a) 1.42 (b) 1.74 (c) 2.06 (d) 2.38 (e) 2.70 (f) 3.02 (g) $3.35 \mathrm{~m} / \mathrm{s}$.

Figure 5-35: Mutual Information plots for model Ltop pressure with secondary air injection, poly-dispersed sand, 3.5 psig feed hopper pressure, $U_{\mathrm{L}}=2.36 \mathrm{~m} / \mathrm{s}, \mathrm{U}_{\mathrm{U}}=$ : (a) 1.42 (b) 1.74 (c) 2.06 (d) 2.38 (e) 2.70 (f) 3.02 (g) $3.35 \mathrm{~m} / \mathrm{s}$. 95 
Figure 5-36: Spectral Density plots for model Lbottom pressure with secondary air injection, poly-dispersed sand, $3.5 \mathrm{psig}$ feed hopper pressure, $\mathrm{U}_{\mathrm{L}}=2.36 \mathrm{~m} / \mathrm{s}, \mathrm{U}_{\mathrm{U}}=$ : (a) 1.42 (b) 1.74 (c) 2.06 (d) 2.38 (e) 2.70 (f) 3.02 (g) $3.35 \mathrm{~m} / \mathrm{s}$. 96 Figure 5-37: Spectral Density plots for model Lmid pressure with secondary air injection, polydispersed sand, $3.5 \mathrm{psig}$ feed hopper pressure, $\mathrm{U}_{\mathrm{L}}=2.36 \mathrm{~m} / \mathrm{s}, \mathrm{U}_{\mathrm{U}}=$ : (a) 1.42 (b) 1.74 (c) 2.06 (d) 2.38 (e) 2.70 (f) 3.02 (g) $3.35 \mathrm{~m} / \mathrm{s}$. 96

Figure 5-38: Spectral Density plots for model Ltop pressure with secondary air injection, polydispersed sand, $3.5 \mathrm{psig}$ feed hopper pressure, $\mathrm{U}_{\mathrm{L}}=2.36 \mathrm{~m} / \mathrm{s}, \mathrm{U}_{\mathrm{U}}=$ : (a) 1.42 (b) 1.74 (c) 2.06 (d) 2.38 (e) 2.70 (f) 3.02 (g) $3.35 \mathrm{~m} / \mathrm{s}$.

Figure 5-39: Relationship between Lbottom standard deviation of pressure and lower riser stage pressure drop for (a) no secondary air injection (b) secondary air injection. 97 Figure 5-40: Relationship between Ubottom standard deviation of pressure and upper riser stage pressure drop for (a) no secondary air injection (b) secondary air injection. 98

Figure 5-41: Still images for lower riser stage mapping (a) slugging (b) turbulent (c) fast fluidization 99

Figure 5-42: Still pictures of upper riser stage mapping (a) turbulent (b) fast fluidization........ 100 Figure 6-1: Layout WADFP fluidized bed ........................................................................ 103

Figure 6-2: Airflow Diagram for WADFP Fluidized Bed ................................................. 105

Figure 6-3: WADFP Steam System Diagram ....................................................................... 106

Figure 6-4: Instrumentation Diagram for WADFP Fluidized Bed ......................................... 107 Figure 7-1: Standard deviation of WADFP lower riser stage pressures (a) Lbottom (b) Lmid (c) Ltop: poly-dispersed sand, feed rate $120 \mathrm{lb} / \mathrm{hr}$, no secondary air injection. 112 Figure 7-2: Shannon entropy of WADFP lower riser stage pressures (a) Lbottom (b) Lmid (c) Ltop: poly-dispersed sand, feed rate $120 \mathrm{lb} / \mathrm{hr}$, no secondary air injection. ........................... 113 Figure 7-3: Autocorrelation of WADFP Lbottom pressure, poly-dispersed sand, feed rate 120 $\mathrm{lb} / \mathrm{hr}$, no secondary air injection, $\mathrm{U}_{\mathrm{L}}=$ : (a) 1.43 (b) 1.65 (c) 1.93 (d) 2.22 (e) 2.79 (f) 3.50 (g) $4.10 \mathrm{~m} / \mathrm{s}$ 114 Figure 7-4: Autocorrelation of WADFP Lmid pressure, poly-dispersed sand, feed rate $120 \mathrm{lb} / \mathrm{hr}$, no secondary air injection, $\mathrm{U}_{\mathrm{L}}=$ : (a) 1.43 (b) 1.65 (c) 1.93 (d) 2.22 (e) 2.79 (f) 3.50 (g) $4.10 \mathrm{~m} / \mathrm{s}$.

Figure 7-5: Autocorrelation of WADFP Ltop pressure, poly-dispersed sand, feed rate $120 \mathrm{lb} / \mathrm{hr}$, no secondary air injection, $\mathrm{U}_{\mathrm{L}}=$ : (a) 1.43 (b) 1.65 (c) 1.93 (d) 2.22 (e) 2.79 (f) 3.50 (g) $4.10 \mathrm{~m} / \mathrm{s}$.

Figure 7-6: Mutual Information of WADFP Lbottom Pressure, poly-dispersed sand, feed rate 120 lb/hr, no secondary air injection, $\mathrm{U}_{\mathrm{L}}=$ : (a) 1.43 (b) 1.65 (c) 1.93 (d) 2.22 (e) 2.79 (f) 3.50 (g) $4.10 \mathrm{~m} / \mathrm{s}$. 116

Figure 7-7: Mutual Information of WADFP Lmid Pressure, poly-dispersed sand, feed rate 120 $\mathrm{lb} / \mathrm{hr}$, no secondary air injection, $\mathrm{U}_{\mathrm{L}}=$ : (a) 1.43 (b) 1.65 (c) 1.93 (d) 2.22 (e) 2.79 (f) 3.50 (g) $4.10 \mathrm{~m} / \mathrm{s}$ 
Figure 7-8: Mutual Information of WADFP Ltop Pressure, poly-dispersed sand, feed rate 120 $\mathrm{lb} / \mathrm{hr}$, no secondary air injection, $\mathrm{U}_{\mathrm{L}}=$ : (a) 1.43 (b) 1.65 (c) 1.93 (d) 2.22 (e) 2.79 (f) 3.50 (g) $4.10 \mathrm{~m} / \mathrm{s}$

Figure 7-9: Spectral Density Plots of WADFP Lbottom Pressure, poly-dispersed sand, feed rate $120 \mathrm{lb} / \mathrm{hr}$, no secondary air injection, $\mathrm{U}_{\mathrm{L}}=$ : (a) 1.43 (b) 1.65 (c) 1.93 (d) 2.22 (e) 2.79 (f) 3.50 (g) $4.10 \mathrm{~m} / \mathrm{s}$.

Figure 7-10: Spectral Density Plots of WADFP Lmid Pressure, poly-dispersed sand, feed rate $120 \mathrm{lb} / \mathrm{hr}$, no secondary air injection, $\mathrm{U}_{\mathrm{L}}=$ : (a) 1.43 (b) 1.65 (c) 1.93 (d) 2.22 (e) 2.79 (f) 3.50 (g) $4.10 \mathrm{~m} / \mathrm{s}$.

Figure 7-11: Spectral Density Plots of WADFP Ltop Pressure, poly-dispersed sand, feed rate 120 $\mathrm{lb} / \mathrm{hr}$, no secondary air injection, $\mathrm{U}_{\mathrm{L}}=$ : (a) 1.43 (b) 1.65 (c) 1.93 (d) 2.22 (e) 2.79 (f) 3.50 (g) $4.10 \mathrm{~m} / \mathrm{s}$ 118

Figure 7-12: Standard deviation of WADFP upper riser stage pressures (a) Ubottom (b) Utop:

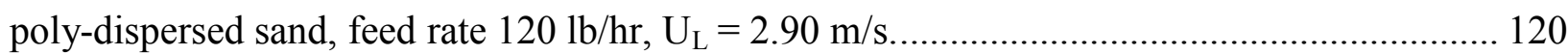
Figure 7-13: Shannon entropy of WADFP upper riser stage pressures (a) Ubottom (b) Utop:

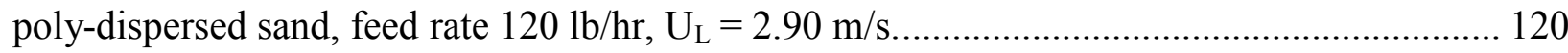
Figure 7-14: Autocorrelation of WADFP Ubottom pressure, poly-dispersed sand, feed rate 120 $\mathrm{lb} / \mathrm{hr}, \mathrm{U}_{\mathrm{L}}=2.90 \mathrm{~m} / \mathrm{s}, \mathrm{U}_{\mathrm{U}}=$ : (a) 1.81 (b) 2.04 (c) 2.27 (d) 2.49 (e) 2.72 (f) 2.95 (g) 3.17 (h) 3.40 $\mathrm{m} / \mathrm{s}$.

Figure 7-15: Autocorrelation of WADFP Ubottom pressure, poly-dispersed sand, feed rate 120 $\mathrm{lb} / \mathrm{hr}, \mathrm{U}_{\mathrm{L}}=2.90 \mathrm{~m} / \mathrm{s}, \mathrm{U}_{\mathrm{U}}=$ : (a) 1.81 (b) 2.04 (c) 2.27 (d) 2.49 (e) 2.72 (f) 2.95 (g) 3.17 (h) 3.40 $\mathrm{m} / \mathrm{s}$.

Figure 7-16: Mutual Information of WADFP Ubottom pressure, poly-dispersed sand, feed rate $120 \mathrm{lb} / \mathrm{hr}, \mathrm{U}_{\mathrm{L}}=2.90 \mathrm{~m} / \mathrm{s}, \mathrm{U}_{\mathrm{U}}=$ : (a) 1.81 (b) 2.04 (c) 2.27 (d) 2.49 (e) 2.72 (f) 2.95 (g) 3.17 (h) $3.40 \mathrm{~m} / \mathrm{s}$.

Figure 7-17: Mutual Information of WADFP Utop pressure, poly-dispersed sand, feed rate 120 $\mathrm{lb} / \mathrm{hr}, \mathrm{U}_{\mathrm{L}}=2.90 \mathrm{~m} / \mathrm{s}, \mathrm{U}_{\mathrm{U}}=$ : (a) 1.81 (b) 2.04 (c) 2.27 (d) 2.49 (e) 2.72 (f) 2.95 (g) 3.17 (h) 3.40 $\mathrm{m} / \mathrm{s}$.

Figure 7-18: Power Spectral Density of WADFP Ubottom pressure, poly-dispersed sand, feed rate $120 \mathrm{lb} / \mathrm{hr}, \mathrm{U}_{\mathrm{L}}=2.90 \mathrm{~m} / \mathrm{s}, \mathrm{U}_{\mathrm{U}}=$ : (a) 1.81 (b) 2.04 (c) 2.27 (d) 2.49 (e) 2.72 (f) 2.95 (g) 3.17 (h) $3.40 \mathrm{~m} / \mathrm{s}$.

Figure 7-19: Power Spectral Density of WADFP Ubottom pressure, poly-dispersed sand, feed rate $120 \mathrm{lb} / \mathrm{hr}, \mathrm{U}_{\mathrm{L}}=2.90 \mathrm{~m} / \mathrm{s}, \mathrm{U}_{\mathrm{U}}=$ : (a) 1.81 (b) 2.04 (c) 2.27 (d) 2.49 (e) 2.72 (f) 2.95 (g) 3.17 (h) $3.40 \mathrm{~m} / \mathrm{s}$.

Figure 7-20: Effects of varying secondary air injection on WADFP lower riser stage average pressures (a) Lbottom $\quad$ (b) Lmid (c) Ltop: poly-dispersed sand, feed rate $120 \mathrm{lb} / \mathrm{hr}, \mathrm{U}_{\mathrm{L}}=2.90$ $\mathrm{m} / \mathrm{s}$. 124

Figure 7-21: Effects of varying secondary air injection on WADFP lower riser stage average voidage: poly-dispersed sand, feed rate $120 \mathrm{lb} / \mathrm{hr}, \mathrm{U}_{\mathrm{L}}=2.90 \mathrm{~m} / \mathrm{s}$. 125 
Figure 7-22: Effects of varying secondary air injection on WADFP lower riser stage standard deviation of pressures (a) Lbottom (b) Lmid (c) Ltop: poly-dispersed sand, feed rate 120 $\mathrm{lb} / \mathrm{hr}, \mathrm{U}_{\mathrm{L}}=2.90 \mathrm{~m} / \mathrm{s}$.

Figure 7-23: Effects of varying secondary air injection on WADFP lower riser stage Shannon entropy of pressures $\quad$ (a) Lbottom (b) Lmid (c) Ltop: poly-dispersed sand, feed rate 120 $\mathrm{lb} / \mathrm{hr}, \mathrm{U}_{\mathrm{L}}=2.90 \mathrm{~m} / \mathrm{s}$.

Figure 7-24: Autocorrelation of WADFP Lbottom pressure, poly-dispersed sand, feed rate 120 $\mathrm{lb} / \mathrm{hr}, \mathrm{U}_{\mathrm{L}}=2.90 \mathrm{~m} / \mathrm{s}, \mathrm{U}_{\mathrm{U}}=$ : (a) 1.81 (b) 2.04 (c) 2.27 (d) 2.49 (e) 2.72 (f) 2.95 (g) 3.17 (h) 3.40.

Figure 7-25: Autocorrelation of WADFP Lmid pressure, poly-dispersed sand, feed rate $120 \mathrm{lb} / \mathrm{hr}$, $\mathrm{U}_{\mathrm{L}}=2.90 \mathrm{~m} / \mathrm{s}, \mathrm{U}_{\mathrm{U}}=$ : (a) 1.81 (b) 2.04 (c) 2.27 (d) 2.49 (e) 2.72 (f) 2.95 (g) 3.17 (h) $3.40 \ldots \ldots 128$ Figure 7-26: Autocorrelation of WADFP Ltop pressure, poly-dispersed sand, feed rate $120 \mathrm{lb} / \mathrm{hr}$, $\mathrm{U}_{\mathrm{L}}=2.90 \mathrm{~m} / \mathrm{s}, \quad \mathrm{U}_{\mathrm{U}}=:$ (a) 1.81 (b) 2.04 (c) 2.27 (d) 2.49 (e) 2.72 (f) 2.95 (g) 3.17 (h) 3.40 128

Figure 7-27: Mutual Information of WADFP Lbottom pressure, poly-dispersed sand, feed rate $120 \mathrm{lb} / \mathrm{hr}, \mathrm{U}_{\mathrm{L}}=2.90 \mathrm{~m} / \mathrm{s}, \mathrm{U}_{\mathrm{U}}=$ : (a) 1.81 (b) 2.04 (c) 2.27 (d) 2.49 (e) 2.72 (f) 2.95 (g) 3.17 (h) 3.40 .

Figure 7-28: Mutual Information of WADFP Lmid pressure, poly-dispersed sand, feed rate 120 $\mathrm{lb} / \mathrm{hr}, \mathrm{U}_{\mathrm{L}}=2.90 \mathrm{~m} / \mathrm{s}, \quad \mathrm{U}_{\mathrm{U}}=$ : (a) 1.81 (b) 2.04 (c) 2.27 (d) 2.49 (e) 2.72 (f) 2.95 (g) 3.17 (h) 3.40

Figure 7-29: Mutual Information of WADFP Ltop pressure, poly-dispersed sand, feed rate 120 $\mathrm{lb} / \mathrm{hr}, \mathrm{U}_{\mathrm{L}}=2.90 \mathrm{~m} / \mathrm{s}, \quad \mathrm{U}_{\mathrm{U}}=$ : (a) 1.81 (b) 2.04 (c) 2.27 (d) 2.49 (e) 2.72 (f) 2.95 (g) 3.17 (h) 3.40

Figure 7-30: Spectral Density Plots of WADFP Lbottom pressure, poly-dispersed sand, feed rate $120 \mathrm{lb} / \mathrm{hr}, \mathrm{U}_{\mathrm{L}}=2.90 \mathrm{~m} / \mathrm{s}, \quad \mathrm{U}_{\mathrm{U}}=$ (a) 1.81 (b) 2.04 (c) 2.27 (d) 2.49 (e) 2.72 (f) 2.95 (g) 3.17 (h) 3.40 .

Figure 7-31: Spectral Density Plots of WADFP Lmid pressure, poly-dispersed sand, feed rate $120 \mathrm{lb} / \mathrm{hr}, \mathrm{U}_{\mathrm{L}}=2.90 \mathrm{~m} / \mathrm{s}, \quad \mathrm{U}_{\mathrm{U}}=$ (a) 1.81 (b) 2.04 (c) 2.27 (d) 2.49 (e) 2.72 (f) 2.95 (g) 3.17 (h) 3.40

Figure 7-32: 7-33: Spectral Density Plots of WADFP Ltop pressure, poly-dispersed sand, feed rate $120 \mathrm{lb} / \mathrm{hr}, \mathrm{U}_{\mathrm{L}}=2.90 \mathrm{~m} / \mathrm{s}, \quad \mathrm{U}_{\mathrm{U}}=$ (a) 1.81 (b) 2.04 (c) 2.27 (d) 2.49 (e) 2.72 (f) 2.95 (g) 3.17 (h) 3.40 .

Figure 7-34: Scaling comparisons of WADFP and scale model standard deviation of pressure as a function of $U_{m f}$-normalized mass flux rate, lower riser mapping: (a) Lbottom (b) Lmid (c) Ltop.

Figure 7-35: Scaling comparisons of WADFP and scale model Shannon entropy of pressure as a function of $U_{m f}$-normalized mass flux rate, lower riser mapping: (a) Lbottom (b) Lmid (c) Ltop. 
Figure 7-36: Scaling comparisons of WADFP and scale model standard deviation of pressure as a function of $U_{m f}$-normalized mass flux rate, upper riser mapping: (a) Lbottom (b) Lmid (c) Ltop (d) Ubottom (e) Utop.

Figure 7-37: Scaling comparisons of WADFP and scale model Shannon entropy of pressure as a function of $\mathrm{U}_{\mathrm{mf}}$-normalized mass flux rate, upper riser mapping: (a) Lbottom (b) Lmid (c) Ltop (d) Ubottom (e) Utop. 138 Figure 7-38: Results of applying scaling method to coal, Lbottom pressure location, cleaned coal, feed rate $55 \mathrm{lb} / \mathrm{hr} ; \quad \mathrm{U}_{\mathrm{L}}=2.0 \mathrm{~m} / \mathrm{s}, \mathrm{U}_{\mathrm{U}}=1.65 \mathrm{~m} / \mathrm{s}$ (a) autocorrelation (b) spectral density; $\mathrm{U}_{\mathrm{L}}=$ $3.13 \mathrm{~m} / \mathrm{s}, \mathrm{U}_{\mathrm{U}}=2.56 \mathrm{~m} / \mathrm{s}$ (c) autocorrelation (d) spectral density. 140 


\section{List of Symbols}

Definition

Units

Equations Used In

(for alternate definitions)

Letters:

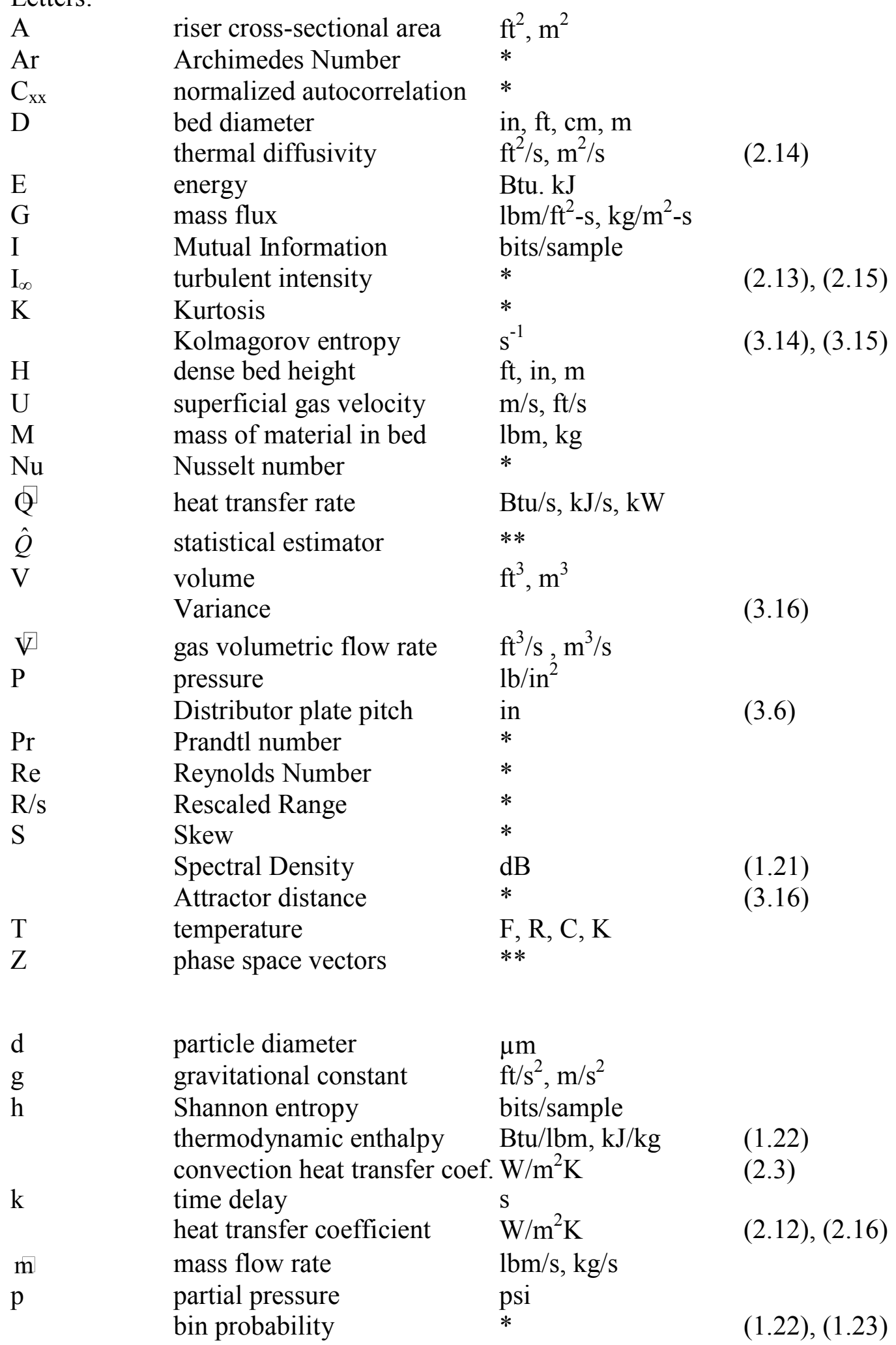




$\begin{array}{lll}\mathrm{u} & \text { internal energy } & \mathrm{kJ} \\ \mathrm{u}_{\mathrm{o}} & \text { superficial gas velocity } & \mathrm{m} / \mathrm{s} \\ \mathrm{u}_{\mathrm{mf}} & \text { min. fluidization velocity } & \mathrm{m} / \mathrm{s} \\ \mathrm{s} & \text { thermodynamic entropy } & \mathrm{Btu} / \mathrm{lbm}-\mathrm{R}, \mathrm{kJ} / \mathrm{kg}-\mathrm{K} \\ \mathrm{t} & \text { time } & \mathrm{s} \\ \mathrm{z} & \text { height } & \text { in } \\ \mathrm{c}_{\mathrm{xx}} & \text { autocorrelation } & * *\end{array}$

Greek:

$\begin{array}{lll}\rho & \text { density } & \mathrm{lbm} / \mathrm{ft}^{3}, \mathrm{~kg} / \mathrm{m}^{3} \\ \varepsilon & \text { bed voidage } & * \\ \mu & \text { gas viscosity } & \mathrm{lbm}-\mathrm{s} / \mathrm{ft}^{2}, \mathrm{~N}-\mathrm{s} / \mathrm{m}^{2} \\ \sigma & \text { standard deviation } & * * \\ \tau & \text { time delay } & \mathrm{s} \\ \xi & \text { moisture content } & * \\ \varpi & \text { specific humidity } & * \\ \eta & \text { efficiency } & * \\ \phi & \text { particle sphericity } & * \\ \Pi_{1} & \text { mass flux scaling term } & \mathrm{lbm} / \mathrm{ft}^{2}-\mathrm{s}, \mathrm{kg} / \mathrm{m}^{2}-\mathrm{s} \\ \Pi_{2} & \text { mass flow rate scaling term } & *\end{array}$

Subscripts:

d droplet

f fluid

ex exergy

$\mathrm{mb} \quad$ minimum bubbling

$\mathrm{mf} \quad$ minimum fluidization

$\mathrm{p} \quad$ particle

s solid

th thermal

tr fast fluidization transition

0 reference state (standard temp, pressure)

* dimensionless

** $\quad$ same units as original time series 


\section{List of Video Files}

Filename:

LRMT1.wmv

LRMT2.wmv

LRMT3.wmv

LRMT4.wmv

LRMT5.wmv

LRMT6.wmv

LRMT7.wmv

LRMT8.wmv

LRMT9.wmv

URMT1.wmv

URMT2.wmv

URMT3.wmV

URMT4.wmV

URMT5.wmV

URMT6.wmv

URMT7.wmv
Description

Lower Riser Mapping Test 1

Lower Riser Mapping Test 2

Lower Riser Mapping Test 3

Lower Riser Mapping Test 4

Lower Riser Mapping Test 5

Lower Riser Mapping Test 6

Lower Riser Mapping Test 7

Lower Riser Mapping Test 8

Lower Riser Mapping Test 9

Upper Riser Mapping Test 1

Upper Riser Mapping Test 2

Upper Riser Mapping Test 3

Upper Riser Mapping Test 4

Upper Riser Mapping Test 5

Upper Riser Mapping Test 6

Upper Riser Mapping Test 7 


\section{Chapter 1 Introduction and Background}

\section{Introduction}

\section{Statement of Objective:}

The objective of this research effort was explore the fluidization characteristics of a novel twostage, variable area fluidized bed riser by constructing a cold-flow, transparent scale model of an existing two-stage, continuous feed, fluidized bed for drying fine coal particles, as well as the development of a simplified set of relationships for scaling the fluidization regime transition points between the two systems. A secondary object was to carry out preliminary experiments with drying coal in the large scale riser to test the feasibility of using a fluidized bed for the drying of coal.

\section{Need for Solution}

Approximately $6-8 \%$ of the energy found in mined coal is located in particle size fractions of less than 150 micron $(\mu \mathrm{m})$, or 100-mesh [1]. Due to the inherent difficulties in dewatering material within this size range, most coal preparation plants discard the minus $150 \mu \mathrm{m}$ size fraction of their run-of-mill coal into slurry ponds will little or no attempt to recover the cleaned coal of this size fraction [2]. In the United States alone, this has resulted in the discharge of approximately 23 billion tons of fine coal into abandoned ponds, as well as 500-800 million tons in active ponds [3]. On an annual basis, coal producers in the U.S. discard roughly 30-40 million tons of fresh fine coal. In addition to the many environmental issues brought about by discarding this material into waste ponds, it has been shown that effective means of recovering fine coal can lead to increased profitability in mining operations. Patwardhan et al. [1] has shown that profits can be increased by as much as $\$ 2.5$ (\$US) million annually for a single $2.4 \mathrm{Mt} /$ year mining operation. 
In addition to the economic aspects associated with recovery of coal fines from waste slurry at preparation plants, drying of small coal particles is also of interest in regards to operation of pulverized coal (PC) boilers used to generate electricity. In a recent report generated for the U.S. Department of Energy's (DOE) National Energy Technology Laboratory (NETL) regarding the use of low rank coals in various gasifier systems, the following information was presented. From an operational perspective, PC boilers can handle fuels with a wide range of moisture content, encompassing both lignite and subbituminous coal with $25-45 \%$ moisture [4]. This report goes on to state that, ultimately, the choice of whether or not to dry the coal is a tradeoff between achieving higher efficiencies within the boiler at the expense of the increased cost inherent in using current drying methods. Historically, coal prices within the United States have been low enough that the "business as usual" approach has been to simply burn the coal as received with minimal attempts to dry it beforehand, and there was little incentive to add cost to improve efficiency. However, the combination of increased coal costs and more stringent emissions requirements has made drying options more common.

Dewatering of fine coal particles is typically accomplished by utilizing either a screen-bowl centrifuge or filters operating under vacuum or pressure [5]. In both cases, wet coal fines from the cleaning process are introduced into these systems in the form of froth from flotation columns. This froth typically has an initial moisture content of approximately $80 \%$, with $20 \%$ solids content [6].

In the case of a screen-bowl centrifuge, a filter cake forms along the side walls of the centrifuge chamber. This filter cake acts like a dense packed bed with parallel capillaries of varying size [5]. The centrifugal force created from the revolution of the centrifugal chamber forces water out 
of the capillaries and through a filter cloth. Because moisture located in small pores is difficult to remove due to high capillary pressure, a flocculant is typically added to the froth. This flocculant causes a "clumping together" of the fine particles, thus increasing the size of the cake pores and decreasing the capillary pressure, resulting in better dewatering. In general, this method results in final cake moistures of $20-25 \%$. Recent innovations in the area of screen-bowl centrifuges have lead to the addition of hyperbaric pressure into the centrifuge chamber during operation $[3,7]$. Laboratory testing of this method has resulted in cake moisture contents as low as $10 \%$ for particle sizes typically found in industrial fine coal dewatering operations.

The second commonly used method for dewatering fine coal particles involves the use of filtration combined with either vacuum or pressure. The two most common applications of this principle are the plate and press filter and the belt filter. In both cases, a cake layer is formed and then pressed between two surfaces (plates in the press filter, belts in the belt filter). The pressure resulting from this compression forces moisture out of the capillaries formed within the cake. Dewatering is typically enhanced by the addition of flocculants and utilization of vacuum filtration in the case of the belt filter $[\mathbf{8 , 2}]$ has lead to results in a mean moisture content of $24 \%$. Similarly, flocculants and pressure filtration are typically incorporated into the plate and press filters, resulting in cake moisture contents between $20-27 \%$ [1].

\section{Experimental Approach}

The basic approach to drying is to heat the particles, thus increasing the water vapor pressure on/in the particle. When the water vapor pressure is greater than that in the surrounding air, the water vapor will then diffuse into the air. Consequently, the higher the particle temperature is and the drier the surrounding air, the quicker the particle will dry. 
For this study, a fluidized bed dryer (referred to from this point on as the WADFP, or Warm Air Dryer of Fine Particles) has been built and utilized for the drying of fine coal particles. Fluidized beds are extremely efficient vehicles for implementation of convective drying of small particles due to several factors; including large heat transfer surfaces, uniform temperature distributions, excellent temperature control and a continuous supply of drying air with low moisture content [9].

The ability to closely control the temperature within a fluidized bed is of great importance when drying fine coal particles. The reason for this being that there is a limit to the temperature that may be employed in the drying process. Temperatures just above $150^{\circ} \mathrm{C}\left(302^{\circ} \mathrm{F}\right)$ may ignite a dust layer $(<75 \mu \mathrm{m}$ diameter) of highly volatile coal [10]. Therefore, it is imperative that any dryer design for coal fines should operate with all dryer surfaces and dry air flows below the lowest ignition temperature. The WADFP concept incorporates all three of these considerations, resulting in an improved coal or mineral drying system.

Figure 1-1 shows the conceptual design of the WADFP system. The WADFP unit consists of 2 riser sections of different diameter; these are referred to as the lower and upper riser stages. The small diameter lower stage will be operated such that a turbulent fluidization regime is maintained. Turbulent fluidization is desired in this portion of the riser based upon the fact that free stream turbulence results in an earlier transition to turbulence in the boundary layer surrounding the individual particles. This earlier onset of turbulence results in higher average convective heat transfer coefficients and thus enhances the drying process $[\mathbf{1 1}, \mathbf{1 2}, \mathbf{1 3}]$. The large diameter upper stage of the WADFP unit will be operated within the fast fluidization, or coreannulus, flow regime. This will allow the drying process to continue until such time as the individual coal particles become light enough (through loss of mass due to evaporation of 
moisture) to be transported out of the riser by the exhaust gases. Once the dry coal particles exit the fluidized bed riser, they will be separated from the fluidizing air by way of a cyclone, and will be deposited into a collection bin for analysis of moisture content.

During operation of the WADFP, dry air enters the system and is divided into three streams. The three steams are heated to about $105^{\circ} \mathrm{C}\left(221^{\circ} \mathrm{F}\right)$ in a heat exchanger using steam as a heat source. Two air streams then enter the bottom of the riser and one stream enters the riser through the injection ring. One of the two bottom streams forms a jet rising up through the riser. The second bottom air stream flows through a distributor plate and dries the particles moving down along the riser wall. The wet coal particles are introduced into the air stream entering the bottom of the riser via a motor-driven auger feed system. The particles are then pneumatically transported via the airstream into the lower stage of the riser, and are then swept upwards by the induced air circulation pattern within the lower turbulent riser stage. As the particles dry, they become lighter and are carried to the upper stage of the riser. Here the particles interact with the drier air from the injection ring. The drying process is completed in the enlarged flow area of the riser.

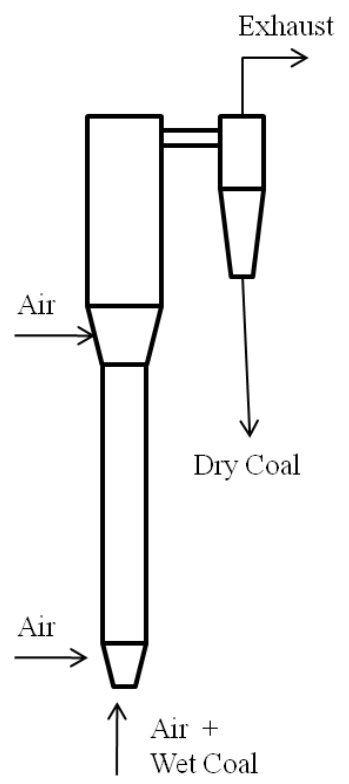

Figure 1-1: WADFP Conceptual Drawing 
In a fluidized bed of constant diameter, the radial introduction of additional feed air creates two regions within the fluidized bed riser whose flow conditions can be maintained such that each section can be treated as separate fluidized beds with distinctly different fluidization regimes [14]. In the WADFP unit, a conical injection ring located between the upper and lower riser stages operates with essentially the same function. By introducing additional air through the injection ring, it is expected that the upper riser stage can be operated at a different superficial velocity that the lower stage, allowing for the possibility of having different fluidization conditions above the injection ring than what exists below it.

In addition to allowing for the establishment of different flow regimes in the upper and lower riser sections, the secondary air injection via the injection ring provides another enhancement to the drying process. As seen in Figure 1-2, as the drying air moves up through the riser, it experiences an increase in specific humidity as moisture evaporates off of the bed particles and is transported upwards along with the rising air. This increase in specific humidity results in a decrease in driving force for the rate of drying higher within the riser. The addition of more dry air via the injection ring lowers the specific humidity, thus increasing the drying rate of particles in the upper stage of the riser.

At this point, it must be noted that operation of the WADFP system depends upon the assumptions of creating and maintaining specific fluidization (regimes) conditions within the riser itself. In order to more easily address this issue, a half-scale transparent model of the WADFP system has been designed and constructed. Initial testing using the scale model system was carried out in order to map out the various fluidization regimes that can exist within the system. These initial tests provided a comprehensive set of visual records of the interactions between particles and fluidizing gas at different gas velocities, as well as time histories of 
pressures and other statistical data that can be used to describe the hydrodynamic characteristics of the WADFP system.

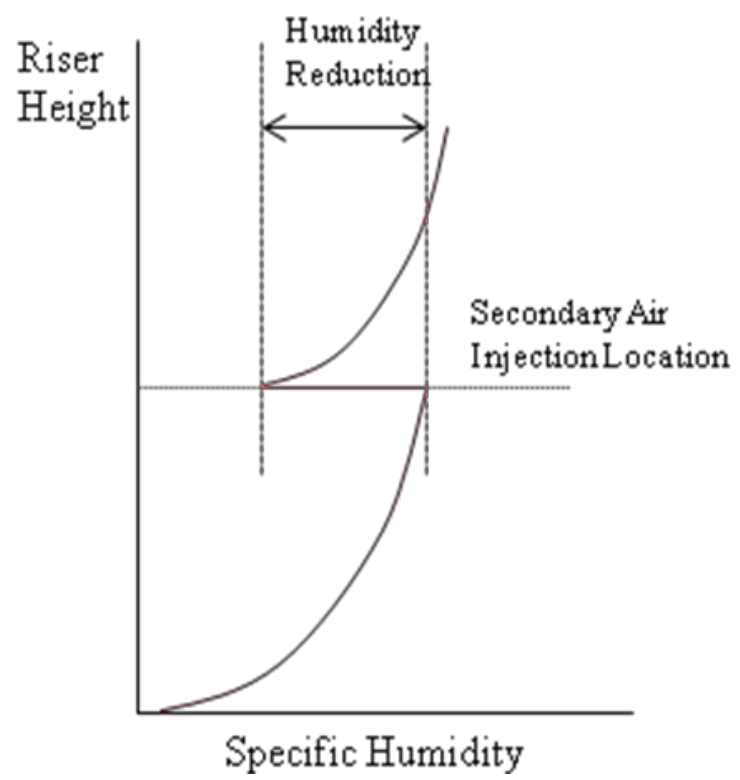

Figure 1-2: Effect of Secondary Air Injection on Specific Humidity

Finally, a method for scaling the laboratory-scale drying process up to industrial use has been developed and verified.

\section{Background}

\section{General types and applications of fluidized bed dryers}

Although not currently used for commercial drying of fine coal particles, many other industries have utilized fluidized beds for the drying of granular materials such as grains, fertilizers and chemicals $[15,16,17,18]$. Fluidized beds possess many advantages over more conventional drying techniques, among these advantages are: better temperature control, more uniform temperature distribution, higher thermal efficiency and intensity of drying, better gas-particle 
contact and less degradation of the particles. Unfortunately, there are also disadvantages associated with fluidized bed drying. These disadvantages include high pressure drops, nonuniform moisture content in the product (when operated in continuous mode) and the inability to adapt to counter-current operations $[\mathbf{1 9 , 2 0 , 2 1 , 1 7 , 9 ]}$.

\section{Literature Review}

While not utilized in commercial coal drying applications, there has been some research conducted to study aspects of fluidized bed drying of coal. Diamond [21] concluded in a study to determine the effects of temperature and particle size on the fluidized bed drying of northern Ireland lignite coal that drying rates increased as air temperatures increases, as well as when particle sizes decreased. Calban [19] obtained similar results while studying the drying characteristics of Turkish lignite in a batch bubbling fluidized bed. In addition to temperature and particle size considerations, Calban determined that the velocity of the drying air had no significant effect on drying rates. In another study, Calban [20] investigated the effects of bed height and initial moisture concentration on drying rates of Turkish lignite. In this study he determined that drying rates increased with decreasing bed height, and decreased with decreasing initial moisture concentration of the coal.

\section{CFD Models for Fluidized Bed Drying of Coal}

The majority of available literature related to numerical models of coal-based applications of fluidized beds deals more with the subject of coal combustion or gasification in fluidized bed combustors than with fluidized bed drying of coal. Chen, Agarwal and Agnew [22] presented a numerical model utilizing a two-phase hydrodynamic model to simulate the drying of coal in a bed fluidized with superheated steam. The use of a superheated steam drying process reduces 
the likelihood of fire and explosive hazards because steam-dried coal is less liable to spontaneous combustion due to a decrease in reactivity to oxygen in the atmosphere. Similarly, Stakic and Tsotsas [23] present a numerical model of a packed bed used for cooling warm coal particles that have been previously dried via a superheated steam drying process.

\section{Basic Principles of Fluidization}

\section{Geldart Particle Classification}

Geldart [24] classified solid particles into four groups based upon average particle diameter and the difference between particle and fluidizing gas densities. The four groups described by Geldart (A, B, C, and D) have been widely adopted for gas-solid fluidized bed design and research and are shown in Figure 1-3.

Group A particles, commonly known as "aeratable" particles, have a typical particle size range of 30-100 microns and are easily fluidized. These particles can be fluidized in the particulate fluidization regime in the absence of bubbling, as well as within the bubbling regime when bubbles are present. This leads to the minimum fluidization velocity being less than the minimum bubbling velocity. [25] In addition, group A particle fluidization exhibits a maximum stable bubble size when the gas velocity is greater than the minimum bubbling velocity.

Group B particles, like Group A, are easily fluidized and are commonly known as "bubbling" particles. However, for Group B particles, the minimum fluidization velocity equals the minimum bubbling velocity so there is no particulate phase. Finally, for Group B, there is not a maximum stable bubble size.

Group C (or "cohesive") consists of small particles which are highly cohesive. These particles are difficult to fluidize due to the dominance of inter-particle and electrostatic forces over 
hydrodynamic forces. Channeling and high bed expansions are characteristics of Group C fluidization.

Group D particles are coarse and do not mix well when fluidized. These particles are normally processed via spouted beds instead of fluidized beds.

In the tests described in later chapters, the poly-dispersed sand and coal was primarily Geldart Type B particles; however, coal particles with diameters smaller than $150 \mu \mathrm{m}$ behaved more like type C (cohesive) particles.

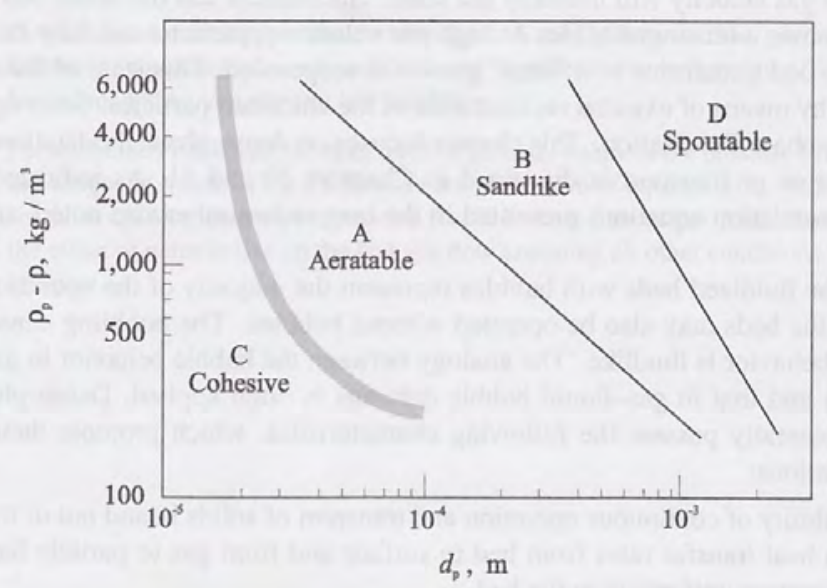

Figure 1-3: Geldart's classification of fluidized particles [24] (reproduced with permission)

\section{Fluidization Regimes}

Gas-solid systems are classified according to the basis of the state of motion of the solid particles within the system [25]. For a batch-solids system, the low velocity gasses percolate through the voids between the packed particles while the particles themselves are motionless. In this situation, the solid particles are said to be in the fixed bed state. As the gas velocity increases, the particles begin to move apart and become suspended. This suspended state is known as fluidization. The term fluidization encompasses a number of categories which exhibit a range of 
different particle motions and behaviors. These different sets of behaviors are commonly referred to as fluidization regimes.

The fluidization regimes of fluidized beds have been the subject of numerous studies and attempts at characterization. Investigations have confirmed the existence of six primary fluidization regimes during fluidized bed operations; these regimes are: particulate fluidization (Geldart A particles only), bubbling, slugging, turbulent, fast fluidization and pneumatic conveying. These regimes are dependent upon such factors as size and density of solid particles, superficial gas velocity, physical properties of the fluidizing gas, temperature, pressure, settled bed height, diameter of the fluidization column, as well as the type of gas distributor and the pressure drop across it $[\mathbf{2 6}, \mathbf{2 7}, \mathbf{2 8}, \mathbf{2 9}, \mathbf{3 0}, \mathbf{3 1}, \mathbf{3 2}]$.

Three of the most commonly used concepts for the characterization and description of fluidization are the superficial gas velocity $(\mathrm{U})$, bed voidage $(\varepsilon)$ and solids circulation (or flux) $\operatorname{rate}\left(\mathrm{G}_{\mathrm{s}) \text {. }}\right.$

The superficial gas velocity $(\mathrm{U})$ is a commonly used reference velocity in fluidization literature. In many cases, it is difficult to measure the actual fluidization gas velocities within a fluidized bed system. In physical terms, the superficial gas velocity is the theoretical velocity at which the fluidization gas would travel in the absence of any solids material. In practice, $\mathrm{U}$ is calculated by dividing volumetric flow rate of gas into the bed by the bed cross-sectional area.

$U=\frac{V_{a i r}}{A_{b e d}}$

In fluidized bed systems, the solid particles are suspended within the bed riser by the fluidizing gas. The spaces between the individual particles can be filled by either the fluidizing gas or bubbles that may form within the bed. The ratio of the volume of these spaces to the volume of 
the fluidized bed is commonly referred as the bed voidage (ع). Monazam and Shadle [29] provide the following relationship between the voidage and the average pressure drop across the fluidized bed:

$$
\frac{d P}{d z}=\rho_{s}(1-\varepsilon) g,
$$

where $g$ is the acceleration due to gravity and $\rho_{\mathrm{s}}$ is the density of the solid. Additionally, the rate at which solid material is being introduced into the bed is known as the solids flux rate $\left(\mathrm{G}_{\mathrm{s})}\right.$. For the study, the solids flux is the product of the solid feed rate and the cross-sectional area of the lower riser stage.

$$
G_{s}=\frac{d W}{d t} / A
$$

Fluidization regimes can be divided into two generic categories: dense-phase and dilute-phase. Dense-phase fluidization is characterized by the existence of a distinct dense bed of solids material and an upper dilute freeboard area. This category of fluidization regimes consists of the particulate (Geldart A only), bubbling, slugging and turbulent regimes (shown in Fig 1-4a-d). The dilute-phase category consists of the fast fluidization and dilute transport (or pneumatic conveying) regimes. 


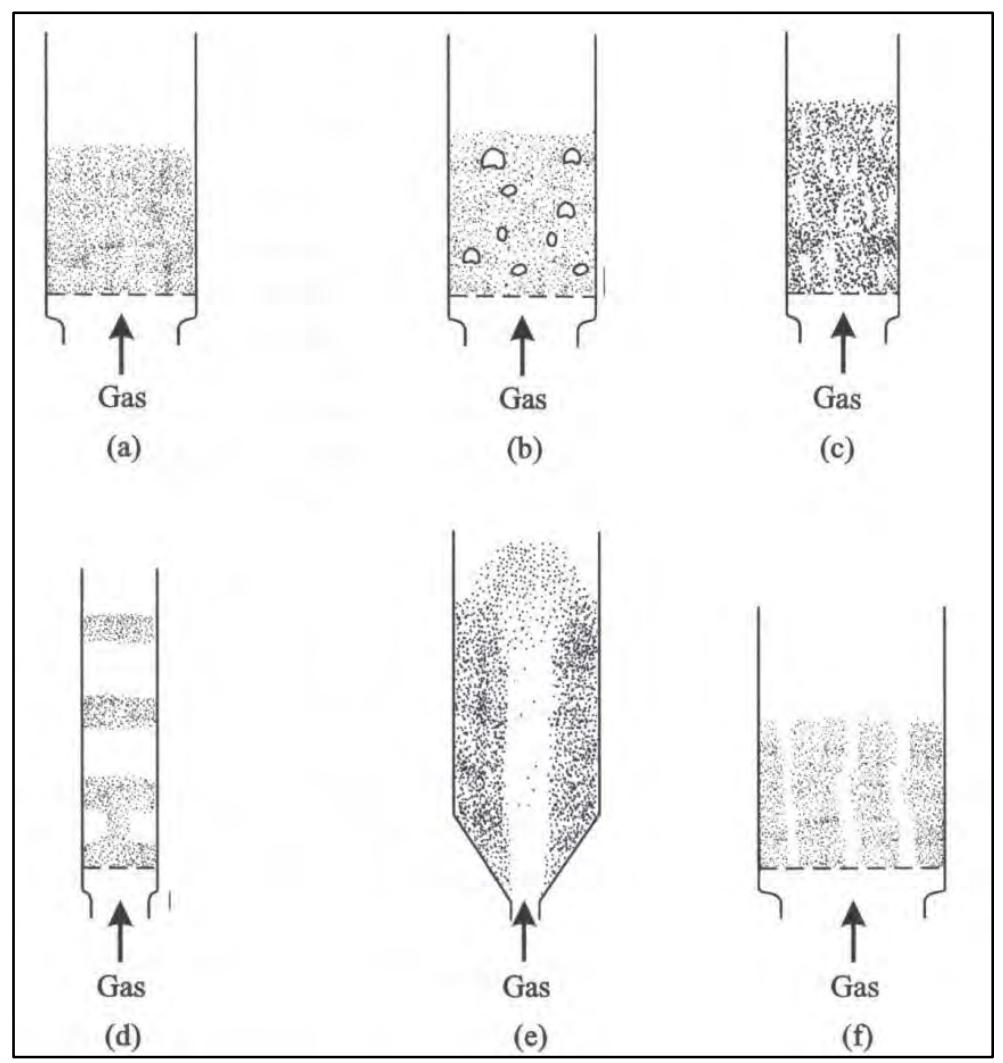

Figure 1-4: Dense fluidization regimes (a) Particulate; (b) Bubbling; (c) Turbulent; (d) Slugging; (e) Spouting; (f) Channeling [25] (reproduced with permission)

\section{Dense Phase Fluidization}

The various dense-phase fluidization regimes are bounded by an array of values of superficial gas velocities. When the bed material consists of Geldart type A particles, particulate fluidization occurs at the minimum fluidization velocity $\left(\mathrm{U}_{\mathrm{mf}}\right)$. In this flow regime, the fluidizing gas passes through the interstitial space between particles without bubbles and the bed appears to be homogeneous in nature [25]. The upper bound of this flow regime is characterized by the minimum superficial gas velocity that leads to the formation of bubbles within the bed. This velocity is known as the minimum bubbling velocity $\left(\mathrm{U}_{\mathrm{mb}}\right)$. For the Geldart type A particles, this velocity range between $U_{m g}$ and $U_{m b}$ is very narrow. For coarser particles, such as Geldart type $\mathrm{B}$ and $\mathrm{D}$ particles, the minimum fluidization velocity equals the minimum bubbling velocity. 
Wen and $\mathrm{Yu}[\mathbf{3 3}]$ proposed the following semi-empirical correlation relating the particle

Reynolds number for minimum fluidization and the Archimedes number Ar (for low pressures):

$$
\operatorname{Re}_{m f}=\sqrt{(33.7)^{2}+0.0408 A r}-33.7,
$$

where the Archimedes number is defined as:

$$
A r=\frac{\rho\left(\rho_{p}-\rho\right) g d_{p}^{3}}{\mu^{2}}
$$

and:

$$
\operatorname{Re}=\frac{\rho U d_{p}}{\mu} .
$$

In addition, the bed voidage at minimum fluidization can be found via the following expression provided by the Ergun equation [25],

$$
A r=150 \frac{\left(1-\varepsilon_{m f}\right)}{\varepsilon_{m f}^{3} \varphi^{2}} \operatorname{Re}_{p m f}+\frac{1.75}{\varepsilon_{m f}^{3} \varphi} \operatorname{Re}_{p m f}^{2}
$$

and the height of the dense bed region at the minimum fluidization velocity is given by [25]

$$
H_{m f}=\frac{M_{p}}{A_{t} \rho_{p}\left(1-\varepsilon_{m f}\right)}
$$

where $\mathrm{M}_{\mathrm{p}}$ is the mass of the solid material in the bed and $\mathrm{A}_{\mathrm{t}}$ is the cross-sectional area of the riser. Additionally, as the gas velocity increases, the dense bin begins to expand, or increase in bed height. The amount of this expansion can be predicted by the following [34]

$$
\frac{H_{f}}{H_{m f}}=1+\frac{21.4\left(U-U_{m f}^{*}\right)^{0.738} d_{p}^{1.006} \rho_{p}^{0.376}}{\left(U_{m f}^{*}\right)^{0.937}\left(w_{g} \frac{p}{p_{a}}\right)^{0.126}}
$$

During the bubbling fluidization regime, the bubbles exhibit a tendency to continually coalesce and break up. As the gas velocity increases, this tendency towards bubble coalescence is 
enhanced and the bed begins to form larger and larger bubbles. Once the bubbles become large enough in size to be comparable to the bed diameter the slugging regime emerges. This slugging phase normally only occurs in fluidized beds with either a small bed diameter or a large heightto-diameter ratio.

Abrahamsen and Geldart [35] determined the following expression for the minimum bubbling velocity:

$$
U_{m b}=2.07 e^{0.716 \phi_{f}} \frac{d_{p} \rho^{0.06}}{\mu^{0.347}}, \quad \text { (MKS units) }
$$

where $v_{\mathrm{f}}$ is the mass fraction of the particles smaller than $45 \mu \mathrm{m}$.

If the superficial velocity of the fluidizing gas continues to increase beyond the slugging velocity $\left(U_{s}\right)$ the bubbles begin to lose their coherency and shape. When this happens, the bubbles are replaced within the bed material by irregularly-shaped voids. These voids undergo rapid change in shapes and locations. This is known as the onset of the turbulent fluidization regime. Cai, et al [36] provided the following correlation for determining the velocity corresponding to the onset of transition to turbulence $\left(\mathrm{U}_{\mathrm{c}}\right)$,

$$
\frac{U_{\underline{c}}}{\sqrt{g d_{p}}}=\left(\frac{\mu_{\underline{a}}}{\mu}\right)^{0.2}\left[\left(\frac{\rho_{a}}{\rho}\right)\left(\frac{\rho_{p}-\rho}{\rho}\right)\left(\frac{K D_{f}}{d_{p}}\right)\right]^{0.27},
$$

where

$$
K D_{f}=d\left(\frac{0.211}{d^{0.27}}+\frac{2.42 \times 10^{-3}}{d^{1.27}}\right)^{\frac{1}{0.27}}, \text { and }
$$

where eqn. 1.12 is applicable for bed diameters $57 \mathrm{~mm} \leq \mathrm{d} \leq 475 \mathrm{~mm}$. Also note that eqn. 1.11 is applicable for group A and B particles within the range of $293<\mathrm{T}<773 \mathrm{~K}$ and $0.1<\mathrm{p}<0.8$ MPa. Equations (1.11) and (1.12) both use MKS units. 
The turbulent fluidization regime is of great interest for many fluidization aspects, as this is the regime in which the greatest amount of mixing and solid-gas heat transfer occurs. Because of this, there have been many methods for predicting or determining the onset of turbulent fluidization proposed in fluidization literature and will be discussed later.

\section{Dilute Phase Fluidization}

As mentioned previously, the second broad category of fluidization regimes is commonly referred to as dilute fluidization. As opposed to dense phase fluidization, dilute fluidization is characterized by the absence of a densely-packed bed of particles. Instead, the dilute phase is characterized by the presence of much more widely spaced particles. These particles can exhibit a circulatory pattern of motion (as in core-annulus flow) or be fully entrained and carried along by the fluidization gas (as in pneumatic transport). For this research project, as in the case of turbulent fluidization, the fast fluidization regime is also of interest as it is intended that the upper, larger diameter riser section of both the WADFP and the small scale model will operate within this fluidization regime.

Fast fluidization occurs as the superficial velocity is increased sufficiently beyond $U_{k}$ that the fluidization column undergoes a transition from a dense bed of particles to a dilute flow pattern commonly known as core-annulus flow. This regime is characterized by a central "core" of rapidly upwards flowing gas and entrained particles, as well as an outer "annulus" counter flow of slower moving particles and gas. It is this circulating flow pattern that gives name to circulating fluidized beds.

The minimum required fluidization velocity for fast fluidization is known as the transport velocity, or $U_{\text {tr. }}$. The transport velocity can be estimated from the following empirical formula provided by Bi and Fan [37]: 


$$
R e_{t r}=2.28 A r^{0.419}
$$

When characterizing the fast fluidization regime, the variables of interest are pressure drop per unit length of the fluidization column $(\Delta \mathrm{P} / \Delta \mathrm{L})$, voidage $(\varepsilon)$ and solids circulation rate $\left(\mathrm{G}_{\mathrm{s}}\right)$. When $\Delta \mathrm{P} / \Delta \mathrm{L}$ is plotted against $\mathrm{G}_{\mathrm{s}}$, the well-documented " $\mathrm{S}$ " curve is generated. According to Monazam and Shadle $[\mathbf{2 9 , 3 8}]$, the fast fluidization regime is characterized by a relatively stable solids circulation rate that shows little dependence upon changes in the pressure drop across the bed riser. This relationship can also be related to the expression for $\mathrm{G}_{\mathrm{s}}$ given in eqn. 1.3 by noting that the solids circulation is only constant when $\mathrm{d} \Delta \mathrm{P} / \mathrm{dt}$ is constant. Also, as shown in Figure 1-5 below, the fast fluidization regime is characterized by a decrease in overall bed pressure drop with increasing superficial velocity. As the pressure drop approaches a minimum value, the bed begins to undergo a transition towards the dilute (or pneumatic) transport regime. Once the bed has fully transitioned to the transport regime, the overall bed pressure drop begins to increase.

The lower portion of Figure 1-5 shows the effect of the gas velocity $U$ and the solids circulation rate $G_{s}\left(J_{p}\right.$ in the figure $)$ on the flow regime. As can be seen in the figure, as the solids circulation rate and gas velocity decrease, the upper and lower bounds of the fast fluidization (core-annulus) flow regime contract towards a critical point denoted by $U_{\text {tr }}$ and $J_{p, t r}$. Any further decrease in either solids circulation rate or gas velocity will result in a collapse back into a dense fluidization regime. 


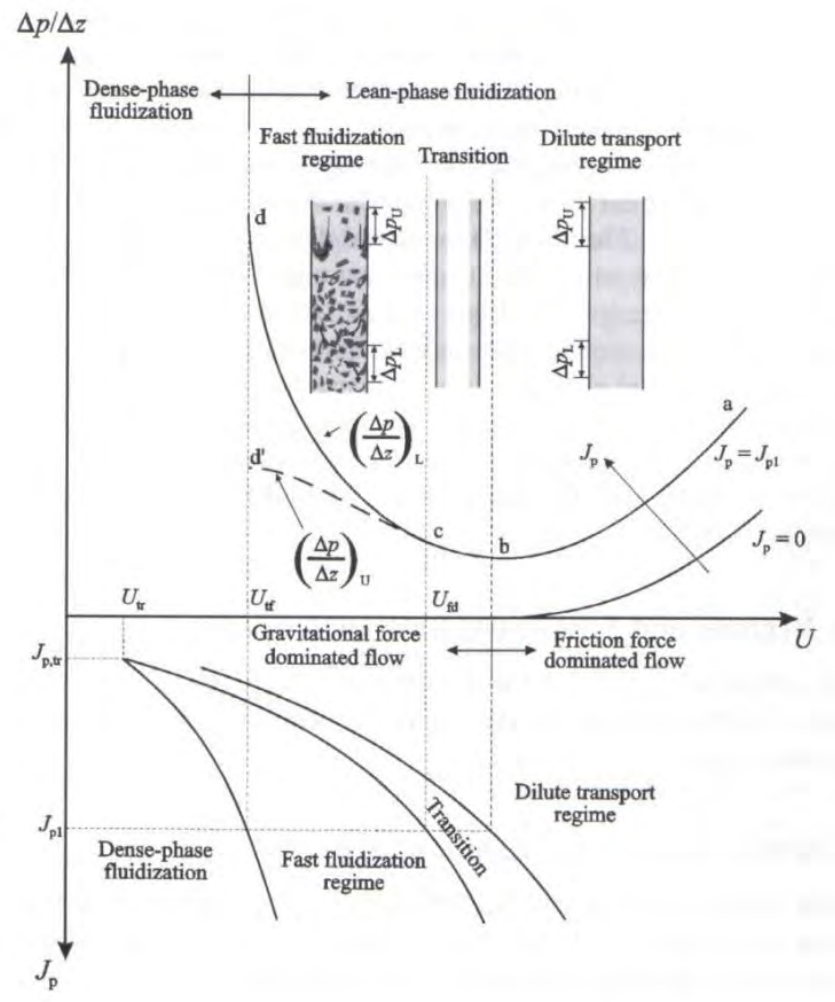

Figure 1-5: Variations of pressure drop per unit riser length with solids circulation rate and gas velocity for various fluidization regimes [39] (reproduced with permission)

\section{Pressure Signal Analysis}

Multiple methods for determining and characterizing the transition points between fluidization regimes via analysis of bed pressure signals have been developed and appear in the fluidization literature. Many of these various methods are presented here and are grouped into the following broad categories: statistical analysis, time domain analysis, frequency domain analysis and chaos analysis.

\section{Statistical Analysis of pressure}

Statistical methods of pressure data analysis include plotting three statistical moments (standard deviation, skewness and kurtosis) against superficial velocity, as well as examination of the autocorrelation function, Rescaled Range and Hurst exponent. 


\section{Standard Deviation}

The amplitude of the signal, $\mathrm{x}$, can be expressed by the standard deviation (or square root of the second-order statistical central moment) $\sigma$, as defined by equation (1.14).

$$
\sigma=\sqrt{\frac{1}{N-1} \sum_{n=1}^{N}(x(n)-\bar{x})^{2}}
$$

where $\mathrm{N}$ is the number of samples and $\bar{x}$ is the sample mean.

In fluidization research, the most common method (proposed by Bi and Fan [37]) for experimentally determining the velocity at which the transition from bubbling to turbulent fluidization begins $\left(U_{c}\right)$ is by plotting the standard deviation of pressure fluctuations versus the superficial velocity (U). Figure 1-6 depicts such a plot. As the value of $U$ increases through the bubbling and slugging regimes towards the turbulent regime, the standard deviation likewise increases until it reaches a maximum value and then begins to decrease. This maximum value of $\sigma$ corresponds to the onset of the transition to turbulence. The corresponding superficial velocity at this point is $\mathrm{U}_{\mathrm{c}}$.

These pressure fluctuations will decrease until the fluidized bed passes through the transition zone and into turbulent fluidization regime itself, where the pressure fluctuations will tend to level off. The velocity at which this occurs is commonly referred to as $U_{k}$.

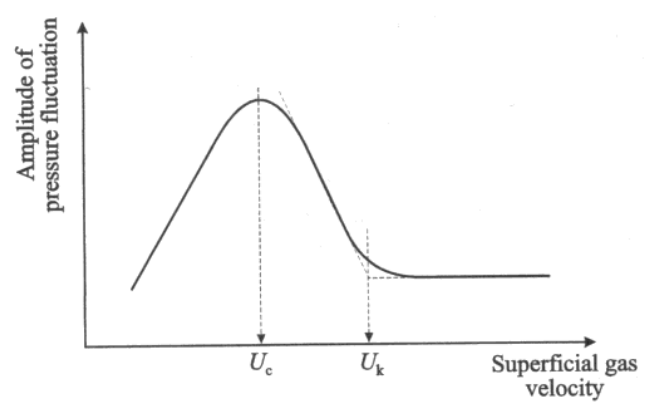

Figure 1-6: Variation of pressure fluctuations with velocity for dense-phase fluidization [40]. (reproduced with permission) 
However, Rhodes and Geldart [41] dispute this method. While valid for Group A particles, Rhodes and Geldart state that for Group B particles, the $U_{k}$ value obtained from the Bi and Fan method above actually yields the velocity at which the dense bed disappears and the bed transitions to dilute fluidization. In other words, for Group B particles, the $U_{k}$ velocity in figure 1-6 is actually the transport velocity, or $\mathrm{U}_{\mathrm{tr}}$.

Additionally, Bi and Grace [42] showed that $\mathrm{U}_{\mathrm{c}}$ is a strong function of the method used to measure bed pressures. Their findings showed that absolute pressure fluctuations differed from differential pressure fluctuations.

\section{Skewness}

The skewness of a set of data is an indicator of the amount of asymmetry about the mean of that data set. Skewness is defined as:

$$
S=\frac{1}{N \sigma^{3}} \sum_{n=1}^{N}(x(n)-\bar{x})^{3}
$$

When the data is normally distributed about the mean, the value of skewness is 0 . However, if the skew is negative, then the majority of the data will be distributed to the right of the sample probability distribution with a long tail on the left side; whereas a positive skew suggests that the majority of the data will be distributed to the left of the distribution with a long tail on the right side.

Lee and Kim [43] examined skew and kurtosis for analysis of pressure signals in a dense bed. They discovered that as the bed transitioned to turbulence, there was a shift from negative to positive skew and a maximum in the flatness (kurtosis) of the data. They considered the point of zero skew to correspond to the transition velocity, $\mathrm{U}_{\mathrm{c}}$. 


\section{Kurtosis}

In statistical analysis, kurtosis (the fourth order statistical moment) is the measure of the "peakedness" of the probability distribution of a real-valued random variable. A high value of kurtosis means that a large portion of the variance of a given data set is due to infrequent but large deviations. Kurtosis is given by the following expression:

$$
K=\frac{1}{N \sigma^{4}} \sum_{n=1}^{N}(x(n)-\bar{x})^{4}
$$

When applied to pressure signals from a fluidized bed, the kurtosis provides a good indication of the presence of large magnitude fluctuations due to large bubbles passing the pressure transducer. The kurtosis can also be seen as a measure of the ratio of time the system remains at a quiescent state to the time it spends at an active state. [44]

\section{Autocorrelation Function}

The autocorrelation function of a signal, given by equation (1.17) [44], depicts the correlation between two points within the signal that are separated by a time lag, $\mathrm{k} \Delta \mathrm{t}$. The autocorrelation function is an excellent tool for determining whether or not a given signal (or data set) is periodic in nature. If the signal is periodic, then the autocorrelation will also be periodic. Additionally, the autocorrelation is additive, so that a signal that consists of combinations of periodic functions will also have an autocorrelation that is a combination of multiple periodicities.

$$
c_{x x}(k)=\sum_{n=0}^{N \dashv|k|-1}(x(n)-\bar{x})(x(n-k)-\bar{x})
$$

When normalized with the autocorrelation value at zero lag, equation (1.17) becomes 


$$
C_{x x}(k)=\frac{c_{x x}(k)}{c_{x x}(0)}
$$

The autocorrelation function has traditionally been used for verification of hydrodynamic scaling between fluidized beds. This application will be discussed further in Chapter 3 .

\section{Rescaled Range and Hurst Exponent}

The Rescaled Range is a statistical measure of the variability of a time series, and how that variability changes as the time period being considered increases. For a given time series $\left\{X_{n}\right\}$ from time $(t)$ to $(t+\tau)$, The Rescaled Range is defined as:

$$
R / s(t, \tau)=\frac{\max \left(\left\{X_{n}\right\}\right)-\min \left(\left\{X_{n}\right\}\right)}{\sigma_{\left\{X_{n}\right\}}},
$$

It has been noted [http://en.wikipedia.org/wiki/Rescaled_range] that, as the sample size, $n$, increases, so too does the value of $R / s$. If $R / s$ is calculated for a range of different sample sizes and plotted against $n$ on a log-log plot, the resulting slope is equal to the Hurst Exponent, $H$.

The Hurst Exponent is an estimate of the predictability of a time series. The Hurst Exponent can take on a value between 0 and 1 . In cases where its value is in the range $0 \geq \mathrm{H}>0.5$, the time series from which it is calculated will exhibit a tendency to reverse trends. In other words, an increase in the value of the series parameter will be followed by a decrease, then an increase, etc. For $0.5>\mathrm{H} \geq 1$, the time series exhibits a tendency to continue a trend (i.e. continue to increase, etc.). A value of $\mathrm{H}=0$ is indicative of a Brownian series, in which one point in the time series has no correlation to future time steps. 


\section{Frequency Domain Analysis}

Given that the hydrodynamic nature of a fluidized bed operating at a given dense fluidization condition is strongly affected by the nature of the bubbling within the bed (i.e. bubble frequency), another tool for analyzing the pressure data from fluidized beds is the Power Spectral Density (PSD) plot. This tool converts the time domain pressure data into the frequency domain and then plots the power associated with a given frequency versus frequency. By examining the distribution of power within the frequency spectrum, the nature of the pressure fluctuations due to bubble frequencies can easily be seen. For instance, a bubbling or slugging fluidization regime is dominated by a primary bubble frequency which will show up in the PSD as the dominant frequency. However, the turbulent regime is characterized by a breakup of the bubbling dynamics as the large bubbles in the earlier regimes are broken apart and lead to a range of different frequencies. This results in a wider range of power distribution and a lack of a dominant frequency in the PSD.

The PSD, or spectral density, is related to the autocorrelation function via the following relationship:

$$
S(f)=\sum c_{x x}(\tau) e^{-2 \pi i f \tau},
$$

where $\tau$ is a given time step in the autocorrelation and $f$ is the frequency.

The spectral density is a commonly used method of verifying hydrodynamic similarity and will be discussed further in Chapter 3. 


\section{Chaos Analysis}

The final broad category of methods of analyzing fluidized bed pressure fluctuations is the application of deterministic chaos theory. Kang et al. [45] utilized the mutual information function, phase-space portraits (or strange attractors) and the correlation dimension to study the effects of gas velocity and pressure on bubble properties (chord length, frequency and rising velocity) in a pressurized gas-water bubble column. Zhong et al. [46] applied Shannon entropy analysis (including entropy increment and increment rate) to differential pressure fluctuations in order to determine the fluidization regime transition velocities in a fluidized bed with cylindrical biomass fuels. Zhong and Zhang [47] applied Shannon entropy analysis to pressure data from multiple locations within a spouted bed in order to study the effects of spouting and fluidizing gas velocities on the fluidization characteristics of a spouted bed of biomass particles. Kang et al. [48] applied the Hurst Exponent, Spectral exponent and Shannon entropy to the study of a three-phase fluidized bed with water, air and glass beads. Zhang and Shi [49] applied the analysis of negative Shannon entropy (or negentropy) to the study of density wave instability in a 200 MW nuclear heating reactor. They compared their entropy calculations, derived from the spectral density of pressure fluctuations from more than 500 operational runs of the reactor that operators had pre-determined were either stable or unstable and found that the unstable operating conditions contained high levels of negentropy. Finally, van den Bleek et al. [50] noted that regime transitions were characterized by a "dip" in entropy. In addition, they suggested that the phase-space portrait (or strange attractor) and entropy be used for scaling and hydrodynamic similarity verification between fluidized beds.

As can be seen from this brief literature review of chaos analysis of fluidized beds, entropy, mutual information and phase-space portraits are common tools. 
Shannon (Kolmogorov) Entropy

When applied to signal processing, entropy is a measure of the uncertainty within a signal. The concept is taken from the Boltzmann relation/definition of entropy from the second law of thermodynamics, which defines entropy in terms of the uncertainty of the energy states of a system. The Boltzmann relationship is given by:

$$
h=-k \sum p \ln (p)
$$

where $k$ is the Boltzmann constant and $p$ is the probability of the occurrence of a given energy state. [51]. Claude Shannon applied the concept of entropy to information theory and signal analysis, thus giving his name to entropy in that field (i.e. Shannon Entropy) [52]. In addition, entropy analysis applied to chaotic systems is commonly referred to as Kolmogorov Entropy.

In fluidized bed literature, both Shannon and Kolmogorov entropy are referenced, and are interchangeable. For the purposes of simplification, it will be referred to as Shannon Entropy throughout the remainder of this dissertation, and is given by:

$$
h=-\sum_{i=1}^{n} p\left(x_{i}\right) \log _{2}\left[p\left(x_{i}\right)\right],
$$

where $x_{i}$ is a given range of values, $n$ is the total number of possible outcomes, and $p$ is the individual bin probabilities of the elements of a histogram of the data/signal probability distribution. When examining a signal, such as pressure data, Shannon Entropy is a function of the probability distribution of the signal and not the magnitude of the signal itself. It is used as both a measure of uncertainty within a signal, as well as a measure of the information contained within that signal. Given that $\log _{2}(0)$ is infinity, the Shannon Entropy is defined as "0" for this 
case. In addition, when the signal contains a constant value (i.e. $\left.\mathrm{p}\left(\mathrm{x}_{\mathrm{i}}\right)=1\right)$ there is no information transmitted in terms of information theory, and Shannon Entropy is " 0 ". The value of Shannon Entropy increases with increasing signal variability or uncertainty.

\section{Mutual Information Function}

Similar to the autocorrelation function, the mutual information function is a measure of the correlation of a signal with itself as a function of time, and is given by:

$$
I(a, b)=h(a)+h(b)-h(a, b)
$$

where $b$ is a subset of $a$, separated by a given time lag $\tau$. In other words, for a given time series

$\mathrm{X}(\mathrm{a})$, then $\mathrm{X}(\mathrm{b})=\mathrm{X}(\mathrm{a}+\tau)$ for a given time lag. Additional, $h(a, b)$ is the entropy of the joint probability of $a$ and $b$.

Phase-Space Portraits

The following definition of a Phase-Space is from http://en.wikipedia.org/wiki/Phase space.

In mathematics and physics, a phase space, introduced by Willard Gibbs in 1901, is a space in which all possible states of a system are represented, with each possible state of the system corresponding to one unique point in the phase space. For mechanical systems, the phase space usually consists of all possible values of position and momentum variables. A plot of position and momentum variables as a function of time is sometimes called a phase plot or a phase diagram. Phase diagram, however, is more usually reserved in the physical sciences for a diagram showing the various regions of stability of the thermodynamic phases of a chemical system, which consists of pressure, temperature, and composition.

In a phase space, every degree of freedom or parameter of the system is represented as an axis of a multidimensional space. For every possible state of the system, or allowed combination of values of the system's parameters, a point is plotted in the multidimensional space. Often this succession of plotted points is analogous to the system's state evolving over time. In the end, the phase diagram represents all that the system can be, and its shape can easily elucidate qualities of the system that might not be obvious otherwise. A phase space may contain 
very many dimensions. For instance, a gas containing many molecules may require a separate dimension for each particle's $x, y$ and $z$ positions and velocities as well as any number of other properties.

Phase-space portraits, also known as strange attractors, are a common tool used to describe a deterministic chaotic system. A number of authors have utilized attractors to visualize the states of fluidization in fluidized beds. $[\mathbf{5 0 , 5 3 , 4 4}]$ In addition, van den Bleek et al. [50] suggest a method for using the attractor as a basis for a feedback control system for reactor operation. This will be discussed in greater detail in Chapter 3.

The phase-space portrait of a pressure fluctuation time series can be constructed by means of the time delay method, as presented by Kang et al. [45]. In this method, the time series $\mathrm{X}(\mathrm{t})$ is digitized with a timestep $\Delta \mathrm{t}$. This results in the following $(\mathrm{m}+1)$ values

$\{\mathrm{X}(0 \Delta \mathrm{t}), \mathrm{X}(1 \Delta \mathrm{t}), \mathrm{X}(2 \Delta \mathrm{t}), \ldots, \mathrm{X}(\mathrm{m} \Delta \mathrm{t})\}$

A series of vectors, $Z_{i}(t)$ can be constructed such that

$\mathrm{Z}_{\mathrm{i}}(\mathrm{t})=[\mathrm{X}(i \Delta t), \mathrm{X}(i \Delta t+\tau), \mathrm{X}(i \Delta t+2 \tau), \ldots, \mathrm{X}(i \Delta t+(p-1) \tau)]$

$i=0,1,2, \ldots,[m-(p-1) k]$

where

$\tau=k \Delta t, k=1,2,3, \ldots$

and $\mathrm{p}$ is the embedded phase-space dimension of the reconstructed attractor, $\mathrm{Z}(\mathrm{t})$.

As a final note, it is a common practice to set the time lag, $\tau$, equal to the time corresponding to the first minimum of the mutual information function. [45] 


\section{Chapter 2 Theoretical Approach for Fluidized Bed Drying}

\section{Principles of Fluidized Bed Drying}

Fluidized beds are commonly found in industrial applications used in drying granular materials such as grains, cereals, fertilizers, crystalline products, minerals and chemicals. Some of the reasons for this include large heat transfer surface areas between the material to be dried and the fluidizing gas and the fact that the high degree of mixing in fluidized beds tends to result in negligible temperature and concentration gradients within the fluidizing column [17]. The drying rates of solids in fluidized beds are much higher than other drying methods, such as conventional hot air drying due to higher heat and mass transfer rates on the surface of the drying materials [18]. There are, however, disadvantages to using a fluidized bed dryer. Among these disadvantages are high-pressure drops, attrition of the solids and erosion of surfaces due to particle-particle and particle-surface collisions, as well as the possibility of non-uniform moisture content in the product as a result of varying residence times of individual particles [54].

Fluidized bed drying of solid materials can be either batch-wise or continuous. Batch operations are preferred for small-scale production as well as for heat-sensitive materials, whereas continuous fluidized beds are normally used for large-scale operations [15]. In cases of batch drying, which is typically carried out in the dense bubbling or slugging regimes, the final moisture content of the product is more uniform due to the fact that the particles tend to be of uniform temperature and residence times, whereas continuously operating fluidized beds vary widely in particle residence times and temperatures due to the continuous introduction of new material into the system. 
Due to the operational nature of fluidized bed dryers (i.e., a continuous flow of warmer gas passing through the drying particles) the primary form of heat transfer between the particles and the fluidizing gas is that of convection heat transfer. According to Nonhebel and Moss [9], if the hot gas used in convection drying is supplied at a constant temperature and humidity, the drying process will occur in two distinct stages.

The initial stage is characterized by a constant drying rate in which moisture is transported to the heat transfer surface from within the solid material being dried at the same rate as moisture is evaporating from this surface. The second stage is characterized by a diminishing drying rate until the particle is completely dry. The moisture content at which the drying rate begins to decrease is referred to as the critical moisture content.

In the constant drying rate period, the controlling factors are the temperature, velocity and humidity of the drying gas; during the falling drying rate period, the factor controlling the drying rate become the rate at which moisture migrates to the drying surface. Figure 2-1 illustrates a set of typical convection drying rate curves showing both the constant and falling rate periods, as well as the critical moisture content region.

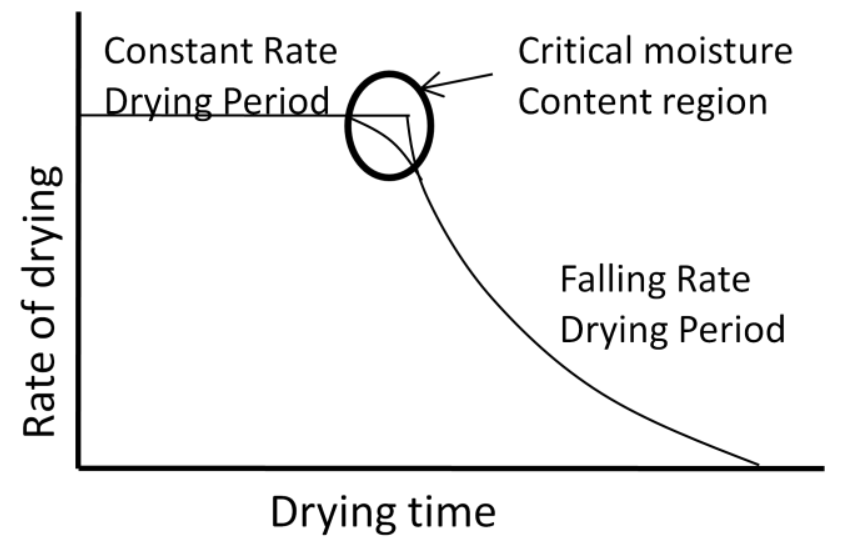

Figure 2-1: Typical convection drying rate curve 
A common method of analyzing the drying rates of fluidized bed dryers in the relevant literature is to plot the normalized ratio of product to initial moisture content, $\mathrm{C} / \mathrm{C}_{\mathrm{o}}$ versus the total drying time. An example of this can be seen in Figure 2-2.

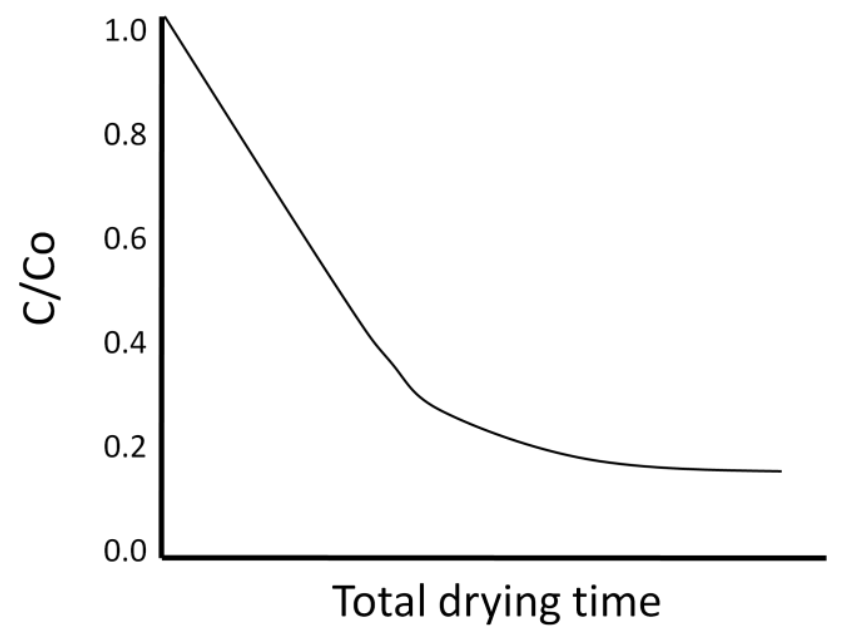

Figure 2-2: Moisture content ratio versus total drying time

\section{Droplet evaporation/film drying model}

The following drying model for a single particle assumes that the particle is at a constant, uniform temperature, and that the moisture resides within a thin film on the particle surface. In addition, it is assumed that drying is taking place during the constant rate period.

To develop this model, one need first examine the nature of the evaporation of a homogenous droplet of fluid at uniform temperature, $T_{d}$, into a surrounding gas at $T_{\infty}$ due to convective heat transfer. An example of such a droplet is shown in Figure 2-3.

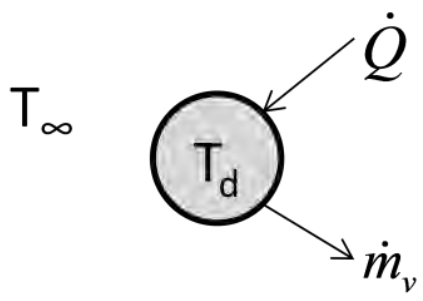

Figure 2-3: Droplet evaporation model 
Neglecting all work except for moving boundary work (simulating the shrinking of the droplet as it evaporates), as well as kinetic and potential energy, the energy balance for this system is given by:

$$
\left.\frac{d E}{d t}\right|_{s y s}=Q-{ }_{v} h_{v}-p \frac{d V_{d}}{d t}
$$

Where

$$
\left.\frac{d E}{d t}\right|_{s y s}=\frac{d\left(m_{d} u_{d}\right)}{d t}=m_{d} \frac{d u_{d}}{d t}+u_{d} \frac{d m_{d}}{d t} \quad ;
$$

where $u_{d}$ and $m_{d}$ are the internal energy (per unit mass) and the mass of the droplet, respectively.

In addition, the heat transfer to the droplet is convective in nature, so

$$
Q=h A_{d}\left(T_{\infty}-T_{d}\right),
$$

and $V_{d}$ is the volume of the droplet, given by:

$$
V_{d}=\frac{m_{d}}{\rho_{d}}
$$

Combining Equations (2.1)-(2.4) and rearranging terms yields:

$$
\begin{gathered}
d \frac{\left(m_{d}\right)}{\mid} m_{d} \frac{d u_{d}}{d t}=h A_{d}\left(T_{\infty}-T_{d}\right)-{ }_{v} h_{v}-p \frac{\left(\rho_{d}\right)}{d t}-u_{d} \frac{d m_{p}}{d t} \\
m
\end{gathered}
$$


Noting that the droplet properties are a function of the temperature and pressure, which are held constant in this model, the density can be pulled out of the partial derivative in the boundary work term in (2.5). In addition, the following substitution can be made:

$$
\frac{d u_{d}}{d t}=c_{p, d} \frac{d T_{d}}{d t}
$$

Now (2.5) takes the following form:

$$
m_{d} c_{p, d} \frac{d T_{d}}{d t}=h A_{d}\left(T_{\infty}-T_{d}\right)-m_{v} h_{v}-\frac{p d m_{d}}{\rho d t}-u_{d} \frac{d m_{p}}{d t}
$$

In addition, by making the following substitutions

$$
\left.v_{d}=\frac{1}{\rho_{d}},-m\right]_{v}=\frac{d m_{p}}{d t}
$$

(2.7) can be rewritten as:

$$
m_{d} c_{p, d} \frac{d T_{d}}{d t}=h A_{d}\left(T_{\infty}-T_{d}\right)-m_{v} h_{v}+m_{v}\left(p v_{d}+u_{d}\right.
$$

The quantity in the parenthesis in the last term of (2.9) is by definition the enthalpy of the droplet, or $h_{d}$. One final simplification making use of the fact that $h_{f g}=h_{v}-h_{d}$, yields:

$$
m_{d} c_{p, d} \frac{d T_{d}}{d t}=h A_{d}\left(T_{\infty}-T_{d}\right)-m \prod_{v} h_{f g}
$$

Assuming a constant droplet temperature, the LHS of (2.10) is zero, leading to the following expression for the evaporation rate of the droplet: 


$$
m_{v}=\frac{h A_{d}\left(T_{\infty}-T_{d}\right)}{h_{f g}}
$$

The convective heat transfer coefficient, h, can be found in terms of the Nusselt number, $\mathrm{Nu}$, such that:

$$
h=\frac{k N u}{d_{d}}
$$

For a droplet exposed to a turbulent flow, Lavender and Pei [12] propose the following empirical expression for the Nusselt number:

$$
N u=2+0.717 \operatorname{Re}^{1 / 2} \operatorname{Pr}^{1 / 3}\left(\operatorname{Re} I_{\infty}\right)^{0.035}
$$

Where Pr is the Prandtl number, or the ratio of momentum diffusivity over thermal diffusivity, and is given by:

$$
\operatorname{Pr}=\frac{\mu / \rho}{D_{t}}
$$

$I_{\infty}$ is the turbulent intensity of the flow field, given by:

$$
I_{\infty}=\frac{u^{\prime}}{U_{\infty}}
$$

Substituting (2.12) and the expression for the surface area of a sphere into (2.11) yields:

$$
m_{v}=\frac{\pi N u k d_{d}\left(T_{\infty}-T_{d}\right)}{h_{f g}}
$$

In addition, 


$$
\frac{d m_{p}}{d t}=\rho_{d} \frac{d}{d t}\left(\frac{4}{3} \pi r_{d}^{3}\right)=v
$$

Combining (2.16) and (2.17) gives:

$$
-\frac{\pi N u k d_{d}\left(T_{\infty}-T_{d}\right)}{h_{f g}}=\rho_{d} \frac{d}{d t}\left(\frac{4}{3} \pi r_{d}^{3}\right)
$$

Upon rearranging

$$
\begin{aligned}
& -\left|\frac{3 \pi N u k\left(T_{\infty}-T_{d}\right)}{\lceil}\right| d t=\frac{1}{r} d\left(r_{d}^{3}\right) \\
& \left\lfloor 2 \rho_{d} h_{f g}\right\rfloor{ }_{d}
\end{aligned}
$$

By making the following substitutions

$$
x=r_{d}^{3}, x^{-1 / 3}=\frac{1}{r_{d}}
$$

(2.19) can be integrated from $\mathrm{t}=0$ to and arbitrary time $\mathrm{t}$ to give the following solution:

$$
r_{d}^{2}(0)-r_{d}^{2}(t)=\frac{N u k\left(T_{\infty}-T_{d}\right)}{\rho_{d} h_{f g}} t
$$

Equation (2.20) is commonly known in combustion literature as the Radius-Squared Rule [55].

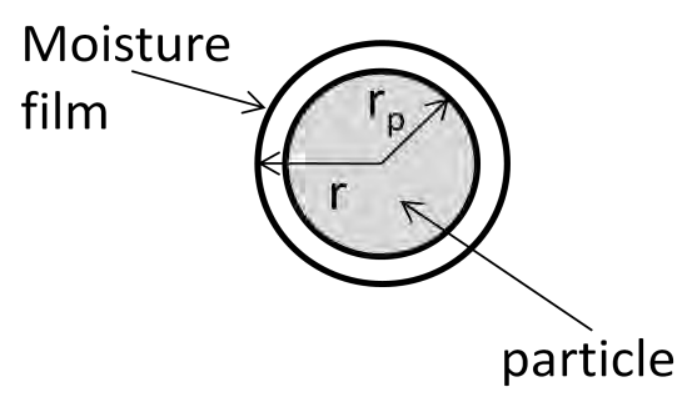


Figure 2-4: Solid particle with thin film of moisture 
Figure 2-4 shows a single solid particle surrounded by a thin film of liquid moisture. If the particle and moisture film are at the same temperature, and $r \geq r_{p}$, then (2.20) can be modified in the following manner:

$$
r_{d}^{2}(0)-r_{p}^{2}=\frac{N u k\left(T_{\infty}-T_{p}\right)}{\rho_{l} h_{f g}} t
$$

Solving (2.21) for $\mathrm{t}$ yields the time required for a moisture film of known thickness to evaporate from the surface of a solid particle.

\section{Thermodynamic Analysis of Fluidized Bed Drying}

The following sections discuss the operation of the WADFP system during steady state operation from a thermodynamic perspective. This analysis is based upon the basic principles of the conservation of mass, energy, thermodynamic entropy and exergy (or availability). For this analysis, the riser and cyclone are treated as a control volume. Entering and leaving this control volume are air, coal and moisture. It is assumed here that all moisture entering the system is with the wet coal, and that the air entering is dry air only. It is further assumed, due to the homogeneous nature of temperature distributions within a fluidized bed, that the air, coal and moisture leaving the system do so at the same temperature.

\section{Conservation of Mass}

From the principles of conservation of mass for a control volume operating at steady state conditions, the rates of airflow, dry coal and moisture entering and exiting the system must be equal. This is expressed in the following equations.

$$
\begin{aligned}
& \left(m_{\text {air }}\right)_{\text {in }}=\left(m_{\text {air }}\right)_{\text {out }} \Rightarrow m m_{a 1}+m_{a 2}+m_{a 3}= \\
& m_{a 4} \\
& \left(m_{\text {coal }}\right)_{\text {in }}=\left(m_{\text {coal }}\right)_{\text {out }} \Rightarrow m m_{\text {coal }, 2}=m_{\text {coal }, 3}
\end{aligned}
$$




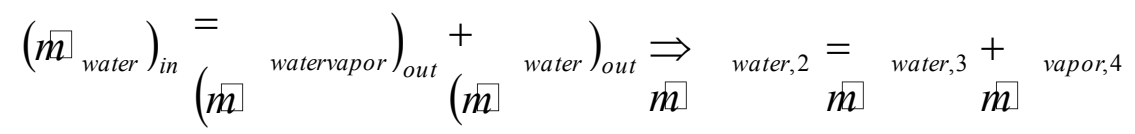

where Figure 2-6 depicts the control volume and associated subscript locations.

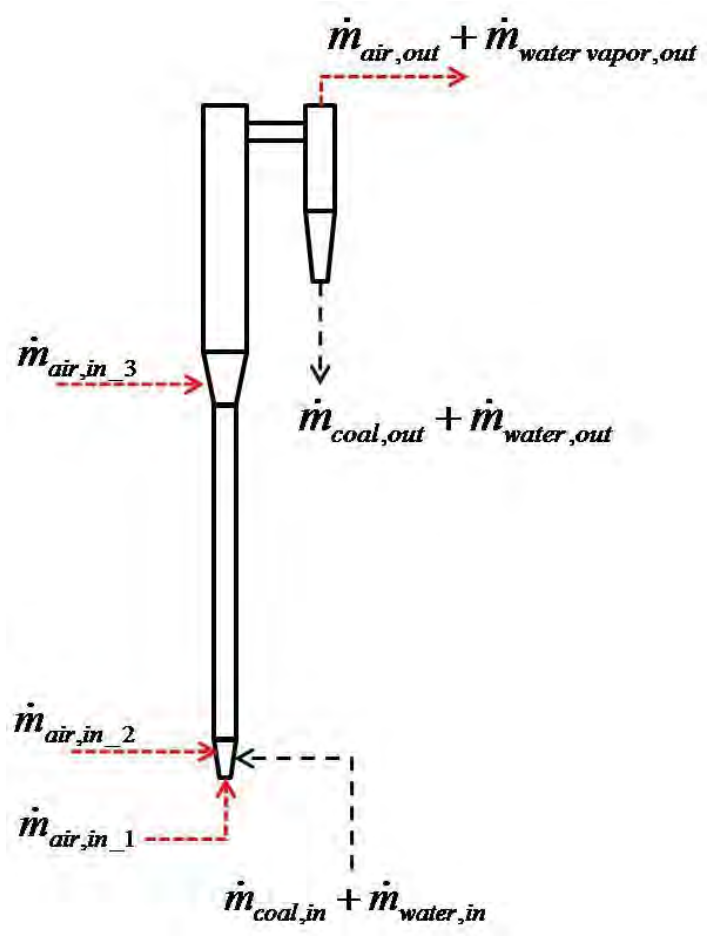

Figure 2-5: WADFP system diagram

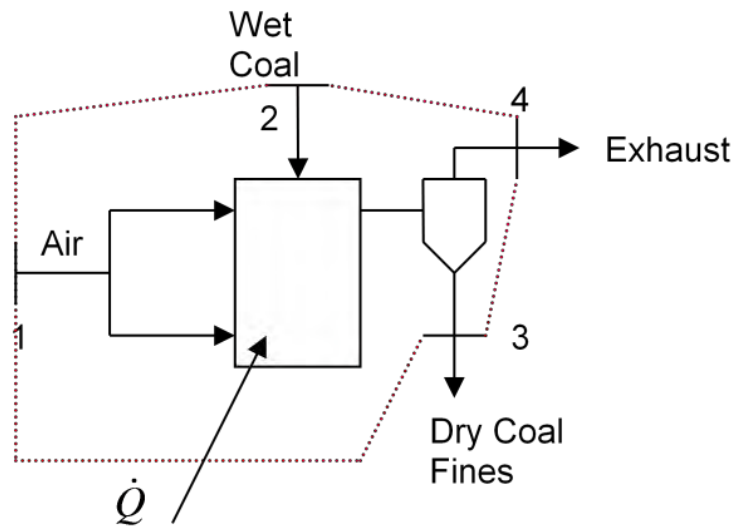

Figure 2-6: Control Volume for thermodynamic analysis 


\section{Energy Analysis}

Based upon the notation used in Figure 2-6, the steady state energy equation for the system is given by:

$$
\begin{aligned}
& 0=Q+m_{1} h_{1}+m_{2} h_{2}-m_{3} h_{3}- \\
& m_{4} h_{4}
\end{aligned}
$$

However, due to the fact that the mass flow rates, $m$, at locations 2 and 3 consist of moisture as well as solids material, it is necessary to decompose the third and fourth terms of the RHS of the above equation into their constituent parts.

$$
\begin{aligned}
& m_{2} h_{2}=\xi_{2} m_{2} h_{f 2}+\left(1-\xi_{2}\right) m_{2} h_{2 c} \\
& m_{3} h_{3}=\xi_{3} m_{3} h_{f 3}+\left(1-\xi_{3}\right) m_{3} h_{3 c}
\end{aligned} ;
$$

where $\xi$ is the percent moisture content at each location, written as a decimal fraction. The subscripts "c" and "f" denote coal and fluid (water), respectively.

Similarly, the exhaust at location 4 must be decomposed into its air and water vapor components.

$$
\begin{aligned}
& m_{4} h_{4}=m_{4 a} h_{4 a}+m m_{4 v} \\
& h_{4 v}
\end{aligned}
$$

However, for steady state operation and assuming that the specific humidity at location 1 is 0 , conservation of mass requires:

$$
\begin{aligned}
& m_{4 a}= \\
& m_{1}
\end{aligned}
$$

In addition, the mass flow of vapor at 4 can be written in terms of the air flow rate into the system at location one by:

$$
\begin{aligned}
& m_{4 v}=\bar{\omega}_{4} \\
& m m_{1}
\end{aligned}
$$


Where $\bar{\omega}_{4}$ is the specific humidity at location 4 .

Substituting these relations into Equation (2.28) leads to the following: 


$$
\begin{aligned}
& m_{4} h_{4}=m_{1} h_{4 a} \overline{+} \omega_{4} \\
& m_{1} h_{4 v}
\end{aligned}
$$

Combining equations (2.25), (2.26), (2.27) and (2.31) yields:

$$
\begin{aligned}
& 0=Q+m_{1} h_{1}+\xi_{2} m_{2}+\left(1-\xi_{2}\right) m_{2} h_{2 c}-\xi_{3} m{ }_{3} h_{3} \\
& h_{2 f} \\
& \quad-\left(1-\xi_{3}\right) m_{3} h_{3 c}-m{ }_{1} h_{4}=\omega_{4} m{ }_{1} h_{4 v}
\end{aligned}
$$

Upon rearranging and solving for the rate of heat transfer, $Q$, this can be rewritten as:

$$
\begin{aligned}
& \left\lceil m_{1}\left(h_{1}-h_{4 a}-\bar{\omega} h\right)+(\xi h+(1-\xi) h)\right\rceil
\end{aligned}
$$

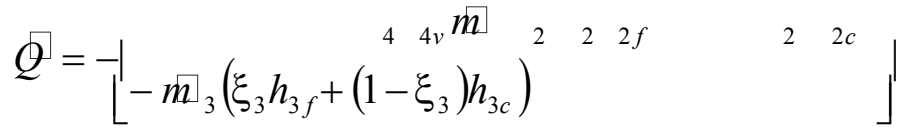

The heat transfer term $Q$ can be further broken down into;

$$
\underbrace{Q}_{\text {loss }}=Q_{\text {evap }}-
$$

$Q_{\text {evap }}$ is the rate of heat transfer due to evaporation of moisture and is given by:

$$
Q_{\text {evap }}=\bar{\omega}_{4} m{ }_{1} h_{f g}
$$

where $\mathrm{h}_{\mathrm{fg}}$ is the latent heat of vaporization of water at the average temperature of the wet material.

$Q_{\text {lo ss }}$ is the rate of heat lost to the system surroundings; in the case of an insulated system with no losses, this term can be neglected.

\section{Energy Efficiency}

Giner and Calvelo [56] defined the thermal efficiency of the fluidized bed drying process as:

$\eta_{t h}=\frac{\text { Energy transmitted to the solid }}{\text { Energy incorporated in the drying air }}$

Syrahrul, et al. [57] propose the following expression (modified for current symbols usage) of 
the thermal efficiency in terms of the energy rate balance equation: 


$$
\eta_{\text {th }}=\frac{m_{\text {coal }}\left[h_{f g}\left(\xi_{2}-\xi_{3}\right)+c_{\text {coal }}\left(T_{\text {coal }, \text { out }}-T_{\text {coal,in }}\right)\right]}{m_{\text {air }}\left(h_{\text {air }, \text { in }}-h_{0}\right)}
$$

\section{Entropy Analysis}

While mass and energy are conserved quantities, this is not true of entropy. To account for the changes in entropy, the entropy balance must be taken into consideration. An analysis of the entropy balance within the fluidized bed drying process can be approached in a manner similar to that provided for the energy analysis provided in the previous section.

For a steady-state, open system based upon the design of the WADFP unit, the entropy balance can be written as:

$$
0=\frac{Q}{T}+m_{{ }_{1}} s_{1}+m_{2} s_{2}-m_{m_{3}} S_{3}-m_{4} s_{4}+
$$

However, as in the previous section, the quantities at locations 2,3 and 4 consist of combinations of solid material, air and moisture; which must be broken down into their constituent components.

Following the same procedure used for the energy balance discussion yields

$$
\begin{aligned}
0= & \left.\frac{Q}{T}+m{ }_{S_{2 f}} s_{1}+\xi_{2} m m_{2}+\left(1-\xi_{2}\right) m_{2} s_{2 c}-\xi_{3} m\right]_{3} s_{3} \\
& -\left(1-\xi_{3}\right) m_{3} s_{3 c}-m{ }_{1} s_{4}=\omega_{4} m{ }_{1} s_{4 v}+S_{g e n}
\end{aligned}
$$

Rearranging and combining terms yields the following expression for the rate of entropy generation within the fluidized bed. 


$$
S_{\text {gen }}=-\begin{gathered}
\left\lceil m_{1}\left(s_{1}-s_{4 a}-\bar{\omega}_{4} s_{4 v}\right)+{ }_{2}\left(\xi_{2} s_{2 f}+\left(1-\xi_{2}\right) s_{2 c}\right)\right. \\
m
\end{gathered} \mid
$$

\section{Exergy Analysis}

Exergy is a term describing the availability of useful work potential within a system without violating any thermodynamic laws. [58] The energy balance equation for a steady state control volume based upon the WADFP fluidized bed is obtained by multiplying the entropy equation (2.40) by a reference temperature, $\mathrm{T}_{0}$, and subtracting it from the energy equation (2.32). This yields the following:

$$
\begin{aligned}
& \left.0=\left.Q\right|^{(}{ }^{1} T_{0}\right)^{\mid+m}{ }_{1}\left[\left(h_{1 a}-h_{4 a}-\omega_{4} h_{4 v}\right)-T_{0}\left(s_{1}-s_{4 a}-\omega_{4} s_{4 v}\right)\right]+ \\
& -\left(\frac{T}{T}\right) \\
& m_{2}\left[\left(\xi_{2} h_{2 f}+\left(1-\xi_{2}\right) h_{2 c}\right)-T_{0}\left(\xi_{2} s_{2 f}+\left(1-\xi_{2}\right) s_{2 c}\right)\right]- \\
& m_{3}\left[\left(\xi_{3} h_{3 f}+\left(1-\xi_{3}\right) h_{3 c}\right)-T_{0}\left(\xi_{3} s_{3 f}+\left(1-\xi_{3}\right) s_{3 c}\right)\right]-T_{0} \text { gen }
\end{aligned}
$$

Or, in a more simplified form:

$$
\begin{aligned}
& 0=E_{2 c}-E_{3 c}+E_{f}+E_{1 a}-E_{4 a}-E_{4 v}+E_{\text {evap }}-E_{D}, \\
& E_{2 f}
\end{aligned}
$$

where $E_{D}$ is the rate of exergy destruction in the dryer.

Syrahrul et al. [59] gives the rate exergy transfer due to evaporation as:

$$
E_{\text {evap }}=\left\lfloor 1-\frac{T_{0}}{T}\right] Q_{\text {evap }}=\left\lfloor 1-T_{0}\right] \bar{\omega}_{4} m_{1} h_{f g}
$$


In addition, Syrahrul et al. states that the exergy efficiency, based upon the exergy rate balance, is given by: 


$$
\eta_{e x}=\frac{\text { rate of exergy transfer due to evaporation }}{\text { rate of exergy transfer of entering air }}
$$

Or

$$
\eta_{e x}=\frac{\left\lfloor 1-\frac{T_{0}}{T}\right] \varpi_{4} \not{ }_{1} h_{f g}}{m_{1}\left[\left(h_{1}-h_{0}\right)-T_{0}\left(s_{1}-s_{0}\right.\right.}
$$




\section{Chapter 3 Fluidized Bed Scaling}

When utilizing a smaller scale fluidized bed operating at low temperatures and atmospheric pressure to model the processes taking place within a larger bed operating at higher temperatures and pressures, one must be careful to accurately account for the differences between the two operating regimes. In addition to ensuring that both fluidized beds have similar geometric features, it is necessary to incorporate the effects of temperature and pressure upon the material properties of fluidizing gas, as changes in density and viscosity can have drastic effects upon the performance characteristics of a fluidized bed.

Over the last couple of decades, there have been many attempts to develop a set of scaling relationships between high temperature and pressure fluidized bed combustors and reactors and smaller "cold" scale models operated at atmospheric conditions. The following sections provide a summary of some of the more well-known works related to hydrodynamic scaling of fluidized beds, including scaling relationships and common techniques for experimental verification of hydrodynamic similitude.

\section{Hydrodynamic Scaling Relationships}

The following sections provide a detailed review of bubbling fluidized bed scaling literature by such authors as Glicksman, Horio, Zhang and Yang $[\mathbf{6 0 , 6 1 , 6 2 , 6 3 , 6 4 , 6 5 ] . ~ I n ~ a d d i t i o n , ~ a n ~}$ alternate approach suggested by van den Bleek et al. [50] utilizing entropy and chaos analysis is presented.

\section{Glicksman's Scaling Relationships.}

Leon Glicksman [60] developed a set of scaling relationships for fluidized beds by nondimensionalizing the governing equations of the conservation of mass and momentum for the fluidizing gas and bed particles. The resulting non-dimensional parameters are: 


$$
\frac{\rho_{s} \rho_{f} d_{p}^{3} g}{\mu^{2}}, \frac{u_{0}^{2}}{g d_{p}}, \frac{\rho_{f}}{\rho_{s}}, \frac{L}{d_{p}}, \frac{D}{d_{p}}, \phi_{s}
$$

Glicksman then goes on to consider a pair of extreme situations in his analysis. The first extreme occurs when particles are closely spaced and the bed approaches a packed bed, resulting in low Reynolds number $(\operatorname{Re} \leq 4)$ and Ergun equation is dominated by the viscous term. In this situation, the effect of the density ratio term in equation 3.1 becomes negligible and the list of scaling parameters can be reduced to the following.

$$
\frac{u_{0}^{2}}{g d_{p}}, \frac{\rho_{s}^{2} d_{p}^{3} g}{\mu^{2}}, \frac{L}{d_{p}}, \frac{D}{d_{p}}, \phi_{s}
$$

For the inertia-driven condition (for high Reynolds number), the governing parameters become:

$$
\frac{u_{0}^{2}}{g d_{p}}, \frac{\rho_{f}}{\rho_{s}}, \frac{L}{d_{p}}, \frac{D}{d_{p}}, \phi_{s} \text { (as well as particle size distribution and bed geometry) }
$$

Finally, between the upper and lower Reynolds number limits, both the viscous and inertial forces are important to the fluid dynamics of the system and no simplifications can be made.

In summary, according to Glicksman, to attain completely similar behavior between a hot bed and a model at atmospheric temperature, the value of each of the non-dimensional parameters must be the same for the two beds. If the hot bed operates in the viscous or inertial dominated regions, then the number of parameters can be reduced to those provided in 3.2 and 3.3, respectively.

In a subsequent paper, Nicastro and Glicksman [63] provided experimental verification of the scaling laws put forth for the low Reynolds number viscous regime [60]. In that study, tests 
carried out on an atmospheric fluidized bed combustor operating at $1050 \mathrm{~K}$ and its scale model operating at ambient temperature showed good agreement between fluid dynamic characteristics measured by minimum fluidization velocity and pressure fluctuations caused by bubbles.

\section{Alternative Glicksman Scaling Laws for the Viscous Limit}

In response to an alternative method suggested by Horio, et al. [64], Glicksman [61] later proposed an alternate set of scaling laws for the viscous-dominated regime. In this alternative method, he proposed non-dimensionalizing all of the length coordinates in the equations of motion and conservation of mass by a bed dimension, $L$, instead of by the particle diameter, $d_{p}$. This method of non-dimensionalization results in the following governing parameters:

$$
\frac{u_{0}^{2}}{g L}, \frac{u_{0}}{u_{m f}}, \frac{L}{D}, \frac{L_{1}}{L_{2}}, \phi_{s} \text {, as well as particle size distribution }
$$

According to Glicksman [61], when applied within the viscous limit, these scaling parameters are identical to those proposed by Horio [64].

\section{Glicksman's Simplified Scaling Relationships}

In his 1993 publication, Glicksman et al. [62] introduced a new, "simplified" approach to fluidized bed scaling. The purpose of this approach was to ease the stricter requirement for similar geometry between the hot and cold beds in order to allow the scaling parameters to cover a wider range of scaling options. Glicksman concludes that for all cases (including viscous and enertial dominant), similititude can be obtained by maintaining constant values for the following list of dimensionless parameters:

$$
\frac{u_{0}^{2}}{g L}, \frac{\rho_{s}}{\rho_{f}}, \frac{u_{0}}{u_{m f}}, \frac{L_{1}}{L_{2}}, \frac{G_{s}}{\rho_{s} u_{0}}, \phi, \text { and particle size distribution. }
$$




\section{Horio's Scaling Parameters.}

Whereas Glicksman $[\mathbf{6 0}, \mathbf{6 1}, \mathbf{6 2}]$ formulated his fluidized bed scaling parameters by nondimensionalizing the basic momentum and mass conservation equations and examining the resulting non-dimensional groupings, Horio et al. [64] proposed an alternative approach to fluidized bed scaling. The set of scaling parameters derived by Horio et al. are based primarily upon maintaining geometric similarity between a large bed operating at high temperatures and pressures and a scale model operating at ambient conditions. Horio et al, approach the issue from the perspective of the bubble behavior within the fluidized bed. This approach attempts to insure similarity in bubble geometry, splitting frequency, rise time as well as similarity of the flow field around each bubble between the two beds under comparison.

With this approach, it is assumed that the bed height $L$, column diameter $D$, distributor orifice diameter $d$, orifice pitch $P$ and other structural aspects of the bed design are changed by the same proportional amount, $m$. In other words, for two beds of differing sizes,

$$
m=\frac{L}{L^{\prime}}=\frac{D}{D^{\prime}}=\frac{P}{P^{\prime}}=\frac{d}{d^{\prime}}
$$

The final scaling parameters proposed by Horio et al. include not only those listed in equation (3.6), but also includes the following:

$$
\begin{aligned}
& \frac{u_{m f}}{\sqrt{g D}}=\frac{u_{m f}^{\prime}}{\sqrt{g D^{\prime}}} \\
& u_{0}-u_{m f}=\sqrt{m}\left(u_{o}-u_{m f}\right)^{\prime}
\end{aligned}
$$

for bubble coalescence, and 


$$
u_{m f}=\sqrt{m} u_{m f}^{\prime}
$$

for geometrically similar flow field around bubble and for similar bubble splitting.

The full derivation method of Horio's scaling parameters is not included as these results are nearly identical to the alternate viscous limit scaling parameters as discussed by Glicksman [61]. In fact, Glicksman states that one of the purposes of his short paper is to show that the Horio scaling parameters can be obtained via non-dimensionalizing the basic momentum and mass conservation equations by a characteristic bed length (such as bed height or column diameter) and simplifying the Ergun equation for the low Reynolds number case.

\section{Zhang and Yang's Scaling Laws}

Another set of dimensionless scaling laws for fluidized bed similarity was proposed by Zhang and Yang [65] for bubbling beds. Following an approach similar to that utilized by Glicksman, Zhang and Yang presented a set of scaling parameters for a bubbling bed operating in the intermediate, viscous and inertial ranges.

For the viscous dominant region, i.e., $\operatorname{Re}<4$, Zhang and Yang concluded that similarity between two fluidized beds could be maintained by keeping the following similarity groups identical:

$$
\begin{gathered}
\frac{g D}{U^{2}} \text { and } \frac{\rho_{s} g\left(\phi_{s} d\right)}{{ }_{2} \mu^{2} D_{4}} \\
{ }_{0}
\end{gathered}
$$

For the inertially-dominant region, i.e., $\operatorname{Re}>400$, the required similarity groups become:

$$
\frac{g D}{U_{0}^{2}} \text { and } \frac{\rho_{f} D}{\rho_{s} \phi_{S} d}
$$


Finally, in the transition region where both viscous and inertial forces are significant, the governing groups are given as:

$$
\begin{aligned}
& g D, \rho_{s} g\left(\phi_{s} d\right) \quad \underline{\rho_{f} D} \\
& \overline{U_{0}^{2}} \frac{{ }^{2}}{\mu_{f}^{2} D}, \rho_{s} \phi_{S} d
\end{aligned}
$$

One major difference between this method proposed by Zhang and Yang and the similarity laws proposed by Glicksman is that the similarity groups of Zhang and Yang do not require a constant particle density to fluid density ratio between the beds being compared. Under the Glicksman approach, maintaining a constant $\rho_{s} / \rho_{f}$ requires the use of a different solids material in the scale model than that used in the full-scale fluidized bed. However, the approach described by Yang and Zhang allow for the same solids material to be used in both beds. This is possible due to the fact that their approach does not require a set particle-to-bed diameter ratio.

Zhang and Yang justify this by noting that $g D \mid U_{0}^{2}$ and $U_{0} U_{m f}$ are equivalent to $g D \mid U_{m f}^{2}$ and $U_{0} U_{m f}$, thus

$$
\frac{U_{m f, A}}{U_{m f, B}}=\left(\frac{D_{A}}{D_{B}}\right)^{\mathbf{U}^{2}},
$$

where the subscripts A and B denote the two fluidized beds being compared. Zhang and Yang utilize equation (3.13) by determining the required minimum fluidization velocity to satisfy (3.13) and selecting an appropriate particle size to obtain that velocity. 


\section{Scaling with Entropy}

During their exploration of the application of chaos analysis to multiphase reactors, van den Bleek et al. [50] demonstrated that application of the Glicksman scaling parameters can lead to drastic differences in Shannon entropy values even when the Glicksman scaling guidelines are followed.

To address this issue, a dimensionless entropy number is introduced:

$$
K_{\sqrt{H_{b} / g}}
$$

Where $\mathrm{K}$ is the Kolmagorov entropy, and

$$
K_{\mathbb{N}} H_{b} / g=\left(\frac{\left(U-U_{m f}\right)^{2}}{g H_{b}}\right)^{m}\left(\frac{H_{b}}{D_{t}}\right)^{n}
$$

In equation (3.15), $H_{b}$ is the dense bed height and $D_{t}$ is the riser diameter. The exponents " $m$ " and " $n$ " are experimentally determined and vary with fluidization regime.

van den Bleek et al. go on to state that scaling for entropy similarity should take priority, and to do so, it is necessary to ensure that the Froude number and bed aspect ratios are maintained constant between fluidized beds. The general approach suggested by van den Bleek is to pick a known scaling method (such as that proposed by Glicksman) and follow it as closely as possible; however give priority to the Froude number and bed aspect ratio, even if doing so means other scaling parameters are not maintained.

\section{Experimental Verification Techniques of Scaling Laws}

Several of the authors mentioned in the previous sections of this Chapter, as well as others $[63,66,67,65]$, have provided experimental verification of the scaling laws previously discussed. 
Nicastro and Glicksman [63] and Westphalen [66] analyzed fluctuating pressure signals to determine the mean, standard deviation, probability density and power spectral density functions for each fluidized bed being tested, and then compared the results between the them. Westphalen [66] also compares the probability density function of the solids fractions between beds. Ellis, et al. [67] utilizes the probability density of the bed voidage, radial voidage profiles and particle velocity versus bed voidage comparisons in determining bed similarity. Zhang and Yang [65] compare bed properties such as collapsed bed height, pressure drop, particle entrainment rate and minimum fluidization velocities to determine similarity.

The most common method of hydrodynamic similarity verification found in literature is to compare the probability distribution function (PDF), autocorrelation and power spectral density (PSD). However, nearly all of the literature pertaining to these methods is given in reference to a fluidized bed operating in the bubbling fluidization regime. In a bubbling bed, the bubble formation is periodic in nature where the frequency of bubble formation is driven by a single dominant frequency. Because of this, the periodic nature of the autocorrelation function can easily be seen, as can the presence of a dominate frequency in the spectral density plot. Unfortunately, these methods are less than ideal for turbulent fluidization conditions. In turbulent fluidization, the bubble formation is non-periodic and occurs over a wide range of frequencies with no single frequency dominant.

To address this, van den Bleek et al. [50] proposes use of the strange attractor and nondimensional entropy number for verification of hydrodynamic similarity in turbulent fluidization. A common method for comparing different attractors in order to determine whether or not they originate from similar dynamic systems is by application of the Diks test [68]. This test calculates a dimensionless distance, $\mathrm{S}$, between two attractors. If the value of " $\mathrm{S}$ " is less than 3 , 
then the attractors are based upon similar physical systems. The dimensionless distance, $\mathrm{S}$, is defined by Diks et al. [69] as:

$$
S=\frac{\hat{Q}}{\sqrt{V_{c}(\hat{Q})}}
$$

Where $\hat{\mathrm{Q}}$ is a statistical estimator of the distance between two distributions, and $V_{c}$ is the variance. This method is described in greater detail by Diks et al. [69]. 


\section{Chapter 4 Scale Model System}

As stated previously, there are two different fluidized beds utilized in this study. The first system is a steam jacketed fluidized bed known as the Warm Air Dryer for Fine Particles, or WADFP. The second system, which is the subject of this chapter, is a small half scale model of the WADFP that was designed and constructed with the intent of being used to first establish the feasibility of operating the WADFP within the desired fluidization regimes, as well as provide data for scaling analysis and verification for the larger fluidized bed.

\section{General Design Concepts}

Both fluidized beds utilize a design that incorporates two multiple-section stages of differing diameters. The lower stage of each bed consists of three removable "small-diameter" pipe sections; while the upper stage of each bed consists of two "large-diameter" pipe sections (one of which is removable). The two stages are connected via a conical injection ring which serves as both a transition from the smaller to larger diameter stages as well as the means by which additional airflow is to be introduced into the upper section of the riser. Similarly, the fluidizing gas (air) is introduced into the lower section of the riser via a distributor section. The fluidizing gas (and entrained particles) exits the top of the riser via an outflow port, where the particulate matter is separated from the exhaust gas via a cyclone. Finally, a feed hopper supplies particulate matter into the riser. Figure 4-1 is a schematic illustration of the basic design of the scale model fluidized bed, as well as shows the general placement of the components discussed above.

A detailed description of the various components of the scale model is given in the following sections; a similar description of the WADFP unit can be found in Chapter Six. 


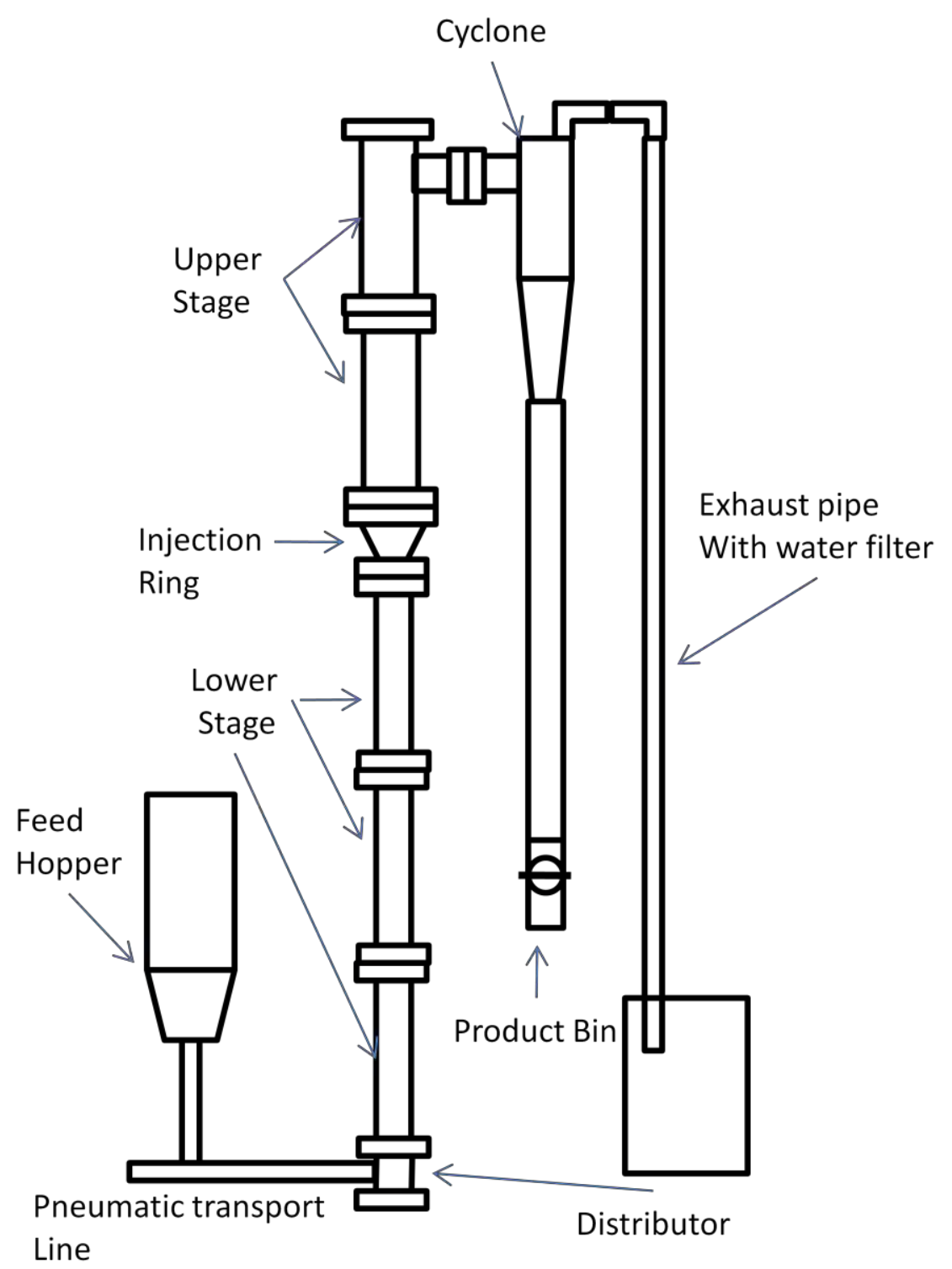

Figure 4-1: Layout for scale model fluidized bed

\section{System Design}

\section{Design Criteria}

The primary consideration behind the design of the scale model fluidized bed was that it was a close physical representation of the larger warm air dryer of fine particles (WADFP) fluidized bed. The scale model riser and cyclone have been designed to maintain a $0.5: 1$ dimensional ratio with the WADFP. The "tube" sections of the lower and upper stages of the riser are constructed 
of transparent acrylic PVC in order to allow for visual inspection and video recording of the fluidization conditions within the riser during operation. The distributor, injection ring and cyclone were unable to be manufactured out of acrylic PVC due to manufacturing limitations and were thus manufactured of carbon steel by Wilson Works in Morgantown, WV.

The scale model fluidized bed experimental system consists of four sections: the riser, cyclone, product bin and feed hopper. Detailed engineering drawings of the scale model system were generated with Autocad and are included on the accompanying dvd.

\section{Riser}

The fluidized bed riser is characterized by two distinct stages of differing diameters, as well as a lower air distributor at the bottom of the lower stage and a conical secondary air injection ring located between the upper and lower stages.

\section{Lower Riser Stage}

The lower stage of the riser consists of the 3 small diameter pipe sections. Each of the pipe sections are approximately 18.375 inches in height and have an inside diameter of 2.29 inches and are made of transparent acrylic pipe. The original design called for 2.5 inch diameters for these sections; however, material availability necessitated the use of 2.29 ID pipe as that was the closest available size. In addition, the top and bottom of each section has 0.5 inch thick flanges

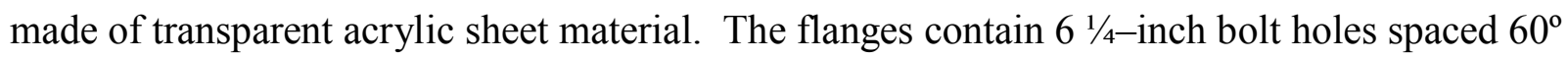
apart so that the sections can be bolted together.

\section{Bottom Air Distributor}

The distributor consists of two components. The lower component is simply a circular flange to which is welded a $3 / 4$-inch steel Tee (positioned vertically). The lower vertical hole of the tee is 
plugged with 3/4-inch plug that can be removed to allow solid particles to drain from the bottom of the riser. The horizontal section of the tee is connected to the air feed/solids feed systems via schedule 80 plastic $3 / 4$ " pipe nipple and serves as one of the two air feeds into the distributor, as well as the location at which the bed solids material enters the riser. The top vertical portion of the tee is welded to the flange so that it matches up to a $3 / 4$-inch hole in the flange.

The upper component of the distributor consists of an inner cone-shaped section with a lower inner diameter of $3 / 4$-inches and an upper inner diameter of 2.29 inches. This inner cone is perforated with three levels of 0.07 inch diameter holes (each level consists of a horizontal plane of holes spaced equidistantly around the cone). The first, second and third sets of holes have 8 , 16 and 32 holes, respectively. In addition, the inner cone is surrounded by an outer cylindrical tube. The second of the two lower air injection ports is located horizontally on this tube. The air entering this port is distributed throughout the cavity between the outer cylindrical shell and the interior cone, and then passes through the perforations in the cone and into the riser. As the perforations are positioned so that each hole is offset radially by an opposing hole, it is assumed that the air entering through these holes will help keep the bottom jet of air aligned with the centerline of the riser.

\section{Secondary Air Injection Ring}

The secondary air injection ring is located at the transition point between the lower and upper riser stages. It consists of two cone-shaped sections, one inside the other with a separation of $1 / 4-$ inch. The inner cone has a lower diameter of 2.29 inches and an upper diameter of 4.0 inches (matching the lower and upper riser stage diameters). Like the distributor section, the inside cone of the injection ring is perforated with two rows of 30 3/16-inch diameter holes close to the top of the cone through which air will enter the riser radially. The outer cone section has a 
horizontally-placed $3 / 4$ " female threaded pipe fitting that that serves as the connection for the secondary air source.

\section{Upper Riser Stage Components}

The upper phase of the riser consists of 2 large diameter pipe sections. Each of the pipe sections are approximately 18.375 inches in height and have an inside diameter of 4.0 inches with 0.5 inch thick flanges and are made of transparent acrylic pvc pipe. The flanges contain $6^{1 / 4-i n c h}$ bolt holes spaced $60^{\circ}$ apart so that the sections can be bolted together. The second pipe section also has a 2.29 inch ID side outflow port located 3 inches below the top of the section. This port attaches to the system's cyclone.

\section{Feed Hopper}

The solid particle material used in this experimental work are introduced into the fluidized bed riser by means of a feed hopper mounted adjacent to the riser. Solids material is passed out of the hopper and into a pneumatic transport line. This pneumatic transport line doubles as one of the lower air feed lines for the lower riser distributor section.

The feed hopper for the scale model system was taken from the experimental system designed and used by a previous graduate student and is described in greater detail in his Thesis [70]. In summary, this feed hopper is constructed of a 36 " long section of 5 " inner diameter, $1 / 2$ " thick clear acrylic tubing. At the bottom of the hopper clay-to-plastic pipe rubber boot connects a $4 \times 2$ inch reducing coupling to the hopper. The coupling uses a $2 x^{1 / 2-i n c h ~ s t r a i g h t ~ t o ~ t h r e a d e d ~ b u s h i n g ~}$ to connect to the pneumatic transport line. In addition, an adapter was required to connect this hopper to the pneumatic transport line. This adapter will be discussed in the next section. 


\section{Pneumatic Transport Line}

The pneumatic transport line (PTL) consists of a $3 / 4$ " schedule 80 plastic tee with a 3" long schedule 80 threaded pipe nipple attached to either end. The left nipple is attached to a $3 / 4$ " threaded coupling with a $3 / 4$ " to $1 / 2$ " brass bushing and a $1 / 2$ " to $1 / 4$ " brass bushing. Inside this final bushing is a $1 / 4 " x 1 "$ pipe nipple to which one of the three air lines is connected via a pipe clamp. A 6" section of 1 "-ID flexible hose connects the pipe nipple to the right of the $3 / 4$ " tee to the $3 / 4$ " pipe nipple connected to the lower distributor of the riser.

In order to make use of the feed hopper discussed previously, an adapter was required to connect the hopper to the PTL. To do this, a schedule $40 \frac{1}{2}$ " threaded ball valve was used in conjunction with a $1 / 2 " x 4 "$ and a $1 / 2 " \times 2 "$ threaded pipe nipple and a $3 / 4 " x 1 / 2 "$ threaded brass bushing. In order to help prevent solids material from clogging this adapter, the 4" pipe nipple was drilled and tapped so that $2 \frac{1 / 4}{4}$ male quick-disconnects could be added. These allow for small amounts of air to be injected into the adapter to aid in solids flow and help break up any clogs that may form.

\section{Cyclone}

The cyclone for the scale model riser was designed so that its major dimensions are $1 / 2$ that of the cyclone for the WADFP system. The cyclone has a 5-inch id at the top, tapering down to 1.5inches at the bottom and an overall length of 24 inches. The cyclone connects to the riser outflow pipe via a 2.5-inch id flanged pipe and is supported via an L-shaped mounting bracket that bolts to the strut channel rack upon which the entire fluidized bed is mounted. A 1.5-inch id pipe elbow exits the top of the cyclone. During operation, particle-laden air enters through the $2 \frac{1 / 2}{2}$ pipe and the particulate matter falls down throw the bottom and the air exits through the top. 


\section{Product Bin}

The product collection bin is constructed of 3 inch id, 60 inch long SCH 80 PVC piping. The top of the product bin connects to the cyclone via a $3 \times 2$ inch reducing coupling. The bottom of the product bin has a 3 inch PVC ball valve to prevent solids and gas flow during testing, as well as allow for easy removal of solids after testing.

\section{Exhaust Gas Filtration}

The exhaust gasses exiting the top of the cyclone are piped away from the cyclone via a $1 \frac{1 / 2}{2}$ SCH 40 PVC pipe, and then through a section of flexible 2" ID discharge hose into a 32 gallon container partially filled with water. As the exhaust gas exits the hose at the bottom of the container, it is assumed that any remaining solids particles not separated by the cyclone will be dissolved into the water reservoir as the air bubbles to the surface of the water.

\section{Air Feed System}

Figure 4-2 demonstrates the general airflow for the scale model fluidized bed riser unit. The supply air is provided via a compressor capable of providing up to 300 SCFM at 120 psi. This house air is regulated to a maximum of 120 psi via a regulator valve and then is introduced into a manifold with three exit ports. At each of the exit ports is a 0-100 SCFM piston style flow meter. High pressure rubber hose connects one of these flow meters to the secondary air injection inlet port in the injection ring located between the upper and lower riser stages. Another of the 0-100 SCFM flow meters is connected to a second manifold, where the air is split into three different flows. One of these feeds a 0-60 SCFM flow meter that controls airflow into the lower air distributor. The second feeds a 0-8 SFCM flow meter that controls airflow into the pneumatic transport line. The final airflow out of the 0-100 SCFM flow meter is regulated to a 
maximum of 15 psig and then split into two further flows. One of these provides back pressure within the feed hopper, and the second can be used for optional aeration air to assist the flow of particles between the feed hopper and pneumatic transport line.

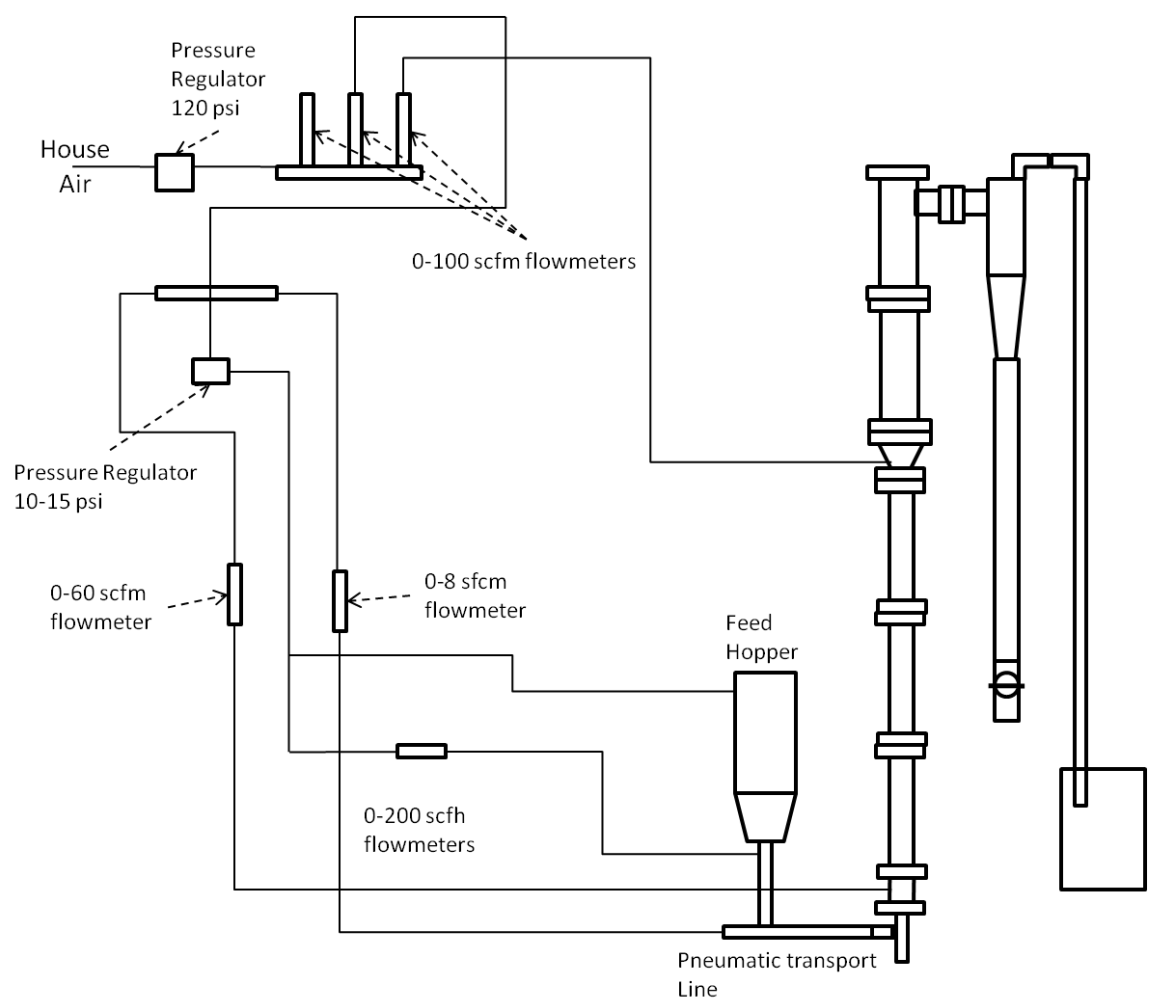

Figure 4-2: Airflow Diagram for Scale Model Riser

\section{Data Acquisition System and Instrumentation}

The following paragraphs detail the data acquisition system and instrumentation utilized in conjunction with the scale model fluidized bed. The data acquisition system and pressure transducers described here were used with the large fluidized bed dryer once testing was completed with the scale model system. 


\section{Data Acquisition System}

The data logging system used to collect and record experimental data includes a Dell GX270 computer with a Intel dual-core Pentium 2.86Gz processor with $3070 \mathrm{MB}$ of memory with an Omega Engineering OMB-DAQ-3000 usb data acquisition system with an Omega Engineering OMB-PDQ30 expansion module. The data acquisition system and expansion module allows for a combined total of 32 differential or 64 single-ended inputs that support input voltages ranging from $+/-31 \mathrm{mV}$ to $+/-10 \mathrm{~V}$, as well as 2 analog output channels. The system incorporates onboard signal conditioning and optional oversampling and 50/60 Hz noise cancellation features. The system also supports several thermocouple types. Finally, the maximum sample rate combined over all channels is 1 million samples per second.

The system utilizes Omega Engineering's Personal Daqview software suite. This software displays all channels in a spreadsheet-like format, allowing the user to activate/deactivate specific channels, as well as assign channel names and engineering units. Sampling rates, number of samples to record, triggering events, oversampling rates and noise cancellation options are also user-selectable.

\section{Pressure Gages and Transducers}

Pressure measurements throughout the riser are made with a series of 4 Omega Engineering PX35K1-030AV (0-30 psia), 1 Omega Engineering PX35K1-050AV (0-50 psia) and 1 Omega Engineering PX35K1-100AV (0-100 psia) pressure transducers. These transducers are capable of operating at temperatures of up to $325^{\circ} \mathrm{F}$ and were purchased for use in the WADFP coal drying fluidized bed. 
For the larger-diameter upper stage of the riser, two of the -030AV are located approximately 1" above the bottom flange and 1" below the outflow port for measuring bed pressures at these locations, as well as determining the pressure drop across the upper stage.

Pressures within the smaller-diameter lower stage of the riser are determined via two of the $030 \mathrm{AV}$ and the $-050 \mathrm{AV}$ transducers. The $-050 \mathrm{AV}$ transducer and one of the remaining $-030 \mathrm{AV}$ transducers are installed approximately 1" above and below the bottom-most and top-most flanges of the transparent tube sections, respectively. The difference between the readings of these transducers provides the total pressure drop across the lower stage. The final -030AV transducer is installed at the midpoint of the lower stage of the riser. The -100AV transducer is installed in the lower air distributor to measure plenum chamber pressure. All of the pressure transducers have a factory specified accuracy of $+/-0.25 \%$ full scale value.

\section{Load Cell}

The feed hopper system is connected to the support frame via an Omegadyne LC101-200 load cell. This load cell has a maximum weight capacity of 200 pounds with an output voltage of 3 $\mathrm{mV} /$ Volt and an excitation voltage of 12 Volts. The solids flux rate into the riser will be calculated from the rate of change in the weight of the feed hopper as recorded by the load cell. 


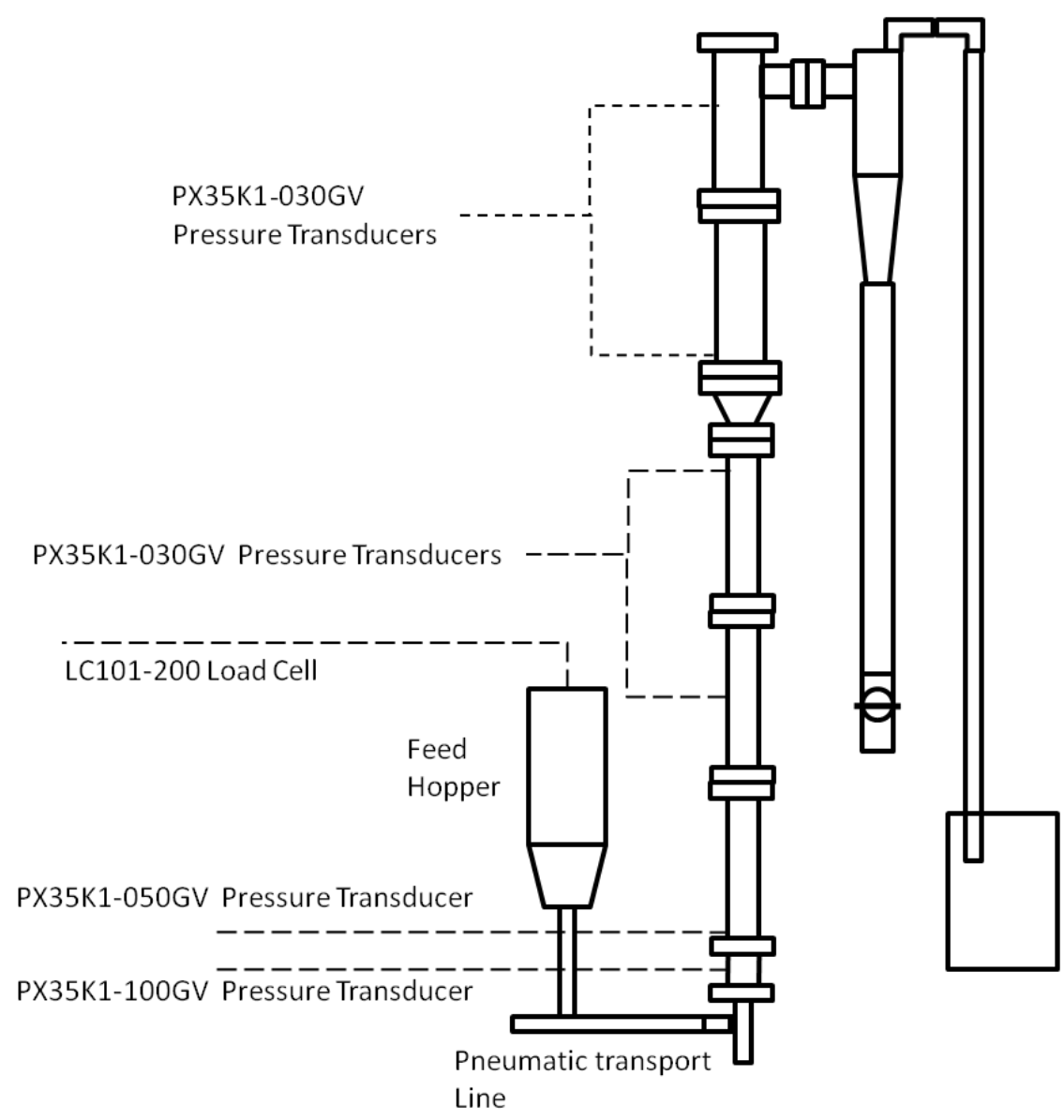

Figure 4-3: Instrumentation Diagram for Small Scale Riser

\section{Procedures}

The following directions describe the startup, shutdown and observation procedures adhered to while operating the small scale model fluidized bed riser.

\section{Start-up Procedure}

The following steps are to be followed when starting the system.

1. Check that all valves are closed.

2. Turn on the computer and open the Daqview software.

3. Turn on the instrumentation power supply.

4. Pour sand into feed hopper. 
5. Open house air supply valve completely.

6. Open air supply valve prior to 100-SCFM flow meter manifold completely.

7. Open valve to allow air to pass through the 100-SCFM flow meter to second manifold.

8. Open second manifold valves to allow air to pass to the 8 and 60 SCFM rotameters.

9. Set flow rates through the 8 and 60 SCFM rotameters to desired level for pneumatic transport line and lower distributer.

10. Set desired flow rate through the 100 SCFM rotameter controlling airflow to injection ring.

11. Set pressure regulator to desired pressure for airflow to feed hopper.

12. Open feed hopper valve

\section{Observations}

For each data point to be collected, the following steps are carried out after the fluidized bed has attained steady state conditions at the desire test conditions.

1. Assign filename, sample rate and number of samples for Daqview data logging.

2. Start Daqview data logging.

3. Acquire video of fluidization conditions within riser.

\section{Shutdown Procedures}

The following steps outline the proper system shutdown procedure.

1. Shut off airflow into feed hopper.

2. Close feed hopper valve.

3. Shut off airflow to riser.

4. Close all valves.

5. Turn off power to instrumentation. 


\section{Calibration}

Prior to the start of testing with the scale model fluidized bed riser, the pressure transducers and load cell were calibrated in order to reduce the amount of error in the measurements taken. In each case, the resulting data were fitted with a linear regression with a resulting minimum $\mathrm{R}^{2}$ value of .996 .

\section{Pressure transducer}

In order to calibrate the 50 and 100 psig pressure transducers, one of the pressure gages installed in the manifold containing the three 100 SCFM flow meters was removed and replaced with a pressure transducer. The pressure regulator upstream of the manifold was then set to a minimum of 5 pressures within the range of the transducers (verified by the gages on the regulator and the other two flow meters). Transducer voltage output was recorded for each pressure. Since the regulator and flow meter pressure gages are given in gage pressure, atmospheric pressure was read from a portable weather station and added to the psig pressure to obtain absolute pressures.

These absolute pressures where then plotted against the output voltage of the transducer to obtain a linear calibration. The same procedure was used with the 30 psia transducers, with the exception on using the $0-15$ psig pressure regulator.

\section{LC101-200 Load Cell}

The feed hopper load cell was calibrated by using a series of known calibration weights and recording the resulting voltage recorded by the data acquisition system. For each known weight, the voltage was plotted and a linear regression was applied to the resulting curve. 


\section{Test Matrix}

The following paragraphs provide a brief description of the solids materials used for testing in the scale model fluidized bed, as well as two primary test procedures for determination of turbulent fluidization in the lower stage of the riser, and core-annular flow in the upper stage.

\section{Initial Particle Sizing}

The solids material used in this study was Quickrete medium sand specified by the manufacturer as being in the 300-500 $\mu \mathrm{m}$ range. However, a 100 gram sample was sieved and table 4-1 shows the resulting particle size distribution. The scale used possessed a resolution of 5 grams, so it can be assumed that the values given below have a $+/-2.5 \mathrm{~g}$ margin of error.

\begin{tabular}{|c|c|}
\hline Screen Size (microns) & \# of grams retained by screen \\
\hline 450 & 5 \\
\hline 425 & 5 \\
\hline 315 & 25 \\
\hline 300 & 5 \\
\hline 250 & 5 \\
\hline 210 & 20 \\
\hline 150 & 40 \\
\hline 75 & 5 \\
\hline
\end{tabular}

Table 4-1: Particle Size Distribution

\section{Turbulent Regime Investigation}

The objective of this portion of the study is to determine the upper and lower bounds of the turbulent fluidization regime in the lower (small diameter) stage of the scale model riser. With air entering the riser from the lower two feeds, the solid material was fed from the feed hopper into the system via the pneumatic transport line. Material was introduced into the system at a constant rate until a dense bed of material is formed. The material feed rate out of the feed 
hopper was controlled by the back pressure in the top of the hopper (as controlled by the $0-15$ psig pressure regulator). Varying the regulator pressure setting leads to different solids flux rates. The volumetric flow rate of air into the pneumatic transport line was held at a constant $2 \mathrm{scfm}$ (corrected for line pressure and atmospheric conditions) while the volumetric flow rate of air into the lower distributor was set to one of the following scfm values for a given test: $1,2,3,4,5,6$, $8,10,12,14,16,18$

For each test condition, pressure and load cell data were recorded for a minimum of 4 minutes to ensure that the bed reached steady state conditions. In addition to collecting system pressure and load cell data, video of the fluidization conditions within the riser was recorded with a handheld digital camcorder.

The pressure data collected during this task was subjected to the analysis methods discussed in Chapter 1 in order to determine the superficial velocities corresponding to the upper and lower bounds of the turbulent fluidization regime.

\section{Fast Fluidization Regime Investigation}

Upon establishing the required operating superficial velocities for the turbulent regime for the lower stage of the riser, the next requirement was to determine the superficial velocities corresponding to the upper and lower bounds for fast fluidization, or core-annulus flow, in the upper stage of the riser.

For this phase of testing, the superficial velocity in the lower stage of the riser was maintained within the upper and lower bounds for turbulent fluidization as determined in the previous section. The operating conditions were held constant within the lower (turbulent) section of the riser while the secondary air inlet volumetric flow rate was set to the following values (in scfm): $10,15,20,25,30,35,40$. As in the fluidization regime testing, digital video of the system was taken 
during the tests and pressure and load cell data was recorded. The recorded data was likewise analyzed via the techniques discussed in Chapter 1. 


\section{Chapter 5 Scale Model Experimental Results}

Presented in this chapter are the experimental results for the small scale transparent model. These results are separated into three sections. The first section discusses the mapping and characterization of the turbulent fluidization regime in the lower riser stage. The second section discusses the mapping and characterization of the fast fluidization, or core-annulus regime in the upper riser stage. The third section examines the effects of varying secondary air injection on the lower riser stage.

\section{Lower Riser Stage Turbulent Fluidization Mapping and Characterization}

The data presented in this section was obtained from experimental runs conducted with the polydispersed sand particles fed continuously with a feed hopper back pressure of 3.5 psi.

Additionally, for the mapping of the turbulent fluidization regime in the lower riser stage only primary air injection into the two lower air inlets was used. No air was introduced into the secondary air injection ring between the lower and upper riser stages for the lower riser stage mapping.

As stated in the Chapter 4, there are a total of 3 pressure transducers located in the lower riser stage between the two air injection rings. Table 5.1 lists these transducers, as well as their designations and distance above the lower injection ring.

\begin{tabular}{|c|c|c|c|}
\hline Transducer & Pressure Range (psi) & Designation & $\begin{array}{c}\text { Distance above lower } \\
\text { injection ring (inches) }\end{array}$ \\
\hline PX35K1-G050AV & $0-50$ & Lbottom & 2.0 \\
\hline PX35K1-G030AV & $0-30$ & Lmid & 27.5 \\
\hline PX35K1-G030AV & $0-30$ & Ltop & 53 \\
\hline
\end{tabular}

Table 5-1: Lower Riser Stage Pressure Transducer Location 
Table 5-2 lists the superficial velocities (lower riser stage) for which data is presented in this section.

\begin{tabular}{|c|c|c|c|c|c|c|c|c|c|}
\hline Test \# & 1 & 2 & 3 & 4 & 5 & 6 & 7 & 8 & 9 \\
\hline $\mathrm{U}_{\mathrm{L}}(\mathrm{m} / \mathrm{s})$ & 0.57 & 0.76 & 1.14 & 1.52 & 1.90 & 2.29 & 2.67 & 3.05 & 3.43 \\
\hline
\end{tabular}

Table 5-2: Lower Riser Stage Superficial Velocity Test Points

\section{Statistical Mapping}

Fluidization regime mapping via statistical methods was carried out upon the experimental data as detailed in Chapter 1. Figure 5-1 depicts the variation of the standard deviation of pressure with the superficial velocity within the lower riser stage. As stated in Chapter 1, the maximum value of the standard deviation of pressure corresponds to the velocity at which a dense bed fluidization system undergoes transition to turbulent fluidization. From the point of maximum value, the magnitude of the standard deviation first decreases sharply and then levels off. For Geldart Type B particles, the superficial velocity at which this leveling off occurs is commonly accepted to be the velocity at which the fluidized bed transitions from turbulence to fast fluidization, or core-annular flow.

In Figure 5-1, the portion of the plots located between the dashed lines (labeled as Region II) depicts the turbulent fluidization regime as determined from analysis of the standard deviation of pressure signals.

Figure 5-2 shows the variation of the skew of the pressure signals from the lower riser stage. While Lee and Kim [43] suggest that the transition from bubbling fluidization to turbulent fluidization is characterized by a transition from negative to positive skew, this does not appear to be the case in this instance. In all but the first data point in the Lbottom skew plot, the values 
of skew are greater than zero and increase towards a maximum value. For each pressure transducer location, there is a sudden decrease in value beyond the maximum value. The location of this lower skew value corresponds to the onset of fast fluidization in Figure 5-1. This suggests that the third order statistical moment (i.e. skew) of a pressure signal can be used to locate the transition from dense fluidization to dilute fluidization regimes.

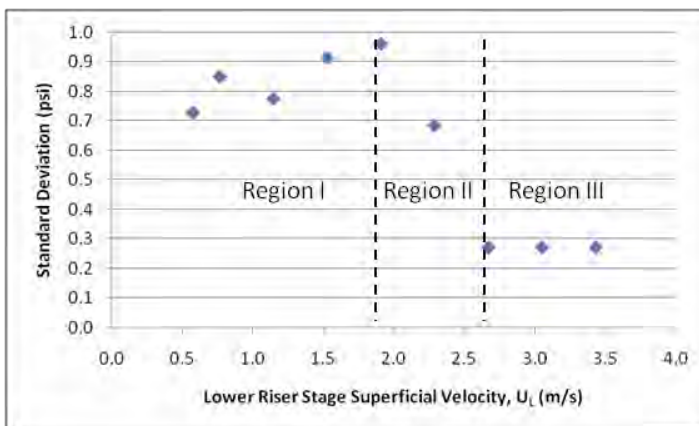

(a)

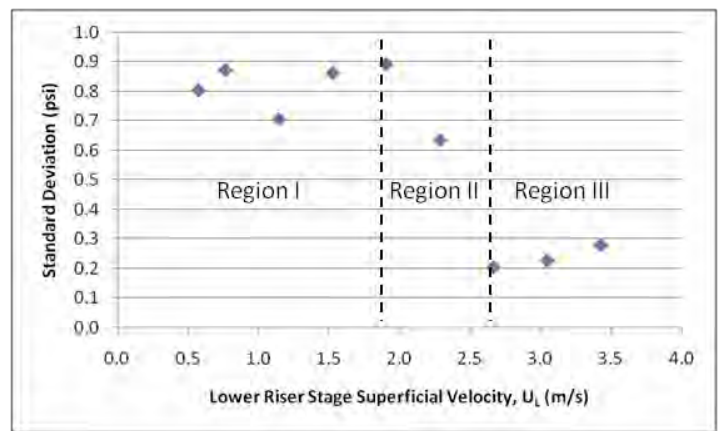

(b)

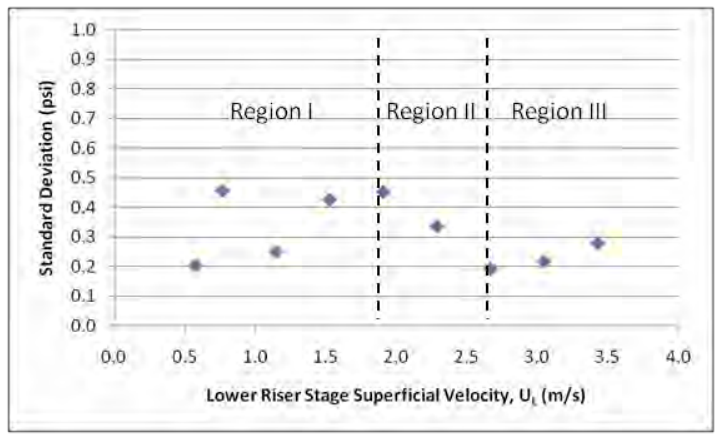

(c)

Figure 5-1 : Standard Deviation vs Superficial Velocity for Lower Riser Stage Pressures: (a) Lbottom (b) Lmid (c) Ltop; poly-dispersed sand, 3.5 psig feed hopper pressure, no secondary air injection. 


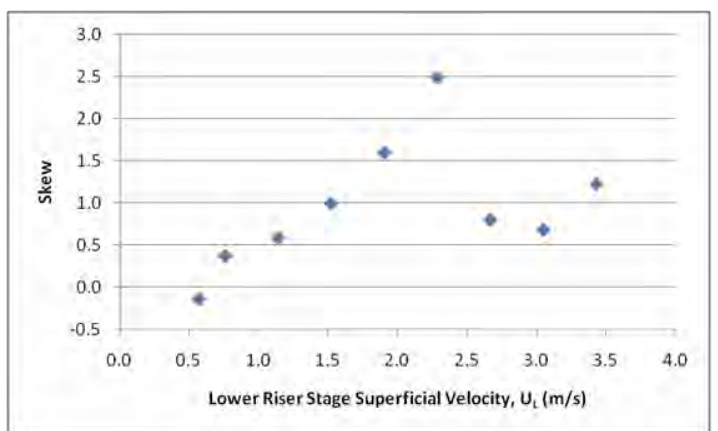

(a)

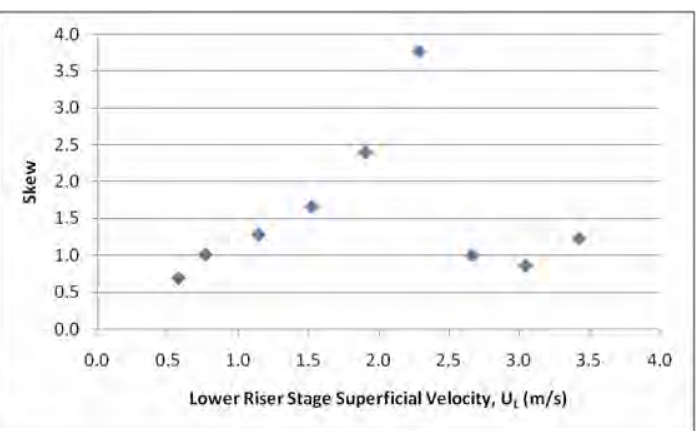

(b)

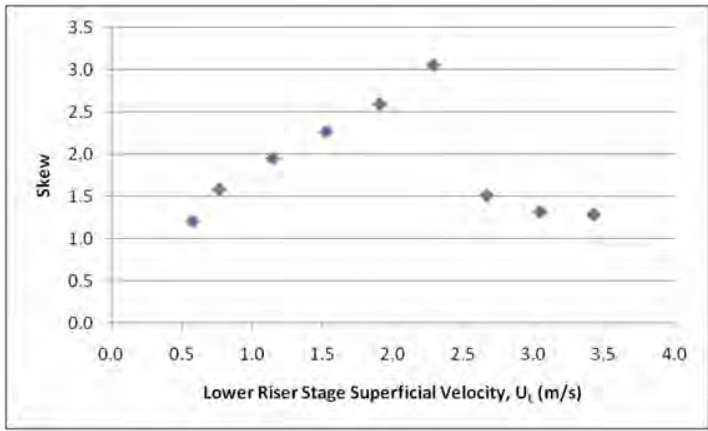

(c)

Figure 5-2: Skew vs Superficial Velocity for Lower Riser Stage Pressures: (a) Lbottom (b) Lmid (c) Ltop; poly-dispersed sand, 3.5 psig feed hopper pressure, no secondary air injection.

Figure 5-3 shows the variation of the fourth statistical moment (kurtosis) of pressure data as a function of the superficial velocity in the lower riser stage of the scale model fluidized bed. In general, the kurtosis follows a trend similar to that seen in the values of skew. Of particular interest is the apparent initial horizontal trend near zero in the Lbottom and Lmid plots. The test points at which this initial linear trend occurs are within the bubbling/slugging fluidization regimes, and the velocity region corresponding to the increasing values of kurtosis is similar to the turbulent region depicted in Figure 5-1. From this it can be inferred that the turbulent fluidization regime is characterized by increased skew. One possible explanation for this might be the fact that the turbulent regime is characterized by the breakup of large bubbles into many smaller ones. This would lead to more rapid pressure fluctuations with smaller magnitudes of fluctuation, and thus a more "spiky" signal. 


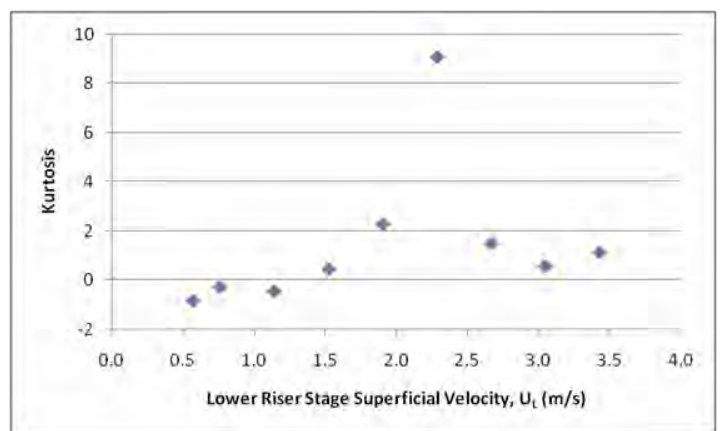

(a)

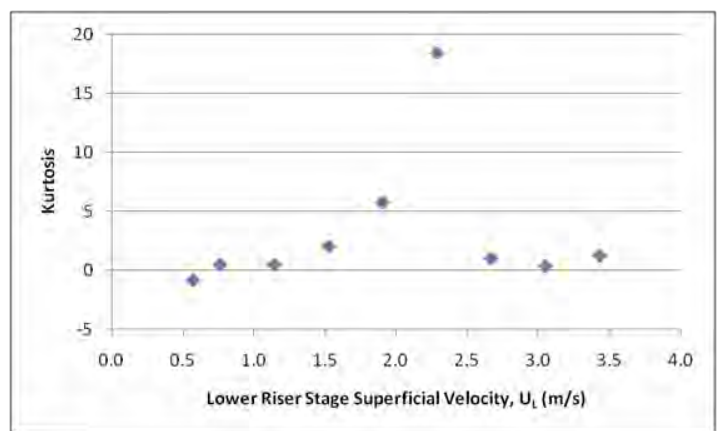

(b)

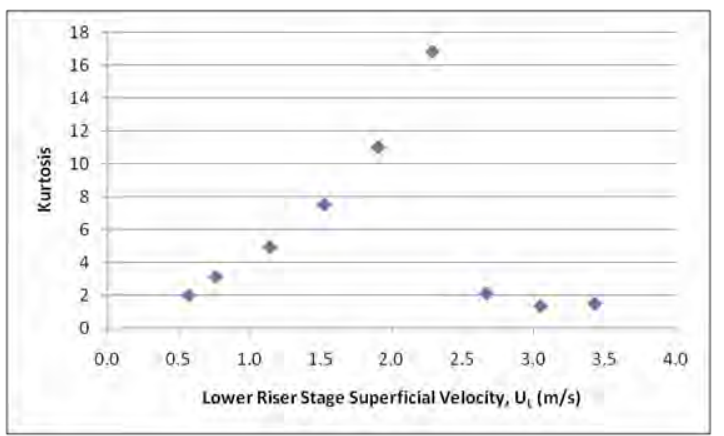

(c)

Figure 5-3: Kurtosis vs Superficial Velocity for Lower Riser Stage Pressures: (a) Lbottom (b) Lmid (c) Ltop; polydispersed sand, 3.5 psig feed hopper pressure, no secondary air injection.

\section{Regime Mapping with Shannon Entropy Analysis}

As previously discussed in Chapter 1, analysis of Shannon entropy has recently been used to map out the different fluidization regimes in spouted beds with non-spherical biomass fuels, bubble columns, three-phase fluidized beds, as well as nuclear reactors. For this study, the Shannon entropy values of each pressure transducer were calculated at each superficial velocity for approximately 1 minute (6000 points) of data using 600 bins. The results are shown in Figure 54.

By dividing the velocity range of each figure into the three regions delineated in Figure 5-1, it becomes apparent that the Shannon entropy plots exhibit differing and unique linear slopes within each of the three areas. This observation agrees with similar trends observed by Zhong et al. $[\mathbf{4 7 , 4 6 ]}$. It must be noted that the results of this study agree with those of Zhong et al. only in 
that each fluidization regime is characterized by a linear trend whose slope differs from that of the adjacent regimes in the Shannon entropy plots. In contrast to their results, the results presented here suggest that entropy is at its highest in the slugging regime, which is characterized by large pressure fluctuations. As the bed transitions to turbulent fluidization, the large bubbles that lead to slugging are broken apart and the magnitude of pressure fluctuations decreases. This decrease results in a corresponding decrease in the entropy values. In each of the figures below, the value of Shannon entropy reaches a minimum value in the fast fluidization, or core-annulus, regime.

On a final note, as can be seen in Figure 5-4, the maximum value of Shannon entropy decreases as the height above the lower injection ring increases. It is speculated that this might be the result of the variation of solids holdup or dampening of pressure fluctuations with vertical distance, but this was not investigated.

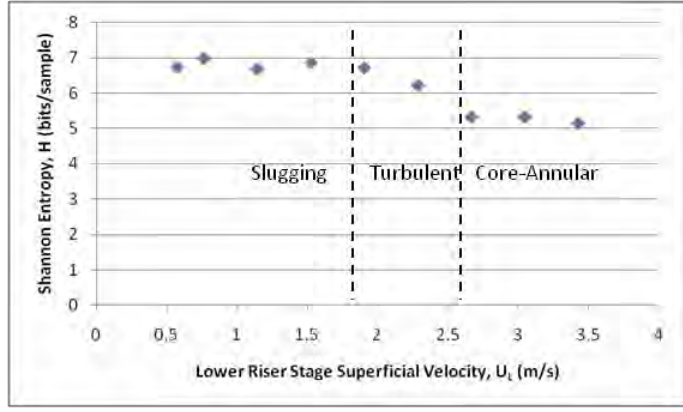

(a)

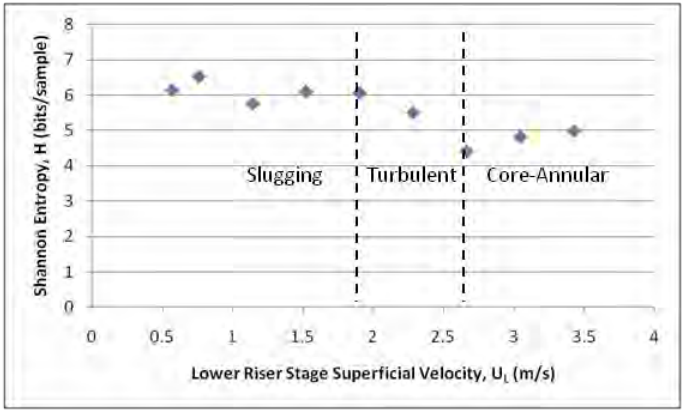

(b)

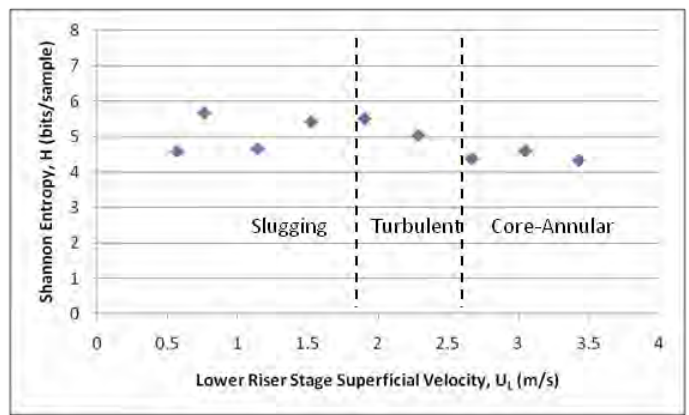

(c)

Figure 5-4: Shannon Entropy vs Superficial Velocity for Lower Riser Stage Pressures: (a) Lbottom (b) Lmid (c) Ltop; poly-dispersed sand, 3.5 psig feed hopper pressure, no secondary air injection. 


\section{Autocorrelation, Mutual Information and Power Spectral Density}

\section{Autocorrelation}

Figures 5-5, 5-6 and 5-7 depict the autocorrelation for each of the three pressure transducers located within the lower riser stage of the scale model fluidized. As can be seen from the figures, the autocorrelations of the Lbottom and Lmid locations exhibit an initial trend of periodicity and smoothness. This trend decays between $U_{L}=1.52 \mathrm{~m} / \mathrm{s}$ and $U_{L}=2.29 \mathrm{~m} / \mathrm{s}$ (the turbulent fluidization region), and is replaced by a pattern of very spiky low magnitude fluctuations between $U_{L}=2.67 \mathrm{~m} / \mathrm{s}$ and $U_{L}=3.43 \mathrm{~m} / \mathrm{s}$ (the fast fluidization, or core-annular, regime). The Ltop location plot (Figure 5-7) exhibits a pattern that more closely matches those seen in the core-annulus regime, suggesting that the upper region of the lower riser stage transitions to that fluidization regime at earlier velocities than the bottom and middle regions.

\section{Mutual Information Function}

Figures 5-8 through 5-10 show the effects varying lower riser stage superficial velocity $\left(\mathrm{U}_{\mathrm{L}}\right)$ on the mutual information function of the three lower riser stage pressures. As can be seen in all three locations, there is an initial increase in mutual information between $U_{L}=0.57 \mathrm{~m} / \mathrm{s}$ and $U_{L}=$ $0.76 \mathrm{~m} / \mathrm{s}$, followed by a sharp drop at $\mathrm{U}_{\mathrm{L}}=1.14 \mathrm{~m} / \mathrm{s}$. This is then followed by a sharp increase at $\mathrm{U}_{\mathrm{L}}=1.52$. The mutual information then proceeds to decline throughout the turbulent range and then hold fairly constant within the fast fluidization regime.

\section{Spectral Density Plots}

As stated in Chapter 1, the Power Spectral Density (PSD) plot depicts the power associated with each frequency contained within a given signal. In fluidized beds, fluctuations in pressure signals are caused primarily by bubbles passing the sensor. The bubbling and slugging regimes 
are characterized by a dominant frequency of bubble formation, but the turbulent regime has no dominant frequency. Figures 5-11 through 5-13 show the PSD plots for the Lbottom, Lmid and Ltop pressure transducer locations for all superficial velocities tested. In the Lbottom and Lmid location plots (Figures 5-11 and 5-12), the presence of one or more narrowly defined dominant frequencies with high levels of associated power can be seen for superficial velocities of 0.57 $\mathrm{m} / \mathrm{s}$ to $1.14 \mathrm{~m} / \mathrm{s}$. Over the turbulent fluidization range of velocities $(1.53 \mathrm{~m} / \mathrm{s}$ through 2.29$)$, the magnitude of power associated with a given frequency steadily decreases, and the range of frequencies over which the power is distributed expands. The core annular velocity range (2.67 $\mathrm{m} / \mathrm{s}$ through $3.43 \mathrm{~m} / \mathrm{s}$ ) is characterized by extremely low levels of power per frequency with the range of frequencies over which the power is distributed is shifted to slightly higher frequencies. The Ltop spectral density plots (Figure 5-13) show slightly different tendencies, but in general is characterized by the decreasing levels of power per frequency distributed over a wider range of frequencies.

While not readily apparent from examination of the standard deviation, skew, kurtosis and Shannon entropy, the autocorrelation and PSD data from the pressure transducers located within the lower riser stage of the scale model fluidized bed show differences between the Ltop location and what is seen in the Lbottom and Lmid locations. Specifically, the plots resulting from analysis of autocorrelation and PSD of the Ltop location is more in line with those seen in both the lower riser stage and upper riser stage fast fluidization data. This leads to the conclusion that lower riser stage contains a dense bed under turbulent fluidization conditions above which is a dilute, fast fluidization (core-annular) region. 


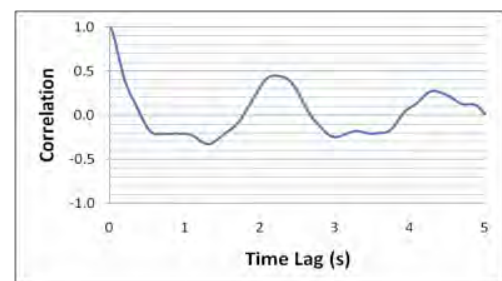

(a)

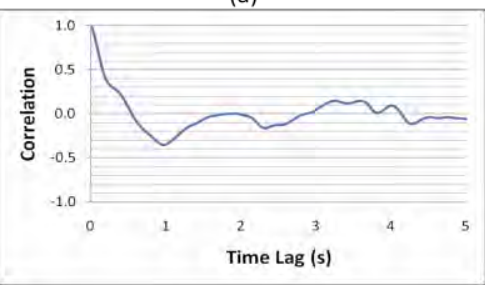

(d)

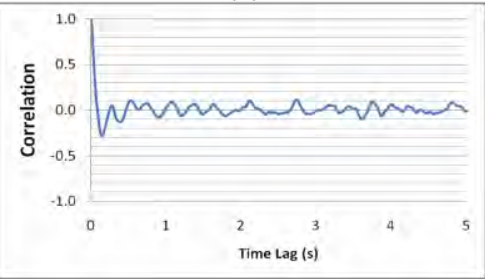

(g)

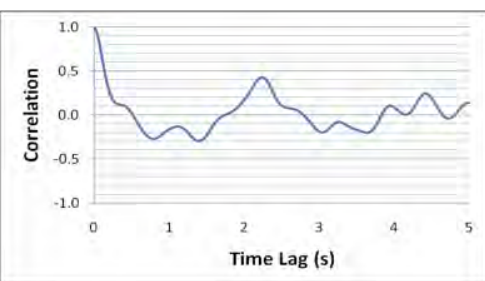

(b)

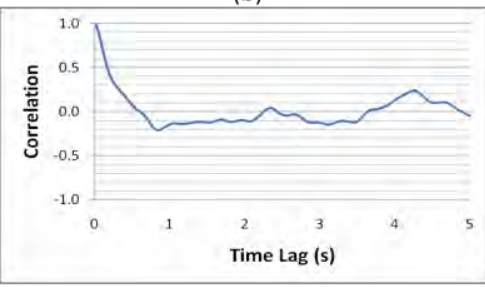

(e)

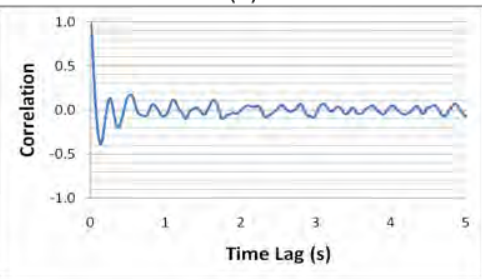

(h)

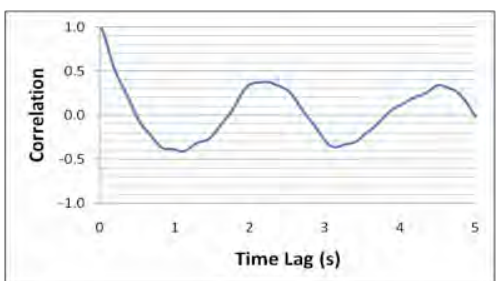

(c)

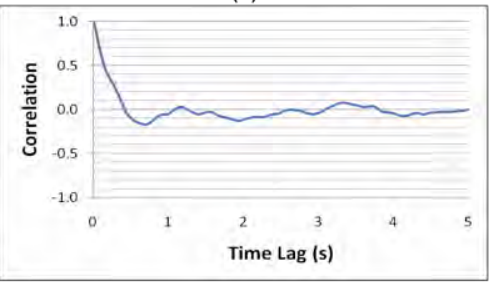

(f)

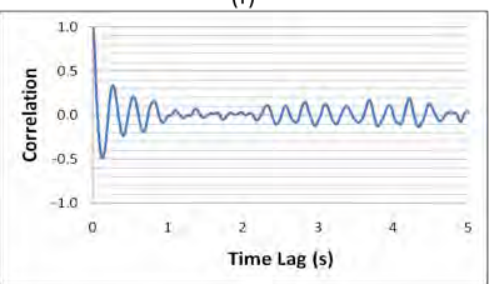

(i)

Figure 5-5: Autocorrelation of scale model Lbottom Pressure: poly-dispersed sand, 3.5 psig feed hopper pressure, no secondary air injection, $U_{L}=:$ (a) 0.57 (b) 0.76 (c) 1.14 (d) 1.52 (e) 1.90 (f) 2.29 (g) 2.67 (h) 3.05 (i) 3.43 m/s.

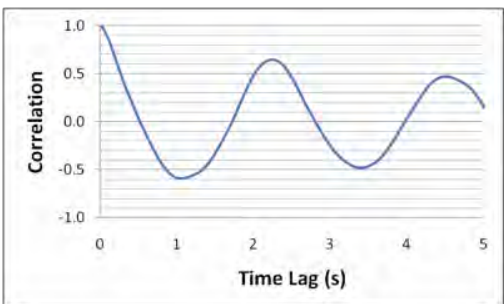

(a)

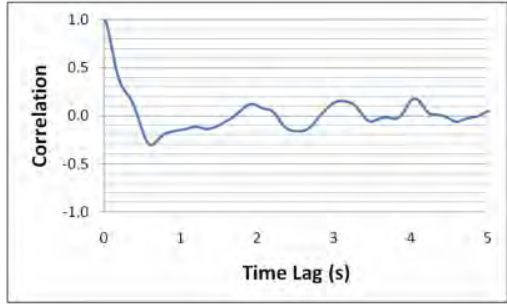

(d)

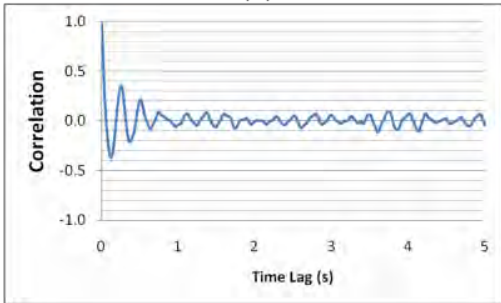

(g)

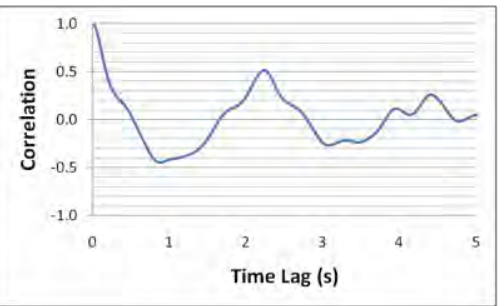

(b)

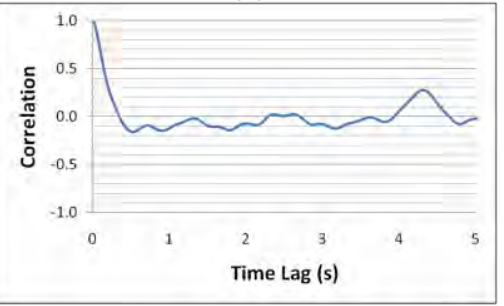

(e)

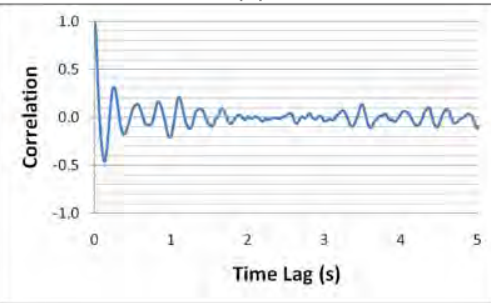

(h)

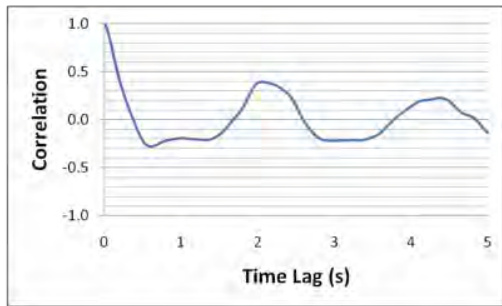

(c)

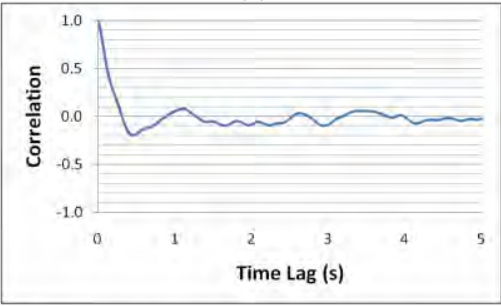

(f)

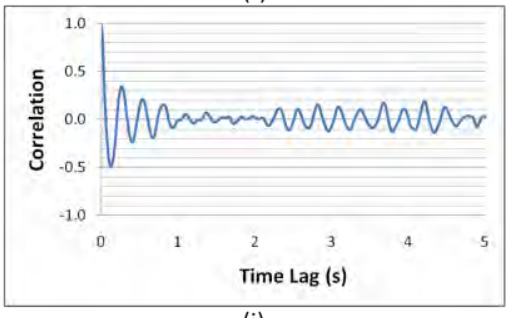

(i)

Figure 5-6: Autocorrelation of scale model Lmid Pressure: poly-dispersed sand, 3.5 psig feed hopper pressure, no secondary air injection, $U_{L}=:$ : (a) 0.57 (b) 0.76 (c) 1.14 (d) 1.52 (e) 1.90 (f) 2.29 (g) 2.67 (h) 3.05 (i) 3.43 m/s. 


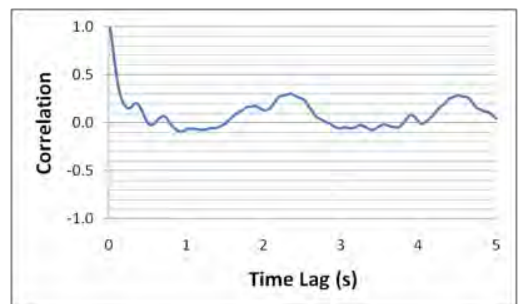

(a)

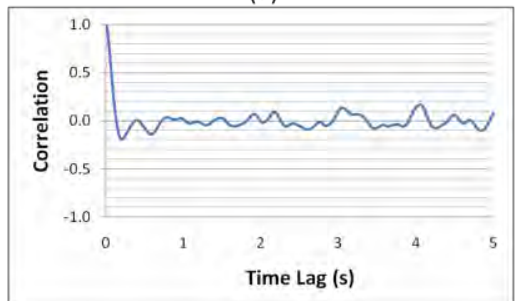

(d)

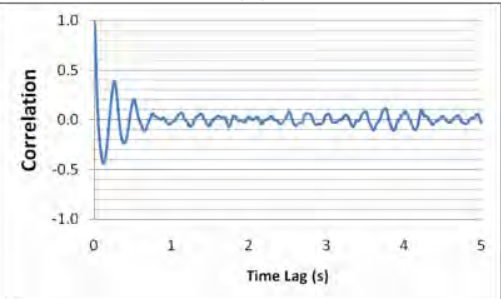

(g)

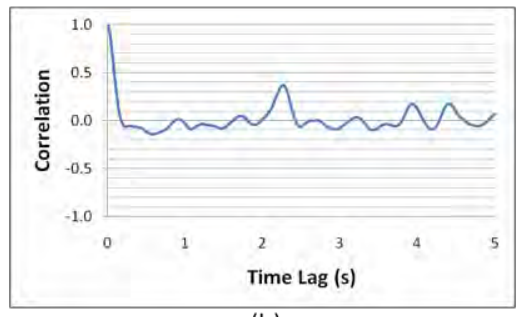

(b)

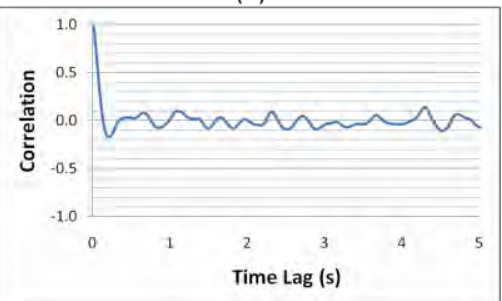

(e)

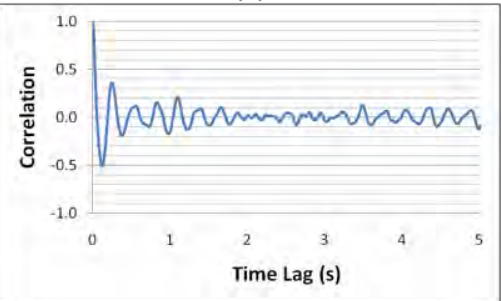

(h)

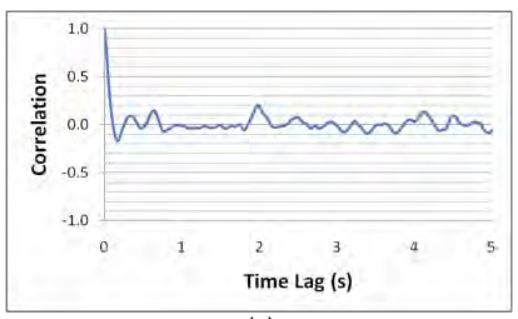

(c)

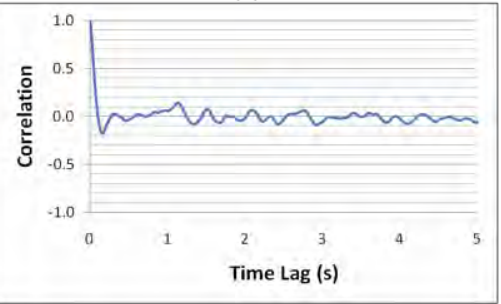

(f)

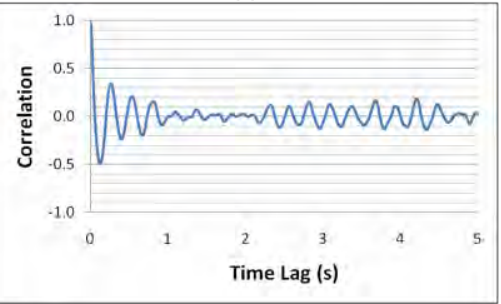

(i)

Figure 5-7: Autocorrelation of scale model Ltop Pressure: poly-dispersed sand, 3.5 psig feed hopper pressure, no secondary air injection, $U_{\mathrm{L}}=:$ : (a) 0.57 (b) 0.76 (c) 1.14 (d) 1.52 (e) 1.90 (f) 2.29 (g) 2.67 (h) 3.05 (i) 3.43 m/s.

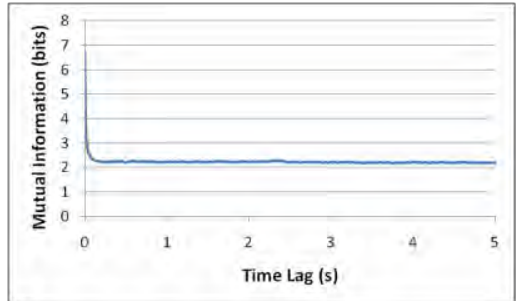

(a)

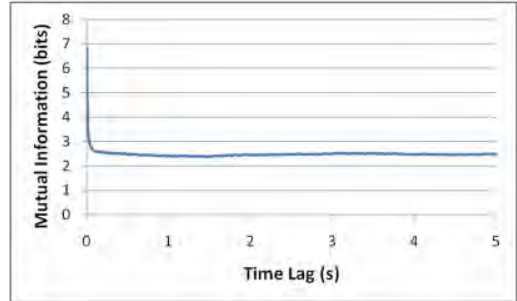

(d)

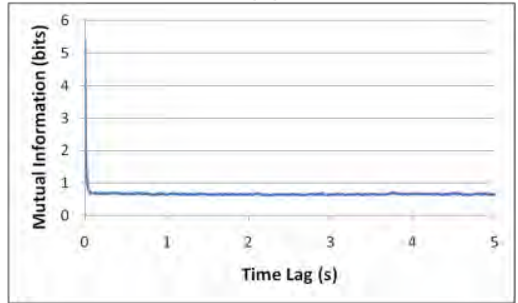

(g)

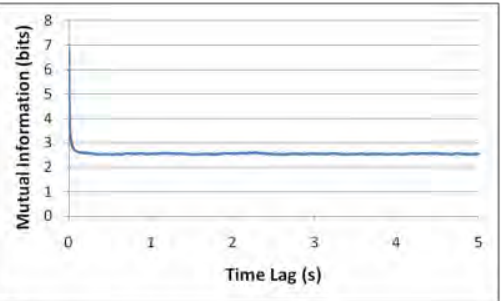

(b)

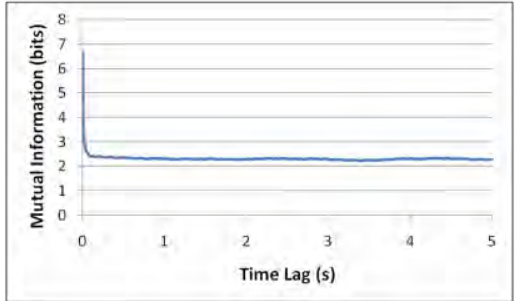

(e)

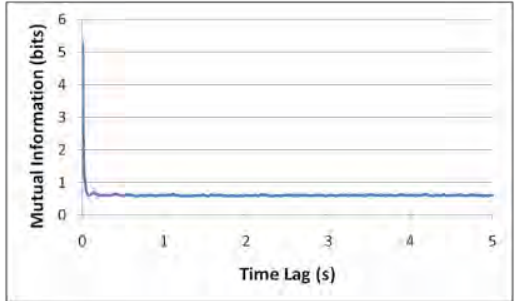

(h)

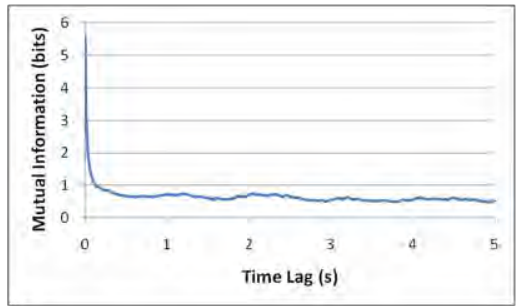

(c)

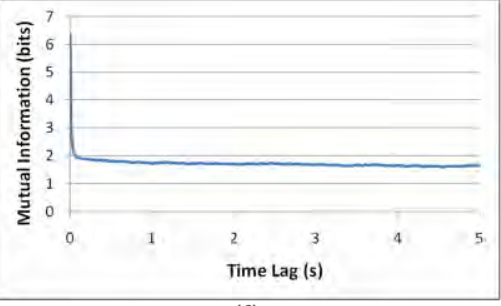

(f)

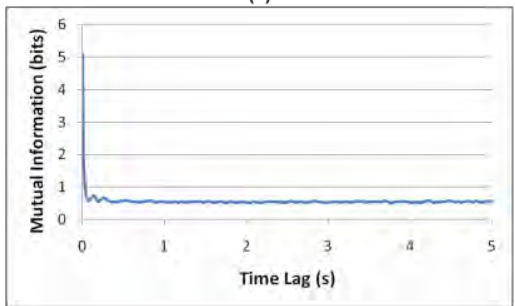

(i)

Figure 5-8: Mutual Information of scale model Lbottom Pressure: poly-dispersed sand, 3.5 psig feed hopper pressure, no secondary air injection, $U_{L}=:$ (a) 0.57 (b) 0.76 (c) 1.14 (d) 1.52 (e) 1.90 (f) 2.29 (g) 2.67 (h) 3.05 (i) 3.43 m/s. 


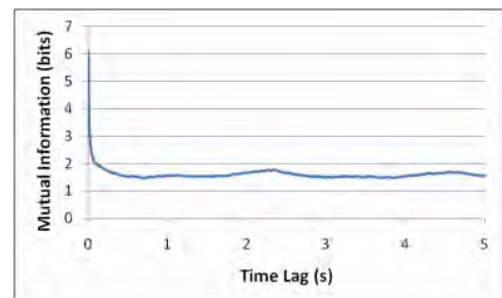

(a)

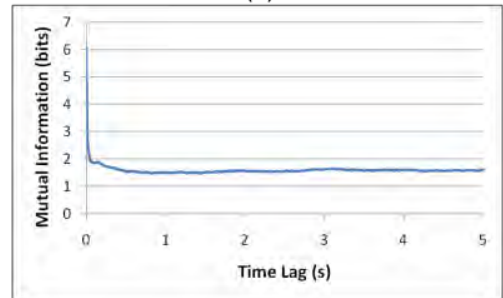

(d)

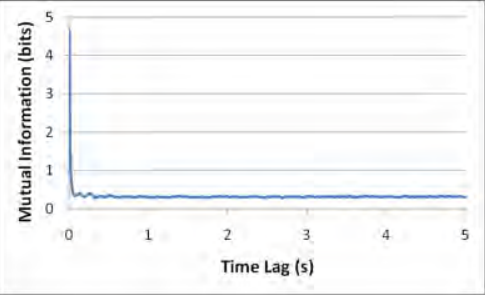

(g)

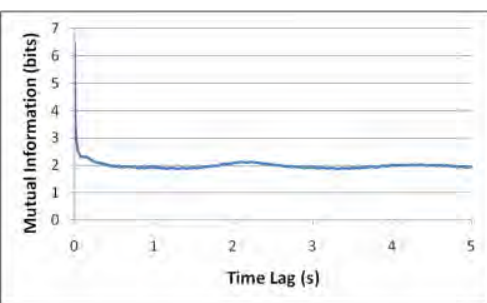

(b)

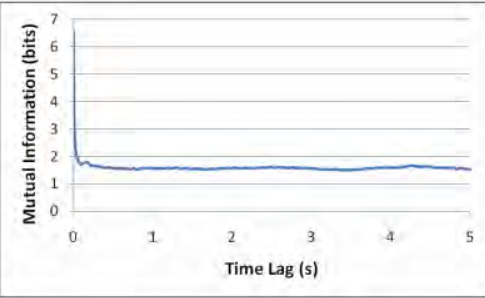

(e)

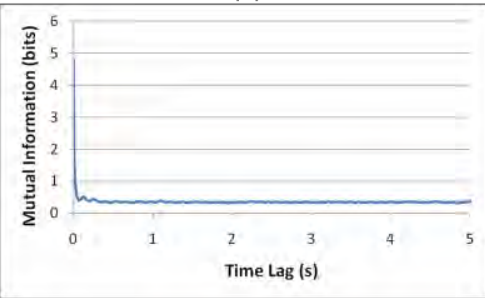

(h)

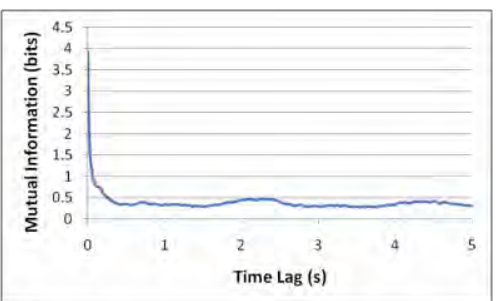

(c)

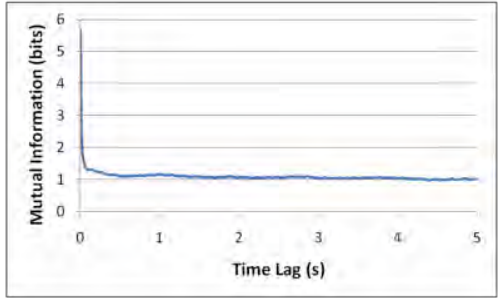

(f)

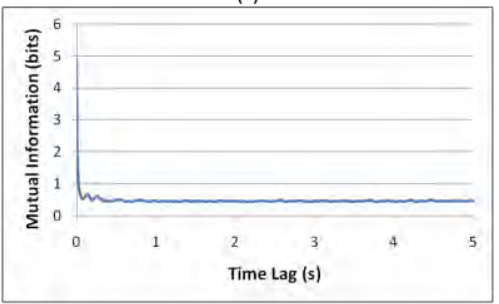

(i)

Figure 5-9: Mutual Information of scale model Lmid Pressure: poly-dispersed sand, 3.5 psig feed hopper pressure, no secondary air injection, $U_{L}=:$ : (a) 0.57 (b) 0.76 (c) 1.14 (d) 1.52 (e) 1.90 (f) 2.29 (g) 2.67 (h) 3.05 (i) 3.43 m/s.

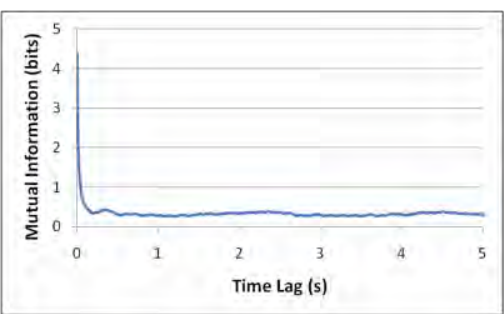

(a)

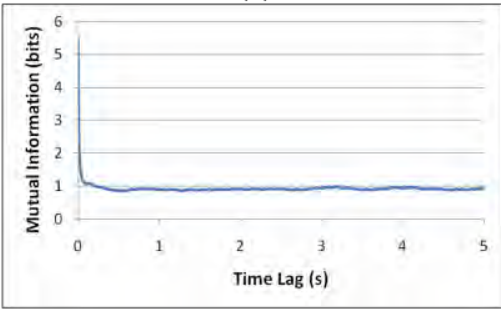

(d)

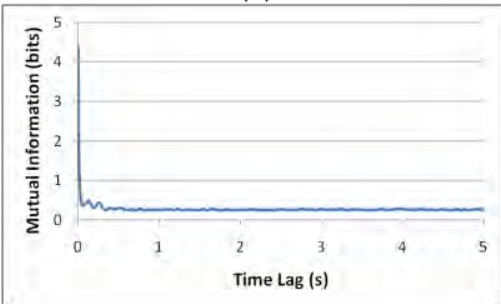

(g)

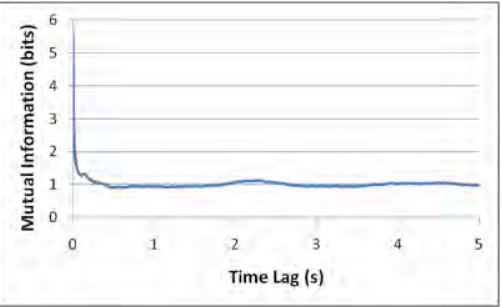

(b)

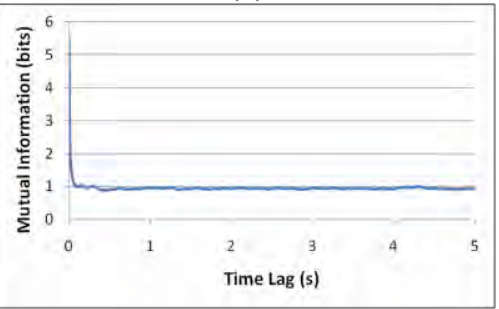

(e)

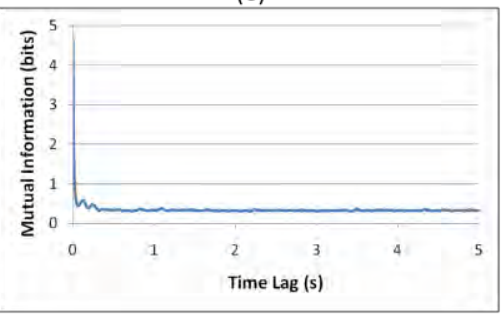

(h)

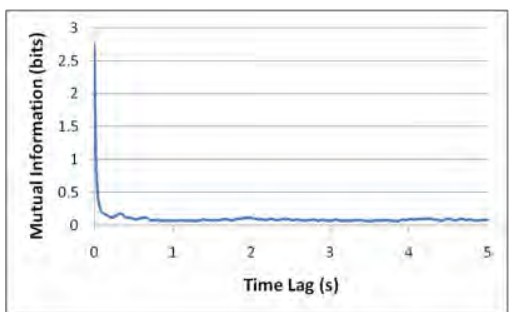

(c)

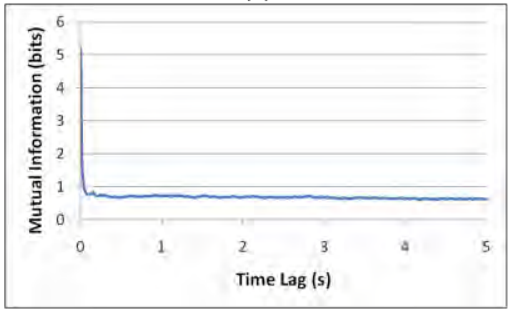

(f)

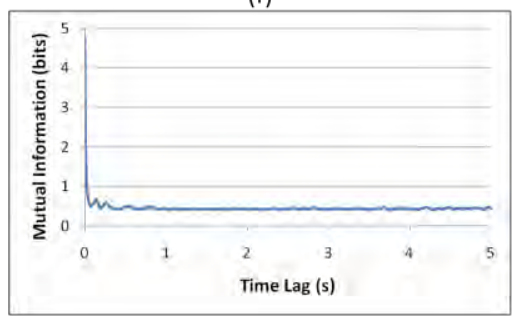

(i)

Figure 5-10: Mutual Information of scale model Ltop Pressure: poly-dispersed sand, 3.5 psig feed hopper pressure, no secondary air injection, $U_{L}=:$ : (a) 0.57 (b) 0.76 (c) 1.14 (d) 1.52 (e) 1.90 (f) 2.29 (g) 2.67 (h) 3.05 (i) 3.43 m/s. 


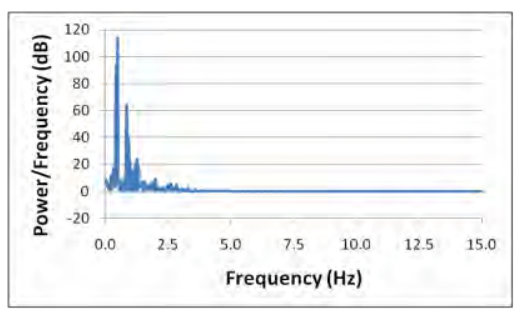

(a)

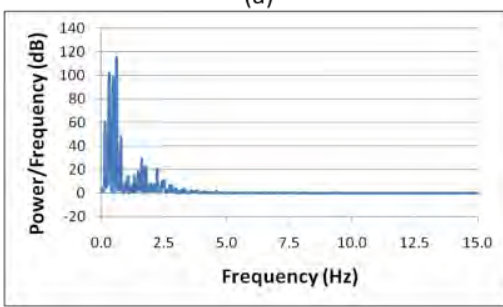

(d)

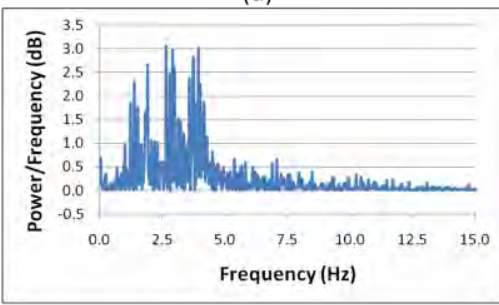

(g)

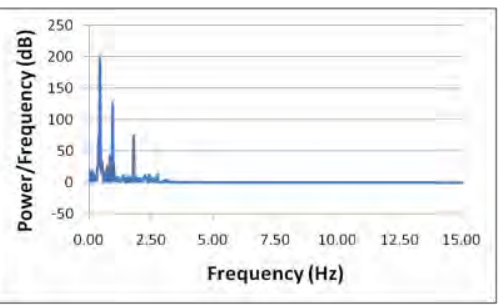

(b)

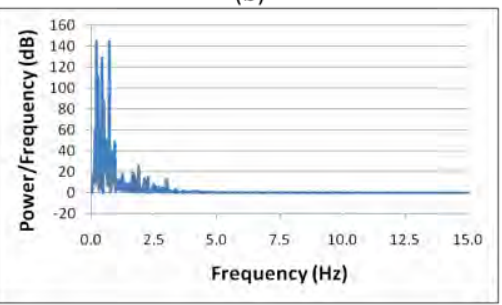

(e)

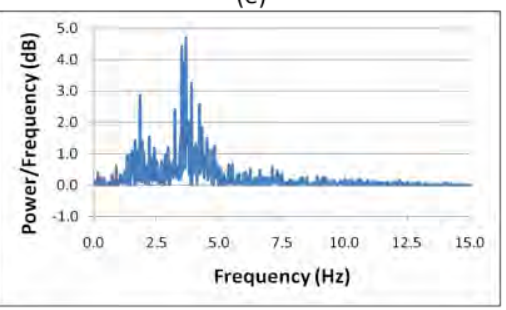

(h)

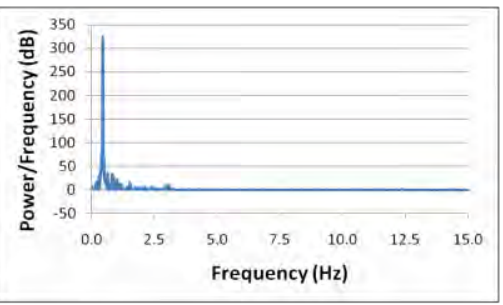

(c)

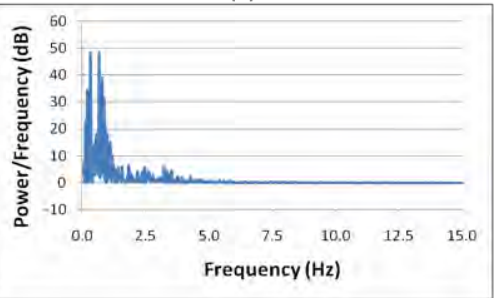

(f)

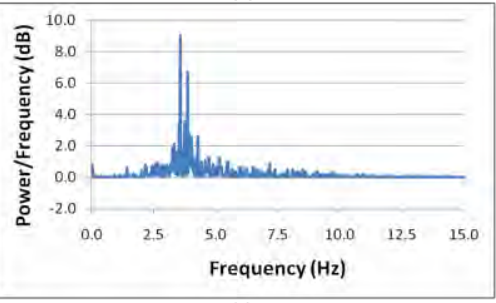

(i)

Figure 5-11: Power Spectral Density Plot of scale model Lbottom Pressure: poly-dispersed sand, 3.5 psig feed hopper pressure, no secondary air injection, $U_{L}=:$ : (a) 0.57 (b) 0.76 (c) 1.14 (d) 1.52 (e) 1.90 (f) 2.29 (g) 2.67 (h) 3.05 (i) 3.43 m/s.

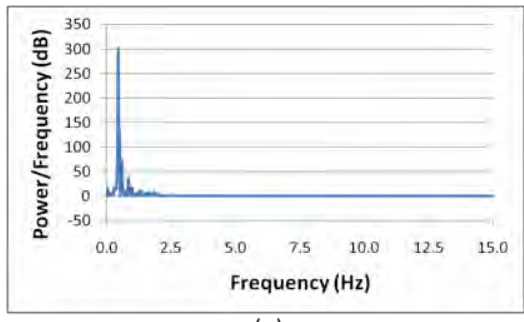

(a)

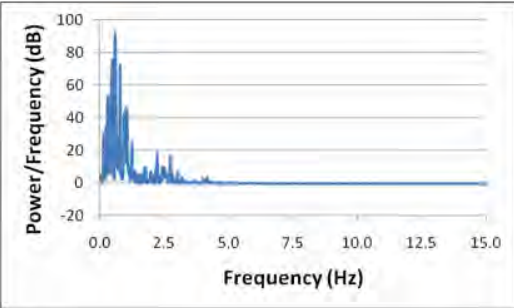

(d)

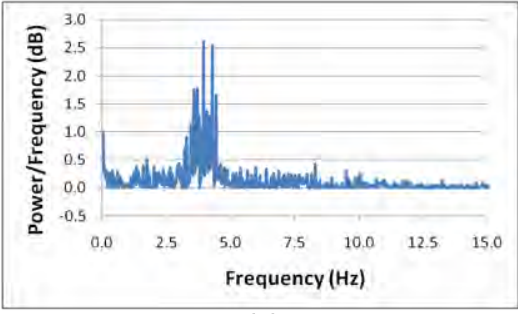

(g)

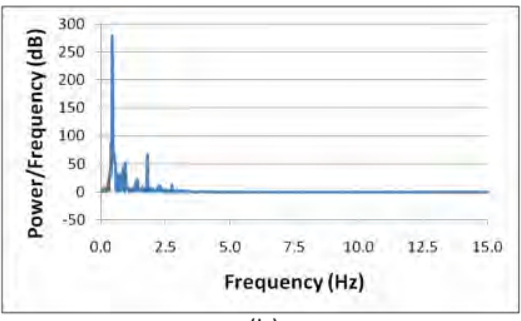

(b)

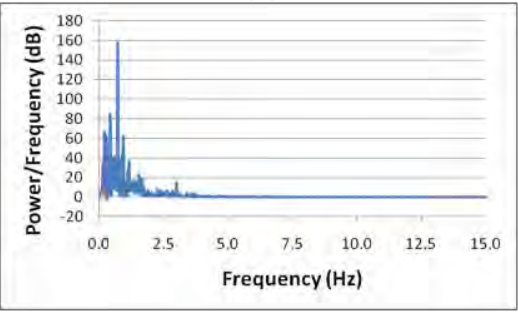

(e)

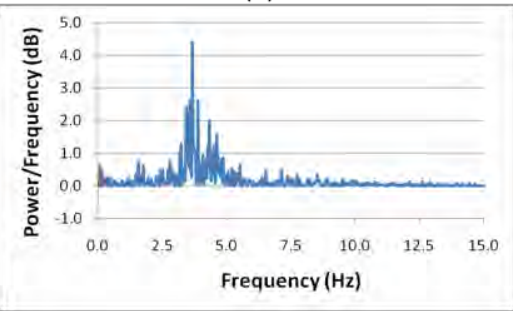

(h)

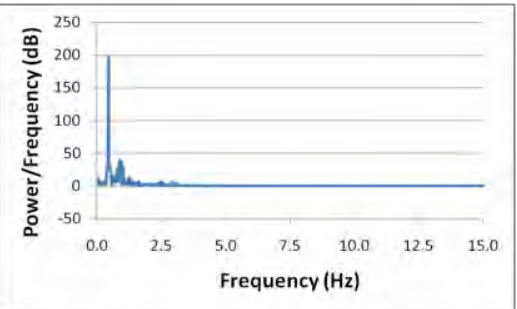

(c)

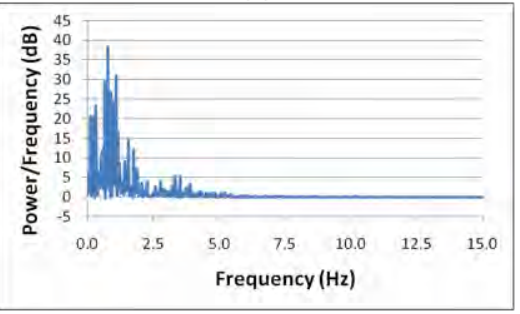

(f)

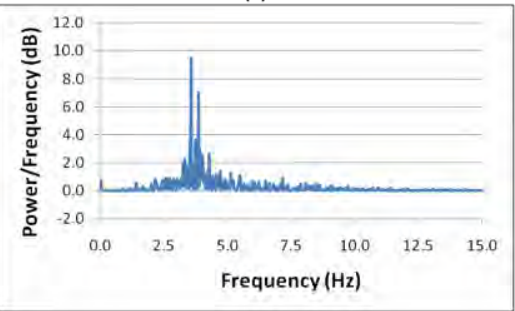

(i)

Figure 5-12: Power Spectral Density Plot of scale model Lmid Pressure: poly-dispersed sand, 3.5 psig feed hopper pressure, no secondary air injection, $U_{L}=:$ : (a) 0.57 (b) 0.76 (c) 1.14 (d) 1.52 (e) 1.90 (f) 2.29 (g) 2.67 (h) 3.05 (i) 3.43 m/s.

78 


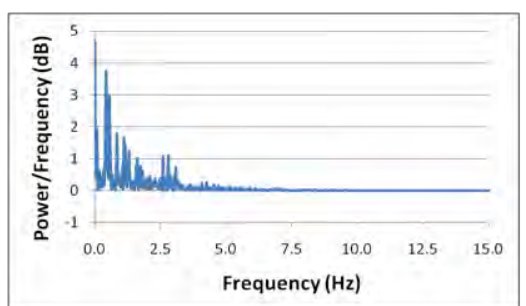

(a)

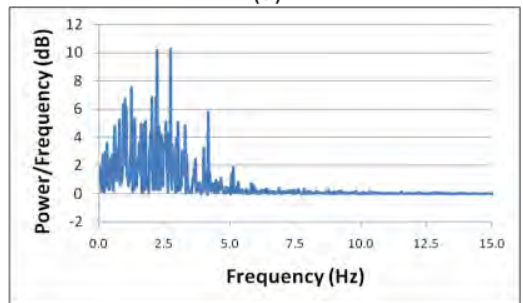

(d)

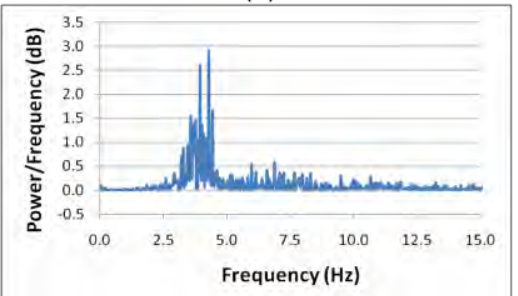

(g)

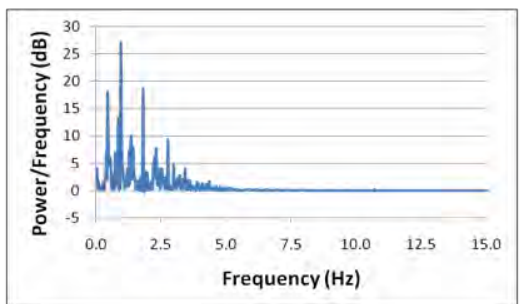

(b)

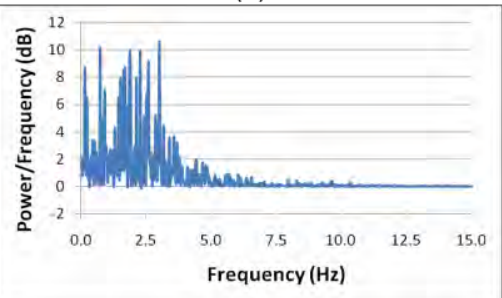

(e)

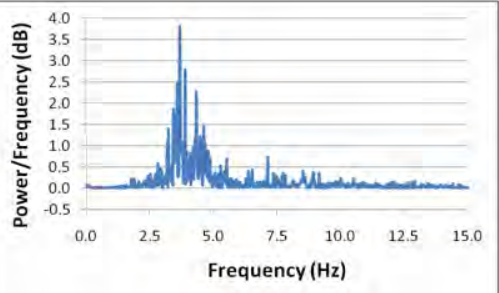

(h)

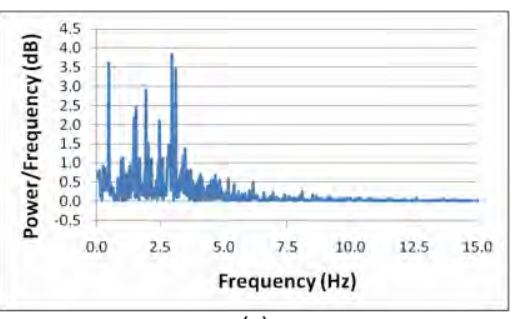

(c)

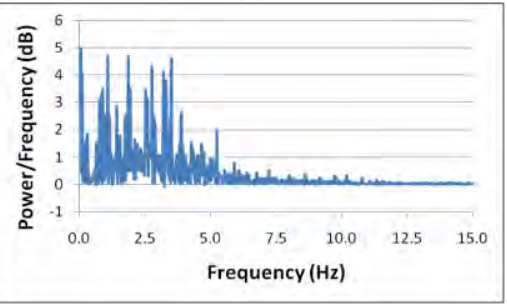

(f)

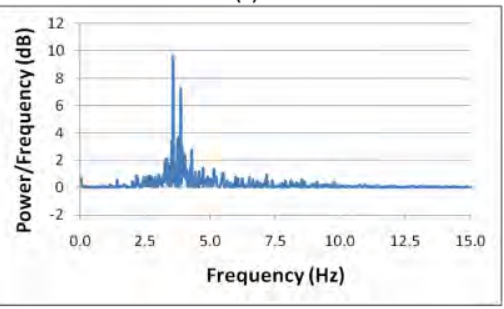

(i)

Figure 5-13: Power Spectral Density Plot of scale model Ltop Pressure: poly-dispersed sand, 3.5 psig feed hopper pressure, no secondary air injection, $U_{L}=:$ : (a) 0.57 (b) 0.76 (c) 1.14 (d) 1.52 (e) 1.90 (f) 2.29 (g) 2.67 (h) 3.05 (i) 3.43 m/s.

\section{Upper Riser Stage Fast Fluidization Mapping and Characterization}

The data presented in this section was obtained from experimental runs conducted with the polydispersed sand particles feed continuously with a feed hopper back pressure of 3.5 psi. The superficial velocity of the lower riser stage $\left(\mathrm{U}_{\mathrm{L}}\right)$ was held constant at $2.36 \mathrm{~m} / \mathrm{s}$ in order to maintain turbulent fluidization in this stage(as determined in the previous section.) The superficial velocity of the upper riser stage $\left(\mathrm{U}_{\mathrm{U}}\right)$ was tested over a range of velocities, as detailed in Table 5-3. As stated in Chapter 4, there are a total of two pressure transducers located in the upper riser stage. Table 5-4 lists these transducers, their designations and distances above the secondary air injection ring and lower injection ring.

\begin{tabular}{|c|c|c|c|c|c|c|c|}
\hline Test \# & 1 & 2 & 3 & 4 & 5 & 6 & 7 \\
\hline $\mathrm{U}_{\mathrm{L}}(\mathrm{m} / \mathrm{s})$ & 2.36 & 2.36 & 2.36 & 2.36 & 2.36 & 2.36 & 2.36 \\
\hline $\mathrm{U}_{\mathrm{U}}(\mathrm{m} / \mathrm{s})$ & 1.42 & 1.74 & 2.06 & 2.38 & 2.70 & 3.02 & 3.35 \\
\hline
\end{tabular}

Table 5-3: Upper Riser Stage Superficial Velocity Test Points 


\begin{tabular}{|c|c|c|c|c|}
\hline Transducer & $\begin{array}{c}\text { Pressure Range } \\
\text { (psi) }\end{array}$ & Designation & $\begin{array}{c}\text { Distance above } \\
\text { secondary air } \\
\text { injection ring } \\
\text { (inches) }\end{array}$ & $\begin{array}{c}\text { Distance above } \\
\text { lower injection } \\
\text { ring (inches) }\end{array}$ \\
\hline PX35K1-G030AV & $0-30$ & Ubottom & 2.0 & 62.0 \\
\hline PX35K1-G030AV & $0-30$ & Utop & 30.0 & 92.0 \\
\hline
\end{tabular}

Table 5-4: Upper Riser Stage Pressure Transducer Location

\section{Statistical Mapping}

Figures 5-14 through 5-17 show the effects of superficial velocity upon the standard deviation, skew and kurtosis of pressure signals of the Ubottom and Utop locations in the upper riser stage.

The standard deviation of pressure shows trends similar to that seen in the mapping of the lower riser section (as presented in the previous section). The peak occurring at $1.74 \mathrm{~m} / \mathrm{s}$ corresponds to the turbulent transition velocity $\left(\mathrm{U}_{\mathrm{C}}\right)$. This is followed by a rapid decline in standard deviation during the turbulent regime. The point at which the slope of the standard deviation curve levels out to nearly horizontal $(2.38 \mathrm{~m} / \mathrm{s})$ corresponds to the transition velocity for fast fluidization $\left(\mathrm{U}_{\mathrm{TR}}\right)$ for Geldart Type B particles.

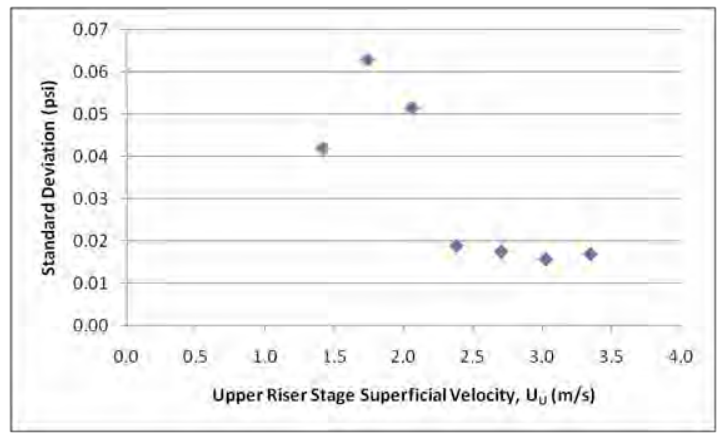

(a)

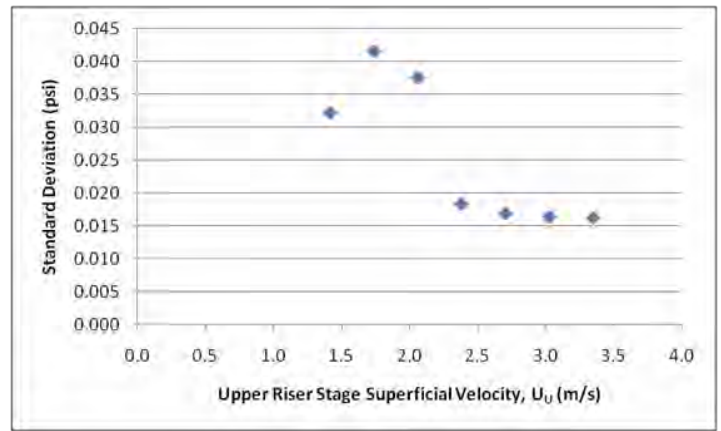

(b)

Figure 5-14: Standard Deviation of model upper riser stage pressures (a) Ubottom (b) Utop: poly-dispersed sand, feed hopper pressure $3.5 \mathrm{psig}, \mathrm{U}_{\mathrm{L}}=2.36 \mathrm{~m} / \mathrm{s}$

As seen with the standard deviation plots in Figure 5-14, similar trends appear in the skew and kurtosis plots (Figures 5-15 and 5-16) to those seen in the previous section for the lower riser stage. As with the standard deviation, the analysis of both the skew and kurtosis of the pressure 
transducer signals indicated that the upper riser stage transitions to fast fluidization at a superficial velocity of approximately $2.38 \mathrm{~m} / \mathrm{s}$.

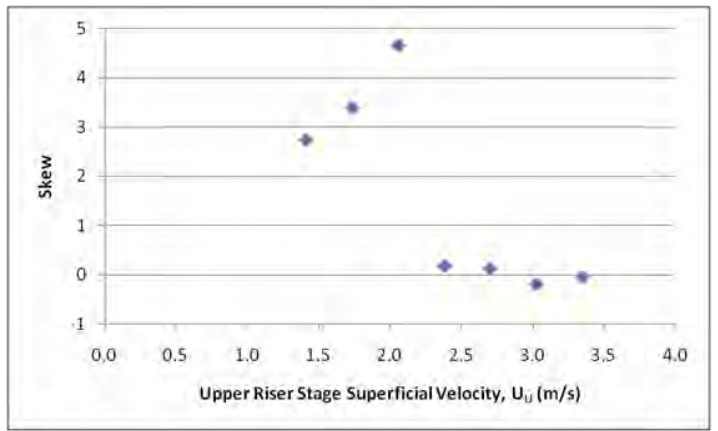

(a)

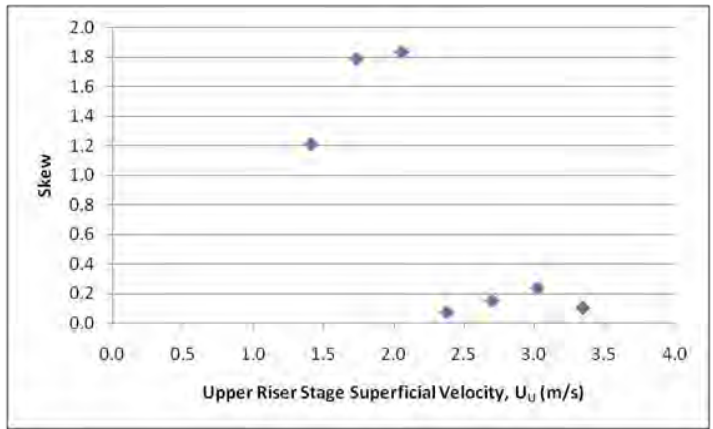

(b)

Figure 5-15: Skew of model upper riser stage pressures (a) Ubottom (b) Utop: poly-dispersed sand, feed hopper pressure $3.5 \mathrm{psig}, \mathrm{U}_{\mathrm{L}}=2.36 \mathrm{~m} / \mathrm{s}$

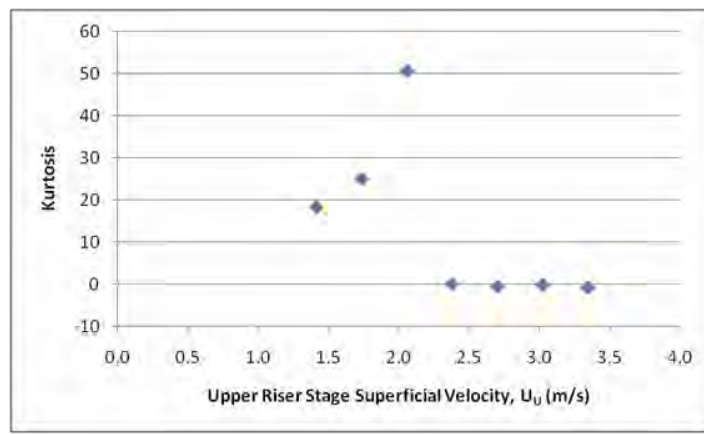

(a)

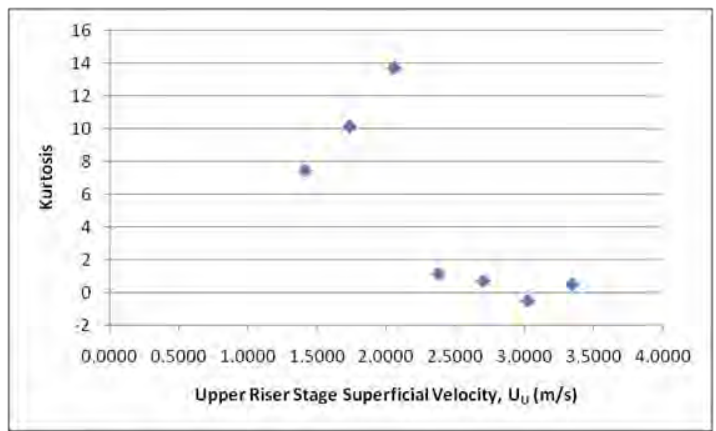

(b)

Figure 5-16: Kurtosis of model upper riser stage pressures (a) Ubottom (b) Utop: poly-dispersed sand, feed hopper pressure $3.5 \mathrm{psig}, \mathrm{U}_{\mathrm{L}}=2.36 \mathrm{~m} / \mathrm{s}$

It can be seen from these results that the upper riser stage begins to exhibit fast fluidization, or core-annular flow, when the superficial velocity in the upper riser stage, $U_{U}$, matches the superficial velocity in the lower riser stage, $U_{L}$. This suggests that the assumption that the addition of secondary air between the two riser stages divides the bed into two distinct regions that can be treated as individual fluidized beds is flawed. The will be discussed further following analysis of the effects of secondary air injection upon the lower riser stage.

\section{Shannon Entropy Analysis}

Figure 5-17 shows the effects of upper riser stage superficial velocity, $\mathrm{U}_{\mathrm{U}}$, on the Shannon entropy of pressure in the upper riser stage. As can be seen, the region bounded by the 